\title{
Quantum Calculation of Proton and Other Charge Transfer Steps in Voltage Sensing in the Kv1.2 Channel
}

Alisher M Kariev, Michael Green

Submitted date: 10/06/2019 Posted date: 12/06/2019

Licence: CC BY-NC 4.0

Citation information: Kariev, Alisher M; Green, Michael (2019): Quantum Calculation of Proton and Other Charge Transfer Steps in Voltage Sensing in the Kv1.2 Channel. ChemRxiv. Preprint.

Quantum calculations on 976 atoms of the voltage sensing domain of the $\mathrm{K}_{\mathrm{v}} 1.2$ channel, with protons in several positions, give energy, charge transfer, and other properties. Motion of the S4 transmembrane segment that accounts for gating current in standard models is shown not to occur; there is $\mathrm{H}^{+}$transfer instead. The potential at which two proton positions cross in energy approximately corresponds to the gating potential for the channel. The charge displacement seems approximately correct for the gating current. Two mutations are accounted for (Y266F, R300cit, cit =citrulline). The primary conclusion is that voltage sensing depends on $\mathrm{H}^{+}$transfer, not motion of arginine charges.

File list (2) 
Alisher M. Kariev and Michael E. Green*

Department of Chemistry and Biochemistry

6

City College of New York

7

New York, NY United States of America

8

9

10

*Corresponding Author:mgreen@ccny.cuny.edu;

Alisher M Kariev: akariev@ccny.cuny.edu

11

12

13

14

Key Words: Ion Channels, Voltage Gating, Proton Transport, Quantum Calculations

15 
ABSTRACT: Ion channels in cell membranes control entry and exit of ions; their gating (opening and closing) is key to their functioning. It is known that protons can pass through the voltage sensing domain (VSD) of channels such as $\mathrm{K}_{\mathrm{v}} 1.2$; quantum calculations for a section of the VSD show the steps protons take in responding to voltage, and show no major displacement of the protein backbone with voltage change; 70 amino acids are included, 42 with side chains (9 directly in the proton path), 28 as backbone only, and 24 water molecules. Protons provide much of the gating current, the capacitative current immediately preceding channel opening, with significant additional contributions from charge transfer to other groups. Most gating models, in contrast, require major protein displacement during gating. Energy terms without classical analogues (exchange plus correlation energy, which are greater than thermal energy) show quantum calculations are required. Energy as a function of voltage for a key proton transfer leads to approximately the correct voltage for channel opening. Calculated total charge transfer (not only protons) for gating is reasonable compared to experimental values. We are also able to account, at least qualitatively, for two mutations, one left shifted, one right shifted, and show the alternate proton paths that are required to account for these. 
Ion channels are an extremely important class of proteins; channels of various types are present in all cells, allowing the transport of $\mathrm{Na}^{+}, \mathrm{K}^{+}$, and $\mathrm{Ca}^{2+}$ through the cell membrane(1); other channels are responsible for transport of other substances, in particular protons $(2,3)$. Channels have even been found in viruses $(4,5)$. Voltage gated ion channels (VGIC) are a particular class of channels, and certain types of these are responsible for the nerve impulse, as well as the properties of other excitable tissue(68). VGIC, both for $\mathrm{Na}^{+}$and $\mathrm{K}^{+}$, have four voltage sensing domains (VSD); for the $\mathrm{K}^{+}$channels, these are identical, while for $\mathrm{Na}^{+}$they are similar (one having a different function). Here we will be concerned with the way a $\mathrm{K}^{+}$channel senses voltage. Each VSD of a $\mathrm{Na}^{+}$or $\mathrm{K}^{+}$channel has four transmembrane (TM) helical segments, linked by extra- or intracellular loops that extend beyond the membrane. The fourth TM helix (labeled S4) is connected via a linker of several amino acids to a domain with 8 TM helices, 2 extending from each VSD, that together form the pore through which the ion passes. Channels of the type we are discussing are closed when there is a voltage of approximately $-70 \mathrm{mV}$, (minus meaning intracellular side negative with respect to the extracellular side), that holds the channel closed; with $0 \mathrm{mV}$ across the channel, it is open. Fig. 1 shows the complete channel. The channel has extensive intracellular sections, and the section closest to the membrane (called T1) is involved with gating(9). S4 has arginines in every third position (except for one lysine near the intracellular end). Gating is preceded by a capacitative current ("gating current"), in which positive charges move in an extracellular direction in response to membrane depolarization. Negative charges moving in an intracellular direction could produce a similar current, but, in spite of the fact that negative charges do exist on the S2 and S3 TM segments, this possibility is not considered in the standard models of gating. These models are based on the motion of the S4 TM segment in an extracellular direction upon membrane depolarization, when its positive charges, assumed to be on three of the arginines, no longer hold it down. 


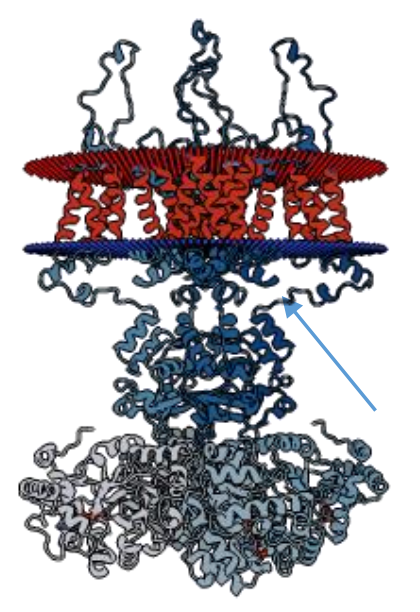

Fig.1: The complete $K_{v} 1.2$ channel, reproduced from the 56 (rcsb) pdb structure 3Lut(10); the red and blue planes mark the membrane boundaries, blue intracellular. The entire blue section below the blue membrane boundary is intracellular; the red helices constitute the VSD and pore. There are four 60 voltage sensing domains, each with four transmembrane 61 segments, between these boundaries; the pore is hidden 62 behind the front VSD. Extracellular loops connecting

alternating transmembrane segments are above the red extracellular membrane boundary. The T1 moiety (arrow) constitutes the upper part of the intracellular section, and is involved in gating(9)

Standard gating models: There are variations in the details of the motion proposed in different versions of the standard model, but as a class, these models are defined by extracellular motion of S4 upon depolarization. The evidence for this type of model was first based on scanning cysteine accessibility mutagenesis (SCAM(11)), and has since been supplemented, primarily by molecular dynamics (MD) (12-16), and FRET (fluorescence quenching) $(17,18)$. We have discussed this evidence in previous reviews (19-22), and concluded that it does not rule out an alternative source of gating current, specifically, proton transport, with S4 remaining stationary. The SCAM method requires the mutation of an amino acid to cysteine. In this case, it was the arginines on S4 that were mutated first. Arginine has a very large side chain, cysteine a very short side chain. The large difference in the molecular volume of these side chains is critical. In the SCAM method, it is assumed that cysteine must move to the membrane surface (either intracellularly or extracellularly) in order to ionize, because the membrane dielectric constant is too low to allow ionization; only the ionized form can react with a methanethiosulfonate (MTS) reagent; the test for whether the cysteine had moved to the membrane surface was that adding an MTS reagent at the side that reacted killed the channel. If the reaction really indicated that the S4 had moved enough as a fairly rigid body to make the cysteine accessible, then 
one would have evidence for the standard model. However, if the cysteine could react in situ, without moving, then the conclusion would have to be that the ability of the MTS reagent to penetrate the membrane had changed, not that the S4 had moved. For the cysteine to ionize, at least two or three water molecules are needed near the cysteine; the presence of water molecules would make the low dielectric assumption no longer valid. The difference in side chain volume is in fact adequate for two or three water molecules. Once ionization occurs, the reactive head of an MTS reagent could replace the water, so that it could react with the ionized cysteine. Therefore, the interpretation of the SCAM data can be reconsidered.

FRET data sometimes show appreciable motion. While we cannot discuss all the data that have been adduced to support the standard model, we note that FRET data showing small S4 motions have also been reported $(18,23-25)$. FRET data may not have high resolution, as the probe is typically approximately $10 \AA$, and the orientation of the probe is important and hard to determine. Most of the computational literature on the VGIC uses molecular dynamics (MD), which is strictly classical. We will discuss below reasons for doubting MD simulations of voltage gating can properly describe this phenomenon.

Proton transport: We have proposed proton transport as the main source of gating current, instead of $\mathrm{S} 4$ motion, in $\mathrm{K}_{\mathrm{v}}$ channels, especially for channels in the Shaker and $\mathrm{K}_{\mathrm{v}} 1$ families, not only in the reviews just cited, but in earlier work as well (26-28). Gating current includes charge transfer on heavy atoms (oxygen and nitrogen), in addition to the proton transfers. These must be added to get the complete gating current. (Charge transfer from water to ions is known to be large, and to require quantum calculations to determine it $(29,30))$ Proton transport is known in a number of systems that are analogous to the $\mathrm{K}_{\mathrm{v}}$ voltage gated potassium family of channels. The discovery that the $\mathrm{H}_{\mathrm{v}} 1$ proton channel is very similar to the VSD (31-33) helps show that proton transport could exist in the VSD. 
Other channels with similarities to the $\mathrm{K}_{\mathrm{v}}$ channels also show proton transport through membranes: Woelke et al give MD calculations on cytochrome $\mathrm{c}$ (cyt c), in which the analogy to the $\mathrm{K}_{\mathrm{v}}$ channel is discussed explicitly (34), shows a very similar sequence to what is present in the $\mathrm{K}_{\mathrm{v}} \operatorname{VSD}(35,36)$; this paper shows a tyrosine ionization essentially similar to what we report here. Additional analogous systems, not all protein, include: the M2 channel in the influenza virus (37-40); a guanidinium-ammonium competition with a crown ether(41); an amino acid-polyether/polyamine macrocycle (42); bacteriorhodopsin (a case involving tyrosine) (43); and arginine-carboxylate interactions(44). Han et al have shown how protonation states control function in a $\mathrm{Na}^{+}, \mathrm{K}^{+}, \mathrm{ATPase}(45)$, in this case relevant to $\mathrm{Na}^{+}$ binding. Mutated VSD of a Shaker potassium channel (46-48), which is essentially the same channel as we are considering, also transmits protons; the key is an $\mathrm{R} \rightarrow \mathrm{H}$ mutation at the end of the path, while the section we calculate here is part of the same path the $\mathrm{H}^{+}$is expected to follow in that case. In WT, the transfer to the extracellular solution is presumably blocked by arginine. Thus, our calculated proton path is consistent with previous experimental results. A proton current through the VSD has also been measured by Zhao and Blunck (49), who showed that the VSD forms a cation channel, with a preference for protons. This appears distinct from the "omega current" $(50,51)$. Motion of the S4 segment would seemingly produce approximately the correct gating current (with $\mathrm{K}_{\mathrm{v}} 1.2$, about 10 charges move across the electric field (52), compared to approximately 13 for Shaker); many standard models assume that there were full +1 charges on the arginines. However, given the distribution of charge, this would not be the case, as we show below; charge on the arginines is $<1$, while charge shifts on other atoms must add to the overall measured charge shift. There is also the question of why the S2 and S3 helices, with their negative charges, should not move in the opposite direction and contribute to the gating current that way; mutational evidence has generally been accepted as showing that only S4 moves. The only time we are aware of that this has been tested, using the SCAM method, the evidence suggested S3 motion in the same direction as the S4 (53); this would be consistent with the cysteine ionizing in situ, not moving to the membrane surface; also, that the access to the cavity with the cysteine was being measured within the membrane, which would be the same for positive and negative charges. If so, basic evidence for the standard model must be reinterpreted. It should also be 
the case that substituting a positive or a negative charge would have opposite effects on gating current; such an experiment was done and it was found that the two charges had the same effect. Those authors interpreted the result in terms of a version of the standard model (54), although it is not clear how nearly compatible this interpretation is with other versions. If the net effect of charge on proton motion is similar, even if by different mechanisms for different charge, this experiment too appears more compatible with proton motion being largely, but not completely, responsible for gating current; we will see that gating current involves charge transfer on heavy atoms $(\mathrm{O}, \mathrm{N})$ as well, together with side chain rotation (Fig. 8 will illustrate this). More details of the evidence for S4 motion could be discussed, but would not add to what we have considered in previous work $(19,21)$.

There is no crystallographic water in the section of the membrane from just below the membrane extracellular side to the lower end of the helix bundle (by convention, consider the orientation of the channel such that extracellular is "up", intracellular "down"). Therefore one section of the proton current which is found, for example in the $\mathrm{R} \rightarrow \mathrm{H}$ mutation case discussed above, is required to consist of protons transferring among amino acids, without water. Proteins contain salt bridges, with a base (arginine or lysine) paired with an acid (glutamate or aspartate); these exist in the VSD. It is assumed in standard models that the two side chains are ionized, and the S4 motion exchanges salt bridge partners as the segment moves. This need not be the case; in fact, without water, salt bridges generally do not ionize (55); however, in groups of three or more ionizable amino acids, even without water, ionization is possible. In addition, we show that a tyrosine, Y266, can ionize in the VSD. Other cases in which tyrosine ionizes within a protein, albeit in the presence of water, have been shown $(56,57)$. Proton transfer may involve the arginine NE atom (here we use pdb notation for $\mathrm{NE}$ and $\mathrm{NH}$ nitrogens on guanidinium, not $\mathrm{N} \varepsilon, \mathrm{N} \eta$ ). The role of individual groups or atoms has, with some exceptions, not been considered in standard models, and the ability of the NE nitrogen to act as a base does not appear to be included in any discussion of the standard model. 
Calculations show proton transfer: We have carried out quantum calculations starting from the X-ray structure of a 976 atom section of the VSD of the $\mathrm{K}_{\mathrm{v}} 1.2$ channel. These show specific proton transfers with voltage. Groups of three to five ionizable amino acids, which may include tyrosine (58), are present in all the analogous channels, cited above, that are known to transfer protons; even salt bridge and hydrogen bond distances are generally similar. There is also a tyrosine dependent voltage sensor in the M2 muscarinic receptor (59). Thus, the results of our calculations are consonant with the properties of a number of proteins well-known to transmit protons; the VSD of a $\mathrm{K}_{\mathrm{v}} 1.2$ potassium channel is similar enough to these other channels that it should not be a surprise that the VSD transmits protons. Other charge transfer in response to membrane depolarization can also contribute. Then the motion of S4 must contribute less. If these contributions are sufficient, it is possible for S4 to not move, and thus not contribute at all. Fig. 6 shows that there is enough charge displacement with no S4 motion to account for the entire gating charge displacement.

RESULTS AND DISCUSSION: Immobility of the S4 backbone: We carried out computations on a segment of the VSD with 976 atoms, of which 904 were protein, from the 3Lut (pdb) X-ray structure with hydrogens added by normal mode analysis (60), and the remainder were 24 water molecules (not crystallographic) that were added in the extracellular cleft. The water, placed at the extracellular ("up") side as a single cluster, was added at bulk density, but at random orientations. Most of the computations shown have all four transmembrane helices anchored by several backbone atoms; 62 atoms in all were fixed. The side chains of 42 amino acids were included, and the backbone of all amino acids (total, 70) in the section were also included; thus less than one atom per amino acid was fixed, on average. However, the key question is whether S4 moves under applied voltage with respect to the remainder of the VSD. Therefore, optimizations were done with S4 free to move (no S4 atoms fixed), with the voltage set to the closed (-70 $\mathrm{mV}$ ) value; the result was compared to the X-ray structure (see Fig. 2), which is considered to be the open state. The S4 backbone does not move in such a way as to provide any gating current; some atoms move about $1 \AA$, in a very few cases, up to $2 \AA$. The exception is one isoleucine, the side chain of which pops up 


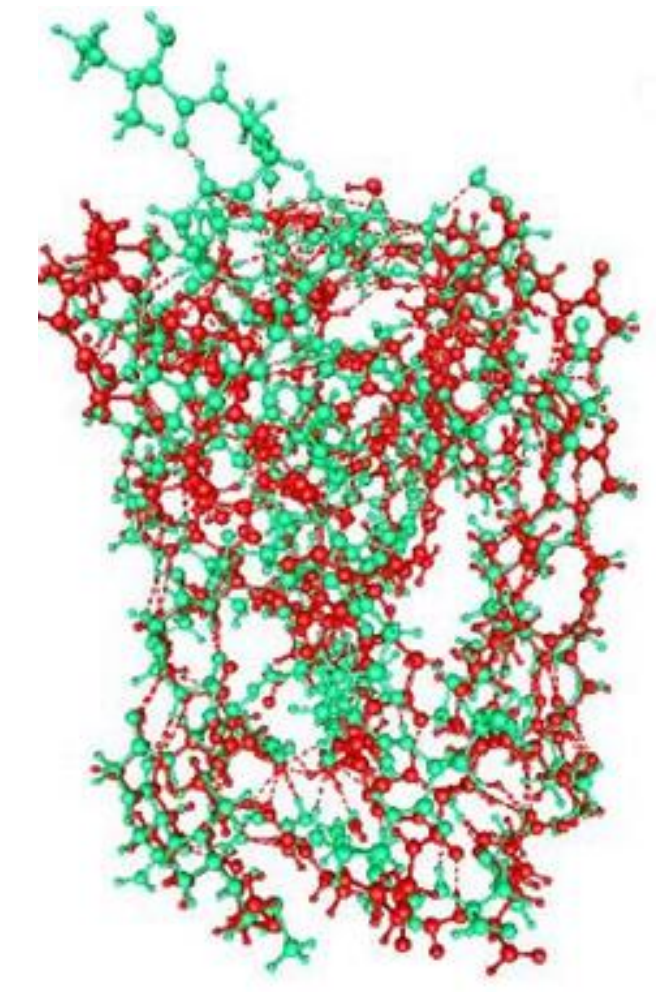

(see Fig. 2), and a somewhat larger movement of the side chain of the first arginine, which rotates to some extent. We also compared the $0 \mathrm{mV}$ (open) optimized fixed and free $\mathrm{S} 4$ cases to the $\mathrm{X}$-ray structure, and found that the structures are quite close; it turned out that the $\mathrm{S} 4$ fixed (i.e., some backbone atoms fixed) structure was slightly closer to the X-ray structure, so we kept S4 fixed thereafter. There is then the question of how the protons contribute to the gating current, and what other charge movement there is. We have done 29 calculations of wild type VSD, with different fields and proton positions (in other words, ionization of different residues), plus a check calculation, and calculations on the two mutations discussed below. The results do not agree with the standard model, but do agree with the experimental results. We have determined the total charge movement, as this is what would be measured, rather than the movement of the protons alone. We do not assume that any residue or atom has nominal charge, but use Natural Bond Orbital $(\mathrm{NBO})(61,62)$ calculations to determine charge. Details of the calculation are described below.

Fig. 2: Comparison of the X-ray (red) and S4 free optimized structure (green), for one VSD, with -70 mV applied, hence what must be the closed structure. The S4 does not move down with respect to the $X$-ray structure, which has the channel open. One amino acid side chain, an isoleucine, pops up. There is practically no displacement of the $S 4$ transmembrane helix backbone, although side chain rotations are extensive, and important for proton transport.

Proton transfer: The calculations show that the entire system acts cooperatively to transfer protons up (closed to open) or down (open to closed). We look primarily at ten amino acids (R297, S184, F222, E183, R300, R303, 
E226, Y266, S176, F180) that are directly involved in proton transport (see Table 6 for the complete list of 42 amino acids in the computation with side chains), although the structural elements of the system are also necessary for the VSD to function. The opening and return paths need not be identical, and because hysteresis in gating has been shown, (63) there must be more than one path. We show a primary path, and show that an alternate path (at least one) exists. We focus further on a triad of amino acids located where it is expected that the main voltage drop occurs(64). This said, the entire group of ten must be understood if we are to explain two mutations that we have calculated, and that have been tested experimentally; these are discussed after the wild type (WT) has been described, as to energy and other properties associated with charge transfer. Non-obvious local transitions, such as the rotation of a serine hydroxyl group, turn out to be important in stabilizing intermediate configurations. A trigger type response followed by a proton cascade would be consistent with the initiation of gating by a very small very fast component of gating current (the "piquito") $(65,66)$. This may not be the only possible interpretation of the "piquito" but it seems the most straightforward. A single proton transfer could produce this result, and once the initial proton had transferred, the others would follow. However, rather than a trigger/cascade mechanism, the overall proton transfer may be a concerted change of state. There are two conformations of the set of ten amino acids, with and without voltage (closed/open), and the entire system may toggle between them. This calculation cannot distinguish an initial state transfer, followed by a cascade (or followed by a concerted shift), from an entirely concerted mechanism. It is almost certainly not important whether there is a slight delay between the first step and the complete transition, or whether the system moves the proton(s) in a concerted transition. Overall, we are able to distinguish two states, open and closed, and under normal circumstances the system toggles between them, but apparently through intermediate states. Because there is hysteresis, and the return may take longer than opening because of inactivation, the switch need not be simple, and may require time for the reverse step to be completed, so that it may be concerted in the forward direction only. Distinguishing these possibilities is left for future work.

The Y266, E183, R300 triad: The $\mathrm{K}_{\mathrm{v}} 1.2$ VSD contains a grouping that includes a tyrosine, a glutamate, and 
an arginine; it is one of several similar groups of three to as many as five amino acids that collectively appear to form a section of a proton pathway (in $\mathrm{K}_{\mathrm{v}} 1.2$ at least one other triad shares one amino acid, the tyrosine, with this triad, making them together a pentad of amino acids, constituting one section of the VSD). These proton transfers do not need water. Arginine may act as an acid in two ways, either losing a proton from the NE nitrogen, or losing one from the two amine groups on the terminal carbon of the guanidinium (i.e., from NH1 or NH2); obviously, the deprotonated forms of these groups can act as bases.

We analyze the Y266, R300, E183 triad of amino acids that transfer a proton in detail, calculating the energy of the possible positions of the proton. In $\mathrm{H}_{\mathrm{v}} 1$ there is a very similar triad to the R300, Y266, E183 group in $\mathrm{K}_{\mathrm{v}} 1.2$ (for $\mathrm{H}_{\mathrm{v}} 1$ in $3 \mathrm{WKV}$ pdb numbering, R207, D170, Y157) which also appears to be involved with proton transfer. It appears that similar groupings occur in bacteriorhodopsin (Y57, R82, E194 in the 1P8H structure, where the $\mathrm{Y}, \mathrm{R}, \mathrm{E}$ distances are similar to those in the $\mathrm{K}_{\mathrm{v}} 1.2$ VSD structure for its key triad) and in cytochrome c (34). Bacteriorhodopsin and cytochrome c are known to transmit protons.

Table 1: Total energies of the three WT proton configurations with and without field

\begin{tabular}{|c|c|c|c|c|}
\hline $\begin{array}{ll}\text { FIELD }(\mathrm{mV}) & \mathrm{H}^{+} \\
\text {transition } & \text { from } \\
\text { standard } & \end{array}$ & $\begin{array}{l}\text { Y266 } \\
\text { charge }\end{array}$ & $\begin{array}{l}\text { R300 } \\
\text { charge }\end{array}$ & $\begin{array}{l}\text { E183 } \\
\text { charge }\end{array}$ & $\Delta$ Energy $(\mathrm{kJ} / \mathrm{mole}) \#(\mathrm{~kJ})$ \\
\hline $0:$ (none) & 0 & + & - & 65.7 \\
\hline-70 & 0 & + & - & 63.7 \\
\hline 0: R300 $\rightarrow$ E183 & 0 & 0 & 0 & 88.5 \\
\hline-70 & 0 & 0 & 0 & 0* (Lowest total energy) \\
\hline $0: \mathrm{Y} 266 \rightarrow \mathrm{E} 183$ & - & + & 0 & $\begin{array}{l}38.3^{* *} \text { (Lowest total energy, for } \\
\text { the open state) }\end{array}$ \\
\hline-70 & - & + & 0 & 78.7 \\
\hline
\end{tabular}


There are additional similar triads in $\mathrm{K}_{\mathrm{v}} 1.2$ itself (e.g., R303, E226, Y266); the Y266 connects this triad to the grouping discussed above, making a pentad. There are other channels with similar triads for which the distances between key atoms are almost identical, with the same H-bond distances between heavy atoms; one such triad in $\mathrm{H}_{\mathrm{v}} 1$, in addition to the triad mentioned above, is its selectivity filter (R201, D108, R204, comparable in dimensions to the $\mathrm{K}_{\mathrm{v}} 1.2 \mathrm{R} 303$, E226, R300, triad (67). Only one case is discussed here, but others are sufficiently similar that the analogy for proton transfer is clear. This result demonstrates the necessity of specifically calculating possible proton transfers, rather than assuming charges based on solution pK values. In addition, water density fluctuations have been suggested as important for the inhibition of the $\mathrm{H}_{\mathrm{v}} 1$ channel(39); there is rotation of the water cluster in our calculation (see Fig. 3) so that we also see a role of water in facilitating or blocking proton transfer; hydrogen bonds in the transfer sequence show linkage to one end of the ten amino acid sequence discussed with Fig. 3, and especially Fig. 4..

Table 1 shows how the energy changes with protonation state and field (corresponding to open and closed states) for the Y266, R300, E183 triad. When a proton is transferred among the three amino acids of a triad, the charges on all three of the amino acids necessarily change. The structure can be optimized, and then the energy calculated, for each configuration resulting from an initial proton placement, with and without an electric field added. As always $-70 \mathrm{mV}$ is taken as the potential drop across the membrane in the closed state. These cases are calculated for wild type. Fig. 4 shows the configurations of the most important subset of the WT optimized structures under the conditions that were calculated. A Y266F mutation is relevant here; the proposed proton path suggests this mutation must be important, and experimental results, for which we thank Dr. Carlos Bassetto and Professor Francisco Bezanilla, confirm this, with an $8.5 \mathrm{mV}$ right shift in gating current. Fig. 5 includes results for this mutation. 


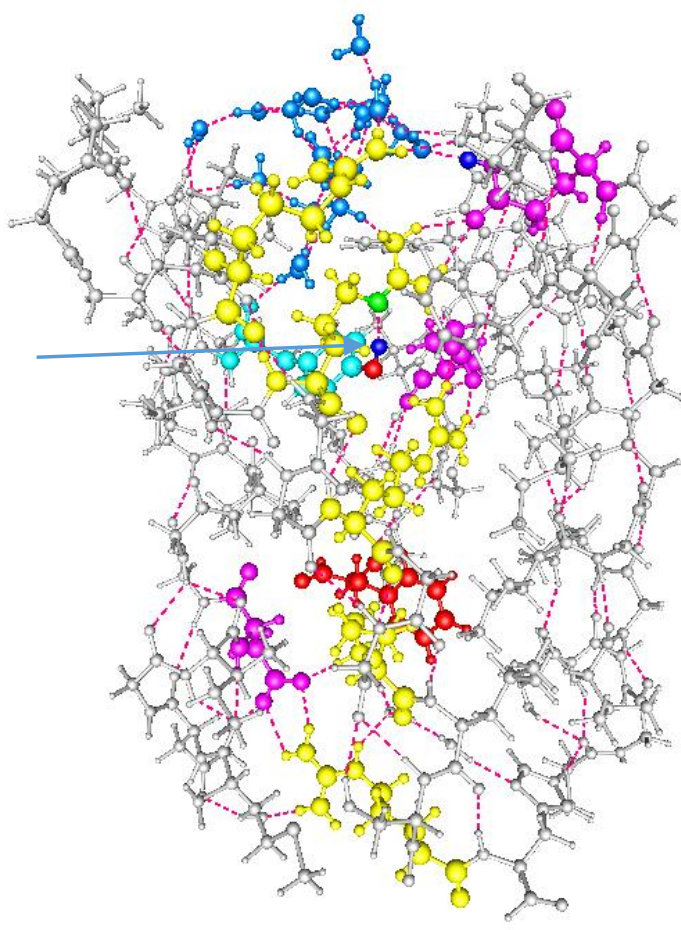

A

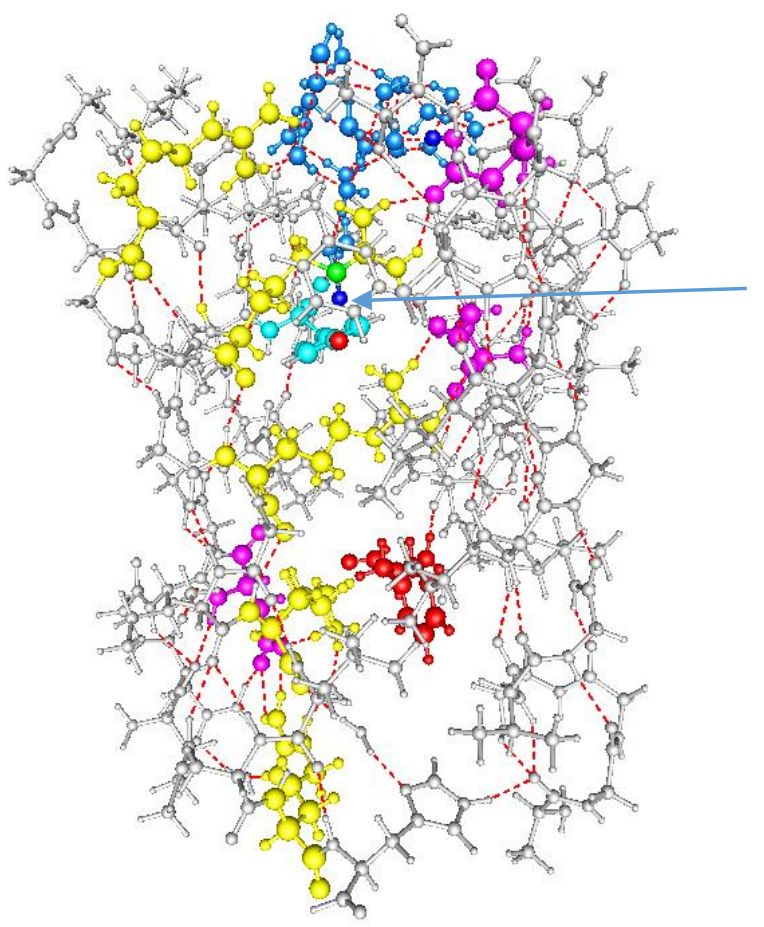

B

Fig. 3: Overview of the 976 atom system shift between closed and open states: A: The lowest energy closed state (-70 mV) Gray: backbone plus most side chain protein atoms. Blue: water cluster at the extracellular (top) end; Yellow: S4 arginines and K306, from R297 to R309.

Magenta: acids, from top E183, E226, D259. Red: F233. Light blue: Y266, the key tyrosine. The arrows point to the (dark blue) proton on the $\mathrm{Y} 266-\mathrm{OH}$ which will ionize to go to the open state, bonded to the red oxygen (Nominal charges R300(0), Y266(0), E183(0)); B: same groups and colors, arrow to same proton, except the proton is now attached to the R300 NE atom (green, just above the dark blue proton in both $A$ and B), instead of the Y266 oxygen (Nominal charges R300(+), Y266(-), E183(0)). Dotted lines indicate hydrogen bonds, including on backbone. For a detail view of the proton transfer, see Fig. 4.

Fig. 3 shows the key shift of a proton from Y266 to R300 (NE atom). In both cases shown here 
E183 is neutral (carboxyl group protonated). When the energy was calculated with the salt bridge R300-E183 ionized, using the X-ray coordinates instead of optimization, the energy was very high, so that the E183 deprotonated state may be transient (also, optimization may lower the energy somewhat). E183 must transfer a proton to water, and accept a proton from R300. These calculations are not sufficient to demonstrate whether the transition is concerted or sequential, but the concerted transition seems likely to have the lower activation energy, as it avoids the high energy ionized state. Comparison of Fig. 3A and 3B shows a number of shifts of side chains, and somewhat as well. Fig. 4 shows the transfer in detail, with rotations of neighboring groups. water molecules. The latter are obvious, as the water spreads out in the open state, and approaches E183, making it easier for water to accept a proton from E183. The R297 side chain rotates slightly up, leaving less space for water; the water cluster rotates and becomes slightly more compact. E226 slides to the right in Fig. 3B, as the Y266 proton to which it was hydrogen bonded in the closed state moves to R300; the E226 - Y266 distance increases by more than $1 \AA$, and the carboxyl group rotates away from Y266. At the lower end, F233 rotates downward and, at the bottom, R309 also rotates downward. The location of cavities in the structure shifts 300 


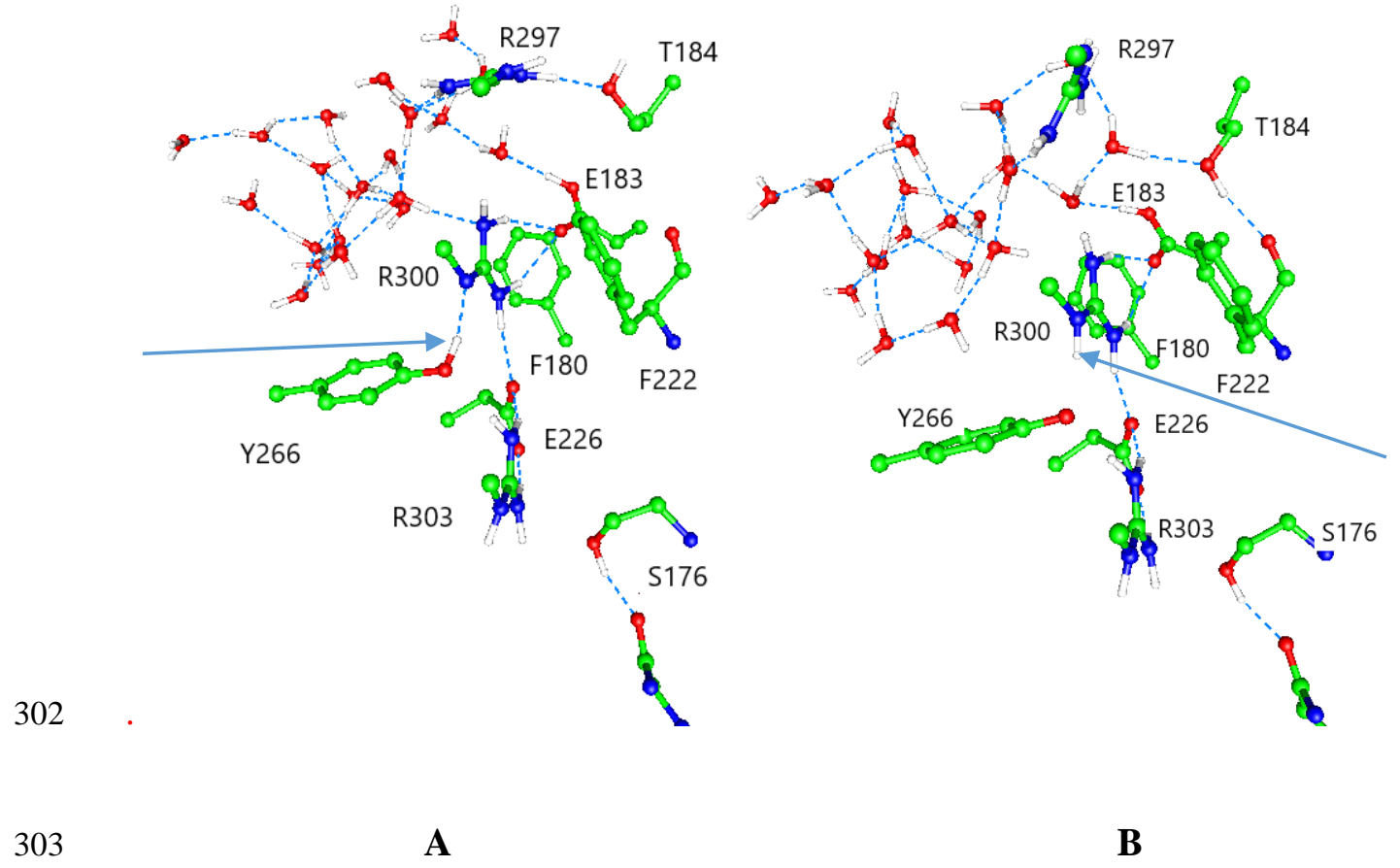

Fig 4: 145 atom detail from Fig. 3, with the amino acid side chains involved in the proton transfer of the system, here showing the key proton transfer Y266 to R300 (arrows). A: closed (cf. Fig. 3A) B: open (cf. Fig. 3B). In the closed case, there is a proton on Y266, nominal charge 0 . The open case has the proton transferred to R300, NE atom, making the nominal charges Y266(-), R300(+). Carbon is green, nitrogen blue, oxygen red, hydrogen white. Atoms not part of the side chains or water are part of the backbone.

In Fig. 4B, the F180 is behind two H-bonds from R300 to E183, not involved in hydrogen bonding itself, but the rotation of the aromatic group may assist in proton transfer(68). The cation- $\pi$ interaction is also affected by $\mathrm{pH}$ (i.e., protonation state), as can be determined spectroscopically $(69,70)$; rotation of the aromatic side chains must affect the protonation state. The differences between closed (Fig. 4A) and open configurations (Fig. 4B) include rotation by almost $90^{\circ}$ of the R297 guanidinium group, and the inclusion of two waters between R297 and E183, completing a plausible proton path from R300 to R297; in the closed state no such path is possible. A nearly linear arrangement of three waters appears near the R297, but not so close to R297 that it can form a path for $\mathrm{H}^{+}$. T184 is hydrogen bonded to R297 in the closed 
317 configuration, but rotates down and hydrogen bonds to water and a backbone carbonyl in the open case.

318 In short, an $\mathrm{H}^{+}$can move through the entire section, reaching $\mathrm{R} 297$, in the open case, but not in the closed case.
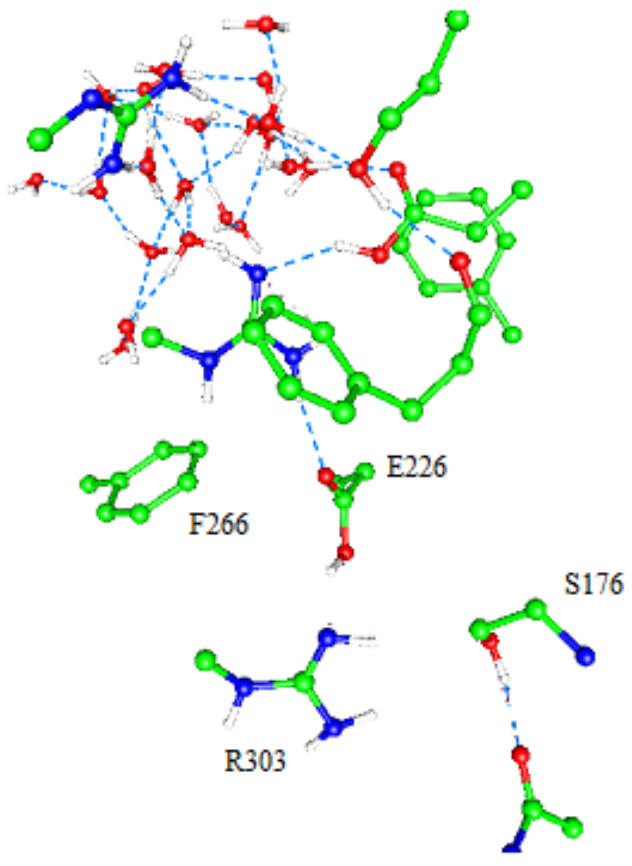

A

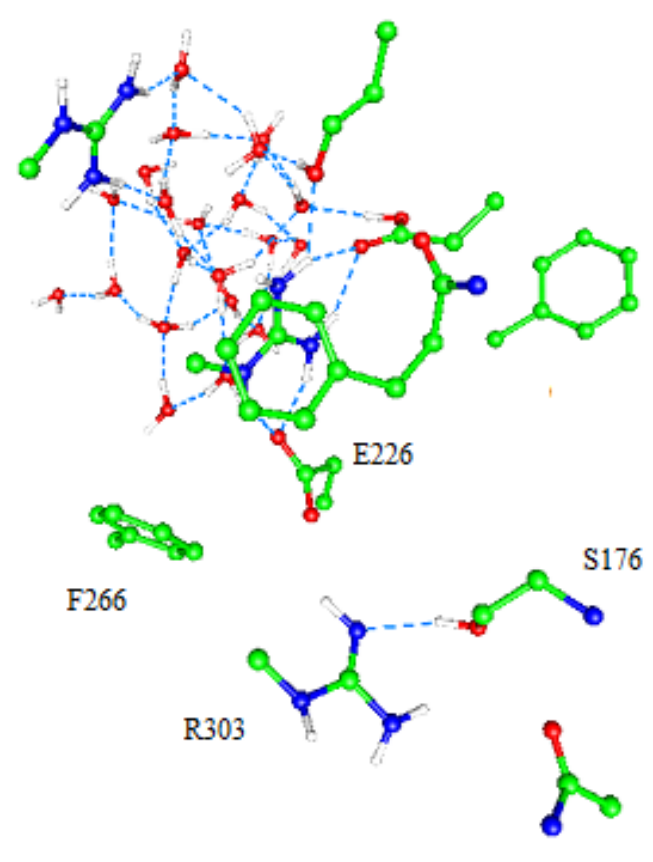

B

Fig. 5: The main changes between closed (A) and open (B) configurations for the Y266F mutant with the same set of atoms as in Fig. 4. Three proton positions each were optimized for closed and for open, and the lowest energy case for each is shown. F266 replaces Y266 in the mutant. The most obvious difference between open and closed here is in the S176-R303-E226 sequence starting at the lower right. In the closed case R303 does not communicate with E226, nor with S176. The orientation of the protons in E226 and S176 leaves the E226 isolated and without hydrogen bonds relevant to the proton path; the S176 hydroxyl is 
oriented down toward the backbone. In the open state, the S176 hydroxyl is hydrogen bonded to R303, which in turn is much closer to E226, in a position to transfer a proton; the S176 hydrogen bond makes this more energetically favorable. From E226 to R300 transfer appears possible in both open and closed configurations, although the voltage may prevent this in the closed case. The F266 ring rotates but does not take part in this transfer. The aromatics (F180 and F222, upper section, not labeled) and the water both move appreciably. A proton path can be found for both open and closed configurations from E226 upward.

The Y266F mutation produces a major rearrangement of side chains in the vicinity of the mutation, as shown by Fig. 5. To the extent that the backbone shifts at all, it is a bit higher in the closed state, the reverse of the standard model. However, the motion is so slight as to be insignificant. In Fig. 5 there is an alternate proton path, via E226, when the tyrosine path is blocked by the Y266F mutation. S176 acts as a switch, which it does not do in WT; in the calculation the $-\mathrm{OH}$ from $\mathrm{S} 176$ rotates so as to be able to $\mathrm{H}$-bond to $\mathrm{R} 303$ when the proton opening transitions start, with R303 becoming neutral. This is very similar to a configuration in bacteriorhodopsin in which a serine (S193) undergoes a rotation when a neighboring glutamate becomes deprotonated (71). Here the path must bypass a break in the proton path that is present in WT. In addition, there exists the possibility of a proton moving along the helical backbone. It was shown by Oliver and Deamer that polyalanine or, better, polyleucine, could conduct protons through a lipid membrane, so it is not possible to rule out steps that include the backbone, even if a much shorter segment than in the Oliver and Deamer case, which brought protons entirely through a membrane (72). In the mutant, the aromatic ring of the phenylalanine (F266) rotates $\approx 90^{\circ}$ under the influence of the field. Since $\mathrm{H}^{+}$-aromatic interactions are generally stronger than $\mathrm{k}_{\mathrm{B}} \mathrm{T}$ (73), this may allow a proton to pass via yet another path in the mutant. We also observe essentially the opposite E226 rotation to that in the WT, forming an H-bond with R300 in the closed state. The role of a dihedral transition of an aromatic (phenylalanine) in assisting proton transport in the $\mathrm{H}_{\mathrm{v}} 1$ channel has been suggested, based on QM/MM calculations, by van Keulen et al (68).

Also, in the mutant, the phenyl ring of F222 shifts approximately $1 \AA$ relative to backbone (alternatively, this could be considered a shift in the phenyl $(\mathrm{CG})-\mathrm{C} \alpha-\mathrm{C} \beta-\mathrm{N}$ dihedral angle (52.9 ${ }^{\circ}$ open, $\left.75.6^{\circ} \mathrm{closed}\right)$, which 
in WT remains almost fixed). The F233 phenyl in the Y266F rotation resembles that in Schwaiger et al(74); here it appears to facilitate transition to the open state. That the Y266 (WT) path is preferred is shown by the Q-V curve being right shifted in the mutant. The calculated energy of the configuration with the E226 protonated is, in WT, approximately $19 \mathrm{k}_{\mathrm{B}} \mathrm{T}$ greater than that with the Y266 protonated. The sign of the energy change is as needed for a right shifted curve. Although the magnitude is somewhat too large, a fairly small shift would bring it within range.

These shifts make several interatomic distances differ from WT by about $1 \AA$, in addition to the F266 ring rotation, with its effect on $\mathrm{H}^{+}-\pi$ interaction; these are shown in Fig. 5. None of the side chain changes is individually large, but collectively they can easily account for a difference of several kвT in energy. One expects that a loss of gating charge would accompany disruption of this system, although this calculation cannot directly show that. Standard models would make an opposite prediction; the removal of the $-\mathrm{OH}$ should make sliding S4 easier, producing a left shift. Y266 ionization in WT is the only apparent explanation for the effect of the Y266F mutation.

A second mutation is also relevant, in which the putative, standard model, gating charges, R297, R300, and R303, are replaced by isosteric, but uncharged, citrulline. Fig. 6 shows the result of a calculation of R303cit, which helps to understand the experimental left shift in the Q - V curve found for this mutation. 

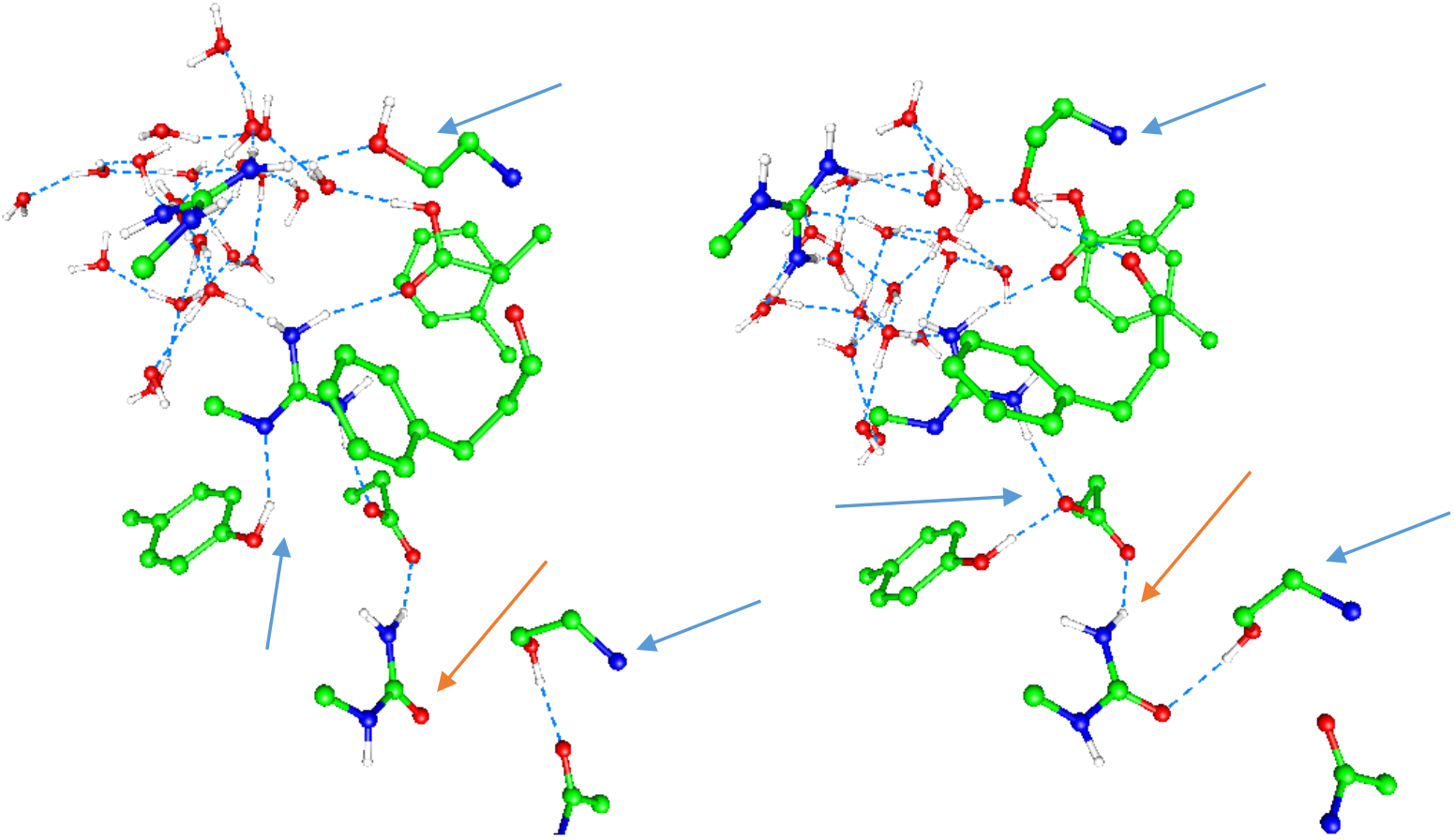

Fig. 6: Two cases of the R303cit mutation, both with the closed proton position (nominal charge Y266(0), R300(0), E226(0)), corresponding to the blue line in Fig. 7). The mutation is marked by the yellow arrow, which points at the carbonyl that replaces the $-\mathrm{NH}_{2}$ of arginine. There are at least three major hydrogen bond shifts marked by blue arrows: T184 rotates (top blue arrow), Y266 switches its hydrogen bond from $R 300(V=-70 \mathrm{mV})$ to E226 (V=0 mV) (middle blue arrow) and the citrulline carbonyl switches from no $H$ bond to an $H$-bond with $S 176$ at $V=0 \mathrm{mV}$ (bottom blue arrow). The largest change is the water cluster at the top, which is pulled up in the $V=-70 \mathrm{mV}$ state (A), interacting only with $R 297$, but in $B$, it interacts 393

394 Also, in the mutant, the phenyl ring of F222 shifts approximately $1 \AA$ A relative to backbone (alternatively, this 
could be considered a shift in the phenyl(CG)-C $\alpha-\mathrm{C} \beta-\mathrm{N}$ dihedral angle $\left(52.9^{\circ}\right.$ open, $75.6^{\circ}$ closed), which in WT remains almost fixed). The F233 phenyl in the Y266F rotation resembles that in Schwaiger et al(74); here it appears to facilitate transition to the open state. That the Y266 (WT) path is preferred is shown by the Q-V curve being right shifted in the mutant. The calculated energy of the configuration with the E226 protonated is, in WT, approximately $19 \mathrm{k}_{\mathrm{B}} \mathrm{T}$ greater than that with the Y266 protonated. The sign of the energy change is as needed for a right shifted curve. Although the magnitude is somewhat too large, a fairly small shift would bring it within range.

These shifts make several interatomic distances differ from WT by about $1 \AA$, in addition to the F266 ring rotation, with its effect on $\mathrm{H}^{+}-\pi$ interaction; these are shown in Fig. 5. None of the side chain changes is individually large, but collectively they can easily account for a difference of several kBT in energy. One expects that a loss of gating charge would accompany disruption of this system, although this calculation cannot directly show that. Standard models would make an opposite prediction; the removal of the $-\mathrm{OH}$ should make sliding S4 easier, producing a left shift. Y266 ionization in WT is the only apparent explanation for the effect of the Y266F mutation.

A second mutation is also relevant, in which the putative, standard model, gating charges, R297, R300, and R303, are replaced by isosteric, but uncharged, citrulline. Fig. 6 shows the result of a calculation of R303cit, which helps to understand the experimental left shift in the $\mathrm{Q}-\mathrm{V}$ curve found for this mutation.

Fig. 6: Two cases of the R303cit mutation, both with the closed proton position (nominal charge Y266(0), R300(0), E226(0)), corresponding to the blue line in Fig. 7). The mutation is marked by the yellow arrow, which points at the carbonyl that replaces the $-\mathrm{NH}_{2}$ of arginine. There are at least three major hydrogen 
bond shifts marked by blue arrows: T184 rotates (top blue arrow), Y266 switches its hydrogen bond from $R 300(V=-70 \mathrm{mV})$ to E226 (V=0 mV) (middle blue arrow) and the citrulline carbonyl switches from no $H$ bond to an H-bond with S176 at V=0 $\mathrm{mV}$ (bottom blue arrow). The largest change is the water cluster at the top, which is pulled up in the $V=-70 \mathrm{mV}$ state (A), interacting only with $R 297$, but in $B$, it interacts strongly with the aromatics and with R300 as well.

In Fig. 6 we see a number of changes with voltage, without shifting a proton. If we compare with the blue line in Fig. 7, representing the energy of the closed state, we see that the $0 \mathrm{mV}$ energy is pulled down by more than $50 \mathrm{~kJ}$, thus reducing the barrier between closed and open by about $10 \mathrm{~kJ}$ (the maximum of the blue curve is pulled down by $>50 \mathrm{~kJ}$ ). Experimentally, the $\mathrm{Q}-\mathrm{V}$ curve is left shifted by over $40 \mathrm{mV}$; this suggests that the energy change is not unreasonable.

Summary of mutations: We should expect that if the proton cannot transfer from the Y266, it should make gating more difficult, and it does; the Y266F mutation leaves a still functional channel, right shifted by approximately $8.5 \mathrm{mV}$ for the gating current curve. Fig. 5 showed the missing $-\mathrm{OH}$ and the possible alternate paths through which the channel could still function. The discussion that accompanied Fig. 5 showed how plausible alternate proton paths could be found.

The second mutation, R303cit, in which a putative (in standard models) gating charge is replaced by the isosteric, but uncharged, citrulline side chain, showed a left shift (75). The energy for the proton transfer dropped in the mutant by approximately $10 \mathrm{~kJ}$, equivalent to $4 \mathrm{k}_{\mathrm{B}} \mathrm{T}$, so that the calculation is consistent with a left shift, as in the experiment; the $>50 \mathrm{~kJ}$ drop is the total for $0 \mathrm{mV}$, while the $10 \mathrm{~kJ}$ refers to the change in the barrier, that is, the energy at which the two curves in Fig. 7 would cross. in this case, the magnitude is quite reasonable. In the wild type, the energy at this point rises, so the mutant favors the open state. Fig. 6 shows the mutant, with the relevant shifts in position of the aromatic rings marked. For lack of computer resources, we did not calculate the R297cit mutation, nor the R300cit mutation, which right shifted the QV curve. Experimentally, R297cit kills the channel; from Fig, 4 it is fairly easy to see why this should be, as both paths for the proton must go through that arginine, and the citrulline does not have the requisite 
$\mathrm{NH}_{2}$ group to transfer a proton; unlike arginine it is not amphoteric. In addition, that is the location where 444 the largest part of the electric field drops(64). The R300cit mutation right shifts the Q-V curve. Given that $\mathrm{R} 300$ is part of the Y266,E183,R300 triad, it is not surprising that R300 behaves like Y266, and removing that path right shifts the curve, similarly to removing Y266; experimentally, it is somewhat larger, but not so much so as to make the interpretation implausible (75). Given that all other mutations and the WT calculations are consistent with the model presented here, the more extensive calculations are left for future work.

Bond orders of hydrogen bonds: The bond orders of certain hydrogen bonds differ in open and closed states.

Bond orders were calculated using NBO, and the results for some hydrogen bonds in WT are shown in Table

2. Not surprisingly there is a relation between bond order and bond length. The small side chain displacements strongly affect the bond strength, since $0.2 \AA$ is enough to change the bond strength appreciably, especially in H-bonds.

Table 2:

\begin{tabular}{|c|c|c|c|c|c|c|c|c|c|}
\hline & $\begin{array}{c}\text { H shif } \\
\rightarrow\end{array}$ & no H shift & no H shift & $\begin{array}{l}\text { R300 } \rightarrow \\
\text { E183 }\end{array}$ & $\begin{array}{l}\text { R300 } \rightarrow \\
\text { E183 }\end{array}$ & $\begin{array}{l}\text { Y266 } \rightarrow \\
\text { E183 }\end{array}$ & $\begin{array}{l}\text { Y266 } \rightarrow \\
\text { E183 }\end{array}$ & $\begin{array}{l}\text { Y266F } \\
\text { mutant }\end{array}$ & $\begin{array}{l}\text { Y266F } \\
\text { mutant }\end{array}$ \\
\hline H-bond & $\mathbf{V}(\mathbf{m V})$ & -70 & 0 & -70 & 0 & -70 & 0 & -70 & 0 \\
\hline R300- & H1-O1 & 0.16 & 0.16 & 0.10 & 0.13 & 0.13 & 0.16 & 0.16 & 0.16 \\
\hline E183 & $\mathrm{H} 2-\mathrm{O} 1$ & 0.24 & 0.24 & 0.11 & 0.09 & 0.13 & 0.10 & 0.21 & 0.23 \\
\hline $\begin{array}{l}\text { R303- } \\
\text { E226 }\end{array}$ & $\begin{array}{l}\mathrm{H} 1-\mathrm{O} 1 \\
\mathrm{H} 2-\mathrm{O} 2\end{array}$ & --+ & - & $\begin{array}{l}0.24 \\
0.31\end{array}$ & - & $\begin{array}{l}0.22 \\
0.30\end{array}$ & $\begin{array}{l}0.22 \\
0.29\end{array}$ & - & $\begin{array}{l}0.19 \\
0.31\end{array}$ \\
\hline $\begin{array}{l}\text { Y266\#- } \\
\text { E226 }\end{array}$ & O-H-O & 0.31 & 0.31 & -- & 0.22 & - & -- & -- & - \\
\hline
\end{tabular}




\begin{tabular}{|c|c|c|c|c|c|c|c|c|c|}
\hline $\begin{array}{l}\text { Y266- } \\
\text { R300(NE) }\end{array}$ & $\begin{array}{l}\mathrm{O}^{-} \\
\mathrm{NE}(\mathrm{H})\end{array}$ & - & -- & -- & $-0.31 *$ & $-0.30 *$ & - & -- & - \\
\hline $\begin{array}{l}\text { Y266- } \\
\text { R303 }\end{array}$ & $\mathrm{O}^{--}-\mathrm{NH}$ & - & - & -- & - & 0.12 & 0.11 & -- & - \\
\hline
\end{tabular}

*: Note that these are fairly strongly anti-bonding: there is at least one node in the electron density between

the two possible positions of the hydrogen atom.

**There is also a strong H-bond (0.40 bond order) between the NE of R300 and the -OH of Y266 in one

case only: (-70 $\mathrm{mV}, \mathrm{R} 300$ proton transferred to E183)

Where there is a blank in Table 2, there is no interaction, generally because the side chain has rotated in such a way that the relevant heavy atoms are either too far apart to form an $\mathrm{H}$-bond, or oriented so as to make the bond impossible by reason of intervening atoms - examination of Fig. 3 through Fig. 6 also shows this. The strongest H-bond bond order, 0.40, almost covalent, (see footnote, Table 2), is between the NE of R300 and the $-\mathrm{OH}$ of $\mathrm{Y} 266$, when the proton has shifted from R300 to E183, neutralizing the salt bridge; this practically locks the structure in the closed state, and has the lowest energy (Fig. 7), about $20 \mathrm{kBT}(\approx 50 \mathrm{~kJ})$ below the crossing point of the two positions, within the error of the calculation and the experimental error of what is found for the activation energy of gating from the temperature dependence of the current. However, this is not so easy to interpret. The fact that individual steps calculated here have different temperature dependences may not be relevant, as the steps from the calculated section to the gate may also be responsible for temperature dependence $(76,77)$, and other steps may contribute. Removing the voltage allows the proton to transfer so that the R300-Y266 H-bond no longer exists, and the side chains can change their local structure. Second, there are hydrogen bonds with bond order near 0.3 between E226 and Y266, and E226 and R303. There is a net of one extra bond with $\mathrm{V}=-70 \mathrm{mV}$. It appears that there are no other 
has lower energy than the open state (with the aid of the voltage) it appears that these strong H-bonds are in

478 the range that could contribute to the lower energy of the closed state. The complication of the system, with

479 multiple possibilities, suggests great caution in interpreting temperature dependence as a consequence of

480 individual steps. It is possible for an individual step to require so large an energy step as to be incompatible

481 with observation. Our result is not incompatible with the observed temperature dependence, but we should 482 not go beyond this.

483

484 The exchange and correlation energies were also calculated; in general, their differences with voltage, as 485 well as proton position, are on the order of tens of $\mathrm{kJ}$ (see Table 3), so that they cannot be neglected.

Table 3:

487

Exchange and correlation energies $(\mathrm{kJ} / \mathrm{mole})^{+}$for the same six cases as Table 1

\begin{tabular}{|c|c|c|c|c|c|c|}
\hline $\begin{array}{l}\text { Nominal charge } \\
\text { and energy } \rightarrow\end{array}$ & $\mathbf{Y 2 6 6}$ & R300 & E183 & $\begin{array}{l}\text { Exchange } \\
\text { energy }\end{array}$ & $\begin{array}{l}\text { Correlation } \\
\text { energy }\end{array}$ & Sum \\
\hline$-70 \mathrm{mV}$ & 0 & + & - & 0 & 2.5 & 2.5 \\
\hline 0 & 0 & + & - & 0 & 0 & 0 \\
\hline-70 & 0 & 0 & 0 & 75.8 & 20.2 & 96.0 \\
\hline 0 & 0 & 0 & 0 & 37.9 & 22.7 & 60.6 \\
\hline-70 & - & + & 0 & 65.7 & 37.9 & 103.6 \\
\hline 0 & - & + & 0 & 12.6 & 12.6 & 25.2 \\
\hline
\end{tabular}

$488+$ Absolute value of energy of the lowest energy state $\left(2^{\text {nd }}\right.$ row $)$ is subtracted from each term. The energies

489 shown are the energy differences between this state and the energy of each configuration. 0, +,- in columns

$490 \quad 2-4$ are the nominal charge states of the amino acid shown for that configuration. To get absolute values

491 of energy, add $6635.04 \mathrm{~kJ}$ to exchange energy, $430.78 \mathrm{~kJ}$ to correlation energy for all cases.

492 Translate the exchange + correlation energies into units of $\mathrm{k}_{\mathrm{B}} \mathrm{T}$ for $\mathrm{T}=300 \mathrm{~K}$, approximately $2.5 \mathrm{~kJ} / \mathrm{k}_{\mathrm{B}} \mathrm{T}$, to 
understand the physiological significance. For the $(0,0,0)$ cases that total $24 \mathrm{kB}_{\mathrm{B}}$ for 0 field, $38 \mathrm{kB}$ for field on, there is a difference of $14 \mathrm{kBT}$; for the $(-,+, 0)$ cases, the energy is $10 \mathrm{kBT}$ ( 0 field) compared to $41.5 \mathrm{kBT}$ (with field), a difference of approximately $31 \mathrm{kBT}$; without the exchange and correlation terms the wrong ionization states would be assigned. The difference of $17 \mathrm{~kJ}$, or about $7 \mathrm{k}_{\mathrm{B}} \mathrm{T}$, makes a considerable difference in the voltage at which the crossing of the two states occurs. These "quantum terms", those with no classical analogue, (the values of kinetic, etc. energy also are quantum terms in this calculation, although classical values exist for these) also bring the "normal" ionization states somewhat closer to the others, although the sum of potential plus kinetic terms still leave them slightly higher in energy. The nominal charges in Table 3 show the location of the proton, while the actual charges, from NBO calculation, are in Table 4

Table 4a: Charges on Y266 and glutamates* as determined by NBO calculations $(61,62,67)$

\begin{tabular}{|c|l|l|l|l|}
\hline Field, R300 charge** & $\mathrm{V}=0, \mathrm{R} 300(+)$ & $\mathrm{V}=0, \mathrm{R} 300(0)$ & $\mathrm{V}<0, \mathrm{R} 300(+)$ & $\mathrm{V}<0, \mathrm{R} 300(0)$ \\
\hline Y266 & -0.843 & -0.247 & -0.843 & -0.248 \\
\hline E183 & -0.014 & -0.016 & -0.009 & -0.013 \\
\hline E226 & -0.733 & -0.729 & -0.732 & -0.728 \\
\hline
\end{tabular}

*For Y266, the charge is either that on the oxygen if ionized (columns 2 and 4), or else small and distributed if it is not ionized; for glutamates, charge is the sum of that on the carboxyl oxygens plus the carbon to which they are bound, plus the proton; the E183 proton comes from the tyrosine if the arginine is charged. E226 is ionized, with constant charge of $\approx-0.73$; none of the charges is close to unity. All these cases have E183 nominally neutral, E226 nominally -1.

**nominal charge (i.e, , $H^{+}$position)

Table $4 b$ Charges on arginines 


\begin{tabular}{|c|c|c|c|c|}
\hline $\begin{array}{c}\text { Field, R300 charge** } \\
\text { (Number of atoms } \\
\text { counted) }\end{array}$ & 0.158 & 0.162 & 0.147 & 0.16 \\
\hline $\mathrm{R} 297(6)$ & 0.838 & 0.842 & 0.835 & 0.840 \\
\hline $\mathrm{R} 297(7)$ & 0.651 & 0.653 & 0.655 & 0.653 \\
\hline $\mathrm{R} 297(9)$ & 0.091 & 0.070 & 0.093 & -0.071 \\
\hline $\mathrm{R} 300(6)$ & 0.778 & 0.572 & 0.781 & 0.570 \\
\hline $\mathrm{R} 300(7)$ & 0.622 & -0.094 & 0.624 & -0.095 \\
\hline $\mathrm{R} 300(9)$ & 0.116 & 0.135 & 0.111 & 0.134 \\
\hline $\mathrm{R} 303(6)$ & 0.798 & 0.817 & 0.793 & 0.816 \\
\hline $\mathrm{R} 303(7)$ & 0.577 & 0.599 & 0.577 & 0.598 \\
\hline $\mathrm{R} 303(9)$ & & & & \\
\hline
\end{tabular}

513 *On each arginine, charges are summed over 6, 7, or 9 atoms: for 6 atoms, the sum is over both $\mathrm{NH}_{2}$

514 groups, or $\mathrm{NH}+\mathrm{NH}_{2}$ if one proton has transferred to another group; 7 atoms, add the central carbon; 9 515 atoms, add the preceding (nearer backbone) $N\left(+H\right.$, if present; this is the $N E$ nitrogen). i.e., if the $H^{+}$from 516 a guanidinium has transferred to another group, the sum over two $\mathrm{NH}_{2}$ groups becomes the sum over $\mathrm{NH}$ $517+\mathrm{NH}_{2}$ and the number of atoms decreases by one to 5, 6, or 8.

518 Table 4 shows the calculated charges on side chain functional groups (guanidinium in arginines, carboxyl in 519 acids, oxygen in tyrosine). Note that these results show a small dependence on applied voltage, and a large 520 dependence on proton position. The charges are not close to +1 and -1, but show the distribution of electron 521 density. We earlier (Table 3, and accompanying discussion) emphasized the importance of including the 522 exchange and correlation terms in order to determine the correct order of states, and thus the ionization states 523 with and without field. The charges in Table 4, and the exchange and correlation energies in Table 3, show 524 quantitatively that the charges on groups depend significantly on the external field and protonation state, 525 and, to a lesser extent, on the hydrogen bonds they participate in. 
The importance of the NE atom on the arginines: As noted in the Table 4 footnotes, it is the NE atom, more than the guanidinium group amines, that is responsible for most of the charge, when the arginine becomes protonated. When protonated it also forms hydrogen bonds that are stronger than most, as shown by the bond orders listed in Table 2. The combination of the NE and guanidinium is thus amphoteric, able to act simultaneously as base and acid, thereby transmitting a proton. Arginine is the only amino acid with this property, which provides an alternate explanation, not involving carrying charge by physical movement, for

532 its conservation on the S4 helix.

\section{ENERGY (kJ)}

\section{ENERGY-VOLTAGE RELATION FOR TWO} PROTONATION STATES

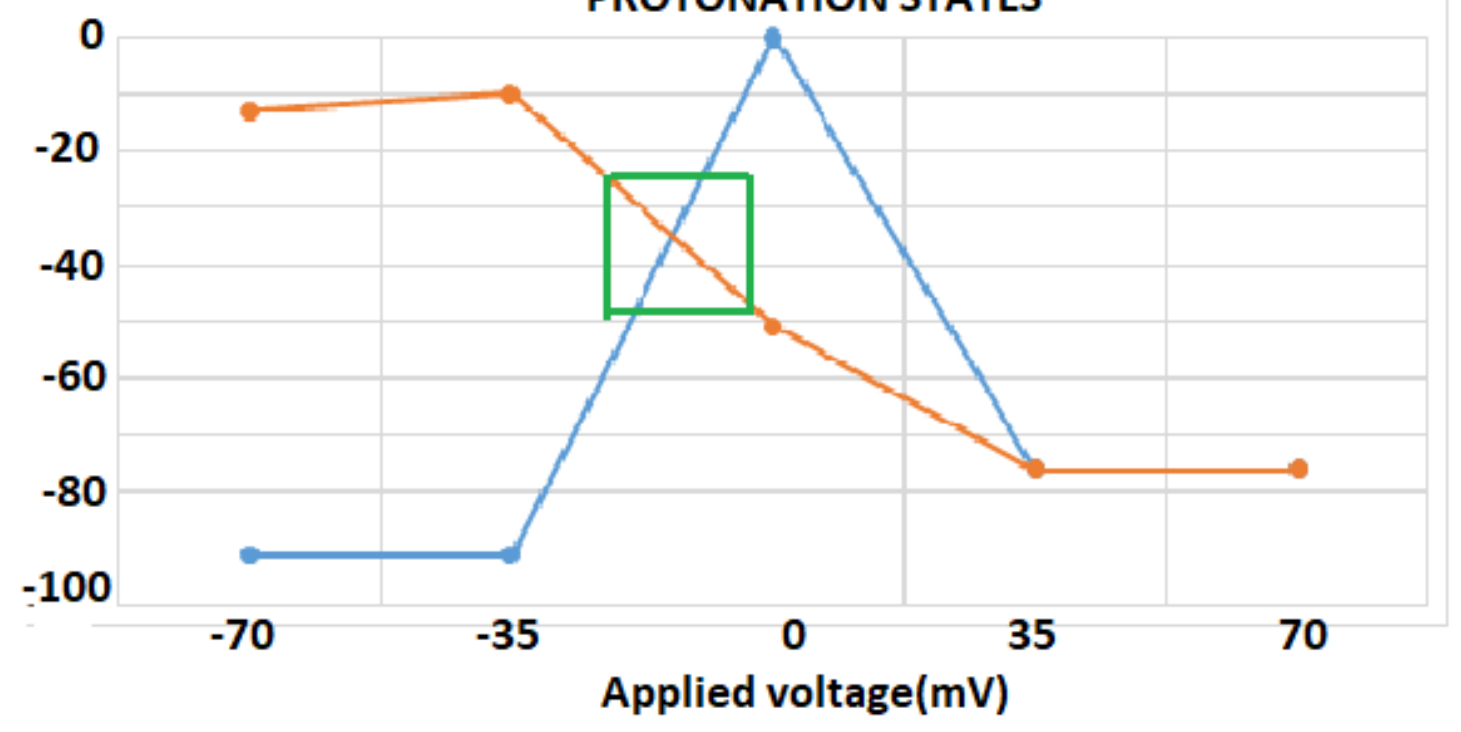

534

BLUE LINE, closed: R300(0), Y266(0), E183(0); ORANGE LINE, open: R300(+), Y266(-), E183(0)

535 Fig. 7: Total energy (entire 976 atom group) as a function of voltage for the two relevant proton configurations. Charges are nominal, indicating only proton position. Energy units are kJ, in relative units; for total energy, add 63419.575 MJ. Calculations were done at $35 \mathrm{mV}$ intervals. The green box indicates the approximate range at which channel open probability goes from near zero (negative voltage) to near one, as well as a rough estimate of calculation error. Conclusions would be essentially unchanged if the crossing were anywhere in the green box, corresponding to an error in relative energy of about $\pm 10 \mathrm{~kJ}( \pm 4$ 
$\left.k_{B} T\right)$, in a range of $\pm 10 \mathrm{mV}$, as large as can reasonably be expected.

542 Fig. 8 shows a calculation of the center of charge in the 976 atom section for an open (i.e., $0 \mathrm{mV}$ ) and a 543 closed $(-70 \mathrm{mV})$ configuration, with the proton shifted, as in Fig. 3 and Fig. 4, from Y266 (closed) to 544 R300 (open); the Fig. 8 red line corresponds to the orange line in Fig. 7, the blue line to the blue line.

545 The center of charge, in coordinates that place the VSD between $0 \AA$ and $33 \AA$, is at $19.5 \AA$ for the open VSD conformation, and $8.2 \AA$ for the closed case, so the center of charge moves $11.3 \AA$ in response to voltage. Because the charges are the sum of all charges in successive $3 \AA$ slices, it includes the effects of all charge transfer, side chain rotation, and hydrogen bond shifts.

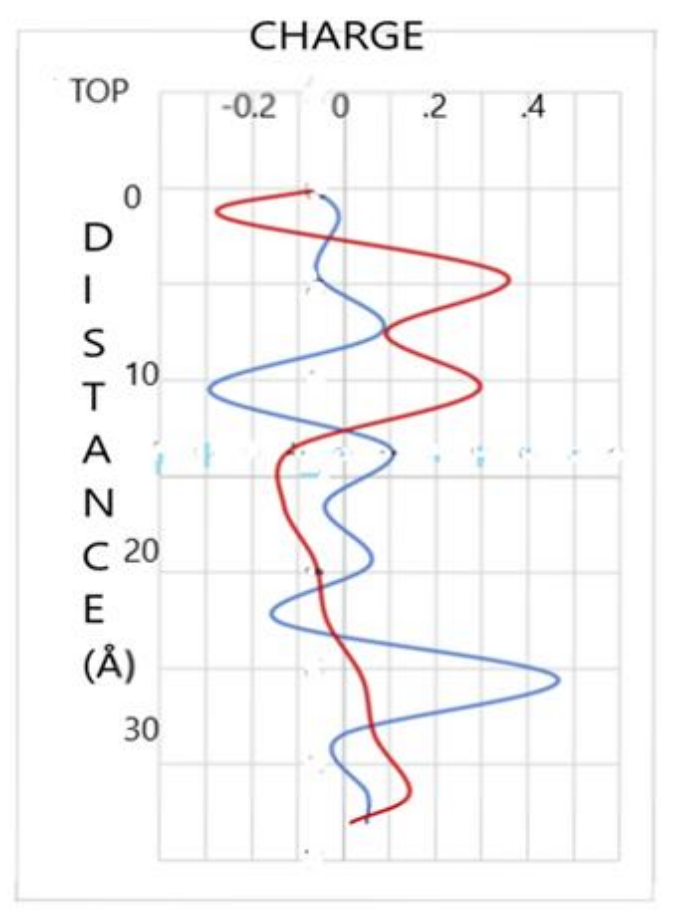

Fig. 8: The sum of the charges on $3 \AA$ slices parallel to the membrane surface; the curves shown are smooth fits to these values. The charge displacement is approximately $11.3 \AA$ between the lowest energy state with $-70 \mathrm{mV}$ and the lowest energy state with $0 \mathrm{mV}$ : "TOP" is the extracellular end, starting with $0 \AA$. (corresponding Fig 7 curves: blue curve to blue curve, red curve to orange curve),

Thus, gating current includes the proton transfer, plus the rotation of the side chains, together with 561 polarization of all groups, which is the collective response of the VSD, including all its atoms. As can 562 be seen from Table 4 , the charges are not +1 on each arginine and zero elsewhere. The same is true for 563 other residues, not in the Table. While most other charges are not large, they add to become non564 negligible. 
566 For the case with the R297 - E183 salt bridge neutral: nominal charge, R297(0), E183(0), and the other two 567 atoms with "normal" nominal charge (R300(+), Y266(0)) in Fig. 5, the energy requirement to transfer a 568 proton from R300 to Y266 is cut by about $15 \mathrm{kBT}(37 \mathrm{~kJ})$ as the transfer lowers the energy when the voltage

569 becomes more positive. That state also showed minimal response to applied electric field, energy changing 570 less than $1 \mathrm{k}_{\mathrm{B}} \mathrm{T}$ in the entire range of voltages. So small an effect is somewhat surprising. Without charge 571 on R297 and E183, this state would not be expected to respond strongly to voltage, for practical purposes, 572 so that it would not take part in gating; the fact that it is as small as it is may be partially fortuitous. On 573 examining the terms that add to give the total energy, we see that the variation in nucleus-nucleus repulsion 574 as the structure shifts slightly very nearly cancels the variation in electron-nuclear attraction, While having $575 \Delta \mathrm{E} \leq \mathrm{k}_{\mathrm{B}} \mathrm{T}$ for this case may be fortuitous, if it were $\pm 4 \mathrm{k}_{\mathrm{b}} \mathrm{T}$, as in the Fig. 7 error estimate, we would still 576 consider this state as not participating in response to voltage, although it is close enough to perhaps offer a 577 secondary path in case of mutation.

While this set of calculations is not compatible with any model in which S4 moves normal to the membrane sufficiently to contribute to gating current or gating, it is still compatible with an intracellular rotation, turning on a hinge below the region calculated, somewhat like that suggested by the bacterial sodium channels studied by Wallace and coworkers(78). Vertical S4 motion does not appear to play a role in those channels either, but the rotation of the helices at the intracellular end of the VSD is central to gating. However, the intracellular region of the $\mathrm{K}_{\mathrm{v}}$ channels is different from that in those channels, so the $\mathrm{K}_{\mathrm{v}}$ channels must use a different mechanism to gate. An extended proton path toward the gate would be possible in the $\mathrm{K}_{\mathrm{v}}$ channel, and would account for gating. In principle, the section not yet calculated, below the 976 atom section discussed here, and near or below the intracellular membrane surface, could add to gating current, but it is expected to make a negligible contribution. This section is also not expected to contribute significantly to voltage dependence, in either standard models, or our model. Experimentally it is 
known that most of the voltage drop across the membrane is localized near R297(64). We expect the continuing proton path to be generated by interaction with the protons that move in response to voltage in 592 the region we have calculated so far. The proton path can be postulated from a position near F233, where these 976 atom calculations end, along the intracellular surface of the membrane, including part of the T1 moiety, to the gate, which is below the transmembrane pore of the channel. There are water molecules in this part of the path. The proton would reach a histidine near the PVPV section at the gate, producing current 596 largely along the membrane surface, rather than perpendicular to it. Protons in this section move in response 597 to the change in protonation state of the section which we have already calculated, hence making little details of the effect of protonating the histidine remain to be calculated. With only a subtle change in the 600 gate diameter required to switch from closed to open, likely as little as 2 to $3 \AA$ for each VSD, the interaction with water at the gate and the interaction with the ion are both crucial. So far, we must restrict ourselves to saying that the protonated histidine will be different from the unprotonated histidine; it would be speculative to go beyond this without doing the calculation, which has just begun.

604

CAlCUlation: All calculations were done using Gaussian09 (D or E versions) (79). The calculations presented here are a section of one domain of the VSD of $\mathrm{K}_{\mathrm{v}} 1.2$; all amino acids are referred to with the 3Lut (pdb) numbering; 976 atoms (904 from protein, plus 24 water molecules) were optimized at HF/6-31G* level; Table 6 shows which amino acids had their side chains included in the calculation; others in the range shown had their backbone atoms included, but the side chains (which in all these cases pointed away from the central path) were truncated, terminated with $\mathrm{H}$. The net charge in all cases was +2 for 611 the entire system. In Fig. 2, all atoms of $\mathrm{S} 4$ were free to move, and two cases were optimized, at $0 \mathrm{mV}$, 612 which should correspond to the X-ray structure, and $-70 \mathrm{mV}$, which should show whether $\mathrm{S} 4$ can move 613 when closing with respect to the other transmembrane segments. In both cases, there was little motion of $614 \mathrm{~S} 4$, and such motion as there was (always $<2 \AA$, rarely $>1 \AA$ ) in the $-70 \mathrm{mV}$ case was in the wrong direction 
for standard models - $\mathrm{S} 4$ showed a very slight rise, rather than moving toward the intracellular end. After the structure was determined at HF/6-31G* level, the energy was calculated at B3LYP/6-31G** level, which was also used for NBO calculations, as single point calculations. The NBO calculations, which give the charges on the atoms, show that the charges depend on the field and the proton position, which would also affect the Coulomb terms in the Hamiltonian. There is also less symmetry than might be expected; for example, the hydrogens on the NH nitrogens on arginine differ sometimes by as much as 0.02 charges, which is not negligible. Ahmadi et al have provided an extensive and careful review of possible uses of QM calculations in multiscale applications, as well as the ways QM cluster calculations can be advantageous, or not(80). HF was not sufficiently accurate for energy and NBO calculations, (for one thing, HF lacks correlation energy), so B3LYP/6-31G** was used in single point computations on the HF optimized structure. Taking these results together, along with the charge transition in Fig. 8, we can conclude that Voltage sensing is a collective response; it is not a single charge, or just three charges, that respond. The possible proton pathways are complex, and alternate pathways exist, involving amino acids that may have only a peripheral role in the lowest energy, hence preferred, path. It is only by including all the relevant amino acids that we can understand the behavior of the channel. This said, it is also true that there are amino acids that play a primarily structural role, and are not directly involved in any proton transfer path.

Validation, with some questions that remain to be resolved. There are problems with these calculations as well. For one thing they find local minima at $0 \mathrm{~K}$, and do not directly allow for any dynamics. If the local minima were shallow, and the probability were high of a system optimizing into a random and unimportant minimum, the meaning of the calculation would be questionable; however, the minima are not shallow compared to $\mathrm{k}_{\mathrm{B}} \mathrm{T}$ at $300 \mathrm{~K}$. As a further test, we started the system from two different configurations (proton positions given in column 1 of Table 5, one of them the X-ray configuration). If there were multiple minima, these would be expected to give different results. If the calculation is reliable, the system should reoptimize with the proton in the $-70 \mathrm{mV}$ position as found starting from the $\mathrm{X}$-ray coordinates; otherwise it will probably give a different minimum. The calculation was done, and the result had the system return to the 
640 same position as it found starting from the X-ray coordinates, with the large distances (atoms separated by

$641>20 \AA$ ) from one TM segment to another changed by $<0.01 \AA$, and hydrogen bonds also the same within

$642<0.01 \AA$ (three are shown in Table 5). The energy differed by approximately $0.0007 \mathrm{Ha}, \approx 1.6 \mathrm{~kJ}$, or about

$6430.7 \mathrm{k}_{\mathrm{B}} \mathrm{T}$. In essence, the system found the same minimum. This was not a short calculation, taking over two

644 months, but the result did show that at least this key minimum is reliable. Limited computer resources

645 prevented testing multiple starting positions, but this case gave as good a chance as any of finding a different 646 minimum. Table 5 summarizes the results.

Table 5

648 Energy and distance comparison of a minimum obtained from different starting coordinates

\begin{tabular}{|l|l|l|l|l|l|l|}
\hline $\begin{array}{l}\text { Energy and } \\
\text { distances }\end{array}$ & $\begin{array}{l}\text { Energy (kJ) } \\
\text { relative to } \\
\text { lowest state }\end{array}$ & $\begin{array}{l}\text { R300/NE } \\
\text { to Y266/O } \\
\text { Angstroms }\end{array}$ & $\begin{array}{l}\text { Y266/O to } \\
\text { E226/O } \\
\text { Angstroms }\end{array}$ & $\begin{array}{l}\text { Y266/O to } \\
\text { R303/NH1 } \\
\text { Angstroms }\end{array}$ & $\begin{array}{l}\text { R297/N to } \\
\text { S169/O } \\
\text { Angstroms }\end{array}$ & $\begin{array}{l}\text { T184/O } \\
\text { S398/O } \\
\text { Angstroms }\end{array}$ \\
\hline $\begin{array}{l}\text { X-ray } \\
\text { R297(+);E183(-); } \\
\text { Y266(0) }\end{array}$ & 57.4 & 3.15 & 2.66 & 3.58 & 24.14 & 22.58 \\
\hline $\begin{array}{l}\text { R297(+);E183(0); } \\
\text { R300(+); Y266(-) }\end{array}$ & 80.2 & 2.74 & 3.85 & 2.76 & 24.74 & 22.88 \\
\hline $\begin{array}{l}\text { R297(+);E183(0); } \\
\text { R300(0); Y266(0) }\end{array}$ & 1.6 & 2.77 & 3.69 & 2.91 & 24.79 & 23.73 \\
\hline $\begin{array}{l}\text { R297(+);E183(0); } \\
\text { R300(0); Y266(0)\# }\end{array}$ & 0.0 & 2.77 & 3.69 & 2.91 & 24.79 & 23.73 \\
\hline
\end{tabular}

649 *Configuration optimized starting from the 3 Lut coordinates (X-ray); \# same configuration. In each case,

650 the starting coordinates are the structure shown two lines above. Energy shown is relative to that in the

651 last line. Both of the last two lines are at $-70 \mathrm{mV}$, and show the same optimized state.

652 Testing reversibility is a stringent test for whether the minima that are found represent actual configurations

653 of the system, and are not random local valleys in the energy landscape. (To the best of our knowledge,

654 there do not appear to be any published MD calculations that attempt to return the system to the starting

655 configuration, except for steered MD.)

656 
A second problem is that these calculations are limited in the number of atoms that can be included. Here, with 976 atoms, we have a very large QM computation, but it is roughly two orders of magnitude smaller than a MD calculation would be. However, once we have determined that the QM exchange and correlation terms are required, along with charge transfer, and the correct kinetic and potential energy terms differ from their classical analogs by more than $\mathrm{k}_{\mathrm{B}} \mathrm{T}$ (the changes in charge in an optimization are already large enough to see this-no classical calculation allows for such changes; including polarization helps, but is not only rarely used but insufficient, while other changes remain to be counted in the total difference), we are obligated to find a means of doing the QM calculation, even without including dynamics. To do so, we try as many as possible of the plausible configurations of the voltage sensing section (including the 3 critical arginines) of the VSD; we do not consider some combinations as plausible, such as anything in which Y266 or E183 is positive, or any guanidinium group negative. If we have enough local minima, we can track a path for the proton as a function of voltage. The region we have calculated includes most of the region in which the field drops(64), and is therefore the relevant region for testing the difference between the proton positions with and without voltage.

Finally, cancellation of errors in the total energy between calculations with different fields and proton positions is likely, to very good accuracy, as the system changes only in local transfers of protons, leaving most of the system unchanged. The lowest energy proton position is different with and without the field applied. We observe that the configuration $(\mathrm{Y}(0), \mathrm{R}(+), \mathrm{E}(-))$ is never the lowest energy; the calculation shows that the proton shifts. Neglecting the exchange and correlation energy would generally assign incorrect charges to the main charged groups; in particular, it would lead to an incorrect result in this case. The limit on the size of the system may, in principle, produce a problem with the boundaries. However, for these calculations, the boundaries are identical for all positions of the proton that shifts internal to the protein. Also, the proton transfer is fairly close to being an isodesmic reaction (one in which the total number of bonds of each type is unchanged); this type of reaction will have the reaction energy essentially correct, even if the 
682

683

684

685

686

687

688

689

690

691

692

693

694

695

696

697

698

699

700

701

absolute value of the total energy is not.(81, 82). If we consider the proton transfer $\mathrm{Y} 266 \rightarrow \mathrm{E} 183$, it is essentially isodesmic, the $-\mathrm{OH}$ from the tyrosine being at least similar to the carboxyl from the glutamate, even though the bonds are not actually identical; all other bonds are unchanged. This neglects the intermediate R300, and only considers the overall transfer, but that is what we need. The atoms at the boundaries, frozen or not, are the same in all cases. None of the amino acids with which we are concerned are at the system boundary; the proton transfer affects only internal amino acids, and effects no change at the boundaries. The error in the crossing energy for the two configurations is then estimated as within $10 \mathrm{~kJ} \approx 4 \mathrm{k}_{\mathrm{B}} \mathrm{T}$. This is a reasonable to generous error estimate; the overall consistency of the results also supports this estimate, as major errors would prevent the independent calculations from giving a coherent result; significant errors would lead to much more scatter, and not a reasonably consistent dependence on voltage, as shown in Fig. 7. We have spent some effort on validation and error estimates as this is a fairly unique calculation, being large for a quantum calculation, and requiring that the errors be within fairly stringent limits. It appears that the results have passed all the tests we were able to subject them to, and the two mutations gave further validation. The conclusions as stated are justified, to the extent possible.

Most amino acids in the calculated region had their side chains included, but those that pointed away were not included. Table 6 shows which side chains were included, representing 42 out of 70 amino acids in the region; those not indicated had their side chains truncated to $\mathrm{H}$ atoms (alternatively, these could be considered replaced by glycine). All backbone atoms were included.

Table 6

42 amino acids with side chains included in the calculation (the other 28, backbone atoms only)

\begin{tabular}{|l|l|l|l|}
\hline S1 & S2 & S3 & S4 \\
\hline A166 & T219 & M255 & I292 \\
\hline S169 & F222 & N256 & I296 \\
\hline V170 & V225 & I258 & R297* \\
\hline
\end{tabular}




\begin{tabular}{|l|l|l|l|}
\hline V172 & E226* & D259** & V299 \\
\hline I173 & T227 & A262 & R300** \\
\hline S176 & C229 & I263 & R303 \\
\hline S179 & I230 & P265 & K306*** \\
\hline F180 & F233 & Y266 & L307 \\
\hline C181 & & T269 & S308 \\
\hline E183* & & L270 & R309*** \\
\hline T184 & & G271 & H310 \\
\hline
\end{tabular}

*salt bridge; **,***members of apparent triads, or larger group

Frozen atoms: The amino acids with truncated side chains would normally have anchored the amino acid against the surroundings (possibly the membrane lipids, not included in the calculation); to replace this, either the backbone carbon, or nitrogen, or, towards the end of the segment, sometimes both, were fixed; in all, 62 atoms out 904 protein atoms were fixed, except that fewer were fixed in the S4 free case (Fig. 2). All water atoms were free. The frozen atoms provided the equivalent to environmental restraints, to a reasonable approximation. In addition to the $\mathrm{S} 4$ free, $\mathrm{V}=-70 \mathrm{mV}$ case, for the sake of completeness one case with $\mathrm{S} 4$ free at $0 \mathrm{mV}$ was also run. Both of these were found to not move enough to produce any gating current; the backbone hardly moved at all $(<1 \AA)$. The water in the calculation is not crystallographic water, but is present at the extracellular surface of the protein, where it comes in contact with water; the cleft in the protein makes it unlikely that there is lipid at this position. There is no crystallographic water within the helix bundle; if any water were present it would be expected to be between charged, or at least polar, residues, and to be held rather tightly; hence it would be expected to be crystallographic water. Since none is seen, it is reasonable to use a model in which one does not insert such water; the water at the top is inserted where the cleft in the extracellular surface of the protein strongly suggests there must be water present. 
The maximum voltage applied is equivalent to $70 \mathrm{mV}$ across a $70 \AA$ membrane (applied as a field of 0.00002 atomic units, $\approx 10^{7} \mathrm{~V} \mathrm{~m}^{-1}$; when the field is shown as $-70 \mathrm{mV}$, it is equivalent to the closed state of the VSD; zero and positive voltages correspond to the open state). The intracellular and extracellular loops are omitted, as is the upper end of S4, including R294, which would be expected to be complexed to the phosphate headgroups in the membrane (36-38); for it to move, this complex would have to be broken, which would cost considerable energy. However, the other three arginines (R297, R300, R303) which in the standard model would be the gating charges, are included. The $35 \mathrm{mV}$ cases were needed to determine the voltage at which the energy curves for the proton transfer crossed (Fig. 7), so the relevant cases were also run with the $35 \mathrm{mV}$ potentials.

SUMMARY: 1) The overall charge transfer in gating is a cooperative transition of at least much of the VSD; proton transfer is a major part of this, with more than one possible proton pathway.

2) In a calculation in which $\mathrm{S} 4$ was free to move, it did not move in response to application of a voltage. (Fig. 2)

3) Proton transfers in response to a change in voltage can be found from a calculation of the energy of several protein configurations with different proton positions as a function of voltage. Two paths in one section of the VSD were found: one through Y266 (preferred), the other through E226; both connect R303 to R300 and then E183 (Fig. 4). All paths then go through R297, which must be able to accept and transfer the proton to the extracellular surface, defined to include the lipid headgroup region, and water in the protein cleft. The energy of the proton transfer as a function of voltage is consistent with the direction of $\mathrm{H}^{+}$motion needed for gating, and with the voltage at which the probability of channel opening increases rapidly. The existence of more than one path is also consistent with hysteresis in gating, which has been shown.

4) The charge displacement in response to the voltage change is consistent with the gating current; it is necessary to include the charges on all atoms to account for charge displacement in the VSD. (Fig. 8)

5) A possible explanation for the conservation of the arginines depends on their amphoteric property, in 
which NE takes part in proton transfer. This is an alternative to accounting for the conservation of the arginines by having them move as positive charges to produce gating current, as in standard models.

6) Hydrogen bonds involving serine, and sometimes threonine, make a significant contribution to the stability of the calculated states via hydrogen bonding; the open case here involves S176, and the closed state, S184. The hydrogen bonds may switch (i.e., -OH rotates) when protons switch positions; this is seen very clearly in the Y266F mutation.

7) These calculations allow for at least qualitative prediction of the non-obvious effects of two mutations; although insufficient information is in the calculation to make quantitative comparison to experiment possible, even the qualitative prediction is not intuitive.

8) It is possible to see a probable continuation of the proton path beyond the region calculated, in the intracellular direction, then along the intracellular surface of the membrane, toward the gate, so that the entire gating transition can be accomplished by proton transfer. It remains to test whether this plausible path is correct.

9) In the one case in which it was tested, the calculation found the same optimized structure from two different starting points, one of which was a reversal of the original proton transfer. This helps confirm the validity of the calculation of the energy minima.

10) The RYE triad of amino acids in the proton transfer step shown in detail in Fig. 4 is similar to groups found in at least several other cases in which protons are known to transfer, so may be a general part of protein proton transfer mechanisms.

SUPPORTING MATERIAL: Supporting Material consists of fourteen coordinate files, the results of the optimizations used in Fig. 7, plus the two for each of the two mutations shown in Fig. 5 and Fig. 6.

ACKNOWLEDGMENTS: We are grateful to Dr. Carlos Bassetto and Professor Francisco Bezanilla for carrying out the experiment with tyrosine mutation, and for permission to use their unpublished data. We thank Miguel Chavez for the calculation of the charge distribution and for providing the accompanying 
Fig. 8. This research used resources of the Center for Functional Nanomaterials, which is a U.S. DOE

Office of Science Facility, and the Scientific Data and Computing Center, a component of the

Computational Science Initiative, at Brookhaven National Laboratory under Contract No. DE-

SC0012704, and resources at the High Performance Computation facility at City University of New York.

\section{References:}

772 1. Hille B. Ion Channels of Excitable Membranes. 3rd ed. Sunderland, MA: Sinauer Associates; 2001.

7732 2. DeCoursey TE. Four varieties of voltage-gated proton channels. Front Biosci. 1998;3:D477-D82.

774 3. DeCoursey TE. The Voltage-Gated Proton Channel: A Riddle, Wrapped in a Mystery, inside an Enigma.

775 Biochemistry. 2015;54(21):3250-68.

776 4. Gazzarrini S, Van EJL, DiFrancesco D, Thiel G, Moroni A. Voltage-dependence of virus-encoded

777 miniature K+ channel Kcv. J Membr Biol. 2002;187(1):15-25.

778 5. Moroni A, Thiel G. Flip-flopping salt bridges gate an ion-channel. Nat Chem Biol. 2006;2:572-3.

7796 6. Hodgkin AL, Huxley AF. Currents carried by sodium and potassium ions through the giant membrane of $780 \quad$ Loligo. J Physiol (Lond). 1952;116:449-72.

781 7. Hodgkin AL, Huxley AF. The components of membrane conductance in the giant axon of Loligo. J Physiol (Lond). 1952;116:473-96.

8. Hodgkin AL, Huxley AF. The dual effect of membrane potential on sodium conductance in the giant axon of Loligo. J Physiol (Lond). 1952;116:497-506.

9. Minor DL, Jr., Lin Y-F, Mobley BC, Avelar A, Jan YN, Jan LY, et al. The polar T1 interface is linked to conformational changes that open the voltage-gated potassium channel. Cell (Cambridge, Mass). 2000;102(5):65770 .

10. Chen X, Wang Q, Ni F, Ma J. Structure of the full-length Shaker potassium channel Kv1.2 by normalmode-based X-ray crystallographic refinement. Proc Natl Acad Sci U S A. 2010;107(25):11352-7.

11. Karlin A, Akabas MA. Substituted-Cysteine Accessibility Method. In: Conn PM, editor. Methods in Enzymology. New York: Academic Press; 1998. p. 123-45.

12. Delemotte L, Klein ML, Tarek M. Molecular dynamics simulations of voltage-gated cation channels: insights on voltage-sensor domain function and modulation. Front Pharmacol Ion Channels Channelopathies. 2012;3(May):97.

13. Delemotte L, Tarek M, Klein ML, Amaral C, Treptow W. Intermediate states of the Kv1.2 voltage sensor from atomistic molecular dynamics simulations. Proc Natl,Acad Sci. 2011.

14. Deyawe A, Kasimova MA, Delemotte L, Tarek M, Loussouarn G, Tarek M. Studying Kv Channels Function using Computational Methods. Methods Mol Biol. 2018;1684:321-41.

15. Jensen MO, Jogini V, Borhani DW, Leffler AE, Dror RO, Shaw DE. Mechanism of voltage gating in potassium channels. Science (Washington, DC, U S). 2012;336(Copyright (C) 2012 American Chemical Society (ACS). All Rights Reserved.):229-33.

16. Schow EV, Freites JA, Gogna K, White SH, Tobias DJ. Down-State Model of the Voltage-Sensing Domain of a Potassium Channel. Biophys J. 2010;98(Copyright (C) 2012 American Chemical Society (ACS). All Rights Reserved.):2857-66.

17. Blunck R, McGuire H, Hyde HC, Bezanilla F. Fluorescence detection of the movement of single KcsA subunits reveals cooperativity. Proc Nat'l Academy of Sciences. 2008;105:20263-8.

18. Sandtner W, Bezanilla F, Correa AM. In vivo measurement of intramolecular distances using genetically encoded reporters. Biophys J. 2007;93:L45-L7.

19. Kariev AM, Green ME. Voltage gated ion channel function: gating, conduction, and the role of water and protons. Int J Mol Sci. 2012;13:1680-709.

20. Kariev AM, Green ME. Caution is required in interpretation of mutations in the voltage sensing domain of voltage gated channels as evidence for gating mechanisms. Int J Mol Sci. 2015;16(1):1627-43. 
813 21. Kariev AM, Green ME. Quantum Calculations on the Kv1.2 Channel Voltage Sensing Domain Show H+ 814 Transfer Provides the Gating Current. arXiv 2017:1712:02866

22. Kariev AM, Green ME. The role of proton transport in gating current in a voltage gated ion channel, as shown by quantum calculations. Sensors. 2018;18(9):3143/1-/29.

23. Cha A, Snyder G, Selvin PR, Bezanilla F. Atomic scale movement of the voltage-sensing region in a potassium channel measured via spectroscopy. Nature. 1999;402:809-13.

24. Chanda B, Asamoah OK, Blunck R, Roux B, Bezanilla F. Gating charge displacement in voltage-gated ion channels involves limited transmembrane movement. Nature. 2005;436:852-6.

25. Posson DJ, Ge P, Miller C, Bezanilla F, Selvin PR. Small vertical movement of a $\mathrm{K}^{+}$channel voltage sensor measured with luminescence energy transfer. Nature. 2005;436:848-51.

26. Lu J, Yin J, Green ME. A model for ion channel voltage gating with static S4 segments. Ferroelectrics. 1999;220:249-71.

27. Sapronova A, Bystrov V, Green ME. Ion channel gating and proton transport. J Mol Struct: THEOCHEM. 2003;630:297-307.

28. Sapronova AV, Bystrov VS, Green ME. Water,proton transfer, and hydrogen bonding in ion channel gating. Frontiers in Bioscience. 2003;8:s1356-s70.

29. Dal Peraro M, Raugei S, Carloni P, Klein ML. Solute-solvent charge transfer in aqueous solution. ChemPhysChem. 2005;6(9):1715-8.

30. Yao Y, Kanai Y, Berkowitz ML. Role of Charge Transfer in Water Diffusivity in Aqueous Ionic Solutions. J Phys Chem Lett. 2014;5(15):2711-6.

31. Ramsey IS, Moran MM, Chong JA, Clapham DE. A voltage-gated proton-selective channel lacking the pore domain. Nature. 2006;440:1213-6.

32. Okamura Y, Fujiwara Y, Sakata S. Gating mechanisms of voltage-gated proton channels. Annu Rev Biochem. 2015;84:685-709.

33. Decoursey TE. Voltage-gated proton channels. Compr Physiol. 2012;2(2):1355-85.

34. Woelke AL, Wagner A, Galstyan G, Meyer T, Knapp E-W. Proton Transfer in the K-Channel Analog of BType Cytochrome c Oxidase from Thermus thermophilus. Biophys J. 2014;107(9):2177-84.

35. Kim YC, Wikstrom M, Hummer G. Kinetic gating of the proton pump in cytochrome c oxidase. Proc Natl Acad Sci U S A. 2009;106(33):13707-12, S/1-S/6.

36. Peng Y, Voth GA. Expanding the view of proton pumping in cytochrome c oxidase through computer simulation. Biochim Biophys Acta, Bioenerg. 2012;1817(4):518-25.

37. Smondyrev AM, Voth GA. Molecular dynamics simulation of proton transport through the influenza A virus M2 channel. Biophys J. 2002;83(4):1987-96.

38. Chen $\mathrm{H}, \mathrm{Wu}$ Y, Voth GA. Proton transport behavior through the influenza A M2 channel: insights from molecular simulation. Biophys J. 2007;93(10):3470-9.

39. Gianti E, Carnevale V, DeGrado WF, Klein ML, Fiorin G. Hydrogen-Bonded Water Molecules in the M2 Channel of the Influenza A Virus Guide the Binding Preferences of Ammonium-Based Inhibitors. J Phys Chem B. 2015;119(3):1173-83.

40. Hu J, Fu R, Nishimura K, Zhang L, Zhou H-X, Busath DD, et al. Histidines, heart of the hydrogen ion channel from influenza A virus: Toward an understanding of conductance and proton selectivity. Proc Natl Acad Sci USA. 2006;103:6865-70.

41. Aviles-Moreno JR, Berden G, Oomens J, Martinez-Haya B. Guanidinium/ammonium competition and proton transfer in the interaction of the amino acid arginine with the tetracarboxylic 18-crown-6 ionophore. Phys Chem Chem Phys. 2018;20(6):4067-73.

42. Aviles-Moreno JR, Berden G, Oomens J, Martinez-Haya B. Isolated complexes of the amino acid arginine with polyether and polyamine macrocycles, the role of proton transfer. Phys Chem Chem Phys. 2017;19(46):3134551.

43. Ma Y, Yu J-G, Sun Q, Li Z, Smith SC. The mechanism of dehydration in chromophore maturation of wildtype green fluorescent protein: A theoretical study. Chem Phys Lett. 2015;631-632:42-6.

44. Melo A, Ramos MJ, Floriano WB, Gomes JANF, Leao JFR, Magalhaes AL, et al. Theoretical study of arginine-carboxylate residues. THEOCHEM. 1999;463:81-90.

45. Han M, Kopec W, Solov'yov IA, Khandelia H. Glutamate Water Gates in the Ion Binding Pocket of Na+ Bound Na+, K+-ATPase. Sci Rep. 2017;7:39829.

46. Starace D, Stefani E, Bezanilla F. Histidine scanning mutagenesis indicates full translocation of two charges of the Shaker K channel voltage sensor. Biophys J. 1998;74:A215. 
47. Starace DM, Stefani E, Bezanilla F. Voltage-dependent proton transport by the voltage sensor of the Shaker $\mathrm{K}^{+}$channel. Neuron. 1997;19:1319-27. S4 segment. Biophys J. 2001;80:217a.

49. Zhao J, Blunck R. The isolated voltage sensing domain of the Shaker potassium channel forms a voltagegated cation channel. Elife. 2016;5.

50. El-Din TMG, Heldstab H, Lehmann C, Greeff NG. Double gaps along Shaker S4 demonstrate omega currents at three different closed states Channels. 2010;4:93-100.

51. Tarek M, Delemotte L. Omega currents in voltage-gated ion channels: What can we learn from uncovering the voltage-sensing mechanism using MD simulations? Acc Chem Res. 2013;46(12):2755-62.

52. Ishida IG, Rangel-Yescas GE, Carrasco-Zanini J, Islas LD. Voltage-dependent gating and gating charge measurements in the Kv1.2 potassium channel. J Gen Physiol. 2015;145(4):345-58.

53. Nguyen TP, Horn R. Movement and crevices around a sodium channel S3 segment. J Gen'l Physiol. 2002;120:419-36.

54. Gonzalez-Perez V, Stack K, Boric K, Naranjo D. Reduced voltage sensitivity in a K ${ }^{+}$-channel voltage sensor by electric field remodeling. Proc Natl Acad Sci. 2010;107:5178-83.

55. Liao S, Green ME. Quantum calculations on salt bridges with water: Potentials, structure, and properties. Comput Theo Chem. 2011;963:207-14.

56. Garczarek F, Brown LS, Lanyi JK, Gerwert K. Proton binding within a membrane protein by a protonated water cluster. Proc Natl Acad Sci U S A. 2005;102(10):3633-8.

57. Garczarek F, Gerwert K. Functional waters in intraprotein proton transfer monitored by FTIR difference spectroscopy. Nature (London, U K). 2006;439(7072):109-12.

58. Takeda M, Jee JG, Ono AM, Terauchi T, Kainosho M. Hydrogen Exchange Rate of Tyrosine Hydroxyl Groups in Proteins As Studied by the Deuterium Isotope Effect on C $\zeta$ Chemical Shifts. J Am Chem Soc.

2009;131(51):18556-62.

59. Barchad-Avitzur O, Priest MF, Dekel N, Bezanilla F, Parnas H, Ben-Chaim Y. A Novel Voltage Sensor in the Orthosteric Binding Site of the M2 Muscarinic Receptor. Biophys J. 2016;111(7):1396-408.

60. Chen X, Wang Q, Ni F, Ma J. Structure of the full-length Shaker potassium channel Kv1.2 by normalmode-based X-ray crystallographic refinement. Proc Natl Acad Sci. 2010;107:11352-7.

61. Weinhold F. NBO 5.0 Program Manual. Madison, WI: Theoretical Chemistry Institute, U. of Wisconsin; 2001.

62. Weinhold F. Natural Bond Orbital Analysis: A Critical Overview of Relationships to Alternative Bonding Perspectives. J Comp Chem. 2012;33:2363-79.

63. Villalba-Galea CA. Hysteresis in voltage-gated channels. Channels (Austin). 2017;11(2):140-55.

64. Asamoah OK, Wuskell JP, Loew LM, Bezanilla F. A Fluorometric Approach to Local Electric Field Measurements in a Voltage-Gated Ion Channel. Neuron. 2003;37:85-97.

65. Stefani E, Bezanilla F. Voltage dependence of the early events in voltage gating. Biophys J. 1997;72:A131. 66. Stefani E, Sigg D, Bezanilla F. Correlation between the early component of gating current and total gating current in Shaker K channels. Biophysical Journal. 2000;78:7A.

67. Dudev T, Musset B, Morgan D, Cherny VV, Smith SME, Mazmanian K, et al. Selectivity Mechanism of the Voltage-gated Proton Channel, HV1. Sci Rep. 2015;5:10320.

68. van Keulen SC, Gianti E, Carnevale V, Klein ML, Rothlisberger U, Delemotte L. Does proton conduction in the voltage-gated $\mathrm{H}+$ channel hHv1 involve Grotthuss-like hopping via acidic residues? J Phys Chem B. 2017; $121: 3340-51$.

69. Eisenberg AS, Juszczak LJ. The Broken Ring: Reduced Aromaticity in Lys-Trp Cations and High pH Tautomer Correlates with Lower Quantum Yield and Shorter Lifetimes. J Phys Chem B. 2014;118(25):7059-69. 70. Juszczak LJ, Eisenberg AS. The Color of Cation- $\pi$ Interactions: Subtleties of Amine-Tryptophan Interaction Energetics Allow for Radical-like Visible Absorbance and Fluorescence. J Am Chem Soc. 2017;139(24):8302-11.

71. Wolf S, Freier E, Gerwert K. A Delocalized Proton-Binding Site within a Membrane Protein. Biophys J. 2014;107(1):174-84.

72. Oliver AE, Deamer DW. $\alpha$-Helical hydrophobic polypeptides form proton-selective channels in lipid bilayers. Biophys J. 1994;66(5):1364-79.

73. Mohajeri A, Karimi E. AIM and NBO analyses of cation- $\pi$ interaction. J Mol Struct: THEOCHEM. 2006;774(1-3):71-6.

74. Schwaiger CS, Liin SI, Elinder F, Lindahl E. The Conserved Phenylalanine in the K+ Channel Voltage- 
Sensor Domain Creates a Barrier with Unidirectional Effects. Biophys J. 2013;104(Copyright (C) 2013 American Chemical Society (ACS). All Rights Reserved.):75-84.

75. Infield DT, Galpin JD, Galles GD, Ahern CA, Lee EEL, Bezanilla F. Replacing voltage sensor arginines with citrulline provides mechanistic insight into charge versus shape. J Gen Physiol. 2018.

76. Chowdhury S, Chanda B. Free-energy relationships in ion channels activated by voltage and ligand. J Gen Physiol. 2013;141(1):11-28.

77. Rodriguez BM, Sigg D, Bezanilla F. Voltage gating of Shaker $\mathrm{K}^{+}$channels: the effect of temperature on ionic and gating currents. J Gen'l Physiol. 1998;112:223-42.

78. McCusker EC, Bagnéris C, E. NC, Cole AR, D'Avanzo N, Nichols CG, et al. Structure of a bacterial voltage-gated sodium channel pore reveals mechanisms of opening and closing. Nature Communications. 2012;3(Art. no. 1102).

79. Frisch MJT, G. W.; Schlegel, H. B.; Scuseria, G. E.; Robb, M. A.; Cheeseman, J. R.; Scalmani, G.; Barone, V.; Mennucci, B.; Petersson, G. A.; Nakatsuji, H.; Caricato, M.; Li, X.; Hratchian, H. P.; Izmaylov, A. F.; Bloino, J.; Zheng, G.; Sonnenberg, J. L.; Hada, M.; Ehara, M.; Toyota, K.; Fukuda, R.; Hasegawa, J.; Ishida, M.; Nakajima, T.; Honda, Y.; Kitao, O.; Nakai, H.; Vreven, T.; Montgomery, J. A., Jr.; Peralta, J. E.; Ogliaro, F.; Bearpark, M.; Heyd, J. J.; Brothers, E.; Kudin, K. N.; Staroverov, V. N.; Kobayashi, R.; Normand, J.; Raghavachari, K.; Rendell, A.; Burant, J. C.; Iyengar, S. S.; Tomasi, J.; Cossi, M.; Rega, N.; Millam, J. M.; Klene, M.; Knox, J. E.; Cross, J. B.; Bakken, V.; Adamo, C.; Jaramillo, J.; Gomperts, R.; Stratmann, R. E.; Yazyev, O.; Austin, A. J.; Cammi, R.; Pomelli, C.; Ochterski, J. W.; Martin, R. L.; Morokuma, K.; Zakrzewski, V. G.; Voth, G. A.; Salvador, P.; Dannenberg, J. J.; Dapprich, S.; Daniels, A. D.; Farkas, Ö.; Foresman, J. B.; Ortiz, J. V.; Cioslowski, J.; Fox, D. J. . Gaussian09, Rev. E01. 2016.

80. Ahmadi S, Barrios Herrera L, Chehelamirani M, Hostas J, Jalife S, Salahub DR. Multiscale modeling of enzymes: QM-cluster, QM/MM, and QM/MM/MD: A tutorial review. Int J Quantum Chem. 2018:Ahead of Print. 81. Dorofeeva OV, Ryzhova ON. Gas-Phase Enthalpies of Formation and Enthalpies of Sublimation of Amino Acids Based on Isodesmic Reaction Calculations. J Phys Chem A. 2014;118(19):3490-502.

82. Hudzik JM, Bozzelli JW. Thermochemistry and Bond Dissociation Energies of Ketones. J Phys Chem A. 2012;116(23):5707-22. 
SUPPORTING INFORMATION: OPTIMIZED COORDINATE FILES

PROTON CONFIGURATION, GIVEN BY NOMINAL CHARGES: R300(0),Y266(0),E183(0)

POTENTIAL: -70mV

N $\quad 15.580944-6.451469 \quad 5.391416$

H $\quad 14.780731-6.200923 \quad 5.939305$

C $\quad 15.509643 \quad-5.856520 \quad 4.054221$

C $\quad 14.783016-6.782962 \quad 3.060452$

O $\quad 14.052228-6.345797 \quad 2.186827$

C $\quad 16.919741-5.576790 \quad 3.520757$

H $\quad \begin{array}{llll}\text { H } & 16.389845 & -6.128186 & 5.884447\end{array}$

H $\quad 14.945059-4.933534 \quad 4.050349$

H $\quad 16.889045 \quad-5.190425 \quad 2.508988$

H $\quad \begin{array}{llll}17.403781 & -4.831921 & 4.142207\end{array}$

H $\quad 17.526994-6.475176 \quad 3.529510$

N $\quad \begin{array}{llll}15.032378 & -8.085604 & 3.186404\end{array}$

H $\quad 15.612369-8.361765 \quad 3.949488$

C $\quad 14.398661 \quad-9.092386 \quad 2.333235$

H $\quad \begin{array}{llll}14.482431 & -8.823798 & 1.292859\end{array}$

H $\quad 14.891368-10.042265 \quad 2.492758$

$\begin{array}{llll}\text { C } & 12.912802 & -9.220816 & 2.671363\end{array}$

O $\quad 12.081430 \quad-9.4247121 .825901$

N $\quad 12.582804 \quad-9.055884 \quad 3.966877$

H $\quad 13.302707 \quad-8.986374 \quad 4.648923$

$\begin{array}{llll}\text { C } & 11.195108 & -9.170814 & 4.421376\end{array}$

H $\quad 11.188268 \quad-9.157965 \quad 5.503131$

H $\quad 10.743743-10.088315 \quad 4.077111$

C $\quad 10.336944 \quad-8.010595 \quad 3.900734$

$\begin{array}{llll}\text { O } & 9.190715 & -8.185973 & 3.584565\end{array}$ 


\begin{tabular}{|c|c|c|c|}
\hline $\mathrm{N}$ & 10.947166 & -6.814584 & 3.855271 \\
\hline C & 10.318595 & -5.634057 & 3.317570 \\
\hline C & 10.126801 & -5.690990 & 1.799352 \\
\hline 0 & 9.096244 & -5.262541 & 1.337380 \\
\hline C & 11.093003 & -4.388030 & 3.776268 \\
\hline 0 & 12.478911 & -4.570920 & 3.769522 \\
\hline $\mathrm{H}$ & 11.905594 & -6.744371 & 4.106682 \\
\hline $\mathrm{H}$ & 9.314756 & -5.553195 & 3.701226 \\
\hline $\mathrm{H}$ & 10.801687 & -3.539751 & 3.167074 \\
\hline $\mathrm{H}$ & 10.827406 & -4.174248 & 4.801233 \\
\hline $\mathrm{H}$ & 12.805998 & -4.755413 & 2.897553 \\
\hline $\mathrm{N}$ & 11.099962 & -6.200428 & 1.023953 \\
\hline C & 10.844256 & -6.396651 & -0.400382 \\
\hline C & 9.693378 & -7.385344 & -0.601380 \\
\hline $\mathrm{O}$ & 8.944764 & -7.279524 & -1.544861 \\
\hline C & 12.132565 & -6.785513 & -1.187311 \\
\hline C & 12.333415 & -8.290910 & -1.404097 \\
\hline C & 12.159258 & -6.060439 & -2.534205 \\
\hline $\mathrm{H}$ & 11.924166 & -6.585903 & 1.431072 \\
\hline $\mathrm{H}$ & 10.474583 & -5.462318 & -0.791691 \\
\hline $\mathrm{H}$ & 12.964681 & -6.415407 & -0.598905 \\
\hline $\mathrm{H}$ & 13.287085 & -8.454107 & -1.895336 \\
\hline $\mathrm{H}$ & 12.332057 & -8.857111 & -0.483479 \\
\hline $\mathrm{H}$ & 11.566150 & -8.698123 & -2.055406 \\
\hline $\mathrm{H}$ & 13.053903 & -6.324343 & -3.087057 \\
\hline $\mathrm{H}$ & 11.298808 & -6.332248 & -3.135779 \\
\hline $\mathrm{H}$ & 12.159266 & -4.985220 & -2.401000 \\
\hline $\mathrm{N}$ & 9.570431 & -8.340344 & 0.328319 \\
\hline $\mathrm{H}$ & 10.315059 & -8.474836 & 0.973641 \\
\hline C & 8.593019 & -9.421180 & 0.241223 \\
\hline
\end{tabular}




\begin{tabular}{|c|c|c|c|}
\hline $\mathrm{H}$ & 8.934634 & -10.218590 & 0.888835 \\
\hline $\mathrm{H}$ & 8.533193 & -9.803631 & -0.766085 \\
\hline$C$ & 7.181698 & -9.045745 & 0.642909 \\
\hline 0 & 6.257483 & -9.502080 & 0.010630 \\
\hline $\mathrm{N}$ & 7.003520 & -8.226545 & 1.685802 \\
\hline$C$ & 5.669684 & -7.739286 & 1.954979 \\
\hline$C$ & 5.251021 & -6.727652 & 0.893553 \\
\hline $\mathrm{O}$ & 4.074248 & -6.630710 & 0.617445 \\
\hline$C$ & 5.415518 & -7.234086 & 3.389334 \\
\hline$C$ & 5.557535 & -8.378469 & 4.397949 \\
\hline$C$ & 6.257448 & -6.025280 & 3.802561 \\
\hline $\mathrm{H}$ & 7.780930 & -7.960683 & 2.250279 \\
\hline $\mathrm{H}$ & 4.986652 & -8.558610 & 1.792682 \\
\hline $\mathrm{H}$ & 4.374715 & -6.925106 & 3.382500 \\
\hline $\mathrm{H}$ & 5.267463 & -8.041397 & 5.387567 \\
\hline $\mathrm{H}$ & 4.919466 & -9.215853 & 4.134954 \\
\hline $\mathrm{H}$ & 6.579689 & -8.735715 & 4.453711 \\
\hline $\mathrm{H}$ & 5.917068 & -5.653359 & 4.763052 \\
\hline $\mathrm{H}$ & 7.299760 & -6.295845 & 3.909491 \\
\hline $\mathrm{H}$ & 6.185275 & -5.210641 & 3.090221 \\
\hline $\mathrm{N}$ & 6.171801 & -6.003043 & 0.249776 \\
\hline$C$ & 5.776269 & -5.184213 & -0.884109 \\
\hline$C$ & 5.343447 & -6.067678 & -2.062536 \\
\hline 0 & 4.319744 & -5.815739 & -2.653842 \\
\hline$C$ & 6.868947 & -4.165014 & -1.264115 \\
\hline$C$ & 7.066958 & -3.148300 & -0.125802 \\
\hline$C$ & 6.498212 & -3.454662 & -2.570627 \\
\hline$C$ & 8.360686 & -2.341733 & -0.239822 \\
\hline $\mathrm{H}$ & 7.133074 & -6.061030 & 0.508390 \\
\hline $\mathrm{H}$ & 4.878561 & -4.647265 & -0.616972 \\
\hline
\end{tabular}




\begin{tabular}{|c|c|c|c|}
\hline $\mathrm{H}$ & 7.798814 & -4.706502 & -1.411874 \\
\hline $\mathrm{H}$ & 6.211977 & -2.473862 & -0.109879 \\
\hline $\mathrm{H}$ & 7.076657 & -3.662085 & 0.826136 \\
\hline $\mathrm{H}$ & 7.203684 & -2.665133 & -2.790646 \\
\hline $\mathrm{H}$ & 6.498225 & -4.129893 & -3.416516 \\
\hline $\mathrm{H}$ & 5.510152 & -3.011194 & -2.503906 \\
\hline $\mathrm{H}$ & 8.461932 & -1.668112 & 0.604440 \\
\hline $\mathrm{H}$ & 9.222078 & -2.999285 & -0.239564 \\
\hline $\mathrm{H}$ & 8.396893 & -1.742148 & -1.142435 \\
\hline$N$ & 6.115624 & -7.109718 & -2.399307 \\
\hline $\mathrm{H}$ & 7.009706 & -7.232401 & -1.972530 \\
\hline C & 5.773400 & -7.949754 & -3.527335 \\
\hline $\mathrm{H}$ & 6.603987 & -8.623480 & -3.697355 \\
\hline $\mathrm{H}$ & 5.618446 & -7.363666 & -4.420900 \\
\hline C & 4.504403 & -8.778232 & -3.325492 \\
\hline $\mathrm{O}$ & 3.666321 & -8.836197 & -4.193943 \\
\hline$N$ & 4.393375 & -9.431857 & -2.162281 \\
\hline $\mathrm{H}$ & 5.131093 & -9.388629 & -1.491888 \\
\hline C & 3.207303 & -10.187596 & -1.834320 \\
\hline r & 2.967719 & -10.900050 & -2.609395 \\
\hline $\mathrm{H}$ & 3.399564 & -10.730343 & -0.917280 \\
\hline C & 1.974183 & -9.306851 & -1.644814 \\
\hline $\mathrm{O}$ & 0.887188 & -9.696141 & -2.004685 \\
\hline$N$ & 2.187877 & -8.107003 & -1.107171 \\
\hline C & 1.062466 & -7.184491 & -1.071036 \\
\hline C & 0.616982 & -6.720560 & -2.460413 \\
\hline $\mathrm{O}$ & -0.565457 & -6.584496 & -2.680891 \\
\hline C & 1.278265 & -5.930431 & -0.230694 \\
\hline O & 1.413888 & -6.189100 & 1.126776 \\
\hline & 3.087713 & -7.817102 & -0.798466 \\
\hline
\end{tabular}




\begin{tabular}{|c|c|c|c|}
\hline $\mathrm{H}$ & 0.211115 & -7.703913 & -0.662271 \\
\hline $\mathrm{H}$ & 2.121256 & -5.365061 & -0.613768 \\
\hline $\mathrm{H}$ & 0.384351 & -5.341622 & -0.351912 \\
\hline $\mathrm{H}$ & 2.307761 & -6.456344 & 1.293756 \\
\hline$N$ & 1.544191 & -6.431998 & -3.383126 \\
\hline C & 1.145560 & -6.024187 & -4.718096 \\
\hline C & 0.378786 & -7.146591 & -5.411039 \\
\hline $\mathrm{O}$ & -0.608559 & -6.891422 & -6.067885 \\
\hline C & 2.355968 & -5.521342 & -5.538583 \\
\hline C & 2.762317 & -4.129358 & -5.026355 \\
\hline C & 2.060425 & -5.496360 & -7.043553 \\
\hline$C$ & 4.087807 & -3.618829 & -5.589442 \\
\hline $\mathrm{H}$ & 2.514587 & -6.487425 & -3.159323 \\
\hline $\mathrm{H}$ & 0.424503 & -5.223000 & -4.638776 \\
\hline $\mathrm{H}$ & 3.176906 & -6.211370 & -5.367887 \\
\hline $\mathrm{H}$ & 1.970489 & -3.423667 & -5.272036 \\
\hline $\mathrm{H}$ & 2.836027 & -4.155457 & -3.946763 \\
\hline $\mathrm{H}$ & 2.918349 & -5.124936 & -7.589997 \\
\hline $\mathrm{H}$ & 1.841543 & -6.483228 & -7.432707 \\
\hline $\mathrm{H}$ & 1.215561 & -4.853593 & -7.270059 \\
\hline $\mathrm{H}$ & 4.381029 & -2.705502 & -5.082374 \\
\hline $\mathrm{H}$ & 4.879679 & -4.345737 & -5.439446 \\
\hline $\mathrm{H}$ & 4.030768 & -3.400397 & -6.649947 \\
\hline$N$ & 0.820049 & -8.397338 & -5.251532 \\
\hline $\mathrm{H}$ & 1.667134 & -8.572954 & -4.752448 \\
\hline C & 0.043277 & -9.504951 & -5.753923 \\
\hline $\mathrm{H}$ & -0.064069 & -9.462747 & -6.827588 \\
\hline $\mathrm{H}$ & 0.556407 & -10.421435 & $5-5.491701$ \\
\hline C & -1.365276 & -9.540103 & -5.166934 \\
\hline 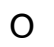 & -2.319135 & -9.771082 & -5.874320 \\
\hline
\end{tabular}




\begin{tabular}{|c|c|c|c|}
\hline$N$ & -1.472639 & -9.318869 & -3.858022 \\
\hline C & -2.776408 & -9.352422 & -3.235418 \\
\hline C & -3.692485 & -8.216308 & -3.692213 \\
\hline 0 & -4.890821 & -8.424043 & -3.732787 \\
\hline C & -2.679029 & -9.329546 & -1.702831 \\
\hline $\mathrm{O}$ & -3.923334 & -9.599034 & -1.118463 \\
\hline $\mathrm{H}$ & -0.656825 & -9.231529 & -3.289008 \\
\hline $\mathrm{H}$ & -3.292832 & -10.256969 & -3.524128 \\
\hline $\mathrm{H}$ & -1.996629 & -10.103273 & -1.379346 \\
\hline $\mathrm{H}$ & -2.290277 & -8.372770 & -1.370431 \\
\hline $\mathrm{H}$ & -4.613441 & -9.195680 & -1.630922 \\
\hline$N$ & -3.143422 & -7.035403 & -3.971064 \\
\hline C & -3.907948 & -5.914822 & -4.493113 \\
\hline C & -4.449216 & -6.233753 & -5.886715 \\
\hline $\mathrm{O}$ & -5.613112 & -6.041171 & -6.155960 \\
\hline$C$ & -3.013316 & -4.655286 & -4.488139 \\
\hline$C$ & -3.472078 & -3.518231 & -5.385380 \\
\hline$C$ & -4.792824 & -3.082106 & -5.426259 \\
\hline$C$ & -2.544447 & -2.883423 & -6.203112 \\
\hline$C$ & -5.168659 & -2.055609 & -6.276261 \\
\hline$C$ & -2.913664 & -1.844149 & -7.041965 \\
\hline$C$ & -4.233507 & -1.431282 & -7.085985 \\
\hline $\mathrm{H}$ & -2.166724 & -6.912808 & -3.802062 \\
\hline $\mathrm{H}$ & -4.776065 & -5.749068 & -3.875653 \\
\hline $\mathrm{H}$ & -2.930369 & -4.319418 & -3.458829 \\
\hline $\mathrm{H}$ & -2.021582 & -4.947355 & -4.803627 \\
\hline $\mathrm{H}$ & -5.537506 & -3.560182 & -4.818737 \\
\hline $\mathrm{H}$ & -1.519848 & -3.211204 & -6.194444 \\
\hline $\mathrm{H}$ & -6.201301 & -1.752629 & -6.312157 \\
\hline $\mathrm{H}$ & -2.174840 & -1.370521 & -7.663061 \\
\hline
\end{tabular}




\begin{tabular}{|c|c|c|c|}
\hline $\mathrm{H}$ & -4.528535 & -0.637244 & -7.749953 \\
\hline$N$ & -3.576116 & -6.724283 & -6.775034 \\
\hline C & -4.019462 & -7.062119 & -8.108086 \\
\hline C & -5.145266 & -8.094859 & -8.085430 \\
\hline 0 & -6.033051 & -8.031072 & -8.895558 \\
\hline C & -2.870758 & -7.593788 & -8.968672 \\
\hline$S$ & -1.652356 & -6.342773 & -9.485001 \\
\hline $\mathrm{H}$ & -2.634887 & -6.908353 & -6.497413 \\
\hline $\mathrm{H}$ & -4.443881 & -6.190243 & -8.582972 \\
\hline $\mathrm{H}$ & -2.366820 & -8.412362 & -8.472689 \\
\hline $\mathrm{H}$ & -3.288935 & -7.972050 & -9.891765 \\
\hline $\mathrm{H}$ & -1.047772 & -6.179428 & -8.318771 \\
\hline $\mathrm{N}$ & -5.078558 & -9.066913 & -7.155619 \\
\hline $\mathrm{H}$ & -4.254248 & -9.192308 & -6.607817 \\
\hline C & -6.085907 & -10.115215 & -7.183050 \\
\hline $\mathrm{H}$ & -6.258813 & -10.452766 & -8.193234 \\
\hline $\mathrm{H}$ & -5.714550 & -10.947558 & -6.596550 \\
\hline C & -7.459140 & -9.722570 & -6.613183 \\
\hline 0 & -8.481540 & -10.086905 & -7.126858 \\
\hline$N$ & -7.417939 & -9.016209 & -5.478356 \\
\hline C & -8.641654 & -8.653168 & -4.788765 \\
\hline C & -9.504927 & -7.658196 & -5.556657 \\
\hline $\mathrm{O}$ & -10.702666 & -7.658824 & -5.450443 \\
\hline C & -8.361797 & -8.103652 & -3.378663 \\
\hline C & -7.519565 & -6.820350 & -3.271137 \\
\hline C & -8.193200 & -5.469023 & -3.256946 \\
\hline $\mathrm{O}$ & -9.495268 & -5.469727 & -3.327708 \\
\hline $\mathrm{H}$ & -9.844193 & -4.571910 & -3.238798 \\
\hline 0 & -7.547134 & -4.455075 & -3.162024 \\
\hline $\mathrm{H}$ & -6.535785 & -8.774572 & -5.078978 \\
\hline
\end{tabular}




\begin{tabular}{|c|c|c|c|}
\hline $\mathrm{H}$ & -9.260681 & -9.533426 & -4.686412 \\
\hline $\mathrm{H}$ & -9.317927 & -7.967214 & -2.894271 \\
\hline $\mathrm{H}$ & -7.836469 & -8.883813 & -2.838322 \\
\hline $\mathrm{H}$ & -6.956763 & -6.846764 & -2.345164 \\
\hline $\mathrm{H}$ & -6.778024 & -6.766612 & -4.050597 \\
\hline$N$ & -8.837301 & -6.733962 & -6.273855 \\
\hline C & -9.555196 & -5.702617 & -7.000840 \\
\hline C & -10.386302 & -6.273665 & -8.141462 \\
\hline $\mathrm{H}$ & -10.409108 & -7.356394 & -8.248911 \\
\hline O & -10.987947 & -5.559205 & -8.887919 \\
\hline C & -8.631217 & -4.564540 & -7.490669 \\
\hline $\mathrm{O}$ & -9.434347 & -3.442192 & -7.832385 \\
\hline C & -7.723201 & -4.928742 & -8.657701 \\
\hline $\mathrm{H}$ & -7.856541 & -6.841277 & -6.421677 \\
\hline $\mathrm{H}$ & -10.268224 & -5.253622 & -6.319020 \\
\hline $\mathrm{H}$ & -8.030048 & -4.257022 & -6.646351 \\
\hline $\mathrm{H}$ & -10.110641 & -3.751079 & -8.429613 \\
\hline $\mathrm{H}$ & -7.045481 & -4.104314 & -8.851716 \\
\hline $\mathrm{H}$ & -7.131392 & -5.809814 & -8.458451 \\
\hline $\mathrm{H}$ & -8.304290 & -5.105593 & -9.556372 \\
\hline $\mathrm{N}$ & -12.952122 & -9.022524 & 3.174171 \\
\hline $\mathrm{H}$ & -13.214938 & -9.550528 & 2.367628 \\
\hline$C$ & -11.773531 & -8.180020 & 2.898781 \\
\hline$C$ & -10.789152 & -8.123178 & 4.050099 \\
\hline 0 & -9.659878 & -7.714257 & 3.857081 \\
\hline$C$ & -12.146694 & -6.722637 & 2.563055 \\
\hline 0 & -12.716169 & -6.204126 & 3.737580 \\
\hline$C$ & -13.114369 & -6.605222 & 1.390979 \\
\hline $\mathrm{H}$ & -13.731500 & -8.453468 & 3.442359 \\
\hline II & -11.222755 & -8.599619 & 2.0682 \\
\hline
\end{tabular}




\begin{tabular}{|c|c|c|c|}
\hline $\mathrm{H}$ & -11.230811 & -6.196532 & 2.328527 \\
\hline $\mathrm{H}$ & -12.850275 & -5.263293 & 3.637750 \\
\hline $\mathrm{H}$ & -13.281620 & -5.557270 & 1.166354 \\
\hline $\mathrm{H}$ & -12.728750 & -7.082400 & 0.497945 \\
\hline $\mathrm{H}$ & -14.074828 & -7.045224 & 1.631540 \\
\hline$N$ & -11.197257 & -8.533635 & 5.241900 \\
\hline $\mathrm{H}$ & -12.119322 & -8.910812 & 5.275372 \\
\hline C & -10.412532 & -8.529004 & 6.451303 \\
\hline $\mathrm{H}$ & -10.336968 & -7.533916 & 6.860733 \\
\hline $\mathrm{H}$ & -10.933725 & -9.138450 & 7.180182 \\
\hline C & -8.974764 & -9.043519 & 6.375023 \\
\hline $\mathrm{O}$ & -8.140210 & -8.478406 & 7.038097 \\
\hline$N$ & -8.658727 & -10.119661 & 5.632233 \\
\hline $\mathrm{H}$ & -9.369329 & -10.599092 & 5.127070 \\
\hline C & -7.270140 & -10.552705 & 5.532084 \\
\hline $\mathrm{H}$ & -7.253507 & -11.532129 & 5.071308 \\
\hline $\mathrm{H}$ & -6.830742 & -10.631426 & 6.513558 \\
\hline$C$ & -6.387460 & -9.608364 & 4.692694 \\
\hline 0 & -5.219627 & -9.468420 & 4.949188 \\
\hline $\mathrm{N}$ & -7.045394 & -9.018012 & 3.701158 \\
\hline$C$ & -6.302000 & -8.005885 & 2.963248 \\
\hline$C$ & -6.261921 & -6.695390 & 3.744988 \\
\hline 0 & -5.267278 & -6.009548 & 3.699390 \\
\hline$C$ & -6.611046 & -7.876391 & 1.459456 \\
\hline$C$ & -7.789973 & -7.050307 & 0.991656 \\
\hline$C$ & -7.776294 & -5.663150 & 1.107344 \\
\hline$C$ & -8.850003 & -7.647187 & 0.322326 \\
\hline$C$ & -8.803632 & -4.895981 & 0.584954 \\
\hline$C$ & -9.869113 & -6.881098 & -0.220160 \\
\hline C & -9.853576 & -5.503358 & -0.083978 \\
\hline
\end{tabular}




\begin{tabular}{|c|c|c|c|}
\hline $\mathrm{H}$ & -8.039939 & -9.013977 & 3.679635 \\
\hline $\mathrm{H}$ & -5.279281 & -8.337534 & 2.997281 \\
\hline $\mathrm{H}$ & -5.714131 & -7.456725 & 1.017445 \\
\hline $\mathrm{H}$ & -6.688005 & -8.886111 & 1.073921 \\
\hline $\mathrm{H}$ & -6.944102 & -5.173253 & 1.579317 \\
\hline $\mathrm{H}$ & -8.862559 & -8.715641 & 0.190486 \\
\hline $\mathrm{H}$ & -8.766571 & -3.824847 & 0.672310 \\
\hline $\mathrm{H}$ & -10.658922 & -7.355970 & -0.772726 \\
\hline $\mathrm{H}$ & -10.635584 & -4.909949 & -0.520432 \\
\hline$N$ & -7.292893 & -6.385747 & 4.534672 \\
\hline C & -7.221569 & -5.273489 & 5.451656 \\
\hline C & -6.102284 & -5.491033 & 6.469414 \\
\hline $\mathrm{O}$ & -5.455956 & -4.539151 & 6.852734 \\
\hline C & -8.598656 & -5.101583 & 6.124751 \\
\hline C & -8.810243 & -3.808954 & 6.883919 \\
\hline C & -8.979045 & -2.606893 & 6.201181 \\
\hline C & -8.906046 & -3.804699 & 8.268743 \\
\hline C & -9.226379 & -1.430441 & 6.886055 \\
\hline C & -9.163887 & -2.629068 & 8.960916 \\
\hline C & -9.323736 & -1.438445 & 8.271779 \\
\hline $\mathrm{H}$ & -8.158167 & -6.869402 & 4.428727 \\
\hline $\mathrm{H}$ & -6.951315 & -4.368588 & 4.926161 \\
\hline $\mathrm{H}$ & -9.342075 & -5.168088 & 5.336903 \\
\hline $\mathrm{H}$ & -8.761766 & -5.940556 & 6.787251 \\
\hline $\mathrm{H}$ & -8.925242 & -2.589088 & 5.126356 \\
\hline $\mathrm{H}$ & -8.781945 & -4.722924 & 8.815886 \\
\hline $\mathrm{H}$ & -9.345858 & -0.512282 & 6.340225 \\
\hline $\mathrm{H}$ & -9.227190 & -2.645511 & 10.034243 \\
\hline $\mathrm{H}$ & -9.506599 & -0.522727 & 8.805758 \\
\hline T. & -5.861756 & -6.728179 & 6.898228 \\
\hline
\end{tabular}




\begin{tabular}{|c|c|c|c|}
\hline $\mathrm{H}$ & -6.516417 & -7.449843 & 6.693430 \\
\hline C & -4.785610 & -7.023263 & 7.817870 \\
\hline $\mathrm{H}$ & -4.824644 & -6.393718 & 8.694135 \\
\hline $\mathrm{H}$ & -4.898019 & -8.053223 & 8.131547 \\
\hline C & -3.389823 & -6.846971 & 7.222572 \\
\hline $\mathrm{O}$ & -2.515980 & -6.352256 & 7.894544 \\
\hline$N$ & -3.189413 & -7.272080 & 5.970444 \\
\hline C & -1.911491 & -7.092406 & 5.298841 \\
\hline C & -1.665164 & -5.620520 & 4.938846 \\
\hline $\mathrm{O}$ & -0.566782 & -5.133818 & 5.107238 \\
\hline C & -1.829952 & -8.027673 & 4.072110 \\
\hline$C$ & -0.664512 & -7.670389 & 3.147846 \\
\hline C & -1.728546 & -9.487857 & 4.527528 \\
\hline $\mathrm{H}$ & -3.922668 & -7.753219 & 5.495583 \\
\hline $\mathrm{H}$ & -1.118126 & -7.338226 & 5.989758 \\
\hline $\mathrm{H}$ & -2.750581 & -7.903937 & 3.506544 \\
\hline $\mathrm{H}$ & -0.600417 & -8.397138 & 2.345039 \\
\hline $\mathrm{H}$ & -0.774998 & -6.696017 & 2.693021 \\
\hline $\mathrm{H}$ & 0.280109 & -7.682140 & 3.682962 \\
\hline $\mathrm{H}$ & -1.741719 & -10.148433 & 3.666644 \\
\hline $\mathrm{H}$ & -0.794904 & -9.654675 & 5.058852 \\
\hline $\mathrm{H}$ & -2.545387 & -9.777676 & 5.175684 \\
\hline $\mathrm{N}$ & -2.700639 & -4.933584 & 4.446697 \\
\hline$C$ & -2.569829 & -3.551378 & 4.028841 \\
\hline$C$ & -2.420113 & -2.639002 & 5.244019 \\
\hline 0 & -1.528605 & -1.820135 & 5.290629 \\
\hline$C$ & -3.758286 & -3.090364 & 3.171672 \\
\hline$C$ & -3.994034 & -3.840510 & 1.850012 \\
\hline$C$ & -2.860081 & -3.730762 & 0.845346 \\
\hline 0 & -1.747217 & -4.135874 & 1.218613 \\
\hline
\end{tabular}




\begin{tabular}{|c|c|c|c|}
\hline $\mathrm{O}$ & -3.112647 & -3.254404 & -0.273866 \\
\hline $\mathrm{H}$ & -3.564207 & -5.403285 & 4.285141 \\
\hline $\mathrm{H}$ & -1.662752 & -3.440843 & 3.465207 \\
\hline $\mathrm{H}$ & -4.667153 & -3.158040 & 3.761700 \\
\hline $\mathrm{H}$ & -3.604929 & -2.035165 & 2.961915 \\
\hline $\mathrm{H}$ & -4.149292 & -4.890266 & 2.048879 \\
\hline $\mathrm{H}$ & -4.899444 & -3.450472 & 1.399192 \\
\hline$N$ & -3.309568 & -2.774884 & 6.244298 \\
\hline C & -3.093978 & -2.024901 & 7.456985 \\
\hline C & -1.778252 & -2.422295 & 8.133804 \\
\hline O & -1.106968 & -1.582694 & 8.694212 \\
\hline C & -4.226302 & -2.004940 & 8.493910 \\
\hline O & -4.436303 & -3.246513 & 9.098559 \\
\hline C & -5.507646 & -1.387323 & 7.948154 \\
\hline $\mathrm{H}$ & -3.988197 & -3.503289 & 6.218708 \\
\hline $\mathrm{H}$ & -2.937574 & -0.995415 & 7.174780 \\
\hline $\mathrm{H}$ & -3.851323 & -1.365655 & 9.283504 \\
\hline $\mathrm{H}$ & -4.889420 & -3.818315 & 8.490671 \\
\hline $\mathrm{H}$ & -6.240042 & -1.327303 & 8.743421 \\
\hline $\mathrm{H}$ & -5.322003 & -0.384111 & 7.576784 \\
\hline $\mathrm{H}$ & -5.928460 & -1.976911 & 7.144841 \\
\hline $\mathrm{N}$ & -1.380397 & -3.700457 & 8.043328 \\
\hline $\mathrm{H}$ & -2.031177 & -4.418219 & 7.811975 \\
\hline$C$ & -0.187160 & -4.107493 & 8.739874 \\
\hline $\mathrm{H}$ & -0.237332 & -3.891849 & 9.797887 \\
\hline $\mathrm{H}$ & -0.075804 & -5.175185 & 8.604000 \\
\hline$C$ & 1.064036 & -3.420003 & 8.218010 \\
\hline 0 & 1.940520 & -3.076040 & 8.977882 \\
\hline $\mathrm{N}$ & 1.153963 & -3.258734 & 6.881772 \\
\hline C & 2.332594 & -2.660245 & 6.317782 \\
\hline
\end{tabular}




\begin{tabular}{|c|c|c|c|}
\hline C & 2.291096 & -1.138359 & 6.164142 \\
\hline $\mathrm{O}$ & 3.359963 & -0.574312 & 6.022983 \\
\hline C & 2.830820 & -3.345975 & 5.045590 \\
\hline$S$ & 1.922866 & -2.976896 & 3.520328 \\
\hline $\mathrm{H}$ & 0.422815 & -3.612332 & 6.296775 \\
\hline $\mathrm{H}$ & 3.116491 & -2.797974 & 7.047090 \\
\hline $\mathrm{H}$ & 3.840685 & -3.007650 & 4.863293 \\
\hline $\mathrm{H}$ & 2.853489 & -4.415035 & 5.205088 \\
\hline $\mathrm{H}$ & 0.859339 & -3.741312 & 3.719541 \\
\hline$N$ & 1.140119 & -0.470673 & 6.273050 \\
\hline C & 1.204068 & 0.968497 & 6.491343 \\
\hline C & 1.755726 & 1.271283 & 7.870550 \\
\hline $\mathrm{O}$ & 2.368197 & 2.289064 & 8.085588 \\
\hline C & -0.095730 & 1.741716 & 6.182264 \\
\hline C & -1.196980 & 1.568389 & 7.238506 \\
\hline C & -0.554707 & 1.440122 & 4.750694 \\
\hline C & -2.466369 & 2.370643 & 6.944548 \\
\hline $\mathrm{H}$ & 0.271804 & -0.965355 & 6.291883 \\
\hline $\mathrm{H}$ & 1.951513 & 1.351097 & 5.814811 \\
\hline $\mathrm{H}$ & 0.208520 & 2.782230 & 6.222188 \\
\hline $\mathrm{H}$ & -1.446000 & 0.523604 & 7.355738 \\
\hline $\mathrm{H}$ & -0.807789 & 1.892646 & 8.199120 \\
\hline $\mathrm{H}$ & -1.310124 & 2.147457 & 4.431579 \\
\hline $\mathrm{H}$ & 0.277973 & 1.520390 & 4.057207 \\
\hline $\mathrm{H}$ & -0.966271 & 0.444389 & 4.656851 \\
\hline $\mathrm{H}$ & -3.136044 & 2.340272 & 7.798665 \\
\hline $\mathrm{H}$ & -2.241293 & 3.414113 & 6.738921 \\
\hline $\mathrm{H}$ & -3.007065 & 1.973406 & 6.092389 \\
\hline$N$ & 1.549788 & 0.323258 & 8.791266 \\
\hline $\mathrm{H}$ & 0.889828 & -0.402217 & 8.613495 \\
\hline
\end{tabular}




\begin{tabular}{|c|c|c|c|}
\hline$C$ & 1.978276 & 0.512612 & 10.160076 \\
\hline $\mathrm{H}$ & 1.470069 & -0.222732 & 10.771000 \\
\hline $\mathrm{H}$ & 1.737711 & 1.502298 & 10.518179 \\
\hline$C$ & 3.482342 & 0.337369 & 10.305033 \\
\hline O & 4.164480 & 1.128924 & 10.896138 \\
\hline $\mathrm{N}$ & 3.999955 & -0.753863 & 9.695360 \\
\hline $\mathrm{H}$ & 3.390737 & -1.466241 & 9.351675 \\
\hline$C$ & 5.425061 & -0.938911 & 9.667405 \\
\hline $\mathrm{H}$ & 5.638287 & -1.909420 & 9.234458 \\
\hline $\mathrm{H}$ & 5.855763 & -0.904784 & 10.657461 \\
\hline$C$ & 6.156882 & 0.119117 & 8.844832 \\
\hline $\mathrm{O}$ & 7.291893 & 0.411950 & 9.129239 \\
\hline $\mathrm{N}$ & 5.502078 & 0.621285 & 7.799030 \\
\hline$C$ & 6.098617 & 1.759274 & 7.146315 \\
\hline$C$ & 5.514803 & 3.076764 & 7.649619 \\
\hline $\mathrm{H}$ & 5.013135 & 3.037215 & 8.615064 \\
\hline $\mathrm{O}$ & 5.636470 & 4.094801 & 7.040848 \\
\hline$C$ & 6.003008 & 1.674981 & 5.620777 \\
\hline$C$ & 6.755411 & 0.506853 & 5.017547 \\
\hline$C$ & 8.121035 & 0.322829 & 5.238389 \\
\hline$C$ & 6.095144 & -0.401675 & 4.195769 \\
\hline$C$ & 8.807450 & -0.722633 & 4.635688 \\
\hline$C$ & 6.779320 & -1.444771 & 3.591585 \\
\hline$C$ & 8.139375 & -1.605749 & 3.801627 \\
\hline $\mathrm{H}$ & 4.575274 & 0.334619 & 7.572613 \\
\hline $\mathrm{H}$ & 7.134062 & 1.774610 & 7.451350 \\
\hline $\mathrm{H}$ & 4.960818 & 1.610760 & 5.334266 \\
\hline $\mathrm{H}$ & 6.381340 & 2.609398 & 5.220643 \\
\hline $\mathrm{H}$ & 8.649059 & 0.978934 & 5.909006 \\
\hline $\mathrm{H}$ & 5.034983 & -0.305689 & 4.057496 \\
\hline
\end{tabular}




\begin{tabular}{|c|c|c|c|}
\hline $\mathrm{H}$ & 9.858141 & -0.849996 & 4.831622 \\
\hline $\mathrm{H}$ & 6.247195 & -2.136641 & 2.963271 \\
\hline $\mathrm{H}$ & 8.659788 & -2.418340 & 3.328667 \\
\hline$N$ & 12.613550 & 9.596234 & -1.248802 \\
\hline $\mathrm{H}$ & 11.873463 & 9.913870 & -1.846454 \\
\hline C & 12.304852 & 8.264740 & -0.719487 \\
\hline$C$ & 11.274100 & 8.450699 & 0.386872 \\
\hline $\mathrm{O}$ & 10.265804 & 7.779082 & 0.416374 \\
\hline C & 13.562726 & 7.580985 & -0.165336 \\
\hline C & 14.645038 & 7.276039 & -1.206574 \\
\hline$S$ & 14.017038 & 6.202095 & -2.529928 \\
\hline C & 15.499712 & 6.053551 & -3.555389 \\
\hline $\mathrm{H}$ & 13.433688 & 9.573142 & -1.821868 \\
\hline $\mathrm{H}$ & 11.834462 & 7.616523 & -1.447350 \\
\hline $\mathrm{H}$ & 14.001313 & 8.205395 & 0.607189 \\
\hline $\mathrm{H}$ & 13.255653 & 6.653816 & 0.304804 \\
\hline $\mathrm{H}$ & 15.045481 & 8.188485 & -1.633895 \\
\hline $\mathrm{H}$ & 15.470190 & 6.783597 & -0.704576 \\
\hline $\mathrm{H}$ & 15.248366 & 5.398907 & -4.375947 \\
\hline $\mathrm{H}$ & 15.798746 & 7.017618 & -3.946054 \\
\hline $\mathrm{H}$ & 16.318070 & 5.616646 & -2.998124 \\
\hline$N$ & 11.504281 & 9.412877 & 1.296997 \\
\hline C & 10.594285 & 9.607741 & 2.408568 \\
\hline C & 9.277001 & 10.274697 & 2.010348 \\
\hline $\mathrm{O}$ & 8.280895 & 10.053125 & 2.652572 \\
\hline C & 11.276205 & 10.369076 & 3.552095 \\
\hline C & 12.447964 & 9.564527 & 4.084498 \\
\hline $\mathrm{O}$ & 13.582765 & 9.826353 & 3.744622 \\
\hline$N$ & 12.144410 & 8.562238 & 4.918949 \\
\hline $\mathrm{H}$ & 12.328099 & 9.967716 & 1.206347 \\
\hline
\end{tabular}




\begin{tabular}{|c|c|c|c|}
\hline $\mathrm{H}$ & 10.295508 & 8.637691 & 2.771304 \\
\hline $\mathrm{H}$ & 11.657189 & 11.326324 & 3.218975 \\
\hline $\mathrm{H}$ & 10.546022 & 10.537651 & 4.333516 \\
\hline $\mathrm{H}$ & 12.888282 & 7.966933 & 5.213303 \\
\hline $\mathrm{H}$ & 11.222912 & 8.176002 & 4.954870 \\
\hline$N$ & 9.263811 & 11.067697 & 0.922328 \\
\hline $\mathrm{H}$ & 10.110810 & 11.229654 & 0.427419 \\
\hline$C$ & 8.003502 & 11.517470 & 0.368859 \\
\hline $\mathrm{H}$ & 8.201251 & 12.260241 & -0.393337 \\
\hline $\mathrm{H}$ & 7.389096 & 11.966669 & 1.131932 \\
\hline$C$ & 7.186384 & 10.384439 & -0.254324 \\
\hline $\mathrm{O}$ & 5.986288 & 10.470076 & -0.291498 \\
\hline $\mathrm{N}$ & 7.862643 & 9.333319 & -0.763272 \\
\hline$C$ & 7.170445 & 8.145634 & -1.233957 \\
\hline$C$ & 6.634883 & 7.300728 & -0.067335 \\
\hline $\mathrm{O}$ & 5.534494 & 6.808351 & -0.148247 \\
\hline$C$ & 8.048947 & 7.344296 & -2.224251 \\
\hline$C$ & 8.343686 & 8.197252 & -3.472933 \\
\hline$C$ & 7.379505 & 6.019216 & -2.608174 \\
\hline$C$ & 9.428319 & 7.625157 & -4.388995 \\
\hline $\mathrm{H}$ & 8.823869 & 9.230816 & -0.536812 \\
\hline $\mathrm{H}$ & 6.279720 & 8.471711 & -1.748418 \\
\hline $\mathrm{H}$ & 8.987987 & 7.117876 & -1.727335 \\
\hline $\mathrm{H}$ & 7.421774 & 8.323284 & -4.036274 \\
\hline $\mathrm{H}$ & 8.645788 & 9.192886 & -3.165432 \\
\hline $\mathrm{H}$ & 7.996920 & 5.478892 & -3.314375 \\
\hline $\mathrm{H}$ & 7.228376 & 5.377920 & -1.748363 \\
\hline $\mathrm{H}$ & 6.409824 & 6.182873 & -3.066713 \\
\hline $\mathrm{H}$ & 9.622358 & 8.305747 & -5.211606 \\
\hline 11 & 10.363628 & 7.479590 & -3.855310 \\
\hline
\end{tabular}




\begin{tabular}{|c|c|c|c|}
\hline $\mathrm{H}$ & 9.148450 & 6.671115 & -4.820162 \\
\hline$N$ & 7.387601 & 7.175215 & 1.043839 \\
\hline C & 6.879055 & 6.458277 & 2.201910 \\
\hline C & 5.527970 & 7.039470 & 2.639634 \\
\hline $\mathrm{O}$ & 4.575502 & 6.332974 & 2.866901 \\
\hline C & 7.833208 & 6.546127 & 3.403444 \\
\hline C & 9.254686 & 5.997273 & 3.244152 \\
\hline $\mathrm{O}$ & 10.145886 & 6.631293 & 3.802334 \\
\hline O & 9.428294 & 4.935727 & 2.595041 \\
\hline $\mathrm{H}$ & 8.347661 & 7.444953 & 1.022551 \\
\hline $\mathrm{H}$ & 6.682440 & 5.429463 & 1.943291 \\
\hline $\mathrm{H}$ & 7.921267 & 7.570736 & 3.733003 \\
\hline $\mathrm{H}$ & 7.375097 & 5.992729 & 4.220654 \\
\hline$N$ & 5.489851 & 8.377405 & 2.786069 \\
\hline $\mathrm{H}$ & 6.320917 & 8.912597 & 2.648950 \\
\hline C & 4.299225 & 9.045885 & 3.249968 \\
\hline $\mathrm{H}$ & 4.538258 & 10.090241 & 3.407632 \\
\hline $\mathrm{H}$ & 3.957292 & 8.638800 & 4.188424 \\
\hline C & 3.136629 & 8.987513 & 2.268583 \\
\hline $\mathrm{O}$ & 1.995144 & 9.109701 & 2.649473 \\
\hline$N$ & 3.470176 & 8.824775 & 0.985192 \\
\hline $\mathrm{H}$ & 4.431315 & 8.829449 & 0.735279 \\
\hline C & 2.517191 & 8.905260 & -0.078305 \\
\hline $\mathrm{H}$ & 3.059863 & 9.091715 & -0.996305 \\
\hline $\mathrm{H}$ & 1.843943 & 9.738046 & 0.071914 \\
\hline C & 1.609133 & 7.687020 & -0.310194 \\
\hline $\mathrm{O}$ & 0.561833 & 7.815066 & -0.879403 \\
\hline$N$ & 2.094474 & 6.538092 & 0.201332 \\
\hline C & 1.289982 & 5.364375 & 0.431021 \\
\hline$C$ & 0.472387 & 5.479549 & 1.721794 \\
\hline
\end{tabular}




\begin{tabular}{|c|c|c|c|}
\hline $\mathrm{O}$ & -0.722708 & 5.301804 & 1.692203 \\
\hline C & 2.166758 & 4.112402 & 0.445792 \\
\hline $\mathrm{H}$ & 3.001528 & 6.575688 & 0.615931 \\
\hline $\mathrm{H}$ & 0.559552 & 5.290356 & -0.357506 \\
\hline $\mathrm{H}$ & 1.566520 & 3.234004 & 0.657550 \\
\hline $\mathrm{H}$ & 2.636496 & 3.991476 & -0.523448 \\
\hline $\mathrm{H}$ & 2.946380 & 4.184428 & 1.196930 \\
\hline$N$ & 1.102514 & 5.751803 & 2.879294 \\
\hline C & 0.364292 & 5.585250 & 4.123057 \\
\hline C & -0.756432 & 6.611697 & 4.274013 \\
\hline $\mathrm{O}$ & -1.792392 & 6.302325 & 4.823364 \\
\hline C & 1.276011 & 5.511847 & 5.372732 \\
\hline C & 1.867809 & 6.857295 & 5.819745 \\
\hline C & 2.362801 & 4.445288 & 5.169506 \\
\hline C & 2.698994 & 6.761005 & 7.101577 \\
\hline $\mathrm{H}$ & 2.090092 & 5.889616 & 2.900944 \\
\hline $\mathrm{H}$ & -0.159830 & 4.642184 & 4.064825 \\
\hline $\mathrm{H}$ & 0.615200 & 5.173522 & 6.165183 \\
\hline $\mathrm{H}$ & 2.478118 & 7.266410 & 5.021408 \\
\hline $\mathrm{H}$ & 1.064424 & 7.567408 & 5.986930 \\
\hline $\mathrm{H}$ & 2.732516 & 4.092368 & 6.121037 \\
\hline $\mathrm{H}$ & 1.969615 & 3.588567 & 4.631398 \\
\hline $\mathrm{H}$ & 3.205855 & 4.834276 & 4.608937 \\
\hline $\mathrm{H}$ & 3.004293 & 7.751938 & 7.424490 \\
\hline $\mathrm{H}$ & 2.121237 & 6.317646 & 7.907208 \\
\hline $\mathrm{H}$ & 3.593843 & 6.165183 & 6.971697 \\
\hline$N$ & -0.542960 & 7.847390 & 3.800966 \\
\hline $\mathrm{H}$ & 0.341472 & 8.089930 & 3.404868 \\
\hline C & -1.513857 & 8.887768 & 4.018661 \\
\hline $\mathrm{H}$ & -1.103669 & 9.816493 & 3.642539 \\
\hline
\end{tabular}




\begin{tabular}{|c|c|c|c|}
\hline $\mathrm{H}$ & -1.724196 & 9.016468 & 5.070588 \\
\hline C & -2.866972 & 8.618762 & 3.365891 \\
\hline $\mathrm{O}$ & -3.871073 & 8.837212 & 4.003256 \\
\hline$N$ & -2.902689 & 8.108966 & 2.120757 \\
\hline C & -4.239977 & 8.013753 & 1.535301 \\
\hline C & -4.984715 & 6.749522 & 1.927679 \\
\hline $\mathrm{O}$ & -6.195865 & 6.757018 & 1.919701 \\
\hline C & -4.021961 & 8.221377 & 0.030789 \\
\hline C & -2.766351 & 9.099777 & 0.020009 \\
\hline C & -1.907226 & 8.456184 & 1.096092 \\
\hline $\mathrm{H}$ & -4.854248 & 8.814662 & 1.917299 \\
\hline $\mathrm{H}$ & -3.842397 & 7.278855 & -0.469760 \\
\hline $\mathrm{H}$ & -4.886376 & 8.684666 & -0.427231 \\
\hline $\mathrm{H}$ & -2.255711 & 9.142462 & -0.923535 \\
\hline $\mathrm{H}$ & -3.027230 & 10.114461 & 0.311403 \\
\hline $\mathrm{H}$ & -1.420606 & 7.566072 & 0.740457 \\
\hline $\mathrm{H}$ & -1.151144 & 9.119009 & 1.484459 \\
\hline$N$ & -4.269046 & 5.673779 & 2.291121 \\
\hline C & -4.938190 & 4.512574 & 2.850520 \\
\hline C & -5.638483 & 4.853566 & 4.168965 \\
\hline $\mathrm{O}$ & -6.741401 & 4.413645 & 4.408446 \\
\hline C & -3.973826 & 3.331470 & 3.086429 \\
\hline C & -3.635815 & 2.505644 & 1.861076 \\
\hline C & -4.332918 & 1.334795 & 1.576597 \\
\hline C & -2.597742 & 2.864590 & 1.013744 \\
\hline C & -4.007257 & 0.547987 & 0.482013 \\
\hline C & -2.270778 & 2.094060 & -0.087910 \\
\hline C & -2.970648 & 0.931871 & -0.361397 \\
\hline O & -2.617894 & 0.205846 & -1.447897 \\
\hline & -3.414708 & -0.117636 & -1.907891 \\
\hline
\end{tabular}




\begin{tabular}{|c|c|c|c|}
\hline $\mathrm{H}$ & -3.275163 & 5.750834 & 2.345475 \\
\hline $\mathrm{H}$ & -5.725584 & 4.209370 & 2.178854 \\
\hline $\mathrm{H}$ & -3.068141 & 3.711073 & 3.548038 \\
\hline $\mathrm{H}$ & -4.443505 & 2.682244 & 3.816470 \\
\hline $\mathrm{H}$ & -5.141527 & 1.028394 & 2.216937 \\
\hline $\mathrm{H}$ & -2.032818 & 3.752495 & 1.204511 \\
\hline $\mathrm{H}$ & -4.536059 & -0.366907 & 0.289642 \\
\hline $\mathrm{H}$ & -1.479397 & 2.397067 & -0.750378 \\
\hline$N$ & -4.970841 & 5.618292 & 5.048293 \\
\hline $\mathrm{H}$ & -4.034958 & 5.911381 & 4.856514 \\
\hline C & -5.582761 & 6.005336 & 6.299177 \\
\hline $\mathrm{H}$ & -4.805951 & 6.385456 & 6.951028 \\
\hline $\mathrm{H}$ & -6.052296 & 5.158934 & 6.774233 \\
\hline C & -6.651500 & 7.084436 & 6.135928 \\
\hline O & -7.706439 & 7.027961 & 6.726190 \\
\hline$N$ & -6.342147 & 8.091247 & 5.313906 \\
\hline $\mathrm{H}$ & -5.445011 & 8.139362 & 4.880103 \\
\hline C & -7.257687 & 9.220267 & 5.085304 \\
\hline $\mathrm{H}$ & -6.781273 & 9.871884 & 4.364233 \\
\hline $\mathrm{H}$ & -7.419732 & 9.775731 & 5.997427 \\
\hline C & -8.632474 & 8.840758 & 4.569496 \\
\hline $\mathrm{O}$ & -9.611456 & 9.429009 & 4.959389 \\
\hline$N$ & -8.690353 & 7.854323 & 3.658142 \\
\hline C & -9.953936 & 7.451763 & 3.088772 \\
\hline C & -10.766367 & 6.542276 & 3.995474 \\
\hline $\mathrm{O}$ & -11.807718 & 6.069059 & 3.559312 \\
\hline C & -9.761225 & 6.816107 & 1.689871 \\
\hline O & -10.954474 & 6.903389 & 0.953235 \\
\hline C & -9.239336 & 5.382290 & 1.729344 \\
\hline 1 & -7.846104 & 7.427544 & 3.334380 \\
\hline
\end{tabular}




\begin{tabular}{|c|c|c|c|}
\hline $\mathrm{H}$ & -10.571605 & 8.331054 & 2.962637 \\
\hline $\mathrm{H}$ & -9.046138 & 7.439464 & 1.169803 \\
\hline $\mathrm{H}$ & -11.641616 & 6.500567 & 1.473253 \\
\hline $\mathrm{H}$ & -8.968511 & 5.061323 & 0.731363 \\
\hline $\mathrm{H}$ & -8.362386 & 5.288810 & 2.354441 \\
\hline $\mathrm{H}$ & -10.001049 & 4.705882 & 2.098831 \\
\hline$N$ & -10.353337 & 6.327943 & 5.240900 \\
\hline C & -11.259067 & 5.732432 & 6.212920 \\
\hline C & -12.445055 & 6.655970 & 6.501978 \\
\hline $\mathrm{O}$ & -13.529692 & 6.188238 & 6.758346 \\
\hline C & -10.538004 & 5.397364 & 7.526365 \\
\hline$C$ & -9.534250 & 4.235087 & 7.447170 \\
\hline C & -8.716902 & 4.184513 & 8.741571 \\
\hline C & -10.219887 & 2.887550 & 7.196651 \\
\hline $\mathrm{H}$ & -9.462967 & 6.657426 & 5.550927 \\
\hline $\mathrm{H}$ & -11.696982 & 4.842273 & 5.794089 \\
\hline $\mathrm{H}$ & -10.028467 & 6.287234 & 7.878934 \\
\hline $\mathrm{H}$ & -11.302465 & 5.156738 & 8.260029 \\
\hline $\mathrm{H}$ & -8.847363 & 4.423679 & 6.629000 \\
\hline $\mathrm{H}$ & -7.988780 & 3.378765 & 8.709922 \\
\hline $\mathrm{H}$ & -8.181943 & 5.114221 & 8.898199 \\
\hline $\mathrm{H}$ & -9.358073 & 4.014626 & 9.603746 \\
\hline $\mathrm{H}$ & -9.479693 & 2.092166 & 7.178789 \\
\hline $\mathrm{H}$ & -10.931085 & 2.662089 & 7.989148 \\
\hline $\mathrm{H}$ & -10.750009 & 2.858102 & 6.251782 \\
\hline$N$ & -12.200522 & 7.978949 & 6.518744 \\
\hline C & -13.273039 & 8.917896 & 6.674532 \\
\hline C & -14.049258 & 9.175384 & 5.400083 \\
\hline $\mathrm{H}$ & -14.889087 & 9.868465 & 5.516222 \\
\hline 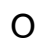 & -13.795693 & 8.696578 & 4.344326 \\
\hline
\end{tabular}




\begin{tabular}{|c|c|c|c|}
\hline $\mathrm{H}$ & -11.339629 & 8.323361 & 6.149409 \\
\hline $\mathrm{H}$ & -13.965271 & 8.567023 & 7.429623 \\
\hline $\mathrm{H}$ & -12.873459 & 9.866487 & 7.019929 \\
\hline$N$ & -11.399969 & 10.603551 & -3.013216 \\
\hline $\mathrm{H}$ & -11.150848 & 10.793386 & -2.062913 \\
\hline C & -11.816457 & 9.206991 & -3.172692 \\
\hline C & -11.420851 & 8.859935 & -4.600828 \\
\hline $\mathrm{O}$ & -10.381233 & 8.297434 & -4.846936 \\
\hline C & -13.274734 & 8.886640 & -2.781030 \\
\hline C & -13.514063 & 9.244538 & -1.302422 \\
\hline$C$ & -13.600995 & 7.414363 & -3.063721 \\
\hline C & -14.953793 & 9.046013 & -0.824864 \\
\hline $\mathrm{H}$ & -12.140699 & 11.235339 & -3.252888 \\
\hline $\mathrm{H}$ & -11.162961 & 8.606955 & -2.555415 \\
\hline $\mathrm{H}$ & -13.942021 & 9.511372 & -3.379459 \\
\hline $\mathrm{H}$ & -12.841528 & 8.656467 & -0.682305 \\
\hline $\mathrm{H}$ & -13.250014 & 10.285347 & -1.142897 \\
\hline $\mathrm{H}$ & -14.652333 & 7.209527 & -2.907474 \\
\hline $\mathrm{H}$ & -13.370472 & 7.129681 & -4.086153 \\
\hline $\mathrm{H}$ & -13.034591 & 6.765561 & -2.402933 \\
\hline $\mathrm{H}$ & -15.063023 & 9.412864 & 0.189451 \\
\hline $\mathrm{H}$ & -15.657185 & 9.588738 & -1.450951 \\
\hline $\mathrm{H}$ & -15.244933 & 8.002168 & -0.821506 \\
\hline$N$ & -12.217787 & 9.294727 & -5.606272 \\
\hline $\mathrm{H}$ & -13.139288 & 9.595964 & -5.384742 \\
\hline C & -11.972872 & 8.843880 & -6.959258 \\
\hline $\mathrm{H}$ & -12.033869 & 7.767144 & -7.039983 \\
\hline $\mathrm{H}$ & -12.729040 & 9.278162 & -7.601904 \\
\hline C & -10.601635 & 9.233244 & -7.517693 \\
\hline $\mathrm{O}$ & -9.996993 & 8.496745 & $-8.24157 \varepsilon$ \\
\hline
\end{tabular}




\begin{tabular}{|c|c|c|c|}
\hline$N$ & -10.162849 & 10.477782 & -7.182627 \\
\hline $\mathrm{H}$ & -10.710967 & 11.007726 & -6.544640 \\
\hline C & -8.894341 & 11.023967 & -7.637100 \\
\hline $\mathrm{H}$ & -8.789701 & 10.891253 & -8.702753 \\
\hline $\mathrm{H}$ & -8.878587 & 12.081461 & -7.407588 \\
\hline C & -7.672564 & 10.359017 & -6.988311 \\
\hline $\mathrm{O}$ & -6.753288 & 9.935059 & -7.630848 \\
\hline$N$ & -7.659101 & 10.352152 & -5.619641 \\
\hline $\mathrm{H}$ & -8.482401 & 10.628144 & -5.133559 \\
\hline C & -6.495253 & 9.961264 & -4.832348 \\
\hline $\mathrm{H}$ & -5.599190 & 10.377760 & -5.264327 \\
\hline $\mathrm{H}$ & -6.616272 & 10.359353 & -3.832338 \\
\hline C & -6.268292 & 8.463912 & -4.714277 \\
\hline $\mathrm{O}$ & -5.133741 & 8.047355 & -4.711552 \\
\hline$N$ & -7.321035 & 7.642772 & -4.555883 \\
\hline C & -7.043209 & 6.284748 & -4.116210 \\
\hline C & -6.395979 & 5.449108 & -5.213283 \\
\hline $\mathrm{O}$ & -5.518963 & 4.674302 & -4.918169 \\
\hline C & -8.232873 & 5.569489 & -3.436346 \\
\hline C & -9.368536 & 5.183245 & -4.399222 \\
\hline C & -8.697051 & 6.392810 & -2.229037 \\
\hline C & -10.549916 & 4.494220 & -3.715501 \\
\hline $\mathrm{H}$ & -8.255734 & 7.997394 & -4.570135 \\
\hline $\mathrm{H}$ & -6.261740 & 6.342785 & -3.373841 \\
\hline $\mathrm{H}$ & -7.807333 & 4.647372 & -3.048883 \\
\hline $\mathrm{H}$ & -9.726245 & 6.052590 & -4.935411 \\
\hline $\mathrm{H}$ & -8.960384 & 4.506207 & -5.145609 \\
\hline $\mathrm{H}$ & -9.400969 & 5.838945 & -1.623718 \\
\hline $\mathrm{H}$ & -7.854514 & 6.643390 & -1.592420 \\
\hline $\mathrm{H}$ & -9.177968 & 7.313947 & -2.53328 \\
\hline
\end{tabular}




\begin{tabular}{|c|c|c|c|}
\hline $\mathrm{H}$ & -11.255488 & 4.128223 & -4.455611 \\
\hline $\mathrm{H}$ & -10.227310 & 3.646433 & -3.122056 \\
\hline $\mathrm{H}$ & -11.086931 & 5.173571 & -3.065645 \\
\hline$N$ & -6.814696 & 5.564734 & -6.493518 \\
\hline C & -6.106090 & 4.822427 & -7.516751 \\
\hline C & -4.760271 & 5.444493 & -7.899241 \\
\hline O & -3.890185 & 4.715921 & -8.316589 \\
\hline C & -6.966879 & 4.499655 & -8.746711 \\
\hline C & -8.036858 & 3.417976 & -8.510429 \\
\hline C & -7.450679 & 2.076806 & -8.041102 \\
\hline$N$ & -8.388348 & 0.950372 & -8.074819 \\
\hline C & -9.198500 & 0.573081 & -7.082223 \\
\hline$N$ & -9.461714 & 1.417389 & -6.096291 \\
\hline$N$ & -9.735184 & -0.627041 & -7.081494 \\
\hline $\mathrm{H}$ & -7.489226 & 6.258287 & -6.735688 \\
\hline $\mathrm{H}$ & -5.795827 & 3.898635 & -7.061643 \\
\hline $\mathrm{H}$ & -7.450821 & 5.399941 & -9.113901 \\
\hline $\mathrm{H}$ & -6.295934 & 4.164635 & -9.530188 \\
\hline $\mathrm{H}$ & -8.777741 & 3.779653 & -7.803319 \\
\hline $\mathrm{H}$ & -8.564457 & 3.255563 & -9.444503 \\
\hline $\mathrm{H}$ & -6.625621 & 1.798488 & -8.683815 \\
\hline $\mathrm{H}$ & -7.050857 & 2.146512 & -7.038324 \\
\hline $\mathrm{H}$ & -8.318670 & 0.320537 & -8.843177 \\
\hline $\mathrm{H}$ & -9.209212 & 2.374113 & -6.170856 \\
\hline $\mathrm{H}$ & -9.975771 & 1.152008 & -5.272487 \\
\hline $\mathrm{H}$ & -9.386628 & -1.392606 & -7.629608 \\
\hline $\mathrm{H}$ & -10.455373 & -0.868109 & -6.419059 \\
\hline $\mathrm{N}$ & -4.571383 & 6.757521 & -7.721942 \\
\hline $\mathrm{H}$ & -5.312315 & 7.350933 & -7.425900 \\
\hline C & -3.257811 & 7.352384 & -8.011367 \\
\hline
\end{tabular}




\begin{tabular}{|c|c|c|c|}
\hline $\mathrm{H}$ & -2.933574 & 7.093301 & -9.006671 \\
\hline $\mathrm{H}$ & -3.359217 & 8.427153 & -7.938226 \\
\hline$C$ & -2.194224 & 6.878592 & -7.032969 \\
\hline $\mathrm{O}$ & -1.067347 & 6.644049 & -7.392535 \\
\hline $\mathrm{N}$ & -2.610525 & 6.772889 & -5.765796 \\
\hline$C$ & -1.723015 & 6.464254 & -4.671163 \\
\hline$C$ & -1.444922 & 4.978007 & -4.544804 \\
\hline $\mathrm{O}$ & -0.316679 & 4.592094 & -4.340327 \\
\hline$C$ & -2.307517 & 7.057597 & -3.382763 \\
\hline$C$ & -1.724815 & 6.352660 & -2.158849 \\
\hline$C$ & -2.065393 & 8.573596 & -3.416044 \\
\hline $\mathrm{H}$ & -3.535125 & 7.069357 & -5.539459 \\
\hline $\mathrm{H}$ & -0.755120 & 6.905332 & -4.861635 \\
\hline $\mathrm{H}$ & -3.376238 & 6.879910 & -3.397953 \\
\hline $\mathrm{H}$ & -1.939327 & 6.893010 & -1.256063 \\
\hline $\mathrm{H}$ & -2.140827 & 5.355759 & -2.055908 \\
\hline $\mathrm{H}$ & -0.652629 & 6.275441 & -2.228523 \\
\hline $\mathrm{H}$ & -2.802863 & 9.103968 & -2.830568 \\
\hline $\mathrm{H}$ & -1.077849 & 8.814563 & -3.037015 \\
\hline $\mathrm{H}$ & -2.144576 & 8.956417 & -4.426975 \\
\hline $\mathrm{N}$ & -2.474279 & 4.124764 & -4.663732 \\
\hline$C$ & -2.164153 & 2.717524 & -4.734152 \\
\hline$C$ & -1.265570 & 2.444041 & -5.932826 \\
\hline O & -0.396160 & 1.606643 & -5.875480 \\
\hline$C$ & -3.408746 & 1.806689 & -4.774910 \\
\hline$C$ & -4.360548 & 1.970537 & -3.579979 \\
\hline$C$ & -5.448860 & 0.884498 & -3.485581 \\
\hline $\mathrm{N}$ & -5.007994 & -0.292803 & -2.757323 \\
\hline$C$ & -5.828029 & -1.254631 & -2.564076 \\
\hline $\mathrm{N}$ & -7.132262 & -1.285043 & -3.042009 \\
\hline
\end{tabular}




\begin{tabular}{|c|c|c|c|}
\hline$N$ & -5.529317 & -2.307063 & -1.768386 \\
\hline $\mathrm{H}$ & -3.401452 & 4.451776 & -4.832759 \\
\hline $\mathrm{H}$ & -1.576330 & 2.442308 & -3.871014 \\
\hline $\mathrm{H}$ & -3.956468 & 1.985261 & -5.698705 \\
\hline $\mathrm{H}$ & -3.044992 & 0.786285 & -4.822080 \\
\hline $\mathrm{H}$ & -3.794850 & 1.985348 & -2.660536 \\
\hline $\mathrm{H}$ & -4.853445 & 2.927338 & -3.654639 \\
\hline $\mathrm{H}$ & -6.318442 & 1.325235 & -2.997340 \\
\hline $\mathrm{H}$ & -5.765388 & 0.631232 & -4.501235 \\
\hline $\mathrm{H}$ & -7.269977 & -0.782391 & -3.890775 \\
\hline $\mathrm{H}$ & -7.547087 & -2.198151 & -3.082629 \\
\hline $\mathrm{H}$ & -4.606193 & -2.417209 & -1.394936 \\
\hline $\mathrm{H}$ & -6.033447 & -3.156481 & -1.906178 \\
\hline$N$ & -1.459759 & 3.193337 & -7.035104 \\
\hline $\mathrm{H}$ & -2.264461 & 3.769652 & -7.144117 \\
\hline C & -0.595841 & 2.983570 & -8.179101 \\
\hline $\mathrm{H}$ & -0.940101 & 3.626229 & -8.979236 \\
\hline $\mathrm{H}$ & -0.614301 & 1.957456 & -8.516738 \\
\hline C & 0.858326 & 3.326035 & -7.861317 \\
\hline $\mathrm{O}$ & 1.786260 & 2.682485 & -8.284293 \\
\hline$N$ & 1.007445 & 4.395989 & -7.064151 \\
\hline $\mathrm{H}$ & 0.213483 & 4.959514 & -6.860698 \\
\hline C & 2.282309 & 4.880648 & -6.640293 \\
\hline $\mathrm{H}$ & 2.918466 & 5.143119 & -7.476200 \\
\hline $\mathrm{H}$ & 2.121619 & 5.773634 & -6.050398 \\
\hline C & 3.094242 & 3.926519 & -5.780065 \\
\hline $\mathrm{O}$ & 4.300265 & 4.045735 & -5.701433 \\
\hline$N$ & 2.428061 & 2.983367 & -5.101940 \\
\hline C & 3.101620 & 2.134935 & -4.154869 \\
\hline C & 4.282205 & 1.343779 & -4.722365 \\
\hline
\end{tabular}




\begin{tabular}{|c|c|c|c|}
\hline $\mathrm{O}$ & 5.201482 & 1.052542 & -3.983650 \\
\hline C & 2.031171 & 1.219401 & -3.563977 \\
\hline C & 2.428250 & 0.354637 & -2.373360 \\
\hline C & 1.133384 & -0.209485 & -1.811071 \\
\hline$N$ & 1.349158 & -1.211023 & -0.786673 \\
\hline C & 0.367638 & -2.053383 & -0.419188 \\
\hline$N$ & -0.791056 & -2.021281 & -1.034301 \\
\hline$N$ & 0.596532 & -2.937674 & 0.523852 \\
\hline $\mathrm{H}$ & 1.433630 & 2.938723 & -5.174896 \\
\hline $\mathrm{H}$ & 3.548660 & 2.735576 & -3.371638 \\
\hline $\mathrm{H}$ & 1.222627 & 1.874467 & -3.257090 \\
\hline $\mathrm{H}$ & 1.623761 & 0.598106 & -4.353093 \\
\hline $\mathrm{H}$ & 3.090803 & -0.451472 & -2.668464 \\
\hline $\mathrm{H}$ & 2.949613 & 0.944398 & -1.622858 \\
\hline $\mathrm{H}$ & 0.514109 & 0.599037 & -1.434189 \\
\hline $\mathrm{H}$ & 0.590668 & -0.683203 & -2.616428 \\
\hline $\mathrm{H}$ & 2.061201 & -1.044576 & -0.110880 \\
\hline $\mathrm{H}$ & -1.129890 & -1.169180 & -1.431149 \\
\hline $\mathrm{H}$ & -1.541493 & -2.653972 & -0.762209 \\
\hline $\mathrm{H}$ & 1.513554 & -3.109966 & 0.865333 \\
\hline $\mathrm{H}$ & -0.183117 & -3.502400 & 0.855326 \\
\hline$N$ & 4.281487 & 0.944899 & -6.004135 \\
\hline $\mathrm{H}$ & 3.540425 & 1.225017 & -6.613436 \\
\hline C & 5.363433 & 0.116651 & -6.504612 \\
\hline $\mathrm{H}$ & 5.138940 & -0.142416 & -7.531832 \\
\hline $\mathrm{H}$ & 5.441318 & -0.797906 & -5.938283 \\
\hline C & 6.736189 & 0.756137 & -6.475992 \\
\hline $\mathrm{O}$ & 7.738501 & 0.089706 & -6.364641 \\
\hline$N$ & 6.766107 & 2.085332 & -6.608300 \\
\hline $\mathrm{H}$ & 5.912164 & 2.604987 & -6.573201 \\
\hline
\end{tabular}




\begin{tabular}{|c|c|c|c|}
\hline C & 8.005490 & 2.823550 & -6.551058 \\
\hline $\mathrm{H}$ & 8.709874 & 2.439443 & -7.275292 \\
\hline $\mathrm{H}$ & 7.800317 & 3.854257 & -6.807692 \\
\hline C & 8.748767 & 2.826982 & -5.213265 \\
\hline $\mathrm{O}$ & 9.889900 & 3.236396 & -5.161819 \\
\hline$N$ & 8.104020 & 2.338761 & -4.145791 \\
\hline C & 8.686068 & 2.174994 & -2.824665 \\
\hline C & 9.950624 & 1.305640 & -2.832450 \\
\hline O & 10.769444 & 1.388760 & -1.931350 \\
\hline C & 7.582249 & 1.508360 & -1.973451 \\
\hline C & 7.781315 & 1.384520 & -0.462949 \\
\hline C & 7.442009 & 2.654382 & 0.326956 \\
\hline C & 7.332442 & 2.387275 & 1.824347 \\
\hline$N$ & 8.672831 & 2.353850 & 2.481175 \\
\hline $\mathrm{O}$ & 11.043759 & 1.617662 & 0.892575 \\
\hline $\mathrm{H}$ & 10.893507 & 1.828893 & -0.029265 \\
\hline $\mathrm{H}$ & 11.522402 & 0.791091 & 0.841458 \\
\hline $\mathrm{H}$ & 7.158111 & 2.035733 & -4.258492 \\
\hline $\mathrm{H}$ & 8.985609 & 3.132478 & -2.419373 \\
\hline $\mathrm{H}$ & 6.666397 & 2.054216 & -2.156485 \\
\hline $\mathrm{H}$ & 7.412186 & 0.521668 & -2.388594 \\
\hline $\mathrm{H}$ & 7.101435 & 0.605609 & -0.131487 \\
\hline $\mathrm{H}$ & 8.776633 & 1.030649 & -0.229089 \\
\hline $\mathrm{H}$ & 8.155686 & 3.454433 & 0.151271 \\
\hline $\mathrm{H}$ & 6.477032 & 3.027453 & -0.000996 \\
\hline $\mathrm{H}$ & 6.772474 & 3.159663 & 2.328139 \\
\hline $\mathrm{H}$ & 6.851243 & 1.441279 & 2.019126 \\
\hline $\mathrm{H}$ & 9.383412 & 1.890053 & 1.923632 \\
\hline $\mathrm{H}$ & 8.992797 & 3.324044 & 2.635486 \\
\hline $\mathrm{H}$ & 8.619521 & 1.875958 & 3.368834 \\
\hline
\end{tabular}




\begin{tabular}{|c|c|c|c|}
\hline$N$ & 10.062466 & 0.448215 & -3.837530 \\
\hline C & 11.109254 & -0.537626 & -4.069489 \\
\hline C & 12.486198 & 0.023151 & -4.427404 \\
\hline $\mathrm{O}$ & 13.443814 & -0.705721 & -4.301297 \\
\hline C & 10.700747 & -1.444010 & -5.252697 \\
\hline C & 9.562911 & -2.442684 & -4.997425 \\
\hline C & 9.130917 & -3.067132 & -6.327922 \\
\hline C & 9.990732 & -3.533414 & -4.015314 \\
\hline $\mathrm{H}$ & 9.324095 & 0.446353 & -4.510534 \\
\hline $\mathrm{H}$ & 11.264786 & -1.125192 & -3.176512 \\
\hline $\mathrm{H}$ & 10.433793 & -0.798369 & -6.084028 \\
\hline $\mathrm{H}$ & 11.583581 & -1.996252 & -5.551753 \\
\hline $\mathrm{H}$ & 8.706525 & -1.911855 & -4.593193 \\
\hline $\mathrm{H}$ & 8.329833 & -3.783547 & -6.173252 \\
\hline $\mathrm{H}$ & 8.773306 & -2.310222 & -7.014278 \\
\hline $\mathrm{H}$ & 9.957889 & -3.595023 & -6.796351 \\
\hline $\mathrm{H}$ & 9.201125 & -4.263650 & -3.875431 \\
\hline $\mathrm{H}$ & 10.860862 & -4.059408 & -4.394208 \\
\hline $\mathrm{H}$ & 10.243855 & -3.136731 & -3.036861 \\
\hline $\mathrm{N}$ & 12.574050 & 1.261829 & -4.919244 \\
\hline$C$ & 13.814631 & 1.844748 & -5.393664 \\
\hline$C$ & 14.958652 & 2.033747 & -4.379133 \\
\hline 0 & 16.013009 & 2.415248 & -4.811960 \\
\hline$C$ & 13.497155 & 3.171312 & -6.087132 \\
\hline 0 & 12.917856 & 4.088294 & -5.202925 \\
\hline $\mathrm{H}$ & 11.756474 & 1.833638 & -4.917124 \\
\hline $\mathrm{H}$ & 14.248647 & 1.177688 & -6.129059 \\
\hline $\mathrm{H}$ & 14.420302 & 3.590289 & -6.455810 \\
\hline $\mathrm{H}$ & 12.846475 & 2.977264 & -6.933908 \\
\hline $\mathrm{H}$ & 11.970501 & 4.079249 & -5.267949 \\
\hline
\end{tabular}




\begin{tabular}{|c|c|c|c|}
\hline$N$ & 14.792295 & 1.722326 & -3.074279 \\
\hline C & 15.928389 & 1.647509 & -2.176950 \\
\hline C & 16.804567 & 0.409888 & -2.402431 \\
\hline O & 17.934351 & 0.393805 & -1.987788 \\
\hline C & 15.495714 & 1.578526 & -0.707339 \\
\hline C & 14.639621 & 2.736041 & -0.198122 \\
\hline C & 14.286898 & 2.490214 & 1.269214 \\
\hline$N$ & 13.261669 & 3.385117 & 1.791185 \\
\hline C & 13.439234 & 4.535908 & 2.419308 \\
\hline$N$ & 14.656891 & 5.011309 & 2.656558 \\
\hline $\mathrm{O}$ & 15.028868 & 7.467212 & 4.211320 \\
\hline $\mathrm{H}$ & 14.745472 & 8.353707 & 3.972084 \\
\hline $\mathrm{H}$ & 15.747678 & 7.573264 & 4.821449 \\
\hline$N$ & 12.389203 & 5.224410 & 2.815850 \\
\hline $\mathrm{H}$ & 13.901122 & 1.419326 & -2.758405 \\
\hline $\mathrm{H}$ & 16.562104 & 2.504313 & -2.346960 \\
\hline $\mathrm{H}$ & 14.954936 & 0.650825 & -0.551362 \\
\hline $\mathrm{H}$ & 16.407795 & 1.509207 & -0.124733 \\
\hline $\mathrm{H}$ & 15.157465 & 3.683640 & -0.315118 \\
\hline $\mathrm{H}$ & 13.720191 & 2.817208 & -0.766111 \\
\hline $\mathrm{H}$ & 13.890572 & 1.490897 & 1.376863 \\
\hline $\mathrm{H}$ & 15.166566 & 2.533534 & 1.900576 \\
\hline $\mathrm{H}$ & 12.321594 & 3.089418 & 1.619820 \\
\hline $\mathrm{H}$ & 15.464852 & 4.518742 & 2.358635 \\
\hline $\mathrm{H}$ & 14.792087 & 5.866171 & 3.168376 \\
\hline $\mathrm{H}$ & 11.433766 & 4.944541 & 2.657797 \\
\hline $\mathrm{H}$ & 12.481544 & 6.067381 & 3.336224 \\
\hline$N$ & 16.200238 & -0.648980 & -2.967241 \\
\hline C & 16.870746 & -1.911547 & -3.168273 \\
\hline 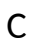 & 17.669182 & -1.889188 & -4.468289 \\
\hline
\end{tabular}




\begin{tabular}{|c|c|c|c|}
\hline $\mathrm{H}$ & 18.064101 & -0.909799 & -4.747558 \\
\hline $\mathrm{O}$ & 17.874238 & -2.852596 & -5.131737 \\
\hline C & 15.867974 & -3.070182 & -3.149443 \\
\hline C & 15.324792 & -3.399919 & -1.791907 \\
\hline$N$ & 14.403950 & -2.627679 & -1.111941 \\
\hline 0 & 12.508282 & -0.434546 & -0.465242 \\
\hline $\mathrm{H}$ & 13.146230 & -1.037812 & -0.850717 \\
\hline $\mathrm{H}$ & 12.028284 & -0.039090 & -1.184792 \\
\hline C & 15.620109 & -4.490418 & -1.049700 \\
\hline C & 14.173035 & -3.251908 & -0.003455 \\
\hline$N$ & 14.883490 & -4.381979 & 0.103203 \\
\hline $\mathrm{H}$ & 15.285820 & -0.550151 & -3.353081 \\
\hline $\mathrm{H}$ & 17.596166 & -2.030815 & -2.371151 \\
\hline $\mathrm{H}$ & 15.050388 & -2.842211 & -3.824572 \\
\hline $\mathrm{H}$ & 16.368621 & -3.944015 & -3.544099 \\
\hline $\mathrm{H}$ & 16.275589 & -5.314100 & -1.235269 \\
\hline $\mathrm{H}$ & 13.494850 & -2.922513 & 0.757658 \\
\hline $\mathrm{H}$ & 14.783037 & -5.064865 & 0.821930 \\
\hline 0 & -14.929722 & -2.440555 & 5.284115 \\
\hline $\mathrm{H}$ & -15.070645 & -1.570308 & 4.920278 \\
\hline $\mathrm{H}$ & -14.426611 & -2.308294 & 6.080648 \\
\hline $\mathrm{O}$ & -12.772073 & -1.570060 & 7.335180 \\
\hline $\mathrm{H}$ & -12.082833 & -2.061380 & 7.768270 \\
\hline $\mathrm{H}$ & -12.982013 & -0.850239 & 7.917504 \\
\hline 0 & -11.882781 & -0.958346 & 4.587550 \\
\hline $\mathrm{H}$ & -11.986054 & -1.109474 & 5.523663 \\
\hline $\mathrm{H}$ & -12.698956 & -0.533300 & 4.317258 \\
\hline 0 & -13.470918 & -1.497777 & 1.153079 \\
\hline $\mathrm{H}$ & -12.611455 & -1.069906 & 1.129696 \\
\hline 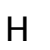 & -13.392863 & -2.234513 & 1.76245 \\
\hline
\end{tabular}




\begin{tabular}{|c|c|c|c|}
\hline 0 & -7.423101 & 2.196895 & -0.103248 \\
\hline $\mathrm{H}$ & -7.555141 & 2.263076 & 0.846437 \\
\hline $\mathrm{H}$ & -6.505869 & 1.972041 & -0.203877 \\
\hline $\mathrm{O}$ & -7.633194 & 2.149865 & 2.721403 \\
\hline $\mathrm{H}$ & -8.411248 & 1.691243 & 3.031058 \\
\hline $\mathrm{H}$ & -7.483551 & 2.881695 & 3.315408 \\
\hline $\mathrm{O}$ & -9.180003 & 0.348640 & -1.275893 \\
\hline $\mathrm{H}$ & -8.589395 & 1.014137 & -0.904100 \\
\hline $\mathrm{H}$ & -8.610225 & -0.340588 & -1.612839 \\
\hline $\mathrm{O}$ & -13.000615 & -1.922159 & -1.654690 \\
\hline $\mathrm{H}$ & -13.314073 & -1.992348 & $\begin{array}{ll}3 & -0.752737\end{array}$ \\
\hline $\mathrm{H}$ & -13.713766 & -2.168422 & -2.234912 \\
\hline $\mathrm{O}$ & -13.813123 & 1.513256 & 0.356362 \\
\hline $\mathrm{H}$ & -14.241874 & 0.686036 & 0.545542 \\
\hline $\mathrm{H}$ & -12.888966 & 1.336987 & 0.542750 \\
\hline $\mathrm{O}$ & -14.680102 & 5.246503 & 4.124659 \\
\hline $\mathrm{H}$ & -14.014772 & 5.838141 & 3.788336 \\
\hline $\mathrm{H}$ & -14.614055 & 5.349535 & 5.069799 \\
\hline $\mathrm{O}$ & -14.703140 & 2.662480 & 2.986863 \\
\hline $\mathrm{H}$ & -14.903586 & 3.540852 & 3.322938 \\
\hline $\mathrm{H}$ & -14.603806 & 2.715437 & 2.041412 \\
\hline 0 & -13.336314 & 0.833938 & -2.396199 \\
\hline $\mathrm{H}$ & -13.632472 & 1.332355 & -1.635709 \\
\hline $\mathrm{H}$ & -13.077163 & -0.016978 & $3-2.039838$ \\
\hline $\mathrm{O}$ & -14.474249 & -1.109944 & -4.141616 \\
\hline $\mathrm{H}$ & -14.414024 & -0.260518 & -3.708060 \\
\hline $\mathrm{H}$ & -15.298233 & -1.129403 & $3-4.610488$ \\
\hline $\mathrm{O}$ & -10.572576 & -2.940057 & $7-3.030987$ \\
\hline $\mathrm{H}$ & -10.892165 & -2.579067 & -3.854251 \\
\hline & -11.254346 & -2.730956 & -2.398086 \\
\hline
\end{tabular}




\begin{tabular}{llll}
$\mathrm{O}$ & -11.266363 & 0.240360 & 0.789619 \\
$\mathrm{H}$ & -10.601802 & 0.163027 & 0.104301 \\
$\mathrm{H}$ & -10.806630 & 0.507530 & 1.590718 \\
$\mathrm{O}$ & -10.209266 & 0.992222 & 3.285589 \\
$\mathrm{H}$ & -10.577648 & 0.309838 & 3.844682 \\
$\mathrm{H}$ & -10.727928 & 1.781921 & 3.462077 \\
$\mathrm{O}$ & -11.957294 & 3.113818 & 3.705388 \\
$\mathrm{H}$ & -11.912013 & 4.064500 & 3.654371 \\
$\mathrm{H}$ & -12.872601 & 2.889280 & 3.553491 \\
$\mathrm{O}$ & -10.746130 & 0.954414 & -3.524643 \\
$\mathrm{H}$ & -11.593027 & 1.223341 & -3.167919 \\
$\mathrm{H}$ & -10.181390 & 0.783597 & -2.763551 \\
$\mathrm{O}$ & -14.430978 & -0.068486 & 3.680545 \\
$\mathrm{H}$ & -14.660585 & 0.857060 & 3.580157 \\
$\mathrm{H}$ & -14.387286 & -0.433740 & 2.802022 \\
$\mathrm{O}$ & -13.081754 & -3.334161 & 3.367518 \\
$\mathrm{H}$ & -13.830590 & -3.189757 & 3.954727 \\
$\mathrm{H}$ & -12.406096 & -2.767751 & 3.737159 \\
$\mathrm{O}$ & -11.696982 & -1.220164 & -5.030317 \\
$\mathrm{H}$ & -12.648753 & -1.298531 & -4.990898 \\
\hline & -11.457902 & -0.551984 & -4.388114
\end{tabular}

PROTON CONFIGURATION, GIVEN BY NOMINAL CHARGES: R300(0),Y266(0),E183(0) POTENTIAL:-35mV
$\begin{array}{llll}N & 15.580944 & -6.451469 & 5.391416\end{array}$
H $\quad 14.780605-6.201176 \quad 5.939239$
C $\quad 15.509567 \quad-5.856494 \quad 4.054239$
C $\quad 14.783016 \quad-6.782962 \quad 3.060452$ 


\begin{tabular}{|c|c|c|c|}
\hline 0 & 14.052292 & -6.345799 & 2.186782 \\
\hline$C$ & 16.919631 & -5.576623 & 3.520772 \\
\hline $\mathrm{H}$ & 16.389693 & -6.127924 & 5.884523 \\
\hline $\mathrm{H}$ & 14.944896 & -4.933563 & 4.050387 \\
\hline $\mathrm{H}$ & 16.888874 & -5.190224 & 2.509018 \\
\hline $\mathrm{H}$ & 17.403616 & -4.831732 & 4.142242 \\
\hline $\mathrm{H}$ & 17.526957 & -6.474962 & 3.529482 \\
\hline $\mathrm{N}$ & 15.032378 & -8.085604 & 3.186404 \\
\hline $\mathrm{H}$ & 15.612322 & -8.361768 & 3.949519 \\
\hline C & 14.398661 & -9.092386 & 2.333235 \\
\hline $\mathrm{H}$ & 14.482433 & -8.823803 & 1.292858 \\
\hline $\mathrm{H}$ & 14.891371 & -10.042262 & 2.492770 \\
\hline C & 12.912807 & -9.220830 & 2.671366 \\
\hline $\mathrm{O}$ & 12.081463 & -9.424755 & 1.825889 \\
\hline $\mathrm{N}$ & 12.582804 & -9.055884 & 3.966877 \\
\hline $\mathrm{H}$ & 13.302703 & -8.986414 & 4.648929 \\
\hline C & 11.195108 & -9.170814 & 4.421376 \\
\hline $\mathrm{H}$ & 11.188270 & -9.157957 & 5.503131 \\
\hline $\mathrm{H}$ & 10.743739 & -10.088316 & 4.077120 \\
\hline C & 10.336944 & -8.010595 & 3.900734 \\
\hline $\mathrm{O}$ & 9.190736 & -8.186000 & 3.584535 \\
\hline$N$ & 10.947160 & -6.814579 & 3.855252 \\
\hline C & 10.318570 & -5.634049 & 3.317564 \\
\hline C & 10.126840 & -5.690931 & 1.799336 \\
\hline $\mathrm{O}$ & 9.096371 & -5.262336 & 1.337324 \\
\hline C & 11.092959 & -4.388035 & 3.776306 \\
\hline $\mathrm{O}$ & 12.478878 & -4.570872 & 3.769343 \\
\hline $\mathrm{H}$ & 11.905570 & -6.744345 & 4.106716 \\
\hline $\mathrm{H}$ & 9.314721 & -5.553204 & 3.701198 \\
\hline $\mathrm{H}$ & 10.801517 & -3.539712 & 3.167236 \\
\hline
\end{tabular}




\begin{tabular}{|c|c|c|c|}
\hline $\mathrm{H}$ & 10.827475 & -4.174374 & 4.801325 \\
\hline $\mathrm{H}$ & 12.805795 & -4.755324 & 2.897303 \\
\hline $\mathrm{N}$ & 11.099999 & -6.200434 & 1.023965 \\
\hline$C$ & 10.844305 & -6.396689 & -0.400370 \\
\hline$C$ & 9.693436 & -7.385377 & -0.601404 \\
\hline 0 & 8.944937 & -7.279603 & -1.544975 \\
\hline$C$ & 12.132613 & -6.785588 & -1.187278 \\
\hline$C$ & 12.333425 & -8.290993 & -1.404044 \\
\hline$C$ & 12.159325 & -6.060542 & -2.534189 \\
\hline $\mathrm{H}$ & 11.924065 & -6.586137 & 1.431140 \\
\hline $\mathrm{H}$ & 10.474649 & -5.462356 & -0.791691 \\
\hline $\mathrm{H}$ & 12.964734 & -6.415498 & -0.598871 \\
\hline $\mathrm{H}$ & 13.287101 & -8.454227 & -1.895265 \\
\hline $\mathrm{H}$ & 12.332025 & -8.857181 & -0.483417 \\
\hline $\mathrm{H}$ & 11.566158 & -8.698188 & -2.055359 \\
\hline $\mathrm{H}$ & 13.053968 & -6.324471 & -3.087038 \\
\hline $\mathrm{H}$ & 11.298871 & -6.332352 & -3.135753 \\
\hline $\mathrm{H}$ & 12.159341 & -4.985320 & -2.401010 \\
\hline $\mathrm{N}$ & 9.570431 & -8.340344 & 0.328319 \\
\hline $\mathrm{H}$ & 10.314998 & -8.474790 & 0.973714 \\
\hline $\mathrm{C}$ & 8.593019 & -9.421180 & 0.241223 \\
\hline $\mathrm{H}$ & 8.934621 & -10.218583 & 0.888853 \\
\hline $\mathrm{H}$ & 8.533220 & -9.803641 & -0.766081 \\
\hline$C$ & 7.181685 & -9.045756 & 0.642870 \\
\hline 0 & 6.257522 & -9.502155 & 0.010573 \\
\hline $\mathrm{N}$ & 7.003461 & -8.226499 & 1.685712 \\
\hline$C$ & 5.669615 & -7.739212 & 1.954813 \\
\hline$C$ & 5.251037 & -6.727566 & 0.893366 \\
\hline 0 & 4.074289 & -6.630604 & 0.617199 \\
\hline C & 5.415370 & -7.234001 & 3.389149 \\
\hline
\end{tabular}




\begin{tabular}{|c|c|c|c|}
\hline C & 5.557241 & -8.378393 & 4.397775 \\
\hline C & 6.257332 & -6.025242 & 3.802446 \\
\hline $\mathrm{H}$ & 7.780838 & -7.960621 & 2.250218 \\
\hline $\mathrm{H}$ & 4.986577 & -8.558522 & 1.792481 \\
\hline $\mathrm{H}$ & 4.374586 & -6.924964 & 3.382236 \\
\hline $\mathrm{H}$ & 5.267122 & -8.041301 & 5.387372 \\
\hline $\mathrm{H}$ & 4.919129 & -9.215730 & 4.134739 \\
\hline $\mathrm{H}$ & 6.579369 & -8.735711 & 4.453607 \\
\hline $\mathrm{H}$ & 5.916866 & -5.653281 & 4.762890 \\
\hline $\mathrm{H}$ & 7.299619 & -6.295871 & 3.909499 \\
\hline $\mathrm{H}$ & 6.185287 & -5.210612 & 3.090082 \\
\hline$N$ & 6.171856 & -6.002978 & 0.249613 \\
\hline C & 5.776344 & -5.184142 & -0.884278 \\
\hline C & 5.343491 & -6.067588 & -2.062706 \\
\hline $\mathrm{O}$ & 4.319796 & -5.815624 & -2.653994 \\
\hline C & 6.869047 & -4.164972 & -1.264289 \\
\hline C & 7.067139 & -3.148304 & -0.125951 \\
\hline C & 6.498301 & -3.454558 & -2.570765 \\
\hline C & 8.360934 & -2.341843 & -0.239949 \\
\hline $\mathrm{H}$ & 7.133119 & -6.060987 & 0.508240 \\
\hline $\mathrm{H}$ & 4.878652 & -4.647170 & -0.617137 \\
\hline $\mathrm{H}$ & 7.798892 & -4.706491 & -1.412092 \\
\hline $\mathrm{H}$ & 6.212216 & -2.473797 & -0.110016 \\
\hline $\mathrm{H}$ & 7.076791 & -3.662111 & 0.825975 \\
\hline $\mathrm{H}$ & 7.203790 & -2.665038 & -2.790771 \\
\hline $\mathrm{H}$ & 6.498266 & -4.129757 & -3.416679 \\
\hline $\mathrm{H}$ & 5.510258 & -3.011068 & -2.504004 \\
\hline $\mathrm{H}$ & 8.462235 & -1.668249 & 0.604329 \\
\hline $\mathrm{H}$ & 9.222273 & -2.999469 & -0.239707 \\
\hline $\mathrm{H}$ & 8.397192 & -1.742241 & -1.142551 \\
\hline
\end{tabular}




\begin{tabular}{|c|c|c|c|}
\hline$N$ & 6.115623 & -7.109673 & -2.399467 \\
\hline $\mathrm{H}$ & 7.009719 & -7.232356 & -1.972736 \\
\hline C & 5.773357 & -7.949703 & -3.527488 \\
\hline $\mathrm{H}$ & 6.603932 & -8.623441 & -3.697534 \\
\hline $\mathrm{H}$ & 5.618384 & -7.363603 & -4.421041 \\
\hline C & 4.504357 & -8.778168 & -3.325618 \\
\hline $\mathrm{O}$ & 3.666280 & -8.836135 & -4.194066 \\
\hline$N$ & 4.393359 & -9.431802 & -2.162407 \\
\hline $\mathrm{H}$ & 5.131091 & -9.388570 & -1.492038 \\
\hline C & 3.207309 & -10.187565 & -1.834412 \\
\hline $\mathrm{H}$ & 2.967702 & -10.899995 & -2.609501 \\
\hline $\mathrm{H}$ & 3.399615 & -10.730343 & -0.917398 \\
\hline C & 1.974183 & -9.306851 & -1.644814 \\
\hline $\mathrm{O}$ & 0.887188 & -9.696194 & -2.004603 \\
\hline$N$ & 2.187877 & -8.107003 & -1.107171 \\
\hline C & 1.062409 & -7.184556 & -1.070943 \\
\hline C & 0.616899 & -6.720548 & -2.460282 \\
\hline $\mathrm{O}$ & -0.565535 & -6.584413 & -2.680697 \\
\hline C & 1.278111 & -5.930572 & -0.230466 \\
\hline O & 1.413950 & -6.189414 & 1.126952 \\
\hline $\mathrm{H}$ & 3.087725 & -7.817064 & -0.798549 \\
\hline $\mathrm{H}$ & 0.211098 & -7.704080 & -0.662224 \\
\hline $\mathrm{H}$ & 2.120966 & -5.365010 & -0.613564 \\
\hline $\mathrm{H}$ & 0.384092 & -5.341882 & -0.351483 \\
\hline $\mathrm{H}$ & 2.307864 & -6.456596 & 1.293773 \\
\hline$N$ & 1.544084 & -6.431961 & -3.383021 \\
\hline C & 1.145401 & -6.024110 & -4.717962 \\
\hline C & 0.378713 & -7.146528 & -5.410977 \\
\hline $\mathrm{O}$ & -0.608617 & -6.891359 & -6.067831 \\
\hline C & 2.355745 & -5.521072 & -5.538423 \\
\hline
\end{tabular}




\begin{tabular}{|c|c|c|c|}
\hline$C$ & 2.761868 & -4.129042 & -5.026137 \\
\hline$C$ & 2.060227 & -5.496091 & -7.043398 \\
\hline$C$ & 4.087304 & -3.618286 & -5.589143 \\
\hline $\mathrm{H}$ & 2.514483 & -6.487418 & -3.159265 \\
\hline $\mathrm{H}$ & 0.424266 & -5.223003 & -4.638574 \\
\hline $\mathrm{H}$ & 3.176787 & -6.210979 & -5.367731 \\
\hline $\mathrm{H}$ & 1.969949 & -3.423461 & -5.271828 \\
\hline $\mathrm{H}$ & 2.835530 & -4.155166 & -3.946542 \\
\hline $\mathrm{H}$ & 2.918116 & -5.124544 & -7.589820 \\
\hline $\mathrm{H}$ & 1.841475 & -6.482977 & -7.432575 \\
\hline $\mathrm{H}$ & 1.215284 & -4.853434 & -7.269908 \\
\hline $\mathrm{H}$ & 4.380365 & -2.704936 & -5.082017 \\
\hline $\mathrm{H}$ & 4.879282 & -4.345086 & -5.439155 \\
\hline $\mathrm{H}$ & 4.030270 & -3.399810 & -6.649639 \\
\hline $\mathrm{N}$ & 0.820021 & -8.397267 & -5.251521 \\
\hline $\mathrm{H}$ & 1.667088 & -8.572880 & -4.752418 \\
\hline$C$ & 0.043272 & -9.504886 & -5.753937 \\
\hline $\mathrm{H}$ & -0.064086 & -9.462635 & -6.827599 \\
\hline $\mathrm{H}$ & 0.556435 & -10.421367 & -5.491762 \\
\hline$C$ & -1.365276 & -9.540103 & -5.166934 \\
\hline $\mathrm{O}$ & -2.319108 & -9.771152 & -5.874321 \\
\hline $\mathrm{N}$ & -1.472639 & -9.318869 & -3.858022 \\
\hline$C$ & -2.776401 & -9.352477 & -3.235399 \\
\hline$C$ & -3.692486 & -8.216370 & -3.692178 \\
\hline 0 & -4.890811 & -8.424118 & -3.732703 \\
\hline$C$ & -2.679003 & -9.329616 & -1.702814 \\
\hline $\mathrm{O}$ & -3.923262 & -9.599234 & -1.118426 \\
\hline $\mathrm{H}$ & -0.656826 & -9.231548 & -3.289012 \\
\hline $\mathrm{H}$ & -3.292805 & -10.257033 & -3.524111 \\
\hline $\mathrm{H}$ & -1.996532 & -10.103288 & -1.379346 \\
\hline
\end{tabular}




\begin{tabular}{|c|c|c|c|}
\hline $\mathrm{H}$ & -2.290336 & -8.372808 & -1.370401 \\
\hline $\mathrm{H}$ & -4.613427 & -9.195876 & -1.630806 \\
\hline$N$ & -3.143423 & -7.035461 & -3.971032 \\
\hline C & -3.907940 & -5.914860 & -4.493056 \\
\hline C & -4.449187 & -6.233746 & -5.886671 \\
\hline $\mathrm{O}$ & -5.613068 & -6.041126 & -6.155912 \\
\hline C & -3.013296 & -4.655333 & -4.488046 \\
\hline C & -3.472029 & -3.518247 & -5.385264 \\
\hline C & -4.792743 & -3.082017 & -5.426058 \\
\hline C & -2.544404 & -2.883530 & -6.203072 \\
\hline C & -5.168555 & -2.055510 & -6.276057 \\
\hline$C$ & -2.913599 & -1.844251 & -7.041929 \\
\hline C & -4.233412 & -1.431280 & -7.085866 \\
\hline $\mathrm{H}$ & -2.166731 & -6.912867 & -3.802026 \\
\hline $\mathrm{H}$ & -4.776058 & -5.749113 & -3.875597 \\
\hline $\mathrm{H}$ & -2.930343 & -4.319494 & -3.458727 \\
\hline $\mathrm{H}$ & -2.021567 & -4.947411 & -4.803548 \\
\hline $\mathrm{H}$ & -5.537422 & -3.560018 & -4.818474 \\
\hline $\mathrm{H}$ & -1.519831 & -3.211402 & -6.194476 \\
\hline $\mathrm{H}$ & -6.201174 & -1.752456 & -6.311892 \\
\hline $\mathrm{H}$ & -2.174785 & -1.370705 & -7.663102 \\
\hline $\mathrm{H}$ & -4.528428 & -0.637244 & -7.749839 \\
\hline$N$ & -3.576093 & -6.724267 & -6.775011 \\
\hline C & -4.019466 & -7.062103 & -8.108057 \\
\hline C & -5.145297 & -8.094816 & -8.085388 \\
\hline $\mathrm{O}$ & -6.033114 & -8.030967 & -8.895470 \\
\hline C & -2.870780 & -7.593811 & -8.968643 \\
\hline$S$ & -1.652311 & -6.342848 & -9.484955 \\
\hline $\mathrm{H}$ & -2.634890 & -6.908431 & -6.497377 \\
\hline & -4.443858 & -6.190215 & -8.582945 \\
\hline
\end{tabular}




\begin{tabular}{|c|c|c|c|}
\hline $\mathrm{H}$ & -2.366882 & -8.412419 & -8.472675 \\
\hline $\mathrm{H}$ & -3.288966 & -7.972037 & -9.891749 \\
\hline $\mathrm{H}$ & -1.047846 & -6.179409 & -8.318677 \\
\hline $\mathrm{N}$ & -5.078558 & -9.066913 & -7.155619 \\
\hline $\mathrm{H}$ & -4.254248 & -9.192320 & -6.607830 \\
\hline$C$ & -6.085910 & -10.115215 & -7.183049 \\
\hline $\mathrm{H}$ & -6.258812 & -10.452778 & -8.193230 \\
\hline $\mathrm{H}$ & -5.714556 & -10.947552 & -6.596537 \\
\hline$C$ & -7.459140 & -9.722570 & -6.613183 \\
\hline 0 & -8.481524 & -10.086878 & -7.126898 \\
\hline $\mathrm{N}$ & -7.417939 & -9.016209 & -5.478356 \\
\hline$C$ & -8.641654 & -8.653167 & -4.788754 \\
\hline$C$ & -9.504928 & -7.658159 & -5.556595 \\
\hline 0 & -10.702650 & -7.658734 & -5.450267 \\
\hline$C$ & -8.361790 & -8.103681 & -3.378645 \\
\hline$C$ & -7.519477 & -6.820431 & -3.271096 \\
\hline$C$ & -8.193038 & -5.469071 & -3.256679 \\
\hline 0 & -9.495079 & -5.469695 & -3.327671 \\
\hline $\mathrm{H}$ & -9.844036 & -4.571891 & -3.238586 \\
\hline 0 & -7.546903 & -4.455184 & -3.161451 \\
\hline $\mathrm{H}$ & -6.535789 & -8.774594 & -5.078968 \\
\hline $\mathrm{H}$ & -9.260690 & -9.533420 & -4.686410 \\
\hline $\mathrm{H}$ & -9.317922 & -7.967172 & -2.894287 \\
\hline $\mathrm{H}$ & -7.836541 & -8.883885 & -2.838288 \\
\hline $\mathrm{H}$ & -6.956541 & -6.846961 & -2.345210 \\
\hline $\mathrm{H}$ & -6.778057 & -6.766643 & -4.050670 \\
\hline $\mathrm{N}$ & -8.837301 & -6.733962 & -6.273855 \\
\hline$C$ & -9.555196 & -5.702617 & -7.000840 \\
\hline C & -10.386046 & -6.273602 & -8.141689 \\
\hline 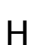 & -10.409089 & -7.356342 & -8.2489 \\
\hline
\end{tabular}




\begin{tabular}{|c|c|c|c|}
\hline $\mathrm{O}$ & -10.987293 & -5.559103 & -8.888423 \\
\hline C & -8.631312 & -4.564298 & -7.490284 \\
\hline $\mathrm{O}$ & -9.434533 & -3.441998 & -7.831927 \\
\hline C & -7.723015 & -4.928132 & -8.657220 \\
\hline $\mathrm{H}$ & -7.856607 & -6.841481 & -6.421929 \\
\hline $\mathrm{H}$ & -10.268410 & -5.253865 & -6.319060 \\
\hline $\mathrm{H}$ & -8.030343 & -4.256844 & -6.645801 \\
\hline $\mathrm{H}$ & -10.110596 & -3.750863 & -8.429428 \\
\hline $\mathrm{H}$ & -7.045359 & -4.103576 & -8.850925 \\
\hline $\mathrm{H}$ & -7.131131 & -5.809168 & -8.458024 \\
\hline $\mathrm{H}$ & -8.303897 & -5.104881 & -9.556039 \\
\hline$N$ & -12.952122 & -9.022524 & 3.174171 \\
\hline $\mathrm{H}$ & -13.215000 & -9.550467 & 2.367604 \\
\hline C & -11.773531 & -8.180020 & 2.898781 \\
\hline C & -10.789122 & -8.123249 & 4.050087 \\
\hline 0 & -9.659793 & -7.714465 & 3.857064 \\
\hline$C$ & -12.146619 & -6.722601 & 2.563120 \\
\hline 0 & -12.715837 & -6.204029 & 3.737738 \\
\hline$C$ & -13.114462 & -6.605062 & 1.391196 \\
\hline $\mathrm{H}$ & -13.731488 & -8.453499 & 3.442454 \\
\hline $\mathrm{H}$ & -11.222783 & -8.599613 & 2.068258 \\
\hline $\mathrm{H}$ & -11.230715 & -6.196590 & 2.328454 \\
\hline $\mathrm{H}$ & -12.850019 & -5.263217 & 3.637899 \\
\hline $\mathrm{H}$ & -13.281661 & -5.557085 & 1.166643 \\
\hline $\mathrm{H}$ & -12.729025 & -7.082233 & 0.498078 \\
\hline $\mathrm{H}$ & -14.074921 & -7.044986 & 1.631883 \\
\hline $\mathrm{N}$ & -11.197257 & -8.533635 & 5.241900 \\
\hline $\mathrm{H}$ & -12.119355 & -8.910736 & 5.275381 \\
\hline C & -10.412545 & -8.529115 & 6.451311 \\
\hline $\mathrm{H}$ & -10.337037 & -7.534078 & 6.860877 \\
\hline
\end{tabular}




\begin{tabular}{|c|c|c|c|}
\hline $\mathrm{H}$ & -10.933713 & -9.138687 & 7.180104 \\
\hline C & -8.974757 & -9.043554 & 6.374947 \\
\hline $\mathrm{O}$ & -8.140154 & -8.478376 & 7.037913 \\
\hline$N$ & -8.658722 & -10.119724 & 5.632203 \\
\hline $\mathrm{H}$ & -9.369346 & -10.599264 & 5.127171 \\
\hline C & -7.270130 & -10.552738 & 5.532057 \\
\hline $\mathrm{H}$ & -7.253485 & -11.532149 & 5.071252 \\
\hline $\mathrm{H}$ & -6.830728 & -10.631478 & 6.513529 \\
\hline C & -6.387460 & -9.608364 & 4.692694 \\
\hline O & -5.219619 & -9.468422 & 4.949191 \\
\hline$N$ & -7.045394 & -9.018012 & 3.701158 \\
\hline C & -6.301973 & -8.005930 & 2.963216 \\
\hline C & -6.261971 & -6.695345 & 3.744811 \\
\hline $\mathrm{O}$ & -5.267301 & -6.009523 & 3.699222 \\
\hline C & -6.610841 & -7.876580 & 1.459383 \\
\hline$C$ & -7.789996 & -7.050923 & 0.991428 \\
\hline$C$ & -7.776654 & -5.663737 & 1.106805 \\
\hline $\mathrm{C}$ & -8.849933 & -7.648230 & 0.322340 \\
\hline$C$ & -8.804228 & -4.896947 & 0.584329 \\
\hline$C$ & -9.869293 & -6.882523 & -0.220215 \\
\hline$C$ & -9.854080 & -5.504749 & -0.084365 \\
\hline $\mathrm{H}$ & -8.039943 & -9.014006 & 3.679598 \\
\hline $\mathrm{H}$ & -5.279248 & -8.337545 & 2.997382 \\
\hline $\mathrm{H}$ & -5.714001 & -7.456606 & 1.017512 \\
\hline $\mathrm{H}$ & -6.687392 & -8.886335 & 1.073854 \\
\hline $\mathrm{H}$ & -6.944552 & -5.173537 & 1.578631 \\
\hline $\mathrm{H}$ & -8.862239 & -8.716719 & 0.190761 \\
\hline $\mathrm{H}$ & -8.767446 & -3.825784 & 0.671445 \\
\hline $\mathrm{H}$ & -10.659064 & -7.357730 & -0.772552 \\
\hline $\mathrm{H}$ & -10.636287 & -4.911642 & -0.520867 \\
\hline
\end{tabular}




\begin{tabular}{|c|c|c|c|}
\hline$N$ & -7.293002 & -6.385610 & 4.534364 \\
\hline C & -7.221730 & -5.273353 & 5.451352 \\
\hline C & -6.102461 & -5.490852 & 6.469145 \\
\hline $\mathrm{O}$ & -5.456099 & -4.538968 & 6.852411 \\
\hline C & -8.598843 & -5.101510 & 6.124410 \\
\hline C & -8.810397 & -3.809065 & 6.883898 \\
\hline C & -8.979288 & -2.606845 & 6.201470 \\
\hline C & -8.906107 & -3.805157 & 8.268734 \\
\hline C & -9.226647 & -1.430576 & 6.886655 \\
\hline C & -9.163955 & -2.629711 & 8.961213 \\
\hline C & -9.323917 & -1.438928 & 8.272379 \\
\hline $\mathrm{H}$ & -8.158220 & -6.869398 & 4.428525 \\
\hline $\mathrm{H}$ & -6.951490 & -4.368444 & 4.925868 \\
\hline $\mathrm{H}$ & -9.342225 & -5.167801 & 5.336512 \\
\hline $\mathrm{H}$ & -8.762024 & -5.940633 & 6.786700 \\
\hline $\mathrm{H}$ & -8.925588 & -2.588776 & 5.126645 \\
\hline $\mathrm{H}$ & -8.781967 & -4.723519 & 8.815638 \\
\hline $\mathrm{H}$ & -9.346256 & -0.512296 & 6.341059 \\
\hline $\mathrm{H}$ & -9.227231 & -2.646428 & 10.034539 \\
\hline $\mathrm{H}$ & -9.506863 & -0.523364 & 8.806590 \\
\hline$N$ & -5.861975 & -6.727968 & 6.898076 \\
\hline $\mathrm{H}$ & -6.516693 & -7.449620 & 6.693395 \\
\hline C & -4.785924 & -7.022971 & 7.817853 \\
\hline $\mathrm{H}$ & -4.825001 & -6.393313 & 8.694036 \\
\hline $\mathrm{H}$ & -4.898403 & -8.052884 & 8.131654 \\
\hline C & -3.390082 & -6.846814 & 7.222645 \\
\hline $\mathrm{O}$ & -2.516229 & -6.352123 & 7.894630 \\
\hline$N$ & -3.189628 & -7.271995 & 5.970549 \\
\hline C & -1.911667 & -7.092411 & 5.299011 \\
\hline C & -1.665261 & -5.620551 & 4.938950 \\
\hline
\end{tabular}




\begin{tabular}{|c|c|c|c|}
\hline $\mathrm{O}$ & -0.566846 & -5.133893 & 5.107319 \\
\hline C & -1.830104 & -8.027759 & 4.072347 \\
\hline C & -0.664591 & -7.670603 & 3.148131 \\
\hline C & -1.728812 & -9.487918 & 4.527864 \\
\hline $\mathrm{H}$ & -3.922885 & -7.753130 & 5.495678 \\
\hline $\mathrm{H}$ & -1.118350 & -7.338224 & 5.989987 \\
\hline $\mathrm{H}$ & -2.750689 & -7.904009 & 3.506718 \\
\hline $\mathrm{H}$ & -0.600501 & -8.397395 & 2.345361 \\
\hline $\mathrm{H}$ & -0.774988 & -6.696245 & 2.693253 \\
\hline $\mathrm{H}$ & 0.280001 & -7.682386 & 3.683300 \\
\hline $\mathrm{H}$ & -1.741975 & -10.148550 & 3.667022 \\
\hline $\mathrm{H}$ & -0.795210 & -9.654755 & 5.059259 \\
\hline $\mathrm{H}$ & -2.545713 & -9.777642 & 5.175983 \\
\hline$N$ & -2.700683 & -4.933602 & 4.446723 \\
\hline C & -2.569791 & -3.551413 & 4.028848 \\
\hline C & -2.420055 & -2.639035 & 5.244026 \\
\hline O & -1.528454 & -1.820255 & 5.290671 \\
\hline C & -3.758179 & -3.090370 & 3.171592 \\
\hline C & -3.993855 & -3.840527 & 1.849922 \\
\hline C & -2.859849 & -3.730828 & 0.845315 \\
\hline 0 & -1.746949 & -4.135755 & 1.218717 \\
\hline O & -3.112387 & -3.254706 & -0.274000 \\
\hline $\mathrm{H}$ & -3.564290 & -5.403253 & 4.285196 \\
\hline $\mathrm{H}$ & -1.662684 & -3.440938 & 3.465257 \\
\hline $\mathrm{H}$ & -4.667090 & -3.158025 & 3.761551 \\
\hline $\mathrm{H}$ & -3.604787 & -2.035175 & 2.961839 \\
\hline $\mathrm{H}$ & -4.149166 & -4.890275 & 2.048787 \\
\hline $\mathrm{H}$ & -4.899225 & -3.450481 & 1.399036 \\
\hline $\mathrm{N}$ & -3.309527 & -2.774871 & 6.244281 \\
\hline$C$ & -3.093978 & -2.024901 & 7.456985 \\
\hline
\end{tabular}




\begin{tabular}{|c|c|c|c|}
\hline C & -1.778227 & -2.422283 & 8.133769 \\
\hline $\mathrm{O}$ & -1.106870 & -1.582682 & 8.694100 \\
\hline C & -4.226306 & -2.005092 & 8.493911 \\
\hline $\mathrm{O}$ & -4.436264 & -3.246772 & 9.098351 \\
\hline C & -5.507665 & -1.387418 & 7.948248 \\
\hline $\mathrm{H}$ & -3.988319 & -3.503125 & 6.218592 \\
\hline $\mathrm{H}$ & -2.937615 & -0.995394 & 7.174834 \\
\hline $\mathrm{H}$ & -3.851340 & -1.365921 & 9.283604 \\
\hline $\mathrm{H}$ & -4.889516 & -3.818426 & 8.490419 \\
\hline $\mathrm{H}$ & -6.240075 & -1.327579 & 8.743512 \\
\hline $\mathrm{H}$ & -5.322056 & -0.384126 & 7.577075 \\
\hline $\mathrm{H}$ & -5.928451 & -1.976867 & 7.144820 \\
\hline$N$ & -1.380397 & -3.700457 & 8.043328 \\
\hline $\mathrm{H}$ & -2.031219 & -4.418216 & 7.812066 \\
\hline C & -0.187162 & -4.107473 & 8.739880 \\
\hline $\mathrm{H}$ & -0.237315 & -3.891790 & 9.797886 \\
\hline $\mathrm{H}$ & -0.075815 & -5.175172 & 8.604040 \\
\hline $\mathrm{C}$ & 1.064024 & -3.420008 & 8.217958 \\
\hline 0 & 1.940562 & -3.076028 & 8.977771 \\
\hline$N$ & 1.153879 & -3.258765 & 6.881711 \\
\hline $\mathrm{C}$ & 2.332480 & -2.660282 & 6.317666 \\
\hline$C$ & 2.291031 & -1.138383 & 6.164108 \\
\hline 0 & 3.359924 & -0.574352 & 6.023012 \\
\hline$C$ & 2.830599 & -3.345982 & 5.045415 \\
\hline$S$ & 1.922615 & -2.976798 & 3.520199 \\
\hline $\mathrm{H}$ & 0.422681 & -3.612342 & 6.296759 \\
\hline $\mathrm{H}$ & 3.116420 & -2.798068 & 7.046915 \\
\hline $\mathrm{H}$ & 3.840481 & -3.007717 & 4.863090 \\
\hline $\mathrm{H}$ & 2.853201 & -4.415051 & 5.204870 \\
\hline $\mathrm{H}$ & 0.859018 & -3.741107 & 3.719458 \\
\hline
\end{tabular}




\begin{tabular}{|c|c|c|c|}
\hline$N$ & 1.140073 & -0.470671 & 6.273042 \\
\hline C & 1.204068 & 0.968497 & 6.491343 \\
\hline C & 1.755867 & 1.271210 & 7.870506 \\
\hline $\mathrm{O}$ & 2.368584 & 2.288860 & 8.085489 \\
\hline C & -0.095735 & 1.741771 & 6.182377 \\
\hline C & -1.196941 & 1.568454 & 7.238669 \\
\hline C & -0.554806 & 1.440244 & 4.750822 \\
\hline C & -2.466310 & 2.370770 & 6.944793 \\
\hline $\mathrm{H}$ & 0.271734 & -0.965324 & 6.291801 \\
\hline $\mathrm{H}$ & 1.951475 & 1.351091 & 5.814764 \\
\hline $\mathrm{H}$ & 0.208555 & 2.782274 & 6.222312 \\
\hline $\mathrm{H}$ & -1.446001 & 0.523677 & 7.355885 \\
\hline $\mathrm{H}$ & -0.807694 & 1.892671 & 8.199275 \\
\hline $\mathrm{H}$ & -1.310205 & 2.147625 & 4.431774 \\
\hline $\mathrm{H}$ & 0.277840 & 1.520488 & 4.057287 \\
\hline $\mathrm{H}$ & -0.966433 & 0.444537 & 4.656977 \\
\hline $\mathrm{H}$ & -3.135946 & 2.340396 & 7.798938 \\
\hline $\mathrm{H}$ & -2.241207 & 3.414239 & 6.739191 \\
\hline $\mathrm{H}$ & -3.007062 & 1.973583 & 6.092649 \\
\hline$N$ & 1.549788 & 0.323258 & 8.791266 \\
\hline $\mathrm{H}$ & 0.889674 & -0.402097 & 8.613548 \\
\hline C & 1.978259 & 0.512664 & 10.160074 \\
\hline $\mathrm{H}$ & 1.470031 & -0.222645 & 10.771021 \\
\hline $\mathrm{H}$ & 1.737710 & 1.502369 & 10.518137 \\
\hline C & 3.482320 & 0.337408 & 10.305053 \\
\hline $\mathrm{O}$ & 4.164481 & 1.128932 & 10.896182 \\
\hline$N$ & 3.999933 & -0.753835 & 9.695408 \\
\hline $\mathrm{H}$ & 3.390718 & -1.466103 & 9.351485 \\
\hline C & 5.425034 & -0.938857 & 9.667453 \\
\hline $\mathrm{H}$ & 5.638280 & -1.909399 & 9.234591 \\
\hline
\end{tabular}




\begin{tabular}{|c|c|c|c|}
\hline $\mathrm{H}$ & 5.855716 & -0.904647 & 10.657516 \\
\hline C & 6.156882 & 0.119117 & 8.844832 \\
\hline $\mathrm{O}$ & 7.291930 & 0.411889 & 9.129191 \\
\hline$N$ & 5.502078 & 0.621285 & 7.799030 \\
\hline C & 6.098617 & 1.759274 & 7.146315 \\
\hline C & 5.514930 & 3.076794 & 7.649682 \\
\hline $\mathrm{H}$ & 5.013174 & 3.037228 & 8.615080 \\
\hline $\mathrm{O}$ & 5.636959 & 4.094891 & 7.041084 \\
\hline C & 6.002880 & 1.675018 & 5.620789 \\
\hline C & 6.755249 & 0.506917 & 5.017467 \\
\hline C & 8.120897 & 0.322917 & 5.238163 \\
\hline C & 6.094905 & -0.401623 & 4.195757 \\
\hline$C$ & 8.807270 & -0.722520 & 4.635365 \\
\hline C & 6.779038 & -1.444694 & 3.591485 \\
\hline C & 8.139125 & -1.605637 & 3.801366 \\
\hline $\mathrm{H}$ & 4.575199 & 0.334750 & 7.572736 \\
\hline $\mathrm{H}$ & 7.134082 & 1.774541 & 7.451288 \\
\hline $\mathrm{H}$ & 4.960665 & 1.610784 & 5.334377 \\
\hline $\mathrm{H}$ & 6.381157 & 2.609453 & 5.220641 \\
\hline $\mathrm{H}$ & 8.648975 & 0.979017 & 5.908743 \\
\hline $\mathrm{H}$ & 5.034721 & -0.305680 & 4.057633 \\
\hline $\mathrm{H}$ & 9.857989 & -0.849857 & 4.831180 \\
\hline $\mathrm{H}$ & 6.246852 & -2.136576 & 2.963236 \\
\hline $\mathrm{H}$ & 8.659502 & -2.418213 & 3.328337 \\
\hline$N$ & 12.613550 & 9.596234 & -1.248802 \\
\hline $\mathrm{H}$ & 11.873251 & 9.914036 & -1.846107 \\
\hline C & 12.304852 & 8.264740 & -0.719487 \\
\hline C & 11.274139 & 8.450687 & 0.386897 \\
\hline O & 10.265877 & 7.779033 & 0.416424 \\
\hline C & 13.562740 & 7.581006 & -0.165338 \\
\hline
\end{tabular}




\begin{tabular}{|c|c|c|c|}
\hline C & 14.645071 & 7.276096 & -1.206565 \\
\hline$S$ & 14.017112 & 6.202108 & -2.529901 \\
\hline C & 15.499794 & 6.053606 & -3.555345 \\
\hline $\mathrm{H}$ & 13.433413 & 9.573022 & -1.822259 \\
\hline $\mathrm{H}$ & 11.834450 & 7.616518 & -1.447338 \\
\hline $\mathrm{H}$ & 14.001304 & 8.205423 & 0.607192 \\
\hline $\mathrm{H}$ & 13.255687 & 6.653825 & 0.304791 \\
\hline $\mathrm{H}$ & 15.045468 & 8.188561 & -1.633889 \\
\hline $\mathrm{H}$ & 15.470243 & 6.783690 & -0.704559 \\
\hline $\mathrm{H}$ & 15.248468 & 5.398939 & -4.375892 \\
\hline $\mathrm{H}$ & 15.798798 & 7.017676 & -3.946031 \\
\hline $\mathrm{H}$ & 16.318169 & 5.616741 & -2.998068 \\
\hline$N$ & 11.504319 & 9.412913 & 1.296974 \\
\hline C & 10.594348 & 9.607808 & 2.408562 \\
\hline$C$ & 9.277067 & 10.274789 & 2.010382 \\
\hline 0 & 8.281020 & 10.053301 & 2.652714 \\
\hline$C$ & 11.276314 & 10.369119 & 3.552080 \\
\hline$C$ & 12.448034 & 9.564515 & 4.084489 \\
\hline 0 & 13.582868 & 9.826309 & 3.744685 \\
\hline $\mathrm{N}$ & 12.144421 & 8.562209 & 4.918899 \\
\hline $\mathrm{H}$ & 12.328109 & 9.967780 & 1.206258 \\
\hline $\mathrm{H}$ & 10.295558 & 8.637760 & 2.771295 \\
\hline $\mathrm{H}$ & 11.657342 & 11.326350 & 3.218957 \\
\hline $\mathrm{H}$ & 10.546139 & 10.537733 & 4.333500 \\
\hline $\mathrm{H}$ & 12.888265 & 7.966850 & 5.213200 \\
\hline $\mathrm{H}$ & 11.222925 & 8.175946 & 4.954709 \\
\hline $\mathrm{N}$ & 9.263831 & 11.067722 & 0.922310 \\
\hline $\mathrm{H}$ & 10.110797 & 11.229597 & 0.427324 \\
\hline$C$ & 8.003502 & 11.517470 & 0.368859 \\
\hline $\mathrm{H}$ & 8.201222 & 12.260245 & -0.3933 \\
\hline
\end{tabular}




\begin{tabular}{|c|c|c|c|}
\hline $\mathrm{H}$ & 7.389103 & 11.966665 & 1.131940 \\
\hline C & 7.186384 & 10.384439 & -0.254324 \\
\hline $\mathrm{O}$ & 5.986296 & 10.470082 & -0.291509 \\
\hline$N$ & 7.862661 & 9.333318 & -0.763260 \\
\hline C & 7.170486 & 8.145603 & -1.233909 \\
\hline C & 6.634992 & 7.300679 & -0.067270 \\
\hline $\mathrm{O}$ & 5.534682 & 6.808152 & -0.148207 \\
\hline C & 8.048994 & 7.344301 & -2.224229 \\
\hline C & 8.343645 & 8.197264 & -3.472928 \\
\hline C & 7.379617 & 6.019178 & -2.608125 \\
\hline C & 9.428312 & 7.625246 & -4.388996 \\
\hline $\mathrm{H}$ & 8.823868 & 9.230810 & -0.536735 \\
\hline $\mathrm{H}$ & 6.279734 & 8.471644 & -1.748344 \\
\hline $\mathrm{H}$ & 8.988067 & 7.117937 & -1.727344 \\
\hline $\mathrm{H}$ & 7.421718 & 8.323212 & -4.036260 \\
\hline $\mathrm{H}$ & 8.645669 & 9.192928 & -3.165447 \\
\hline $\mathrm{H}$ & 7.997037 & 5.478886 & -3.314349 \\
\hline $\mathrm{H}$ & 7.228554 & 5.377877 & -1.748308 \\
\hline $\mathrm{H}$ & 6.409910 & 6.182774 & -3.066625 \\
\hline $\mathrm{H}$ & 9.622269 & 8.305829 & -5.211634 \\
\hline $\mathrm{H}$ & 10.363646 & 7.479783 & -3.855321 \\
\hline $\mathrm{H}$ & 9.148522 & 6.671165 & -4.820127 \\
\hline$N$ & 7.387690 & 7.175308 & 1.043947 \\
\hline C & 6.879166 & 6.458329 & 2.201997 \\
\hline C & 5.528064 & 7.039473 & 2.639720 \\
\hline $\mathrm{O}$ & 4.575678 & 6.332911 & 2.867084 \\
\hline C & 7.833311 & 6.546165 & 3.403537 \\
\hline C & 9.254785 & 5.997325 & 3.244210 \\
\hline $\mathrm{O}$ & 10.146034 & 6.631439 & 3.802226 \\
\hline 0 & 9.428387 & 4.935707 & 2.595214 \\
\hline
\end{tabular}




\begin{tabular}{|c|c|c|c|}
\hline $\mathrm{H}$ & 8.347749 & 7.445033 & 1.022646 \\
\hline $\mathrm{H}$ & 6.682584 & 5.429517 & 1.943351 \\
\hline $\mathrm{H}$ & 7.921361 & 7.570767 & 3.733117 \\
\hline $\mathrm{H}$ & 7.375197 & 5.992739 & 4.220726 \\
\hline$N$ & 5.489887 & 8.377412 & 2.786093 \\
\hline $\mathrm{H}$ & 6.320922 & 8.912627 & 2.648912 \\
\hline C & 4.299247 & 9.045888 & 3.249974 \\
\hline $\mathrm{H}$ & 4.538272 & 10.090246 & 3.407640 \\
\hline $\mathrm{H}$ & 3.957307 & 8.638803 & 4.188427 \\
\hline C & 3.136661 & 8.987517 & 2.268580 \\
\hline O & 1.995196 & 9.109753 & 2.649482 \\
\hline$N$ & 3.470198 & 8.824750 & 0.985184 \\
\hline $\mathrm{H}$ & 4.431331 & 8.829481 & 0.735262 \\
\hline C & 2.517191 & 8.905260 & -0.078305 \\
\hline $\mathrm{H}$ & 3.059845 & 9.091730 & -0.996314 \\
\hline $\mathrm{H}$ & 1.843947 & 9.738043 & 0.071942 \\
\hline C & 1.609133 & 7.687020 & -0.310194 \\
\hline $\mathrm{O}$ & 0.561873 & 7.815080 & -0.879456 \\
\hline$N$ & 2.094450 & 6.538077 & 0.201341 \\
\hline C & 1.289915 & 5.364391 & 0.431052 \\
\hline C & 0.472366 & 5.479579 & 1.721851 \\
\hline $\mathrm{O}$ & -0.722723 & 5.301849 & 1.692290 \\
\hline C & 2.166640 & 4.112382 & 0.445787 \\
\hline $\mathrm{H}$ & 3.001458 & 6.575677 & 0.616027 \\
\hline $\mathrm{H}$ & 0.559459 & 5.290403 & -0.357453 \\
\hline $\mathrm{H}$ & 1.566371 & 3.234011 & 0.657559 \\
\hline $\mathrm{H}$ & 2.636342 & 3.991439 & -0.523469 \\
\hline $\mathrm{H}$ & 2.946287 & 4.184370 & 1.196906 \\
\hline$N$ & 1.102526 & 5.751848 & 2.879338 \\
\hline$c$ & 0.364319 & 5.585295 & 4.123115 \\
\hline
\end{tabular}




\begin{tabular}{|c|c|c|c|}
\hline C & -0.756395 & 6.611745 & 4.274095 \\
\hline 0 & -1.792311 & 6.302365 & 4.823511 \\
\hline C & 1.276044 & 5.511889 & 5.372777 \\
\hline C & 1.867890 & 6.857325 & 5.819756 \\
\hline C & 2.362792 & 4.445281 & 5.169577 \\
\hline C & 2.699032 & 6.761047 & 7.101617 \\
\hline $\mathrm{H}$ & 2.090115 & 5.889556 & 2.900976 \\
\hline $\mathrm{H}$ & -0.159805 & 4.642232 & 4.064891 \\
\hline $\mathrm{H}$ & 0.615224 & 5.173609 & 6.165237 \\
\hline $\mathrm{H}$ & 2.478240 & 7.266383 & 5.021419 \\
\hline $\mathrm{H}$ & 1.064534 & 7.567480 & 5.986889 \\
\hline $\mathrm{H}$ & 2.732497 & 4.092365 & 6.121113 \\
\hline $\mathrm{H}$ & 1.969567 & 3.588565 & 4.631492 \\
\hline $\mathrm{H}$ & 3.205860 & 4.834223 & 4.608992 \\
\hline $\mathrm{H}$ & 3.004371 & 7.751979 & 7.424500 \\
\hline $\mathrm{H}$ & 2.121225 & 6.317754 & 7.907248 \\
\hline $\mathrm{H}$ & 3.593851 & 6.165168 & 6.971790 \\
\hline $\mathrm{N}$ & -0.542944 & 7.847430 & 3.801020 \\
\hline $\mathrm{H}$ & 0.341450 & 8.089943 & 3.404838 \\
\hline$C$ & -1.513861 & 8.887808 & 4.018652 \\
\hline $\mathrm{H}$ & -1.103685 & 9.816518 & 3.642483 \\
\hline $\mathrm{H}$ & -1.724196 & 9.016557 & 5.070574 \\
\hline$C$ & -2.866972 & 8.618762 & 3.365891 \\
\hline 0 & -3.871054 & 8.837162 & 4.003288 \\
\hline $\mathrm{N}$ & -2.902689 & 8.108966 & 2.120757 \\
\hline$C$ & -4.239994 & 8.013726 & 1.535324 \\
\hline$C$ & -4.984708 & 6.749465 & 1.927653 \\
\hline O & -6.195848 & 6.756948 & 1.919618 \\
\hline$C$ & -4.022033 & 8.221434 & 0.030819 \\
\hline C & -2.766445 & 9.099865 & 0.020044 \\
\hline
\end{tabular}




\begin{tabular}{|c|c|c|c|}
\hline C & -1.907276 & 8.456243 & 1.096073 \\
\hline $\mathrm{H}$ & -4.854277 & 8.814601 & 1.917373 \\
\hline $\mathrm{H}$ & -3.842473 & 7.278944 & -0.469789 \\
\hline $\mathrm{H}$ & -4.886472 & 8.684732 & -0.427141 \\
\hline $\mathrm{H}$ & -2.255835 & 9.142614 & -0.923514 \\
\hline $\mathrm{H}$ & -3.027341 & 10.114526 & 0.311498 \\
\hline $\mathrm{H}$ & -1.420659 & 7.566150 & 0.740384 \\
\hline $\mathrm{H}$ & -1.151189 & 9.119065 & 1.484439 \\
\hline$N$ & -4.269045 & 5.673722 & 2.291129 \\
\hline C & -4.938229 & 4.512552 & 2.850562 \\
\hline C & -5.638496 & 4.853563 & 4.169019 \\
\hline $\mathrm{O}$ & -6.741410 & 4.413657 & 4.408508 \\
\hline C & -3.973914 & 3.331409 & 3.086460 \\
\hline C & -3.635868 & 2.505617 & 1.861093 \\
\hline C & -4.333057 & 1.334853 & 1.576479 \\
\hline C & -2.597692 & 2.864521 & 1.013868 \\
\hline C & -4.007367 & 0.548080 & 0.481876 \\
\hline C & -2.270704 & 2.094030 & -0.087806 \\
\hline C & -2.970649 & 0.931914 & -0.361421 \\
\hline $\mathrm{O}$ & -2.617869 & 0.205930 & -1.447938 \\
\hline $\mathrm{H}$ & -3.414679 & -0.117521 & -1.907982 \\
\hline $\mathrm{H}$ & -3.275174 & 5.750810 & 2.345605 \\
\hline $\mathrm{H}$ & -5.725643 & 4.209378 & 2.178910 \\
\hline $\mathrm{H}$ & -3.068234 & 3.710965 & 3.548120 \\
\hline $\mathrm{H}$ & -4.443647 & 2.682173 & 3.816457 \\
\hline $\mathrm{H}$ & -5.141779 & 1.028511 & 2.216703 \\
\hline $\mathrm{H}$ & -2.032745 & 3.752393 & 1.204716 \\
\hline $\mathrm{H}$ & -4.536277 & -0.366724 & 0.289378 \\
\hline $\mathrm{H}$ & -1.479270 & 2.397027 & -0.750216 \\
\hline $\mathrm{N}$ & -4.970839 & 5.618277 & 5.048350 \\
\hline
\end{tabular}




\begin{tabular}{|c|c|c|c|}
\hline $\mathrm{H}$ & -4.034974 & 5.911393 & 4.856556 \\
\hline C & -5.582770 & 6.005349 & 6.299222 \\
\hline $\mathrm{H}$ & -4.805964 & 6.385465 & 6.951082 \\
\hline $\mathrm{H}$ & -6.052320 & 5.158954 & 6.774277 \\
\hline C & -6.651496 & 7.084455 & 6.135958 \\
\hline $\mathrm{O}$ & -7.706406 & 7.028002 & 6.726261 \\
\hline$N$ & -6.342147 & 8.091247 & 5.313906 \\
\hline $\mathrm{H}$ & -5.445016 & 8.139354 & 4.880110 \\
\hline C & -7.257687 & 9.220267 & 5.085304 \\
\hline $\mathrm{H}$ & -6.781278 & 9.871869 & 4.364214 \\
\hline $\mathrm{H}$ & -7.419712 & 9.775739 & 5.997421 \\
\hline C & -8.632477 & 8.840767 & 4.569520 \\
\hline $\mathrm{O}$ & -9.611434 & 9.429008 & 4.959464 \\
\hline$N$ & -8.690359 & 7.854349 & 3.658146 \\
\hline C & -9.953936 & 7.451763 & 3.088772 \\
\hline C & -10.766323 & 6.542229 & 3.995444 \\
\hline $\mathrm{O}$ & -11.807588 & 6.068902 & 3.559164 \\
\hline C & -9.761142 & 6.816141 & 1.689857 \\
\hline $\mathrm{O}$ & -10.954305 & 6.903463 & 0.953096 \\
\hline C & -9.239228 & 5.382323 & 1.729283 \\
\hline $\mathrm{H}$ & -7.846118 & 7.427536 & 3.334427 \\
\hline $\mathrm{H}$ & -10.571638 & 8.331037 & 2.962658 \\
\hline $\mathrm{H}$ & -9.046018 & 7.439524 & 1.169869 \\
\hline $\mathrm{H}$ & -11.641554 & 6.500755 & 1.473063 \\
\hline $\mathrm{H}$ & -8.968246 & 5.061445 & 0.731314 \\
\hline $\mathrm{H}$ & -8.362371 & 5.288787 & 2.354506 \\
\hline $\mathrm{H}$ & -10.000981 & 4.705865 & 2.098583 \\
\hline $\mathrm{N}$ & -10.353337 & 6.327943 & 5.240900 \\
\hline C & -11.259093 & 5.732447 & 6.212924 \\
\hline$C$ & -12.445055 & 6.656022 & 6.502021 \\
\hline
\end{tabular}




\begin{tabular}{|c|c|c|c|}
\hline $\mathrm{O}$ & -13.529726 & 6.188271 & 6.758159 \\
\hline C & -10.538025 & 5.397307 & 7.526339 \\
\hline C & -9.534251 & 4.235052 & 7.447051 \\
\hline C & -8.716965 & 4.184331 & 8.741485 \\
\hline C & -10.219852 & 2.887533 & 7.196333 \\
\hline $\mathrm{H}$ & -9.463052 & 6.657570 & 5.550986 \\
\hline $\mathrm{H}$ & -11.697039 & 4.842319 & 5.794053 \\
\hline $\mathrm{H}$ & -10.028513 & 6.287162 & 7.878980 \\
\hline $\mathrm{H}$ & -11.302479 & 5.156613 & 8.259987 \\
\hline $\mathrm{H}$ & -8.847326 & 4.423758 & 6.628936 \\
\hline $\mathrm{H}$ & -7.988825 & 3.378599 & 8.709769 \\
\hline $\mathrm{H}$ & -8.182037 & 5.114034 & 8.898260 \\
\hline $\mathrm{H}$ & -9.358174 & 4.014320 & 9.603606 \\
\hline $\mathrm{H}$ & -9.479647 & 2.092158 & 7.178431 \\
\hline $\mathrm{H}$ & -10.931103 & 2.661970 & 7.988749 \\
\hline $\mathrm{H}$ & -10.749907 & 2.858178 & 6.251424 \\
\hline$N$ & -12.200391 & 7.978986 & 6.519086 \\
\hline C & -13.272865 & 8.918063 & 6.674440 \\
\hline C & -14.047138 & 9.177161 & 5.399140 \\
\hline $\mathrm{H}$ & -14.886714 & 9.870642 & 5.514692 \\
\hline $\mathrm{O}$ & -13.792339 & 8.699104 & 4.343338 \\
\hline $\mathrm{H}$ & -11.339485 & 8.323326 & 6.149711 \\
\hline $\mathrm{H}$ & -13.966260 & 8.566385 & 7.428079 \\
\hline $\mathrm{H}$ & -12.873616 & 9.866157 & 7.021585 \\
\hline$N$ & -11.400139 & 10.603601 & -3.013218 \\
\hline $\mathrm{H}$ & -11.151156 & 10.793474 & -2.062887 \\
\hline C & -11.816457 & 9.206991 & -3.172692 \\
\hline C & -11.420798 & 8.859979 & -4.600829 \\
\hline $\mathrm{O}$ & -10.381146 & 8.297520 & -4.846936 \\
\hline C & -13.274697 & 8.886451 & -2.781041 \\
\hline
\end{tabular}




\begin{tabular}{|c|c|c|c|}
\hline C & -13.514119 & 9.244426 & -1.302467 \\
\hline C & -13.600730 & 7.414103 & -3.063630 \\
\hline C & -14.953833 & 9.045731 & -0.824926 \\
\hline $\mathrm{H}$ & -12.140937 & 11.235286 & $6-3.252954$ \\
\hline $\mathrm{H}$ & -11.162892 & 8.607041 & -2.555403 \\
\hline $\mathrm{H}$ & -13.942062 & 9.511037 & -3.379532 \\
\hline $\mathrm{H}$ & -12.841512 & 8.656501 & -0.682289 \\
\hline $\mathrm{H}$ & -13.250226 & 10.285287 & $7-1.143016$ \\
\hline $\mathrm{H}$ & -14.652033 & 7.209116 & -2.907368 \\
\hline $\mathrm{H}$ & -13.370171 & 7.129386 & -4.086046 \\
\hline $\mathrm{H}$ & -13.034226 & 6.765433 & -2.402795 \\
\hline $\mathrm{H}$ & -15.063132 & 9.412642 & 0.189358 \\
\hline $\mathrm{H}$ & -15.657291 & 9.588307 & -1.451064 \\
\hline $\mathrm{H}$ & -15.244825 & 8.001845 & -0.821495 \\
\hline$N$ & -12.217767 & 9.294713 & -5.606266 \\
\hline $\mathrm{H}$ & -13.139261 & 9.595970 & -5.384728 \\
\hline C & -11.972872 & 8.843880 & -6.959258 \\
\hline $\mathrm{H}$ & -12.033858 & 7.767145 & -7.039985 \\
\hline $\mathrm{H}$ & -12.729057 & 9.278149 & -7.601893 \\
\hline C & -10.601635 & 9.233244 & -7.517693 \\
\hline $\mathrm{O}$ & -9.996941 & 8.496732 & -8.241527 \\
\hline$N$ & -10.162845 & 10.477780 & -7.182625 \\
\hline $\mathrm{H}$ & -10.711033 & 11.007782 & $2-6.544746$ \\
\hline C & -8.894341 & 11.023967 & -7.637100 \\
\hline $\mathrm{H}$ & -8.789698 & 10.891248 & -8.702753 \\
\hline $\mathrm{H}$ & -8.878586 & 12.081461 & -7.407589 \\
\hline C & -7.672564 & 10.359017 & -6.988311 \\
\hline $\mathrm{O}$ & -6.753256 & 9.935092 & -7.630831 \\
\hline$N$ & -7.659106 & 10.352139 & -5.619638 \\
\hline $\mathrm{H}$ & -8.482447 & 10.628022 & -5.133559 \\
\hline
\end{tabular}




\begin{tabular}{|c|c|c|c|}
\hline C & -6.495253 & 9.961264 & -4.832348 \\
\hline $\mathrm{H}$ & -5.599197 & 10.377765 & -5.264338 \\
\hline $\mathrm{H}$ & -6.616278 & 10.359356 & -3.832341 \\
\hline C & -6.268292 & 8.463912 & -4.714277 \\
\hline $\mathrm{O}$ & -5.133740 & 8.047342 & -4.711620 \\
\hline$N$ & -7.321026 & 7.642803 & -4.555739 \\
\hline C & -7.043152 & 6.284788 & -4.116082 \\
\hline C & -6.395851 & 5.449242 & -5.213188 \\
\hline $\mathrm{O}$ & -5.518651 & 4.674607 & -4.918154 \\
\hline C & -8.232787 & 5.569469 & -3.436228 \\
\hline C & -9.368455 & 5.183222 & -4.399094 \\
\hline C & -8.696989 & 6.392754 & -2.228906 \\
\hline C & -10.549754 & 4.494052 & -3.715373 \\
\hline $\mathrm{H}$ & -8.255737 & 7.997406 & -4.569992 \\
\hline $\mathrm{H}$ & -6.261691 & 6.342851 & -3.373705 \\
\hline $\mathrm{H}$ & -7.807220 & 4.647354 & -3.048787 \\
\hline $\mathrm{H}$ & -9.726255 & 6.052590 & -4.935186 \\
\hline $\mathrm{H}$ & -8.960269 & 4.506274 & -5.145546 \\
\hline $\mathrm{H}$ & -9.400871 & 5.838844 & -1.623588 \\
\hline $\mathrm{H}$ & -7.854458 & 6.643370 & -1.592293 \\
\hline $\mathrm{H}$ & -9.177962 & 7.313866 & -2.533136 \\
\hline $\mathrm{H}$ & -11.255362 & 4.128107 & -4.455475 \\
\hline $\mathrm{H}$ & -10.227063 & 3.646217 & -3.122038 \\
\hline $\mathrm{H}$ & -11.086774 & 5.173295 & -3.065412 \\
\hline $\mathrm{N}$ & -6.814645 & 5.564832 & -6.493391 \\
\hline $\mathrm{C}$ & -6.106146 & 4.822474 & -7.516656 \\
\hline$C$ & -4.760276 & 5.444472 & -7.899124 \\
\hline 0 & -3.890142 & 4.715858 & -8.316310 \\
\hline $\mathrm{C}$ & -6.967005 & 4.499892 & -8.746620 \\
\hline C & -8.037268 & 3.418486 & -8.510343 \\
\hline
\end{tabular}




$\begin{array}{llll}\mathrm{C} & -7.451480 & 2.077185 & -8.040920 \\ \mathrm{~N} & -8.389453 & 0.950985 & -8.074694 \\ \mathrm{C} & -9.199263 & 0.573556 & -7.081841 \\ \mathrm{~N} & -9.462412 & 1.417955 & -6.095954 \\ \mathrm{~N} & -9.735558 & -0.626717 & -7.080755 \\ \mathrm{H} & -7.489451 & 6.258135 & -6.735507 \\ \mathrm{H} & -5.795978 & 3.898611 & -7.061617 \\ \mathrm{H} & -7.450710 & 5.400293 & -9.113841 \\ \mathrm{H} & -6.296125 & 4.164688 & -9.530075 \\ \mathrm{H} & -8.778088 & 3.780379 & -7.803276 \\ \mathrm{H} & -8.564870 & 3.256187 & -9.444433 \\ \mathrm{H} & -6.626428 & 1.798640 & -8.683547 \\ \mathrm{H} & -7.051772 & 2.146842 & -7.038095 \\ \mathrm{H} & -8.319794 & 0.321084 & -8.843001 \\ \mathrm{H} & -9.316663 & 4.592108 & -4.340345 \\ \mathrm{H} & -9.210871 & 2.374884 & -6.171143 \\ \mathrm{H} & -1.723040 & 6.464253 & -4.671160 \\ \mathrm{H} & -9.976329 & 1.152578 & -5.272038 \\ \mathrm{H} & -9.387084 & -1.392206 & -7.629023 \\ \mathrm{H} & -10.455807 & -0.867823 & -6.418365 \\ \mathrm{H} & -4.571383 & 6.757521 & -7.721942 \\ \mathrm{H} & -5.312316 & 7.350933 & -7.425893 \\ \mathrm{H} & -3.257811 & 7.352384 & -8.011367 \\ \mathrm{H} & -2.933574 & 7.093271 & -9.006665 \\ \mathrm{H} & -3.359220 & 8.427155 & -7.938262 \\ \mathrm{H} & -1.067310 & 6.644126 & -7.392501 \\ \mathrm{H} & -6.057556 & -3.382764\end{array}$




\begin{tabular}{|c|c|c|c|}
\hline C & -1.724829 & 6.352659 & -2.158853 \\
\hline C & -2.065567 & 8.573569 & -3.416052 \\
\hline $\mathrm{H}$ & -3.535171 & 7.069255 & -5.539484 \\
\hline $\mathrm{H}$ & -0.755152 & 6.905360 & -4.861604 \\
\hline $\mathrm{H}$ & -3.376293 & 6.879800 & -3.397955 \\
\hline $\mathrm{H}$ & -1.939342 & 6.893014 & -1.256070 \\
\hline $\mathrm{H}$ & -2.140806 & 5.355748 & -2.055884 \\
\hline $\mathrm{H}$ & -0.652641 & 6.275472 & -2.228560 \\
\hline $\mathrm{H}$ & -2.803049 & 9.103886 & -2.830544 \\
\hline $\mathrm{H}$ & -1.078020 & 8.814606 & -3.037070 \\
\hline $\mathrm{H}$ & -2.144829 & 8.956383 & -4.426977 \\
\hline$N$ & -2.474273 & 4.124764 & -4.663714 \\
\hline C & -2.164153 & 2.717524 & -4.734152 \\
\hline C & -1.265510 & 2.444099 & -5.932795 \\
\hline $\mathrm{O}$ & -0.395981 & 1.606821 & -5.875409 \\
\hline$C$ & -3.408743 & 1.806684 & -4.774960 \\
\hline$C$ & -4.360591 & 1.970565 & -3.580069 \\
\hline$C$ & -5.448858 & 0.884496 & -3.485617 \\
\hline$N$ & -5.007890 & -0.292811 & -2.757433 \\
\hline$C$ & -5.827923 & -1.254618 & -2.564041 \\
\hline $\mathrm{N}$ & -7.132237 & -1.284998 & -3.041769 \\
\hline$N$ & -5.529119 & -2.307063 & -1.768413 \\
\hline $\mathrm{H}$ & -3.401435 & 4.451781 & -4.832822 \\
\hline $\mathrm{H}$ & -1.576351 & 2.442291 & -3.871004 \\
\hline $\mathrm{H}$ & -3.956426 & 1.985242 & -5.698780 \\
\hline $\mathrm{H}$ & -3.044991 & 0.786278 & -4.822098 \\
\hline $\mathrm{H}$ & -3.794925 & 1.985472 & -2.660604 \\
\hline $\mathrm{H}$ & -4.853529 & 2.927337 & -3.654816 \\
\hline $\mathrm{H}$ & -6.318404 & 1.325198 & -2.997289 \\
\hline $\mathrm{H}$ & -5.765468 & 0.631256 & -4.501251 \\
\hline
\end{tabular}




\begin{tabular}{|c|c|c|c|}
\hline $\mathrm{H}$ & -7.270081 & -0.782411 & -3.890557 \\
\hline $\mathrm{H}$ & -7.547128 & -2.198086 & -3.082229 \\
\hline $\mathrm{H}$ & -4.605959 & -2.417238 & -1.395079 \\
\hline $\mathrm{H}$ & -6.033341 & -3.156447 & -1.906095 \\
\hline$N$ & -1.459759 & 3.193337 & -7.035104 \\
\hline $\mathrm{H}$ & -2.264571 & 3.769495 & -7.144196 \\
\hline C & -0.595832 & 2.983550 & -8.179089 \\
\hline $\mathrm{H}$ & -0.940093 & 3.626187 & -8.979240 \\
\hline $\mathrm{H}$ & -0.614272 & 1.957428 & -8.516706 \\
\hline C & 0.858332 & 3.326033 & -7.861294 \\
\hline $\mathrm{O}$ & 1.786295 & 2.682497 & -8.284242 \\
\hline$N$ & 1.007444 & 4.396007 & -7.064155 \\
\hline $\mathrm{H}$ & 0.213477 & 4.959530 & -6.860689 \\
\hline C & 2.282309 & 4.880648 & -6.640293 \\
\hline $\mathrm{H}$ & 2.918466 & 5.143147 & -7.476195 \\
\hline $\mathrm{H}$ & 2.121614 & 5.773614 & -6.050368 \\
\hline C & 3.094243 & 3.926494 & -5.780092 \\
\hline $\mathrm{O}$ & 4.300279 & 4.045687 & -5.701465 \\
\hline$N$ & 2.428061 & 2.983367 & -5.101940 \\
\hline C & 3.101641 & 2.134963 & -4.154867 \\
\hline C & 4.282232 & 1.343822 & -4.722385 \\
\hline $\mathrm{O}$ & 5.201595 & 1.052678 & -3.983728 \\
\hline C & 2.031200 & 1.219459 & -3.563912 \\
\hline C & 2.428279 & 0.354765 & -2.373247 \\
\hline C & 1.133390 & -0.209096 & -1.810740 \\
\hline$N$ & 1.349169 & -1.210787 & -0.786501 \\
\hline C & 0.367647 & -2.053261 & -0.419186 \\
\hline$N$ & -0.791012 & -2.021153 & -1.034330 \\
\hline$N$ & 0.596603 & -2.937678 & 0.523714 \\
\hline & 1.433627 & 2.938694 & -5.174913 \\
\hline
\end{tabular}




\begin{tabular}{|c|c|c|c|}
\hline $\mathrm{H}$ & 3.548704 & 2.735628 & -3.371667 \\
\hline $\mathrm{H}$ & 1.222666 & 1.874552 & -3.257062 \\
\hline $\mathrm{H}$ & 1.623769 & 0.598124 & -4.352985 \\
\hline $\mathrm{H}$ & 3.090659 & -0.451492 & -2.668342 \\
\hline $\mathrm{H}$ & 2.949837 & 0.944516 & -1.622870 \\
\hline $\mathrm{H}$ & 0.514363 & 0.599529 & -1.433668 \\
\hline $\mathrm{H}$ & 0.590414 & -0.682622 & -2.616027 \\
\hline $\mathrm{H}$ & 2.060887 & -1.044140 & -0.110411 \\
\hline $\mathrm{H}$ & -1.129920 & -1.169038 & -1.431095 \\
\hline $\mathrm{H}$ & -1.541386 & -2.654010 & -0.762410 \\
\hline $\mathrm{H}$ & 1.513623 & -3.109826 & 0.865267 \\
\hline $\mathrm{H}$ & -0.183083 & -3.502307 & 0.855343 \\
\hline$N$ & 4.281487 & 0.944901 & -6.004141 \\
\hline $\mathrm{H}$ & 3.540371 & 1.224938 & -6.613418 \\
\hline C & 5.363433 & 0.116651 & -6.504612 \\
\hline $\mathrm{H}$ & 5.138944 & -0.142419 & -7.531832 \\
\hline $\mathrm{H}$ & 5.441320 & -0.797906 & -5.938283 \\
\hline C & 6.736191 & 0.756138 & -6.475981 \\
\hline $\mathrm{O}$ & 7.738526 & 0.089729 & -6.364633 \\
\hline$N$ & 6.766107 & 2.085332 & -6.608300 \\
\hline $\mathrm{H}$ & 5.912156 & 2.604985 & -6.573201 \\
\hline C & 8.005486 & 2.823552 & -6.551065 \\
\hline $\mathrm{H}$ & 8.709854 & 2.439480 & -7.275335 \\
\hline $\mathrm{H}$ & 7.800291 & 3.854267 & -6.807648 \\
\hline C & 8.748798 & 2.826930 & -5.213288 \\
\hline $\mathrm{O}$ & 9.889951 & 3.236315 & -5.161832 \\
\hline$N$ & 8.104016 & 2.338735 & -4.145826 \\
\hline C & 8.685994 & 2.174934 & -2.824675 \\
\hline C & 9.950570 & 1.305606 & -2.832425 \\
\hline 0 & 10.769365 & 1.388724 & -1.931295 \\
\hline
\end{tabular}




\begin{tabular}{|c|c|c|c|}
\hline C & 7.582130 & 1.508244 & -1.973555 \\
\hline C & 7.780996 & 1.384533 & -0.463019 \\
\hline C & 7.441450 & 2.654429 & 0.326731 \\
\hline C & 7.331647 & 2.387428 & 1.824123 \\
\hline$N$ & 8.671949 & 2.354031 & 2.481140 \\
\hline $\mathrm{O}$ & 11.043286 & 1.617612 & 0.892719 \\
\hline $\mathrm{H}$ & 10.893052 & 1.828799 & -0.029133 \\
\hline $\mathrm{H}$ & 11.521828 & 0.790985 & 0.841668 \\
\hline $\mathrm{H}$ & 7.158097 & 2.035734 & -4.258562 \\
\hline $\mathrm{H}$ & 8.985493 & 3.132409 & -2.419326 \\
\hline $\mathrm{H}$ & 6.666251 & 2.053993 & -2.156744 \\
\hline $\mathrm{H}$ & 7.412207 & 0.521513 & -2.388659 \\
\hline $\mathrm{H}$ & 7.101135 & 0.605589 & -0.131601 \\
\hline $\mathrm{H}$ & 8.776310 & 1.030766 & -0.228981 \\
\hline $\mathrm{H}$ & 8.155104 & 3.454515 & 0.151101 \\
\hline $\mathrm{H}$ & 6.476503 & 3.027407 & -0.001413 \\
\hline $\mathrm{H}$ & 6.771589 & 3.159838 & 2.327782 \\
\hline $\mathrm{H}$ & 6.850425 & 1.441442 & 2.018892 \\
\hline $\mathrm{H}$ & 9.382571 & 1.890136 & 1.923742 \\
\hline $\mathrm{H}$ & 8.991993 & 3.324207 & 2.635424 \\
\hline $\mathrm{H}$ & 8.618503 & 1.876206 & 3.368828 \\
\hline$N$ & 10.062466 & 0.448215 & -3.837530 \\
\hline C & 11.109251 & -0.537627 & -4.069489 \\
\hline C & 12.486201 & 0.023149 & -4.427400 \\
\hline $\mathrm{O}$ & 13.443843 & -0.705691 & -4.301267 \\
\hline C & 10.700734 & -1.443986 & -5.252716 \\
\hline C & 9.562929 & -2.442696 & -4.997444 \\
\hline C & 9.130895 & -3.067095 & -6.327950 \\
\hline C & 9.990812 & -3.533456 & -4.015394 \\
\hline $\mathrm{H}$ & 9.324093 & 0.446344 & -4.510536 \\
\hline
\end{tabular}




\begin{tabular}{|c|c|c|c|}
\hline $\mathrm{H}$ & 11.264778 & -1.125208 & -3.176521 \\
\hline $\mathrm{H}$ & 10.433734 & -0.798323 & -6.084015 \\
\hline $\mathrm{H}$ & 11.583574 & -1.996197 & -5.551819 \\
\hline $\mathrm{H}$ & 8.706548 & -1.911906 & -4.593160 \\
\hline $\mathrm{H}$ & 8.329842 & -3.783539 & -6.173274 \\
\hline $\mathrm{H}$ & 8.773221 & -2.310164 & -7.014248 \\
\hline $\mathrm{H}$ & 9.957864 & -3.594938 & -6.796442 \\
\hline $\mathrm{H}$ & 9.201223 & -4.263707 & -3.875504 \\
\hline $\mathrm{H}$ & 10.860934 & -4.059422 & -4.394350 \\
\hline $\mathrm{H}$ & 10.243969 & -3.136808 & -3.036935 \\
\hline$N$ & 12.574050 & 1.261829 & -4.919244 \\
\hline C & 13.814624 & 1.844749 & -5.393663 \\
\hline C & 14.958706 & 2.033567 & -4.379164 \\
\hline $\mathrm{O}$ & 16.013158 & 2.414783 & -4.812030 \\
\hline C & 13.497165 & 3.171410 & -6.086959 \\
\hline $\mathrm{O}$ & 12.917964 & 4.088335 & -5.202640 \\
\hline $\mathrm{H}$ & 11.756425 & 1.833577 & -4.917248 \\
\hline $\mathrm{H}$ & 14.248583 & 1.177768 & -6.129163 \\
\hline $\mathrm{H}$ & 14.420310 & 3.590389 & -6.455646 \\
\hline $\mathrm{H}$ & 12.846429 & 2.977487 & -6.933720 \\
\hline $\mathrm{H}$ & 11.970594 & 4.079224 & -5.267509 \\
\hline$N$ & 14.792295 & 1.722326 & -3.074279 \\
\hline C & 15.928362 & 1.647498 & -2.176930 \\
\hline C & 16.804572 & 0.409919 & -2.402499 \\
\hline $\mathrm{O}$ & 17.934404 & 0.393867 & -1.987966 \\
\hline C & 15.495618 & 1.578402 & -0.707341 \\
\hline C & 14.639479 & 2.735871 & -0.198089 \\
\hline C & 14.286573 & 2.489893 & 1.269178 \\
\hline$N$ & 13.261352 & 3.384797 & 1.791148 \\
\hline C & 13.438934 & 4.535615 & 2.4192 \\
\hline
\end{tabular}




\begin{tabular}{|c|c|c|c|}
\hline$N$ & 14.656613 & 5.010999 & 2.656427 \\
\hline $\mathrm{O}$ & 15.029030 & 7.467122 & 4.210782 \\
\hline $\mathrm{H}$ & 14.745418 & 8.353611 & 3.971764 \\
\hline $\mathrm{H}$ & 15.747617 & 7.573218 & 4.821163 \\
\hline$N$ & 12.388927 & 5.224132 & 2.815771 \\
\hline $\mathrm{H}$ & 13.901038 & 1.419628 & -2.758344 \\
\hline $\mathrm{H}$ & 16.562064 & 2.504331 & -2.346854 \\
\hline $\mathrm{H}$ & 14.954846 & 0.650683 & -0.551461 \\
\hline $\mathrm{H}$ & 16.407670 & 1.509060 & -0.124688 \\
\hline $\mathrm{H}$ & 15.157351 & 3.683476 & -0.314932 \\
\hline $\mathrm{H}$ & 13.720110 & 2.817115 & -0.766163 \\
\hline $\mathrm{H}$ & 13.890152 & 1.490595 & 1.376643 \\
\hline $\mathrm{H}$ & 15.166171 & 2.533074 & 1.900653 \\
\hline $\mathrm{H}$ & 12.321274 & 3.089093 & 1.619810 \\
\hline $\mathrm{H}$ & 15.464549 & 4.518437 & 2.358435 \\
\hline $\mathrm{H}$ & 14.791850 & 5.865912 & 3.168142 \\
\hline $\mathrm{H}$ & 11.433471 & 4.944259 & 2.657765 \\
\hline $\mathrm{H}$ & 12.481277 & 6.067154 & 3.336059 \\
\hline$N$ & 16.200238 & -0.648980 & -2.967241 \\
\hline C & 16.870796 & -1.911508 & -3.168197 \\
\hline C & 17.669503 & -1.889228 & -4.468054 \\
\hline $\mathrm{H}$ & 18.063310 & -0.909605 & -4.748073 \\
\hline $\mathrm{O}$ & 17.875929 & -2.852970 & -5.130598 \\
\hline C & 15.868043 & -3.070177 & -3.149486 \\
\hline C & 15.324788 & -3.399916 & -1.791982 \\
\hline$N$ & 14.403993 & -2.627607 & -1.112039 \\
\hline $\mathrm{O}$ & 12.508231 & -0.434478 & -0.465182 \\
\hline $\mathrm{H}$ & 13.146153 & -1.037701 & -0.850756 \\
\hline $\mathrm{H}$ & 12.028078 & -0.039074 & -1.184664 \\
\hline C & 15.619975 & -4.490463 & -1.049798 \\
\hline
\end{tabular}




\begin{tabular}{|c|c|c|c|}
\hline$C$ & 14.172980 & -3.251840 & -0.003576 \\
\hline $\mathrm{N}$ & 14.883327 & -4.381982 & 0.103080 \\
\hline $\mathrm{H}$ & 15.285740 & -0.550231 & -3.352920 \\
\hline $\mathrm{H}$ & 17.596095 & -2.030812 & -2.370971 \\
\hline $\mathrm{H}$ & 15.050489 & -2.842195 & -3.824652 \\
\hline $\mathrm{H}$ & 16.368753 & -3.943989 & -3.544107 \\
\hline $\mathrm{H}$ & 16.275364 & -5.314216 & -1.235376 \\
\hline $\mathrm{H}$ & 13.494777 & -2.922413 & 0.757508 \\
\hline $\mathrm{H}$ & 14.782798 & -5.064870 & 0.821794 \\
\hline 0 & -14.930263 & -2.440032 & 5.283656 \\
\hline $\mathrm{H}$ & -15.070599 & -1.569671 & 4.919845 \\
\hline $\mathrm{H}$ & -14.427377 & -2.308097 & 6.080381 \\
\hline 0 & -12.772909 & -1.570133 & 7.335218 \\
\hline $\mathrm{H}$ & -12.084057 & -2.061818 & 7.768504 \\
\hline $\mathrm{H}$ & -12.983745 & -0.851100 & 7.918196 \\
\hline 0 & -11.882749 & -0.958225 & 4.588006 \\
\hline $\mathrm{H}$ & -11.986359 & -1.108925 & 5.524154 \\
\hline $\mathrm{H}$ & -12.698520 & -0.532607 & 4.317364 \\
\hline 0 & -13.470540 & -1.498157 & 1.152937 \\
\hline $\mathrm{H}$ & -12.611059 & -1.070319 & 1.129708 \\
\hline $\mathrm{H}$ & -13.392767 & -2.234664 & 1.762625 \\
\hline 0 & -7.422954 & 2.197725 & -0.103554 \\
\hline $\mathrm{H}$ & -7.555241 & 2.263681 & 0.846107 \\
\hline $\mathrm{H}$ & -6.505638 & 1.973138 & -0.203986 \\
\hline 0 & -7.633101 & 2.150031 & 2.721242 \\
\hline $\mathrm{H}$ & -8.410996 & 1.691130 & 3.030900 \\
\hline $\mathrm{H}$ & -7.483601 & 2.881792 & 3.315363 \\
\hline 0 & -9.179938 & 0.349107 & -1.275921 \\
\hline $\mathrm{H}$ & -8.589391 & 1.014686 & -0.904213 \\
\hline H & -8.610112 & -0.340096 & -1.612837 \\
\hline
\end{tabular}




\begin{tabular}{|c|c|c|c|}
\hline 0 & -13.000534 & -1.922754 & -1.654893 \\
\hline $\mathrm{H}$ & -13.314173 & -1.992742 & -0.752983 \\
\hline $\mathrm{H}$ & -13.713485 & -2.169468 & -2.235174 \\
\hline 0 & -13.813492 & 1.512328 & 0.356300 \\
\hline $\mathrm{H}$ & -14.241974 & 0.684947 & 0.545414 \\
\hline $\mathrm{H}$ & -12.889308 & 1.336384 & 0.542820 \\
\hline 0 & -14.679677 & 5.246409 & 4.124678 \\
\hline $\mathrm{H}$ & -14.013136 & 5.837092 & 3.789073 \\
\hline $\mathrm{H}$ & -14.614470 & 5.349385 & 5.069876 \\
\hline 0 & -14.702604 & 2.662671 & 2.986968 \\
\hline $\mathrm{H}$ & -14.903288 & 3.540962 & 3.323119 \\
\hline $\mathrm{H}$ & -14.603995 & 2.715563 & 2.041436 \\
\hline 0 & -13.336717 & 0.833135 & -2.396289 \\
\hline $\mathrm{H}$ & -13.633173 & 1.331508 & -1.635880 \\
\hline $\mathrm{H}$ & -13.077609 & -0.017755 & -2.039831 \\
\hline 0 & -14.474167 & -1.111029 & -4.142076 \\
\hline $\mathrm{H}$ & -14.414266 & -0.261514 & -3.708659 \\
\hline $\mathrm{H}$ & -15.298045 & -1.130810 & -4.611127 \\
\hline 0 & -10.572453 & -2.940303 & -3.030550 \\
\hline $\mathrm{H}$ & -10.892042 & -2.579323 & -3.853822 \\
\hline $\mathrm{H}$ & -11.254298 & -2.731306 & -2.397683 \\
\hline 0 & -11.266099 & 0.240199 & 0.789947 \\
\hline $\mathrm{H}$ & -10.601653 & 0.163090 & 0.104503 \\
\hline $\mathrm{H}$ & -10.806266 & 0.507340 & 1.591006 \\
\hline 0 & -10.208600 & 0.991664 & 3.285826 \\
\hline $\mathrm{H}$ & -10.576781 & 0.309085 & 3.844813 \\
\hline $\mathrm{H}$ & -10.727483 & 1.781199 & 3.462469 \\
\hline 0 & -11.956667 & 3.113274 & 3.704819 \\
\hline $\mathrm{H}$ & -11.911088 & 4.063934 & 3.653883 \\
\hline $\mathrm{H}$ & -12.872018 & 2.889008 & 3.552708 \\
\hline
\end{tabular}




$\begin{array}{llll}\mathrm{O} & -10.746486 & 0.954037 & -3.524517 \\ \mathrm{H} & -11.593341 & 1.223036 & -3.167728 \\ \mathrm{H} & -10.181604 & 0.783524 & -2.763460 \\ \mathrm{O} & -14.430372 & -0.068004 & 3.680697 \\ \mathrm{H} & -14.660205 & 0.857483 & 3.580218 \\ \mathrm{H} & -14.386823 & -0.433381 & 2.802221 \\ \mathrm{O} & -13.081569 & -3.333714 & 3.367965 \\ \mathrm{H} & -13.830636 & -3.189182 & 3.954855 \\ \mathrm{H} & -12.406101 & -2.767136 & 3.737728 \\ \mathrm{O} & -11.697057 & -1.220663 & -5.029854 \\ \mathrm{H} & -12.648835 & -1.299146 & -4.990487 \\ \mathrm{H} & -11.458123 & -0.552442 & -4.387631\end{array}$

PROTON CONFIGURATION, GIVEN BY NOMINAL CHARGES: R300(0),Y266(0),E183(0) POTENTIAL: $0 \mathrm{mV}$

$\begin{array}{llll}\mathrm{N} & 15.802748 & -6.133741 & 5.261069 \\ \mathrm{H} & 15.000474 & -5.906329 & 5.815964 \\ \mathrm{C} & 15.709624 & -5.529811 & 3.929295 \\ \mathrm{C} & 15.001670 & -6.466409 & 2.931375 \\ \mathrm{O} & 14.254140 & -6.041260 & 2.065796 \\ \mathrm{C} & 17.109416 & -5.209681 & 3.391311 \\ \mathrm{H} & 16.607142 & -5.796305 & 5.751991 \\ \mathrm{H} & 15.121687 & -4.621509 & 3.935920 \\ \mathrm{H} & 17.063954 & -4.816033 & 2.382904 \\ \mathrm{H} & 17.577240 & -4.457711 & 4.016567 \\ \mathrm{H} & 17.739550 & -6.092207 & 3.389811 \\ \mathrm{~N} & 15.285550 & -7.763112 & 3.044991 \\ \mathrm{H} & 15.877215 & -8.030363 & 3.802303\end{array}$




\begin{tabular}{|c|c|c|c|}
\hline$C$ & 14.673853 & -8.778914 & 2.186456 \\
\hline $\mathrm{H}$ & 14.746006 & -8.500238 & 1.147858 \\
\hline $\mathrm{H}$ & 15.190942 & -9.717305 & 2.336458 \\
\hline$C$ & 13.193214 & -8.946854 & 2.530571 \\
\hline $\mathrm{O}$ & 12.362027 & -9.161640 & 1.687330 \\
\hline $\mathrm{N}$ & 12.866158 & -8.803500 & 3.829326 \\
\hline $\mathrm{H}$ & 13.587727 & -8.723517 & 4.508537 \\
\hline$C$ & 11.484286 & -8.958399 & 4.289736 \\
\hline $\mathrm{H}$ & 11.482590 & -8.954602 & 5.371574 \\
\hline $\mathrm{H}$ & 11.055077 & -9.884466 & 3.940086 \\
\hline$C$ & 10.593529 & -7.816666 & 3.783179 \\
\hline $\mathrm{O}$ & 9.450421 & -8.019874 & 3.471227 \\
\hline $\mathrm{N}$ & 11.170827 & -6.604632 & 3.745066 \\
\hline$C$ & 10.507953 & -5.436464 & 3.221573 \\
\hline$C$ & 10.309540 & -5.486674 & 1.704078 \\
\hline 0 & 9.264435 & -5.084642 & 1.249788 \\
\hline$C$ & 11.252668 & -4.174356 & 3.686533 \\
\hline $\mathrm{O}$ & 12.642423 & -4.322692 & 3.673183 \\
\hline $\mathrm{H}$ & 12.127772 & -6.510127 & 3.994548 \\
\hline $\mathrm{H}$ & 9.504595 & -5.385282 & 3.611648 \\
\hline $\mathrm{H}$ & 10.937622 & -3.329139 & 3.084844 \\
\hline $\mathrm{H}$ & 10.985755 & -3.974887 & 4.713991 \\
\hline $\mathrm{H}$ & 12.971644 & -4.488421 & 2.798266 \\
\hline $\mathrm{N}$ & 11.292688 & -5.962586 & 0.920590 \\
\hline$C$ & 11.037128 & -6.153732 & -0.504232 \\
\hline$C$ & 9.910206 & -7.168917 & -0.707747 \\
\hline 0 & 9.151731 & -7.071253 & -1.644702 \\
\hline$C$ & 12.331963 & -6.504276 & -1.299021 \\
\hline$C$ & 12.565671 & -8.002012 & -1.535208 \\
\hline 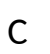 & 12.339033 & -5.762081 & -2.6368 \\
\hline
\end{tabular}




\begin{tabular}{|c|c|c|c|}
\hline $\mathrm{H}$ & 12.130428 & -6.325788 & 1.320764 \\
\hline $\mathrm{H}$ & 10.642682 & -5.226036 & -0.887172 \\
\hline $\mathrm{H}$ & 13.157179 & -6.123167 & -0.707998 \\
\hline $\mathrm{H}$ & 13.520521 & -8.137387 & -2.032441 \\
\hline $\mathrm{H}$ & 12.581601 & -8.579208 & -0.621618 \\
\hline $\mathrm{H}$ & 11.805032 & -8.418528 & -2.188472 \\
\hline $\mathrm{H}$ & 13.238657 & -5.997828 & -3.194163 \\
\hline $\mathrm{H}$ & 11.484320 & -6.047105 & -3.240534 \\
\hline $\mathrm{H}$ & 12.313833 & -4.688852 & -2.490505 \\
\hline$N$ & 9.817331 & -8.136373 & 0.212020 \\
\hline $\mathrm{H}$ & 10.569257 & -8.257922 & 0.851718 \\
\hline C & 8.868022 & -9.241609 & 0.120497 \\
\hline $\mathrm{H}$ & 9.237689 & -10.037783 & 0.754129 \\
\hline $\mathrm{H}$ & 8.809266 & -9.613071 & -0.890983 \\
\hline C & 7.451044 & -8.911795 & 0.540059 \\
\hline $\mathrm{O}$ & 6.533445 & -9.381790 & -0.092114 \\
\hline$N$ & 7.258889 & -8.114639 & 1.597322 \\
\hline C & 5.914032 & -7.670855 & 1.882615 \\
\hline C & 5.458354 & -6.661318 & 0.834886 \\
\hline $\mathrm{O}$ & 4.274943 & -6.583092 & 0.580055 \\
\hline C & 5.655596 & -7.187100 & 3.323779 \\
\hline C & 5.841147 & -8.335144 & 4.321046 \\
\hline C & 6.461916 & -5.955941 & 3.742489 \\
\hline $\mathrm{H}$ & 8.032219 & -7.837947 & 2.162579 \\
\hline $\mathrm{H}$ & 5.253503 & -8.507948 & 1.717107 \\
\hline $\mathrm{H}$ & 4.605128 & -6.912388 & 3.327921 \\
\hline $\mathrm{H}$ & 5.548252 & -8.015614 & 5.315475 \\
\hline $\mathrm{H}$ & 5.227594 & -9.189776 & 4.055306 \\
\hline $\mathrm{H}$ & 6.874357 & -8.660447 & 4.365866 \\
\hline $\mathrm{H}$ & 6.110686 & -5.597222 & 4.704005 \\
\hline
\end{tabular}




\begin{tabular}{|c|c|c|c|}
\hline $\mathrm{H}$ & 7.511554 & -6.196499 & 3.848301 \\
\hline $\mathrm{H}$ & 6.368667 & -5.140781 & 3.032580 \\
\hline$N$ & 6.354726 & -5.914430 & 0.181730 \\
\hline C & 5.931727 & -5.102530 & -0.947122 \\
\hline C & 5.527744 & -5.988931 & -2.133641 \\
\hline $\mathrm{O}$ & 4.492212 & -5.764840 & -2.716587 \\
\hline C & 6.987312 & -4.041396 & -1.317312 \\
\hline C & 7.147864 & -3.028089 & -0.170286 \\
\hline C & 6.594155 & -3.335295 & -2.619478 \\
\hline C & 8.408132 & -2.169551 & -0.276491 \\
\hline $\mathrm{H}$ & 7.320806 & -5.958333 & 0.425317 \\
\hline $\mathrm{H}$ & 5.013931 & -4.602217 & -0.677309 \\
\hline $\mathrm{H}$ & 7.936597 & -4.546701 & -1.467448 \\
\hline $\mathrm{H}$ & 6.267486 & -2.384975 & -0.148062 \\
\hline $\mathrm{H}$ & 7.177721 & -3.549449 & 0.777112 \\
\hline $\mathrm{H}$ & 7.269413 & -2.518046 & -2.832373 \\
\hline $\mathrm{H}$ & 6.620301 & -4.003373 & -3.470330 \\
\hline $\mathrm{H}$ & 5.588856 & -2.930740 & -2.553669 \\
\hline $\mathrm{H}$ & 8.482391 & -1.500994 & 0.574242 \\
\hline $\mathrm{H}$ & 9.293835 & -2.793589 & -0.281833 \\
\hline $\mathrm{H}$ & 8.420624 & -1.561022 & -1.173564 \\
\hline$N$ & 6.331194 & -7.000322 & -2.485061 \\
\hline $\mathrm{H}$ & 7.231876 & -7.098295 & -2.065280 \\
\hline C & 6.006648 & -7.842851 & -3.616850 \\
\hline $\mathrm{H}$ & 6.851172 & -8.498178 & -3.789603 \\
\hline $\mathrm{H}$ & 5.839077 & -7.256977 & -4.508291 \\
\hline C & 4.755158 & -8.697411 & -3.415597 \\
\hline O & 3.916274 & -8.770019 & -4.282039 \\
\hline$N$ & 4.658416 & -9.353786 & -2.252550 \\
\hline 11 & 5.399685 & -9.303325 & -1.586413 \\
\hline
\end{tabular}




\begin{tabular}{|c|c|c|c|}
\hline C & 3.490006 & -10.137156 & -1.926906 \\
\hline $\mathrm{H}$ & 3.264766 & -10.851309 & -2.704675 \\
\hline $\mathrm{H}$ & 3.694665 & -10.679454 & -1.012301 \\
\hline C & 2.238767 & -9.284259 & -1.731649 \\
\hline O & 1.159870 & -9.694837 & -2.091310 \\
\hline$N$ & 2.423893 & -8.083818 & -1.184805 \\
\hline C & 1.273961 & -7.190680 & -1.156699 \\
\hline C & 0.821010 & -6.740694 & -2.548666 \\
\hline $\mathrm{O}$ & -0.363806 & -6.622665 & -2.764319 \\
\hline C & 1.484533 & -5.917358 & -0.353069 \\
\hline O & 1.626429 & -6.121778 & 1.018217 \\
\hline $\mathrm{H}$ & 3.320025 & -7.766764 & -0.892777 \\
\hline $\mathrm{H}$ & 0.434883 & -7.723825 & -0.740868 \\
\hline $\mathrm{H}$ & 2.328249 & -5.376915 & -0.756689 \\
\hline $\mathrm{H}$ & 0.592172 & -5.322698 & -0.482288 \\
\hline $\mathrm{H}$ & 2.493379 & -6.471572 & 1.180093 \\
\hline$N$ & 1.741980 & -6.433439 & -3.470856 \\
\hline C & 1.337628 & -6.029174 & -4.806418 \\
\hline C & 0.583618 & -7.161829 & -5.495852 \\
\hline $\mathrm{O}$ & -0.414651 & -6.924998 & -6.142244 \\
\hline C & 2.542054 & -5.518012 & -5.630954 \\
\hline C & 2.941030 & -4.121456 & -5.125238 \\
\hline C & 2.240675 & -5.499481 & -7.135097 \\
\hline C & 4.263526 & -3.607619 & -5.693153 \\
\hline $\mathrm{H}$ & 2.713252 & -6.475322 & -3.248263 \\
\hline $\mathrm{H}$ & 0.609644 & -5.234427 & -4.726866 \\
\hline $\mathrm{H}$ & 3.368439 & -6.201764 & -5.461649 \\
\hline $\mathrm{H}$ & 2.145325 & -3.420994 & -5.372929 \\
\hline $\mathrm{H}$ & 3.016607 & -4.143456 & -4.045455 \\
\hline $\mathrm{H}$ & 3.093572 & -5.123035 & -7.685782 \\
\hline
\end{tabular}




\begin{tabular}{|c|c|c|c|}
\hline $\mathrm{H}$ & 2.028242 & -6.489108 & -7.520646 \\
\hline $\mathrm{H}$ & 1.390108 & -4.863974 & -7.360555 \\
\hline $\mathrm{H}$ & 4.553952 & -2.689064 & -5.193861 \\
\hline $\mathrm{H}$ & 5.059399 & -4.329581 & -5.539671 \\
\hline $\mathrm{H}$ & 4.204307 & -3.396494 & -6.754782 \\
\hline$N$ & 1.052900 & -8.403395 & -5.341579 \\
\hline $\mathrm{H}$ & 1.906718 & -8.561590 & -4.848372 \\
\hline C & 0.292540 & -9.526508 & -5.833032 \\
\hline $\mathrm{H}$ & 0.178789 & -9.493322 & -6.906357 \\
\hline $\mathrm{H}$ & 0.820525 & -10.433212 & -5.566498 \\
\hline C & -1.111732 & -9.575076 & -5.239009 \\
\hline $\mathrm{O}$ & -2.067570 & -9.804500 & -5.945007 \\
\hline$N$ & -1.217901 & -9.367827 & -3.927608 \\
\hline C & -2.522875 & -9.413595 & -3.306249 \\
\hline C & -3.454420 & -8.284898 & -3.755120 \\
\hline 0 & -4.649155 & -8.508635 & -3.796956 \\
\hline $\mathrm{C}$ & -2.424017 & -9.396615 & -1.773672 \\
\hline 0 & -3.663373 & -9.680314 & -1.186800 \\
\hline $\mathrm{H}$ & -0.402368 & -9.276158 & -3.358981 \\
\hline $\mathrm{H}$ & -3.030589 & -10.321873 & -3.598442 \\
\hline $\mathrm{H}$ & -1.733119 & -10.164896 & -1.454967 \\
\hline $\mathrm{H}$ & -2.043728 & -8.437411 & -1.438209 \\
\hline $\mathrm{H}$ & -4.360911 & -9.288971 & -1.698526 \\
\hline $\mathrm{N}$ & -2.922992 & -7.092240 & -4.019939 \\
\hline$C$ & -3.699120 & -5.963151 & -4.507090 \\
\hline$C$ & -4.266581 & -6.254021 & -5.894860 \\
\hline 0 & -5.431084 & -6.023322 & -6.151709 \\
\hline$C$ & -2.797331 & -4.703446 & -4.499222 \\
\hline$C$ & -3.209253 & -3.587394 & -5.442368 \\
\hline C & -4.494906 & -3.057994 & -5.451093 \\
\hline
\end{tabular}




\begin{tabular}{|c|c|c|c|}
\hline C & -2.278404 & -3.070113 & -6.334911 \\
\hline C & -4.840068 & -2.054184 & -6.339080 \\
\hline C & -2.613330 & -2.051179 & -7.212352 \\
\hline C & -3.900316 & -1.543837 & -7.220504 \\
\hline $\mathrm{H}$ & -1.947778 & -6.960565 & -3.851005 \\
\hline $\mathrm{H}$ & -4.557863 & -5.807294 & -3.872755 \\
\hline $\mathrm{H}$ & -2.750007 & -4.341042 & -3.477091 \\
\hline $\mathrm{H}$ & -1.797726 & -5.011419 & -4.768673 \\
\hline $\mathrm{H}$ & -5.237413 & -3.445645 & -4.779343 \\
\hline $\mathrm{H}$ & -1.281404 & -3.473773 & -6.353442 \\
\hline $\mathrm{H}$ & -5.848912 & -1.682233 & -6.350090 \\
\hline $\mathrm{H}$ & -1.874235 & -1.666058 & -7.891564 \\
\hline $\mathrm{H}$ & -4.168910 & -0.763907 & -7.911241 \\
\hline$N$ & -3.424976 & -6.765524 & -6.794730 \\
\hline C & -3.901721 & -7.102960 & -8.117293 \\
\hline C & -5.015665 & -8.146642 & -8.051272 \\
\hline O & -5.986586 & -8.038891 & -8.755275 \\
\hline C & -2.770058 & -7.620611 & -9.008468 \\
\hline$S$ & -1.576341 & -6.355968 & -9.547156 \\
\hline $\mathrm{H}$ & -2.484376 & -6.973635 & -6.530493 \\
\hline $\mathrm{H}$ & -4.350060 & -6.233736 & -8.573782 \\
\hline $\mathrm{H}$ & -2.246139 & -8.436966 & -8.529932 \\
\hline $\mathrm{H}$ & -3.206103 & -7.999536 & -9.923341 \\
\hline $\mathrm{H}$ & -0.946915 & -6.192580 & -8.393968 \\
\hline$N$ & -4.846012 & -9.182107 & -7.204948 \\
\hline $\mathrm{H}$ & -3.988537 & -9.313012 & -6.711513 \\
\hline C & -5.828424 & -10.255158 & -7.239410 \\
\hline $\mathrm{H}$ & -6.001956 & -10.584056 & -8.252976 \\
\hline $\mathrm{H}$ & -5.430288 & -11.085676 & -6.668447 \\
\hline $\mathrm{C}$ & -7.207602 & -9.905697 & -6.656110 \\
\hline
\end{tabular}




\begin{tabular}{|c|c|c|c|}
\hline 0 & -8.221584 & -10.297350 & -7.164233 \\
\hline$N$ & -7.176471 & -9.204614 & -5.516534 \\
\hline C & -8.405087 & -8.888694 & -4.813583 \\
\hline C & -9.295053 & -7.892144 & -5.549413 \\
\hline $\mathrm{O}$ & -10.487679 & -7.899805 & -5.403543 \\
\hline C & -8.123801 & -8.374777 & -3.392059 \\
\hline C & -7.335396 & -7.061709 & -3.261420 \\
\hline C & -8.076275 & -5.748480 & -3.272464 \\
\hline $\mathrm{O}$ & -9.376603 & -5.830778 & -3.312950 \\
\hline $\mathrm{H}$ & -9.777446 & -4.958579 & -3.223018 \\
\hline 0 & -7.492178 & -4.695781 & -3.211041 \\
\hline $\mathrm{H}$ & -6.297958 & -8.952920 & -5.115385 \\
\hline $\mathrm{H}$ & -9.002589 & -9.785822 & -4.732765 \\
\hline $\mathrm{H}$ & -9.073941 & -8.290667 & -2.885504 \\
\hline $\mathrm{H}$ & -7.556829 & -9.148504 & -2.885423 \\
\hline $\mathrm{H}$ & -6.809518 & -7.062844 & -2.312834 \\
\hline $\mathrm{H}$ & -6.568258 & -6.978572 & -4.012532 \\
\hline$N$ & -8.659483 & -6.956499 & -6.285211 \\
\hline C & -9.413283 & -5.938871 & -6.996586 \\
\hline C & -10.435645 & -6.574016 & -7.933625 \\
\hline $\mathrm{H}$ & -10.226589 & -7.596577 & -8.250185 \\
\hline 0 & -11.392722 & -5.979786 & -8.314266 \\
\hline C & -8.412166 & -5.020115 & -7.727878 \\
\hline 0 & -7.712074 & -4.259095 & -6.766165 \\
\hline C & -9.057410 & -4.040272 & -8.697726 \\
\hline $\mathrm{H}$ & -7.703276 & -7.105189 & -6.523395 \\
\hline $\mathrm{H}$ & -9.976016 & -5.342482 & -6.289748 \\
\hline $\mathrm{H}$ & -7.706396 & -5.637513 & -8.273658 \\
\hline $\mathrm{H}$ & -6.962397 & -4.744461 & -6.429734 \\
\hline $\mathrm{H}$ & -8.292053 & -3.357331 & -9.048877 \\
\hline
\end{tabular}




\begin{tabular}{|c|c|c|c|}
\hline $\mathrm{H}$ & -9.491093 & -4.546015 & -9.549108 \\
\hline $\mathrm{H}$ & -9.844202 & -3.472736 & -8.213192 \\
\hline$N$ & -12.664563 & -9.429889 & 3.164222 \\
\hline $\mathrm{H}$ & -12.922766 & -9.946953 & 2.349065 \\
\hline C & -11.516487 & -8.546511 & 2.893191 \\
\hline C & -10.519175 & -8.500792 & 4.031525 \\
\hline 0 & -9.392645 & -8.085782 & 3.829688 \\
\hline C & -11.932060 & -7.082274 & 2.633439 \\
\hline $\mathrm{O}$ & -12.474845 & -6.633573 & 3.848899 \\
\hline C & -12.935884 & -6.926080 & 1.497481 \\
\hline $\mathrm{H}$ & -13.458516 & -8.892653 & 3.454407 \\
\hline $\mathrm{H}$ & -10.971693 & -8.917900 & 2.037330 \\
\hline $\mathrm{H}$ & -11.033097 & -6.527056 & 2.397600 \\
\hline $\mathrm{H}$ & -12.541560 & -5.680343 & 3.845233 \\
\hline $\mathrm{H}$ & -13.128231 & -5.872036 & 1.327427 \\
\hline $\mathrm{H}$ & -12.569422 & -7.352180 & 0.570999 \\
\hline $\mathrm{H}$ & -13.881137 & -7.394637 & 1.743802 \\
\hline $\mathrm{N}$ & -10.912409 & -8.912490 & 5.227241 \\
\hline $\mathrm{H}$ & -11.836108 & -9.282675 & 5.278305 \\
\hline$C$ & -10.117759 & -8.874740 & 6.429074 \\
\hline $\mathrm{H}$ & -10.065494 & -7.874086 & 6.829758 \\
\hline $\mathrm{H}$ & -10.611782 & -9.494111 & 7.168415 \\
\hline$C$ & -8.667437 & -9.345701 & 6.335156 \\
\hline 0 & -7.838228 & -8.745009 & 6.973389 \\
\hline $\mathrm{N}$ & -8.330303 & -10.424052 & 5.604750 \\
\hline $\mathrm{H}$ & -9.032680 & -10.929901 & 5.114057 \\
\hline$C$ & -6.931824 & -10.817710 & 5.495285 \\
\hline $\mathrm{H}$ & -6.890673 & -11.799038 & 5.039984 \\
\hline $\mathrm{H}$ & -6.481718 & -10.878653 & 6.473277 \\
\hline 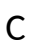 & -6.079123 & -9.857234 & 4.64436 \\
\hline
\end{tabular}




\begin{tabular}{|c|c|c|c|}
\hline $\mathrm{O}$ & -4.909138 & -9.699485 & 4.884645 \\
\hline$N$ & -6.757552 & -9.275458 & 3.661449 \\
\hline C & -6.038043 & -8.278086 & 2.880352 \\
\hline C & -6.032951 & -6.932366 & 3.598651 \\
\hline $\mathrm{O}$ & -5.071207 & -6.205153 & 3.495938 \\
\hline C & -6.365409 & -8.211815 & 1.375404 \\
\hline C & -7.563889 & -7.414372 & 0.907779 \\
\hline C & -7.554388 & -6.022942 & 0.960824 \\
\hline C & -8.650173 & -8.045961 & 0.317469 \\
\hline C & -8.615467 & -5.285316 & 0.463498 \\
\hline C & -9.703604 & -7.310186 & -0.200852 \\
\hline C & -9.695539 & -5.927888 & -0.119682 \\
\hline $\mathrm{H}$ & -7.751975 & -9.304257 & 3.637764 \\
\hline $\mathrm{H}$ & -5.007752 & -8.584803 & 2.922330 \\
\hline $\mathrm{H}$ & -5.479883 & -7.798417 & 0.905878 \\
\hline $\mathrm{H}$ & -6.438147 & -9.235389 & 1.027308 \\
\hline $\mathrm{H}$ & -6.703622 & -5.508566 & 1.370048 \\
\hline $\mathrm{H}$ & -8.660485 & -9.119306 & 0.232669 \\
\hline $\mathrm{H}$ & -8.582834 & -4.211204 & 0.502953 \\
\hline $\mathrm{H}$ & -10.517229 & -7.813391 & -0.690783 \\
\hline $\mathrm{H}$ & -10.505638 & -5.358031 & -0.535137 \\
\hline$N$ & -7.068614 & -6.639245 & 4.386842 \\
\hline C & -7.049148 & -5.501546 & 5.271473 \\
\hline C & -5.961075 & -5.671111 & 6.331199 \\
\hline $\mathrm{O}$ & -5.372862 & -4.692340 & 6.740093 \\
\hline C & -8.450381 & -5.333199 & 5.899359 \\
\hline C & -8.751341 & -3.960707 & 6.461252 \\
\hline C & -8.965609 & -2.885350 & 5.600933 \\
\hline C & -8.874792 & -3.754368 & 7.827316 \\
\hline C & -9.286704 & -1.632532 & 6.093501 \\
\hline
\end{tabular}




\begin{tabular}{|c|c|c|c|}
\hline C & -9.195603 & -2.500391 & 8.327972 \\
\hline C & -9.398100 & -1.436530 & 7.465149 \\
\hline $\mathrm{H}$ & -7.903950 & -7.178532 & 4.319669 \\
\hline $\mathrm{H}$ & -6.779097 & -4.608454 & 4.727083 \\
\hline $\mathrm{H}$ & -9.172151 & -5.551465 & 5.119998 \\
\hline $\mathrm{H}$ & -8.582433 & -6.083469 & 6.668561 \\
\hline $\mathrm{H}$ & -8.872206 & -3.025792 & 4.536719 \\
\hline $\mathrm{H}$ & -8.718599 & -4.572081 & 8.508817 \\
\hline $\mathrm{H}$ & -9.448415 & -0.817091 & 5.412546 \\
\hline $\mathrm{H}$ & -9.275206 & -2.356397 & 9.390682 \\
\hline $\mathrm{H}$ & -9.617167 & -0.457767 & 7.853658 \\
\hline$N$ & -5.685488 & -6.900498 & 6.761219 \\
\hline $\mathrm{H}$ & -6.315177 & -7.639717 & 6.543351 \\
\hline C & -4.631720 & -7.174764 & 7.711953 \\
\hline $\mathrm{H}$ & -4.686018 & -6.524319 & 8.571651 \\
\hline $\mathrm{H}$ & -4.754942 & -8.196190 & 8.048555 \\
\hline$C$ & -3.227715 & -7.014292 & 7.133762 \\
\hline 0 & -2.367537 & -6.481107 & 7.793480 \\
\hline $\mathrm{N}$ & -3.003625 & -7.484309 & 5.900788 \\
\hline$C$ & -1.725803 & -7.275557 & 5.240277 \\
\hline$C$ & -1.513059 & -5.794499 & 4.908379 \\
\hline 0 & -0.427999 & -5.287363 & 5.091746 \\
\hline$C$ & -1.611442 & -8.184485 & 3.996883 \\
\hline$C$ & -0.441793 & -7.783753 & 3.093626 \\
\hline$C$ & -1.478461 & -9.650672 & 4.424243 \\
\hline $\mathrm{H}$ & -3.713061 & -8.010858 & 5.437440 \\
\hline $\mathrm{H}$ & -0.929713 & -7.514148 & 5.930938 \\
\hline $\mathrm{H}$ & -2.527989 & -8.070681 & 3.423080 \\
\hline $\mathrm{H}$ & -0.359090 & -8.489264 & 2.273441 \\
\hline $\mathrm{H}$ & -0.559254 & -6.798317 & 2.663451 \\
\hline
\end{tabular}




\begin{tabular}{|c|c|c|c|}
\hline $\mathrm{H}$ & 0.496694 & -7.796616 & 3.639845 \\
\hline $\mathrm{H}$ & -1.469878 & -10.294669 & 3.550747 \\
\hline $\mathrm{H}$ & -0.544786 & -9.805130 & 4.959131 \\
\hline $\mathrm{H}$ & -2.292382 & -9.972110 & 5.060412 \\
\hline$N$ & -2.558118 & -5.116075 & 4.420471 \\
\hline C & -2.426588 & -3.732439 & 4.010585 \\
\hline C & -2.324387 & -2.803144 & 5.221773 \\
\hline $\mathrm{O}$ & -1.475246 & -1.932802 & 5.235110 \\
\hline C & -3.575238 & -3.292506 & 3.091832 \\
\hline C & -3.628136 & -3.979870 & 1.713639 \\
\hline C & -2.479503 & -3.590899 & 0.790552 \\
\hline $\mathrm{O}$ & -1.342591 & -3.954180 & 1.143503 \\
\hline $\mathrm{O}$ & -2.739628 & -2.938473 & -0.234984 \\
\hline $\mathrm{H}$ & -3.410076 & -5.596120 & 4.225320 \\
\hline $\mathrm{H}$ & -1.497605 & -3.622503 & 3.481084 \\
\hline $\mathrm{H}$ & -4.519995 & -3.463047 & 3.596899 \\
\hline $\mathrm{H}$ & -3.484559 & -2.219278 & 2.954851 \\
\hline $\mathrm{H}$ & -3.614135 & -5.053802 & 1.840203 \\
\hline $\mathrm{H}$ & -4.563888 & -3.711327 & 1.237959 \\
\hline$N$ & -3.177610 & -2.969248 & 6.241614 \\
\hline C & -2.974605 & -2.214912 & 7.461881 \\
\hline C & -1.640742 & -2.582382 & 8.119778 \\
\hline $\mathrm{O}$ & -0.980839 & -1.722762 & 8.662099 \\
\hline C & -4.109964 & -2.244122 & 8.495206 \\
\hline $\mathrm{O}$ & -4.313550 & -3.515362 & 9.037236 \\
\hline C & -5.394365 & -1.610222 & 7.972251 \\
\hline $\mathrm{H}$ & -3.839910 & -3.713084 & 6.219782 \\
\hline $\mathrm{H}$ & -2.847728 & -1.180019 & 7.184399 \\
\hline $\mathrm{H}$ & -3.742723 & -1.638615 & 9.314637 \\
\hline & -4.785192 & -4.049063 & 8.409040 \\
\hline
\end{tabular}




\begin{tabular}{|c|c|c|c|}
\hline $\mathrm{H}$ & -6.133761 & -1.601223 & 8.763995 \\
\hline $\mathrm{H}$ & -5.216438 & -0.585765 & 7.660682 \\
\hline $\mathrm{H}$ & -5.803610 & -2.157134 & 7.133035 \\
\hline$N$ & -1.210544 & -3.848625 & 8.020389 \\
\hline $\mathrm{H}$ & -1.843776 & -4.589353 & 7.811082 \\
\hline C & 0.050815 & -4.201498 & 8.622318 \\
\hline $\mathrm{H}$ & 0.083492 & -3.965337 & 9.676576 \\
\hline $\mathrm{H}$ & 0.189537 & -5.267439 & 8.496148 \\
\hline C & 1.221575 & -3.477235 & 7.972298 \\
\hline $\mathrm{O}$ & 2.158276 & -3.080226 & 8.626546 \\
\hline$N$ & 1.159934 & -3.329648 & 6.637167 \\
\hline C & 2.250933 & -2.710311 & 5.932569 \\
\hline C & 2.307638 & -1.197139 & 6.114518 \\
\hline $\mathrm{O}$ & 3.391932 & -0.651233 & 6.088007 \\
\hline$C$ & 2.246399 & -3.076065 & 4.437518 \\
\hline$S$ & 1.378895 & -1.930579 & 3.318776 \\
\hline $\mathrm{H}$ & 0.431044 & -3.787567 & 6.128394 \\
\hline $\mathrm{H}$ & 3.178921 & -3.066031 & 6.357491 \\
\hline $\mathrm{H}$ & 3.270579 & -3.062558 & 4.087529 \\
\hline $\mathrm{H}$ & 1.859735 & -4.077278 & 4.311634 \\
\hline $\mathrm{H}$ & 0.164234 & -1.986988 & 3.842867 \\
\hline$N$ & 1.164542 & -0.526618 & 6.278802 \\
\hline C & 1.235250 & 0.904035 & 6.497816 \\
\hline C & 1.821366 & 1.192585 & 7.867627 \\
\hline $\mathrm{O}$ & 2.444194 & 2.205491 & 8.080769 \\
\hline C & -0.076753 & 1.680972 & 6.250513 \\
\hline C & -1.140346 & 1.495259 & 7.344063 \\
\hline C & -0.598007 & 1.407534 & 4.835329 \\
\hline C & -2.418168 & 2.302508 & 7.103229 \\
\hline $\mathrm{H}$ & 0.290780 & -1.009638 & 6.2593 \\
\hline
\end{tabular}




\begin{tabular}{|c|c|c|c|}
\hline $\mathrm{H}$ & 1.960746 & 1.295620 & 5.801259 \\
\hline $\mathrm{H}$ & 0.231373 & 2.719880 & 6.294890 \\
\hline $\mathrm{H}$ & -1.387546 & 0.448886 & 7.459256 \\
\hline $\mathrm{H}$ & -0.718301 & 1.808024 & 8.294147 \\
\hline $\mathrm{H}$ & -1.340271 & 2.142554 & 4.552689 \\
\hline $\mathrm{H}$ & 0.207234 & 1.461563 & 4.108774 \\
\hline $\mathrm{H}$ & -1.053415 & 0.429760 & 4.751920 \\
\hline $\mathrm{H}$ & -3.057469 & 2.263117 & 7.979825 \\
\hline $\mathrm{H}$ & -2.199018 & 3.347843 & 6.901398 \\
\hline $\mathrm{H}$ & -2.989606 & 1.916613 & 6.265996 \\
\hline$N$ & 1.617625 & 0.243785 & 8.787960 \\
\hline $\mathrm{H}$ & 0.981755 & -0.499819 & 8.600173 \\
\hline C & 2.050768 & 0.429130 & 10.154569 \\
\hline $\mathrm{H}$ & 1.549636 & -0.311413 & 10.765252 \\
\hline $\mathrm{H}$ & 1.802070 & 1.414861 & 10.518231 \\
\hline C & 3.555361 & 0.267358 & 10.306953 \\
\hline $\mathrm{O}$ & 4.214056 & 1.036300 & 10.953169 \\
\hline$N$ & 4.098247 & -0.786619 & 9.658822 \\
\hline $\mathrm{H}$ & 3.511996 & -1.446043 & 9.192314 \\
\hline$c$ & 5.527090 & -0.933177 & 9.619740 \\
\hline $\mathrm{H}$ & 5.763342 & -1.888623 & 9.166057 \\
\hline $\mathrm{H}$ & 5.955740 & -0.908823 & 10.610386 \\
\hline C & 6.228689 & 0.159508 & 8.816932 \\
\hline $\mathrm{O}$ & 7.355392 & 0.482952 & 9.101689 \\
\hline $\mathrm{N}$ & 5.555629 & 0.653155 & 7.778697 \\
\hline C & 6.120025 & 1.811755 & 7.133439 \\
\hline C & 5.515616 & 3.112176 & 7.655833 \\
\hline $\mathrm{H}$ & 5.026430 & 3.053767 & 8.626533 \\
\hline O & 5.611046 & 4.137864 & 7.055268 \\
\hline 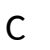 & 6.008296 & 1.737836 & 5.608566 \\
\hline
\end{tabular}




\begin{tabular}{|c|c|c|c|}
\hline C & 6.784554 & 0.591938 & 4.994027 \\
\hline C & 8.162248 & 0.463588 & 5.177200 \\
\hline C & 6.135465 & -0.354819 & 4.207908 \\
\hline C & 8.871836 & -0.563028 & 4.569974 \\
\hline C & 6.843548 & -1.379535 & 3.599952 \\
\hline C & 8.214360 & -1.484291 & 3.769247 \\
\hline $\mathrm{H}$ & 4.630602 & 0.352148 & 7.562127 \\
\hline $\mathrm{H}$ & 7.158632 & 1.845458 & 7.426406 \\
\hline $\mathrm{H}$ & 4.964569 & 1.648830 & 5.333407 \\
\hline $\mathrm{H}$ & 6.358299 & 2.683683 & 5.209822 \\
\hline $\mathrm{H}$ & 8.682240 & 1.149650 & 5.824100 \\
\hline $\mathrm{H}$ & 5.068106 & -0.303880 & 4.103691 \\
\hline $\mathrm{H}$ & 9.931830 & -0.647230 & 4.736986 \\
\hline $\mathrm{H}$ & 6.321910 & -2.105105 & 3.001245 \\
\hline $\mathrm{H}$ & 8.752085 & -2.283556 & 3.292809 \\
\hline$N$ & 12.383857 & 9.886082 & -1.227636 \\
\hline $\mathrm{H}$ & 11.630001 & 10.191693 & -1.814265 \\
\hline C & 12.112715 & 8.542590 & -0.708150 \\
\hline C & 11.085237 & 8.693381 & 0.406429 \\
\hline O & 10.095792 & 7.994430 & 0.438015 \\
\hline C & 13.391383 & 7.886848 & -0.167975 \\
\hline C & 14.475049 & 7.620190 & -1.218230 \\
\hline$S$ & 13.868045 & 6.540985 & -2.547125 \\
\hline C & 15.347148 & 6.442829 & -3.583754 \\
\hline $\mathrm{H}$ & 13.198078 & 9.888251 & -1.809547 \\
\hline $\mathrm{H}$ & 11.654092 & 7.888713 & -1.438492 \\
\hline $\mathrm{H}$ & 13.818114 & 8.515339 & 0.607837 \\
\hline $\mathrm{H}$ & 13.111438 & 6.947613 & 0.295098 \\
\hline $\mathrm{H}$ & 14.847990 & 8.546716 & -1.640023 \\
\hline $\mathrm{H}$ & 15.316364 & 7.145977 & -0.725587 \\
\hline
\end{tabular}




\begin{tabular}{|c|c|c|c|}
\hline $\mathrm{H}$ & 15.107796 & 5.789869 & -4.409240 \\
\hline $\mathrm{H}$ & 15.617264 & 7.418448 & -3.966705 \\
\hline $\mathrm{H}$ & 16.180919 & 6.022870 & -3.036382 \\
\hline$N$ & 11.295982 & 9.655610 & 1.320992 \\
\hline C & 10.388487 & 9.820145 & 2.439510 \\
\hline C & 9.050916 & 10.452758 & 2.053155 \\
\hline $\mathrm{O}$ & 8.065149 & 10.198791 & 2.699204 \\
\hline C & 11.057152 & 10.594026 & 3.582483 \\
\hline C & 12.252313 & 9.817485 & 4.104239 \\
\hline $\mathrm{O}$ & 13.377804 & 10.107592 & 3.756372 \\
\hline$N$ & 11.979720 & 8.806247 & 4.938560 \\
\hline $\mathrm{H}$ & 12.103934 & 10.232935 & 1.227910 \\
\hline $\mathrm{H}$ & 10.118612 & 8.840609 & 2.799267 \\
\hline $\mathrm{H}$ & 11.411283 & 11.562274 & $\quad 3.251529$ \\
\hline $\mathrm{H}$ & 10.327490 & 10.740272 & 4.368871 \\
\hline $\mathrm{H}$ & 12.739835 & 8.228462 & 5.226151 \\
\hline $\mathrm{H}$ & 11.068087 & 8.398111 & 4.980888 \\
\hline$N$ & 9.009229 & 11.252354 & 0.970760 \\
\hline $\mathrm{H}$ & 9.848677 & 11.443606 & 0.473489 \\
\hline C & 7.733763 & 11.672740 & 0.429119 \\
\hline $\mathrm{H}$ & 7.906541 & 12.427355 & -0.327496 \\
\hline $\mathrm{H}$ & 7.112401 & 12.098598 & 1.199931 \\
\hline C & 6.943222 & 10.524008 & -0.199576 \\
\hline $\mathrm{O}$ & 5.741296 & 10.577846 & -0.230249 \\
\hline$N$ & 7.645152 & 9.495791 & -0.720961 \\
\hline C & 6.983405 & 8.293529 & -1.197890 \\
\hline C & 6.479952 & 7.422750 & -0.035888 \\
\hline $\mathrm{O}$ & 5.395673 & 6.896153 & -0.116478 \\
\hline C & 7.877311 & 7.526978 & -2.201847 \\
\hline C & 8.140504 & 8.400451 & -3.443380 \\
\hline
\end{tabular}




\begin{tabular}{|c|c|c|c|}
\hline C & 7.242399 & 6.187890 & -2.595638 \\
\hline C & 9.233707 & 7.867386 & -4.372701 \\
\hline $\mathrm{H}$ & 8.609818 & 9.417835 & -0.499519 \\
\hline $\mathrm{H}$ & 6.080261 & 8.599108 & -1.703230 \\
\hline $\mathrm{H}$ & 8.825439 & 7.321574 & -1.712961 \\
\hline $\mathrm{H}$ & 7.211700 & 8.507628 & -3.999226 \\
\hline $\mathrm{H}$ & 8.417932 & 9.400511 & -3.127307 \\
\hline $\mathrm{H}$ & 7.870034 & 5.672220 & -3.311277 \\
\hline $\mathrm{H}$ & 7.114159 & 5.534008 & -1.741643 \\
\hline $\mathrm{H}$ & 6.265695 & 6.329547 & -3.046508 \\
\hline $\mathrm{H}$ & 9.404524 & 8.562101 & -5.188613 \\
\hline $\mathrm{H}$ & 10.175992 & 7.740289 & -3.846574 \\
\hline $\mathrm{H}$ & 8.975894 & 6.911343 & -4.813183 \\
\hline$N$ & 7.241240 & 7.312631 & 1.071146 \\
\hline C & 6.758910 & 6.575094 & 2.227283 \\
\hline C & 5.394786 & 7.116569 & 2.675935 \\
\hline $\mathrm{O}$ & 4.464116 & 6.382429 & 2.904781 \\
\hline C & 7.717131 & 6.680898 & 3.424162 \\
\hline C & 9.152789 & 6.174196 & 3.253817 \\
\hline O & 10.028205 & 6.826612 & 3.815162 \\
\hline $\mathrm{O}$ & 9.354669 & 5.124504 & 2.592663 \\
\hline $\mathrm{H}$ & 8.192235 & 7.612284 & 1.048095 \\
\hline $\mathrm{H}$ & 6.588370 & 5.543074 & 1.962990 \\
\hline $\mathrm{H}$ & 7.778016 & 7.705052 & 3.761116 \\
\hline $\mathrm{H}$ & 7.279348 & 6.108991 & 4.239792 \\
\hline$N$ & 5.320965 & 8.452235 & 2.829043 \\
\hline $\mathrm{H}$ & 6.136771 & 9.010091 & 2.691104 \\
\hline C & 4.116095 & 9.086744 & 3.304149 \\
\hline $\mathrm{H}$ & 4.329186 & 10.135870 & 3.467203 \\
\hline - & 3.790535 & 8.665205 & 4.241985 \\
\hline
\end{tabular}




\begin{tabular}{|c|c|c|c|}
\hline$C$ & 2.949105 & 9.005195 & 2.329710 \\
\hline $\mathrm{O}$ & 1.807928 & 9.098765 & 2.718544 \\
\hline $\mathrm{N}$ & 3.277772 & 8.856085 & 1.043021 \\
\hline $\mathrm{H}$ & 4.236666 & 8.887923 & 0.786687 \\
\hline$C$ & 2.315110 & 8.922066 & -0.012995 \\
\hline $\mathrm{H}$ & 2.845449 & 9.132165 & -0.933090 \\
\hline $\mathrm{H}$ & 1.621128 & 9.734982 & 0.150817 \\
\hline$C$ & 1.437959 & 7.682495 & -0.250768 \\
\hline $\mathrm{O}$ & 0.387946 & 7.788238 & -0.819563 \\
\hline $\mathrm{N}$ & 1.951160 & 6.542334 & 0.253240 \\
\hline$C$ & 1.171912 & 5.351145 & 0.482825 \\
\hline$C$ & 0.361371 & 5.446887 & 1.779559 \\
\hline 0 & -0.831956 & 5.257420 & 1.757791 \\
\hline$C$ & 2.073747 & 4.117060 & 0.486749 \\
\hline $\mathrm{H}$ & 2.855588 & 6.600485 & 0.670987 \\
\hline $\mathrm{H}$ & 0.438235 & 5.266224 & -0.301549 \\
\hline $\mathrm{H}$ & 1.494563 & 3.225435 & 0.701115 \\
\hline $\mathrm{H}$ & 2.537735 & 4.009248 & -0.486920 \\
\hline $\mathrm{H}$ & 2.858441 & 4.202792 & 1.231184 \\
\hline $\mathrm{N}$ & 0.998212 & 5.717252 & 2.934108 \\
\hline$C$ & 0.275445 & 5.525141 & 4.183080 \\
\hline$C$ & -0.868864 & 6.522241 & 4.349713 \\
\hline $\mathrm{O}$ & -1.890221 & 6.183620 & 4.908404 \\
\hline$C$ & 1.199852 & 5.466570 & 5.423842 \\
\hline$C$ & 1.765598 & 6.822965 & 5.871129 \\
\hline$C$ & 2.308303 & 4.425232 & 5.206004 \\
\hline$C$ & 2.607500 & 6.740673 & 7.146901 \\
\hline $\mathrm{H}$ & 1.984395 & 5.864619 & 2.948433 \\
\hline $\mathrm{H}$ & -0.225898 & 4.569811 & 4.123881 \\
\hline $\mathrm{H}$ & 0.553566 & 5.110669 & 6.220525 \\
\hline
\end{tabular}




\begin{tabular}{|c|c|c|c|}
\hline $\mathrm{H}$ & 2.361547 & 7.246916 & 5.069795 \\
\hline $\mathrm{H}$ & 0.948472 & 7.515283 & 6.045784 \\
\hline $\mathrm{H}$ & 2.693298 & 4.073751 & 6.152106 \\
\hline $\mathrm{H}$ & 1.930509 & 3.563554 & 4.664756 \\
\hline $\mathrm{H}$ & 3.138550 & 4.836578 & 4.642139 \\
\hline $\mathrm{H}$ & 2.895374 & 7.736762 & 7.469958 \\
\hline $\mathrm{H}$ & 2.044140 & 6.284611 & 7.955656 \\
\hline $\mathrm{H}$ & 3.513073 & 6.162905 & 7.009565 \\
\hline$N$ & -0.692923 & 7.764629 & 3.879136 \\
\hline $\mathrm{H}$ & 0.180600 & 8.032433 & 3.475516 \\
\hline C & -1.694154 & 8.774355 & 4.102975 \\
\hline $\mathrm{H}$ & -1.314008 & 9.716363 & 3.728426 \\
\hline $\mathrm{H}$ & -1.904515 & 8.893212 & 5.155959 \\
\hline C & -3.041855 & 8.466288 & 3.455299 \\
\hline O & -4.047221 & 8.649548 & 4.101027 \\
\hline$N$ & -3.070667 & 7.966250 & 2.206026 \\
\hline C & -4.406233 & 7.836078 & 1.623995 \\
\hline C & -5.094550 & 6.531640 & 1.984415 \\
\hline $\mathrm{O}$ & -6.304689 & 6.488564 & 1.983499 \\
\hline C & -4.208045 & 8.094939 & 0.123981 \\
\hline C & -2.974220 & 9.003829 & 0.124771 \\
\hline C & -2.092246 & 8.357384 & 1.180601 \\
\hline $\mathrm{H}$ & -5.048940 & 8.599905 & 2.033488 \\
\hline $\mathrm{H}$ & -4.012536 & 7.172667 & -0.407075 \\
\hline $\mathrm{H}$ & -5.088412 & 8.550702 & -0.310665 \\
\hline $\mathrm{H}$ & -2.471857 & 9.079763 & -0.821686 \\
\hline $\mathrm{H}$ & -3.257158 & 10.005150 & 0.440630 \\
\hline $\mathrm{H}$ & -1.588773 & 7.486191 & 0.802424 \\
\hline $\mathrm{H}$ & -1.347523 & 9.028405 & 1.576536 \\
\hline IV & -4.330088 & 5.480246 & 2.318004 \\
\hline
\end{tabular}




\begin{tabular}{|c|c|c|c|}
\hline C & -4.939773 & 4.287578 & 2.879019 \\
\hline C & -5.662807 & 4.598426 & 4.194002 \\
\hline $\mathrm{O}$ & -6.734970 & 4.091019 & 4.436276 \\
\hline C & -3.908856 & 3.172631 & 3.151819 \\
\hline C & -3.498995 & 2.288368 & 1.988751 \\
\hline C & -4.070440 & 1.031025 & 1.821627 \\
\hline$C$ & -2.471099 & 2.643734 & 1.124587 \\
\hline C & -3.608548 & 0.142446 & 0.863177 \\
\hline C & -1.993049 & 1.765001 & 0.169450 \\
\hline C & -2.533348 & 0.493957 & 0.054352 \\
\hline $\mathrm{O}$ & -1.980588 & -0.355925 & -0.831761 \\
\hline $\mathrm{H}$ & -3.339965 & 5.599635 & 2.365121 \\
\hline $\mathrm{H}$ & -5.702994 & 3.930468 & 2.205395 \\
\hline $\mathrm{H}$ & -3.030555 & 3.626387 & 3.598977 \\
\hline $\mathrm{H}$ & -4.338682 & 2.532664 & 3.914101 \\
\hline $\mathrm{H}$ & -4.883486 & 0.726258 & 2.458948 \\
\hline $\mathrm{H}$ & -2.011482 & 3.605910 & 1.209833 \\
\hline $\mathrm{H}$ & -4.057421 & -0.825899 & 0.753641 \\
\hline $\mathrm{H}$ & -1.186879 & 2.052809 & -0.482149 \\
\hline $\mathrm{H}$ & -2.410248 & -1.214754 & -0.789572 \\
\hline$N$ & -5.041506 & 5.405707 & 5.070182 \\
\hline $\mathrm{H}$ & -4.118312 & 5.738167 & 4.882226 \\
\hline C & -5.658300 & 5.744123 & 6.333700 \\
\hline $\mathrm{H}$ & -4.888942 & 6.133471 & 6.989071 \\
\hline $\mathrm{H}$ & -6.095539 & 4.871295 & 6.791254 \\
\hline C & -6.764388 & 6.791694 & 6.209783 \\
\hline $\mathrm{O}$ & -7.810298 & 6.680503 & 6.806277 \\
\hline$N$ & -6.491976 & 7.831950 & 5.415786 \\
\hline $\mathrm{H}$ & -5.600377 & 7.910776 & 4.975696 \\
\hline C & -7.437641 & 8.938719 & 5.201742 \\
\hline
\end{tabular}




\begin{tabular}{|c|c|c|c|}
\hline $\mathrm{H}$ & -6.977888 & 9.611436 & 4.489175 \\
\hline $\mathrm{H}$ & -7.611958 & 9.479724 & 6.120432 \\
\hline C & -8.800673 & 8.534415 & 4.682785 \\
\hline $\mathrm{O}$ & -9.791069 & 9.104447 & 5.066729 \\
\hline$N$ & -8.835835 & 7.546389 & 3.772404 \\
\hline C & -10.092268 & 7.121937 & 3.209379 \\
\hline C & -10.929987 & 6.249957 & 4.136488 \\
\hline $\mathrm{O}$ & -12.007398 & 5.854152 & 3.729266 \\
\hline C & -9.867420 & 6.401904 & 1.860625 \\
\hline $\mathrm{O}$ & -11.079957 & 6.338429 & 1.139119 \\
\hline C & -9.255115 & 5.015759 & 2.015190 \\
\hline $\mathrm{H}$ & -7.982515 & 7.141712 & 3.446454 \\
\hline $\mathrm{H}$ & -10.703100 & 7.995526 & 3.023360 \\
\hline $\mathrm{H}$ & -9.200640 & 7.024912 & 1.279211 \\
\hline $\mathrm{H}$ & -11.780557 & 6.166429 & 1.764307 \\
\hline $\mathrm{H}$ & -9.038669 & 4.594942 & 1.039850 \\
\hline $\mathrm{H}$ & -8.328106 & 5.042938 & 2.566355 \\
\hline $\mathrm{H}$ & -9.940372 & 4.350556 & 2.524124 \\
\hline$N$ & -10.456258 & 5.965807 & 5.348047 \\
\hline C & -11.302903 & 5.322569 & 6.337304 \\
\hline C & -12.343941 & 6.267083 & 6.945964 \\
\hline $\mathrm{O}$ & -13.316478 & 5.802174 & 7.481217 \\
\hline C & -10.474461 & 4.700681 & 7.469339 \\
\hline C & -9.476803 & 3.616299 & 7.037939 \\
\hline C & -8.687130 & 3.140127 & 8.261023 \\
\hline C & -10.151012 & 2.434062 & 6.338097 \\
\hline $\mathrm{H}$ & -9.553545 & 6.284560 & 5.629687 \\
\hline $\mathrm{H}$ & -11.883924 & 4.561948 & 5.842119 \\
\hline $\mathrm{H}$ & -9.932887 & 5.490667 & 7.979045 \\
\hline $\mathrm{H}$ & -11.180362 & 4.285920 & 8.181983 \\
\hline
\end{tabular}




\begin{tabular}{|c|c|c|c|}
\hline $\mathrm{H}$ & -8.769630 & 4.051118 & 6.342279 \\
\hline $\mathrm{H}$ & -7.940700 & 2.402325 & 7.980096 \\
\hline $\mathrm{H}$ & -8.173974 & 3.968077 & 8.739364 \\
\hline $\mathrm{H}$ & -9.342523 & 2.685460 & 9.001295 \\
\hline $\mathrm{H}$ & -9.412913 & 1.679941 & 6.082584 \\
\hline $\mathrm{H}$ & -10.901186 & 1.971142 & 6.977240 \\
\hline $\mathrm{H}$ & -10.637981 & 2.725219 & 5.414616 \\
\hline$N$ & -12.075339 & 7.588921 & 6.914073 \\
\hline C & -13.044269 & 8.545836 & 7.358486 \\
\hline C & -13.963462 & 9.038987 & 6.261640 \\
\hline $\mathrm{H}$ & -14.736248 & 9.737014 & 6.603628 \\
\hline $\mathrm{O}$ & -13.879623 & 8.735855 & 5.118199 \\
\hline $\mathrm{H}$ & -11.334800 & 7.927312 & 6.339070 \\
\hline $\mathrm{H}$ & -13.647074 & 8.109472 & 8.143917 \\
\hline $\mathrm{H}$ & -12.541807 & 9.412364 & 7.780728 \\
\hline$N$ & -11.774269 & 10.301570 & -2.867425 \\
\hline $\mathrm{H}$ & -11.550479 & 10.511687 & -1.915085 \\
\hline C & -12.057381 & 8.873136 & -3.031538 \\
\hline C & -11.637701 & 8.561088 & -4.461752 \\
\hline $\mathrm{O}$ & -10.573975 & 8.047280 & -4.711819 \\
\hline C & -13.477255 & 8.411683 & -2.638576 \\
\hline C & -13.738860 & 8.720923 & -1.152848 \\
\hline C & -13.669542 & 6.921041 & -2.946100 \\
\hline C & -15.150582 & 8.382236 & -0.670443 \\
\hline $\mathrm{H}$ & -12.569189 & 10.862537 & -3.109816 \\
\hline $\mathrm{H}$ & -11.346835 & 8.335175 & -2.419433 \\
\hline $\mathrm{H}$ & -14.202226 & 8.982667 & -3.223557 \\
\hline $\mathrm{H}$ & -13.012665 & 8.183499 & -0.547093 \\
\hline $\mathrm{H}$ & -13.568194 & 9.778381 & -0.975725 \\
\hline & -14.695271 & 6.617766 & -2.780854 \\
\hline
\end{tabular}




\begin{tabular}{|c|c|c|c|}
\hline $\mathrm{H}$ & -13.428081 & 6.677733 & -3.976698 \\
\hline $\mathrm{H}$ & -13.037964 & 6.313969 & -2.305421 \\
\hline $\mathrm{H}$ & -15.290557 & 8.734224 & 0.345229 \\
\hline $\mathrm{H}$ & -15.905575 & 8.856387 & -1.292304 \\
\hline $\mathrm{H}$ & -15.336959 & 7.314989 & -0.667488 \\
\hline$N$ & -12.454351 & 8.971177 & -5.463497 \\
\hline $\mathrm{H}$ & -13.386990 & 9.230152 & -5.234506 \\
\hline C & -12.203840 & 8.540113 & -6.822271 \\
\hline $\mathrm{H}$ & -12.239052 & 7.462990 & -6.914349 \\
\hline $\mathrm{H}$ & -12.972240 & 8.963496 & -7.457590 \\
\hline C & -10.843941 & 8.968308 & -7.379885 \\
\hline $\mathrm{O}$ & -10.218054 & 8.251090 & -8.106106 \\
\hline$N$ & -10.441202 & 10.224953 & $\begin{array}{ll}3 & -7.045037\end{array}$ \\
\hline $\mathrm{H}$ & -11.000653 & 10.735764 & $4 \quad-6.401013$ \\
\hline C & -9.186693 & 10.805485 & -7.496849 \\
\hline $\mathrm{H}$ & -9.082878 & 10.689732 & -8.564582 \\
\hline $\mathrm{H}$ & -9.192905 & 11.859428 & -7.251726 \\
\hline C & -7.950004 & 10.154849 & -6.859773 \\
\hline $\mathrm{O}$ & -7.030748 & 9.742790 & -7.510898 \\
\hline$N$ & -7.924642 & 10.148202 & -5.491991 \\
\hline $\mathrm{H}$ & -8.750879 & 10.403576 & -4.999946 \\
\hline C & -6.746635 & 9.782074 & -4.713273 \\
\hline $\mathrm{H}$ & -5.864645 & 10.225397 & -5.147240 \\
\hline $\mathrm{H}$ & -6.874295 & 10.170116 & -3.710183 \\
\hline C & -6.476702 & 8.292089 & -4.610371 \\
\hline $\mathrm{O}$ & -5.330600 & 7.909733 & -4.664048 \\
\hline$N$ & -7.492467 & 7.436523 & -4.399787 \\
\hline C & -7.153435 & 6.077348 & -3.999693 \\
\hline C & -6.570537 & 5.265334 & -5.149732 \\
\hline O & -5.743984 & 4.414192 & -4.923375 \\
\hline
\end{tabular}




\begin{tabular}{|c|c|c|c|}
\hline C & -8.275357 & 5.318294 & -3.252192 \\
\hline C & -9.452254 & 4.883170 & -4.142003 \\
\hline C & -8.705067 & 6.123713 & -2.020850 \\
\hline C & -10.516487 & 4.070834 & -3.400982 \\
\hline $\mathrm{H}$ & -8.439857 & 7.756500 & -4.382877 \\
\hline $\mathrm{H}$ & -6.326213 & 6.147364 & -3.309479 \\
\hline $\mathrm{H}$ & -7.789055 & 4.415569 & -2.893135 \\
\hline $\mathrm{H}$ & -9.915572 & 5.746170 & -4.604973 \\
\hline $\mathrm{H}$ & -9.065322 & 4.267012 & -4.949600 \\
\hline $\mathrm{H}$ & -9.336642 & 5.531938 & -1.373288 \\
\hline $\mathrm{H}$ & -7.842089 & 6.427344 & -1.436729 \\
\hline $\mathrm{H}$ & -9.259547 & 7.013582 & -2.296123 \\
\hline $\mathrm{H}$ & -11.254875 & 3.692881 & -4.100414 \\
\hline $\mathrm{H}$ & -10.083257 & 3.214659 & -2.897101 \\
\hline $\mathrm{H}$ & -11.040818 & 4.667261 & -2.664049 \\
\hline$N$ & -6.998006 & 5.503105 & -6.407942 \\
\hline C & -6.362224 & 4.816119 & -7.512847 \\
\hline C & -4.962937 & 5.348416 & -7.824774 \\
\hline $\mathrm{O}$ & -4.110131 & 4.591822 & -8.224643 \\
\hline C & -7.258994 & 4.807640 & -8.759840 \\
\hline C & -8.375610 & 3.758623 & -8.670076 \\
\hline C & -7.828880 & 2.341058 & -8.860548 \\
\hline$N$ & -8.821775 & 1.285910 & -8.671057 \\
\hline C & -9.140480 & 0.701623 & -7.518305 \\
\hline$N$ & -8.653572 & 1.117996 & -6.365612 \\
\hline$N$ & -9.980252 & -0.325030 & -7.514862 \\
\hline $\mathrm{H}$ & -7.655215 & 6.233623 & -6.576107 \\
\hline $\mathrm{H}$ & -6.167801 & 3.806302 & -7.192415 \\
\hline $\mathrm{H}$ & -7.691418 & 5.791967 & -8.909186 \\
\hline $\mathrm{H}$ & -6.641402 & 4.606136 & -9.629449 \\
\hline
\end{tabular}




\begin{tabular}{|c|c|c|c|}
\hline $\mathrm{H}$ & -8.889923 & 3.841983 & -7.717773 \\
\hline $\mathrm{H}$ & -9.118264 & 3.951122 & -9.436925 \\
\hline $\mathrm{H}$ & -7.456656 & 2.226713 & -9.869675 \\
\hline $\mathrm{H}$ & -6.987218 & 2.139202 & -8.211451 \\
\hline $\mathrm{H}$ & -9.195402 & 0.868814 & -9.493519 \\
\hline $\mathrm{H}$ & -8.081304 & 1.928561 & -6.314791 \\
\hline $\mathrm{H}$ & -9.071247 & 0.799664 & -5.496124 \\
\hline $\mathrm{H}$ & -10.263431 & -0.722632 & -8.382475 \\
\hline $\mathrm{H}$ & -9.927012 & -0.971018 & -6.738523 \\
\hline$N$ & -4.754067 & 6.653678 & -7.639028 \\
\hline $\mathrm{H}$ & -5.497497 & 7.241151 & -7.336808 \\
\hline C & -3.458658 & 7.285624 & -7.930073 \\
\hline $\mathrm{H}$ & -3.131748 & 7.045217 & -8.929764 \\
\hline $\mathrm{H}$ & -3.589236 & 8.356009 & -7.845070 \\
\hline C & -2.379194 & 6.827494 & -6.960669 \\
\hline $\mathrm{O}$ & -1.247966 & 6.625809 & -7.326991 \\
\hline$N$ & -2.790477 & 6.693948 & -5.694638 \\
\hline C & -1.893827 & 6.400219 & -4.603203 \\
\hline C & -1.572135 & 4.921459 & -4.490809 \\
\hline $\mathrm{O}$ & -0.430339 & 4.569941 & -4.297964 \\
\hline C & -2.493377 & 6.963548 & -3.308186 \\
\hline C & -1.882206 & 6.271030 & -2.091065 \\
\hline C & -2.303139 & 8.486730 & -3.327439 \\
\hline $\mathrm{H}$ & -3.721010 & 6.968701 & -5.464970 \\
\hline $\mathrm{H}$ & -0.939124 & 6.870919 & -4.789568 \\
\hline $\mathrm{H}$ & -3.555952 & 6.750370 & -3.321073 \\
\hline $\mathrm{H}$ & -2.113193 & 6.798492 & -1.184326 \\
\hline $\mathrm{H}$ & -2.261283 & 5.259152 & -1.992765 \\
\hline $\mathrm{H}$ & -0.808109 & 6.233310 & -2.164805 \\
\hline & -3.049481 & 8.984000 & -2.72444 \\
\hline
\end{tabular}




\begin{tabular}{|c|c|c|c|}
\hline $\mathrm{H}$ & -1.319034 & 8.756132 & -2.958611 \\
\hline $\mathrm{H}$ & -2.409773 & 8.878835 & -4.332153 \\
\hline$N$ & -2.575259 & 4.037305 & -4.605964 \\
\hline C & -2.222951 & 2.639263 & -4.694383 \\
\hline C & -1.324197 & 2.410477 & -5.902598 \\
\hline $\mathrm{O}$ & -0.418234 & 1.611602 & -5.856302 \\
\hline C & -3.442005 & 1.700715 & -4.705460 \\
\hline C & -4.272908 & 1.753024 & -3.415302 \\
\hline C & -5.392390 & 0.695236 & -3.355790 \\
\hline$N$ & -4.964371 & -0.487506 & -2.638029 \\
\hline C & -5.736553 & -1.483163 & -2.534551 \\
\hline$N$ & -7.015059 & -1.592161 & -3.091903 \\
\hline$N$ & -5.426364 & -2.549541 & -1.735825 \\
\hline $\mathrm{H}$ & -3.511859 & 4.333369 & -4.781694 \\
\hline $\mathrm{H}$ & -1.608930 & 2.380591 & -3.844315 \\
\hline $\mathrm{H}$ & -4.073469 & 1.938191 & -5.559538 \\
\hline $\mathrm{H}$ & -3.068722 & 0.693524 & -4.856035 \\
\hline $\mathrm{H}$ & -3.627602 & 1.600034 & -2.559519 \\
\hline $\mathrm{H}$ & -4.713334 & 2.737240 & -3.320084 \\
\hline $\mathrm{H}$ & -6.258872 & 1.141773 & -2.868512 \\
\hline $\mathrm{H}$ & -5.707339 & 0.465385 & -4.378258 \\
\hline $\mathrm{H}$ & -7.156105 & -1.056987 & -3.921307 \\
\hline $\mathrm{H}$ & -7.328802 & -2.535277 & -3.219982 \\
\hline $\mathrm{H}$ & -4.495864 & -2.589562 & -1.369950 \\
\hline $\mathrm{H}$ & -5.831305 & -3.430454 & -1.968264 \\
\hline$N$ & -1.546899 & 3.166383 & -6.996490 \\
\hline $\mathrm{H}$ & -2.384008 & 3.695807 & -7.104179 \\
\hline C & -0.684621 & 2.988588 & -8.146855 \\
\hline $\mathrm{H}$ & -1.048786 & 3.629593 & -8.939611 \\
\hline H & -0.677718 & 1.965414 & -8.494111 \\
\hline
\end{tabular}




\begin{tabular}{|c|c|c|c|}
\hline C & 0.761414 & 3.366436 & -7.833196 \\
\hline $\mathrm{O}$ & 1.704631 & 2.751445 & -8.266111 \\
\hline$N$ & 0.886570 & 4.434447 & -7.030068 \\
\hline $\mathrm{H}$ & 0.077947 & 4.970822 & -6.811251 \\
\hline C & 2.150711 & 4.947380 & -6.607804 \\
\hline $\mathrm{H}$ & 2.774349 & 5.237305 & -7.444073 \\
\hline $\mathrm{H}$ & 1.969938 & 5.828493 & -6.005802 \\
\hline C & 2.988861 & 4.006517 & -5.765766 \\
\hline $\mathrm{O}$ & 4.192980 & 4.151955 & -5.695960 \\
\hline$N$ & 2.354379 & 3.042206 & -5.086466 \\
\hline C & 3.081572 & 2.233818 & -4.149153 \\
\hline C & 4.274256 & 1.480442 & -4.743854 \\
\hline $\mathrm{O}$ & 5.224074 & 1.228848 & -4.027510 \\
\hline C & 2.091308 & 1.277985 & -3.483307 \\
\hline C & 2.701082 & 0.445943 & -2.366101 \\
\hline C & 1.630090 & -0.376493 & -1.677118 \\
\hline$N$ & 2.267514 & -1.276559 & -0.727735 \\
\hline C & 1.605599 & -2.275340 & -0.135155 \\
\hline$N$ & 0.319277 & -2.373148 & -0.211845 \\
\hline$N$ & 2.349633 & -3.204568 & 0.493186 \\
\hline $\mathrm{H}$ & 1.359993 & 2.965328 & -5.140961 \\
\hline $\mathrm{H}$ & 3.535366 & 2.865429 & -3.394631 \\
\hline $\mathrm{H}$ & 1.287387 & 1.888614 & -3.085004 \\
\hline $\mathrm{H}$ & 1.642934 & 0.639218 & -4.236031 \\
\hline $\mathrm{H}$ & 3.460178 & -0.218313 & -2.766251 \\
\hline $\mathrm{H}$ & 3.194403 & 1.086214 & -1.640124 \\
\hline $\mathrm{H}$ & 0.929690 & 0.260970 & -1.154057 \\
\hline $\mathrm{H}$ & 1.071727 & -0.947326 & -2.412459 \\
\hline $\mathrm{H}$ & 3.242728 & -1.428775 & -0.853500 \\
\hline $\mathrm{H}$ & -0.224512 & -1.676438 & -0.678853 \\
\hline
\end{tabular}




\begin{tabular}{|c|c|c|c|}
\hline $\mathrm{H}$ & -0.238034 & -3.061896 & 0.324159 \\
\hline $\mathrm{H}$ & 3.185036 & -2.867870 & 0.917627 \\
\hline $\mathrm{H}$ & 1.859640 & -3.863432 & 1.066685 \\
\hline$N$ & 4.255160 & 1.062184 & -6.017655 \\
\hline $\mathrm{H}$ & 3.495761 & 1.315850 & -6.616364 \\
\hline C & 5.356056 & 0.264885 & -6.527831 \\
\hline $\mathrm{H}$ & 5.134253 & 0.006589 & -7.555780 \\
\hline $\mathrm{H}$ & 5.460038 & -0.651013 & -5.967442 \\
\hline C & 6.711559 & 0.940334 & -6.498776 \\
\hline $\mathrm{O}$ & 7.730666 & 0.298281 & -6.395134 \\
\hline$N$ & 6.706442 & 2.270224 & -6.621776 \\
\hline $\mathrm{H}$ & 5.839990 & 2.768362 & -6.583816 \\
\hline C & 7.927348 & 3.039685 & -6.568360 \\
\hline $\mathrm{H}$ & 8.635467 & 2.679711 & -7.301300 \\
\hline $\mathrm{H}$ & 7.693344 & 4.066636 & -6.815194 \\
\hline C & 8.681407 & 3.052067 & -5.236507 \\
\hline $\mathrm{O}$ & 9.813889 & 3.485641 & -5.192020 \\
\hline$N$ & 8.055645 & 2.544411 & -4.166993 \\
\hline C & 8.650716 & 2.387740 & -2.850913 \\
\hline$c$ & 9.934176 & 1.546812 & -2.871461 \\
\hline $\mathrm{O}$ & 10.756469 & 1.643148 & -1.974849 \\
\hline C & 7.568167 & 1.691852 & -1.995590 \\
\hline C & 7.779636 & 1.560365 & -0.487081 \\
\hline C & 7.404784 & 2.811082 & 0.317200 \\
\hline C & 7.314321 & 2.526708 & 1.812828 \\
\hline$N$ & 8.659764 & 2.532590 & 2.459248 \\
\hline $\mathrm{O}$ & 11.041439 & 1.863703 & 0.850900 \\
\hline $\mathrm{H}$ & 10.883284 & 2.079267 & -0.068494 \\
\hline $\mathrm{H}$ & 11.538746 & 1.048805 & 0.791572 \\
\hline & 7.115177 & 2.222033 & -4.274544 \\
\hline
\end{tabular}




\begin{tabular}{|c|c|c|c|}
\hline $\mathrm{H}$ & 8.931345 & 3.349542 & -2.442288 \\
\hline $\mathrm{H}$ & 6.639722 & 2.219138 & -2.168699 \\
\hline $\mathrm{H}$ & 7.417572 & 0.704827 & -2.417773 \\
\hline $\mathrm{H}$ & 7.125707 & 0.758072 & -0.158624 \\
\hline $\mathrm{H}$ & 8.786470 & 1.234814 & -0.261518 \\
\hline $\mathrm{H}$ & 8.091564 & 3.634946 & 0.144614 \\
\hline $\mathrm{H}$ & 6.426425 & 3.156445 & -0.001087 \\
\hline $\mathrm{H}$ & 6.732013 & 3.275340 & 2.327000 \\
\hline $\mathrm{H}$ & 6.865679 & 1.563523 & 2.002905 \\
\hline $\mathrm{H}$ & 9.380389 & 2.094429 & 1.894021 \\
\hline $\mathrm{H}$ & 8.951158 & 3.512073 & 2.617746 \\
\hline $\mathrm{H}$ & 8.627893 & 2.047850 & 3.343990 \\
\hline$N$ & 10.058650 & 0.696606 & -3.881387 \\
\hline C & 11.129472 & -0.259667 & -4.125770 \\
\hline C & 12.489143 & 0.339165 & -4.489112 \\
\hline 0 & 13.465331 & -0.366932 & -4.376726 \\
\hline$C$ & 10.735989 & -1.171172 & -5.309690 \\
\hline$C$ & 9.624889 & -2.198394 & -5.049916 \\
\hline$C$ & 9.198826 & -2.829031 & -6.379392 \\
\hline$C$ & 10.086508 & -3.281167 & -4.074183 \\
\hline $\mathrm{H}$ & 9.318266 & 0.683740 & -4.552079 \\
\hline $\mathrm{H}$ & 11.306775 & -0.846992 & -3.236697 \\
\hline $\mathrm{H}$ & 10.447111 & -0.529551 & -6.136758 \\
\hline $\mathrm{H}$ & 11.630117 & -1.700232 & -5.616872 \\
\hline $\mathrm{H}$ & 8.758645 & -1.689694 & -4.637881 \\
\hline $\mathrm{H}$ & 8.416909 & -3.565845 & -6.221833 \\
\hline $\mathrm{H}$ & 8.817637 & -2.078794 & -7.060355 \\
\hline $\mathrm{H}$ & 10.035197 & -3.334490 & -6.855692 \\
\hline $\mathrm{H}$ & 9.317612 & -4.033170 & -3.933316 \\
\hline П & 10.968253 & -3.782358 & -4.45 \\
\hline
\end{tabular}




\begin{tabular}{|c|c|c|c|}
\hline $\mathrm{H}$ & 10.334401 & -2.881562 & -3.095488 \\
\hline$N$ & 12.542524 & 1.584484 & -4.968577 \\
\hline C & 13.765787 & 2.202097 & -5.444716 \\
\hline C & 14.910696 & 2.409630 & -4.434670 \\
\hline 0 & 15.954544 & 2.815639 & -4.870377 \\
\hline C & 13.411961 & 3.526644 & -6.124218 \\
\hline O & 12.813856 & 4.419940 & -5.228417 \\
\hline $\mathrm{H}$ & 11.710868 & 2.135565 & -4.957977 \\
\hline $\mathrm{H}$ & 14.211829 & 1.552525 & -6.188459 \\
\hline $\mathrm{H}$ & 14.322623 & 3.972013 & -6.493133 \\
\hline $\mathrm{H}$ & 12.762343 & 3.324321 & -6.969834 \\
\hline $\mathrm{H}$ & 11.866893 & 4.390685 & -5.292755 \\
\hline $\mathrm{N}$ & 14.757554 & 2.087271 & -3.130783 \\
\hline$C$ & 15.898589 & 2.034411 & -2.238236 \\
\hline$C$ & 16.805271 & 0.821235 & -2.476649 \\
\hline 0 & 17.935779 & 0.830173 & -2.063942 \\
\hline$C$ & 15.472907 & 1.943926 & -0.767740 \\
\hline$C$ & 14.595386 & 3.079522 & -0.245368 \\
\hline$C$ & 14.258499 & 2.817660 & 1.223026 \\
\hline $\mathrm{N}$ & 13.216154 & 3.685176 & 1.757003 \\
\hline$C$ & 13.370553 & 4.832917 & 2.397392 \\
\hline $\mathrm{N}$ & 14.577967 & 5.334231 & 2.632910 \\
\hline 0 & 14.887347 & 7.785931 & 4.204727 \\
\hline $\mathrm{H}$ & 14.578776 & 8.665622 & 3.971311 \\
\hline $\mathrm{H}$ & 15.604143 & 7.908461 & 4.814163 \\
\hline$N$ & 12.306995 & 5.491581 & 2.807899 \\
\hline $\mathrm{H}$ & 13.873120 & 1.768387 & -2.811523 \\
\hline $\mathrm{H}$ & 16.510192 & 2.908101 & -2.403639 \\
\hline $\mathrm{H}$ & 14.952133 & 1.003743 & -0.617587 \\
\hline $\mathrm{H}$ & 16.388419 & 1.888623 & -0.189083 \\
\hline
\end{tabular}




\begin{tabular}{|c|c|c|c|}
\hline $\mathrm{H}$ & 15.091918 & 4.038693 & -0.360045 \\
\hline $\mathrm{H}$ & 13.670505 & 3.144588 & -0.806631 \\
\hline $\mathrm{H}$ & 13.886981 & 1.808507 & 1.328228 \\
\hline $\mathrm{H}$ & 15.141340 & 2.878477 & 1.848384 \\
\hline $\mathrm{H}$ & 12.282184 & 3.369420 & 1.588875 \\
\hline $\mathrm{H}$ & 15.395644 & 4.865869 & 2.322748 \\
\hline $\mathrm{H}$ & 14.695290 & 6.187779 & 3.151491 \\
\hline $\mathrm{H}$ & 11.357379 & 5.190863 & 2.652281 \\
\hline $\mathrm{H}$ & 12.383061 & 6.331278 & 3.336222 \\
\hline$N$ & 16.227348 & -0.247290 & -3.050912 \\
\hline C & 16.929294 & -1.490185 & -3.266240 \\
\hline C & 17.718560 & -1.436230 & -4.570917 \\
\hline $\mathrm{H}$ & 18.090134 & -0.445400 & -4.841683 \\
\hline $\mathrm{O}$ & 17.940201 & -2.387623 & -5.246250 \\
\hline C & 15.957168 & -2.674677 & -3.251207 \\
\hline C & 15.430677 & -3.029401 & -1.893381 \\
\hline$N$ & 14.492872 & -2.287890 & -1.202385 \\
\hline $\mathrm{O}$ & 12.530502 & -0.160862 & -0.526063 \\
\hline $\mathrm{H}$ & 13.189211 & -0.736126 & -0.919235 \\
\hline $\mathrm{H}$ & 12.046009 & 0.237110 & -1.241214 \\
\hline C & 15.759939 & -4.117039 & -1.161279 \\
\hline C & 14.285604 & -2.926542 & -0.097458 \\
\hline$N$ & 15.027110 & -4.037674 & -0.003582 \\
\hline $\mathrm{H}$ & 15.309545 & -0.168152 & -3.433238 \\
\hline $\mathrm{H}$ & 17.662417 & -1.596689 & -2.474392 \\
\hline $\mathrm{H}$ & 15.130048 & -2.462611 & -3.919868 \\
\hline $\mathrm{H}$ & 16.477984 & -3.531995 & -3.655863 \\
\hline $\mathrm{H}$ & 16.436989 & -4.920770 & -1.356654 \\
\hline $\mathrm{H}$ & 13.603393 & -2.621376 & 0.670151 \\
\hline & 14.950024 & -4.728121 & 0.710725 \\
\hline
\end{tabular}




\begin{tabular}{|c|c|c|c|}
\hline 0 & -14.438086 & -2.383573 & 5.091980 \\
\hline $\mathrm{H}$ & -14.767973 & -1.787825 & 4.422158 \\
\hline $\mathrm{H}$ & -14.099199 & -1.827430 & 5.786474 \\
\hline 0 & -12.890300 & -0.584917 & 6.951697 \\
\hline $\mathrm{H}$ & -12.132422 & -1.045627 & 7.292992 \\
\hline $\mathrm{H}$ & -13.246819 & -0.080253 & 7.672366 \\
\hline 0 & -12.135746 & 0.209585 & 4.158463 \\
\hline $\mathrm{H}$ & -12.314066 & 0.221434 & 5.092147 \\
\hline $\mathrm{H}$ & -12.957235 & -0.047264 & 3.739165 \\
\hline 0 & -12.837848 & -2.314602 & 1.292744 \\
\hline $\mathrm{H}$ & -12.098380 & -1.737748 & 1.093041 \\
\hline $\mathrm{H}$ & -12.582524 & -2.869011 & 2.028398 \\
\hline 0 & -7.036657 & 1.814962 & -0.038654 \\
\hline $\mathrm{H}$ & -7.225215 & 1.880872 & 0.900906 \\
\hline $\mathrm{H}$ & -6.141776 & 1.502057 & -0.098539 \\
\hline 0 & -7.717176 & 1.874269 & 2.715620 \\
\hline $\mathrm{H}$ & -8.670871 & 1.824102 & 2.688340 \\
\hline $\mathrm{H}$ & -7.496990 & 2.582716 & 3.315511 \\
\hline $\mathrm{O}$ & -8.793847 & 0.063963 & -1.356228 \\
\hline $\mathrm{H}$ & -8.229756 & 0.699752 & -0.902017 \\
\hline $\mathrm{H}$ & -8.202043 & -0.592126 & -1.723694 \\
\hline $\mathrm{O}$ & -13.028528 & -2.718408 & -1.533118 \\
\hline $\mathrm{H}$ & -13.194345 & -2.680124 & -0.593613 \\
\hline $\mathrm{H}$ & -12.154913 & -3.082244 & -1.625007 \\
\hline $\mathrm{O}$ & -13.061449 & 1.290371 & -0.778209 \\
\hline $\mathrm{H}$ & -12.441591 & 0.650155 & -0.422362 \\
\hline $\mathrm{H}$ & -12.639838 & 2.134677 & -0.618408 \\
\hline 0 & -14.098216 & 4.428300 & 2.099492 \\
\hline $\mathrm{H}$ & -13.767483 & 4.776763 & 2.922080 \\
\hline H & -14.582774 & 3.631437 & 2.285102 \\
\hline
\end{tabular}




\begin{tabular}{|c|c|c|c|}
\hline 0 & -15.111912 & 1.660826 & 1.203236 \\
\hline $\mathrm{H}$ & -15.948448 & 1.770159 & 0.769981 \\
\hline $\mathrm{H}$ & -14.468988 & 1.521245 & 0.502477 \\
\hline $\mathrm{O}$ & -12.668751 & -0.443425 & -3.150057 \\
\hline $\mathrm{H}$ & -12.982382 & 0.332983 & -2.692212 \\
\hline $\mathrm{H}$ & -12.818264 & -1.162455 & -2.528201 \\
\hline $\mathrm{O}$ & -12.299681 & -2.511599 & -5.076945 \\
\hline $\mathrm{H}$ & -12.624584 & -1.709556 & -4.666812 \\
\hline $\mathrm{H}$ & -13.058730 & -3.041389 & -5.287997 \\
\hline $\mathrm{O}$ & -10.592271 & -3.361633 & -2.937916 \\
\hline $\mathrm{H}$ & -9.930690 & -2.692983 & -3.079320 \\
\hline $\mathrm{H}$ & -11.253597 & -3.197991 & -3.610429 \\
\hline $\mathrm{O}$ & -11.005167 & -0.364898 & 0.396741 \\
\hline $\mathrm{H}$ & -10.270494 & -0.380491 & -0.219277 \\
\hline $\mathrm{H}$ & -10.747130 & 0.248030 & 1.089289 \\
\hline $\mathrm{O}$ & -10.582475 & 1.634128 & 2.322356 \\
\hline $\mathrm{H}$ & -11.130577 & 1.312913 & 3.041652 \\
\hline $\mathrm{H}$ & -11.058205 & 2.324770 & 1.862869 \\
\hline $\mathrm{O}$ & -11.974884 & 3.550962 & 0.543654 \\
\hline $\mathrm{H}$ & -12.747957 & 3.850999 & 1.031936 \\
\hline $\mathrm{H}$ & -11.464680 & 4.343137 & 0.405926 \\
\hline $\mathrm{O}$ & -9.971490 & 0.540979 & -3.903732 \\
\hline $\mathrm{H}$ & -10.851526 & 0.198577 & -3.758063 \\
\hline $\mathrm{H}$ & -9.549248 & 0.514014 & -3.044481 \\
\hline $\mathrm{O}$ & -14.525230 & -0.619052 & 2.842211 \\
\hline $\mathrm{H}$ & -14.890310 & 0.126340 & 2.366871 \\
\hline $\mathrm{H}$ & -14.119313 & -1.189423 & 2.188264 \\
\hline $\mathrm{O}$ & -12.385611 & -3.733866 & 3.807224 \\
\hline $\mathrm{H}$ & -13.142009 & -3.345255 & 4.266564 \\
\hline $\mathrm{H}$ & -11.623186 & -3.381574 & 4.250543 \\
\hline
\end{tabular}




\begin{tabular}{|c|c|c|}
\hline $\mathrm{O}$ & $-9.420324-2.321268$ & -5.567122 \\
\hline $\mathrm{H}$ & $\begin{array}{ll}-10.278427 & -2.728276\end{array}$ & -5.512558 \\
\hline & $\begin{array}{lll}-8.803969 & -2.991078\end{array}$ & -5.8714 \\
\hline
\end{tabular}

PROTON CONFIGURATION, GIVEN BY NOMINAL CHARGES: R300(0),Y266(0),E183(0) POTENTIAL:mV

$\mathrm{N}$

$15.580942 \quad-6.451471 \quad 5.391415$

$\mathrm{H}$

$14.780479-6.2015115 .939215$

C

$15.509435 \quad-5.856447 \quad 4.054287$

C

$14.783016-6.782958 \quad 3.060460$

O

$\begin{array}{lll}14.052429 & -6.345779 & 2.186689\end{array}$

C

$\begin{array}{lll}16.919450 & -5.576363 & 3.520804\end{array}$

H $\quad 16.389532-6.127655 \quad 5.884604$

H $\quad 14.944632-4.933596 \quad 4.050480$

H $\quad 16.888621-5.189909 \quad 2.509075$

H $\quad 17.403352-4.831448 \quad 4.142312$

H $\quad \begin{array}{llll}\text { H } & 17.526880 & -6.474634 & 3.529458\end{array}$

N $\quad \begin{array}{llll}15.032384 & -8.085605 & 3.186393\end{array}$

H $\quad \begin{array}{llll}15.612145 & -8.361766 & 3.949651\end{array}$

C $\quad 14.398655-9.092385 \quad 2.333235$

H $\quad \begin{array}{llll}14.482424 & -8.823789 & 1.292862\end{array}$

H $\quad 14.891358-10.042266 \quad 2.492764$

C $\quad 12.912790 \quad-9.220804 \quad 2.671360$

$\begin{array}{llll}\text { O } & 12.081437 & -9.424628 & 1.825872\end{array}$

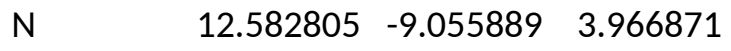

H $\quad \begin{array}{llll}13.302707 & -8.986601 & 4.648937\end{array}$

C $\quad 11.195100 \quad-9.170804 \quad 4.421405$

H $\quad 11.188266-9.157888 \quad 5.503158$ 


\begin{tabular}{|c|c|c|c|}
\hline $\mathrm{H}$ & 10.743721 & -10.088321 & 4.077202 \\
\hline$C$ & 10.336963 & -8.010603 & 3.900697 \\
\hline 0 & 9.190766 & -8.186036 & 3.584551 \\
\hline $\mathrm{N}$ & 10.947138 & -6.814584 & 3.855238 \\
\hline$C$ & 10.318531 & -5.634101 & 3.317471 \\
\hline$C$ & 10.126974 & -5.691219 & 1.799240 \\
\hline O & 9.096466 & -5.262968 & 1.337009 \\
\hline$C$ & 11.092846 & -4.388030 & 3.776178 \\
\hline O & 12.478788 & -4.570848 & 3.769250 \\
\hline $\mathrm{H}$ & 11.905504 & -6.744284 & 4.106854 \\
\hline $\mathrm{H}$ & 9.314641 & -5.553287 & 3.701015 \\
\hline $\mathrm{H}$ & 10.801417 & -3.539736 & 3.167069 \\
\hline $\mathrm{H}$ & 10.827334 & -4.174335 & 4.801180 \\
\hline $\mathrm{H}$ & 12.805733 & -4.755148 & 2.897195 \\
\hline $\mathrm{N}$ & 11.100353 & -6.200682 & 1.024103 \\
\hline$C$ & 10.845034 & -6.397096 & -0.400261 \\
\hline$C$ & 9.694185 & -7.385714 & -0.601587 \\
\hline $\mathrm{O}$ & 8.946438 & -7.280155 & -1.545785 \\
\hline$C$ & 12.133522 & -6.786225 & -1.186799 \\
\hline$C$ & 12.333825 & -8.291648 & -1.403956 \\
\hline$C$ & 12.161035 & -6.060793 & -2.533486 \\
\hline $\mathrm{H}$ & 11.924441 & -6.586132 & 1.431466 \\
\hline $\mathrm{H}$ & 10.475519 & -5.462799 & -0.791815 \\
\hline $\mathrm{H}$ & 12.965563 & -6.416658 & -0.597953 \\
\hline $\mathrm{H}$ & 13.287629 & -8.455103 & -1.894858 \\
\hline $\mathrm{H}$ & 12.331845 & -8.858100 & -0.483496 \\
\hline $\mathrm{H}$ & 11.566654 & -8.698326 & -2.055702 \\
\hline $\mathrm{H}$ & 13.055861 & -6.324799 & -3.086002 \\
\hline $\mathrm{H}$ & 11.300786 & -6.332239 & -3.135504 \\
\hline $\mathrm{H}$ & 12.161253 & -4.985603 & -2.400022 \\
\hline
\end{tabular}




\begin{tabular}{|c|c|c|c|}
\hline$N$ & 9.570408 & -8.340330 & 0.328361 \\
\hline $\mathrm{H}$ & 10.314554 & -8.474508 & 0.974296 \\
\hline C & 8.593032 & -9.421193 & 0.241195 \\
\hline $\mathrm{H}$ & 8.935836 & -10.219581 & 0.886993 \\
\hline $\mathrm{H}$ & 8.532029 & -9.802164 & -0.766590 \\
\hline C & 7.181949 & -9.047511 & 0.645327 \\
\hline $\mathrm{O}$ & 6.257502 & -9.505198 & 0.014371 \\
\hline$N$ & 7.004066 & -8.228535 & 1.688395 \\
\hline C & 5.670100 & -7.742121 & 1.958461 \\
\hline C & 5.250597 & -6.730659 & 0.897218 \\
\hline $\mathrm{O}$ & 4.073671 & -6.634113 & 0.621668 \\
\hline C & 5.416252 & -7.237171 & 3.392948 \\
\hline C & 5.559846 & -8.381368 & 4.401542 \\
\hline C & 6.257073 & -6.027437 & 3.805773 \\
\hline $\mathrm{H}$ & 7.781739 & -7.961854 & 2.252086 \\
\hline $\mathrm{H}$ & 4.987440 & -8.561786 & 1.796420 \\
\hline $\mathrm{H}$ & 4.375092 & -6.929353 & 3.386541 \\
\hline $\mathrm{H}$ & 5.269940 & -8.044537 & 5.391283 \\
\hline $\mathrm{H}$ & 4.922452 & -9.219365 & 4.138886 \\
\hline $\mathrm{H}$ & 6.582366 & -8.737628 & 4.456781 \\
\hline $\mathrm{H}$ & 5.916524 & -5.655616 & 4.766245 \\
\hline $\mathrm{H}$ & 7.299674 & -6.296965 & 3.912576 \\
\hline $\mathrm{H}$ & 6.184061 & -5.213007 & 3.093247 \\
\hline$N$ & 6.171141 & -6.006024 & 0.253015 \\
\hline C & 5.775821 & -5.188189 & -0.881627 \\
\hline C & 5.344902 & -6.072718 & -2.059952 \\
\hline $\mathrm{O}$ & 4.321527 & -5.821692 & -2.652240 \\
\hline C & 6.867622 & -4.167921 & -1.261341 \\
\hline C & 7.064018 & -3.150665 & -0.123252 \\
\hline 6 & 6.496716 & -3.458381 & -2.568268 \\
\hline
\end{tabular}




\begin{tabular}{|c|c|c|c|}
\hline C & 8.356690 & -2.342411 & -0.237303 \\
\hline $\mathrm{H}$ & 7.132427 & -6.063826 & 0.511597 \\
\hline $\mathrm{H}$ & 4.877378 & -4.652001 & -0.615495 \\
\hline $\mathrm{H}$ & 7.798150 & -4.708422 & -1.408517 \\
\hline $\mathrm{H}$ & 6.208140 & -2.477374 & -0.107600 \\
\hline $\mathrm{H}$ & 7.074268 & -3.664218 & 0.828805 \\
\hline $\mathrm{H}$ & 7.201530 & -2.668254 & -2.788286 \\
\hline $\mathrm{H}$ & 6.497673 & -4.133880 & -3.413917 \\
\hline $\mathrm{H}$ & 5.508189 & -3.015822 & -2.502062 \\
\hline $\mathrm{H}$ & 8.456989 & -1.668587 & 0.606900 \\
\hline $\mathrm{H}$ & 9.218939 & -2.998846 & -0.236964 \\
\hline $\mathrm{H}$ & 8.392128 & -1.742864 & -1.139973 \\
\hline$N$ & 6.117667 & -7.114687 & -2.395326 \\
\hline $\mathrm{H}$ & 7.012122 & -7.235793 & -1.968905 \\
\hline C & 5.776732 & -7.955836 & -3.522959 \\
\hline $\mathrm{H}$ & 6.607343 & -8.630035 & -3.690966 \\
\hline $\mathrm{H}$ & 5.623352 & -7.370778 & -4.417476 \\
\hline C & 4.507108 & -8.783602 & -3.322011 \\
\hline $\mathrm{O}$ & 3.670412 & -8.842385 & -4.191720 \\
\hline$N$ & 4.393861 & -9.435378 & -2.157989 \\
\hline $\mathrm{H}$ & 5.130915 & -9.392171 & -1.486873 \\
\hline C & 3.206588 & -10.189414 & -1.830430 \\
\hline $\mathrm{H}$ & 2.967436 & -10.903132 & -2.604468 \\
\hline $\mathrm{H}$ & 3.396868 & -10.730498 & -0.911998 \\
\hline C & 1.974201 & -9.306854 & -1.644807 \\
\hline $\mathrm{O}$ & 0.887577 & -9.694694 & -2.007244 \\
\hline$N$ & 2.187865 & -8.107004 & -1.107166 \\
\hline C & 1.065926 & -7.180031 & -1.078543 \\
\hline C & 0.623280 & -6.721154 & -2.470727 \\
\hline 0 & -0.558603 & -6.582579 & -2.692724 \\
\hline
\end{tabular}




\begin{tabular}{|c|c|c|c|}
\hline C & 1.286930 & -5.920607 & -0.247529 \\
\hline $\mathrm{O}$ & 1.414503 & -6.167183 & 1.112812 \\
\hline $\mathrm{H}$ & 3.087790 & -7.818090 & -0.797813 \\
\hline $\mathrm{H}$ & 0.211762 & -7.692804 & -0.667171 \\
\hline $\mathrm{H}$ & 2.135110 & -5.363953 & -0.632026 \\
\hline $\mathrm{H}$ & 0.397794 & -5.326624 & -0.378159 \\
\hline $\mathrm{H}$ & 2.304989 & -6.441424 & 1.286538 \\
\hline$N$ & 1.552222 & -6.437210 & -3.393005 \\
\hline C & 1.156378 & -6.032032 & -4.729704 \\
\hline C & 0.385351 & -7.153770 & -5.418695 \\
\hline $\mathrm{O}$ & -0.601544 & -6.897828 & -6.075722 \\
\hline C & 2.369642 & -5.538780 & -5.551809 \\
\hline C & 2.784754 & -4.147329 & -5.045158 \\
\hline C & 2.073800 & -5.517412 & -7.056823 \\
\hline C & 4.115057 & -3.649297 & -5.608316 \\
\hline $\mathrm{H}$ & 2.522139 & -6.493774 & -3.167551 \\
\hline $\mathrm{H}$ & 0.439087 & -5.227080 & -4.653887 \\
\hline $\mathrm{H}$ & 3.186448 & -6.233131 & -5.378830 \\
\hline $\mathrm{H}$ & 1.998478 & -3.437158 & -5.295842 \\
\hline $\mathrm{H}$ & 2.856259 & -4.168626 & -3.965333 \\
\hline $\mathrm{H}$ & 2.933338 & -5.151974 & -7.604763 \\
\hline $\mathrm{H}$ & 1.849929 & -6.504431 & -7.442677 \\
\hline $\mathrm{H}$ & 1.231979 & -4.871320 & -7.285177 \\
\hline $\mathrm{H}$ & 4.414579 & -2.735892 & -5.105135 \\
\hline $\mathrm{H}$ & 4.901158 & -4.381480 & -5.453470 \\
\hline $\mathrm{H}$ & 4.061695 & -3.435596 & -6.669960 \\
\hline$N$ & 0.823537 & -8.405147 & -5.255465 \\
\hline $\mathrm{H}$ & 1.669692 & -8.581375 & -4.755081 \\
\hline C & 0.043375 & -9.512147 & -5.753635 \\
\hline $\mathrm{H}$ & -0.063611 & -9.473771 & -6.827473 \\
\hline
\end{tabular}




\begin{tabular}{|c|c|c|c|}
\hline $\mathrm{H}$ & 0.553342 & -10.429288 & -5.487588 \\
\hline C & -1.365316 & -9.540114 & -5.166944 \\
\hline $\mathrm{O}$ & -2.319964 & -9.767304 & -5.874452 \\
\hline$N$ & -1.472594 & -9.318871 & -3.858013 \\
\hline C & -2.776797 & -9.346644 & -3.236459 \\
\hline C & -3.690587 & -8.211648 & -3.700405 \\
\hline $\mathrm{O}$ & -4.888661 & -8.419507 & -3.743653 \\
\hline C & -2.680550 & -9.315430 & -1.703970 \\
\hline $\mathrm{O}$ & -3.925580 & -9.579847 & -1.118911 \\
\hline $\mathrm{H}$ & -0.656813 & -9.233311 & -3.288688 \\
\hline $\mathrm{H}$ & -3.294811 & -10.251763 & -3.520575 \\
\hline $\mathrm{H}$ & -1.999603 & -10.088451 & -1.375710 \\
\hline $\mathrm{H}$ & -2.290578 & -8.357443 & -1.376562 \\
\hline $\mathrm{H}$ & -4.614968 & -9.178369 & -1.633771 \\
\hline$N$ & -3.140655 & -7.031662 & -3.981788 \\
\hline C & -3.902769 & -5.911621 & -4.509797 \\
\hline C & -4.444447 & -6.236110 & -5.902240 \\
\hline $\mathrm{O}$ & -5.608389 & -6.045075 & -6.172188 \\
\hline C & -3.003095 & -4.655420 & -4.508645 \\
\hline C & -3.446380 & -3.519790 & -5.415705 \\
\hline C & -4.768321 & -3.093150 & -5.495271 \\
\hline C & -2.499073 & -2.874260 & -6.202304 \\
\hline C & -5.125934 & -2.067125 & -6.354129 \\
\hline C & -2.850183 & -1.834735 & -7.048233 \\
\hline C & -4.171537 & -1.432443 & -7.132354 \\
\hline $\mathrm{H}$ & -2.163927 & -6.909817 & -3.812392 \\
\hline $\mathrm{H}$ & -4.770871 & -5.740594 & -3.893622 \\
\hline $\mathrm{H}$ & -2.925229 & -4.313042 & -3.481049 \\
\hline $\mathrm{H}$ & -2.010643 & -4.954531 & -4.814990 \\
\hline $\mathrm{H}$ & -5.527473 & -3.578761 & -4.912377 \\
\hline
\end{tabular}




\begin{tabular}{|c|c|c|c|}
\hline $\mathrm{H}$ & -1.472579 & -3.193931 & -6.163025 \\
\hline $\mathrm{H}$ & -6.159656 & -1.773327 & -6.422551 \\
\hline $\mathrm{H}$ & -2.096146 & -1.352477 & -7.643889 \\
\hline $\mathrm{H}$ & -4.452436 & -0.638994 & -7.803045 \\
\hline$N$ & -3.570652 & -6.729299 & -6.788369 \\
\hline C & -4.012526 & -7.071457 & -8.120709 \\
\hline C & -5.136406 & -8.106133 & -8.097629 \\
\hline 0 & -6.015097 & -8.052808 & -8.918295 \\
\hline C & -2.862124 & -7.602866 & -8.979106 \\
\hline$S$ & -1.645366 & -6.350795 & -9.496697 \\
\hline $\mathrm{H}$ & -2.629940 & -6.913399 & -6.509064 \\
\hline $\mathrm{H}$ & -4.438351 & -6.201492 & -8.597927 \\
\hline $\mathrm{H}$ & -2.357426 & -8.419574 & -8.480780 \\
\hline $\mathrm{H}$ & -3.278871 & -7.983616 & -9.901770 \\
\hline $\mathrm{H}$ & -1.045043 & -6.180632 & -8.329226 \\
\hline$N$ & -5.078532 & -9.066905 & -7.155631 \\
\hline $\mathrm{H}$ & -4.257160 & -9.188229 & -6.602657 \\
\hline$C$ & -6.085167 & -10.116169 & -7.180925 \\
\hline $\mathrm{H}$ & -6.256477 & -10.456664 & -8.190310 \\
\hline $\mathrm{H}$ & -5.713987 & -10.946522 & -6.591454 \\
\hline $\mathrm{C}$ & -7.459147 & -9.722831 & -6.613014 \\
\hline 0 & -8.481320 & -10.087395 & -7.126865 \\
\hline $\mathrm{N}$ & -7.418017 & -9.015655 & -5.478475 \\
\hline$C$ & -8.641670 & -8.655601 & -4.788458 \\
\hline $\mathrm{C}$ & -9.503017 & -7.657777 & -5.554102 \\
\hline 0 & -10.700968 & -7.657543 & -5.447825 \\
\hline$C$ & -8.362855 & -8.115633 & -3.374865 \\
\hline$C$ & -7.517860 & -6.836014 & -3.258320 \\
\hline $\mathrm{C}$ & -8.186753 & -5.483364 & -3.283175 \\
\hline O & -9.487551 & -5.484227 & -3.352543 \\
\hline
\end{tabular}




\begin{tabular}{|c|c|c|c|}
\hline $\mathrm{H}$ & -9.837256 & -4.582248 & -3.306502 \\
\hline $\mathrm{O}$ & -7.537304 & -4.468857 & -3.215812 \\
\hline $\mathrm{H}$ & -6.535762 & -8.773452 & -5.079871 \\
\hline $\mathrm{H}$ & -9.261405 & -9.536031 & -4.692017 \\
\hline $\mathrm{H}$ & -9.318911 & -7.979418 & -2.890214 \\
\hline $\mathrm{H}$ & -7.839687 & -8.900248 & -2.838717 \\
\hline $\mathrm{H}$ & -6.984941 & -6.853282 & -2.314174 \\
\hline $\mathrm{H}$ & -6.752283 & -6.796836 & -4.014541 \\
\hline $\mathrm{N}$ & -8.837317 & -6.734064 & -6.273863 \\
\hline$C$ & -9.562353 & -5.706192 & -6.995284 \\
\hline$C$ & -10.429122 & -6.285710 & -8.105065 \\
\hline $\mathrm{H}$ & -10.447462 & -7.368398 & -8.211424 \\
\hline 0 & -11.058251 & -5.576097 & -8.833344 \\
\hline$C$ & -8.635007 & -4.592758 & -7.533829 \\
\hline 0 & -9.429395 & -3.468152 & -7.883445 \\
\hline$C$ & -7.759880 & -4.998445 & -8.712315 \\
\hline $\mathrm{H}$ & -7.855288 & -6.834373 & -6.418161 \\
\hline $\mathrm{H}$ & -10.250398 & -5.232968 & -6.304218 \\
\hline $\mathrm{H}$ & -8.009309 & -4.274530 & -6.711386 \\
\hline $\mathrm{H}$ & -10.132133 & -3.782359 & -8.446361 \\
\hline $\mathrm{H}$ & -7.080861 & -4.185601 & -8.946726 \\
\hline $\mathrm{H}$ & -7.169989 & -5.878957 & -8.504625 \\
\hline $\mathrm{H}$ & -8.364799 & -5.194171 & -9.591307 \\
\hline $\mathrm{N}$ & -12.952321 & -9.022071 & 3.174379 \\
\hline $\mathrm{H}$ & -13.222146 & -9.545387 & 2.367154 \\
\hline$C$ & -11.777508 & -8.184389 & 2.899330 \\
\hline$C$ & -10.788057 & -8.138211 & 4.046012 \\
\hline 0 & -9.654089 & -7.745134 & 3.846077 \\
\hline C & -12.130679 & -6.717804 & 2.577699 \\
\hline 0 & -12.657772 & -6.188815 & 3.767602 \\
\hline
\end{tabular}




\begin{tabular}{|c|c|c|c|}
\hline C & -13.123009 & -6.574425 & 1.429801 \\
\hline $\mathrm{H}$ & -13.731297 & -8.457322 & 3.452923 \\
\hline $\mathrm{H}$ & -11.232578 & -8.601504 & 2.063676 \\
\hline $\mathrm{H}$ & -11.209169 & -6.212258 & 2.322468 \\
\hline $\mathrm{H}$ & -12.680856 & -5.236061 & 3.711671 \\
\hline $\mathrm{H}$ & -13.268370 & -5.522651 & 1.207567 \\
\hline $\mathrm{H}$ & -12.771076 & -7.061543 & 0.527784 \\
\hline $\mathrm{H}$ & -14.088587 & -6.989138 & 1.693548 \\
\hline$N$ & -11.197267 & -8.533701 & 5.241859 \\
\hline $\mathrm{H}$ & -12.124297 & -8.897000 & 5.283782 \\
\hline C & -10.409179 & -8.524195 & 6.449430 \\
\hline $\mathrm{H}$ & -10.331005 & -7.527003 & 6.853274 \\
\hline $\mathrm{H}$ & -10.928917 & -9.129397 & 7.182851 \\
\hline C & -8.972216 & -9.040204 & 6.371429 \\
\hline O & -8.135522 & -8.471439 & 7.028538 \\
\hline $\mathrm{N}$ & -8.657864 & -10.121159 & 5.634789 \\
\hline $\mathrm{H}$ & -9.368751 & -10.601812 & 5.131275 \\
\hline$C$ & -7.269066 & -10.552649 & 5.533214 \\
\hline $\mathrm{H}$ & -7.251942 & -11.532377 & 5.073068 \\
\hline $\mathrm{H}$ & -6.828289 & -10.630327 & 6.514174 \\
\hline$C$ & -6.387526 & -9.608322 & 4.692701 \\
\hline 0 & -5.219513 & -9.466974 & 4.948500 \\
\hline $\mathrm{N}$ & -7.045373 & -9.017976 & 3.701159 \\
\hline$C$ & -6.304936 & -8.012553 & 2.952685 \\
\hline$C$ & -6.268136 & -6.697733 & 3.725430 \\
\hline 0 & -5.276217 & -6.007839 & 3.677062 \\
\hline$C$ & -6.623050 & -7.894358 & 1.449040 \\
\hline $\mathrm{C}$ & -7.795906 & -7.059156 & 0.980215 \\
\hline$C$ & -7.766186 & -5.671085 & 1.083039 \\
\hline$C$ & -8.866659 & -7.649456 & 0.32200 \\
\hline
\end{tabular}




\begin{tabular}{|c|c|c|c|}
\hline C & -8.788086 & -4.896873 & 0.560016 \\
\hline C & -9.880568 & -6.876670 & -0.221238 \\
\hline C & -9.849027 & -5.497956 & -0.097356 \\
\hline $\mathrm{H}$ & -8.039864 & -9.019892 & 3.675724 \\
\hline $\mathrm{H}$ & -5.281581 & -8.342668 & 2.985222 \\
\hline $\mathrm{H}$ & -5.724586 & -7.489365 & 0.996656 \\
\hline $\mathrm{H}$ & -6.714094 & -8.906804 & 1.073831 \\
\hline $\mathrm{H}$ & -6.926224 & -5.186208 & 1.546229 \\
\hline $\mathrm{H}$ & -8.892439 & -8.718846 & 0.200032 \\
\hline $\mathrm{H}$ & -8.738598 & -3.825486 & 0.637859 \\
\hline $\mathrm{H}$ & -10.679453 & -7.347689 & -0.764169 \\
\hline $\mathrm{H}$ & -10.627349 & -4.898939 & -0.533061 \\
\hline$N$ & -7.302432 & -6.389941 & 4.510455 \\
\hline C & -7.241876 & -5.272633 & 5.420616 \\
\hline C & -6.127028 & -5.479086 & 6.445287 \\
\hline $\mathrm{O}$ & -5.497120 & -4.519354 & 6.836475 \\
\hline C & -8.622432 & -5.098381 & 6.088825 \\
\hline C & -8.872758 & -3.754050 & 6.737521 \\
\hline C & -9.064858 & -2.621777 & 5.950879 \\
\hline C & -8.980392 & -3.631623 & 8.116053 \\
\hline C & -9.345681 & -1.395909 & 6.527569 \\
\hline C & -9.269866 & -2.406899 & 8.700123 \\
\hline C & -9.450149 & -1.284429 & 7.908656 \\
\hline $\mathrm{H}$ & -8.162406 & -6.883368 & 4.408389 \\
\hline $\mathrm{H}$ & -6.972227 & -4.370832 & 4.889855 \\
\hline $\mathrm{H}$ & -9.367772 & -5.249049 & 5.314885 \\
\hline $\mathrm{H}$ & -8.757597 & -5.887147 & 6.816788 \\
\hline $\mathrm{H}$ & -8.996785 & -2.697078 & 4.879017 \\
\hline $\mathrm{H}$ & -8.840090 & -4.495318 & 8.742128 \\
\hline $\mathrm{H}$ & -9.481069 & -0.533084 & 5.901567 \\
\hline
\end{tabular}




\begin{tabular}{|c|c|c|c|}
\hline $\mathrm{H}$ & -9.342478 & -2.330740 & 9.770197 \\
\hline $\mathrm{H}$ & -9.648834 & -0.328309 & 8.360311 \\
\hline$N$ & -5.873230 & -6.714979 & 6.870111 \\
\hline $\mathrm{H}$ & -6.521864 & -7.441240 & 6.663048 \\
\hline C & -4.799079 & -7.005524 & 7.793367 \\
\hline $\mathrm{H}$ & -4.839922 & -6.373376 & 8.667599 \\
\hline $\mathrm{H}$ & -4.911843 & -8.034232 & 8.110938 \\
\hline C & -3.402203 & -6.831989 & 7.199980 \\
\hline $\mathrm{O}$ & -2.527231 & -6.342434 & 7.874409 \\
\hline$N$ & -3.202368 & -7.252154 & 5.946254 \\
\hline C & -1.926484 & -7.066532 & 5.272865 \\
\hline C & -1.685057 & -5.591986 & 4.920271 \\
\hline $\mathrm{O}$ & -0.587038 & -5.103739 & 5.087820 \\
\hline C & -1.845247 & -7.995910 & 4.041724 \\
\hline C & -0.684044 & -7.630164 & 3.115619 \\
\hline C & -1.736978 & -9.457662 & 4.490480 \\
\hline $\mathrm{H}$ & -3.935846 & -7.731711 & 5.470360 \\
\hline $\mathrm{H}$ & -1.130903 & -7.313510 & 5.960817 \\
\hline $\mathrm{H}$ & -2.767910 & -7.872757 & 3.479479 \\
\hline$r$ & -0.619196 & -8.353490 & 2.309793 \\
\hline $\mathrm{H}$ & -0.800420 & -6.654424 & 2.665166 \\
\hline $\mathrm{H}$ & 0.262242 & -7.639889 & 3.647725 \\
\hline $\mathrm{H}$ & -1.751175 & -10.114617 & 3.626812 \\
\hline $\mathrm{H}$ & -0.800585 & -9.623373 & 5.017337 \\
\hline $\mathrm{H}$ & -2.550182 & -9.753032 & 5.140610 \\
\hline$N$ & -2.723927 & -4.903732 & 4.437466 \\
\hline C & -2.597893 & -3.516499 & 4.035543 \\
\hline C & -2.446034 & -2.619079 & 5.261650 \\
\hline O & -1.559470 & -1.794995 & 5.313931 \\
\hline & -3.790408 & -3.045701 & 3.189295 \\
\hline
\end{tabular}




\begin{tabular}{|c|c|c|c|}
\hline C & -4.037981 & -3.782445 & 1.862420 \\
\hline C & -2.904492 & -3.687105 & 0.855819 \\
\hline $\mathrm{O}$ & -1.795440 & -4.103132 & 1.227785 \\
\hline $\mathrm{O}$ & -3.154514 & -3.213727 & -0.265368 \\
\hline $\mathrm{H}$ & -3.585464 & -5.375494 & 4.270725 \\
\hline $\mathrm{H}$ & -1.693303 & -3.396975 & 3.469887 \\
\hline $\mathrm{H}$ & -4.695986 & -3.115585 & 3.784333 \\
\hline $\mathrm{H}$ & -3.635064 & -1.989098 & 2.988351 \\
\hline $\mathrm{H}$ & -4.209536 & -4.830885 & 2.054288 \\
\hline $\mathrm{H}$ & -4.937934 & -3.375541 & 1.415391 \\
\hline$N$ & -3.328777 & -2.774748 & 6.264400 \\
\hline C & -3.116859 & -2.037315 & 7.484959 \\
\hline C & -1.791667 & -2.427115 & 8.149577 \\
\hline $\mathrm{O}$ & -1.125609 & -1.586204 & 8.713719 \\
\hline C & -4.245943 & -2.040807 & 8.526022 \\
\hline $\mathrm{O}$ & -4.452008 & -3.295287 & 9.104614 \\
\hline C & -5.530736 & -1.412400 & 7.998357 \\
\hline $\mathrm{H}$ & -4.008785 & -3.501260 & 6.228593 \\
\hline $\mathrm{H}$ & -2.972317 & -1.002434 & 7.215561 \\
\hline $\mathrm{H}$ & -3.869434 & -1.417281 & 9.327417 \\
\hline $\mathrm{H}$ & -4.908584 & -3.852181 & 8.485616 \\
\hline $\mathrm{H}$ & -6.261222 & -1.373425 & 8.797095 \\
\hline $\mathrm{H}$ & -5.347553 & -0.399450 & 7.653245 \\
\hline $\mathrm{H}$ & -5.953407 & -1.981174 & 7.180933 \\
\hline$N$ & -1.380408 & -3.700466 & 8.043355 \\
\hline $\mathrm{H}$ & -2.027708 & -4.421554 & 7.811933 \\
\hline C & -0.184091 & -4.103747 & 8.736407 \\
\hline $\mathrm{H}$ & -0.230724 & -3.886177 & 9.794168 \\
\hline $\mathrm{H}$ & -0.071074 & -5.171591 & 8.602276 \\
\hline C & 1.063838 & -3.415919 & 8.208175 \\
\hline
\end{tabular}




\begin{tabular}{|c|c|c|c|}
\hline 0 & 1.943307 & -3.068518 & 8.963123 \\
\hline$N$ & 1.148181 & -3.258361 & 6.871275 \\
\hline C & 2.325631 & -2.662470 & 6.301979 \\
\hline C & 2.289707 & -1.139358 & 6.162426 \\
\hline $\mathrm{O}$ & 3.359923 & -0.576622 & 6.026584 \\
\hline C & 2.805207 & -3.337765 & 5.017039 \\
\hline$S$ & 1.888426 & -2.938262 & 3.504732 \\
\hline $\mathrm{H}$ & 0.413889 & -3.611959 & 6.290052 \\
\hline $\mathrm{H}$ & 3.116264 & -2.811866 & 7.021712 \\
\hline $\mathrm{H}$ & 3.817303 & -3.008412 & 4.830443 \\
\hline $\mathrm{H}$ & 2.817064 & -4.409033 & 5.162202 \\
\hline $\mathrm{H}$ & 0.819662 & -3.695520 & 3.703153 \\
\hline$N$ & 1.139734 & -0.470510 & 6.273420 \\
\hline C & 1.204805 & 0.968264 & 6.492091 \\
\hline$C$ & 1.755965 & 1.271304 & 7.871007 \\
\hline $\mathrm{O}$ & 2.368723 & 2.288945 & 8.086022 \\
\hline$C$ & -0.095215 & 1.740145 & 6.181627 \\
\hline$C$ & -1.202794 & 1.552827 & 7.228616 \\
\hline C & -0.544654 & 1.447358 & 4.745150 \\
\hline$C$ & -2.470463 & 2.358748 & 6.937163 \\
\hline $\mathrm{H}$ & 0.270186 & -0.963421 & 6.287491 \\
\hline $\mathrm{H}$ & 1.952683 & 1.350982 & 5.815981 \\
\hline $\mathrm{H}$ & 0.205170 & 2.781457 & 6.231659 \\
\hline $\mathrm{H}$ & -1.452823 & 0.506505 & 7.328410 \\
\hline $\mathrm{H}$ & -0.820147 & 1.863386 & 8.196292 \\
\hline $\mathrm{H}$ & -1.301520 & 2.153743 & 4.427573 \\
\hline $\mathrm{H}$ & 0.291333 & 1.536440 & 4.056705 \\
\hline $\mathrm{H}$ & -0.951844 & 0.450705 & 4.642208 \\
\hline $\mathrm{H}$ & -3.144870 & 2.318451 & 7.787086 \\
\hline $\mathrm{H}$ & -2.244335 & 3.404566 & 6.744903 \\
\hline
\end{tabular}




\begin{tabular}{|c|c|c|c|}
\hline $\mathrm{H}$ & -3.006485 & 1.971194 & 6.077663 \\
\hline $\mathrm{N}$ & 1.549780 & 0.323275 & 8.791255 \\
\hline $\mathrm{H}$ & 0.890658 & -0.402710 & 8.612969 \\
\hline$C$ & 1.977859 & 0.512213 & 10.160290 \\
\hline $\mathrm{H}$ & 1.469235 & -0.223036 & 10.770952 \\
\hline $\mathrm{H}$ & 1.737340 & 1.501890 & 10.518399 \\
\hline$C$ & 3.481799 & 0.336565 & 10.305798 \\
\hline 0 & 4.163615 & 1.126810 & 10.899127 \\
\hline $\mathrm{N}$ & 3.999641 & -0.753619 & 9.694705 \\
\hline $\mathrm{H}$ & 3.390695 & -1.463403 & 9.345242 \\
\hline$C$ & 5.424653 & -0.938721 & 9.667238 \\
\hline $\mathrm{H}$ & 5.638022 & -1.909234 & 9.234361 \\
\hline $\mathrm{H}$ & 5.854686 & -0.904700 & 10.657563 \\
\hline$C$ & 6.156890 & 0.119123 & 8.844832 \\
\hline 0 & 7.292087 & 0.411119 & 9.129513 \\
\hline $\mathrm{N}$ & 5.502074 & 0.621268 & 7.799018 \\
\hline $\mathrm{C}$ & 6.105378 & 1.756770 & 7.150115 \\
\hline$C$ & 5.525641 & 3.075937 & 7.654194 \\
\hline $\mathrm{H}$ & 5.023288 & 3.036911 & 8.619393 \\
\hline 0 & 5.649980 & 4.094244 & 7.046402 \\
\hline$C$ & 6.011878 & 1.676263 & 5.624076 \\
\hline$C$ & 6.760826 & 0.506536 & 5.019756 \\
\hline$C$ & 8.126630 & 0.320636 & 5.237924 \\
\hline$C$ & 6.097432 & -0.401574 & 4.200043 \\
\hline$C$ & 8.810266 & -0.726233 & 4.634553 \\
\hline$C$ & 6.778853 & -1.446088 & 3.595216 \\
\hline$C$ & 8.139103 & -1.608939 & 3.802541 \\
\hline $\mathrm{H}$ & 4.574672 & 0.337152 & 7.571829 \\
\hline $\mathrm{H}$ & 7.140254 & 1.766956 & 7.456616 \\
\hline$\Pi$ & 4.969867 & 1.616766 & 5.335682 \\
\hline
\end{tabular}




\begin{tabular}{|c|c|c|c|}
\hline $\mathrm{H}$ & 6.394472 & 2.610041 & 5.226532 \\
\hline $\mathrm{H}$ & 8.656907 & 0.976659 & 5.906885 \\
\hline $\mathrm{H}$ & 5.037127 & -0.304115 & 4.063932 \\
\hline $\mathrm{H}$ & 9.861208 & -0.854931 & 4.828272 \\
\hline $\mathrm{H}$ & 6.244460 & -2.137561 & 2.968384 \\
\hline $\mathrm{H}$ & 8.657307 & -2.422587 & 3.328959 \\
\hline$N$ & 12.613548 & 3 9.596235 & -1.248797 \\
\hline $\mathrm{H}$ & 11.873073 & 39.914179 & -1.845810 \\
\hline C & 12.304855 & 8.264733 & -0.719494 \\
\hline C & 11.274028 & 8.450598 & 0.386818 \\
\hline $\mathrm{O}$ & 10.265751 & 7.778972 & 0.416160 \\
\hline C & 13.562725 & 7.581082 & -0.165205 \\
\hline C & 14.645173 & 3.276181 & -1.206308 \\
\hline$S$ & 14.017431 & 6.202019 & -2.529609 \\
\hline C & 15.500221 & 6.053543 & -3.554886 \\
\hline $\mathrm{H}$ & 13.433195 & 9.572927 & -1.822563 \\
\hline $\mathrm{H}$ & 11.834545 & 7.616477 & -1.447377 \\
\hline $\mathrm{H}$ & 14.001191 & 8.205558 & 0.607330 \\
\hline $\mathrm{H}$ & 13.255689 & 6.653907 & 0.304954 \\
\hline $\mathrm{H}$ & 15.045510 & 8.188653 & -1.633679 \\
\hline $\mathrm{H}$ & 15.470345 & 6.783887 & -0.704191 \\
\hline $\mathrm{H}$ & 15.249053 & 5.398756 & -4.375389 \\
\hline $\mathrm{H}$ & 15.799178 & 7.017593 & -3.945666 \\
\hline $\mathrm{H}$ & 16.318592 & 5.616834 & -2.997477 \\
\hline$N$ & 11.504154 & 9.412695 & 1.297047 \\
\hline C & 10.594052 & 9.607451 & 2.408568 \\
\hline C & 9.276854 & 10.274549 & 2.010327 \\
\hline $\mathrm{O}$ & 8.280717 & 10.052953 & 2.652477 \\
\hline C & 11.275896 & 10.368514 & 3.552323 \\
\hline$C$ & 12.447318 & 3.563602 & 4.084891 \\
\hline
\end{tabular}




\begin{tabular}{|c|c|c|c|}
\hline O & 13.582290 & 9.825107 & 3.745252 \\
\hline $\mathrm{N}$ & 12.143320 & 8.561357 & 4.919198 \\
\hline $\mathrm{H}$ & 12.327994 & 9.967518 & 1.206545 \\
\hline $\mathrm{H}$ & 10.295156 & 8.637352 & 2.771082 \\
\hline $\mathrm{H}$ & 11.657172 & 11.325715 & 3.219399 \\
\hline $\mathrm{H}$ & 10.545575 & 10.537170 & 4.333596 \\
\hline $\mathrm{H}$ & 12.886915 & 7.965736 & 5.213588 \\
\hline $\mathrm{H}$ & 11.221689 & 8.175412 & 4.954942 \\
\hline $\mathrm{N}$ & 9.263775 & 11.067655 & 0.922390 \\
\hline $\mathrm{H}$ & 10.110811 & 11.229659 & 0.427571 \\
\hline C & 8.003507 & 11.517472 & 0.368847 \\
\hline $\mathrm{H}$ & 8.201320 & 12.260188 & -0.393384 \\
\hline $\mathrm{H}$ & 7.389092 & 11.966751 & 1.131867 \\
\hline C & 7.186380 & 10.384432 & -0.254307 \\
\hline 0 & 5.986305 & 10.470120 & -0.291584 \\
\hline$N$ & 7.862638 & 9.333265 & -0.763205 \\
\hline C & 7.170367 & 8.145651 & -1.233959 \\
\hline C & 6.634520 & 7.300821 & -0.067416 \\
\hline 0 & 5.534028 & 6.808746 & -0.148451 \\
\hline C & 8.048860 & 7.344203 & -2.224163 \\
\hline C & 8.343758 & 8.197099 & -3.472847 \\
\hline C & 7.379302 & 6.019178 & -2.608086 \\
\hline C & 9.428431 & 7.624913 & -4.388803 \\
\hline $\mathrm{H}$ & 8.823805 & 9.230641 & -0.536565 \\
\hline $\mathrm{H}$ & 6.279740 & 8.471836 & -1.748518 \\
\hline $\mathrm{H}$ & 8.987847 & 7.117705 & -1.727184 \\
\hline $\mathrm{H}$ & 7.421907 & 8.323173 & -4.036275 \\
\hline $\mathrm{H}$ & 8.645895 & 9.192725 & -3.165352 \\
\hline $\mathrm{H}$ & 7.996709 & 5.478750 & -3.314220 \\
\hline $\mathrm{H}$ & 7.228034 & 5.377937 & -1.748260 \\
\hline
\end{tabular}




\begin{tabular}{|c|c|c|c|}
\hline $\mathrm{H}$ & 6.409672 & 6.182905 & -3.066700 \\
\hline $\mathrm{H}$ & 9.622568 & 8.305455 & -5.211433 \\
\hline $\mathrm{H}$ & 10.363692 & 7.479321 & -3.855031 \\
\hline $\mathrm{H}$ & 9.148543 & 6.670865 & -4.819945 \\
\hline $\mathrm{N}$ & 7.387113 & 7.175020 & 1.043841 \\
\hline$C$ & 6.878130 & 6.458284 & 2.201865 \\
\hline$C$ & 5.527274 & 7.040164 & 2.639452 \\
\hline 0 & 4.574360 & 6.334191 & 2.866542 \\
\hline$C$ & 7.832261 & 6.545612 & 3.403471 \\
\hline$C$ & 9.253675 & 5.996725 & 3.243850 \\
\hline $\mathrm{O}$ & 10.145147 & 6.631019 & 3.801318 \\
\hline 0 & 9.427075 & 4.934907 & 2.595110 \\
\hline $\mathrm{H}$ & 8.347195 & 7.444671 & 1.022750 \\
\hline $\mathrm{H}$ & 6.681020 & 5.429582 & 1.943173 \\
\hline $\mathrm{H}$ & 7.920449 & 7.570104 & 3.733357 \\
\hline $\mathrm{H}$ & 7.374114 & 5.991983 & 4.220501 \\
\hline $\mathrm{N}$ & 5.489857 & 8.378108 & 2.785881 \\
\hline $\mathrm{H}$ & 6.321223 & 8.912873 & 2.648924 \\
\hline$C$ & 4.299553 & 9.047240 & 3.249676 \\
\hline $\mathrm{H}$ & 4.538928 & 10.091629 & 3.406586 \\
\hline $\mathrm{H}$ & 3.957729 & 8.640894 & 4.188486 \\
\hline$C$ & 3.136800 & 8.988549 & 2.268521 \\
\hline 0 & 1.995347 & 9.111078 & 2.649457 \\
\hline $\mathrm{N}$ & 3.470143 & 8.825044 & 0.985192 \\
\hline $\mathrm{H}$ & 4.431256 & 8.829047 & 0.735185 \\
\hline$C$ & 2.517174 & 8.905264 & -0.078282 \\
\hline $\mathrm{H}$ & 3.059647 & 9.091911 & -0.996340 \\
\hline $\mathrm{H}$ & 1.843672 & 9.737837 & 0.071976 \\
\hline$C$ & 1.609145 & 7.687007 & -0.310220 \\
\hline 0 & 0.563844 & 7.814918 & -0.883302 \\
\hline
\end{tabular}




\begin{tabular}{|c|c|c|c|}
\hline$N$ & 2.090551 & 6.538698 & 0.206399 \\
\hline C & 1.283461 & 5.366493 & 0.435216 \\
\hline C & 0.467909 & 5.480602 & 1.727559 \\
\hline O & -0.727375 & 5.301066 & 1.700446 \\
\hline C & 2.156825 & 4.112013 & 0.446620 \\
\hline $\mathrm{H}$ & 2.996944 & 6.575258 & 0.622557 \\
\hline $\mathrm{H}$ & 0.552025 & 5.295917 & -0.352648 \\
\hline $\mathrm{H}$ & 1.554542 & 3.234765 & 0.657298 \\
\hline $\mathrm{H}$ & 2.625134 & 3.991727 & -0.523375 \\
\hline $\mathrm{H}$ & 2.937416 & 4.180555 & 1.197084 \\
\hline$N$ & 1.100153 & 5.752901 & 2.883514 \\
\hline C & 0.363855 & 5.588889 & 4.128797 \\
\hline C & -0.756499 & 6.616005 & 4.277630 \\
\hline $\mathrm{O}$ & -1.793405 & 6.307721 & 4.825433 \\
\hline C & 1.276925 & 5.517244 & 5.377594 \\
\hline$C$ & 1.869158 & 6.863261 & 5.822215 \\
\hline$C$ & 2.363409 & 4.450295 & 5.174675 \\
\hline$C$ & 2.702750 & 6.768435 & 7.102599 \\
\hline $\mathrm{H}$ & 2.087537 & 5.892449 & 2.903121 \\
\hline $\mathrm{H}$ & -0.160444 & 4.645841 & 4.073045 \\
\hline $\mathrm{H}$ & 0.616881 & 5.179865 & 6.171099 \\
\hline $\mathrm{H}$ & 2.477873 & 7.271798 & 5.022356 \\
\hline $\mathrm{H}$ & 1.065866 & 7.573300 & 5.990218 \\
\hline $\mathrm{H}$ & 2.734254 & 4.098677 & 6.126275 \\
\hline $\mathrm{H}$ & 1.969468 & 3.592745 & 4.638542 \\
\hline $\mathrm{H}$ & 3.205721 & 4.838364 & 4.612320 \\
\hline $\mathrm{H}$ & 3.008373 & 7.759737 & 7.424027 \\
\hline $\mathrm{H}$ & 2.126664 & 6.325645 & 7.909712 \\
\hline $\mathrm{H}$ & 3.597555 & 6.172743 & 6.971574 \\
\hline$N$ & -0.542379 & 7.851102 & 3.802898 \\
\hline
\end{tabular}




\begin{tabular}{|c|c|c|c|}
\hline $\mathrm{H}$ & 0.342671 & 8.093786 & 3.408281 \\
\hline C & -1.514248 & 8.891015 & 4.018121 \\
\hline $\mathrm{H}$ & -1.105089 & 9.819419 & 3.640139 \\
\hline $\mathrm{H}$ & -1.725162 & 9.021732 & 5.069675 \\
\hline C & -2.867021 & 8.618723 & 3.365915 \\
\hline $\mathrm{O}$ & -3.871201 & 8.835194 & 4.003704 \\
\hline$N$ & -2.902653 & 8.109029 & 2.120712 \\
\hline C & -4.238304 & 8.007140 & 1.534087 \\
\hline C & -4.968813 & 6.730657 & 1.912238 \\
\hline $\mathrm{O}$ & -6.180395 & 6.724062 & 1.899541 \\
\hline C & -4.023360 & 8.231437 & 0.030870 \\
\hline$C$ & -2.767508 & 9.109534 & 0.024558 \\
\hline C & -1.907940 & 8.459272 & 1.096327 \\
\hline $\mathrm{H}$ & -4.860614 & 8.797324 & 1.924965 \\
\hline $\mathrm{H}$ & -3.845773 & 7.294410 & -0.480499 \\
\hline $\mathrm{H}$ & -4.888841 & 8.699932 & -0.419823 \\
\hline $\mathrm{H}$ & -2.258022 & 9.157414 & -0.919586 \\
\hline $\mathrm{H}$ & -3.027490 & 10.122568 & 0.322266 \\
\hline $\mathrm{H}$ & -1.423282 & 7.569977 & 0.735735 \\
\hline $\mathrm{H}$ & -1.149998 & 9.118592 & 1.486782 \\
\hline$N$ & -4.242062 & 5.660723 & 2.268993 \\
\hline C & -4.898274 & 4.489751 & 2.822160 \\
\hline C & -5.601877 & 4.809738 & 4.143779 \\
\hline $\mathrm{O}$ & -6.682132 & 4.319340 & 4.387400 \\
\hline C & -3.922450 & 3.315696 & 3.044939 \\
\hline C & -3.602503 & 2.490692 & 1.814569 \\
\hline C & -4.319708 & 1.332976 & 1.526721 \\
\hline C & -2.563174 & 2.838102 & 0.963972 \\
\hline C & -4.010612 & 0.545503 & 0.427976 \\
\hline$C$ & -2.253167 & 2.067810 & -0.142660 \\
\hline
\end{tabular}




\begin{tabular}{|c|c|c|c|}
\hline C & -2.971075 & 0.916975 & -0.417876 \\
\hline $\mathrm{O}$ & -2.631644 & 0.190555 & -1.507482 \\
\hline $\mathrm{H}$ & -3.433245 & -0.132650 & -1.959688 \\
\hline $\mathrm{H}$ & -3.248636 & 5.746562 & 2.321847 \\
\hline $\mathrm{H}$ & -5.684950 & 4.182806 & 2.150951 \\
\hline $\mathrm{H}$ & -3.011371 & 3.702003 & 3.489514 \\
\hline $\mathrm{H}$ & -4.374445 & 2.664801 & 3.784727 \\
\hline $\mathrm{H}$ & -5.129104 & 1.032648 & 2.170014 \\
\hline $\mathrm{H}$ & -1.985680 & 3.717778 & 1.156763 \\
\hline $\mathrm{H}$ & -4.552287 & -0.361740 & 0.235736 \\
\hline $\mathrm{H}$ & -1.460646 & 2.361894 & -0.807782 \\
\hline$N$ & -4.963871 & 5.610892 & 5.012293 \\
\hline $\mathrm{H}$ & -4.035546 & 5.925060 & 4.817543 \\
\hline$C$ & -5.577344 & 5.988661 & 6.265812 \\
\hline $\mathrm{H}$ & -4.801079 & 6.365747 & 6.920127 \\
\hline $\mathrm{H}$ & -6.043692 & 5.137099 & 6.734369 \\
\hline$C$ & -6.651448 & 7.065798 & 6.114413 \\
\hline $\mathrm{O}$ & -7.709569 & 6.987260 & 6.695029 \\
\hline$N$ & -6.341993 & 8.091462 & 5.314137 \\
\hline $\mathrm{H}$ & -5.441180 & 8.148207 & 4.889102 \\
\hline C & -7.257699 & 9.220084 & 5.085090 \\
\hline $\mathrm{H}$ & -6.773822 & 9.881153 & 4.377801 \\
\hline $\mathrm{H}$ & -7.436849 & 9.765732 & 5.999939 \\
\hline C & -8.620671 & 8.834785 & 4.545740 \\
\hline $\mathrm{O}$ & -9.605844 & 9.430471 & 4.903626 \\
\hline$N$ & -8.666119 & 7.828890 & 3.652672 \\
\hline C & -9.928735 & 7.427001 & 3.085873 \\
\hline C & -10.814208 & 6.631357 & 4.032647 \\
\hline $\mathrm{O}$ & -11.920018 & 6.304751 & 3.637635 \\
\hline C & -9.727470 & 6.646850 & 1.766838 \\
\hline
\end{tabular}




\begin{tabular}{|c|c|c|c|}
\hline $\mathrm{O}$ & -10.949281 & 6.583909 & 1.057384 \\
\hline$C$ & -9.151222 & 5.252050 & 1.967219 \\
\hline $\mathrm{H}$ & -7.818177 & 7.408817 & 3.331702 \\
\hline $\mathrm{H}$ & -10.503055 & 8.315241 & 2.856243 \\
\hline $\mathrm{H}$ & -9.047561 & 7.228395 & 1.158214 \\
\hline $\mathrm{H}$ & -11.656044 & 6.539440 & 1.697476 \\
\hline $\mathrm{H}$ & -8.976819 & 4.784915 & 1.004375 \\
\hline $\mathrm{H}$ & -8.208806 & 5.278256 & 2.490847 \\
\hline $\mathrm{H}$ & -9.838715 & 4.627531 & 2.524461 \\
\hline $\mathrm{N}$ & -10.353503 & 6.328342 & 5.241144 \\
\hline C & -11.230801 & 5.723599 & 6.228936 \\
\hline C & -12.252343 & 6.706083 & 6.809021 \\
\hline 0 & -13.255900 & 6.278141 & 7.316874 \\
\hline C & -10.435120 & 5.093126 & 7.379532 \\
\hline C & -9.488846 & 3.950511 & 6.981945 \\
\hline C & -8.704722 & 3.491845 & 8.215332 \\
\hline C & -10.221807 & 2.767264 & 6.343005 \\
\hline $\mathrm{H}$ & -9.434313 & 6.600061 & 5.520595 \\
\hline $\mathrm{H}$ & -11.827697 & 4.972732 & 5.737374 \\
\hline $\mathrm{H}$ & -9.858702 & 5.870268 & 7.869945 \\
\hline $\mathrm{H}$ & -11.161036 & 4.730162 & 8.100289 \\
\hline $\mathrm{H}$ & -8.772377 & 4.325185 & 6.260399 \\
\hline $\mathrm{H}$ & -7.994884 & 2.710804 & 7.958003 \\
\hline $\mathrm{H}$ & -8.150028 & 4.315991 & 8.651707 \\
\hline $\mathrm{H}$ & -9.369896 & 3.098314 & 8.981175 \\
\hline $\mathrm{H}$ & -9.518995 & 1.971602 & 6.115290 \\
\hline $\mathrm{H}$ & -10.981145 & 2.365577 & 7.011897 \\
\hline $\mathrm{H}$ & -10.707761 & 3.037695 & 5.411874 \\
\hline $\mathrm{N}$ & -11.934887 & 8.016946 & 6.787785 \\
\hline$C$ & -12.883747 & 9.001314 & 7.216481 \\
\hline
\end{tabular}




\begin{tabular}{|c|c|c|c|}
\hline C & -13.851804 & 9.433132 & 6.135411 \\
\hline $\mathrm{H}$ & -14.611514 & 10.147443 & 6.472740 \\
\hline $\mathrm{O}$ & -13.817023 & 9.067938 & 5.007695 \\
\hline $\mathrm{H}$ & -11.174354 & 8.331188 & 6.225258 \\
\hline $\mathrm{H}$ & -13.453358 & 8.617522 & 8.052914 \\
\hline $\mathrm{H}$ & -12.358801 & 9.887276 & 7.563048 \\
\hline$N$ & -11.450257 & 10.622822 & -3.030063 \\
\hline $\mathrm{H}$ & -11.209401 & 10.836207 & -2.082680 \\
\hline C & -11.805542 & 9.208255 & -3.171992 \\
\hline$C$ & -11.410578 & 8.856581 & -4.599606 \\
\hline $\mathrm{O}$ & -10.372866 & 8.290255 & -4.846274 \\
\hline C & -13.244634 & 8.824411 & -2.765598 \\
\hline C & -13.482045 & 9.162735 & -1.282253 \\
\hline C & -13.513530 & 7.341796 & -3.054282 \\
\hline C & -14.912049 & 8.922018 & -0.794870 \\
\hline $\mathrm{H}$ & -12.216615 & 11.220134 & -3.277471 \\
\hline $\mathrm{H}$ & -11.119531 & 8.644244 & -2.555653 \\
\hline $\mathrm{H}$ & -13.943185 & 9.424308 & -3.353434 \\
\hline $\mathrm{H}$ & -12.790636 & 8.583990 & -0.673589 \\
\hline $\mathrm{H}$ & -13.242353 & 10.208066 & -1.113989 \\
\hline $\mathrm{H}$ & -14.550493 & 7.090409 & -2.872697 \\
\hline $\mathrm{H}$ & -13.297926 & 7.076618 & -4.085117 \\
\hline $\mathrm{H}$ & -12.902994 & 6.712089 & -2.414610 \\
\hline $\mathrm{H}$ & -15.024746 & 9.283026 & 0.221026 \\
\hline $\mathrm{H}$ & -15.634259 & 9.446908 & -1.414658 \\
\hline $\mathrm{H}$ & -15.172536 & 7.870289 & -0.791612 \\
\hline$N$ & -12.211998 & 9.288077 & -5.602655 \\
\hline $\mathrm{H}$ & -13.130162 & 9.596732 & -5.377231 \\
\hline C & -11.972873 & 8.843875 & -6.959238 \\
\hline $\mathrm{H}$ & -12.037085 & 7.767788 & -7.04562 \\
\hline
\end{tabular}




\begin{tabular}{|c|c|c|c|}
\hline $\mathrm{H}$ & -12.729643 & 9.284213 & -7.596911 \\
\hline C & -10.601375 & 9.232961 & -7.517198 \\
\hline $\mathrm{O}$ & -9.996310 & 8.496530 & -8.240798 \\
\hline$N$ & -10.162436 & 10.477354 & - -7.181606 \\
\hline $\mathrm{H}$ & -10.711595 & 11.008624 & -6.545545 \\
\hline C & -8.894348 & 11.024005 & -7.637086 \\
\hline $\mathrm{H}$ & -8.790095 & 10.891080 & -8.702745 \\
\hline $\mathrm{H}$ & -8.878879 & 12.081542 & -7.407784 \\
\hline C & -7.672362 & 10.359334 & -6.988510 \\
\hline $\mathrm{O}$ & -6.752675 & 9.936618 & -7.631252 \\
\hline$N$ & -7.659715 & 10.350056 & -5.619875 \\
\hline $\mathrm{H}$ & -8.482644 & 10.627868 & -5.134444 \\
\hline C & -6.495283 & 9.961236 & -4.832291 \\
\hline $\mathrm{H}$ & -5.599767 & 10.376146 & -5.266668 \\
\hline $\mathrm{H}$ & -6.615421 & 10.362681 & -3.833478 \\
\hline C & -6.266714 & 8.464815 & -4.710673 \\
\hline $\mathrm{O}$ & -5.130749 & 8.052024 & -4.722058 \\
\hline$N$ & -7.313248 & 7.638944 & -4.532851 \\
\hline C & -7.014154 & 6.281817 & -4.104237 \\
\hline C & -6.385456 & 5.453663 & -5.218210 \\
\hline $\mathrm{O}$ & -5.500989 & 4.680577 & -4.941478 \\
\hline C & -8.169246 & 5.547930 & -3.385353 \\
\hline C & -9.334491 & 5.136850 & -4.300200 \\
\hline C & -8.608671 & 6.363499 & -2.163462 \\
\hline C & -10.433624 & 4.357136 & -3.576042 \\
\hline $\mathrm{H}$ & -8.251731 & 7.983895 & -4.545473 \\
\hline $\mathrm{H}$ & -6.212394 & 6.347979 & -3.384898 \\
\hline $\mathrm{H}$ & -7.712488 & 4.634768 & -3.012760 \\
\hline $\mathrm{H}$ & -9.766675 & 6.006171 & -4.779698 \\
\hline $\mathrm{H}$ & -8.937059 & 4.509640 & -5.09455 \\
\hline
\end{tabular}




\begin{tabular}{|c|c|c|c|}
\hline $\mathrm{H}$ & -9.268033 & 5.788314 & -1.527947 \\
\hline $\mathrm{H}$ & -7.750844 & 6.646609 & -1.561923 \\
\hline $\mathrm{H}$ & -9.133340 & 7.266292 & -2.452937 \\
\hline $\mathrm{H}$ & -11.164286 & 3.980870 & -4.285180 \\
\hline $\mathrm{H}$ & -10.029718 & 3.505141 & L -3.040149 \\
\hline $\mathrm{H}$ & -10.964625 & 4.978458 & $3-2.865183$ \\
\hline$N$ & -6.821804 & 5.574681 & -6.491828 \\
\hline C & -6.122650 & 4.837516 & -7.526359 \\
\hline C & -4.766933 & 5.445622 & -7.897427 \\
\hline $\mathrm{O}$ & -3.900287 & 4.709945 & -8.309122 \\
\hline C & -6.985446 & 4.553700 & -8.764525 \\
\hline C & -8.063468 & 3.474445 & -8.557259 \\
\hline C & -7.485599 & 2.123282 & -8.106835 \\
\hline$N$ & -8.413266 & 0.992326 & -8.200115 \\
\hline C & -9.246595 & 0.580570 & -7.239923 \\
\hline $\mathrm{N}$ & -9.565574 & 1.404866 & -6.254236 \\
\hline $\mathrm{N}$ & -9.750218 & -0.632824 & -7.273985 \\
\hline $\mathrm{H}$ & -7.500345 & 6.268083 & -6.723064 \\
\hline $\mathrm{H}$ & -5.828110 & 3.900653 & -7.087703 \\
\hline $\mathrm{H}$ & -7.460724 & 5.467152 & -9.109803 \\
\hline $\mathrm{H}$ & -6.316866 & 4.232021 & -9.555641 \\
\hline $\mathrm{H}$ & -8.808023 & 3.827661 & -7.849474 \\
\hline $\mathrm{H}$ & -8.583770 & 3.331754 & -9.498531 \\
\hline $\mathrm{H}$ & -6.639228 & 1.867242 & -8.730931 \\
\hline $\mathrm{H}$ & -7.117893 & 2.168777 & -7.090395 \\
\hline $\mathrm{H}$ & -8.305494 & 0.376873 & -8.975587 \\
\hline $\mathrm{H}$ & -9.343070 & 2.370149 & -6.313647 \\
\hline $\mathrm{H}$ & -10.096875 & 1.113067 & $7-5.449591$ \\
\hline $\mathrm{H}$ & -9.353943 & -1.381802 & -7.810369 \\
\hline 11 & -10.478063 & -0.911624 & $4 \quad-6.634207$ \\
\hline
\end{tabular}




\begin{tabular}{|c|c|c|c|}
\hline$N$ & -4.571397 & 6.757500 & -7.722194 \\
\hline $\mathrm{H}$ & -5.311839 & 7.353646 & -7.430197 \\
\hline C & -3.257798 & 7.352383 & -8.011132 \\
\hline $\mathrm{H}$ & -2.933328 & 7.094293 & -9.006716 \\
\hline $\mathrm{H}$ & -3.359263 & 8.427000 & -7.936787 \\
\hline C & -2.193925 & 6.877266 & -7.033153 \\
\hline $\mathrm{O}$ & -1.066831 & 6.644365 & -7.393204 \\
\hline$N$ & -2.610444 & 6.768791 & -5.766313 \\
\hline C & -1.723879 & 6.459944 & -4.670821 \\
\hline C & -1.446884 & 4.973443 & -4.542387 \\
\hline O & -0.318980 & 4.587434 & -4.335975 \\
\hline C & -2.308725 & 7.054951 & -3.383215 \\
\hline C & -1.727750 & 6.351465 & -2.157691 \\
\hline C & -2.065842 & 8.570665 & -3.416545 \\
\hline $\mathrm{H}$ & -3.535779 & 7.063300 & -5.540733 \\
\hline $\mathrm{H}$ & -0.755531 & 6.900104 & -4.861077 \\
\hline $\mathrm{H}$ & -3.377571 & 6.877876 & -3.399277 \\
\hline $\mathrm{H}$ & -1.942860 & 6.894108 & -1.256264 \\
\hline $\mathrm{H}$ & -2.144386 & 5.355004 & -2.053182 \\
\hline $\mathrm{H}$ & -0.655538 & 6.273649 & -2.226193 \\
\hline $\mathrm{H}$ & -2.801574 & 9.100883 & -2.828668 \\
\hline $\mathrm{H}$ & -1.077337 & 8.810805 & -3.039524 \\
\hline $\mathrm{H}$ & -2.147406 & 8.954165 & -4.426999 \\
\hline $\mathrm{N}$ & -2.476495 & 4.120597 & -4.661333 \\
\hline$C$ & -2.166304 & 2.713538 & -4.735604 \\
\hline$C$ & -1.263088 & 2.445083 & -5.932189 \\
\hline O & -0.390986 & 1.610467 & -5.875037 \\
\hline$C$ & -3.409265 & 1.801838 & -4.796464 \\
\hline$C$ & -4.384645 & 1.957318 & -3.620059 \\
\hline C & -5.461595 & 0.858245 & -3.5519 \\
\hline
\end{tabular}




\begin{tabular}{|c|c|c|c|}
\hline$N$ & -5.024496 & -0.309681 & -2.806600 \\
\hline C & -5.834253 & -1.285002 & -2.637217 \\
\hline$N$ & -7.120260 & -1.337914 & -3.162348 \\
\hline$N$ & -5.545493 & -2.328910 & -1.829167 \\
\hline $\mathrm{H}$ & -3.402222 & 4.448079 & -4.837172 \\
\hline $\mathrm{H}$ & -1.584944 & 2.432904 & -3.869959 \\
\hline $\mathrm{H}$ & -3.941466 & 1.981175 & -5.729108 \\
\hline $\mathrm{H}$ & -3.042275 & 0.782435 & -4.840131 \\
\hline $\mathrm{H}$ & -3.838600 & 1.977226 & -2.688909 \\
\hline $\mathrm{H}$ & -4.885377 & 2.909031 & -3.704561 \\
\hline $\mathrm{H}$ & -6.351110 & 1.287881 & -3.090705 \\
\hline $\mathrm{H}$ & -5.745251 & 0.597613 & -4.575295 \\
\hline $\mathrm{H}$ & -7.226831 & -0.855575 & -4.027498 \\
\hline $\mathrm{H}$ & -7.521314 & -2.257482 & -3.202541 \\
\hline $\mathrm{H}$ & -4.636504 & -2.422368 & -1.418150 \\
\hline $\mathrm{H}$ & -6.040973 & -3.182962 & -1.968369 \\
\hline$N$ & -1.459772 & 3.193335 & -7.035054 \\
\hline $\mathrm{H}$ & -2.267073 & 3.766053 & -7.144654 \\
\hline C & -0.596025 & 2.984495 & -8.179304 \\
\hline $\mathrm{H}$ & -0.940339 & 3.627838 & -8.978877 \\
\hline $\mathrm{H}$ & -0.614408 & 1.958680 & -8.517967 \\
\hline C & 0.858091 & 3.326704 & -7.861354 \\
\hline 0 & 1.786002 & 2.683366 & -8.284673 \\
\hline $\mathrm{N}$ & 1.007247 & 4.396226 & -7.063566 \\
\hline $\mathrm{H}$ & 0.213454 & 4.960291 & -6.860760 \\
\hline $\mathrm{C}$ & 2.282316 & 4.880650 & -6.640295 \\
\hline $\mathrm{H}$ & 2.918451 & 5.142291 & -7.476484 \\
\hline $\mathrm{H}$ & 2.122218 & 5.774110 & -6.050950 \\
\hline $\mathrm{C}$ & 3.094585 & 3.926538 & -5.779514 \\
\hline 0 & 4.300619 & 4.045709 & -5.700965 \\
\hline
\end{tabular}




\begin{tabular}{|c|c|c|c|}
\hline$N$ & 2.428034 & 2.983354 & -5.101941 \\
\hline C & 3.098463 & 2.130525 & -4.156529 \\
\hline C & 4.281958 & 1.344186 & -4.723576 \\
\hline $\mathrm{O}$ & 5.201586 & 1.054233 & -3.984871 \\
\hline C & 2.026251 & 1.207917 & -3.579545 \\
\hline C & 2.417358 & 0.331843 & -2.395340 \\
\hline C & 1.121318 & -0.252054 & -1.856988 \\
\hline$N$ & 1.333465 & -1.247445 & -0.825635 \\
\hline C & 0.341907 & -2.073104 & -0.445731 \\
\hline$N$ & -0.814703 & -2.039920 & -1.064747 \\
\hline$N$ & 0.558570 & -2.942123 & 0.514014 \\
\hline $\mathrm{H}$ & 1.433644 & 2.939389 & -5.176189 \\
\hline $\mathrm{H}$ & 3.540141 & 2.726312 & -3.366558 \\
\hline $\mathrm{H}$ & 1.214753 & 1.858392 & -3.271114 \\
\hline $\mathrm{H}$ & 1.624538 & 0.593943 & -4.377136 \\
\hline $\mathrm{H}$ & 3.092421 & -0.463484 & -2.691511 \\
\hline $\mathrm{H}$ & 2.922225 & 0.917788 & -1.630589 \\
\hline $\mathrm{H}$ & 0.483874 & 0.548064 & -1.492865 \\
\hline $\mathrm{H}$ & 0.600009 & -0.735144 & -2.671209 \\
\hline $\mathrm{H}$ & 2.042994 & -1.075112 & -0.148596 \\
\hline $\mathrm{H}$ & -1.141136 & -1.193131 & -1.482368 \\
\hline $\mathrm{H}$ & -1.575371 & -2.653224 & -0.776534 \\
\hline $\mathrm{H}$ & 1.472959 & -3.119916 & 0.859965 \\
\hline $\mathrm{H}$ & -0.227421 & -3.495280 & 0.850676 \\
\hline $\mathrm{N}$ & 4.281635 & 0.945291 & -6.005325 \\
\hline $\mathrm{H}$ & 3.540573 & 1.224875 & -6.614868 \\
\hline $\mathrm{C}$ & 5.363426 & 0.116658 & -6.504603 \\
\hline $\mathrm{H}$ & 5.139409 & -0.143537 & -7.531646 \\
\hline $\mathrm{H}$ & 5.441115 & -0.797352 & -5.937286 \\
\hline$C$ & 6.736239 & 0.756118 & -6.476126 \\
\hline
\end{tabular}




\begin{tabular}{|c|c|c|c|}
\hline $\mathrm{O}$ & 7.738555 & 0.089656 & -6.364875 \\
\hline$N$ & 6.766121 & 2.085332 & -6.608323 \\
\hline $\mathrm{H}$ & 5.912181 & 2.604987 & -6.573038 \\
\hline C & 8.005506 & 2.823554 & -6.551101 \\
\hline $\mathrm{H}$ & 8.709856 & 2.439513 & -7.275404 \\
\hline $\mathrm{H}$ & 7.800297 & 3.854280 & -6.807629 \\
\hline C & 8.748820 & 2.826845 & -5.213322 \\
\hline 0 & 9.890039 & 3.236074 & -5.161844 \\
\hline$N$ & 8.103915 & 2.338674 & -4.145947 \\
\hline C & 8.685626 & 2.174737 & -2.824696 \\
\hline C & 9.950262 & 1.305501 & -2.832336 \\
\hline $\mathrm{O}$ & 10.768881 & 1.388557 & -1.931030 \\
\hline C & 7.581575 & 1.507829 & -1.973953 \\
\hline C & 7.779817 & 1.384037 & -0.463337 \\
\hline C & 7.439605 & 2.653774 & 0.326391 \\
\hline C & 7.329383 & 2.386651 & 1.823737 \\
\hline$N$ & 8.669523 & 2.353504 & 2.481094 \\
\hline $\mathrm{O}$ & 11.041612 & 1.616871 & 0.893228 \\
\hline $\mathrm{H}$ & 10.891632 & 1.828025 & -0.028672 \\
\hline $\mathrm{H}$ & 11.520020 & 0.790173 & 0.842419 \\
\hline $\mathrm{H}$ & 7.157877 & 2.036062 & -4.258768 \\
\hline $\mathrm{H}$ & 8.984963 & 3.132165 & -2.419114 \\
\hline $\mathrm{H}$ & 6.665683 & 2.053445 & -2.157437 \\
\hline $\mathrm{H}$ & 7.411974 & 0.521088 & -2.389189 \\
\hline $\mathrm{H}$ & 7.099968 & 0.604930 & -0.132299 \\
\hline $\mathrm{H}$ & 8.775100 & 1.030447 & -0.228885 \\
\hline $\mathrm{H}$ & 8.153081 & 3.454089 & 0.151070 \\
\hline $\mathrm{H}$ & 6.474658 & 3.026456 & -0.002074 \\
\hline $\mathrm{H}$ & 6.769015 & 3.158916 & 2.327285 \\
\hline $\mathrm{H}$ & 6.848286 & 1.440553 & 2.018338 \\
\hline
\end{tabular}




\begin{tabular}{|c|c|c|c|}
\hline $\mathrm{H}$ & 9.380292 & 1.889581 & 1.923937 \\
\hline $\mathrm{H}$ & 8.989521 & 3.323710 & 2.635327 \\
\hline $\mathrm{H}$ & 8.615971 & 1.875762 & 3.368830 \\
\hline$N$ & 10.062478 & 0.448235 & -3.837531 \\
\hline C & 11.109302 & -0.537622 & -4.069126 \\
\hline C & 12.486186 & 0.023064 & -4.427545 \\
\hline O & 13.443828 & -0.705843 & -4.301729 \\
\hline C & 10.700491 & -1.444770 & -5.251616 \\
\hline C & 9.562626 & -2.443148 & -4.995255 \\
\hline C & 9.130006 & -3.068388 & -6.325173 \\
\hline C & 9.990725 & -3.533279 & -4.012591 \\
\hline $\mathrm{H}$ & 9.324254 & 0.446347 & -4.510701 \\
\hline $\mathrm{H}$ & 11.265043 & -1.124641 & -3.175827 \\
\hline $\mathrm{H}$ & 10.433337 & -0.799693 & -6.083324 \\
\hline $\mathrm{H}$ & 11.583200 & -1.997286 & -5.550529 \\
\hline $\mathrm{H}$ & 8.706467 & -1.911957 & -4.590989 \\
\hline $\mathrm{H}$ & 8.328910 & -3.784615 & -6.169730 \\
\hline $\mathrm{H}$ & 8.772190 & -2.311859 & -7.011845 \\
\hline $\mathrm{H}$ & 9.956732 & -3.596651 & -6.793621 \\
\hline $\mathrm{H}$ & 9.201166 & -4.263427 & -3.872016 \\
\hline $\mathrm{H}$ & 10.860730 & -4.059506 & -4.391441 \\
\hline $\mathrm{H}$ & 10.244155 & -3.135993 & -3.034455 \\
\hline$N$ & 12.574038 & 1.261815 & -4.919239 \\
\hline C & 13.814611 & 1.844699 & -5.393667 \\
\hline C & 14.958796 & 2.033213 & -4.379222 \\
\hline $\mathrm{O}$ & 16.013406 & 2.413914 & -4.812180 \\
\hline C & 13.497216 & 3.171519 & -6.086692 \\
\hline $\mathrm{O}$ & 12.918143 & 4.088316 & -5.202169 \\
\hline $\mathrm{H}$ & 11.756430 & 1.833595 & -4.917107 \\
\hline $\mathrm{H}$ & 14.248463 & 1.177819 & -6.129320 \\
\hline
\end{tabular}




\begin{tabular}{|c|c|c|c|}
\hline $\mathrm{H}$ & 14.420374 & 3.590493 & -6.455363 \\
\hline $\mathrm{H}$ & 12.846416 & 2.977796 & -6.933449 \\
\hline $\mathrm{H}$ & 11.970767 & 4.079321 & -5.267015 \\
\hline$N$ & 14.792294 & 1.722322 & -3.074271 \\
\hline C & 15.928290 & 1.647471 & -2.176854 \\
\hline C & 16.804559 & 0.409966 & -2.402567 \\
\hline O & 17.934463 & 0.393973 & -1.988198 \\
\hline C & 15.495410 & 1.578098 & -0.707307 \\
\hline C & 14.638887 & 2.735265 & -0.197980 \\
\hline C & 14.285572 & 2.488812 & 1.269118 \\
\hline$N$ & 13.260121 & 3.383439 & 1.791074 \\
\hline$C$ & 13.437415 & 4.534331 & 2.419116 \\
\hline$N$ & 14.655014 & 5.009909 & 2.656431 \\
\hline $\mathrm{O}$ & 15.027888 & 7.465698 & 4.211033 \\
\hline $\mathrm{H}$ & 14.744161 & 8.352217 & 3.972230 \\
\hline $\mathrm{H}$ & 15.746003 & 7.571727 & 4.821972 \\
\hline$N$ & 12.387261 & 5.222685 & 2.815466 \\
\hline $\mathrm{H}$ & 13.900860 & 1.420265 & -2.758213 \\
\hline $\mathrm{H}$ & 16.561977 & 2.504360 & -2.346580 \\
\hline $\mathrm{H}$ & 14.954873 & 0.650222 & -0.551603 \\
\hline $\mathrm{H}$ & 16.407420 & 1.508933 & -0.124562 \\
\hline $\mathrm{H}$ & 15.156582 & 3.683014 & -0.314429 \\
\hline $\mathrm{H}$ & 13.719649 & 2.816474 & -0.766270 \\
\hline $\mathrm{H}$ & 13.889203 & 1.489447 & 1.376121 \\
\hline $\mathrm{H}$ & 15.164998 & 2.531855 & 1.900852 \\
\hline $\mathrm{H}$ & 12.320099 & 3.087488 & 1.619789 \\
\hline $\mathrm{H}$ & 15.463034 & 4.517248 & 2.358844 \\
\hline $\mathrm{H}$ & 14.790124 & 5.864747 & 3.168310 \\
\hline $\mathrm{H}$ & 11.431842 & 4.942742 & 2.657259 \\
\hline & 12.479311 & 6.065747 & 3.335753 \\
\hline
\end{tabular}




\begin{tabular}{|c|c|c|c|}
\hline$N$ & 16.200243 & -0.648980 & -2.967249 \\
\hline C & 16.870870 & -1.911472 & -3.168108 \\
\hline C & 17.669797 & -1.889273 & -4.467827 \\
\hline $\mathrm{H}$ & 18.062651 & -0.909448 & -4.748489 \\
\hline $\mathrm{O}$ & 17.877414 & -2.853311 & -5.129576 \\
\hline C & 15.868175 & -3.070204 & -3.149457 \\
\hline C & 15.324743 & -3.399835 & -1.791995 \\
\hline$N$ & 14.403816 & -2.627471 & -1.112311 \\
\hline $\mathrm{O}$ & 12.508299 & -0.434059 & -0.465262 \\
\hline $\mathrm{H}$ & 13.145756 & -1.037495 & -0.851236 \\
\hline $\mathrm{H}$ & 12.027967 & -0.038276 & -1.184423 \\
\hline C & 15.619899 & -4.490240 & -1.049586 \\
\hline C & 14.172673 & -3.251535 & -0.003779 \\
\hline$N$ & 14.883079 & -4.381616 & 0.103171 \\
\hline $\mathrm{H}$ & 15.285669 & -0.550324 & -3.352780 \\
\hline $\mathrm{H}$ & 17.596080 & -2.030752 & -2.370797 \\
\hline $\mathrm{H}$ & 15.050696 & -2.842318 & -3.824751 \\
\hline $\mathrm{H}$ & 16.369011 & -3.944015 & -3.543925 \\
\hline $\mathrm{H}$ & 16.275354 & -5.313992 & -1.234936 \\
\hline $\mathrm{H}$ & 13.494351 & -2.922007 & 0.757153 \\
\hline $\mathrm{H}$ & 14.782435 & -5.064439 & 0.821938 \\
\hline 0 & -14.798044 & -2.305754 & 5.077409 \\
\hline $\mathrm{H}$ & -15.090905 & -1.616880 & 4.485558 \\
\hline $\mathrm{H}$ & -14.377340 & -1.863123 & 5.807280 \\
\hline 0 & -12.885843 & -0.761308 & 6.903392 \\
\hline $\mathrm{H}$ & -12.159022 & -1.250959 & 7.273172 \\
\hline $\mathrm{H}$ & -13.152096 & -0.138636 & 7.568679 \\
\hline 0 & -12.140923 & -0.228943 & 4.090862 \\
\hline $\mathrm{H}$ & -12.285262 & -0.224152 & 5.032934 \\
\hline $\mathrm{H}$ & -13.017632 & -0.218848 & 3.703235 \\
\hline
\end{tabular}




\begin{tabular}{|c|c|c|c|}
\hline 0 & -13.359540 & -2.023823 & 0.907578 \\
\hline $\mathrm{H}$ & -12.590514 & -1.456197 & 0.826825 \\
\hline $\mathrm{H}$ & -13.169463 & -2.625755 & 1.627177 \\
\hline $\mathrm{O}$ & -7.462981 & 2.078119 & -0.250589 \\
\hline $\mathrm{H}$ & -7.582864 & 2.166507 & 0.698314 \\
\hline $\mathrm{H}$ & -6.548782 & 1.844730 & -0.359079 \\
\hline $\mathrm{O}$ & -7.781876 & 2.148195 & 2.588044 \\
\hline $\mathrm{H}$ & -8.712592 & 1.982084 & 2.721336 \\
\hline $\mathrm{H}$ & -7.530711 & 2.835493 & 3.200300 \\
\hline $\mathrm{O}$ & -9.219699 & 0.267820 & -1.542912 \\
\hline $\mathrm{H}$ & -8.654544 & 0.920588 & -1.115801 \\
\hline $\mathrm{H}$ & -8.625703 & -0.395486 & -1.892490 \\
\hline $\mathrm{O}$ & -13.105016 & -2.225681 & -1.907724 \\
\hline $\mathrm{H}$ & -13.351473 & -2.375355 & -0.993383 \\
\hline $\mathrm{H}$ & -13.802370 & -2.556482 & -2.463211 \\
\hline $\mathrm{O}$ & -13.484751 & 1.744874 & -0.190583 \\
\hline $\mathrm{H}$ & -12.857725 & 1.055718 & 0.037437 \\
\hline $\mathrm{H}$ & -12.999711 & 2.554839 & -0.018400 \\
\hline $\mathrm{O}$ & -14.181219 & 4.914623 & 2.450307 \\
\hline $\mathrm{H}$ & -13.801617 & 5.401629 & 3.176341 \\
\hline $\mathrm{H}$ & -14.711518 & 4.211966 & 2.807283 \\
\hline $\mathrm{O}$ & -15.446010 & 2.146062 & 1.862203 \\
\hline $\mathrm{H}$ & -16.298712 & 2.345649 & 1.498153 \\
\hline $\mathrm{H}$ & -14.850012 & 2.052006 & 1.116081 \\
\hline $\mathrm{O}$ & -13.497394 & 0.499191 & -2.728085 \\
\hline $\mathrm{H}$ & -13.706526 & 1.081279 & -1.997199 \\
\hline $\mathrm{H}$ & -13.279600 & -0.342387 & -2.323820 \\
\hline $\mathrm{O}$ & -14.513808 & -1.463214 & -4.510948 \\
\hline $\mathrm{H}$ & -14.515842 & -0.619392 & -4.063504 \\
\hline $\mathrm{H}$ & -15.310723 & -1.512896 & -5.022209 \\
\hline
\end{tabular}




\begin{tabular}{llll}
$\mathrm{O}$ & -10.574806 & -2.969291 & -3.202223 \\
$\mathrm{H}$ & -10.866863 & -2.657694 & -4.055438 \\
$\mathrm{H}$ & -11.319224 & -2.817613 & -2.624595 \\
$\mathrm{O}$ & -11.340807 & -0.095887 & 0.389376 \\
$\mathrm{H}$ & -10.664017 & -0.135631 & -0.288250 \\
$\mathrm{H}$ & -10.954649 & 0.397056 & 1.116747 \\
$\mathrm{O}$ & -10.645993 & 1.634979 & 2.510579 \\
$\mathrm{H}$ & -11.151044 & 1.180499 & 3.185947 \\
$\mathrm{H}$ & -11.153168 & 2.384140 & 2.200282 \\
$\mathrm{O}$ & -12.086443 & 3.782053 & 1.051736 \\
$\mathrm{H}$ & -12.806674 & 4.185137 & 1.549897 \\
$\mathrm{H}$ & -11.540687 & 4.511513 & 0.773464 \\
$\mathrm{O}$ & -10.887995 & 0.832534 & -3.747225 \\
$\mathrm{H}$ & -11.765775 & 0.977393 & -3.390389 \\
$\mathrm{H}$ & -10.308430 & 0.682653 & -2.994348 \\
$\mathrm{O}$ & -14.735692 & -0.392839 & 2.923980 \\
$\mathrm{H}$ & -15.121422 & 0.439458 & 2.650263 \\
$\mathrm{H}$ & -14.531649 & -0.883577 & 2.129567 \\
$\mathrm{O}$ & -12.750308 & -3.277875 & 3.454294 \\
$\mathrm{H}$ & -13.513474 & -3.074762 & 4.007107 \\
$\mathrm{H}$ & -12.113285 & -2.610857 & 3.692400 \\
\hline & -11.716682 & -1.375674 & -5.293692 \\
$\mathrm{H}$ & -12.662486 & -1.515867 & -5.271991 \\
\hline & -11.531033 & -0.699389 & -4.643671
\end{tabular}

PROTON CONFIGURATION, GIVEN BY NOMINAL CHARGES: R300(0),Y266(0),E183(0) POTENTIAL:+70mV

$\mathrm{N}$

$15.580942-6.451471 \quad 5.391415$ 


\begin{tabular}{|c|c|c|c|}
\hline $\mathrm{H}$ & 14.780349 & -6.201766 & 5.939146 \\
\hline C & 15.509367 & -5.856426 & 4.054303 \\
\hline C & 14.783016 & -6.782958 & 3.060460 \\
\hline $\mathrm{O}$ & 14.052521 & -6.345772 & 2.186629 \\
\hline C & 16.919361 & -5.576248 & 3.520821 \\
\hline $\mathrm{H}$ & 16.389372 & -6.127384 & 5.884687 \\
\hline $\mathrm{H}$ & 14.944498 & -4.933617 & 4.050508 \\
\hline $\mathrm{H}$ & 16.888494 & -5.189753 & 2.509108 \\
\hline $\mathrm{H}$ & 17.403232 & -4.831330 & 4.142353 \\
\hline $\mathrm{H}$ & 17.526831 & -6.474492 & 3.529436 \\
\hline$N$ & 15.032384 & -8.085605 & 3.186393 \\
\hline $\mathrm{H}$ & 15.612054 & -8.361765 & 3.949721 \\
\hline C & 14.398655 & -9.092385 & 2.333235 \\
\hline $\mathrm{H}$ & 14.482396 & -8.823759 & 1.292869 \\
\hline $\mathrm{H}$ & 14.891390 & -10.042255 & 2.492729 \\
\hline$C$ & 12.912798 & -9.220863 & 2.671372 \\
\hline 0 & 12.081474 & -9.424771 & 1.825885 \\
\hline $\mathrm{N}$ & 12.582805 & -9.055889 & 3.966871 \\
\hline $\mathrm{H}$ & 13.302698 & -8.986571 & 4.648940 \\
\hline$C$ & 11.195100 & -9.170804 & 4.421405 \\
\hline $\mathrm{H}$ & 11.188267 & -9.157896 & 5.503158 \\
\hline $\mathrm{H}$ & 10.743722 & -10.088319 & 4.077196 \\
\hline$C$ & 10.336963 & -8.010603 & 3.900697 \\
\hline 0 & 9.190772 & -8.186042 & 3.584573 \\
\hline $\mathrm{N}$ & 10.947108 & -6.814557 & 3.855284 \\
\hline$C$ & 10.318434 & -5.634083 & 3.317568 \\
\hline$C$ & 10.126894 & -5.691133 & 1.799331 \\
\hline $\mathrm{O}$ & 9.096452 & -5.262764 & 1.337093 \\
\hline$C$ & 11.092693 & -4.388012 & 3.776312 \\
\hline 0 & 12.478661 & -4.570737 & 3.769134 \\
\hline
\end{tabular}




\begin{tabular}{|c|c|c|c|}
\hline $\mathrm{H}$ & 11.905473 & -6.744232 & 4.106887 \\
\hline $\mathrm{H}$ & 9.314542 & -5.553322 & 3.701121 \\
\hline $\mathrm{H}$ & 10.801112 & -3.539672 & 3.167344 \\
\hline $\mathrm{H}$ & 10.827326 & -4.174458 & 4.801380 \\
\hline $\mathrm{H}$ & 12.805421 & -4.755040 & 2.897012 \\
\hline$N$ & 11.100275 & -6.200613 & 1.024177 \\
\hline C & 10.844930 & -6.396984 & -0.400192 \\
\hline C & 9.694191 & -7.385718 & -0.601590 \\
\hline O & 8.946504 & -7.280192 & -1.545833 \\
\hline C & 12.133418 & -6.785894 & -1.186814 \\
\hline C & 12.333972 & -8.291280 & -1.403984 \\
\hline C & 12.160705 & -6.060462 & -2.533508 \\
\hline $\mathrm{H}$ & 11.924268 & -6.586240 & 1.431556 \\
\hline $\mathrm{H}$ & 10.475301 & -5.462697 & -0.791660 \\
\hline $\mathrm{H}$ & 12.965434 & -6.416169 & -0.598032 \\
\hline $\mathrm{H}$ & 13.287800 & -8.454577 & -1.894898 \\
\hline $\mathrm{H}$ & 12.332078 & -8.857756 & -0.483538 \\
\hline $\mathrm{H}$ & 11.566859 & -8.698071 & -2.055726 \\
\hline $\mathrm{H}$ & 13.055508 & -6.324363 & -3.086116 \\
\hline $\mathrm{H}$ & 11.300428 & -6.332005 & -3.135436 \\
\hline $\mathrm{H}$ & 12.160806 & -4.985273 & -2.400041 \\
\hline $\mathrm{N}$ & 9.570408 & -8.340330 & 0.328361 \\
\hline $\mathrm{H}$ & 10.314480 & -8.474432 & 0.974388 \\
\hline$C$ & 8.593032 & -9.421193 & 0.241195 \\
\hline $\mathrm{H}$ & 8.935861 & -10.219590 & 0.886968 \\
\hline $\mathrm{H}$ & 8.532017 & -9.802139 & -0.766597 \\
\hline$C$ & 7.181944 & -9.047549 & 0.645357 \\
\hline 0 & 6.257521 & -9.505331 & 0.014451 \\
\hline $\mathrm{N}$ & 7.004056 & -8.228491 & 1.688362 \\
\hline C & 5.670081 & -7.742066 & 1.958390 \\
\hline
\end{tabular}




\begin{tabular}{|c|c|c|c|}
\hline C & 5.250599 & -6.730681 & 0.897072 \\
\hline $\mathrm{O}$ & 4.073692 & -6.634174 & 0.621488 \\
\hline C & 5.416214 & -7.237024 & 3.392842 \\
\hline C & 5.559791 & -8.381152 & 4.401516 \\
\hline C & 6.257028 & -6.027261 & 3.805597 \\
\hline $\mathrm{H}$ & 7.781723 & -7.961726 & 2.252011 \\
\hline $\mathrm{H}$ & 4.987432 & -8.561748 & 1.796393 \\
\hline $\mathrm{H}$ & 4.375059 & -6.929201 & 3.386405 \\
\hline $\mathrm{H}$ & 5.269882 & -8.044251 & 5.391231 \\
\hline $\mathrm{H}$ & 4.922390 & -9.219159 & 4.138918 \\
\hline $\mathrm{H}$ & 6.582310 & -8.737422 & 4.456785 \\
\hline $\mathrm{H}$ & 5.916438 & -5.655352 & 4.766019 \\
\hline $\mathrm{H}$ & 7.299625 & -6.296792 & 3.912474 \\
\hline $\mathrm{H}$ & 6.184055 & -5.212892 & 3.092997 \\
\hline$N$ & 6.171151 & -6.006054 & 0.252849 \\
\hline C & 5.775815 & -5.188296 & -0.881848 \\
\hline C & 5.344935 & -6.072896 & -2.060131 \\
\hline $\mathrm{O}$ & 4.321599 & -5.821913 & -2.652478 \\
\hline C & 6.867606 & -4.168045 & -1.261631 \\
\hline C & 7.064145 & -3.150823 & -0.123538 \\
\hline C & 6.496595 & -3.458456 & -2.568499 \\
\hline C & 8.356860 & -2.342647 & -0.237676 \\
\hline $\mathrm{H}$ & 7.132427 & -6.063805 & 0.511463 \\
\hline $\mathrm{H}$ & 4.877360 & -4.652106 & -0.615760 \\
\hline $\mathrm{H}$ & 7.798107 & -4.708570 & -1.408911 \\
\hline $\mathrm{H}$ & 6.208311 & -2.477482 & -0.107810 \\
\hline $\mathrm{H}$ & 7.074430 & -3.664394 & 0.828510 \\
\hline $\mathrm{H}$ & 7.201410 & -2.668340 & -2.788559 \\
\hline $\mathrm{H}$ & 6.497445 & -4.133933 & -3.414165 \\
\hline $\mathrm{H}$ & 5.508088 & -3.015876 & -2.502185 \\
\hline
\end{tabular}




\begin{tabular}{|c|c|c|c|}
\hline $\mathrm{H}$ & 8.457266 & -1.668840 & 0.606528 \\
\hline $\mathrm{H}$ & 9.219071 & -2.999136 & -0.237414 \\
\hline $\mathrm{H}$ & 8.392266 & -1.743091 & -1.140341 \\
\hline$N$ & 6.117711 & -7.114918 & -2.395362 \\
\hline $\mathrm{H}$ & 7.012137 & -7.235987 & -1.968883 \\
\hline C & 5.776849 & -7.956143 & -3.522959 \\
\hline $\mathrm{H}$ & 6.607482 & -8.630335 & -3.690883 \\
\hline $\mathrm{H}$ & 5.623490 & -7.371145 & -4.417519 \\
\hline C & 4.507232 & -8.783923 & -3.322025 \\
\hline $\mathrm{O}$ & 3.670649 & -8.842852 & -4.191822 \\
\hline$N$ & 4.393877 & -9.435505 & -2.157905 \\
\hline $\mathrm{H}$ & 5.130851 & -9.392153 & -1.486720 \\
\hline C & 3.206567 & -10.189471 & -1.830312 \\
\hline $\mathrm{H}$ & 2.967419 & -10.903250 & -2.604295 \\
\hline $\mathrm{H}$ & 3.396800 & -10.730478 & -0.911826 \\
\hline C & 1.974201 & -9.306854 & -1.644807 \\
\hline 0 & 0.887592 & -9.694727 & -2.007218 \\
\hline$N$ & 2.187865 & -8.107004 & -1.107166 \\
\hline C & 1.065875 & -7.180082 & -1.078547 \\
\hline C & 0.623278 & -6.721166 & -2.470731 \\
\hline $\mathrm{O}$ & -0.558585 & -6.582566 & -2.692751 \\
\hline C & 1.286703 & -5.920711 & -0.247405 \\
\hline $\mathrm{O}$ & 1.414449 & -6.167423 & 1.112892 \\
\hline $\mathrm{H}$ & 3.087781 & -7.818076 & -0.797810 \\
\hline $\mathrm{H}$ & 0.211730 & -7.692961 & -0.667269 \\
\hline $\mathrm{H}$ & 2.134735 & -5.363828 & -0.631909 \\
\hline $\mathrm{H}$ & 0.397434 & -5.326895 & -0.377869 \\
\hline $\mathrm{H}$ & 2.304978 & -6.441587 & 1.286480 \\
\hline$N$ & 1.552246 & -6.437224 & -3.393002 \\
\hline C & 1.156398 & -6.032021 & -4.729693 \\
\hline
\end{tabular}




\begin{tabular}{|c|c|c|c|}
\hline C & 0.385423 & -7.153760 & -5.418744 \\
\hline $\mathrm{O}$ & -0.601390 & -6.897794 & -6.075867 \\
\hline C & 2.369640 & -5.538668 & -5.551767 \\
\hline C & 2.784659 & -4.147221 & -5.045031 \\
\hline C & 2.073813 & -5.517231 & -7.056783 \\
\hline C & 4.114937 & -3.649070 & -5.608139 \\
\hline $\mathrm{H}$ & 2.522156 & -6.493743 & -3.167532 \\
\hline $\mathrm{H}$ & 0.439066 & -5.227110 & -4.653854 \\
\hline $\mathrm{H}$ & 3.186486 & -6.232980 & -5.378815 \\
\hline $\mathrm{H}$ & 1.998346 & -3.437083 & -5.295685 \\
\hline $\mathrm{H}$ & 2.856144 & -4.168576 & -3.965206 \\
\hline $\mathrm{H}$ & 2.933347 & -5.151736 & -7.604695 \\
\hline $\mathrm{H}$ & 1.849975 & -6.504239 & -7.442685 \\
\hline $\mathrm{H}$ & 1.231972 & -4.871160 & -7.285116 \\
\hline $\mathrm{H}$ & 4.414406 & -2.735690 & -5.104875 \\
\hline $\mathrm{H}$ & 4.901076 & -4.381227 & -5.453355 \\
\hline $\mathrm{H}$ & 4.061563 & -3.435277 & -6.669765 \\
\hline$N$ & 0.823559 & -8.405150 & -5.255441 \\
\hline $\mathrm{H}$ & 1.669666 & -8.581384 & -4.754991 \\
\hline C & 0.043390 & -9.512153 & -5.753610 \\
\hline $\mathrm{H}$ & -0.063568 & -9.473792 & -6.827452 \\
\hline $\mathrm{H}$ & 0.553346 & -10.429292 & -5.487533 \\
\hline C & -1.365316 & -9.540114 & -5.166944 \\
\hline $\mathrm{O}$ & -2.319938 & -9.767288 & -5.874474 \\
\hline$N$ & -1.472594 & -9.318871 & -3.858013 \\
\hline C & -2.776806 & -9.346623 & -3.236465 \\
\hline C & -3.690591 & -8.211640 & -3.700453 \\
\hline $\mathrm{O}$ & -4.888648 & -8.419516 & -3.743742 \\
\hline C & -2.680572 & -9.315384 & -1.703977 \\
\hline 0 & -3.925575 & -9.579870 & -1.118921 \\
\hline
\end{tabular}




\begin{tabular}{|c|c|c|c|}
\hline $\mathrm{H}$ & -0.656820 & -9.233313 & -3.288687 \\
\hline $\mathrm{H}$ & -3.294817 & -10.251748 & -3.520567 \\
\hline $\mathrm{H}$ & -1.999587 & -10.088365 & -1.375700 \\
\hline $\mathrm{H}$ & -2.290650 & -8.357371 & -1.376577 \\
\hline $\mathrm{H}$ & -4.614992 & -9.178316 & -1.633687 \\
\hline$N$ & -3.140671 & -7.031631 & -3.981802 \\
\hline C & -3.902800 & -5.911611 & -4.509848 \\
\hline C & -4.444401 & -6.236116 & -5.902315 \\
\hline $\mathrm{O}$ & -5.608320 & -6.045095 & -6.172322 \\
\hline C & -3.003179 & -4.655368 & -4.508644 \\
\hline C & -3.446430 & -3.519778 & -5.415770 \\
\hline$C$ & -4.768369 & -3.093147 & -5.495387 \\
\hline C & -2.499107 & -2.874292 & -6.202386 \\
\hline C & -5.125967 & -2.067161 & -6.354301 \\
\hline C & -2.850203 & -1.834810 & -7.048372 \\
\hline C & -4.171554 & -1.432517 & -7.132535 \\
\hline $\mathrm{H}$ & -2.163960 & -6.909757 & -3.812366 \\
\hline $\mathrm{H}$ & -4.770939 & -5.740614 & -3.893718 \\
\hline $\mathrm{H}$ & -2.925428 & -4.312966 & -3.481048 \\
\hline $\mathrm{H}$ & -2.010683 & -4.954438 & -4.814888 \\
\hline $\mathrm{H}$ & -5.527531 & -3.578735 & -4.912488 \\
\hline $\mathrm{H}$ & -1.472615 & -3.193968 & -6.163074 \\
\hline $\mathrm{H}$ & -6.159685 & -1.773371 & -6.422763 \\
\hline $\mathrm{H}$ & -2.096161 & -1.352588 & -7.644053 \\
\hline $\mathrm{H}$ & -4.452444 & -0.639105 & -7.803271 \\
\hline$N$ & -3.570568 & -6.729386 & -6.788376 \\
\hline C & -4.012389 & -7.071542 & -8.120729 \\
\hline C & -5.136283 & -8.106206 & -8.097705 \\
\hline $\mathrm{O}$ & -6.014865 & -8.052897 & -8.918488 \\
\hline C & -2.861979 & -7.603003 & -8.979078 \\
\hline
\end{tabular}




\begin{tabular}{|c|c|c|c|}
\hline$S$ & -1.645138 & -6.351001 & -9.496656 \\
\hline $\mathrm{H}$ & -2.629879 & -6.913493 & -6.509016 \\
\hline $\mathrm{H}$ & -4.438183 & -6.201578 & -8.597974 \\
\hline $\mathrm{H}$ & -2.357333 & -8.419729 & -8.480725 \\
\hline $\mathrm{H}$ & -3.278715 & -7.983739 & -9.901750 \\
\hline $\mathrm{H}$ & -1.045060 & -6.180606 & -8.329095 \\
\hline$N$ & -5.078532 & -9.066905 & -7.155631 \\
\hline $\mathrm{H}$ & -4.257250 & -9.188153 & -6.602517 \\
\hline C & -6.085178 & -10.116164 & -7.180937 \\
\hline $\mathrm{H}$ & -6.256484 & -10.456631 & -8.190330 \\
\hline $\mathrm{H}$ & -5.714002 & -10.946528 & -6.591481 \\
\hline C & -7.459147 & -9.722831 & -6.613014 \\
\hline $\mathrm{O}$ & -8.481305 & -10.087403 & -7.126876 \\
\hline$N$ & -7.418017 & -9.015655 & -5.478475 \\
\hline C & -8.641664 & -8.655583 & -4.788433 \\
\hline C & -9.503025 & -7.657707 & -5.553989 \\
\hline $\mathrm{O}$ & -10.700950 & -7.657359 & -5.447580 \\
\hline C & -8.362789 & -8.115649 & -3.374852 \\
\hline C & -7.517819 & -6.836019 & -3.258320 \\
\hline C & -8.186702 & -5.483367 & -3.283259 \\
\hline $\mathrm{O}$ & -9.487484 & -5.484213 & -3.352610 \\
\hline $\mathrm{H}$ & -9.837195 & -4.582227 & -3.306516 \\
\hline $\mathrm{O}$ & -7.537218 & -4.468877 & -3.215821 \\
\hline $\mathrm{H}$ & -6.535760 & -8.773291 & -5.079991 \\
\hline $\mathrm{H}$ & -9.261399 & -9.536011 & -4.691970 \\
\hline $\mathrm{H}$ & -9.318829 & -7.979455 & -2.890166 \\
\hline $\mathrm{H}$ & -7.839592 & -8.900272 & -2.838741 \\
\hline $\mathrm{H}$ & -6.984949 & -6.853250 & -2.314144 \\
\hline $\mathrm{H}$ & -6.752209 & -6.796859 & -4.014506 \\
\hline$N$ & -8.837317 & -6.734064 & -6.273863 \\
\hline
\end{tabular}




\begin{tabular}{|c|c|c|c|}
\hline C & -9.562353 & -5.706192 & -6.995284 \\
\hline C & -10.429282 & -6.285649 & -8.104962 \\
\hline $\mathrm{H}$ & -10.447834 & -7.368339 & -8.211261 \\
\hline 0 & -11.058382 & -5.575959 & -8.833188 \\
\hline C & -8.634934 & -4.592890 & -7.533973 \\
\hline $\mathrm{O}$ & -9.429234 & -3.468192 & -7.883582 \\
\hline C & -7.760018 & -4.998713 & -8.712559 \\
\hline $\mathrm{H}$ & -7.855346 & -6.834553 & -6.418399 \\
\hline $\mathrm{H}$ & -10.250263 & -5.232842 & -6.304175 \\
\hline $\mathrm{H}$ & -8.009124 & -4.274700 & -6.711601 \\
\hline $\mathrm{H}$ & -10.132138 & -3.782364 & -8.446313 \\
\hline $\mathrm{H}$ & -7.080829 & -4.186024 & -8.947006 \\
\hline $\mathrm{H}$ & -7.170316 & -5.879379 & -8.504966 \\
\hline $\mathrm{H}$ & -8.365083 & -5.194266 & -9.591490 \\
\hline$N$ & -12.952321 & -9.022071 & 3.174379 \\
\hline $\mathrm{H}$ & -13.222242 & -9.545279 & 2.367114 \\
\hline C & -11.777508 & -8.184389 & 2.899330 \\
\hline C & -10.788044 & -8.138268 & 4.046003 \\
\hline $\mathrm{O}$ & -9.654030 & -7.745285 & 3.846063 \\
\hline C & -12.130734 & -6.717775 & 2.577797 \\
\hline $\mathrm{O}$ & -12.657555 & -6.188825 & 3.767839 \\
\hline C & -13.123326 & -6.574357 & 1.430132 \\
\hline $\mathrm{H}$ & -13.731255 & -8.457326 & 3.453056 \\
\hline $\mathrm{H}$ & -11.232605 & -8.601484 & 2.063655 \\
\hline $\mathrm{H}$ & -11.209285 & -6.212204 & 2.322373 \\
\hline $\mathrm{H}$ & -12.680798 & -5.236074 & 3.711916 \\
\hline $\mathrm{H}$ & -13.268773 & -5.522576 & 1.207991 \\
\hline $\mathrm{H}$ & -12.771579 & -7.061413 & 0.528011 \\
\hline $\mathrm{H}$ & -14.088830 & -6.989115 & 1.694065 \\
\hline $\mathrm{N}$ & -11.197267 & -8.533701 & 5.241859 \\
\hline
\end{tabular}




\begin{tabular}{|c|c|c|c|}
\hline $\mathrm{H}$ & -12.124328 & -8.896923 & 5.283816 \\
\hline C & -10.409167 & -8.524250 & 6.449423 \\
\hline $\mathrm{H}$ & -10.331035 & -7.527086 & 6.853346 \\
\hline $\mathrm{H}$ & -10.928864 & -9.129539 & 7.182800 \\
\hline C & -8.972188 & -9.040182 & 6.371330 \\
\hline O & -8.135436 & -8.471324 & 7.028298 \\
\hline$N$ & -8.657852 & -10.121190 & 5.634760 \\
\hline $\mathrm{H}$ & -9.368763 & -10.601945 & 5.131375 \\
\hline C & -7.269059 & -10.552673 & 5.533184 \\
\hline $\mathrm{H}$ & -7.251934 & -11.532386 & 5.073001 \\
\hline $\mathrm{H}$ & -6.828276 & -10.630382 & 6.514139 \\
\hline C & -6.387526 & -9.608322 & 4.692701 \\
\hline $\mathrm{O}$ & -5.219497 & -9.466997 & 4.948475 \\
\hline$N$ & -7.045373 & -9.017976 & 3.701159 \\
\hline C & -6.304931 & -8.012558 & 2.952689 \\
\hline C & -6.268090 & -6.697759 & 3.725473 \\
\hline 0 & -5.276112 & -6.007922 & 3.677171 \\
\hline C & -6.623074 & -7.894316 & 1.449060 \\
\hline C & -7.795882 & -7.059029 & 0.980280 \\
\hline C & -7.766000 & -5.670957 & 1.082999 \\
\hline C & -8.866773 & -7.649269 & 0.322235 \\
\hline C & -8.787869 & -4.896672 & 0.560020 \\
\hline C & -9.880657 & -6.876416 & -0.220950 \\
\hline C & -9.848945 & -5.497693 & -0.097189 \\
\hline $\mathrm{H}$ & -8.039868 & -9.019905 & 3.675706 \\
\hline $\mathrm{H}$ & -5.281581 & -8.342691 & 2.985193 \\
\hline $\mathrm{H}$ & -5.724588 & -7.489368 & 0.996675 \\
\hline $\mathrm{H}$ & -6.714187 & -8.906752 & 1.073838 \\
\hline $\mathrm{H}$ & -6.925939 & -5.186139 & 1.546078 \\
\hline & -8.892693 & -8.718667 & 0.200367 \\
\hline
\end{tabular}




\begin{tabular}{|c|c|c|c|}
\hline $\mathrm{H}$ & -8.738272 & -3.825285 & 0.637789 \\
\hline $\mathrm{H}$ & -10.679672 & -7.347393 & -0.763725 \\
\hline $\mathrm{H}$ & -10.627261 & -4.898628 & -0.532836 \\
\hline$N$ & -7.302440 & -6.389884 & 4.510376 \\
\hline C & -7.241904 & -5.272589 & 5.420553 \\
\hline C & -6.127059 & -5.479045 & 6.445228 \\
\hline $\mathrm{O}$ & -5.497086 & -4.519332 & 6.836380 \\
\hline C & -8.622454 & -5.098367 & 6.088776 \\
\hline C & -8.872837 & -3.753989 & 6.737360 \\
\hline$C$ & -9.064940 & -2.621771 & 5.950646 \\
\hline C & -8.980564 & -3.631491 & 8.115883 \\
\hline C & -9.345869 & -1.395886 & 6.527256 \\
\hline C & -9.270126 & -2.406751 & 8.699869 \\
\hline$C$ & -9.450419 & -1.284332 & 7.908326 \\
\hline $\mathrm{H}$ & -8.162416 & -6.883321 & 4.408297 \\
\hline $\mathrm{H}$ & -6.972272 & -4.370777 & 4.889804 \\
\hline $\mathrm{H}$ & -9.367819 & -5.249171 & 5.314884 \\
\hline $\mathrm{H}$ & -8.757533 & -5.887072 & 6.816818 \\
\hline $\mathrm{H}$ & -8.996843 & -2.697121 & 4.878790 \\
\hline $\mathrm{H}$ & -8.840289 & -4.495153 & 8.742009 \\
\hline $\mathrm{H}$ & -9.481307 & -0.533112 & 5.901194 \\
\hline $\mathrm{H}$ & -9.342837 & -2.330540 & 9.769933 \\
\hline $\mathrm{H}$ & -9.649214 & -0.328205 & 8.359915 \\
\hline$N$ & -5.873327 & -6.714919 & 6.870140 \\
\hline $\mathrm{H}$ & -6.522006 & -7.441169 & 6.663147 \\
\hline C & -4.799226 & -7.005427 & 7.793460 \\
\hline $\mathrm{H}$ & -4.840126 & -6.373275 & 8.667686 \\
\hline $\mathrm{H}$ & -4.911987 & -8.034134 & 8.111036 \\
\hline C & -3.402321 & -6.831884 & 7.200144 \\
\hline 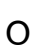 & -2.527365 & -6.342320 & 7.874602 \\
\hline
\end{tabular}




\begin{tabular}{|c|c|c|c|}
\hline$N$ & -3.202406 & -7.252132 & 5.946462 \\
\hline C & -1.926492 & -7.066544 & 5.273132 \\
\hline C & -1.685041 & -5.592029 & 4.920417 \\
\hline O & -0.587000 & -5.103790 & 5.087924 \\
\hline C & -1.845186 & -7.996026 & 4.042074 \\
\hline C & -0.683925 & -7.630368 & 3.116008 \\
\hline C & -1.736960 & -9.457739 & 4.490962 \\
\hline $\mathrm{H}$ & -3.935891 & -7.731633 & 5.470520 \\
\hline $\mathrm{H}$ & -1.130946 & -7.313462 & 5.961147 \\
\hline $\mathrm{H}$ & -2.767813 & -7.872914 & 3.479766 \\
\hline $\mathrm{H}$ & -0.619047 & -8.353756 & 2.310238 \\
\hline $\mathrm{H}$ & -0.800258 & -6.654658 & 2.665480 \\
\hline $\mathrm{H}$ & 0.262332 & -7.640071 & 3.648170 \\
\hline $\mathrm{H}$ & -1.751106 & -10.114767 & 3.627348 \\
\hline $\mathrm{H}$ & -0.800602 & -9.623412 & 5.017899 \\
\hline $\mathrm{H}$ & -2.550214 & -9.753041 & 5.141056 \\
\hline $\mathrm{N}$ & -2.723898 & -4.903800 & 4.437579 \\
\hline$C$ & -2.597873 & -3.516586 & 4.035586 \\
\hline$C$ & -2.446008 & -2.619124 & 5.261663 \\
\hline 0 & -1.559369 & -1.795104 & 5.313952 \\
\hline$C$ & -3.790373 & -3.045871 & 3.189272 \\
\hline$C$ & -4.037835 & -3.782688 & 1.862411 \\
\hline$C$ & -2.904281 & -3.687319 & 0.855894 \\
\hline 0 & -1.795219 & -4.103278 & 1.227963 \\
\hline $\mathrm{O}$ & -3.154229 & -3.214010 & -0.265332 \\
\hline $\mathrm{H}$ & -3.585472 & -5.375546 & 4.270945 \\
\hline $\mathrm{H}$ & -1.693274 & -3.397091 & 3.469942 \\
\hline $\mathrm{H}$ & -4.695979 & -3.115782 & 3.784263 \\
\hline $\mathrm{H}$ & -3.635079 & -1.989268 & 2.988289 \\
\hline $\mathrm{H}$ & -4.209364 & -4.831126 & 2.054315 \\
\hline
\end{tabular}




\begin{tabular}{|c|c|c|c|}
\hline $\mathrm{H}$ & -4.937775 & -3.375838 & 1.415311 \\
\hline$N$ & -3.328770 & -2.774724 & 6.264389 \\
\hline C & -3.116859 & -2.037315 & 7.484959 \\
\hline C & -1.791620 & -2.427091 & 8.149505 \\
\hline $\mathrm{O}$ & -1.125457 & -1.586158 & 8.713508 \\
\hline C & -4.245902 & -2.040988 & 8.526077 \\
\hline $\mathrm{O}$ & -4.451830 & -3.295548 & 9.104538 \\
\hline C & -5.530771 & -1.412628 & 7.998546 \\
\hline $\mathrm{H}$ & -4.008877 & -3.501147 & 6.228544 \\
\hline $\mathrm{H}$ & -2.972408 & -1.002401 & 7.215625 \\
\hline $\mathrm{H}$ & -3.869398 & -1.417516 & 9.327519 \\
\hline $\mathrm{H}$ & -4.908463 & -3.852384 & 8.485526 \\
\hline $\mathrm{H}$ & -6.261226 & -1.373828 & 8.797319 \\
\hline $\mathrm{H}$ & -5.347703 & -0.399615 & 7.653558 \\
\hline $\mathrm{H}$ & -5.953429 & -1.981335 & 7.181070 \\
\hline $\mathrm{N}$ & -1.380408 & -3.700466 & 8.043355 \\
\hline $\mathrm{H}$ & -2.027778 & -4.421552 & 7.812090 \\
\hline$C$ & -0.184104 & -4.103740 & 8.736422 \\
\hline $\mathrm{H}$ & -0.230722 & -3.886129 & 9.794177 \\
\hline $\mathrm{H}$ & -0.071111 & -5.171594 & 8.602335 \\
\hline$C$ & 1.063837 & -3.415977 & 8.208148 \\
\hline 0 & 1.943392 & -3.068667 & 8.963047 \\
\hline $\mathrm{N}$ & 1.148099 & -3.258383 & 6.871248 \\
\hline$C$ & 2.325512 & -2.662498 & 6.301899 \\
\hline$C$ & 2.289608 & -1.139382 & 6.162378 \\
\hline 0 & 3.359845 & -0.576666 & 6.026494 \\
\hline$C$ & 2.805039 & -3.337807 & 5.016948 \\
\hline$S$ & 1.888257 & -2.938296 & 3.504645 \\
\hline $\mathrm{H}$ & 0.413717 & -3.611876 & 6.290069 \\
\hline $\mathrm{H}$ & 3.116173 & -2.811897 & 7.021600 \\
\hline
\end{tabular}




\begin{tabular}{|c|c|c|c|}
\hline $\mathrm{H}$ & 3.817145 & -3.008490 & 4.830336 \\
\hline $\mathrm{H}$ & 2.816852 & -4.409073 & 5.162126 \\
\hline $\mathrm{H}$ & 0.819535 & -3.695637 & 3.703000 \\
\hline$N$ & 1.139670 & -0.470506 & 6.273406 \\
\hline C & 1.204805 & 0.968264 & 6.492091 \\
\hline C & 1.756128 & 1.271223 & 7.870957 \\
\hline $\mathrm{O}$ & 2.369161 & 2.288717 & 8.085908 \\
\hline C & -0.095209 & 1.740211 & 6.181737 \\
\hline C & -1.202670 & 1.553130 & 7.228888 \\
\hline C & -0.544879 & 1.447272 & 4.745363 \\
\hline C & -2.470298 & 2.359126 & 6.937464 \\
\hline $\mathrm{H}$ & 0.270099 & -0.963389 & 6.287514 \\
\hline $\mathrm{H}$ & 1.952635 & 1.350962 & 5.815916 \\
\hline $\mathrm{H}$ & 0.205266 & 2.781508 & 6.231591 \\
\hline $\mathrm{H}$ & -1.452777 & 0.506845 & 7.328849 \\
\hline $\mathrm{H}$ & -0.819884 & 1.863788 & 8.196479 \\
\hline $\mathrm{H}$ & -1.301750 & 2.153662 & 4.427816 \\
\hline $\mathrm{H}$ & 0.291009 & 1.536211 & 4.056776 \\
\hline $\mathrm{H}$ & -0.952151 & 0.450631 & 4.642613 \\
\hline r & -3.144614 & 2.318997 & 7.787465 \\
\hline $\mathrm{H}$ & -2.244102 & 3.404899 & 6.745042 \\
\hline $\mathrm{H}$ & -3.006451 & 1.971509 & 6.078076 \\
\hline$N$ & 1.549780 & 0.323275 & 8.791255 \\
\hline $\mathrm{H}$ & 0.890444 & -0.402546 & 8.613047 \\
\hline C & 1.977874 & 0.512251 & 10.160280 \\
\hline $\mathrm{H}$ & 1.469252 & -0.222981 & 10.770964 \\
\hline $\mathrm{H}$ & 1.737371 & 1.501937 & 10.518378 \\
\hline C & 3.481809 & 0.336581 & 10.305781 \\
\hline $\mathrm{O}$ & 4.163662 & 1.126803 & 10.899107 \\
\hline $\mathrm{N}$ & 3.999635 & -0.753621 & 9.694705 \\
\hline
\end{tabular}




\begin{tabular}{|c|c|c|c|}
\hline $\mathrm{H}$ & 3.390680 & -1.463356 & 9.345149 \\
\hline C & 5.424640 & -0.938719 & 9.667230 \\
\hline $\mathrm{H}$ & 5.638010 & -1.909229 & 9.234347 \\
\hline $\mathrm{H}$ & 5.854673 & -0.904705 & 10.657557 \\
\hline C & 6.156890 & 0.119123 & 8.844832 \\
\hline O & 7.292104 & 0.411098 & 9.129501 \\
\hline$N$ & 5.502074 & 0.621268 & 7.799018 \\
\hline C & 6.105378 & 1.756770 & 7.150115 \\
\hline C & 5.525754 & 3.075976 & 7.654228 \\
\hline $\mathrm{H}$ & 5.023096 & 3.036937 & 8.619263 \\
\hline $\mathrm{O}$ & 5.650481 & 4.094321 & 7.046575 \\
\hline C & 6.011748 & 1.676271 & 5.624080 \\
\hline C & 6.760831 & 0.506655 & 5.019708 \\
\hline C & 8.126684 & 0.320974 & 5.237773 \\
\hline C & 6.097513 & -0.401591 & 4.200082 \\
\hline C & 8.810437 & -0.725805 & 4.634383 \\
\hline C & 6.779052 & -1.446022 & 3.595243 \\
\hline C & 8.139346 & -1.608653 & 3.802465 \\
\hline $\mathrm{H}$ & 4.574642 & 0.337206 & 7.571870 \\
\hline $\mathrm{H}$ & 7.140280 & 1.766917 & 7.456520 \\
\hline $\mathrm{H}$ & 4.969721 & 1.616607 & 5.335781 \\
\hline $\mathrm{H}$ & 6.394175 & 2.610107 & 5.226508 \\
\hline $\mathrm{H}$ & 8.656905 & 0.977099 & 5.906681 \\
\hline $\mathrm{H}$ & 5.037177 & -0.304328 & 4.064082 \\
\hline $\mathrm{H}$ & 9.861415 & -0.854335 & 4.828031 \\
\hline $\mathrm{H}$ & 6.244709 & -2.137611 & 2.968497 \\
\hline $\mathrm{H}$ & 8.657635 & -2.422252 & 3.328888 \\
\hline $\mathrm{N}$ & 12.613548 & 9.596235 & -1.248797 \\
\hline $\mathrm{H}$ & 11.872888 & 9.914331 & -1.845504 \\
\hline C & 12.304855 & 8.264733 & -0.719494 \\
\hline
\end{tabular}




\begin{tabular}{|c|c|c|c|}
\hline$C$ & 11.274118 & 8.450599 & 0.386898 \\
\hline O & 10.265933 & 7.778869 & 0.416405 \\
\hline$C$ & 13.562743 & 7.581057 & -0.165268 \\
\hline$C$ & 14.645171 & 7.276174 & -1.206399 \\
\hline$S$ & 14.017413 & 6.202010 & -2.529687 \\
\hline$C$ & 15.500160 & 6.053615 & -3.555024 \\
\hline $\mathrm{H}$ & 13.432955 & 9.572824 & -1.822901 \\
\hline $\mathrm{H}$ & 11.834482 & 7.616492 & -1.447350 \\
\hline $\mathrm{H}$ & 14.001230 & 8.205515 & 0.607268 \\
\hline $\mathrm{H}$ & 13.255717 & 6.653873 & 0.304879 \\
\hline $\mathrm{H}$ & 15.045479 & 8.188658 & -1.633772 \\
\hline $\mathrm{H}$ & 15.470364 & 6.783888 & -0.704308 \\
\hline $\mathrm{H}$ & 15.248997 & 5.398790 & -4.375499 \\
\hline $\mathrm{H}$ & 15.799031 & 7.017675 & -3.945849 \\
\hline $\mathrm{H}$ & 16.318596 & 5.616979 & -2.997646 \\
\hline $\mathrm{N}$ & 11.504255 & 9.412828 & 1.296996 \\
\hline$C$ & 10.594230 & 9.607677 & 2.408576 \\
\hline$C$ & 9.277006 & 10.274769 & 2.010397 \\
\hline O & 8.280961 & 10.053319 & 2.652723 \\
\hline$C$ & 11.276203 & 10.368772 & 3.552226 \\
\hline$C$ & 12.447520 & 9.563777 & 4.084885 \\
\hline 0 & 13.582550 & 9.825193 & 3.745343 \\
\hline $\mathrm{N}$ & 12.143330 & 8.561481 & 4.919061 \\
\hline $\mathrm{H}$ & 12.328022 & 9.967725 & 1.206308 \\
\hline $\mathrm{H}$ & 10.295334 & 8.637602 & 2.771156 \\
\hline $\mathrm{H}$ & 11.657595 & 11.325890 & 3.219193 \\
\hline $\mathrm{H}$ & 10.545941 & 10.537611 & 4.333516 \\
\hline $\mathrm{H}$ & 12.886830 & 7.965718 & 5.213395 \\
\hline $\mathrm{H}$ & 11.221666 & 8.175583 & 4.954638 \\
\hline$T$ & 9.263820 & 11.067708 & 0.922336 \\
\hline
\end{tabular}




\begin{tabular}{|c|c|c|c|}
\hline $\mathrm{H}$ & 10.110806 & 11.229613 & 0.427402 \\
\hline$C$ & 8.003507 & 11.517472 & 0.368847 \\
\hline $\mathrm{H}$ & 8.201268 & 12.260205 & -0.393381 \\
\hline $\mathrm{H}$ & 7.389112 & 11.966735 & 1.131893 \\
\hline$C$ & 7.186380 & 10.384432 & -0.254307 \\
\hline 0 & 5.986325 & 10.470163 & -0.291674 \\
\hline $\mathrm{N}$ & 7.862646 & 9.333243 & -0.763184 \\
\hline$C$ & 7.170360 & 8.145621 & -1.233907 \\
\hline$C$ & 6.634551 & 7.300767 & -0.067358 \\
\hline 0 & 5.534115 & 6.808599 & -0.148417 \\
\hline$C$ & 8.048803 & 7.344196 & -2.224169 \\
\hline$C$ & 8.343623 & 8.197115 & -3.472857 \\
\hline$C$ & 7.379229 & 6.019179 & -2.608087 \\
\hline $\mathrm{C}$ & 9.428272 & 7.624966 & -4.388863 \\
\hline $\mathrm{H}$ & 8.823800 & 9.230617 & -0.536510 \\
\hline $\mathrm{H}$ & 6.279715 & 8.471812 & -1.748434 \\
\hline $\mathrm{H}$ & 8.987821 & 7.117690 & -1.727244 \\
\hline $\mathrm{H}$ & 7.421746 & 8.323158 & -4.036246 \\
\hline $\mathrm{H}$ & 8.645736 & 9.192750 & -3.165369 \\
\hline $\mathrm{H}$ & 7.996585 & 5.478783 & -3.314292 \\
\hline $\mathrm{H}$ & 7.228035 & 5.377911 & -1.748270 \\
\hline $\mathrm{H}$ & 6.409560 & 6.182911 & -3.066614 \\
\hline $\mathrm{H}$ & 9.622315 & 8.305493 & -5.211529 \\
\hline $\mathrm{H}$ & 10.363575 & 7.479448 & -3.855143 \\
\hline $\mathrm{H}$ & 9.148417 & 6.670885 & -4.819956 \\
\hline $\mathrm{N}$ & 7.387121 & 7.175059 & 1.043940 \\
\hline$C$ & 6.878148 & 6.458328 & 2.201978 \\
\hline$C$ & 5.527294 & 7.040202 & 2.639577 \\
\hline 0 & 4.574441 & 6.334199 & 2.866783 \\
\hline$c$ & 7.832292 & 6.545691 & 3.403562 \\
\hline
\end{tabular}




\begin{tabular}{|c|c|c|c|}
\hline C & 9.253664 & 5.996720 & 3.243963 \\
\hline $\mathrm{O}$ & 10.145200 & 6.631053 & 3.801319 \\
\hline $\mathrm{O}$ & 9.427037 & 4.934897 & 2.595238 \\
\hline $\mathrm{H}$ & 8.347205 & 7.444682 & 1.022838 \\
\hline $\mathrm{H}$ & 6.681042 & 5.429620 & 1.943308 \\
\hline $\mathrm{H}$ & 7.920530 & 7.570200 & 3.733380 \\
\hline $\mathrm{H}$ & 7.374144 & 5.992143 & 4.220647 \\
\hline$N$ & 5.489861 & 8.378154 & 2.785912 \\
\hline $\mathrm{H}$ & 6.321206 & 8.912914 & 2.648845 \\
\hline C & 4.299565 & 9.047333 & 3.249675 \\
\hline $\mathrm{H}$ & 4.538969 & 10.091718 & 3.406566 \\
\hline $\mathrm{H}$ & 3.957722 & 8.641020 & 4.188490 \\
\hline C & 3.136817 & 8.988654 & 2.268517 \\
\hline $\mathrm{O}$ & 1.995394 & 9.111339 & 2.649451 \\
\hline$N$ & 3.470151 & 8.825006 & 0.985194 \\
\hline $\mathrm{H}$ & 4.431261 & 8.828978 & 0.735190 \\
\hline C & 2.517174 & 8.905264 & -0.078282 \\
\hline $\mathrm{H}$ & 3.059635 & 9.091925 & -0.996343 \\
\hline $\mathrm{H}$ & 1.843680 & 9.737834 & 0.072013 \\
\hline C & 1.609145 & 7.687007 & -0.310220 \\
\hline $\mathrm{O}$ & 0.563858 & 7.814945 & -0.883301 \\
\hline$N$ & 2.090528 & 6.538674 & 0.206384 \\
\hline C & 1.283381 & 5.366494 & 0.435195 \\
\hline C & 0.467875 & 5.480594 & 1.727562 \\
\hline $\mathrm{O}$ & -0.727393 & 5.301042 & 1.700501 \\
\hline C & 2.156685 & 4.111974 & 0.446513 \\
\hline $\mathrm{H}$ & 2.996864 & 6.575239 & 0.622653 \\
\hline $\mathrm{H}$ & 0.551923 & 5.295989 & -0.352655 \\
\hline $\mathrm{H}$ & 1.554370 & 3.234745 & 0.657176 \\
\hline $\mathrm{H}$ & 2.624951 & 3.991708 & -0.523505 \\
\hline
\end{tabular}




\begin{tabular}{|c|c|c|c|}
\hline $\mathrm{H}$ & 2.937305 & 4.180443 & 1.196957 \\
\hline$N$ & 1.100166 & 5.752938 & 2.883496 \\
\hline C & 0.363908 & 5.588936 & 4.128803 \\
\hline C & -0.756441 & 6.616054 & 4.277688 \\
\hline $\mathrm{O}$ & -1.793301 & 6.307749 & 4.825540 \\
\hline C & 1.277017 & 5.517294 & 5.377563 \\
\hline C & 1.869171 & 6.863332 & 5.822217 \\
\hline C & 2.363575 & 4.450435 & 5.174566 \\
\hline C & 2.702782 & 6.768521 & 7.102590 \\
\hline $\mathrm{H}$ & 2.087549 & 5.892476 & 2.903068 \\
\hline $\mathrm{H}$ & -0.160392 & 4.645887 & 4.073071 \\
\hline $\mathrm{H}$ & 0.617019 & 5.179838 & 6.171072 \\
\hline $\mathrm{H}$ & 2.477859 & 7.271919 & 5.022362 \\
\hline $\mathrm{H}$ & 1.065842 & 7.573320 & 5.990239 \\
\hline $\mathrm{H}$ & 2.734478 & 4.098798 & 6.126137 \\
\hline $\mathrm{H}$ & 1.969683 & 3.592879 & 4.638409 \\
\hline $\mathrm{H}$ & 3.205836 & 4.838596 & 4.612193 \\
\hline $\mathrm{H}$ & 3.008334 & 7.759834 & 7.424055 \\
\hline $\mathrm{H}$ & 2.126734 & 6.325663 & 7.909692 \\
\hline $\mathrm{H}$ & 3.597632 & 6.172900 & 6.971536 \\
\hline $\mathrm{N}$ & -0.542353 & 7.851148 & 3.802925 \\
\hline $\mathrm{H}$ & 0.342661 & 8.093820 & 3.408237 \\
\hline$C$ & -1.514248 & 8.891052 & 4.018116 \\
\hline $\mathrm{H}$ & -1.105100 & 9.819454 & 3.640120 \\
\hline $\mathrm{H}$ & -1.725168 & 9.021780 & 5.069665 \\
\hline$C$ & -2.867021 & 8.618723 & 3.365915 \\
\hline 0 & -3.871177 & 8.835136 & 4.003743 \\
\hline $\mathrm{N}$ & -2.902653 & 8.109029 & 2.120712 \\
\hline$C$ & -4.238324 & 8.007130 & 1.534100 \\
\hline 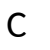 & -4.968810 & 6.730607 & 1.912165 \\
\hline
\end{tabular}




\begin{tabular}{|c|c|c|c|}
\hline $\mathrm{O}$ & -6.180377 & 6.723992 & 1.899404 \\
\hline C & -4.023439 & 8.231576 & 0.030898 \\
\hline C & -2.767606 & 9.109700 & 0.024624 \\
\hline C & -1.907994 & 8.459364 & 1.096316 \\
\hline $\mathrm{H}$ & -4.860642 & 8.797264 & 1.925061 \\
\hline $\mathrm{H}$ & -3.845864 & 7.294605 & -0.480577 \\
\hline $\mathrm{H}$ & -4.888944 & 8.700104 & -0.419709 \\
\hline $\mathrm{H}$ & -2.258156 & 9.157674 & -0.919537 \\
\hline $\mathrm{H}$ & -3.027601 & 10.122702 & 0.322427 \\
\hline $\mathrm{H}$ & -1.423342 & 7.570099 & 0.735637 \\
\hline $\mathrm{H}$ & -1.150039 & 9.118658 & 1.486793 \\
\hline$N$ & -4.242059 & 5.660668 & 2.268948 \\
\hline C & -4.898311 & 4.489696 & 2.822075 \\
\hline C & -5.601942 & 4.809620 & 4.143697 \\
\hline $\mathrm{O}$ & -6.682156 & 4.319140 & 4.387286 \\
\hline C & -3.922513 & 3.315617 & 3.044840 \\
\hline C & -3.602555 & 2.490632 & 1.814460 \\
\hline C & -4.319968 & 1.333086 & 1.526420 \\
\hline C & -2.563037 & 2.837884 & 0.964040 \\
\hline C & -4.010876 & 0.545635 & 0.427664 \\
\hline C & -2.253031 & 2.067610 & -0.142613 \\
\hline C & -2.971130 & 0.916942 & -0.418016 \\
\hline O & -2.631708 & 0.190538 & -1.507631 \\
\hline $\mathrm{H}$ & -3.433330 & -0.132565 & -1.959934 \\
\hline $\mathrm{H}$ & -3.248637 & 5.746501 & 2.321824 \\
\hline $\mathrm{H}$ & -5.684982 & 4.182788 & 2.150843 \\
\hline $\mathrm{H}$ & -3.011435 & 3.701906 & 3.489434 \\
\hline $\mathrm{H}$ & -4.374530 & 2.664717 & 3.784611 \\
\hline $\mathrm{H}$ & -5.129536 & 1.032893 & 2.169558 \\
\hline $\mathrm{H}$ & -1.985421 & 3.717452 & 1.156964 \\
\hline
\end{tabular}




\begin{tabular}{|c|c|c|c|}
\hline $\mathrm{H}$ & -4.552745 & -0.361458 & 0.235267 \\
\hline $\mathrm{H}$ & -1.460382 & 2.361604 & -0.807624 \\
\hline$N$ & -4.963985 & 5.610804 & 5.012229 \\
\hline $\mathrm{H}$ & -4.035727 & 5.925115 & 4.817424 \\
\hline C & -5.577479 & 5.988549 & 6.265746 \\
\hline $\mathrm{H}$ & -4.801211 & 6.365451 & 6.920165 \\
\hline $\mathrm{H}$ & -6.043964 & 5.136989 & 6.734166 \\
\hline C & -6.651474 & 7.065805 & 6.114413 \\
\hline O & -7.709535 & 6.987358 & 6.695136 \\
\hline$N$ & -6.341993 & 8.091462 & 5.314137 \\
\hline $\mathrm{H}$ & -5.441195 & 8.148173 & 4.889085 \\
\hline C & -7.257699 & 9.220084 & 5.085090 \\
\hline $\mathrm{H}$ & -6.773806 & 9.881174 & 4.377832 \\
\hline $\mathrm{H}$ & -7.436862 & 9.765711 & 5.999948 \\
\hline C & -8.620673 & 8.834837 & 4.545706 \\
\hline $\mathrm{O}$ & -9.605806 & 9.430594 & 4.903538 \\
\hline$N$ & -8.666130 & 7.828883 & 3.652697 \\
\hline$c$ & -9.928735 & 7.427001 & 3.085873 \\
\hline C & -10.814144 & 6.631211 & 4.032578 \\
\hline$c$ & -11.919862 & 6.304410 & 3.637505 \\
\hline C & -9.727464 & 6.646934 & 1.766796 \\
\hline $\mathrm{O}$ & -10.949256 & 6.584046 & 1.057330 \\
\hline$c$ & -9.151248 & 5.252108 & 1.967152 \\
\hline $\mathrm{H}$ & -7.818197 & 7.408776 & 3.331769 \\
\hline $\mathrm{H}$ & -10.503099 & 8.315235 & 2.856328 \\
\hline $\mathrm{H}$ & -9.047528 & 7.228489 & 1.158208 \\
\hline $\mathrm{H}$ & -11.656023 & 6.539435 & 1.697411 \\
\hline $\mathrm{H}$ & -8.976723 & 4.785046 & 1.004296 \\
\hline $\mathrm{H}$ & -8.208905 & 5.278259 & 2.490920 \\
\hline & -9.838835 & 4.627571 & 2.524251 \\
\hline
\end{tabular}




\begin{tabular}{|c|c|c|c|}
\hline$N$ & -10.353503 & 6.328342 & 5.241144 \\
\hline C & -11.230850 & 5.723473 & 6.228817 \\
\hline C & -12.252751 & 6.705790 & 6.808551 \\
\hline $\mathrm{O}$ & -13.256428 & 6.277675 & 7.316005 \\
\hline C & -10.435281 & 5.093264 & 7.379626 \\
\hline C & -9.488829 & 3.950679 & 6.982356 \\
\hline C & -8.704761 & 3.492370 & 8.215910 \\
\hline C & -10.221569 & 2.767193 & 6.343598 \\
\hline $\mathrm{H}$ & -9.434347 & 6.600099 & 5.520645 \\
\hline $\mathrm{H}$ & -11.827490 & 4.972438 & 5.737201 \\
\hline $\mathrm{H}$ & -9.859024 & 5.870540 & 7.870016 \\
\hline $\mathrm{H}$ & -11.161263 & 4.730326 & 8.100330 \\
\hline $\mathrm{H}$ & -8.772341 & 4.325287 & 6.260793 \\
\hline $\mathrm{H}$ & -7.994812 & 2.711349 & 7.958815 \\
\hline $\mathrm{H}$ & -8.150205 & 4.316678 & 8.652154 \\
\hline $\mathrm{H}$ & -9.369959 & 3.098927 & 8.981775 \\
\hline $\mathrm{H}$ & -9.518635 & 1.971544 & 6.116205 \\
\hline $\mathrm{H}$ & -10.980974 & 2.365598 & 7.012467 \\
\hline $\mathrm{H}$ & -10.707399 & 3.037330 & 5.412318 \\
\hline$N$ & -11.935445 & 8.016698 & 6.787516 \\
\hline C & -12.884695 & 9.000950 & 7.215647 \\
\hline C & -13.851947 & 9.432812 & 6.133866 \\
\hline $\mathrm{H}$ & -14.612175 & 10.146788 & 6.470712 \\
\hline $\mathrm{O}$ & -13.816147 & 9.067787 & 5.006124 \\
\hline $\mathrm{H}$ & -11.174724 & 8.331014 & 6.225290 \\
\hline $\mathrm{H}$ & -13.454872 & 8.617012 & 8.051621 \\
\hline $\mathrm{H}$ & -12.360078 & 9.886922 & 7.562692 \\
\hline$N$ & -11.450004 & 10.622759 & -3.030048 \\
\hline $\mathrm{H}$ & -11.209347 & 10.836129 & -2.082610 \\
\hline$C$ & -11.805542 & 9.208255 & -3.171992 \\
\hline
\end{tabular}




\begin{tabular}{|c|c|c|c|}
\hline C & -11.410598 & 8.856561 & -4.599609 \\
\hline $\mathrm{O}$ & -10.372854 & 8.290278 & -4.846281 \\
\hline C & -13.244706 & 8.824652 & -2.765620 \\
\hline C & -13.482122 & 9.163105 & -1.282303 \\
\hline C & -13.513810 & 7.342058 & -3.054218 \\
\hline C & -14.912145 & 8.922492 & -0.794921 \\
\hline $\mathrm{H}$ & -12.216211 & 11.220192 & -3.277637 \\
\hline $\mathrm{H}$ & -11.119634 & 8.644120 & -2.555652 \\
\hline $\mathrm{H}$ & -13.943154 & 9.424616 & -3.353506 \\
\hline $\mathrm{H}$ & -12.790744 & 8.584378 & -0.673583 \\
\hline $\mathrm{H}$ & -13.242391 & 10.208439 & -1.114120 \\
\hline $\mathrm{H}$ & -14.550839 & 7.090861 & -2.872760 \\
\hline $\mathrm{H}$ & -13.298105 & 7.076750 & -4.084999 \\
\hline $\mathrm{H}$ & -12.903473 & 6.712301 & -2.414402 \\
\hline $\mathrm{H}$ & -15.024839 & 9.283592 & 0.220942 \\
\hline $\mathrm{H}$ & -15.634322 & 9.447362 & -1.414760 \\
\hline $\mathrm{H}$ & -15.172680 & 7.870776 & -0.791579 \\
\hline$N$ & -12.211997 & 9.288083 & -5.602657 \\
\hline $\mathrm{H}$ & -13.130153 & 9.596772 & -5.377247 \\
\hline C & -11.972873 & 8.843875 & -6.959238 \\
\hline $\mathrm{H}$ & -12.037064 & 7.767786 & -7.045600 \\
\hline $\mathrm{H}$ & -12.729661 & 9.284178 & -7.596912 \\
\hline C & -10.601375 & 9.232961 & -7.517198 \\
\hline $\mathrm{O}$ & -9.996255 & 8.496520 & -8.240744 \\
\hline$N$ & -10.162429 & 10.477356 & -7.181602 \\
\hline $\mathrm{H}$ & -10.711675 & 11.008704 & -6.545682 \\
\hline C & -8.894348 & 11.024005 & -7.637086 \\
\hline $\mathrm{H}$ & -8.790089 & 10.891089 & -8.702746 \\
\hline $\mathrm{H}$ & -8.878869 & 12.081540 & -7.407775 \\
\hline C & -7.672362 & 10.359334 & -6.988510 \\
\hline
\end{tabular}




\begin{tabular}{|c|c|c|c|}
\hline $\mathrm{O}$ & -6.752644 & 9.936648 & -7.631237 \\
\hline$N$ & -7.659719 & 10.350048 & -5.619869 \\
\hline $\mathrm{H}$ & -8.482692 & 10.627738 & -5.134437 \\
\hline C & -6.495283 & 9.961236 & -4.832291 \\
\hline $\mathrm{H}$ & -5.599773 & 10.376146 & -5.266681 \\
\hline $\mathrm{H}$ & -6.615414 & 10.362680 & -3.833476 \\
\hline C & -6.266714 & 8.464815 & -4.710673 \\
\hline 0 & -5.130741 & 8.052021 & -4.722029 \\
\hline$N$ & -7.313250 & 7.638968 & -4.532827 \\
\hline C & -7.014200 & 6.281823 & -4.104247 \\
\hline C & -6.385416 & 5.453733 & -5.218218 \\
\hline $\mathrm{O}$ & -5.500749 & 4.680846 & -4.941532 \\
\hline C & -8.169386 & 5.547942 & -3.385495 \\
\hline C & -9.334503 & 5.136864 & -4.300510 \\
\hline C & -8.608947 & 6.363530 & -2.163665 \\
\hline$C$ & -10.433979 & 4.357572 & -3.576425 \\
\hline $\mathrm{H}$ & -8.251741 & 7.983907 & -4.545552 \\
\hline $\mathrm{H}$ & -6.212499 & 6.347950 & -3.384840 \\
\hline $\mathrm{H}$ & -7.712696 & 4.634775 & -3.012828 \\
\hline $\mathrm{H}$ & -9.766409 & 6.006148 & -4.780323 \\
\hline $\mathrm{H}$ & -8.936982 & 4.509370 & -5.094599 \\
\hline $\mathrm{H}$ & -9.268349 & 5.788334 & -1.528201 \\
\hline $\mathrm{H}$ & -7.751178 & 6.646670 & -1.562057 \\
\hline $\mathrm{H}$ & -9.133613 & 7.266301 & -2.453200 \\
\hline $\mathrm{H}$ & -11.164557 & 3.981301 & -4.285647 \\
\hline $\mathrm{H}$ & -10.030402 & 3.505615 & -3.040225 \\
\hline $\mathrm{H}$ & -10.965010 & 4.979204 & -2.865860 \\
\hline $\mathrm{N}$ & -6.821822 & 5.574666 & -6.491817 \\
\hline $\mathrm{C}$ & -6.122655 & 4.837517 & -7.526349 \\
\hline C & -4.766885 & 5.445593 & -7.897275 \\
\hline
\end{tabular}




\begin{tabular}{|c|c|c|c|}
\hline $\mathrm{O}$ & -3.900145 & 4.709900 & -8.308774 \\
\hline C & -6.985394 & 4.553869 & -8.764595 \\
\hline C & -8.063608 & 3.474780 & -8.557474 \\
\hline C & -7.486040 & 2.123528 & -8.106951 \\
\hline$N$ & -8.413891 & 0.992753 & -8.200354 \\
\hline C & -9.247023 & 0.580826 & -7.240055 \\
\hline$N$ & -9.565917 & 1.405081 & -6.254299 \\
\hline$N$ & -9.750419 & -0.632651 & -7.274070 \\
\hline $\mathrm{H}$ & -7.500531 & 6.267906 & -6.723047 \\
\hline $\mathrm{H}$ & -5.828205 & 3.900592 & -7.087760 \\
\hline $\mathrm{H}$ & -7.460499 & 5.467402 & -9.109894 \\
\hline $\mathrm{H}$ & -6.316779 & 4.232109 & -9.555651 \\
\hline $\mathrm{H}$ & -8.808220 & 3.828074 & -7.849781 \\
\hline $\mathrm{H}$ & -8.583797 & 3.332195 & -9.498826 \\
\hline $\mathrm{H}$ & -6.639544 & 1.867317 & -8.730819 \\
\hline $\mathrm{H}$ & -7.118616 & 2.168991 & -7.090414 \\
\hline $\mathrm{H}$ & -8.306663 & 0.377692 & -8.976215 \\
\hline $\mathrm{H}$ & -9.344245 & 2.370532 & -6.314187 \\
\hline $\mathrm{H}$ & -10.097070 & 1.113256 & -5.449550 \\
\hline $\mathrm{H}$ & -9.353985 & -1.381590 & -7.810395 \\
\hline $\mathrm{H}$ & -10.478295 & -0.911510 & -6.634346 \\
\hline$N$ & -4.571397 & 6.757500 & -7.722194 \\
\hline $\mathrm{H}$ & -5.311895 & 7.353674 & -7.430382 \\
\hline C & -3.257798 & 7.352383 & -8.011132 \\
\hline $\mathrm{H}$ & -2.933305 & 7.094249 & -9.006697 \\
\hline $\mathrm{H}$ & -3.359275 & 8.427002 & -7.936831 \\
\hline C & -2.193936 & 6.877304 & -7.033115 \\
\hline $\mathrm{O}$ & -1.066806 & 6.644459 & -7.393122 \\
\hline$N$ & -2.610473 & 6.768838 & -5.766290 \\
\hline C & -1.723951 & 6.459952 & -4.670781 \\
\hline
\end{tabular}




\begin{tabular}{|c|c|c|c|}
\hline C & -1.446916 & 4.973453 & -4.542400 \\
\hline $\mathrm{O}$ & -0.318988 & 4.587468 & -4.336016 \\
\hline C & -2.308861 & 7.054869 & -3.383162 \\
\hline C & -1.727790 & 6.351443 & -2.157648 \\
\hline C & -2.066160 & 8.570611 & -3.416451 \\
\hline $\mathrm{H}$ & -3.535867 & 7.063198 & -5.540745 \\
\hline $\mathrm{H}$ & -0.755605 & 6.900148 & -4.860973 \\
\hline $\mathrm{H}$ & -3.377683 & 6.877671 & -3.399219 \\
\hline $\mathrm{H}$ & -1.942950 & 6.894074 & -1.256222 \\
\hline $\mathrm{H}$ & -2.144316 & 5.354939 & -2.053116 \\
\hline $\mathrm{H}$ & -0.655569 & 6.273740 & -2.226170 \\
\hline $\mathrm{H}$ & -2.801922 & 9.100706 & -2.828503 \\
\hline $\mathrm{H}$ & -1.077657 & 8.810853 & -3.039486 \\
\hline $\mathrm{H}$ & -2.147842 & 8.954143 & -4.426881 \\
\hline$N$ & -2.476509 & 4.120594 & -4.661332 \\
\hline C & -2.166304 & 2.713538 & -4.735604 \\
\hline C & -1.263021 & 2.445154 & -5.932153 \\
\hline $\mathrm{O}$ & -0.390765 & 1.610691 & -5.874936 \\
\hline C & -3.409253 & 1.801827 & -4.796543 \\
\hline C & -4.384750 & 1.957396 & -3.620250 \\
\hline C & -5.461605 & 0.858243 & -3.552073 \\
\hline$N$ & -5.024352 & -0.309686 & -2.806819 \\
\hline C & -5.834088 & -1.285026 & -2.637352 \\
\hline$N$ & -7.120142 & -1.337912 & -3.162328 \\
\hline$N$ & -5.545208 & -2.328950 & -1.829388 \\
\hline $\mathrm{H}$ & -3.402241 & 4.448062 & -4.837198 \\
\hline $\mathrm{H}$ & -1.584983 & 2.432899 & -3.869933 \\
\hline $\mathrm{H}$ & -3.941363 & 1.981098 & -5.729249 \\
\hline $\mathrm{H}$ & -3.042255 & 0.782421 & -4.840102 \\
\hline $\mathrm{H}$ & -3.838795 & 1.977506 & -2.689048 \\
\hline
\end{tabular}




\begin{tabular}{|c|c|c|c|}
\hline $\mathrm{H}$ & -4.885565 & 2.909052 & -3.704949 \\
\hline $\mathrm{H}$ & -6.351102 & 1.287798 & -3.090722 \\
\hline $\mathrm{H}$ & -5.745348 & 0.597643 & -4.575397 \\
\hline $\mathrm{H}$ & -7.226829 & -0.855583 & -4.027469 \\
\hline $\mathrm{H}$ & -7.521277 & -2.257450 & -3.202406 \\
\hline $\mathrm{H}$ & -4.636224 & -2.422398 & -1.418379 \\
\hline $\mathrm{H}$ & -6.040804 & -3.182966 & -1.968389 \\
\hline$N$ & -1.459772 & 3.193335 & -7.035054 \\
\hline $\mathrm{H}$ & -2.267192 & 3.765881 & -7.144744 \\
\hline C & -0.596016 & 2.984490 & -8.179296 \\
\hline $\mathrm{H}$ & -0.940318 & 3.627846 & -8.978864 \\
\hline $\mathrm{H}$ & -0.614398 & 1.958679 & -8.517973 \\
\hline C & 0.858094 & 3.326702 & -7.861330 \\
\hline O & 1.786035 & 2.683375 & -8.284624 \\
\hline $\mathrm{N}$ & 1.007247 & 4.396249 & -7.063579 \\
\hline $\mathrm{H}$ & 0.213446 & 4.960297 & -6.860737 \\
\hline C & 2.282316 & 4.880650 & -6.640295 \\
\hline $\mathrm{H}$ & 2.918456 & 5.142312 & -7.476474 \\
\hline $\mathrm{H}$ & 2.122221 & 5.774091 & -6.050920 \\
\hline C & 3.094582 & 3.926515 & -5.779533 \\
\hline 0 & 4.300635 & 4.045656 & -5.701005 \\
\hline $\mathrm{N}$ & 2.428034 & 2.983354 & -5.101941 \\
\hline C & 3.098460 & 2.130521 & -4.156539 \\
\hline C & 4.281984 & 1.344235 & -4.723594 \\
\hline O & 5.201684 & 1.054362 & -3.984935 \\
\hline C & 2.026255 & 1.207884 & -3.579586 \\
\hline C & 2.417310 & 0.331931 & -2.395276 \\
\hline C & 1.121230 & -0.251754 & -1.856780 \\
\hline$N$ & 1.333350 & -1.247206 & -0.825494 \\
\hline C & 0.341807 & -2.072958 & -0.445718 \\
\hline
\end{tabular}




\begin{tabular}{|c|c|c|c|}
\hline$N$ & -0.814787 & -2.039801 & -1.064745 \\
\hline$N$ & 0.558512 & -2.942064 & 0.513940 \\
\hline $\mathrm{H}$ & 1.433643 & 2.939341 & -5.176232 \\
\hline $\mathrm{H}$ & 3.540110 & 2.726300 & -3.366546 \\
\hline $\mathrm{H}$ & 1.214694 & 1.858336 & -3.271280 \\
\hline $\mathrm{H}$ & 1.624632 & 0.593830 & -4.377160 \\
\hline $\mathrm{H}$ & 3.092263 & -0.463522 & -2.691370 \\
\hline $\mathrm{H}$ & 2.922286 & 0.917915 & -1.630625 \\
\hline $\mathrm{H}$ & 0.483946 & 0.548459 & -1.492586 \\
\hline $\mathrm{H}$ & 0.599758 & -0.734748 & -2.670949 \\
\hline $\mathrm{H}$ & 2.042725 & -1.074796 & -0.148314 \\
\hline $\mathrm{H}$ & -1.141342 & -1.192989 & -1.482243 \\
\hline $\mathrm{H}$ & -1.575377 & -2.653242 & -0.776559 \\
\hline $\mathrm{H}$ & 1.472900 & -3.119781 & 0.859926 \\
\hline $\mathrm{H}$ & -0.227485 & -3.495225 & 0.850629 \\
\hline$N$ & 4.281642 & 0.945295 & -6.005331 \\
\hline $\mathrm{H}$ & 3.540514 & 1.224776 & -6.614846 \\
\hline C & 5.363426 & 0.116658 & -6.504603 \\
\hline $\mathrm{H}$ & 5.139423 & -0.143515 & -7.531654 \\
\hline $\mathrm{H}$ & 5.441111 & -0.797366 & -5.937306 \\
\hline C & 6.736237 & 0.756120 & -6.476105 \\
\hline $\mathrm{O}$ & 7.738575 & 0.089675 & -6.364842 \\
\hline$N$ & 6.766121 & 2.085332 & -6.608323 \\
\hline $\mathrm{H}$ & 5.912179 & 2.604995 & -6.573019 \\
\hline C & 8.005509 & 2.823538 & -6.551101 \\
\hline $\mathrm{H}$ & 8.709834 & 2.439534 & -7.275449 \\
\hline $\mathrm{H}$ & 7.800300 & 3.854283 & -6.807558 \\
\hline C & 8.748871 & 2.826732 & -5.213347 \\
\hline O & 9.890154 & 3.235828 & -5.161891 \\
\hline $\mathbf{I N}$ & 8.103920 & 2.338653 & -4.145971 \\
\hline
\end{tabular}




\begin{tabular}{|c|c|c|c|}
\hline C & 8.685569 & 2.174685 & -2.824698 \\
\hline C & 9.950210 & 1.305456 & -2.832305 \\
\hline $\mathrm{O}$ & 10.768807 & 1.388487 & -1.930967 \\
\hline C & 7.581462 & 1.507752 & -1.974036 \\
\hline C & 7.779545 & 1.384045 & -0.463395 \\
\hline C & 7.439220 & 2.653824 & 0.326219 \\
\hline C & 7.328841 & 2.386785 & 1.823564 \\
\hline$N$ & 8.668931 & 2.353589 & 2.481038 \\
\hline $\mathrm{O}$ & 11.041280 & 1.616744 & 0.893292 \\
\hline $\mathrm{H}$ & 10.891336 & 1.827886 & -0.028619 \\
\hline $\mathrm{H}$ & 11.519641 & 0.790022 & 0.842503 \\
\hline $\mathrm{H}$ & 7.157843 & 2.036144 & -4.258798 \\
\hline $\mathrm{H}$ & 8.984887 & 3.132101 & -2.419072 \\
\hline $\mathrm{H}$ & 6.665568 & 2.053317 & -2.157635 \\
\hline $\mathrm{H}$ & 7.411935 & 0.520992 & -2.389253 \\
\hline $\mathrm{H}$ & 7.099674 & 0.604947 & -0.132388 \\
\hline $\mathrm{H}$ & 8.774806 & 1.030482 & -0.228813 \\
\hline $\mathrm{H}$ & 8.152705 & 3.454140 & 0.150925 \\
\hline $\mathrm{H}$ & 6.474304 & 3.026469 & -0.002372 \\
\hline $\mathrm{H}$ & 6.768461 & 3.159097 & 2.327029 \\
\hline $\mathrm{H}$ & 6.847680 & 1.440721 & 2.018165 \\
\hline $\mathrm{H}$ & 9.379709 & 1.889596 & 1.923959 \\
\hline $\mathrm{H}$ & 8.989016 & 3.323756 & 2.635285 \\
\hline $\mathrm{H}$ & 8.615271 & 1.875867 & 3.368782 \\
\hline$N$ & 10.062478 & 0.448235 & -3.837531 \\
\hline C & 11.109288 & -0.537620 & -4.069164 \\
\hline C & 12.486185 & 0.023066 & -4.427535 \\
\hline O & 13.443856 & -0.705807 & $7-4.301670$ \\
\hline C & 10.700469 & -1.444643 & $3-5.251751$ \\
\hline C & 9.562495 & -2.442934 & -4.995550 \\
\hline
\end{tabular}




\begin{tabular}{|c|c|c|c|}
\hline C & 9.129826 & -3.067907 & -6.325579 \\
\hline C & 9.990453 & -3.533264 & -4.013046 \\
\hline $\mathrm{H}$ & 9.324245 & 0.446354 & -4.510697 \\
\hline $\mathrm{H}$ & 11.265001 & -1.124722 & -3.175914 \\
\hline $\mathrm{H}$ & 10.433405 & -0.799467 & -6.083409 \\
\hline $\mathrm{H}$ & 11.583144 & -1.997215 & -5.550670 \\
\hline $\mathrm{H}$ & 8.706388 & -1.911705 & -4.591231 \\
\hline $\mathrm{H}$ & 8.328652 & -3.784069 & -6.170262 \\
\hline $\mathrm{H}$ & 8.772094 & -2.311226 & -7.012127 \\
\hline $\mathrm{H}$ & 9.956502 & -3.596183 & -6.794105 \\
\hline $\mathrm{H}$ & 9.200793 & -4.263319 & -3.872571 \\
\hline $\mathrm{H}$ & 10.860385 & -4.059559 & -4.391976 \\
\hline $\mathrm{H}$ & 10.243938 & -3.136158 & -3.034849 \\
\hline$N$ & 12.574038 & 1.261815 & -4.919239 \\
\hline C & 13.814598 & 1.844700 & -5.393665 \\
\hline C & 14.958833 & 2.033070 & -4.379249 \\
\hline 0 & 16.013518 & 2.413551 & -4.812238 \\
\hline C & 13.497199 & 3.171601 & -6.086541 \\
\hline 0 & 12.918242 & 4.088351 & -5.201903 \\
\hline $\mathrm{H}$ & 11.756392 & 1.833549 & -4.917202 \\
\hline $\mathrm{H}$ & 14.248406 & 1.177890 & -6.129408 \\
\hline $\mathrm{H}$ & 14.420350 & 3.590566 & -6.455251 \\
\hline $\mathrm{H}$ & 12.846329 & 2.977977 & -6.933265 \\
\hline $\mathrm{H}$ & 11.970851 & 4.079335 & -5.266599 \\
\hline$N$ & 14.792294 & 1.722322 & -3.074271 \\
\hline C & 15.928267 & 1.647463 & -2.176843 \\
\hline C & 16.804560 & 0.409991 & -2.402623 \\
\hline 0 & 17.934510 & 0.394028 & -1.988350 \\
\hline C & 15.495334 & 1.578022 & -0.707310 \\
\hline$C$ & 14.638736 & 2.735142 & -0.197993 \\
\hline
\end{tabular}




\begin{tabular}{|c|c|c|c|}
\hline C & 14.285265 & 2.488615 & 1.269059 \\
\hline$N$ & 13.259778 & 3.383220 & 1.790963 \\
\hline C & 13.437031 & 4.534134 & 2.418972 \\
\hline$N$ & 14.654643 & 5.009739 & 2.656236 \\
\hline $\mathrm{O}$ & 15.027936 & 7.465602 & 4.210742 \\
\hline $\mathrm{H}$ & 14.744204 & 8.352164 & 3.972083 \\
\hline $\mathrm{H}$ & 15.745893 & 7.571535 & 4.821879 \\
\hline$N$ & 12.386879 & 5.222483 & 2.815285 \\
\hline $\mathrm{H}$ & 13.900806 & 1.420456 & -2.758176 \\
\hline $\mathrm{H}$ & 16.561947 & 2.504372 & -2.346507 \\
\hline $\mathrm{H}$ & 14.954826 & 0.650121 & -0.551663 \\
\hline $\mathrm{H}$ & 16.407320 & 1.508875 & -0.124521 \\
\hline $\mathrm{H}$ & 15.156423 & 3.682909 & -0.314349 \\
\hline $\mathrm{H}$ & 13.719550 & 2.816364 & -0.766363 \\
\hline $\mathrm{H}$ & 13.888865 & 1.489253 & 1.375952 \\
\hline $\mathrm{H}$ & 15.164630 & 2.531614 & 1.900891 \\
\hline $\mathrm{H}$ & 12.319759 & 3.087225 & 1.619716 \\
\hline 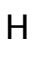 & 15.462651 & 4.517082 & 2.358613 \\
\hline $\mathrm{H}$ & 14.789755 & 5.864579 & 3.168103 \\
\hline $\mathrm{H}$ & 11.431450 & 4.942520 & 2.657106 \\
\hline $\mathrm{H}$ & 12.478890 & 6.065589 & 3.335508 \\
\hline$N$ & 16.200243 & -0.648980 & -2.967249 \\
\hline C & 16.870911 & -1.911444 & -3.168063 \\
\hline C & 17.670040 & -1.889279 & -4.467661 \\
\hline $\mathrm{H}$ & 18.062080 & -0.909278 & -4.748851 \\
\hline $\mathrm{O}$ & 17.878681 & -2.853544 & -5.128762 \\
\hline C & 15.868239 & -3.070209 & -3.149497 \\
\hline C & 15.324776 & -3.399867 & -1.792053 \\
\hline$N$ & 14.403859 & -2.627488 & -1.112382 \\
\hline O & 12.508213 & -0.434098 & -0.465247 \\
\hline
\end{tabular}




\begin{tabular}{|c|c|c|c|}
\hline $\mathrm{H}$ & 13.145673 & -1.037493 & -0.851266 \\
\hline $\mathrm{H}$ & 12.027814 & -0.038330 & -1.184376 \\
\hline C & 15.619918 & -4.490272 & -1.049640 \\
\hline C & 14.172683 & -3.251551 & -0.003857 \\
\hline$N$ & 14.883081 & -4.381637 & 0.103104 \\
\hline $\mathrm{H}$ & 15.285615 & -0.550381 & -3.352678 \\
\hline $\mathrm{H}$ & 17.596037 & -2.030736 & -2.370677 \\
\hline $\mathrm{H}$ & 15.050771 & -2.842318 & -3.824804 \\
\hline $\mathrm{H}$ & 16.369122 & -3.943997 & -3.543957 \\
\hline $\mathrm{H}$ & 16.275343 & -5.314047 & -1.234992 \\
\hline $\mathrm{H}$ & 13.494340 & -2.922022 & 0.757056 \\
\hline $\mathrm{H}$ & 14.782430 & -5.064459 & 0.821874 \\
\hline 0 & -14.798003 & -2.305642 & 5.077112 \\
\hline $\mathrm{H}$ & -15.090888 & -1.616867 & 4.485156 \\
\hline $\mathrm{H}$ & -14.377476 & -1.862811 & 5.806964 \\
\hline 0 & -12.886313 & -0.760382 & 6.902943 \\
\hline $\mathrm{H}$ & -12.159802 & -1.250025 & 7.273334 \\
\hline $\mathrm{H}$ & -13.153007 & -0.137559 & 7.567914 \\
\hline 0 & -12.140772 & -0.228791 & 4.090461 \\
\hline $\mathrm{H}$ & -12.285224 & -0.223791 & 5.032520 \\
\hline $\mathrm{H}$ & -13.017444 & -0.218849 & 3.702739 \\
\hline 0 & -13.359306 & -2.024100 & 0.907285 \\
\hline $\mathrm{H}$ & -12.590290 & -1.456457 & 0.826721 \\
\hline $\mathrm{H}$ & -13.169281 & -2.625977 & 1.626936 \\
\hline 0 & -7.462894 & 2.078515 & -0.250981 \\
\hline $\mathrm{H}$ & -7.582969 & 2.166579 & 0.697926 \\
\hline $\mathrm{H}$ & -6.548672 & 1.845155 & -0.359334 \\
\hline 0 & -7.781878 & 2.147925 & 2.587834 \\
\hline $\mathrm{H}$ & -8.712664 & 1.982022 & 2.720968 \\
\hline H & -7.530710 & 2.835293 & 3.200006 \\
\hline
\end{tabular}




\begin{tabular}{|c|c|c|c|}
\hline $\mathrm{O}$ & -9.219556 & 0.268046 & -1.543300 \\
\hline $\mathrm{H}$ & -8.654417 & 0.920861 & -1.116260 \\
\hline $\mathrm{H}$ & -8.625594 & -0.395291 & -1.892853 \\
\hline 0 & -13.104983 & -2.225893 & -1.908178 \\
\hline $\mathrm{H}$ & -13.351355 & -2.375510 & -0.993819 \\
\hline $\mathrm{H}$ & -13.802447 & -2.556578 & -2.463598 \\
\hline O & -13.484588 & 1.744872 & -0.191105 \\
\hline $\mathrm{H}$ & -12.857556 & 1.055739 & 0.036926 \\
\hline $\mathrm{H}$ & -12.999598 & 2.554874 & -0.018958 \\
\hline $\mathrm{O}$ & -14.181231 & 4.914670 & 2.449534 \\
\hline $\mathrm{H}$ & -13.801872 & 5.401488 & 3.175814 \\
\hline $\mathrm{H}$ & -14.711660 & 4.211922 & 2.806152 \\
\hline $\mathrm{O}$ & -15.446011 & 2.145884 & 1.861697 \\
\hline $\mathrm{H}$ & -16.298797 & 2.345407 & 1.497799 \\
\hline $\mathrm{H}$ & -14.850135 & 2.051818 & 1.115481 \\
\hline 0 & -13.497749 & 0.498958 & -2.728436 \\
\hline $\mathrm{H}$ & -13.706908 & 1.081122 & -1.997612 \\
\hline $\mathrm{H}$ & -13.279855 & -0.342565 & -2.324130 \\
\hline 0 & -14.513559 & -1.463913 & -4.511132 \\
\hline $\mathrm{H}$ & -14.515837 & -0.619962 & -4.063929 \\
\hline $\mathrm{H}$ & -15.310446 & -1.513964 & -5.022410 \\
\hline 0 & -10.574390 & -2.969205 & -3.202248 \\
\hline $\mathrm{H}$ & -10.866490 & -2.657676 & -4.055481 \\
\hline $\mathrm{H}$ & -11.318892 & -2.817667 & -2.624688 \\
\hline 0 & -11.340505 & -0.095921 & 0.389263 \\
\hline $\mathrm{H}$ & -10.663811 & -0.135502 & -0.288446 \\
\hline $\mathrm{H}$ & -10.954446 & 0.397240 & 1.116547 \\
\hline O & -10.645926 & 1.635178 & 2.510120 \\
\hline H & -11.151041 & 1.180674 & 3.185435 \\
\hline & -11.153101 & 2.384321 & 2.199776 \\
\hline
\end{tabular}




$\begin{array}{llll}\mathrm{O} & -12.086338 & 3.782159 & 1.051177 \\ \mathrm{H} & -12.806634 & 4.185138 & 1.549342 \\ \mathrm{H} & -11.540705 & 4.511703 & 0.772887 \\ \mathrm{O} & -10.888322 & 0.832962 & -3.747451 \\ \mathrm{H} & -11.766127 & 0.977606 & -3.390586 \\ \mathrm{H} & -10.308683 & 0.683062 & -2.994652 \\ \mathrm{O} & -14.735511 & -0.392918 & 2.923478 \\ \mathrm{H} & -15.121325 & 0.439349 & 2.649763 \\ \mathrm{H} & -14.531491 & -0.883649 & 2.129065 \\ \mathrm{O} & -12.750242 & -3.277893 & 3.454330 \\ \mathrm{H} & -13.513525 & -3.074626 & 4.006952 \\ \mathrm{H} & -12.113276 & -2.610803 & 3.692382 \\ \mathrm{O} & -11.716553 & -1.375614 & -5.293525 \\ \mathrm{H} & -12.662337 & -1.516011 & -5.271893 \\ \mathrm{H} & -11.531061 & -0.699335 & -4.643453\end{array}$

PROTON CONFIGURATION, GIVEN BY NOMINAL CHARGES: R300(+),Y266(-),E183(0) POTENTIAL:-70mV

$\begin{array}{llll}\mathrm{N} & 15.580944 & -6.451469 & 5.391416 \\ \mathrm{H} & 14.780173 & -6.202168 & 5.939086 \\ \mathrm{C} & 15.508993 & -5.856291 & 4.054355 \\ \mathrm{C} & 14.783016 & -6.782962 & 3.060452 \\ \mathrm{O} & 14.052852 & -6.345866 & 2.186400 \\ \mathrm{C} & 16.918802 & -5.575378 & 3.520809 \\ \mathrm{H} & 16.389097 & -6.126718 & 5.884712 \\ \mathrm{H} & 14.943644 & -4.933781 & 4.050735 \\ \mathrm{H} & 16.887619 & -5.188754 & 2.509159 \\ \mathrm{H} & 17.402401 & -4.830320 & 4.142393 \\ \mathrm{H} & 17.526676 & -6.473359 & 3.529226\end{array}$




\begin{tabular}{|c|c|c|c|}
\hline$N$ & 15.032378 & -8.085605 & 3.186405 \\
\hline $\mathrm{H}$ & 15.611823 & -8.361771 & 3.949887 \\
\hline$C$ & 14.398660 & -9.092385 & 2.333233 \\
\hline $\mathrm{H}$ & 14.482014 & -8.823398 & 1.292932 \\
\hline $\mathrm{H}$ & 14.891843 & -10.042103 & $\begin{array}{ll}3 & 2.492271\end{array}$ \\
\hline$C$ & 12.912965 & -9.221837 & 2.671557 \\
\hline 0 & 12.082056 & -9.427871 & 1.826217 \\
\hline $\mathrm{N}$ & 12.582804 & -9.055884 & 3.966878 \\
\hline $\mathrm{H}$ & 13.302600 & -8.983985 & 4.648750 \\
\hline$C$ & 11.195108 & -9.170814 & 4.421375 \\
\hline $\mathrm{H}$ & 11.188284 & -9.158108 & 5.503134 \\
\hline $\mathrm{H}$ & 10.743835 & -10.088273 & $\begin{array}{ll}3 & 4.076932\end{array}$ \\
\hline$C$ & 10.336943 & -8.010596 & 3.900736 \\
\hline 0 & 9.191046 & -8.186327 & 3.583715 \\
\hline $\mathrm{N}$ & 10.946934 & -6.814391 & 3.855551 \\
\hline$C$ & 10.317888 & -5.633853 & 3.318203 \\
\hline$C$ & 10.125898 & -5.690235 & 1.799945 \\
\hline 0 & 9.095707 & -5.261140 & 1.338159 \\
\hline$C$ & 11.092037 & -4.387866 & 3.777168 \\
\hline 0 & 12.478184 & -4.570170 & 3.768239 \\
\hline $\mathrm{H}$ & 11.905264 & -6.743798 & 4.107138 \\
\hline $\mathrm{H}$ & 9.314131 & -5.553344 & 3.702087 \\
\hline $\mathrm{H}$ & 10.799505 & -3.539122 & 3.169275 \\
\hline $\mathrm{H}$ & 10.827762 & -4.175240 & 4.802705 \\
\hline $\mathrm{H}$ & 12.803731 & -4.753415 & 2.895470 \\
\hline $\mathrm{N}$ & 11.098972 & -6.199760 & 1.024173 \\
\hline$C$ & 10.842818 & -6.395674 & -0.400144 \\
\hline C & 9.692691 & -7.385274 & -0.601544 \\
\hline 0 & 8.944478 & -7.280481 & -1.545340 \\
\hline 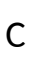 & 12.131172 & -6.783268 & -1.18742 \\
\hline
\end{tabular}




\begin{tabular}{|c|c|c|c|}
\hline C & 12.334410 & -8.288589 & -1.402440 \\
\hline C & 12.155830 & -6.059826 & -2.535219 \\
\hline $\mathrm{H}$ & 11.922742 & -6.586169 & 1.431210 \\
\hline $\mathrm{H}$ & 10.472399 & -5.461424 & -0.790919 \\
\hline $\mathrm{H}$ & 12.963052 & -6.411050 & -0.600020 \\
\hline $\mathrm{H}$ & 13.288349 & -8.450898 & -1.893511 \\
\hline $\mathrm{H}$ & 12.333844 & -8.853758 & -0.481177 \\
\hline $\mathrm{H}$ & 11.567700 & -8.697764 & -2.053167 \\
\hline $\mathrm{H}$ & 13.050308 & -6.323364 & -3.088571 \\
\hline $\mathrm{H}$ & 11.295100 & -6.333226 & -3.135653 \\
\hline $\mathrm{H}$ & 12.154727 & -4.984474 & -2.403241 \\
\hline $\mathrm{N}$ & 9.570430 & -8.340342 & 0.328312 \\
\hline $\mathrm{H}$ & 10.314649 & -8.474146 & 0.974145 \\
\hline C & 8.593019 & -9.421182 & 0.241228 \\
\hline $\mathrm{H}$ & 8.932305 & -10.216770 & 0.892269 \\
\hline $\mathrm{H}$ & 8.535637 & -9.806255 & -0.765234 \\
\hline C & 7.181203 & -9.042464 & 0.638009 \\
\hline 0 & 6.257797 & -9.497564 & 0.003641 \\
\hline$N$ & 7.002492 & -8.221880 & 1.679685 \\
\hline C & 5.669019 & -7.732021 & 1.946137 \\
\hline C & 5.252621 & -6.721873 & 0.882411 \\
\hline O & 4.076751 & -6.627532 & 0.602378 \\
\hline C & 5.413666 & -7.224142 & 3.379357 \\
\hline C & 5.551149 & -8.367537 & 4.389728 \\
\hline C & 6.257926 & -6.016989 & 3.792493 \\
\hline $\mathrm{H}$ & 7.779501 & -7.956116 & 2.244709 \\
\hline $\mathrm{H}$ & 4.985221 & -8.550829 & 1.784424 \\
\hline $\mathrm{H}$ & 4.373722 & -6.912400 & 3.370605 \\
\hline $\mathrm{H}$ & 5.260481 & -8.028316 & 5.378435 \\
\hline $\mathrm{H}$ & 4.911189 & -9.203555 & 4.126944 \\
\hline
\end{tabular}




\begin{tabular}{|c|c|c|c|}
\hline $\mathrm{H}$ & 6.572303 & -8.727453 & 4.447510 \\
\hline $\mathrm{H}$ & 5.916474 & -5.642739 & 4.751679 \\
\hline $\mathrm{H}$ & 7.299292 & -6.290260 & 3.902023 \\
\hline $\mathrm{H}$ & 6.189352 & -5.203276 & 3.078847 \\
\hline$N$ & 6.173165 & -5.995446 & 0.240637 \\
\hline C & 5.775650 & -5.174300 & -0.891224 \\
\hline C & 5.339778 & -6.055846 & -2.069924 \\
\hline $\mathrm{O}$ & 4.316554 & -5.802555 & -2.661062 \\
\hline C & 6.869646 & -4.156740 & -1.271726 \\
\hline C & 7.070893 & -3.141320 & -0.132717 \\
\hline C & 6.498936 & -3.444256 & -2.577032 \\
\hline C & 8.367774 & -2.339676 & -0.245832 \\
\hline $\mathrm{H}$ & 7.134029 & -6.050096 & 0.501393 \\
\hline $\mathrm{H}$ & 4.879069 & -4.636632 & -0.621597 \\
\hline $\mathrm{H}$ & 7.798320 & -4.700086 & -1.420947 \\
\hline $\mathrm{H}$ & 6.218484 & -2.463887 & -0.116855 \\
\hline $\mathrm{H}$ & 7.078334 & -3.655467 & 0.819086 \\
\hline $\mathrm{H}$ & 7.205586 & -2.655636 & -2.796593 \\
\hline $\mathrm{H}$ & 6.497377 & -4.118719 & -3.423668 \\
\hline $\mathrm{H}$ & 5.511966 & -2.998986 & -2.509092 \\
\hline $\mathrm{H}$ & 8.470914 & -1.666203 & 0.598331 \\
\hline $\mathrm{H}$ & 9.226825 & -3.000371 & -0.244817 \\
\hline $\mathrm{H}$ & 8.406844 & -1.740406 & -1.148542 \\
\hline$N$ & 6.110937 & -7.098823 & -2.408166 \\
\hline $\mathrm{H}$ & 7.004041 & -7.224504 & -1.980349 \\
\hline C & 5.766224 & -7.936500 & -3.536910 \\
\hline $\mathrm{H}$ & 6.596769 & -8.609256 & -3.711161 \\
\hline $\mathrm{H}$ & 5.607735 & -7.348312 & -4.428476 \\
\hline C & 4.498689 & -8.766736 & -3.333203 \\
\hline 0 & 3.658210 & -8.824187 & -4.199480 \\
\hline
\end{tabular}




\begin{tabular}{|c|c|c|c|}
\hline$N$ & 4.392527 & -9.424027 & -2.171602 \\
\hline $\mathrm{H}$ & 5.130655 & -9.378505 & -1.501821 \\
\hline C & 3.209146 & -10.183380 & -1.842411 \\
\hline $\mathrm{H}$ & 2.968932 & -10.893506 & -2.619442 \\
\hline $\mathrm{H}$ & 3.405813 & -10.729233 & -0.928135 \\
\hline C & 1.974189 & -9.306856 & -1.644805 \\
\hline $\mathrm{O}$ & 0.886786 & -9.700155 & -2.000101 \\
\hline$N$ & 2.187869 & -8.107001 & -1.107183 \\
\hline C & 1.052634 & -7.196951 & -1.048103 \\
\hline C & 0.601552 & -6.713526 & -2.428651 \\
\hline $\mathrm{O}$ & -0.582310 & -6.579102 & -2.644204 \\
\hline C & 1.251457 & -5.970574 & -0.162525 \\
\hline $\mathrm{O}$ & 1.412772 & -6.294255 & 1.178745 \\
\hline $\mathrm{H}$ & 3.086709 & -7.815605 & -0.796908 \\
\hline $\mathrm{H}$ & 0.208799 & -7.739346 & -0.654199 \\
\hline $\mathrm{H}$ & 2.078030 & -5.371688 & -0.529123 \\
\hline $\mathrm{H}$ & 0.344443 & -5.394923 & -0.244733 \\
\hline $\mathrm{H}$ & 2.319777 & -6.527902 & 1.321835 \\
\hline$N$ & 1.523733 & -6.420954 & -3.355538 \\
\hline C & 1.118171 & -6.006582 & -4.686094 \\
\hline C & 0.366591 & -7.131210 & -5.393853 \\
\hline $\mathrm{O}$ & -0.616579 & -6.878354 & -6.058668 \\
\hline C & 2.319655 & -5.472227 & -5.499986 \\
\hline C & 2.696594 & -4.078811 & -4.969418 \\
\hline C & 2.025603 & -5.435939 & -7.004976 \\
\hline C & 4.006520 & -3.527492 & -5.529793 \\
\hline $\mathrm{H}$ & 2.495346 & -6.472438 & -3.135669 \\
\hline $\mathrm{H}$ & 0.385122 & -5.217788 & -4.597028 \\
\hline $\mathrm{H}$ & 3.153949 & -6.147638 & -5.335701 \\
\hline $\mathrm{H}$ & 1.887508 & -3.387959 & -5.200378 \\
\hline
\end{tabular}




\begin{tabular}{|c|c|c|c|}
\hline $\mathrm{H}$ & 2.775597 & -4.119379 & -3.890688 \\
\hline $\mathrm{H}$ & 2.876429 & -5.040229 & -7.545503 \\
\hline $\mathrm{H}$ & 1.828531 & -6.423019 & -7.405382 \\
\hline $\mathrm{H}$ & 1.167262 & -4.809177 & -7.225534 \\
\hline $\mathrm{H}$ & 4.277310 & -2.614058 & -5.010545 \\
\hline $\mathrm{H}$ & 4.817030 & -4.236127 & -5.392936 \\
\hline $\mathrm{H}$ & 3.939404 & -3.295428 & -6.586923 \\
\hline$N$ & 0.812661 & -8.380776 & -5.239064 \\
\hline $\mathrm{H}$ & 1.658723 & -8.555543 & -4.737799 \\
\hline C & 0.043838 & -9.489011 & -5.752955 \\
\hline $\mathrm{H}$ & -0.063094 & -9.437045 & -6.826236 \\
\hline $\mathrm{H}$ & 0.563431 & -10.404330 & -5.499466 \\
\hline C & -1.365267 & -9.540096 & -5.166931 \\
\hline $\mathrm{O}$ & -2.317049 & -9.778914 & -5.874508 \\
\hline$N$ & -1.472644 & -9.318876 & -3.858022 \\
\hline C & -2.773861 & -9.358770 & -3.234318 \\
\hline C & -3.688320 & -8.224570 & -3.694876 \\
\hline $\mathrm{O}$ & -4.883588 & -8.440681 & -3.757278 \\
\hline C & -2.678486 & -9.337286 & -1.701918 \\
\hline $\mathrm{O}$ & -3.924134 & -9.612073 & -1.121424 \\
\hline $\mathrm{H}$ & -0.656065 & -9.230217 & -3.289993 \\
\hline $\mathrm{H}$ & -3.288400 & -10.263844 & -3.524706 \\
\hline $\mathrm{H}$ & -1.994466 & -10.109348 & -1.378045 \\
\hline $\mathrm{H}$ & -2.294276 & -8.379929 & -1.366622 \\
\hline $\mathrm{H}$ & -4.614315 & -9.215993 & -1.638945 \\
\hline$N$ & -3.140832 & -7.038300 & -3.955706 \\
\hline C & -3.899323 & -5.915086 & -4.482726 \\
\hline C & -4.451814 & -6.249649 & -5.867267 \\
\hline $\mathrm{O}$ & -5.624687 & -6.088408 & -6.120172 \\
\hline C & -2.987397 & -4.668975 & -4.499857 \\
\hline
\end{tabular}




\begin{tabular}{|c|c|c|c|}
\hline C & -3.418161 & -3.519203 & -5.398608 \\
\hline C & -4.746416 & -3.185602 & -5.638959 \\
\hline C & -2.430755 & -2.759753 & -6.021257 \\
\hline C & -5.071658 & -2.147370 & -6.500352 \\
\hline C & -2.750066 & -1.710393 & -6.864747 \\
\hline C & -4.078759 & -1.406114 & -7.116393 \\
\hline $\mathrm{H}$ & -2.168236 & -6.911483 & -3.766018 \\
\hline $\mathrm{H}$ & -4.761149 & -5.732637 & -3.860641 \\
\hline $\mathrm{H}$ & -2.882581 & -4.329553 & -3.473769 \\
\hline $\mathrm{H}$ & -2.005629 & -4.982126 & -4.824976 \\
\hline $\mathrm{H}$ & -5.540598 & -3.751901 & -5.194369 \\
\hline $\mathrm{H}$ & -1.395513 & -2.998702 & -5.851834 \\
\hline $\mathrm{H}$ & -6.110294 & -1.946391 & -6.699659 \\
\hline $\mathrm{H}$ & -1.964466 & -1.141973 & -7.329659 \\
\hline $\mathrm{H}$ & -4.330924 & -0.608516 & -7.793667 \\
\hline$N$ & -3.582681 & -6.715106 & -6.770418 \\
\hline C & -4.048127 & -7.046157 & -8.097915 \\
\hline C & -5.186090 & -8.063944 & -8.050111 \\
\hline $\mathrm{O}$ & -6.114137 & -7.965980 & -8.809499 \\
\hline C & -2.916689 & -7.590810 & -8.972520 \\
\hline$S$ & -1.688348 & -6.353753 & -9.500059 \\
\hline $\mathrm{H}$ & -2.632397 & -6.879591 & -6.510637 \\
\hline $\mathrm{H}$ & -4.472223 & -6.170046 & -8.565992 \\
\hline $\mathrm{H}$ & -2.417255 & -8.416480 & -8.483860 \\
\hline $\mathrm{H}$ & -3.349076 & -7.962399 & -9.891961 \\
\hline $\mathrm{H}$ & -1.067496 & -6.199102 & -8.340924 \\
\hline$N$ & -5.078560 & -9.066910 & -7.155609 \\
\hline $\mathrm{H}$ & -4.238851 & -9.204214 & -6.634586 \\
\hline C & -6.086025 & -10.114377 & -7.182687 \\
\hline $\mathrm{H}$ & -6.261083 & -10.452751 & -8.192667 \\
\hline
\end{tabular}




\begin{tabular}{|c|c|c|c|}
\hline $\mathrm{H}$ & -5.714273 & -10.946867 & -6.596749 \\
\hline C & -7.459139 & -9.722627 & -6.613157 \\
\hline 0 & -8.480633 & -10.092015 & -7.125244 \\
\hline$N$ & -7.417940 & -9.016150 & -5.478415 \\
\hline C & -8.636115 & -8.653971 & -4.781504 \\
\hline C & -9.495657 & -7.633536 & -5.521422 \\
\hline $\mathrm{O}$ & -10.688492 & -7.607651 & -5.361495 \\
\hline C & -8.345059 & -8.144470 & -3.358873 \\
\hline C & -7.426847 & -6.915813 & -3.231955 \\
\hline C & -8.012652 & -5.535819 & -3.104155 \\
\hline 0 & -9.305154 & -5.428966 & -3.235643 \\
\hline $\mathrm{H}$ & -9.566081 & -4.511826 & -3.102773 \\
\hline $\mathrm{O}$ & -7.307092 & -4.582490 & -2.878392 \\
\hline $\mathrm{H}$ & -6.532786 & -8.774357 & -5.086263 \\
\hline $\mathrm{H}$ & -9.265048 & -9.529213 & -4.698554 \\
\hline $\mathrm{H}$ & -9.298086 & -7.962025 & -2.882575 \\
\hline $\mathrm{H}$ & -7.870967 & -8.961678 & -2.826041 \\
\hline $\mathrm{H}$ & -6.797836 & -7.023546 & -2.358218 \\
\hline $\mathrm{H}$ & -6.742023 & -6.849089 & -4.060794 \\
\hline $\mathrm{N}$ & -8.837281 & -6.733946 & -6.273830 \\
\hline $\mathrm{C}$ & -9.555208 & -5.702634 & -7.000846 \\
\hline$C$ & -10.241246 & -6.303328 & -8.225725 \\
\hline $\mathrm{H}$ & -9.886965 & -7.288064 & -8.536094 \\
\hline 0 & -11.123214 & -5.743558 & -8.795675 \\
\hline$C$ & -8.611510 & -4.505222 & -7.297455 \\
\hline 0 & -8.597017 & -3.622202 & -6.192752 \\
\hline$C$ & -8.932695 & -3.726903 & -8.570353 \\
\hline $\mathrm{H}$ & -7.859762 & -6.848467 & -6.436592 \\
\hline $\mathrm{H}$ & -10.361686 & -5.346750 & -6.373685 \\
\hline $\mathrm{H}$ & -7.600579 & -4.876156 & -7.370234 \\
\hline
\end{tabular}




\begin{tabular}{|c|c|c|c|}
\hline $\mathrm{H}$ & -9.438003 & -3.183621 & -6.139031 \\
\hline $\mathrm{H}$ & -8.252828 & -2.882799 & -8.630093 \\
\hline $\mathrm{H}$ & -8.801458 & -4.331156 & -9.458851 \\
\hline $\mathrm{H}$ & -9.956879 & -3.366625 & -8.569319 \\
\hline$N$ & -12.952099 & -9.022565 & 3.174142 \\
\hline $\mathrm{H}$ & -13.213067 & -9.553349 & 2.368879 \\
\hline C & -11.773563 & -8.180068 & 2.898826 \\
\hline C & -10.790797 & -8.116813 & 4.051837 \\
\hline O & -9.663991 & -7.701334 & 3.858866 \\
\hline C & -12.146833 & -6.729545 & 2.544429 \\
\hline O & -12.732347 & -6.198257 & 3.704599 \\
\hline C & -13.100838 & -6.633808 & 1.358713 \\
\hline $\mathrm{H}$ & -13.732180 & -8.453398 & 3.440187 \\
\hline $\mathrm{H}$ & -11.218895 & -8.607381 & 2.074186 \\
\hline $\mathrm{H}$ & -11.231022 & -6.202436 & 2.314759 \\
\hline $\mathrm{H}$ & -12.851647 & -5.257655 & 3.590089 \\
\hline $\mathrm{H}$ & -13.263333 & -5.590693 & 1.110282 \\
\hline $\mathrm{H}$ & -12.705959 & -7.131727 & 0.480806 \\
\hline $\mathrm{H}$ & -14.064686 & -7.067140 & 1.597799 \\
\hline $\mathrm{N}$ & -11.197245 & -8.533612 & 5.241880 \\
\hline $\mathrm{H}$ & -12.116436 & -8.918186 & 5.272036 \\
\hline$C$ & -10.412669 & -8.535205 & 6.452135 \\
\hline $\mathrm{H}$ & -10.331223 & -7.540879 & 6.861664 \\
\hline $\mathrm{H}$ & -10.939610 & -9.140480 & 7.180255 \\
\hline$C$ & -8.977467 & -9.059643 & 6.382018 \\
\hline 0 & -8.146753 & -8.509831 & 7.062118 \\
\hline $\mathrm{N}$ & -8.659576 & -10.125588 & 5.624873 \\
\hline $\mathrm{H}$ & -9.368442 & -10.596078 & 5.109017 \\
\hline$C$ & -7.270783 & -10.559509 & 5.524535 \\
\hline $\mathrm{H}$ & -7.254061 & -11.535705 & 5.0569 \\
\hline
\end{tabular}




\begin{tabular}{|c|c|c|c|}
\hline $\mathrm{H}$ & -6.833680 & -10.645053 & 6.506363 \\
\hline C & -6.387435 & -9.608346 & 4.692696 \\
\hline $\mathrm{O}$ & -5.221533 & -9.463486 & 4.952964 \\
\hline$N$ & -7.045410 & -9.018041 & 3.701147 \\
\hline C & -6.310791 & -7.982307 & 2.986439 \\
\hline C & -6.265892 & -6.696323 & 3.808100 \\
\hline 0 & -5.273688 & -6.006844 & 3.776808 \\
\hline C & -6.639029 & -7.804216 & 1.490455 \\
\hline C & -7.802289 & -6.937044 & 1.054349 \\
\hline C & -7.783366 & -5.559917 & 1.256384 \\
\hline C & -8.849303 & -7.481404 & 0.321962 \\
\hline C & -8.793900 & -4.754281 & 0.759851 \\
\hline C & -9.849025 & -6.675063 & -0.197929 \\
\hline C & -9.829848 & -5.309104 & 0.027269 \\
\hline $\mathrm{H}$ & -8.039932 & -9.013245 & 3.684374 \\
\hline $\mathrm{H}$ & -5.287260 & -8.313220 & 2.998176 \\
\hline $\mathrm{H}$ & -5.738656 & -7.393325 & 1.047002 \\
\hline $\mathrm{H}$ & -6.744883 & -8.800772 & 1.078490 \\
\hline $\mathrm{H}$ & -6.960450 & -5.105017 & 1.776080 \\
\hline $\mathrm{H}$ & -8.866993 & -8.539436 & 0.124331 \\
\hline $\mathrm{H}$ & -8.754232 & -3.691639 & 0.921090 \\
\hline $\mathrm{H}$ & -10.626298 & -7.108815 & -0.799445 \\
\hline $\mathrm{H}$ & -10.600457 & -4.682964 & -0.384771 \\
\hline $\mathrm{N}$ & -7.294316 & -6.408986 & 4.609639 \\
\hline$C$ & -7.227561 & -5.302045 & 5.534434 \\
\hline$C$ & -6.096903 & -5.516946 & 6.539673 \\
\hline 0 & -5.444831 & -4.565812 & 6.913838 \\
\hline$C$ & -8.600103 & -5.148622 & 6.222600 \\
\hline$C$ & -8.841720 & -3.835506 & 6.935796 \\
\hline$C$ & -9.097852 & -2.677055 & 6.207847 \\
\hline
\end{tabular}




\begin{tabular}{|c|c|c|c|}
\hline C & -8.880551 & -3.771106 & 8.322263 \\
\hline C & -9.376988 & -1.483019 & 6.849914 \\
\hline C & -9.169184 & -2.579369 & 8.971176 \\
\hline C & -9.417835 & -1.431224 & 8.237381 \\
\hline $\mathrm{H}$ & -8.162423 & -6.882997 & 4.485443 \\
\hline $\mathrm{H}$ & -6.971403 & -4.390630 & 5.012884 \\
\hline $\mathrm{H}$ & -9.352249 & -5.262344 & 5.448756 \\
\hline $\mathrm{H}$ & -8.730460 & -5.968286 & 6.915933 \\
\hline $\mathrm{H}$ & -9.085555 & -2.706831 & 5.131767 \\
\hline $\mathrm{H}$ & -8.689596 & -4.656063 & 8.903829 \\
\hline $\mathrm{H}$ & -9.561263 & -0.597156 & 6.269776 \\
\hline $\mathrm{H}$ & -9.190687 & -2.548983 & 10.045647 \\
\hline $\mathrm{H}$ & -9.622741 & -0.501325 & 8.737904 \\
\hline$N$ & -5.855191 & -6.753756 & 6.970338 \\
\hline $\mathrm{H}$ & -6.508096 & -7.477079 & 6.765111 \\
\hline C & -4.773232 & -7.044576 & 7.884499 \\
\hline $\mathrm{H}$ & -4.809152 & -6.412003 & 8.758724 \\
\hline $\mathrm{H}$ & -4.881918 & -8.073724 & 8.202392 \\
\hline C & -3.380280 & -6.867559 & 7.282620 \\
\hline O & -2.505144 & -6.368755 & 7.949534 \\
\hline$N$ & -3.183362 & -7.298853 & 6.031826 \\
\hline C & -1.904254 & -7.129798 & 5.359139 \\
\hline C & -1.649722 & -5.662443 & 4.986657 \\
\hline $\mathrm{O}$ & -0.548371 & -5.181647 & 5.149540 \\
\hline C & -1.826576 & -8.075697 & 4.140312 \\
\hline C & -0.660442 & -7.730824 & 3.212267 \\
\hline C & -1.730441 & -9.532267 & 4.608212 \\
\hline $\mathrm{H}$ & -3.919237 & -7.777663 & 5.558602 \\
\hline $\mathrm{H}$ & -1.112616 & -7.373855 & 6.052609 \\
\hline $\mathrm{H}$ & -2.747525 & -7.954226 & 3.574625 \\
\hline
\end{tabular}




\begin{tabular}{|c|c|c|c|}
\hline $\mathrm{H}$ & -0.600446 & -8.463360 & 2.414463 \\
\hline $\mathrm{H}$ & -0.765634 & -6.759153 & 2.750581 \\
\hline $\mathrm{H}$ & 0.284622 & -7.743232 & 3.746384 \\
\hline $\mathrm{H}$ & -1.747066 & -10.200284 & 3.753147 \\
\hline $\mathrm{H}$ & -0.796988 & -9.698143 & 5.140118 \\
\hline $\mathrm{H}$ & -2.547872 & -9.813172 & 5.259657 \\
\hline$N$ & -2.681891 & -4.974529 & 4.488449 \\
\hline C & -2.544729 & -3.598248 & 4.052412 \\
\hline C & -2.409400 & -2.667629 & 5.255735 \\
\hline $\mathrm{O}$ & -1.517012 & -1.850248 & 5.300323 \\
\hline C & -3.719613 & -3.148952 & 3.171265 \\
\hline C & -3.946461 & -3.930458 & 1.866772 \\
\hline C & -2.815028 & -3.839906 & 0.855659 \\
\hline $\mathrm{O}$ & -1.692692 & -4.192297 & 1.246563 \\
\hline O & -3.087204 & -3.435732 & -0.288369 \\
\hline $\mathrm{H}$ & -3.550456 & -5.439000 & 4.341003 \\
\hline $\mathrm{H}$ & -1.630408 & -3.497602 & 3.498921 \\
\hline $\mathrm{H}$ & -4.635599 & -3.195680 & 3.752390 \\
\hline $\mathrm{H}$ & -3.554492 & -2.100768 & 2.937811 \\
\hline $\mathrm{H}$ & -4.095971 & -4.976696 & 2.089065 \\
\hline $\mathrm{H}$ & -4.855834 & -3.559717 & 1.407102 \\
\hline $\mathrm{N}$ & -3.309499 & -2.786699 & 6.250263 \\
\hline$C$ & -3.093962 & -2.024874 & 7.456968 \\
\hline$C$ & -1.781427 & -2.422854 & 8.139373 \\
\hline 0 & -1.112920 & -1.584727 & 8.704382 \\
\hline$C$ & -4.230130 & -1.984010 & 8.489110 \\
\hline 0 & -4.436969 & -3.210485 & 9.125309 \\
\hline$C$ & -5.512456 & -1.384185 & 7.924365 \\
\hline $\mathrm{H}$ & -3.985337 & -3.517943 & 6.232720 \\
\hline 11 & -2.930707 & -0.999966 & 7.162568 \\
\hline
\end{tabular}




\begin{tabular}{|c|c|c|c|}
\hline $\mathrm{H}$ & -3.859592 & -1.324027 & 9.263496 \\
\hline $\mathrm{H}$ & -4.880343 & -3.802171 & 8.529640 \\
\hline $\mathrm{H}$ & -6.246415 & -1.303123 & 8.716555 \\
\hline $\mathrm{H}$ & -5.327951 & -0.391537 & 7.525320 \\
\hline $\mathrm{H}$ & -5.931295 & -1.996477 & 7.136884 \\
\hline$N$ & -1.380404 & -3.700488 & 8.043333 \\
\hline $\mathrm{H}$ & -2.031405 & -4.418664 & 7.815121 \\
\hline C & -0.187103 & -4.107119 & 8.740481 \\
\hline $\mathrm{H}$ & -0.237745 & -3.891376 & 9.798432 \\
\hline $\mathrm{H}$ & -0.075398 & -5.174668 & 8.604493 \\
\hline C & 1.064398 & -3.419527 & 8.218984 \\
\hline $\mathrm{O}$ & 1.940509 & -3.075711 & 8.979614 \\
\hline$N$ & 1.154868 & -3.258584 & 6.883072 \\
\hline C & 2.333323 & -2.661209 & 6.317654 \\
\hline C & 2.291863 & -1.139270 & 6.162009 \\
\hline $\mathrm{O}$ & 3.361073 & -0.575733 & 6.019687 \\
\hline C & 2.833850 & -3.351996 & 5.048951 \\
\hline$S$ & 1.917574 & -3.010657 & 3.521828 \\
\hline $\mathrm{H}$ & 0.424128 & -3.613200 & 6.298452 \\
\hline $\mathrm{H}$ & 3.116994 & -2.796536 & 7.047750 \\
\hline $\mathrm{H}$ & 3.839399 & -3.004126 & 4.860731 \\
\hline $\mathrm{H}$ & 2.869582 & -4.419143 & 5.218859 \\
\hline $\mathrm{H}$ & 0.871393 & -3.797789 & 3.723145 \\
\hline$N$ & 1.141585 & -0.471381 & 6.273705 \\
\hline C & 1.204073 & 0.968482 & 6.491382 \\
\hline C & 1.754778 & 1.271816 & 7.870572 \\
\hline $\mathrm{O}$ & 2.367382 & 2.289418 & 8.086251 \\
\hline C & -0.097125 & 1.738345 & 6.177633 \\
\hline C & -1.199708 & 1.569150 & 7.233116 \\
\hline$C$ & -0.554722 & 1.428947 & 4.747046 \\
\hline
\end{tabular}




\begin{tabular}{|c|c|c|c|}
\hline C & -2.468607 & 2.370110 & 6.932475 \\
\hline $\mathrm{H}$ & 0.273305 & -0.966275 & 6.289291 \\
\hline $\mathrm{H}$ & 1.951674 & 1.351075 & 5.815093 \\
\hline $\mathrm{H}$ & 0.205235 & 2.779403 & 6.212995 \\
\hline $\mathrm{H}$ & -1.448695 & 0.524663 & 7.354476 \\
\hline $\mathrm{H}$ & -0.812453 & 1.897582 & 8.193255 \\
\hline $\mathrm{H}$ & -1.310148 & 2.133330 & 4.421954 \\
\hline $\mathrm{H}$ & 0.278296 & 1.505066 & 4.053675 \\
\hline $\mathrm{H}$ & -0.965854 & 0.432503 & 4.657950 \\
\hline $\mathrm{H}$ & -3.141183 & 2.341901 & 7.784491 \\
\hline $\mathrm{H}$ & -2.243043 & 3.412937 & 6.724540 \\
\hline $\mathrm{H}$ & -3.006175 & 1.971272 & 6.079117 \\
\hline$N$ & 1.549774 & 0.323287 & 8.791241 \\
\hline $\mathrm{H}$ & 0.889446 & -0.401834 & 8.613645 \\
\hline C & 1.977963 & 0.512992 & 10.159942 \\
\hline $\mathrm{H}$ & 1.469328 & -0.221878 & 10.771109 \\
\hline $\mathrm{H}$ & 1.737815 & 1.502921 & 10.517661 \\
\hline C & 3.481890 & 0.337003 & 10.305708 \\
\hline $\mathrm{O}$ & 4.163989 & 1.127275 & 10.898753 \\
\hline$N$ & 3.999613 & -0.753731 & 9.695240 \\
\hline $\mathrm{H}$ & 3.390273 & -1.464888 & 9.349179 \\
\hline C & 5.424647 & -0.938945 & 9.667146 \\
\hline $\mathrm{H}$ & 5.637679 & -1.909414 & 9.233983 \\
\hline $\mathrm{H}$ & 5.855301 & -0.905126 & 10.657241 \\
\hline C & 6.156885 & 0.119116 & 8.844830 \\
\hline $\mathrm{O}$ & 7.291893 & 0.411921 & 9.129599 \\
\hline$N$ & 5.502087 & 0.621274 & 7.799020 \\
\hline C & 6.098611 & 1.759282 & 7.146322 \\
\hline C & 5.516989 & 3.077428 & 7.650625 \\
\hline $\mathrm{H}$ & 5.008623 & 3.037109 & 8.612516 \\
\hline
\end{tabular}




\begin{tabular}{|c|c|c|c|}
\hline $\mathrm{O}$ & 5.647525 & 4.096724 & 7.045855 \\
\hline C & 6.000490 & 1.675605 & 5.620851 \\
\hline C & 6.752868 & 0.508291 & 5.015840 \\
\hline C & 8.118857 & 0.324860 & 5.234851 \\
\hline C & 6.092155 & -0.399758 & 4.193756 \\
\hline C & 8.805238 & -0.719444 & 4.630036 \\
\hline C & 6.776271 & -1.441664 & 3.587522 \\
\hline C & 8.136741 & -1.601990 & 3.795788 \\
\hline $\mathrm{H}$ & 4.575437 & 0.334273 & 7.572148 \\
\hline $\mathrm{H}$ & 7.134546 & 1.773642 & 7.449604 \\
\hline $\mathrm{H}$ & 4.957923 & 1.610670 & 5.335968 \\
\hline $\mathrm{H}$ & 6.377511 & 2.610542 & 5.220655 \\
\hline $\mathrm{H}$ & 8.647345 & 0.980755 & 5.905308 \\
\hline $\mathrm{H}$ & 5.031812 & -0.304257 & 4.056810 \\
\hline $\mathrm{H}$ & 9.856335 & -0.846128 & 4.824330 \\
\hline $\mathrm{H}$ & 6.243756 & -2.132916 & 2.958885 \\
\hline $\mathrm{H}$ & 8.657125 & -2.413641 & 3.321104 \\
\hline$N$ & 12.613550 & 9.596234 & -1.248801 \\
\hline $\mathrm{H}$ & 11.871931 & 9.914842 & -1.844065 \\
\hline C & 12.304852 & 8.264740 & -0.719487 \\
\hline C & 11.274074 & 8.450488 & 0.386932 \\
\hline $\mathrm{O}$ & 10.266460 & 7.778079 & 0.416885 \\
\hline C & 13.562635 & 7.581254 & -0.164773 \\
\hline C & 14.645809 & 7.276762 & -1.205210 \\
\hline$S$ & 14.019107 & 6.202959 & -2.529293 \\
\hline C & 15.503560 & 6.052522 & -3.551813 \\
\hline $\mathrm{H}$ & 13.431761 & 9.572127 & -1.824612 \\
\hline $\mathrm{H}$ & 11.834595 & 7.616402 & -1.447338 \\
\hline $\mathrm{H}$ & 14.000549 & 8.205693 & 0.608096 \\
\hline & 13.255556 & 6.653940 & 0.305079 \\
\hline
\end{tabular}




\begin{tabular}{|c|c|c|c|}
\hline $\mathrm{H}$ & 15.046417 & 8.189438 & -1.631929 \\
\hline $\mathrm{H}$ & 15.470590 & 6.784241 & -0.702674 \\
\hline $\mathrm{H}$ & 15.253538 & 5.397039 & -4.372129 \\
\hline $\mathrm{H}$ & 15.803961 & 7.015967 & -3.943018 \\
\hline $\mathrm{H}$ & 16.320662 & 5.615815 & -2.992518 \\
\hline $\mathrm{N}$ & 11.503854 & 9.413387 & 1.296494 \\
\hline$C$ & 10.594376 & 9.608173 & 2.408605 \\
\hline$C$ & 9.277505 & 10.276976 & 2.011975 \\
\hline 0 & 8.282490 & 10.058380 & 2.656594 \\
\hline$C$ & 11.277548 & 10.367649 & 3.552607 \\
\hline$C$ & 12.448646 & 9.561571 & 4.084067 \\
\hline 0 & 13.583823 & 9.823178 & 3.744914 \\
\hline $\mathrm{N}$ & 12.144241 & 8.558465 & 4.917144 \\
\hline $\mathrm{H}$ & 12.327517 & 9.968356 & 1.205447 \\
\hline $\mathrm{H}$ & 10.294804 & 8.638030 & 2.770465 \\
\hline $\mathrm{H}$ & 11.659335 & 11.324850 & 3.220226 \\
\hline $\mathrm{H}$ & 10.547717 & 10.536155 & 4.334353 \\
\hline $\mathrm{H}$ & 12.887664 & 7.961865 & 5.209970 \\
\hline $\mathrm{H}$ & 11.222682 & 8.171961 & 4.951082 \\
\hline $\mathrm{N}$ & 9.263954 & 11.068441 & 0.922631 \\
\hline $\mathrm{H}$ & 10.110243 & 11.226365 & 0.425284 \\
\hline$C$ & 8.003503 & 11.517472 & 0.368854 \\
\hline $\mathrm{H}$ & 8.201018 & 12.260056 & -0.393596 \\
\hline $\mathrm{H}$ & 7.389140 & 11.966802 & 1.131876 \\
\hline$C$ & 7.186384 & 10.384437 & -0.254318 \\
\hline 0 & 5.986775 & 10.472231 & -0.295113 \\
\hline $\mathrm{N}$ & 7.862978 & 9.332464 & -0.761465 \\
\hline$C$ & 7.170138 & 8.144936 & -1.231517 \\
\hline$C$ & 6.633636 & 7.301781 & -0.064246 \\
\hline 0 & 5.533035 & 6.810543 & -0.144947 \\
\hline
\end{tabular}




\begin{tabular}{|c|c|c|c|}
\hline C & 8.047996 & 7.341936 & -2.220961 \\
\hline C & 8.344874 & 8.194047 & -3.469685 \\
\hline C & 7.376586 & 6.017832 & -2.604920 \\
\hline C & 9.428444 & 7.619508 & -4.385483 \\
\hline $\mathrm{H}$ & 8.823031 & 9.228200 & -0.530946 \\
\hline $\mathrm{H}$ & 6.279797 & 8.471393 & -1.746362 \\
\hline $\mathrm{H}$ & 8.986449 & 7.114217 & -1.723515 \\
\hline $\mathrm{H}$ & 7.423341 & 8.321927 & -4.033247 \\
\hline $\mathrm{H}$ & 8.649049 & 9.189073 & -3.162211 \\
\hline $\mathrm{H}$ & 7.994109 & 5.475714 & -3.309702 \\
\hline $\mathrm{H}$ & 7.222745 & 5.377564 & -1.744888 \\
\hline $\mathrm{H}$ & 6.407909 & 6.182798 & -3.065074 \\
\hline $\mathrm{H}$ & 9.624223 & 8.299583 & -5.208170 \\
\hline $\mathrm{H}$ & 10.363320 & 7.471946 & -3.851578 \\
\hline $\mathrm{H}$ & 9.146586 & 6.666005 & -4.816531 \\
\hline$N$ & 7.386090 & 7.177064 & 1.047533 \\
\hline C & 6.878058 & 6.458044 & 2.204615 \\
\hline C & 5.525417 & 7.035242 & 2.642676 \\
\hline $\mathrm{O}$ & 4.575698 & 6.325710 & 2.869639 \\
\hline C & 7.831823 & 6.546133 & 3.406336 \\
\hline C & 9.253242 & 5.997872 & 3.245998 \\
\hline $\mathrm{O}$ & 10.145552 & 6.633626 & 3.800850 \\
\hline $\mathrm{O}$ & 9.426538 & 4.934993 & 2.599048 \\
\hline $\mathrm{H}$ & 8.346713 & 7.444503 & 1.025500 \\
\hline $\mathrm{H}$ & 6.683575 & 5.429172 & 1.944750 \\
\hline $\mathrm{H}$ & 7.919361 & 7.570733 & 3.736175 \\
\hline $\mathrm{H}$ & 7.374017 & 5.992117 & 4.223285 \\
\hline$N$ & 5.483175 & 8.373301 & 2.790396 \\
\hline $\mathrm{H}$ & 6.312418 & 8.911141 & 2.653236 \\
\hline C & 4.290495 & 9.037052 & 3.256214 \\
\hline
\end{tabular}




\begin{tabular}{|c|c|c|c|}
\hline $\mathrm{H}$ & 4.528240 & 10.079997 & 3.424687 \\
\hline $\mathrm{H}$ & 3.946128 & 8.620644 & 4.189680 \\
\hline C & 3.130157 & 8.987765 & 2.271344 \\
\hline 0 & 1.988696 & 9.117162 & 2.649945 \\
\hline$N$ & 3.466368 & 8.825158 & 0.988734 \\
\hline $\mathrm{H}$ & 4.428136 & 8.815095 & 0.742262 \\
\hline C & 2.517188 & 8.905263 & -0.078309 \\
\hline $\mathrm{H}$ & 3.062191 & 9.089692 & -0.995140 \\
\hline $\mathrm{H}$ & 1.843547 & 9.738082 & 0.069355 \\
\hline C & 1.609135 & 7.687017 & -0.310189 \\
\hline $\mathrm{O}$ & 0.576528 & 7.817136 & -0.907598 \\
\hline$N$ & 2.067321 & 6.540452 & 0.227893 \\
\hline C & 1.247478 & 5.370795 & 0.435183 \\
\hline C & 0.429320 & 5.471011 & 1.727210 \\
\hline $\mathrm{O}$ & -0.764889 & 5.294461 & 1.701385 \\
\hline C & 2.105543 & 4.106074 & 0.428589 \\
\hline $\mathrm{H}$ & 2.967725 & 6.569127 & 0.657195 \\
\hline $\mathrm{H}$ & 0.519337 & 5.320351 & -0.356785 \\
\hline $\mathrm{H}$ & 1.488821 & 3.234769 & 0.619684 \\
\hline $\mathrm{H}$ & 2.577659 & 3.996865 & -0.540870 \\
\hline $\mathrm{H}$ & 2.882861 & 4.150965 & 1.184548 \\
\hline$N$ & 1.068144 & 5.733430 & 2.884289 \\
\hline C & 0.338240 & 5.567665 & 4.132151 \\
\hline C & -0.773547 & 6.602325 & 4.288484 \\
\hline $\mathrm{O}$ & -1.809091 & 6.306242 & 4.844108 \\
\hline C & 1.259390 & 5.493095 & 5.375049 \\
\hline C & 1.849954 & 6.839293 & 5.821976 \\
\hline C & 2.348874 & 4.431293 & 5.161096 \\
\hline$C$ & 2.694150 & 6.741593 & 7.095152 \\
\hline $\mathrm{H}$ & 2.056666 & 5.861681 & 2.900722 \\
\hline
\end{tabular}




\begin{tabular}{|c|c|c|c|}
\hline $\mathrm{H}$ & -0.191263 & 4.627355 & 4.077572 \\
\hline $\mathrm{H}$ & 0.606028 & 5.150646 & 6.171767 \\
\hline $\mathrm{H}$ & 2.450286 & 7.255113 & 5.019340 \\
\hline $\mathrm{H}$ & 1.045016 & 7.544810 & 6.000464 \\
\hline $\mathrm{H}$ & 2.725099 & 4.075955 & 6.109256 \\
\hline $\mathrm{H}$ & 1.956046 & 3.575736 & 4.621135 \\
\hline $\mathrm{H}$ & 3.187566 & 4.825930 & 4.597762 \\
\hline $\mathrm{H}$ & 2.997228 & 7.732711 & 7.419839 \\
\hline $\mathrm{H}$ & 2.126660 & 6.291255 & 7.904203 \\
\hline $\mathrm{H}$ & 3.590948 & 6.151261 & 6.954100 \\
\hline$N$ & -0.550448 & 7.835331 & 3.810099 \\
\hline $\mathrm{H}$ & 0.334165 & 8.069694 & 3.410291 \\
\hline$C$ & -1.514985 & 8.881093 & 4.023532 \\
\hline $\mathrm{H}$ & -1.098684 & 9.807618 & 3.648530 \\
\hline $\mathrm{H}$ & -1.729449 & 9.011353 & 5.074384 \\
\hline C & -2.866971 & 8.618756 & 3.365893 \\
\hline $\mathrm{O}$ & -3.871298 & 8.845797 & 4.000033 \\
\hline$N$ & -2.902686 & 8.108974 & 2.120752 \\
\hline C & -4.238908 & 8.033144 & 1.526312 \\
\hline$c$ & -4.983016 & 6.752713 & 1.867113 \\
\hline $\mathrm{O}$ & -6.195326 & 6.753512 & 1.841256 \\
\hline C & -4.018977 & 8.294981 & 0.029374 \\
\hline C & -2.743956 & 9.145315 & 0.042208 \\
\hline C & -1.900516 & 8.455929 & 1.102605 \\
\hline $\mathrm{H}$ & -4.854125 & 8.818720 & 1.936371 \\
\hline $\mathrm{H}$ & -3.864510 & 7.368756 & -0.508147 \\
\hline $\mathrm{H}$ & -4.874696 & 8.795099 & -0.406124 \\
\hline $\mathrm{H}$ & -2.231365 & 9.201413 & -0.900536 \\
\hline $\mathrm{H}$ & -2.982282 & 10.157674 & 0.359873 \\
\hline & -1.435726 & 7.562413 & 0.726469 \\
\hline
\end{tabular}




\begin{tabular}{|c|c|c|c|}
\hline $\mathrm{H}$ & -1.127588 & 9.090184 & 1.504427 \\
\hline$N$ & -4.262083 & 5.675638 & 2.205270 \\
\hline C & -4.913756 & 4.487561 & 2.728202 \\
\hline C & -5.629669 & 4.783442 & 4.049083 \\
\hline 0 & -6.704184 & 4.276882 & 4.284658 \\
\hline C & -3.920509 & 3.321935 & 2.930975 \\
\hline C & -3.593074 & 2.501948 & 1.699298 \\
\hline C & -4.103263 & 1.217462 & 1.542430 \\
\hline C & -2.725310 & 2.957968 & 0.709553 \\
\hline C & -3.773125 & 0.420881 & 0.456677 \\
\hline C & -2.385976 & 2.179111 & -0.376871 \\
\hline C & -2.891591 & 0.873949 & -0.548713 \\
\hline 0 & -2.575077 & 0.154040 & -1.578985 \\
\hline $\mathrm{H}$ & -3.268415 & 5.756907 & 2.260692 \\
\hline $\mathrm{H}$ & -5.692121 & 4.185194 & 2.045196 \\
\hline $\mathrm{H}$ & -3.015997 & 3.722001 & 3.380431 \\
\hline $\mathrm{H}$ & -4.359600 & 2.660499 & 3.669923 \\
\hline $\mathrm{H}$ & -4.769433 & 0.819062 & 2.291192 \\
\hline $\mathrm{H}$ & -2.286245 & 3.931681 & 0.795370 \\
\hline $\mathrm{H}$ & -4.149608 & -0.585843 & 0.393077 \\
\hline $\mathrm{H}$ & -1.708846 & 2.562527 & -1.121988 \\
\hline $\mathrm{N}$ & -5.003831 & 5.588445 & 4.927061 \\
\hline $\mathrm{H}$ & -4.072496 & 5.898401 & 4.741623 \\
\hline C & -5.608129 & 5.941694 & 6.191342 \\
\hline $\mathrm{H}$ & -4.823434 & 6.281171 & 6.856408 \\
\hline $\mathrm{H}$ & -6.091579 & 5.085964 & 6.633940 \\
\hline C & -6.660989 & 7.045619 & 6.082282 \\
\hline 0 & -7.715594 & 6.968719 & 6.671221 \\
\hline $\mathrm{N}$ & -6.342145 & 8.091259 & 5.313948 \\
\hline $\mathrm{H}$ & -5.447584 & 8.140444 & 4.874543 \\
\hline
\end{tabular}




\begin{tabular}{|c|c|c|c|}
\hline$C$ & -7.257648 & 9.220183 & 5.085240 \\
\hline $\mathrm{H}$ & -6.772996 & 9.882451 & 4.379837 \\
\hline $\mathrm{H}$ & -7.440908 & 9.763387 & 6.000489 \\
\hline$C$ & -8.621434 & 8.828988 & 4.542963 \\
\hline $\mathrm{O}$ & -9.606154 & 9.421356 & 4.911824 \\
\hline $\mathrm{N}$ & -8.673304 & 7.827129 & 3.645035 \\
\hline$C$ & -9.943657 & 7.435616 & 3.076809 \\
\hline$C$ & -10.836112 & 6.654154 & 4.038280 \\
\hline $\mathrm{O}$ & -11.951366 & 6.350768 & 3.656646 \\
\hline$C$ & -9.775355 & 6.671601 & 1.743354 \\
\hline $\mathrm{O}$ & -10.966221 & 6.755487 & 0.999702 \\
\hline$C$ & -9.335359 & 5.222831 & 1.911865 \\
\hline $\mathrm{H}$ & -7.828428 & 7.411577 & 3.308851 \\
\hline $\mathrm{H}$ & -10.508949 & 8.330803 & 2.856257 \\
\hline $\mathrm{H}$ & -9.027794 & 7.206342 & 1.172417 \\
\hline $\mathrm{H}$ & -11.678807 & 6.479207 & 1.568244 \\
\hline $\mathrm{H}$ & -9.141346 & 4.784207 & 0.940478 \\
\hline $\mathrm{H}$ & -8.432174 & 5.142045 & 2.500323 \\
\hline $\mathrm{H}$ & -10.115390 & 4.638921 & 2.389369 \\
\hline $\mathrm{N}$ & -10.364858 & 6.344445 & 5.246952 \\
\hline$C$ & -11.209900 & 5.754972 & 6.261796 \\
\hline$C$ & -12.250233 & 6.739905 & 6.800707 \\
\hline 0 & -13.299761 & 6.324066 & 7.234959 \\
\hline$C$ & -10.393766 & 5.201063 & 7.438215 \\
\hline$C$ & -9.459862 & 4.028309 & 7.101350 \\
\hline$C$ & -8.608341 & 3.695033 & 8.330318 \\
\hline$C$ & -10.218984 & 2.785840 & 6.625799 \\
\hline $\mathrm{H}$ & -9.431732 & 6.593809 & 5.498784 \\
\hline $\mathrm{H}$ & -11.788480 & 4.964027 & 5.813640 \\
\hline 11 & -9.808421 & 6.007047 & 7.86512 \\
\hline
\end{tabular}




\begin{tabular}{|c|c|c|c|}
\hline $\mathrm{H}$ & -11.106374 & 4.885791 & 8.194963 \\
\hline $\mathrm{H}$ & -8.787056 & 4.333148 & 6.307967 \\
\hline $\mathrm{H}$ & -7.910733 & 2.890830 & 8.114203 \\
\hline $\mathrm{H}$ & -8.035275 & 4.558331 & 8.650941 \\
\hline $\mathrm{H}$ & -9.230083 & 3.379019 & 9.165326 \\
\hline $\mathrm{H}$ & -9.522406 & 1.974178 & 6.436950 \\
\hline $\mathrm{H}$ & -10.930118 & 2.450629 & 7.378911 \\
\hline $\mathrm{H}$ & -10.765335 & 2.958213 & 5.705507 \\
\hline$N$ & -11.913914 & 8.040303 & 6.829253 \\
\hline$C$ & -12.864986 & 9.029249 & 7.245767 \\
\hline$C$ & -13.878678 & 9.396399 & 6.182266 \\
\hline $\mathrm{H}$ & -14.628944 & 10.123921 & 6.511973 \\
\hline $\mathrm{O}$ & -13.888666 & 8.967770 & 5.077159 \\
\hline $\mathrm{H}$ & -11.120801 & 8.352755 & 6.310483 \\
\hline $\mathrm{H}$ & -13.401643 & 8.681093 & 8.119589 \\
\hline $\mathrm{H}$ & -12.337839 & 9.934594 & 7.531090 \\
\hline$N$ & -11.495258 & 10.629858 & -3.034777 \\
\hline $\mathrm{H}$ & -11.248077 & 10.848333 & -2.090127 \\
\hline C & -11.816457 & 9.206991 & -3.172691 \\
\hline C & -11.414026 & 8.858194 & -4.598774 \\
\hline $\mathrm{O}$ & -10.375345 & 8.293058 & -4.845137 \\
\hline C & -13.245719 & 8.788261 & -2.767124 \\
\hline C & -13.495134 & 9.129862 & -1.286499 \\
\hline C & -13.470822 & 7.296728 & -3.047345 \\
\hline C & -14.921044 & 8.858600 & -0.803354 \\
\hline $\mathrm{H}$ & -12.278577 & 11.208827 & -3.272327 \\
\hline $\mathrm{H}$ & -11.118046 & 8.661113 & -2.553749 \\
\hline $\mathrm{H}$ & -13.958980 & 9.364155 & -3.361464 \\
\hline $\mathrm{H}$ & -12.791593 & 8.573797 & -0.671323 \\
\hline $\mathrm{H}$ & -13.282903 & 10.182143 & -1.125066 \\
\hline
\end{tabular}




\begin{tabular}{|c|c|c|c|}
\hline $\mathrm{H}$ & -14.499942 & 7.015742 & -2.863719 \\
\hline $\mathrm{H}$ & -13.248956 & 7.033394 & -4.077557 \\
\hline $\mathrm{H}$ & -12.840088 & 6.689705 & -2.405270 \\
\hline $\mathrm{H}$ & -15.044255 & 9.216650 & 0.212390 \\
\hline $\mathrm{H}$ & -15.653383 & 9.367357 & -1.424742 \\
\hline $\mathrm{H}$ & -15.159411 & 7.801297 & -0.802281 \\
\hline$N$ & -12.214010 & 9.288635 & -5.604063 \\
\hline $\mathrm{H}$ & -13.131104 & 9.601756 & -5.380554 \\
\hline C & -11.972873 & 8.843878 & -6.959262 \\
\hline $\mathrm{H}$ & -12.035595 & 7.767552 & -7.044834 \\
\hline $\mathrm{H}$ & -12.729370 & 9.282577 & -7.598478 \\
\hline C & -10.601634 & 9.233249 & -7.517685 \\
\hline 0 & -9.997336 & 8.495992 & -8.242649 \\
\hline$N$ & -10.162498 & 10.476538 & $3-7.182377$ \\
\hline $\mathrm{H}$ & -10.709239 & 11.005111 & -6.541746 \\
\hline$C$ & -8.894338 & 11.023969 & -7.637102 \\
\hline $\mathrm{H}$ & -8.790001 & 10.891226 & -8.702742 \\
\hline $\mathrm{H}$ & -8.880302 & 12.081585 & -7.408178 \\
\hline$C$ & -7.672571 & 10.358998 & -6.988330 \\
\hline O & -6.759069 & 9.923372 & -7.632194 \\
\hline$N$ & -7.657768 & 10.354083 & -5.620722 \\
\hline $\mathrm{H}$ & -8.475389 & 10.646641 & -5.135096 \\
\hline C & -6.495249 & 9.961279 & -4.832307 \\
\hline $\mathrm{H}$ & -5.598013 & 10.374782 & -5.264527 \\
\hline $\mathrm{H}$ & -6.616236 & 10.361980 & -3.833380 \\
\hline C & -6.268288 & 8.463922 & -4.714299 \\
\hline O & -5.135168 & 8.046485 & -4.751247 \\
\hline $\mathrm{N}$ & -7.314744 & 7.639629 & -4.521267 \\
\hline C & -7.020319 & 6.271198 & -4.124299 \\
\hline C & -6.465820 & 5.440474 & -5.27517 \\
\hline
\end{tabular}




\begin{tabular}{|c|c|c|c|}
\hline $\mathrm{O}$ & -5.668775 & 4.563531 & -5.043674 \\
\hline C & -8.156724 & 5.541156 & -3.368995 \\
\hline C & -9.345704 & 5.122690 & -4.249903 \\
\hline C & -8.567333 & 6.358545 & -2.138727 \\
\hline$C$ & -10.386214 & 4.279190 & -3.509429 \\
\hline $\mathrm{H}$ & -8.251834 & 7.988603 & -4.514215 \\
\hline $\mathrm{H}$ & -6.188423 & 6.314123 & -3.437886 \\
\hline $\mathrm{H}$ & -7.685830 & 4.629992 & -3.010188 \\
\hline $\mathrm{H}$ & -9.825096 & 5.993879 & -4.678492 \\
\hline $\mathrm{H}$ & -8.968657 & 4.536094 & -5.084857 \\
\hline $\mathrm{H}$ & -9.222673 & 5.791693 & -1.491862 \\
\hline $\mathrm{H}$ & -7.697748 & 6.634611 & -1.551106 \\
\hline $\mathrm{H}$ & -9.089360 & 7.266113 & -2.418740 \\
\hline $\mathrm{H}$ & -11.138813 & 3.914238 & -4.202010 \\
\hline $\mathrm{H}$ & -9.930522 & 3.416713 & -3.033396 \\
\hline $\mathrm{H}$ & -10.898388 & 4.848340 & -2.743523 \\
\hline $\mathrm{N}$ & -6.882017 & 5.679794 & -6.537956 \\
\hline$C$ & -6.242877 & 4.969527 & -7.628380 \\
\hline$C$ & -4.821659 & 5.460691 & -7.917387 \\
\hline 0 & -3.990473 & 4.676202 & -8.307804 \\
\hline$C$ & -7.108198 & 4.971753 & -8.897586 \\
\hline$C$ & -8.277947 & 3.978321 & -8.838637 \\
\hline$C$ & -7.800139 & 2.523672 & -8.902302 \\
\hline $\mathrm{N}$ & -8.881613 & 1.534874 & -8.891460 \\
\hline$C$ & -9.434749 & 0.982245 & -7.816715 \\
\hline $\mathrm{N}$ & -9.166716 & 1.438150 & -6.605629 \\
\hline $\mathrm{N}$ & -10.274741 & -0.031477 & $7 \quad-7.966914$ \\
\hline $\mathrm{H}$ & -7.493953 & 6.446051 & -6.719521 \\
\hline $\mathrm{H}$ & -6.080804 & 3.958127 & -7.296394 \\
\hline $\mathrm{H}$ & -7.494577 & 5.969805 & -9.079059 \\
\hline
\end{tabular}




\begin{tabular}{|c|c|c|c|}
\hline $\mathrm{H}$ & -6.473446 & 4.727161 & -9.743172 \\
\hline $\mathrm{H}$ & -8.868341 & 4.149107 & -7.943185 \\
\hline $\mathrm{H}$ & -8.939450 & 4.160827 & -9.678931 \\
\hline $\mathrm{H}$ & -7.254451 & 2.358428 & -9.821294 \\
\hline $\mathrm{H}$ & -7.115529 & 2.281763 & -8.100881 \\
\hline $\mathrm{H}$ & -9.153769 & 1.158705 & -9.772140 \\
\hline $\mathrm{H}$ & -8.640528 & 2.272789 & -6.487212 \\
\hline $\mathrm{H}$ & -9.639822 & 1.093020 & -5.784688 \\
\hline $\mathrm{H}$ & -10.333485 & -0.492681 & -8.847730 \\
\hline $\mathrm{H}$ & -10.589868 & -0.568969 & -7.173564 \\
\hline$N$ & -4.571419 & 6.757537 & -7.721986 \\
\hline $\mathrm{H}$ & -5.302822 & 7.370440 & -7.441701 \\
\hline C & -3.257792 & 7.352338 & -8.011302 \\
\hline $\mathrm{H}$ & -2.929120 & 7.090538 & -9.004951 \\
\hline $\mathrm{H}$ & -3.360599 & 8.426642 & -7.939669 \\
\hline C & -2.202314 & 6.874116 & -7.024551 \\
\hline $\mathrm{O}$ & -1.073683 & 6.639153 & -7.377141 \\
\hline$N$ & -2.634097 & 6.758718 & -5.763275 \\
\hline C & -1.761616 & 6.448145 & -4.656398 \\
\hline C & -1.491280 & 4.960888 & -4.524187 \\
\hline $\mathrm{O}$ & -0.363855 & 4.577069 & -4.319321 \\
\hline C & -2.350380 & 7.048033 & -3.373471 \\
\hline C & -1.763409 & 6.354104 & -2.145479 \\
\hline C & -2.113584 & 8.563997 & -3.410440 \\
\hline $\mathrm{H}$ & -3.557786 & 7.064290 & -5.547223 \\
\hline $\mathrm{H}$ & -0.788704 & 6.881447 & -4.838418 \\
\hline $\mathrm{H}$ & -3.418831 & 6.866648 & -3.387816 \\
\hline $\mathrm{H}$ & -1.980538 & 6.901562 & -1.247451 \\
\hline $\mathrm{H}$ & -2.173217 & 5.356047 & -2.033377 \\
\hline & -0.690739 & 6.283819 & -2.215385 \\
\hline
\end{tabular}




\begin{tabular}{|c|c|c|c|}
\hline $\mathrm{H}$ & -2.838959 & 9.089537 & -2.805586 \\
\hline $\mathrm{H}$ & -1.118867 & 8.805764 & -3.051243 \\
\hline $\mathrm{H}$ & -2.215567 & 8.949902 & -4.418163 \\
\hline $\mathrm{N}$ & -2.517791 & 4.099989 & -4.645961 \\
\hline$C$ & -2.183943 & 2.693865 & -4.750294 \\
\hline$C$ & -1.220401 & 2.507869 & -5.924342 \\
\hline 0 & -0.293656 & 1.732576 & -5.842545 \\
\hline$C$ & -3.407935 & 1.776689 & -4.871585 \\
\hline$C$ & -4.365142 & 1.887237 & -3.676898 \\
\hline$C$ & -5.406639 & 0.763185 & -3.611684 \\
\hline $\mathrm{N}$ & -4.878991 & -0.427510 & -2.949865 \\
\hline$C$ & -5.583335 & -1.519506 & -2.748470 \\
\hline $\mathrm{N}$ & -6.812937 & -1.655781 & -3.294816 \\
\hline $\mathrm{N}$ & -5.154037 & -2.493880 & -1.972978 \\
\hline $\mathrm{H}$ & -3.439509 & 4.421220 & -4.850424 \\
\hline $\mathrm{H}$ & -1.629168 & 2.394292 & -3.874460 \\
\hline $\mathrm{H}$ & -3.944310 & 1.990555 & -5.794151 \\
\hline $\mathrm{H}$ & -3.028749 & 0.764647 & -4.951019 \\
\hline $\mathrm{H}$ & -3.802460 & 1.900235 & -2.756953 \\
\hline $\mathrm{H}$ & -4.904655 & 2.820111 & -3.734796 \\
\hline $\mathrm{H}$ & -6.276650 & 1.112905 & -3.065381 \\
\hline $\mathrm{H}$ & -5.727669 & 0.511016 & -4.619263 \\
\hline $\mathrm{H}$ & -3.984166 & -0.324710 & -2.456363 \\
\hline $\mathrm{H}$ & -6.974347 & -1.177487 & -4.151327 \\
\hline $\mathrm{H}$ & -7.208358 & -2.578475 & -3.266250 \\
\hline $\mathrm{H}$ & -4.300366 & -2.472857 & -1.440328 \\
\hline $\mathrm{H}$ & -5.664429 & -3.351690 & -1.949454 \\
\hline $\mathrm{N}$ & -1.459790 & 3.193298 & -7.035093 \\
\hline $\mathrm{H}$ & -2.285219 & 3.739318 & -7.148812 \\
\hline$C$ & -0.596550 & 2.997474 & -8.183376 \\
\hline
\end{tabular}




\begin{tabular}{|c|c|c|c|}
\hline $\mathrm{H}$ & -0.949630 & 3.640655 & -8.979121 \\
\hline $\mathrm{H}$ & -0.611029 & 1.972250 & -8.524591 \\
\hline$C$ & 0.855931 & 3.344672 & -7.871776 \\
\hline 0 & 1.781598 & 2.711140 & -8.313085 \\
\hline $\mathrm{N}$ & 1.005371 & 4.405090 & -7.063925 \\
\hline $\mathrm{H}$ & 0.212146 & 4.964338 & -6.846605 \\
\hline$C$ & 2.282350 & 4.880661 & -6.640316 \\
\hline $\mathrm{H}$ & 2.919001 & 5.137295 & -7.477590 \\
\hline $\mathrm{H}$ & 2.128458 & 5.777142 & -6.053742 \\
\hline$C$ & 3.092840 & 3.928367 & -5.775178 \\
\hline 0 & 4.297669 & 4.059457 & -5.688187 \\
\hline $\mathrm{N}$ & 2.428015 & 2.983364 & -5.101894 \\
\hline$C$ & 3.096572 & 2.136985 & -4.148066 \\
\hline$C$ & 4.270175 & 1.327688 & -4.711094 \\
\hline 0 & 5.184059 & 1.025872 & -3.970246 \\
\hline$C$ & 2.008341 & 1.259942 & -3.526779 \\
\hline$C$ & 2.372020 & 0.441550 & -2.293976 \\
\hline$C$ & 1.055758 & 0.031691 & -1.642286 \\
\hline $\mathrm{N}$ & 1.235378 & -1.004799 & -0.647430 \\
\hline$C$ & 0.298798 & -1.926790 & -0.373999 \\
\hline $\mathrm{N}$ & -0.855083 & -1.920612 & -0.991963 \\
\hline $\mathrm{N}$ & 0.588351 & -2.879746 & 0.489881 \\
\hline $\mathrm{H}$ & 1.438713 & 2.900979 & -5.211350 \\
\hline $\mathrm{H}$ & 3.557525 & 2.746655 & -3.380324 \\
\hline $\mathrm{H}$ & 1.210708 & 1.943596 & -3.257011 \\
\hline $\mathrm{H}$ & 1.592591 & 0.612085 & -4.289465 \\
\hline $\mathrm{H}$ & 2.946770 & -0.439481 & -2.558613 \\
\hline $\mathrm{H}$ & 2.977062 & 1.024247 & -1.603012 \\
\hline $\mathrm{H}$ & 0.561443 & 0.897278 & -1.214220 \\
\hline $\mathrm{H}$ & 0.398150 & -0.361469 & -2.402202 \\
\hline
\end{tabular}




\begin{tabular}{|c|c|c|c|}
\hline $\mathrm{H}$ & 1.939830 & -0.874312 & 0.043445 \\
\hline $\mathrm{H}$ & -1.298717 & -1.062577 & -1.309484 \\
\hline $\mathrm{H}$ & -1.527138 & -2.646256 & -0.776918 \\
\hline $\mathrm{H}$ & 1.506800 & -2.990418 & 0.850720 \\
\hline $\mathrm{H}$ & -0.161269 & -3.479286 & 0.821989 \\
\hline$N$ & 4.280002 & 0.941732 & -5.998662 \\
\hline $\mathrm{H}$ & 3.540833 & 1.225199 & -6.608051 \\
\hline C & 5.363424 & 0.116638 & -6.504622 \\
\hline $\mathrm{H}$ & 5.137151 & -0.137697 & -7.532686 \\
\hline $\mathrm{H}$ & 5.442866 & -0.800473 & -5.942810 \\
\hline C & 6.736121 & 0.755923 & -6.476823 \\
\hline $\mathrm{O}$ & 7.738867 & 0.089514 & -6.368823 \\
\hline$N$ & 6.766124 & 2.085339 & -6.608293 \\
\hline $\mathrm{H}$ & 5.911903 & 2.604351 & -6.566390 \\
\hline C & 8.004993 & 2.823920 & -6.549089 \\
\hline $\mathrm{H}$ & 8.710109 & 2.440732 & -7.273087 \\
\hline $\mathrm{H}$ & 7.799709 & 3.854876 & -6.804647 \\
\hline C & 8.746900 & 2.826001 & -5.210612 \\
\hline 0 & 9.887685 & 3.236397 & -5.157394 \\
\hline$N$ & 8.101358 & 2.335228 & -4.144928 \\
\hline C & 8.680903 & 2.169883 & -2.822859 \\
\hline C & 9.947179 & 1.302961 & -2.830367 \\
\hline 0 & 10.764503 & 1.385616 & -1.927871 \\
\hline$C$ & 7.576267 & 1.499495 & -1.975518 \\
\hline$C$ & 7.769338 & 1.380008 & -0.464046 \\
\hline $\mathrm{C}$ & 7.422858 & 2.651184 & 0.320627 \\
\hline$C$ & 7.309505 & 2.388446 & 1.818384 \\
\hline $\mathrm{N}$ & 8.648575 & 2.358116 & 2.478467 \\
\hline 0 & 11.029331 & 1.615187 & 0.898018 \\
\hline $\mathrm{H}$ & 10.881146 & 1.825071 & -0.024448 \\
\hline
\end{tabular}




\begin{tabular}{|c|c|c|c|}
\hline $\mathrm{H}$ & 11.506317 & 0.787662 & 0.849543 \\
\hline $\mathrm{H}$ & 7.155638 & 2.032457 & -4.259047 \\
\hline $\mathrm{H}$ & 8.977804 & 3.127170 & -2.415175 \\
\hline $\mathrm{H}$ & 6.658632 & 2.040632 & -2.163162 \\
\hline $\mathrm{H}$ & 7.411904 & 0.511411 & -2.389546 \\
\hline $\mathrm{H}$ & 7.090270 & 0.600060 & -0.133568 \\
\hline $\mathrm{H}$ & 8.764776 & 1.029803 & -0.225093 \\
\hline $\mathrm{H}$ & 8.134561 & 3.453008 & 0.144581 \\
\hline $\mathrm{H}$ & 6.457656 & 3.019727 & -0.011584 \\
\hline $\mathrm{H}$ & 6.747001 & 3.161349 & 2.318600 \\
\hline $\mathrm{H}$ & 6.829051 & 1.442376 & 2.014474 \\
\hline $\mathrm{H}$ & 9.360209 & 1.892163 & 1.924320 \\
\hline $\mathrm{H}$ & 8.969585 & 3.328263 & 2.631120 \\
\hline $\mathrm{H}$ & 8.593559 & 1.882790 & 3.367436 \\
\hline$N$ & 10.062468 & 0.448216 & -3.837538 \\
\hline C & 11.109355 & -0.537517 & -4.069387 \\
\hline C & 12.486379 & 0.023157 & -4.427133 \\
\hline $\mathrm{O}$ & 13.444198 & -0.705546 & -4.301401 \\
\hline C & 10.701431 & -1.443076 & -5.253560 \\
\hline$c$ & 9.563249 & -2.441793 & -5.000147 \\
\hline C & 9.132350 & -3.064984 & -6.331588 \\
\hline C & 9.990084 & -3.533487 & -4.018744 \\
\hline r & 9.324190 & 0.445767 & -4.510612 \\
\hline $\mathrm{H}$ & 11.264635 & -1.125579 & -3.176687 \\
\hline $\mathrm{H}$ & 10.435176 & -0.796633 & -6.084514 \\
\hline $\mathrm{H}$ & 11.584405 & -1.995209 & -5.552449 \\
\hline $\mathrm{H}$ & 8.706564 & -1.911333 & -4.596274 \\
\hline $\mathrm{H}$ & 8.331207 & -3.781573 & -6.178100 \\
\hline $\mathrm{H}$ & 8.775123 & -2.307430 & -7.017426 \\
\hline & 9.959751 & -3.592416 & -6.799865 \\
\hline
\end{tabular}




\begin{tabular}{|c|c|c|c|}
\hline $\mathrm{H}$ & 9.199967 & -4.263282 & -3.879665 \\
\hline $\mathrm{H}$ & 10.860108 & -4.059658 & -4.397726 \\
\hline $\mathrm{H}$ & 10.242959 & -3.137666 & -3.039915 \\
\hline$N$ & 12.574050 & 1.261829 & -4.919242 \\
\hline C & 13.814406 & 1.845262 & -5.393366 \\
\hline C & 14.958878 & 2.033284 & -4.379087 \\
\hline $\mathrm{O}$ & 16.013534 & 2.413871 & -4.812175 \\
\hline C & 13.496569 & 3.172498 & -6.085440 \\
\hline O & 12.918096 & 4.089114 & -5.200443 \\
\hline $\mathrm{H}$ & 11.756268 & 1.833402 & -4.916258 \\
\hline $\mathrm{H}$ & 14.248272 & 1.179028 & -6.129607 \\
\hline $\mathrm{H}$ & 14.419539 & 3.591691 & -6.454352 \\
\hline $\mathrm{H}$ & 12.845354 & 2.979136 & -6.931993 \\
\hline $\mathrm{H}$ & 11.970528 & 4.078373 & -5.262637 \\
\hline $\mathrm{N}$ & 14.792294 & 1.722326 & -3.074279 \\
\hline C & 15.927713 & 1.647124 & -2.176233 \\
\hline C & 16.804430 & 0.410056 & -2.402579 \\
\hline $\mathrm{O}$ & 17.934558 & 0.394323 & -1.988747 \\
\hline C & 15.493359 & 1.575964 & -0.707144 \\
\hline C & 14.634954 & 2.731677 & -0.197567 \\
\hline C & 14.278275 & 2.482164 & 1.268234 \\
\hline$N$ & 13.252919 & 3.376635 & 1.790435 \\
\hline C & 13.430800 & 4.528269 & 2.417003 \\
\hline$N$ & 14.648910 & 5.003840 & 2.652460 \\
\hline $\mathrm{O}$ & 15.028274 & 7.461559 & 4.203992 \\
\hline $\mathrm{H}$ & 14.743853 & 8.348419 & 3.967260 \\
\hline $\mathrm{H}$ & 15.746592 & 7.566788 & 4.814797 \\
\hline$N$ & 12.381272 & 5.217168 & 2.813488 \\
\hline $\mathrm{H}$ & 13.900680 & 1.420521 & -2.758456 \\
\hline 11 & 16.561246 & 2.504414 & -2.344561 \\
\hline
\end{tabular}




\begin{tabular}{|c|c|c|c|}
\hline $\mathrm{H}$ & 14.953644 & 0.647366 & -0.552984 \\
\hline $\mathrm{H}$ & 16.404775 & 1.507146 & -0.123410 \\
\hline $\mathrm{H}$ & 15.152401 & 3.679935 & -0.311134 \\
\hline $\mathrm{H}$ & 13.716902 & 2.813455 & -0.767682 \\
\hline $\mathrm{H}$ & 13.880352 & 1.483080 & 1.371699 \\
\hline $\mathrm{H}$ & 15.156521 & 2.522513 & 1.901889 \\
\hline $\mathrm{H}$ & 12.312653 & 3.080244 & 1.620849 \\
\hline $\mathrm{H}$ & 15.456406 & 4.510167 & 2.355170 \\
\hline $\mathrm{H}$ & 14.784908 & 5.859087 & 3.163319 \\
\hline $\mathrm{H}$ & 11.425389 & 4.937390 & 2.656475 \\
\hline $\mathrm{H}$ & 12.473703 & 6.061002 & 3.332453 \\
\hline$N$ & 16.200238 & -0.648980 & -2.967242 \\
\hline C & 16.870752 & -1.911587 & -3.167518 \\
\hline C & 17.670006 & -1.889781 & -4.467008 \\
\hline $\mathrm{H}$ & 18.062386 & -0.909889 & -4.748118 \\
\hline 0 & 17.878402 & -2.854052 & -5.128230 \\
\hline$C$ & 15.867932 & -3.070228 & -3.148719 \\
\hline$C$ & 15.323739 & -3.398988 & -1.791343 \\
\hline $\mathrm{N}$ & 14.402798 & -2.625854 & -1.112620 \\
\hline 0 & 12.505039 & -0.433470 & -0.461488 \\
\hline $\mathrm{H}$ & 13.142221 & -1.035718 & -0.849492 \\
\hline $\mathrm{H}$ & 12.022768 & -0.037399 & -1.179250 \\
\hline$C$ & 15.618082 & -4.489097 & -1.048180 \\
\hline$C$ & 14.170786 & -3.249195 & -0.003865 \\
\hline $\mathrm{N}$ & 14.880727 & -4.379499 & 0.104143 \\
\hline $\mathrm{H}$ & 15.285196 & -0.550771 & -3.351807 \\
\hline $\mathrm{H}$ & 17.595790 & -2.030750 & -2.370018 \\
\hline $\mathrm{H}$ & 15.050801 & -2.842595 & -3.824522 \\
\hline $\mathrm{H}$ & 16.368880 & -3.944319 & -3.542447 \\
\hline $\mathrm{H}$ & 16.273237 & -5.313268 & -1.232733 \\
\hline
\end{tabular}




\begin{tabular}{|c|c|c|c|}
\hline $\mathrm{H}$ & 13.492091 & -2.918983 & 0.756432 \\
\hline $\mathrm{H}$ & 14.779374 & -5.062039 & 0.823108 \\
\hline $\mathrm{O}$ & -15.022332 & -2.472904 & 4.971587 \\
\hline $\mathrm{H}$ & -15.215300 & -1.663969 & 4.504968 \\
\hline $\mathrm{H}$ & -14.530546 & -2.214795 & 5.744220 \\
\hline $\mathrm{O}$ & -12.909024 & -1.269273 & 6.930563 \\
\hline $\mathrm{H}$ & -12.222363 & -1.721546 & 7.408263 \\
\hline $\mathrm{H}$ & -13.143100 & -0.515308 & 7.458159 \\
\hline $\mathrm{O}$ & -12.057046 & -0.850324 & 4.159021 \\
\hline $\mathrm{H}$ & -12.178973 & -0.901868 & 5.104213 \\
\hline $\mathrm{H}$ & -12.869358 & -0.460195 & 3.833426 \\
\hline $\mathrm{O}$ & -13.394460 & -1.861170 & 0.708884 \\
\hline $\mathrm{H}$ & -12.619910 & -1.296729 & 0.765568 \\
\hline $\mathrm{H}$ & -13.316722 & -2.508005 & 1.414714 \\
\hline $\mathrm{O}$ & -7.286814 & 1.298494 & -0.241241 \\
\hline $\mathrm{H}$ & -7.499537 & 1.634533 & 0.633103 \\
\hline $\mathrm{H}$ & -6.357823 & 1.097608 & -0.204946 \\
\hline 0 & -7.887148 & 2.075832 & 2.453266 \\
\hline $\mathrm{H}$ & -8.798915 & 1.921491 & 2.688293 \\
\hline $\mathrm{H}$ & -7.560872 & 2.752423 & 3.040734 \\
\hline 0 & -9.343424 & -0.025500 & -1.609512 \\
\hline $\mathrm{H}$ & -8.581061 & 0.392263 & -1.188839 \\
\hline $\mathrm{H}$ & -9.094469 & -0.923898 & -1.806345 \\
\hline 0 & -12.844866 & -2.342602 & -2.020416 \\
\hline $\mathrm{H}$ & -13.121070 & -2.378383 & -1.101268 \\
\hline $\mathrm{H}$ & -13.457236 & -2.853965 & -2.536527 \\
\hline $\mathrm{O}$ & -14.033873 & 0.923434 & -0.583660 \\
\hline $\mathrm{H}$ & -14.378102 & 0.061001 & -0.376872 \\
\hline $\mathrm{H}$ & -13.123337 & 0.882180 & -0.275039 \\
\hline O & -14.251532 & 4.927624 & 4.735036 \\
\hline
\end{tabular}




\begin{tabular}{|c|c|c|c|}
\hline $\mathrm{H}$ & -13.682965 & 5.569178 & 4.315612 \\
\hline $\mathrm{H}$ & -14.290144 & 5.212331 & 5.643516 \\
\hline $\mathrm{O}$ & -15.231526 & 2.152017 & 1.699077 \\
\hline $\mathrm{H}$ & -15.858627 & 2.863324 & 1.685254 \\
\hline $\mathrm{H}$ & -14.946794 & 1.987269 & 0.801499 \\
\hline $\mathrm{O}$ & -13.156822 & 0.240589 & -3.245781 \\
\hline $\mathrm{H}$ & -13.606222 & 0.770980 & -2.590586 \\
\hline $\mathrm{H}$ & -12.938664 & -0.563428 & -2.772435 \\
\hline $\mathrm{O}$ & -13.770736 & -1.932656 & -4.984021 \\
\hline $\mathrm{H}$ & -13.939482 & -1.069812 & -4.612480 \\
\hline $\mathrm{H}$ & -14.497007 & -2.146080 & -5.555548 \\
\hline $\mathrm{O}$ & -10.158816 & -2.763951 & -2.954684 \\
\hline $\mathrm{H}$ & -10.300592 & -2.610371 & -3.882439 \\
\hline $\mathrm{H}$ & -11.021513 & -2.687230 & -2.548996 \\
\hline $\mathrm{O}$ & -11.486467 & 0.211533 & 0.369975 \\
\hline $\mathrm{H}$ & -10.759889 & 0.130538 & -0.249436 \\
\hline $\mathrm{H}$ & -11.146307 & 0.652745 & 1.155701 \\
\hline 0 & -10.714271 & 1.340138 & 2.829264 \\
\hline $\mathrm{H}$ & -10.880652 & 0.580670 & 3.384821 \\
\hline $\mathrm{H}$ & -11.395603 & 1.966258 & 3.090879 \\
\hline 0 & -13.057024 & 2.613282 & 3.631853 \\
\hline $\mathrm{H}$ & -13.358808 & 3.352220 & 4.162904 \\
\hline $\mathrm{H}$ & -13.713452 & 2.533207 & 2.947629 \\
\hline 0 & -10.472382 & 0.624522 & -4.111174 \\
\hline $\mathrm{H}$ & -11.377476 & 0.788837 & -3.840270 \\
\hline $\mathrm{H}$ & -9.985111 & 0.491478 & -3.290428 \\
\hline 0 & -14.689701 & -0.343782 & 3.041473 \\
\hline $\mathrm{H}$ & -15.011369 & 0.525584 & 2.812373 \\
\hline $\mathrm{H}$ & -14.564724 & -0.802618 & 2.217301 \\
\hline O & -13.086346 & -3.361956 & 3.14808 \\
\hline
\end{tabular}




$\begin{array}{llll}\mathrm{H} & -13.858824 & -3.221180 & 3.705238 \\ \mathrm{H} & -12.460173 & -2.723946 & 3.489388 \\ \mathrm{O} & -11.006560 & -1.654552 & -5.649329 \\ \mathrm{H} & -11.936215 & -1.878970 & -5.566085 \\ \mathrm{H} & -10.849057 & -0.947846 & -5.019580\end{array}$

PROTON CONFIGURATION, GIVEN BY NOMINAL CHARGES: R300(+),Y266(-),E183(0) POTENTIAL:-35mV

$\begin{array}{llll}\mathrm{N} & 15.580944 & -6.451469 & 5.391416 \\ \mathrm{H} & 14.781091 & -6.200103 & 5.939456 \\ \mathrm{C} & 15.509751 & -5.856564 & 4.054164 \\ \mathrm{C} & 14.783016 & -6.782962 & 3.060452 \\ \mathrm{O} & 14.052231 & -6.345849 & 2.186818 \\ \mathrm{C} & 16.919877 & -5.577004 & 3.520677 \\ \mathrm{H} & 16.390237 & -6.128756 & 5.884185 \\ \mathrm{H} & 14.945257 & -4.933528 & 4.050277 \\ \mathrm{H} & 16.889205 & -5.190740 & 2.508870 \\ \mathrm{H} & 17.403974 & -4.832107 & 4.142045 \\ \mathrm{H} & 17.527065 & -6.475436 & 3.529506 \\ \mathrm{~N} & 15.032378 & -8.085605 & 3.186405 \\ \mathrm{H} & 15.612411 & -8.361775 & 3.949450 \\ \mathrm{C} & 14.398660 & -9.092385 & 2.333233 \\ \mathrm{H} & 14.482160 & -8.823545 & 1.292899 \\ \mathrm{H} & 14.891694 & -10.042153 & 2.492416 \\ \mathrm{C} & 12.912930 & -9.221560 & 2.671507 \\ \mathrm{O} & 12.081797 & -9.427103 & 1.826194 \\ \mathrm{~N} & 12.582804 & -9.055884 & 3.966878 \\ \mathrm{H} & 13.302624 & -8.984073 & 4.648759\end{array}$




\begin{tabular}{|c|c|c|c|}
\hline C & 11.195108 & -9.170814 & 4.421375 \\
\hline $\mathrm{H}$ & 11.188291 & -9.158045 & 5.503134 \\
\hline $\mathrm{H}$ & 10.743837 & -10.088296 & 4.076981 \\
\hline C & 10.336943 & -8.010596 & 3.900736 \\
\hline $\mathrm{O}$ & 9.190873 & -8.186196 & 3.583978 \\
\hline$N$ & 10.946985 & -6.814479 & 3.855537 \\
\hline C & 10.318198 & -5.633870 & 3.318106 \\
\hline C & 10.125791 & -5.690670 & 1.799932 \\
\hline $\mathrm{O}$ & 9.095030 & -5.262426 & 1.338387 \\
\hline C & 11.092794 & -4.387934 & 3.776743 \\
\hline 0 & 12.478717 & -4.571005 & 3.769569 \\
\hline $\mathrm{H}$ & 11.905387 & -6.744053 & 4.106970 \\
\hline $\mathrm{H}$ & 9.314538 & -5.552986 & 3.702167 \\
\hline $\mathrm{H}$ & 10.801350 & -3.539534 & 3.167806 \\
\hline $\mathrm{H}$ & 10.827492 & -4.174283 & 4.801806 \\
\hline $\mathrm{H}$ & 12.805484 & -4.754836 & 2.897355 \\
\hline $\mathrm{N}$ & 11.098814 & -6.199773 & 1.024010 \\
\hline$C$ & 10.842605 & -6.395616 & -0.400299 \\
\hline$C$ & 9.692264 & -7.385027 & -0.601336 \\
\hline 0 & 8.943209 & -7.279826 & -1.544493 \\
\hline$C$ & 12.130941 & -6.783382 & -1.187602 \\
\hline$C$ & 12.333878 & -8.288693 & -1.402909 \\
\hline$C$ & 12.155886 & -6.059627 & -2.535221 \\
\hline $\mathrm{H}$ & 11.923312 & -6.585041 & 1.430728 \\
\hline $\mathrm{H}$ & 10.472291 & -5.461328 & -0.791112 \\
\hline $\mathrm{H}$ & 12.962862 & -6.411440 & -0.600078 \\
\hline $\mathrm{H}$ & 13.287694 & -8.451074 & -1.894160 \\
\hline $\mathrm{H}$ & 12.333414 & -8.854024 & -0.481751 \\
\hline $\mathrm{H}$ & 11.567004 & -8.697623 & -2.053617 \\
\hline $\mathrm{H}$ & 13.050400 & -6.323168 & -3.088487 \\
\hline
\end{tabular}




\begin{tabular}{|c|c|c|c|}
\hline $\mathrm{H}$ & 11.295217 & $7-6.332776$ & -3.135875 \\
\hline $\mathrm{H}$ & 12.154970 & -4.984307 & -2.402985 \\
\hline$N$ & 9.570430 & -8.340342 & 0.328312 \\
\hline $\mathrm{H}$ & 10.315146 & -8.474590 & 0.973538 \\
\hline C & 8.593019 & -9.421182 & 0.241228 \\
\hline $\mathrm{H}$ & 8.932376 & -10.216809 & 0.892183 \\
\hline $\mathrm{H}$ & 8.535552 & -9.806223 & -0.765248 \\
\hline C & 7.181275 & -9.042549 & 0.638193 \\
\hline $\mathrm{O}$ & 6.257642 & -9.497245 & 0.003736 \\
\hline$N$ & 7.002656 & -8.222457 & 1.680250 \\
\hline C & 5.669209 & -7.732900 & 1.947061 \\
\hline C & 5.252360 & -6.722864 & 0.883408 \\
\hline $\mathrm{O}$ & 4.076325 & -6.628877 & 0.603483 \\
\hline C & 5.414160 & -7.225036 & 3.380337 \\
\hline C & 5.552431 & -8.368373 & 4.390677 \\
\hline C & 6.258144 & -6.017592 & 3.793165 \\
\hline $\mathrm{H}$ & 7.779778 & -7.956827 & 2.245245 \\
\hline $\mathrm{H}$ & 4.985483 & -8.551816 & 1.785555 \\
\hline $\mathrm{H}$ & 4.374079 & -6.913683 & 3.371905 \\
\hline $\mathrm{H}$ & 5.261938 & -8.029263 & 5.379481 \\
\hline $\mathrm{H}$ & 4.912756 & -9.204679 & 4.128075 \\
\hline $\mathrm{H}$ & 6.573742 & -8.727837 & 4.448151 \\
\hline $\mathrm{H}$ & 5.917098 & -5.643641 & 4.752620 \\
\hline $\mathrm{H}$ & 7.299669 & -6.290423 & 3.902014 \\
\hline $\mathrm{H}$ & 6.188808 & -5.203807 & 3.079677 \\
\hline$N$ & 6.172629 & -5.996260 & 0.241633 \\
\hline C & 5.775138 & -5.175126 & -0.890224 \\
\hline C & 5.339353 & -6.056734 & -2.068895 \\
\hline $\mathrm{O}$ & 4.315679 & -5.803894 & -2.659636 \\
\hline$C$ & 6.869138 & -4.157520 & -1.270669 \\
\hline
\end{tabular}




\begin{tabular}{|c|c|c|c|}
\hline C & 7.070399 & -3.142261 & -0.131528 \\
\hline C & 6.498412 & -3.444914 & -2.575899 \\
\hline C & 8.367244 & -2.340569 & -0.244584 \\
\hline $\mathrm{H}$ & 7.133553 & -6.050773 & 0.502348 \\
\hline $\mathrm{H}$ & 4.878541 & -4.637464 & -0.620622 \\
\hline $\mathrm{H}$ & 7.797785 & -4.700839 & -1.419964 \\
\hline $\mathrm{H}$ & 6.217942 & -2.464864 & -0.115509 \\
\hline $\mathrm{H}$ & 7.077954 & -3.656580 & 0.820178 \\
\hline $\mathrm{H}$ & 7.205179 & -2.656424 & -2.795501 \\
\hline $\mathrm{H}$ & 6.496710 & -4.119325 & -3.422578 \\
\hline $\mathrm{H}$ & 5.511513 & -2.999455 & -2.507823 \\
\hline $\mathrm{H}$ & 8.470448 & -1.667319 & 0.599745 \\
\hline $\mathrm{H}$ & 9.226289 & -3.001250 & -0.243796 \\
\hline $\mathrm{H}$ & 8.406234 & -1.741067 & -1.147137 \\
\hline$N$ & 6.110770 & -7.099307 & -2.407461 \\
\hline $\mathrm{H}$ & 7.004114 & -7.224662 & -1.979936 \\
\hline C & 5.766130 & -7.937018 & -3.536188 \\
\hline $\mathrm{H}$ & 6.596660 & -8.609801 & -3.710364 \\
\hline $\mathrm{H}$ & 5.607740 & -7.348885 & -4.427809 \\
\hline C & 4.498577 & -8.767159 & -3.332400 \\
\hline $\mathrm{O}$ & 3.657706 & -8.824310 & -4.198409 \\
\hline$N$ & 4.392510 & -9.424584 & -2.170883 \\
\hline $\mathrm{H}$ & 5.130817 & -9.379417 & -1.501210 \\
\hline C & 3.209017 & -10.183686 & -1.841694 \\
\hline $\mathrm{H}$ & 2.968869 & -10.894126 & -2.618465 \\
\hline $\mathrm{H}$ & 3.405377 & -10.729151 & -0.927119 \\
\hline C & 1.974189 & -9.306856 & -1.644805 \\
\hline $\mathrm{O}$ & 0.886706 & -9.699804 & -2.000519 \\
\hline$N$ & 2.187869 & -8.107001 & -1.107183 \\
\hline$C$ & 1.053184 & -7.196290 & -1.049133 \\
\hline
\end{tabular}




\begin{tabular}{|c|c|c|c|}
\hline C & 0.602296 & -6.713916 & -2.430128 \\
\hline $\mathrm{O}$ & -0.581645 & -6.579853 & -2.645997 \\
\hline C & 1.252922 & -5.968692 & -0.165429 \\
\hline $\mathrm{O}$ & 1.412852 & -6.289994 & 1.176558 \\
\hline $\mathrm{H}$ & 3.086662 & -7.815834 & -0.796443 \\
\hline $\mathrm{H}$ & 0.208948 & -7.737541 & -0.654474 \\
\hline $\mathrm{H}$ & 2.080444 & -5.371463 & -0.532552 \\
\hline $\mathrm{H}$ & 0.346632 & -5.392140 & -0.249368 \\
\hline $\mathrm{H}$ & 2.319532 & -6.524414 & 1.320781 \\
\hline$N$ & 1.524545 & -6.421357 & -3.356796 \\
\hline C & 1.119340 & -6.007312 & -4.687560 \\
\hline C & 0.366941 & -7.131822 & -5.394547 \\
\hline O & -0.616594 & -6.878978 & -6.058973 \\
\hline C & 2.321339 & -5.474700 & -5.501861 \\
\hline C & 2.700149 & -4.081492 & -4.972089 \\
\hline$C$ & 2.027002 & -5.438763 & -7.006801 \\
\hline$C$ & 4.010930 & -3.532444 & -5.532708 \\
\hline $\mathrm{H}$ & 2.496147 & -6.472601 & -3.136624 \\
\hline $\mathrm{H}$ & 0.386994 & -5.217779 & -4.599022 \\
\hline $\mathrm{H}$ & 3.154808 & -6.151073 & -5.337426 \\
\hline $\mathrm{H}$ & 1.892034 & -3.389647 & -5.203569 \\
\hline $\mathrm{H}$ & 2.779037 & -4.121497 & -3.893337 \\
\hline $\mathrm{H}$ & 2.878141 & -5.044252 & -7.547683 \\
\hline $\mathrm{H}$ & 1.828756 & -6.425797 & -7.406757 \\
\hline $\mathrm{H}$ & 1.169328 & -4.811086 & -7.227436 \\
\hline $\mathrm{H}$ & 4.283091 & -2.619153 & -5.013969 \\
\hline $\mathrm{H}$ & 4.820371 & -4.242192 & -5.395364 \\
\hline $\mathrm{H}$ & 3.944286 & -3.300907 & -6.589979 \\
\hline $\mathrm{N}$ & 0.812833 & -8.381381 & -5.239583 \\
\hline $\mathrm{H}$ & 1.659106 & -8.556154 & -4.738586 \\
\hline
\end{tabular}




\begin{tabular}{|c|c|c|c|}
\hline C & 0.043777 & -9.489598 & -5.753025 \\
\hline $\mathrm{H}$ & -0.063177 & -9.438059 & -6.826330 \\
\hline $\mathrm{H}$ & 0.563129 & -10.404949 & -5.499179 \\
\hline C & -1.365267 & -9.540096 & -5.166931 \\
\hline $\mathrm{O}$ & -2.317300 & -9.778481 & -5.874449 \\
\hline$N$ & -1.472644 & -9.318876 & -3.858022 \\
\hline C & -2.773824 & -9.358721 & -3.234357 \\
\hline C & -3.688263 & -8.224473 & -3.694805 \\
\hline O & -4.883636 & -8.440525 & -3.757268 \\
\hline C & -2.678523 & -9.337272 & -1.701948 \\
\hline $\mathrm{O}$ & -3.924501 & -9.611371 & -1.121610 \\
\hline $\mathrm{H}$ & -0.656034 & -9.230247 & -3.289963 \\
\hline $\mathrm{H}$ & -3.288434 & -10.263746 & -3.524794 \\
\hline $\mathrm{H}$ & -1.994932 & -10.109663 & -1.377982 \\
\hline $\mathrm{H}$ & -2.293917 & -8.380107 & -1.366616 \\
\hline $\mathrm{H}$ & -4.614336 & -9.215571 & -1.639796 \\
\hline$N$ & -3.140792 & -7.038199 & -3.955302 \\
\hline C & -3.899230 & -5.914910 & -4.482136 \\
\hline C & -4.452023 & -6.249501 & -5.866547 \\
\hline $\mathrm{O}$ & -5.625075 & -6.088448 & -6.119169 \\
\hline C & -2.987087 & -4.668947 & -4.499399 \\
\hline C & -3.417506 & -3.519152 & -5.398291 \\
\hline C & -4.745639 & -3.185576 & -5.639365 \\
\hline C & -2.429784 & -2.759580 & -6.020311 \\
\hline C & -5.070431 & -2.147296 & -6.500877 \\
\hline C & -2.748658 & -1.710165 & -6.863894 \\
\hline C & -4.077226 & -1.405944 & -7.116296 \\
\hline $\mathrm{H}$ & -2.168081 & -6.911521 & -3.765855 \\
\hline $\mathrm{H}$ & -4.760930 & -5.732366 & -3.859883 \\
\hline $\mathrm{H}$ & -2.882251 & -4.329419 & -3.473346 \\
\hline
\end{tabular}




\begin{tabular}{|c|c|c|c|}
\hline $\mathrm{H}$ & -2.005354 & -4.982331 & -4.824377 \\
\hline $\mathrm{H}$ & -5.540022 & -3.751971 & -5.195256 \\
\hline $\mathrm{H}$ & -1.394633 & -2.998469 & -5.850315 \\
\hline $\mathrm{H}$ & -6.108971 & -1.946338 & -6.700821 \\
\hline $\mathrm{H}$ & -1.962807 & -1.141650 & -7.328248 \\
\hline $\mathrm{H}$ & -4.329005 & -0.608301 & -7.793668 \\
\hline$N$ & -3.583096 & -6.714759 & -6.769885 \\
\hline C & -4.048698 & -7.045628 & -8.097358 \\
\hline C & -5.186661 & -8.063368 & -8.049386 \\
\hline 0 & -6.115432 & -7.964920 & -8.807918 \\
\hline C & -2.917288 & -7.590043 & -8.972181 \\
\hline$S$ & -1.689356 & -6.352640 & -9.499737 \\
\hline $\mathrm{H}$ & -2.632651 & -6.879001 & -6.510381 \\
\hline $\mathrm{H}$ & -4.472881 & -6.169452 & -8.565247 \\
\hline $\mathrm{H}$ & -2.417606 & -8.415632 & -8.483638 \\
\hline $\mathrm{H}$ & -3.349733 & -7.961716 & -9.891567 \\
\hline $\mathrm{H}$ & -1.067005 & -6.199593 & -8.341190 \\
\hline $\mathrm{N}$ & -5.078560 & -9.066910 & -7.155609 \\
\hline $\mathrm{H}$ & -4.238567 & -9.204541 & -6.635057 \\
\hline$C$ & -6.086052 & -10.114294 & -7.182808 \\
\hline $\mathrm{H}$ & -6.261190 & -10.452571 & -8.192815 \\
\hline $\mathrm{H}$ & -5.714335 & -10.946880 & -6.596984 \\
\hline$C$ & -7.459139 & -9.722627 & -6.613157 \\
\hline 0 & -8.480744 & -10.092011 & -7.125161 \\
\hline $\mathrm{N}$ & -7.417940 & -9.016150 & -5.478415 \\
\hline $\mathrm{C}$ & -8.636054 & -8.653965 & -4.781521 \\
\hline$C$ & -9.495623 & -7.633624 & -5.521555 \\
\hline 0 & -10.688582 & -7.608002 & -5.362047 \\
\hline $\mathrm{C}$ & -8.344878 & -8.144478 & -3.358911 \\
\hline C & -7.426713 & -6.915793 & -3.232024 \\
\hline
\end{tabular}




\begin{tabular}{|c|c|c|c|}
\hline C & -8.012745 & -5.535991 & -3.103580 \\
\hline 0 & -9.305367 & -5.429296 & -3.235178 \\
\hline $\mathrm{H}$ & -9.566295 & -4.512283 & -3.101628 \\
\hline $\mathrm{O}$ & -7.307557 & -4.582583 & -2.877283 \\
\hline $\mathrm{H}$ & -6.532739 & -8.774317 & -5.086305 \\
\hline $\mathrm{H}$ & -9.265015 & -9.529190 & -4.698509 \\
\hline $\mathrm{H}$ & -9.297852 & -7.962122 & -2.882458 \\
\hline $\mathrm{H}$ & -7.870667 & -8.961722 & -2.826246 \\
\hline $\mathrm{H}$ & -6.797312 & -7.023712 & -2.358611 \\
\hline $\mathrm{H}$ & -6.742167 & -6.848728 & -4.061081 \\
\hline$N$ & -8.837281 & -6.733946 & -6.273830 \\
\hline C & -9.555208 & -5.702634 & -7.000846 \\
\hline C & -10.241444 & -6.303436 & -8.225512 \\
\hline $\mathrm{H}$ & -9.886122 & -7.287502 & -8.536798 \\
\hline $\mathrm{O}$ & -11.124482 & -5.744364 & -8.794575 \\
\hline C & -8.611262 & -4.505520 & -7.297856 \\
\hline $\mathrm{O}$ & -8.596367 & -3.622330 & -6.193337 \\
\hline C & -8.932608 & -3.727460 & -8.570875 \\
\hline $\mathrm{H}$ & -7.859620 & -6.848089 & -6.436168 \\
\hline $\mathrm{H}$ & -10.361529 & -5.346458 & -6.373644 \\
\hline $\mathrm{H}$ & -7.600419 & -4.876651 & -7.370771 \\
\hline $\mathrm{H}$ & -9.437618 & -3.184416 & -6.138697 \\
\hline $\mathrm{H}$ & -8.252636 & -2.883477 & -8.630984 \\
\hline $\mathrm{H}$ & -8.801590 & -4.331958 & -9.459248 \\
\hline $\mathrm{H}$ & -9.956788 & -3.367087 & -8.569729 \\
\hline$N$ & -12.952099 & -9.022565 & 3.174142 \\
\hline $\mathrm{H}$ & -13.212983 & -9.553275 & 2.368796 \\
\hline C & -11.773563 & -8.180068 & 2.898826 \\
\hline C & -10.790963 & -8.116278 & 4.051923 \\
\hline $\mathrm{O}$ & -9.664563 & -7.699910 & 3.8590 \\
\hline
\end{tabular}




\begin{tabular}{|c|c|c|c|}
\hline C & -12.147103 & -6.729778 & 2.543795 \\
\hline 0 & -12.733777 & -6.198346 & 3.703356 \\
\hline C & -13.100358 & -6.634674 & 1.357417 \\
\hline $\mathrm{H}$ & -13.732123 & -8.453221 & 3.439978 \\
\hline $\mathrm{H}$ & -11.218696 & -8.607536 & 2.074398 \\
\hline $\mathrm{H}$ & -11.231354 & -6.202371 & 2.314642 \\
\hline $\mathrm{H}$ & -12.852378 & -5.257660 & 3.588813 \\
\hline $\mathrm{H}$ & -13.263234 & -5.591661 & 1.108782 \\
\hline $\mathrm{H}$ & -12.704662 & -7.132439 & 0.479807 \\
\hline $\mathrm{H}$ & -14.064139 & -7.068482 & 1.595973 \\
\hline$N$ & -11.197245 & -8.533612 & 5.241880 \\
\hline $\mathrm{H}$ & -12.116114 & -8.918914 & 5.271876 \\
\hline C & -10.412641 & -8.534803 & 6.452134 \\
\hline $\mathrm{H}$ & -10.330954 & -7.540281 & 6.861132 \\
\hline $\mathrm{H}$ & -10.939748 & -9.139572 & 7.180551 \\
\hline$C$ & -8.977528 & -9.059639 & 6.382431 \\
\hline 0 & -8.147112 & -8.510269 & 7.063166 \\
\hline $\mathrm{N}$ & -8.659647 & -10.125342 & 5.624950 \\
\hline $\mathrm{H}$ & -9.368430 & -10.595275 & 5.108491 \\
\hline$C$ & -7.270846 & -10.559425 & 5.524585 \\
\hline $\mathrm{H}$ & -7.254236 & -11.535653 & 5.057095 \\
\hline $\mathrm{H}$ & -6.833782 & -10.644954 & 6.506426 \\
\hline$C$ & -6.387435 & -9.608346 & 4.692696 \\
\hline 0 & -5.221622 & -9.463504 & 4.953059 \\
\hline $\mathrm{N}$ & -7.045410 & -9.018041 & 3.701147 \\
\hline$C$ & -6.310644 & -7.982221 & 2.986667 \\
\hline$C$ & -6.265755 & -6.696391 & 3.808545 \\
\hline 0 & -5.273914 & -6.006573 & 3.776925 \\
\hline$C$ & -6.638572 & -7.803893 & 1.490680 \\
\hline C & -7.801944 & -6.936852 & 1.054646 \\
\hline
\end{tabular}




\begin{tabular}{|c|c|c|c|}
\hline C & -7.783273 & -5.559758 & 1.256976 \\
\hline C & -8.848770 & -7.481221 & 0.321991 \\
\hline C & -8.793848 & -4.754182 & 0.760441 \\
\hline C & -9.848520 & -6.674923 & -0.197906 \\
\hline C & -9.829590 & -5.309009 & 0.027572 \\
\hline $\mathrm{H}$ & -8.039897 & -9.013221 & 3.684354 \\
\hline $\mathrm{H}$ & -5.287134 & -8.313207 & 2.998513 \\
\hline $\mathrm{H}$ & -5.738222 & -7.392705 & 1.047483 \\
\hline $\mathrm{H}$ & -6.744121 & -8.800366 & 1.078440 \\
\hline $\mathrm{H}$ & -6.960446 & -5.104826 & 1.776764 \\
\hline $\mathrm{H}$ & -8.866169 & -8.539197 & 0.124031 \\
\hline $\mathrm{H}$ & -8.754263 & -3.691552 & 0.921788 \\
\hline $\mathrm{H}$ & -10.625514 & -7.108648 & -0.799807 \\
\hline $\mathrm{H}$ & -10.600112 & -4.682866 & -0.384637 \\
\hline$N$ & -7.293899 & -6.409573 & 4.610793 \\
\hline C & -7.226774 & -5.302748 & 5.535658 \\
\hline C & -6.096069 & -5.517837 & 6.540834 \\
\hline 0 & -5.444773 & -4.566561 & 6.915796 \\
\hline C & -8.599152 & -5.148978 & 6.224076 \\
\hline C & -8.840646 & -3.835326 & 6.936330 \\
\hline C & -9.095369 & -2.677111 & 6.207499 \\
\hline C & -8.880576 & -3.770076 & 8.322719 \\
\hline C & -9.374103 & -1.482488 & 6.848635 \\
\hline C & -9.168904 & -2.577761 & 8.970707 \\
\hline C & -9.416084 & -1.429845 & 8.236054 \\
\hline $\mathrm{H}$ & -8.162020 & -6.883468 & 4.486674 \\
\hline $\mathrm{H}$ & -6.970431 & -4.391381 & 5.014129 \\
\hline $\mathrm{H}$ & -9.351528 & -5.263245 & 5.450526 \\
\hline $\mathrm{H}$ & -8.729316 & -5.968136 & 6.918061 \\
\hline $\mathrm{H}$ & -9.081971 & -2.707479 & 5.131451 \\
\hline
\end{tabular}




\begin{tabular}{|c|c|c|c|}
\hline $\mathrm{H}$ & -8.690468 & -4.654767 & 8.904957 \\
\hline $\mathrm{H}$ & -9.556950 & -0.596768 & 6.267818 \\
\hline $\mathrm{H}$ & -9.191023 & -2.546691 & 10.045147 \\
\hline $\mathrm{H}$ & -9.620419 & -0.499437 & 8.735873 \\
\hline$N$ & -5.853788 & -6.754869 & 6.970525 \\
\hline $\mathrm{H}$ & -6.506301 & -7.478288 & 6.764593 \\
\hline C & -4.771469 & -7.046114 & 7.884182 \\
\hline $\mathrm{H}$ & -4.807238 & -6.414045 & 8.758773 \\
\hline $\mathrm{H}$ & -4.879903 & -8.075481 & 8.201436 \\
\hline C & -3.378763 & -6.868401 & 7.281895 \\
\hline $\mathrm{O}$ & -2.503741 & -6.369400 & 7.948712 \\
\hline$N$ & -3.182169 & -7.299127 & 6.030851 \\
\hline C & -1.903339 & -7.129367 & 5.357699 \\
\hline C & -1.649485 & -5.661756 & 4.985820 \\
\hline $\mathrm{O}$ & -0.548494 & -5.180483 & 5.149104 \\
\hline$C$ & -1.825810 & -8.074680 & 4.138419 \\
\hline C & -0.659949 & -7.729133 & 3.210260 \\
\hline C & -1.729287 & -9.531460 & 4.605591 \\
\hline $\mathrm{H}$ & -3.917955 & -7.778217 & 5.557820 \\
\hline $\mathrm{H}$ & -1.111378 & -7.373486 & 6.050772 \\
\hline $\mathrm{H}$ & -2.746930 & -7.953082 & 3.572991 \\
\hline $\mathrm{H}$ & -0.599900 & -8.461363 & 2.412187 \\
\hline $\mathrm{H}$ & -0.765505 & -6.757335 & 2.748919 \\
\hline $\mathrm{H}$ & 0.285210 & -7.741500 & 3.744188 \\
\hline $\mathrm{H}$ & -1.745998 & -10.199062 & 3.750208 \\
\hline $\mathrm{H}$ & -0.795673 & -9.697428 & 5.137152 \\
\hline $\mathrm{H}$ & -2.546467 & -9.812858 & 5.257167 \\
\hline$N$ & -2.682005 & -4.974019 & 4.487882 \\
\hline C & -2.545164 & -3.597631 & 4.052069 \\
\hline C & -2.409867 & -2.667118 & 5.255465 \\
\hline
\end{tabular}




\begin{tabular}{|c|c|c|c|}
\hline $\mathrm{O}$ & -1.518125 & -1.849152 & 5.299780 \\
\hline C & -3.720352 & -3.148186 & 3.171366 \\
\hline C & -3.947925 & -3.929451 & 1.866852 \\
\hline C & -2.816703 & -3.839267 & 0.855428 \\
\hline $\mathrm{O}$ & -1.694637 & -4.192671 & 1.245875 \\
\hline $\mathrm{O}$ & -3.088981 & -3.434340 & -0.288347 \\
\hline $\mathrm{H}$ & -3.550187 & -5.438910 & 4.339790 \\
\hline $\mathrm{H}$ & -1.630955 & -3.496690 & 3.498427 \\
\hline $\mathrm{H}$ & -4.636093 & -3.194818 & 3.752918 \\
\hline $\mathrm{H}$ & -3.555192 & -2.100004 & 2.937947 \\
\hline $\mathrm{H}$ & -4.097726 & -4.975661 & 2.089032 \\
\hline $\mathrm{H}$ & -4.857302 & -3.558258 & 1.407512 \\
\hline$N$ & -3.309689 & -2.786764 & 6.250329 \\
\hline C & -3.093962 & -2.024874 & 7.456968 \\
\hline C & -1.781672 & -2.422988 & 8.139766 \\
\hline O & -1.113697 & -1.584913 & 8.705368 \\
\hline C & -4.230241 & -1.983044 & 8.488898 \\
\hline O & -4.437644 & -3.208907 & 9.126153 \\
\hline C & -5.512339 & -1.383379 & 7.923457 \\
\hline $\mathrm{H}$ & -3.984636 & -3.518795 & 6.233181 \\
\hline $\mathrm{H}$ & -2.930236 & -1.000161 & 7.162253 \\
\hline $\mathrm{H}$ & -3.859682 & -1.322574 & 9.262834 \\
\hline $\mathrm{H}$ & -4.879909 & -3.801439 & 8.530532 \\
\hline $\mathrm{H}$ & -6.246381 & -1.301433 & 8.715494 \\
\hline $\mathrm{H}$ & -5.327528 & -0.391141 & 7.523527 \\
\hline $\mathrm{H}$ & -5.931168 & -1.996298 & 7.136449 \\
\hline $\mathrm{N}$ & -1.380404 & -3.700488 & 8.043333 \\
\hline $\mathrm{H}$ & -2.031003 & -4.418665 & 7.814160 \\
\hline C & -0.186948 & -4.107261 & 8.740209 \\
\hline $\mathrm{H}$ & -0.237554 & -3.891848 & 9.798220 \\
\hline
\end{tabular}




\begin{tabular}{|c|c|c|c|}
\hline $\mathrm{H}$ & -0.075182 & -5.174752 & 8.603858 \\
\hline$C$ & 1.064490 & -3.419348 & 8.218883 \\
\hline 0 & 1.940102 & -3.075052 & 8.979783 \\
\hline $\mathrm{N}$ & 1.155313 & -3.258516 & 6.882966 \\
\hline$C$ & 2.333919 & -2.661015 & 6.317786 \\
\hline$C$ & 2.292315 & -1.139101 & 6.162096 \\
\hline 0 & 3.361308 & -0.575434 & 6.019565 \\
\hline$C$ & 2.834703 & -3.351539 & 5.049051 \\
\hline$S$ & 1.918436 & -3.009871 & 3.521955 \\
\hline $\mathrm{H}$ & 0.424945 & -3.613498 & 6.298139 \\
\hline $\mathrm{H}$ & 3.117469 & -2.796350 & 7.048011 \\
\hline $\mathrm{H}$ & 3.840189 & -3.003438 & 4.861002 \\
\hline $\mathrm{H}$ & 2.870646 & -4.418722 & 5.218663 \\
\hline $\mathrm{H}$ & 0.872619 & -3.797578 & 3.722815 \\
\hline $\mathrm{N}$ & 1.141841 & -0.471357 & 6.273787 \\
\hline$C$ & 1.204073 & 0.968482 & 6.491382 \\
\hline$C$ & 1.753690 & 1.272376 & 7.870910 \\
\hline $\mathrm{O}$ & 2.364358 & 2.290991 & 8.087017 \\
\hline$C$ & -0.097046 & 1.738100 & 6.176832 \\
\hline$C$ & -1.200206 & 1.568193 & 7.231597 \\
\hline$C$ & -0.553630 & 1.428961 & 4.745869 \\
\hline$C$ & -2.469273 & 2.368698 & 6.930463 \\
\hline $\mathrm{H}$ & 0.273681 & -0.966378 & 6.289418 \\
\hline $\mathrm{H}$ & 1.952058 & 1.351106 & 5.815539 \\
\hline $\mathrm{H}$ & 0.204996 & 2.779216 & 6.212705 \\
\hline $\mathrm{H}$ & -1.448849 & 0.523567 & 7.352494 \\
\hline $\mathrm{H}$ & -0.813653 & 1.896478 & 8.192054 \\
\hline $\mathrm{H}$ & -1.309057 & 2.133214 & 4.420446 \\
\hline $\mathrm{H}$ & 0.279830 & 1.505532 & 4.053097 \\
\hline $\mathrm{H}$ & -0.964394 & 0.432418 & 4.656221 \\
\hline
\end{tabular}




\begin{tabular}{|c|c|c|c|}
\hline $\mathrm{H}$ & -3.142326 & 2.340022 & 7.782109 \\
\hline $\mathrm{H}$ & -2.243999 & 3.411664 & 6.722902 \\
\hline $\mathrm{H}$ & -3.006174 & 1.969830 & 6.076689 \\
\hline$N$ & 1.549774 & 0.323287 & 8.791241 \\
\hline $\mathrm{H}$ & 0.890691 & -0.402779 & 8.613169 \\
\hline C & 1.977802 & 0.512702 & 10.160033 \\
\hline $\mathrm{H}$ & 1.469272 & -0.222454 & 10.770953 \\
\hline $\mathrm{H}$ & 1.737385 & 1.502491 & 10.517938 \\
\hline C & 3.481795 & 0.337028 & 10.305837 \\
\hline $\mathrm{O}$ & 4.163620 & 1.127585 & 10.898746 \\
\hline$N$ & 3.999649 & -0.753675 & 9.695463 \\
\hline $\mathrm{H}$ & 3.390372 & -1.464979 & 9.349663 \\
\hline C & 5.424736 & -0.938883 & 9.667283 \\
\hline $\mathrm{H}$ & 5.637718 & -1.909422 & 9.234270 \\
\hline $\mathrm{H}$ & 5.855436 & -0.904929 & 10.657348 \\
\hline C & 6.156885 & 0.119116 & 8.844830 \\
\hline $\mathrm{O}$ & 7.291754 & 0.412091 & 9.129721 \\
\hline$N$ & 5.502087 & 0.621274 & 7.799020 \\
\hline C & 6.098611 & 1.759282 & 7.146322 \\
\hline C & 5.515755 & 3.077093 & 7.649950 \\
\hline $\mathrm{H}$ & 5.009274 & 3.036938 & 8.612868 \\
\hline $\mathrm{O}$ & 5.642887 & 4.095919 & 7.043695 \\
\hline C & 6.001799 & 1.675241 & 5.620806 \\
\hline C & 6.754289 & 0.507592 & 5.016621 \\
\hline C & 8.119984 & 0.323554 & 5.236933 \\
\hline C & 6.093947 & -0.400178 & 4.193981 \\
\hline C & 8.806411 & -0.721137 & 4.632864 \\
\hline C & 6.778104 & -1.442485 & 3.588462 \\
\hline C & 8.138271 & -1.603448 & 3.798047 \\
\hline & 4.575710 & 0.333815 & 7.571699 \\
\hline
\end{tabular}




\begin{tabular}{|c|c|c|c|}
\hline $\mathrm{H}$ & 7.134274 & 1.774212 & 7.450535 \\
\hline $\mathrm{H}$ & 4.959444 & 1.610593 & 5.335055 \\
\hline $\mathrm{H}$ & 6.379367 & 2.609976 & 5.220685 \\
\hline $\mathrm{H}$ & 8.648202 & 0.979222 & 5.907817 \\
\hline $\mathrm{H}$ & 5.033800 & -0.304076 & 4.055849 \\
\hline $\mathrm{H}$ & 9.857250 & -0.848320 & 4.828159 \\
\hline $\mathrm{H}$ & 6.245884 & -2.133503 & 2.959315 \\
\hline $\mathrm{H}$ & 8.658738 & -2.415364 & 3.323940 \\
\hline$N$ & 12.613550 & 9.596234 & -1.248801 \\
\hline $\mathrm{H}$ & 11.873304 & 9.913777 & -1.846311 \\
\hline C & 12.304852 & 8.264740 & -0.719487 \\
\hline C & 11.273689 & 8.450546 & 0.386591 \\
\hline $\mathrm{O}$ & 10.265530 & 7.778767 & 0.415804 \\
\hline C & 13.562549 & 7.581221 & -0.164596 \\
\hline C & 14.645707 & 7.276548 & -1.205017 \\
\hline$S$ & 14.018810 & 6.203150 & -2.529344 \\
\hline C & 15.503354 & 6.052419 & -3.551773 \\
\hline $\mathrm{H}$ & 13.433529 & 9.572929 & -1.822100 \\
\hline $\mathrm{H}$ & 11.834820 & 7.616386 & -1.447461 \\
\hline $\mathrm{H}$ & 14.000496 & 8.205656 & 0.608263 \\
\hline $\mathrm{H}$ & 13.255359 & 6.653971 & 0.305291 \\
\hline $\mathrm{H}$ & 15.046679 & 8.189118 & -1.631593 \\
\hline $\mathrm{H}$ & 15.470297 & 6.783724 & -0.702487 \\
\hline $\mathrm{H}$ & 15.253298 & 5.397008 & -4.372129 \\
\hline $\mathrm{H}$ & 15.803968 & 7.015827 & -3.942864 \\
\hline $\mathrm{H}$ & 16.320271 & 5.615506 & -2.992399 \\
\hline$N$ & 11.503479 & 9.412865 & 1.296722 \\
\hline C & 10.593734 & 9.607380 & 2.408635 \\
\hline C & 9.276928 & 10.276086 & 2.011659 \\
\hline 4 & 8.281457 & 10.056921 & 2.655491 \\
\hline
\end{tabular}




\begin{tabular}{|c|c|c|c|}
\hline C & 11.276507 & 10.366957 & 3.552816 \\
\hline C & 12.448227 & 9.561470 & 4.083903 \\
\hline $\mathrm{O}$ & 13.582992 & 9.823427 & 3.743914 \\
\hline$N$ & 12.144690 & 8.558477 & 4.917500 \\
\hline $\mathrm{H}$ & 12.327486 & 9.967466 & 1.206418 \\
\hline $\mathrm{H}$ & 10.294206 & 8.637194 & 2.770427 \\
\hline $\mathrm{H}$ & 11.657728 & 11.324458 & 3.220662 \\
\hline $\mathrm{H}$ & 10.546636 & 10.534855 & 4.334660 \\
\hline $\mathrm{H}$ & 12.888577 & 7.962509 & 5.210493 \\
\hline $\mathrm{H}$ & 11.223226 & 8.171947 & 4.952502 \\
\hline$N$ & 9.263775 & 11.068227 & 0.922800 \\
\hline $\mathrm{H}$ & 10.110275 & 11.226553 & 0.425903 \\
\hline C & 8.003503 & 11.517472 & 0.368854 \\
\hline $\mathrm{H}$ & 8.201268 & 12.259938 & -0.393645 \\
\hline $\mathrm{H}$ & 7.389040 & 11.966937 & 1.131722 \\
\hline C & 7.186384 & 10.384437 & -0.254318 \\
\hline 0 & 5.986674 & 10.472166 & -0.294965 \\
\hline$N$ & 7.862838 & 9.332497 & -0.761515 \\
\hline C & 7.169921 & 8.145082 & -1.231698 \\
\hline C & 6.632700 & 7.302320 & -0.064480 \\
\hline $\mathrm{O}$ & 5.531496 & 6.812191 & -0.145111 \\
\hline C & 8.048023 & 7.341579 & -2.220518 \\
\hline C & 8.345826 & 8.193291 & -3.469295 \\
\hline C & 7.376281 & 6.017628 & -2.604436 \\
\hline C & 9.429443 & 7.618030 & -4.384588 \\
\hline $\mathrm{H}$ & 8.822974 & 9.228191 & -0.531268 \\
\hline $\mathrm{H}$ & 6.279891 & 8.471639 & -1.747020 \\
\hline $\mathrm{H}$ & 8.986130 & 7.113644 & -1.722574 \\
\hline $\mathrm{H}$ & 7.424555 & 8.321605 & -4.033217 \\
\hline $\mathrm{H}$ & 8.650434 & 9.188198 & -3.161869 \\
\hline
\end{tabular}




\begin{tabular}{|c|c|c|c|}
\hline $\mathrm{H}$ & 7.993928 & 5.475068 & -3.308756 \\
\hline $\mathrm{H}$ & 7.221789 & 5.377635 & -1.744299 \\
\hline $\mathrm{H}$ & 6.407889 & 6.182861 & -3.065124 \\
\hline $\mathrm{H}$ & 9.625971 & 8.297919 & -5.207242 \\
\hline $\mathrm{H}$ & 10.364005 & 7.469971 & -3.850308 \\
\hline $\mathrm{H}$ & 9.147214 & 6.664653 & -4.815676 \\
\hline$N$ & 7.385225 & 7.176512 & 1.047012 \\
\hline C & 6.877042 & 6.457524 & 2.204057 \\
\hline C & 5.524559 & 7.035060 & 2.642101 \\
\hline $\mathrm{O}$ & 4.574308 & 6.325967 & 2.868652 \\
\hline C & 7.830848 & 6.545496 & 3.405779 \\
\hline C & 9.252392 & 5.997242 & 3.245545 \\
\hline $\mathrm{O}$ & 10.144252 & 6.632337 & 3.801686 \\
\hline O & 9.425757 & 4.935103 & 2.597434 \\
\hline $\mathrm{H}$ & 8.346014 & 7.443499 & 1.024955 \\
\hline $\mathrm{H}$ & 6.682390 & 5.428675 & 1.944175 \\
\hline $\mathrm{H}$ & 7.918404 & 7.570066 & 3.735686 \\
\hline $\mathrm{H}$ & 7.373052 & 5.991421 & 4.222696 \\
\hline $\mathrm{N}$ & 5.482753 & 8.373085 & 2.790280 \\
\hline $\mathrm{H}$ & 6.312206 & 8.910754 & 2.653481 \\
\hline$C$ & 4.290219 & 9.036913 & 3.256193 \\
\hline $\mathrm{H}$ & 4.527987 & 10.079896 & 3.424426 \\
\hline $\mathrm{H}$ & 3.945919 & 8.620697 & 4.189781 \\
\hline$C$ & 3.129854 & 8.987455 & 2.271387 \\
\hline O & 1.988204 & 9.116366 & 2.649901 \\
\hline $\mathrm{N}$ & 3.466171 & 8.825313 & 0.988812 \\
\hline $\mathrm{H}$ & 4.427981 & 8.815249 & 0.742385 \\
\hline$C$ & 2.517188 & 8.905263 & -0.078309 \\
\hline $\mathrm{H}$ & 3.062362 & 9.089581 & -0.995062 \\
\hline $\mathrm{H}$ & 1.843510 & 9.738111 & 0.069104 \\
\hline
\end{tabular}




\begin{tabular}{|c|c|c|c|}
\hline$C$ & 1.609135 & 7.687017 & -0.310189 \\
\hline O & 0.575988 & 7.817118 & -0.906827 \\
\hline $\mathrm{N}$ & 2.067776 & 6.540439 & 0.227359 \\
\hline$C$ & 1.248377 & 5.370549 & 0.434794 \\
\hline$C$ & 0.429813 & 5.470884 & 1.726571 \\
\hline $\mathrm{O}$ & -0.764475 & 5.294262 & 1.700373 \\
\hline$C$ & 2.107097 & 4.106256 & 0.428929 \\
\hline $\mathrm{H}$ & 2.968602 & 6.569054 & 0.655859 \\
\hline $\mathrm{H}$ & 0.520431 & 5.319457 & -0.357322 \\
\hline $\mathrm{H}$ & 1.490830 & 3.234670 & 0.620250 \\
\hline $\mathrm{H}$ & 2.579505 & 3.996917 & -0.540369 \\
\hline $\mathrm{H}$ & 2.884237 & 4.151893 & 1.184999 \\
\hline $\mathrm{N}$ & 1.068191 & 5.733375 & 2.883787 \\
\hline$C$ & 0.338029 & 5.567457 & 4.131461 \\
\hline$C$ & -0.773870 & 6.602006 & 4.287546 \\
\hline $\mathrm{O}$ & -1.809965 & 6.305745 & 4.842241 \\
\hline$C$ & 1.258920 & 5.492817 & 5.374590 \\
\hline$C$ & 1.849360 & 6.838951 & 5.821881 \\
\hline$C$ & 2.348493 & 4.431102 & 5.160687 \\
\hline$C$ & 2.693295 & 6.740957 & 7.095211 \\
\hline $\mathrm{H}$ & 2.056692 & 5.862019 & 2.900468 \\
\hline $\mathrm{H}$ & -0.191362 & 4.627095 & 4.076667 \\
\hline $\mathrm{H}$ & 0.605403 & 5.150203 & 6.171125 \\
\hline $\mathrm{H}$ & 2.449867 & 7.254926 & 5.019470 \\
\hline $\mathrm{H}$ & 1.044378 & 7.544444 & 6.000363 \\
\hline $\mathrm{H}$ & 2.724610 & 4.075758 & 6.108871 \\
\hline $\mathrm{H}$ & 1.955797 & 3.575567 & 4.620583 \\
\hline $\mathrm{H}$ & 3.187257 & 4.825824 & 4.597549 \\
\hline $\mathrm{H}$ & 2.996390 & 7.731996 & 7.420105 \\
\hline $\mathrm{H}$ & 2.125610 & 6.290510 & 7.904070 \\
\hline
\end{tabular}




\begin{tabular}{|c|c|c|c|}
\hline $\mathrm{H}$ & 3.590054 & 6.150574 & 6.954232 \\
\hline$N$ & -0.550527 & 7.835200 & 3.809811 \\
\hline $\mathrm{H}$ & 0.334405 & 8.069848 & 3.410757 \\
\hline C & -1.515000 & 8.880907 & 4.023560 \\
\hline $\mathrm{H}$ & -1.098733 & 9.807511 & 3.648703 \\
\hline $\mathrm{H}$ & -1.729412 & 9.010996 & 5.074454 \\
\hline C & -2.866971 & 8.618756 & 3.365893 \\
\hline $\mathrm{O}$ & -3.871476 & 8.845921 & 3.999867 \\
\hline$N$ & -2.902686 & 8.108974 & 2.120752 \\
\hline C & -4.238787 & 8.033194 & 1.526231 \\
\hline C & -4.983202 & 6.753155 & 1.867818 \\
\hline $\mathrm{O}$ & -6.195626 & 6.754318 & 1.842582 \\
\hline C & -4.018520 & 8.294005 & 0.029162 \\
\hline C & -2.743446 & 9.144276 & 0.041792 \\
\hline C & -1.900223 & 8.455342 & 1.102623 \\
\hline $\mathrm{H}$ & -4.853889 & 8.819190 & 1.935695 \\
\hline $\mathrm{H}$ & -3.863914 & 7.367408 & -0.507685 \\
\hline $\mathrm{H}$ & -4.874122 & 8.793873 & -0.406893 \\
\hline $\mathrm{H}$ & -2.230657 & 9.199871 & -0.900853 \\
\hline $\mathrm{H}$ & -2.981774 & 10.156806 & 0.358919 \\
\hline $\mathrm{H}$ & -1.435443 & 7.561610 & 0.727030 \\
\hline $\mathrm{H}$ & -1.127335 & 9.089719 & 1.504288 \\
\hline$N$ & -4.262497 & 5.675999 & 2.205931 \\
\hline C & -4.914197 & 4.488141 & 2.729283 \\
\hline C & -5.629842 & 4.784577 & 4.050116 \\
\hline $\mathrm{O}$ & -6.704975 & 4.279094 & 4.285713 \\
\hline C & -3.921052 & 3.322434 & 2.932266 \\
\hline C & -3.594390 & 2.501999 & 1.700733 \\
\hline C & -4.106842 & 1.218509 & 1.543310 \\
\hline$C$ & -2.725103 & 2.956670 & 0.711682 \\
\hline
\end{tabular}




\begin{tabular}{|c|c|c|c|}
\hline C & -3.777512 & 0.421568 & 0.457558 \\
\hline C & -2.386559 & 2.177468 & -0.374700 \\
\hline C & -2.894361 & 0.873216 & -0.547092 \\
\hline 0 & -2.578338 & 0.152933 & -1.577154 \\
\hline $\mathrm{H}$ & -3.268757 & 5.756994 & 2.260898 \\
\hline $\mathrm{H}$ & -5.692673 & 4.185650 & 2.046458 \\
\hline $\mathrm{H}$ & -3.016284 & 3.722511 & 3.381139 \\
\hline $\mathrm{H}$ & -4.359935 & 2.661397 & 3.671706 \\
\hline $\mathrm{H}$ & -4.774113 & 0.821132 & 2.291646 \\
\hline $\mathrm{H}$ & -2.284183 & 3.929499 & 0.798091 \\
\hline $\mathrm{H}$ & -4.155513 & -0.584569 & 0.393644 \\
\hline $\mathrm{H}$ & -1.708100 & 2.559722 & -1.119194 \\
\hline$N$ & -5.003331 & 5.588851 & 4.928170 \\
\hline $\mathrm{H}$ & -4.071671 & 5.898081 & 4.742882 \\
\hline C & -5.607624 & 5.942419 & 6.192324 \\
\hline $\mathrm{H}$ & -4.823026 & 6.282676 & 6.857091 \\
\hline $\mathrm{H}$ & -6.090651 & 5.086742 & 6.635504 \\
\hline$C$ & -6.660906 & 7.045825 & 6.082512 \\
\hline 0 & -7.715840 & 6.968813 & 6.671022 \\
\hline $\mathrm{N}$ & -6.342145 & 8.091259 & 5.313948 \\
\hline $\mathrm{H}$ & -5.447376 & 8.140725 & 4.874874 \\
\hline$C$ & -7.257648 & 9.220183 & 5.085240 \\
\hline $\mathrm{H}$ & -6.773142 & 9.882285 & 4.379589 \\
\hline $\mathrm{H}$ & -7.440761 & 9.763554 & 6.000424 \\
\hline$C$ & -8.621452 & 8.828637 & 4.543285 \\
\hline 0 & -9.606440 & 9.420459 & 4.912576 \\
\hline $\mathrm{N}$ & -8.673257 & 7.827086 & 3.645025 \\
\hline$C$ & -9.943614 & 7.435666 & 3.076848 \\
\hline $\mathrm{C}$ & -10.836112 & 6.654154 & 4.038280 \\
\hline 0 & -11.951735 & 6.351439 & 3.656934 \\
\hline
\end{tabular}




\begin{tabular}{|c|c|c|c|}
\hline C & -9.775573 & 6.671908 & 1.743215 \\
\hline $\mathrm{O}$ & -10.966834 & 6.755852 & 1.000025 \\
\hline C & -9.335384 & 5.223187 & 1.911330 \\
\hline $\mathrm{H}$ & -7.828303 & 7.411929 & 3.308412 \\
\hline $\mathrm{H}$ & -10.508955 & 8.330879 & 2.856496 \\
\hline $\mathrm{H}$ & -9.028293 & 7.206830 & 1.172109 \\
\hline $\mathrm{H}$ & -11.679080 & 6.479466 & 1.568919 \\
\hline $\mathrm{H}$ & -9.141858 & 4.784639 & 0.939817 \\
\hline $\mathrm{H}$ & -8.431870 & 5.142469 & 2.499257 \\
\hline $\mathrm{H}$ & -10.115082 & 4.639142 & 2.389249 \\
\hline$N$ & -10.364715 & 6.343808 & 5.246677 \\
\hline C & -11.209900 & 5.754972 & 6.261796 \\
\hline C & -12.249540 & 6.740696 & 6.800523 \\
\hline $\mathrm{O}$ & -13.299314 & 6.325844 & 7.235299 \\
\hline C & -10.393773 & 5.201421 & 7.438426 \\
\hline C & -9.459963 & 4.028445 & 7.102085 \\
\hline C & -8.608442 & 3.695712 & 8.331194 \\
\hline C & -10.219219 & 2.785819 & 6.627183 \\
\hline $\mathrm{H}$ & -9.431308 & 6.592549 & 5.498266 \\
\hline $\mathrm{H}$ & -11.788811 & 4.964004 & 5.814091 \\
\hline$r$ & -9.808306 & 6.007527 & 7.864915 \\
\hline $\mathrm{H}$ & -11.106388 & 4.886558 & 8.195353 \\
\hline $\mathrm{H}$ & -8.787143 & 4.332846 & 6.308556 \\
\hline $\mathrm{H}$ & -7.910898 & 2.891370 & 8.115457 \\
\hline $\mathrm{H}$ & -8.035281 & 4.559110 & 8.651339 \\
\hline $\mathrm{H}$ & -9.230183 & 3.380162 & 9.166390 \\
\hline $\mathrm{H}$ & -9.522693 & 1.974053 & 6.438632 \\
\hline $\mathrm{H}$ & -10.930266 & 2.450990 & 7.380572 \\
\hline $\mathrm{H}$ & -10.765692 & 2.957793 & 5.706887 \\
\hline $\mathrm{N}$ & -11.912361 & 8.040824 & 6.828433 \\
\hline
\end{tabular}




\begin{tabular}{|c|c|c|c|}
\hline C & -12.861882 & 9.030441 & 7.246619 \\
\hline C & -13.878980 & 9.396814 & 6.186155 \\
\hline $\mathrm{H}$ & -14.627930 & 10.124808 & 6.517977 \\
\hline $\mathrm{O}$ & -13.893172 & 8.967294 & 5.081459 \\
\hline $\mathrm{H}$ & -11.118791 & 8.352760 & 6.310016 \\
\hline $\mathrm{H}$ & -13.395984 & 8.683690 & 8.122608 \\
\hline $\mathrm{H}$ & -12.333526 & 9.935970 & 7.529092 \\
\hline$N$ & -11.496190 & 10.630062 & -3.035009 \\
\hline $\mathrm{H}$ & -11.247733 & 10.848697 & -2.090743 \\
\hline C & -11.816457 & 9.206991 & -3.172691 \\
\hline C & -11.414235 & 8.858125 & -4.598813 \\
\hline $\mathrm{O}$ & -10.375790 & 8.292762 & -4.845254 \\
\hline C & -13.245339 & 8.787459 & -2.766642 \\
\hline C & -13.494301 & 9.128697 & -1.285863 \\
\hline C & -13.469848 & 7.295863 & -3.047016 \\
\hline C & -14.919980 & 8.857049 & -0.802254 \\
\hline $\mathrm{H}$ & -12.280122 & 11.208570 & -3.271591 \\
\hline $\mathrm{H}$ & -11.117532 & 8.661615 & -2.553899 \\
\hline $\mathrm{H}$ & -13.959090 & 9.363117 & -3.360659 \\
\hline $\mathrm{H}$ & -12.790450 & 8.572654 & -0.671044 \\
\hline $\mathrm{H}$ & -13.282206 & 10.180979 & -1.124282 \\
\hline $\mathrm{H}$ & -14.498742 & 7.014321 & -2.862893 \\
\hline $\mathrm{H}$ & -13.248375 & 7.032851 & -4.077384 \\
\hline $\mathrm{H}$ & -12.838464 & 6.689036 & -2.405414 \\
\hline $\mathrm{H}$ & -15.042877 & 9.214780 & 0.213647 \\
\hline $\mathrm{H}$ & -15.652653 & 9.365844 & -1.423251 \\
\hline $\mathrm{H}$ & -15.158148 & 7.799696 & -0.801399 \\
\hline$N$ & -12.214200 & 9.288719 & -5.604122 \\
\hline $\mathrm{H}$ & -13.131169 & 9.602190 & -5.380670 \\
\hline C & -11.972873 & 8.843878 & -6.959262 \\
\hline
\end{tabular}




\begin{tabular}{|c|c|c|c|}
\hline $\mathrm{H}$ & -12.035531 & 7.767542 & -7.044753 \\
\hline $\mathrm{H}$ & -12.729290 & 9.282532 & -7.598613 \\
\hline$r$ & -10.601634 & 9.233249 & -7.517685 \\
\hline $\mathrm{O}$ & -9.997808 & 8.496125 & -8.243133 \\
\hline$N$ & -10.162516 & 10.476492 & $2-7.182350$ \\
\hline $\mathrm{H}$ & -10.708599 & 11.004447 & $7-6.540654$ \\
\hline C & -8.894338 & 11.023969 & -7.637102 \\
\hline $\mathrm{H}$ & -8.790084 & 10.891100 & -8.702725 \\
\hline $\mathrm{H}$ & -8.880399 & 12.081618 & -7.408308 \\
\hline C & -7.672571 & 10.358998 & -6.988330 \\
\hline 0 & -6.759378 & 9.922967 & -7.632279 \\
\hline$N$ & -7.657747 & 10.354147 & -5.620749 \\
\hline $\mathrm{H}$ & -8.474939 & 10.647805 & -5.135105 \\
\hline C & -6.495249 & 9.961279 & -4.832307 \\
\hline $\mathrm{H}$ & -5.597967 & 10.374767 & -5.264456 \\
\hline $\mathrm{H}$ & -6.616235 & 10.362002 & -3.833391 \\
\hline$C$ & -6.268288 & 8.463922 & -4.714299 \\
\hline $\mathrm{O}$ & -5.135244 & 8.046505 & -4.751044 \\
\hline$N$ & -7.314867 & 7.639575 & -4.521584 \\
\hline$C$ & -7.020622 & 6.271133 & -4.124525 \\
\hline C & -6.467326 & 5.439528 & -5.275336 \\
\hline $\mathrm{O}$ & -5.672345 & 4.560888 & -5.043417 \\
\hline$c$ & -8.156626 & 5.541630 & -3.368127 \\
\hline C & -9.346222 & 5.122976 & -4.248120 \\
\hline C & -8.566288 & 6.359529 & -2.137897 \\
\hline C & -10.386105 & 4.279503 & -3.506728 \\
\hline $\mathrm{H}$ & -8.251879 & 7.988654 & -4.514505 \\
\hline $\mathrm{H}$ & -6.188186 & 6.314002 & -3.438769 \\
\hline $\mathrm{H}$ & -7.685647 & 4.630542 & -3.009289 \\
\hline & -9.825976 & 5.994079 & -4.676481 \\
\hline
\end{tabular}




\begin{tabular}{|c|c|c|c|}
\hline $\mathrm{H}$ & -8.969838 & 4.536264 & -5.083273 \\
\hline $\mathrm{H}$ & -9.221411 & 5.793059 & -1.490471 \\
\hline $\mathrm{H}$ & -7.696283 & 6.635527 & -1.550876 \\
\hline $\mathrm{H}$ & -9.088194 & 7.267188 & -2.417891 \\
\hline $\mathrm{H}$ & -11.139265 & 3.914479 & -4.198687 \\
\hline $\mathrm{H}$ & -9.929940 & 3.417091 & -3.031033 \\
\hline $\mathrm{H}$ & -10.897643 & 4.848657 & -2.740383 \\
\hline $\mathrm{N}$ & -6.882379 & 5.679822 & -6.538388 \\
\hline$C$ & -6.242751 & 4.969450 & -7.628480 \\
\hline$C$ & -4.821713 & 5.460812 & -7.917777 \\
\hline $\mathrm{O}$ & -3.990853 & 4.676350 & -8.308830 \\
\hline$C$ & -7.107766 & 4.970248 & -8.897881 \\
\hline$C$ & -8.275025 & 3.973956 & -8.839301 \\
\hline $\mathrm{C}$ & -7.793513 & 2.520527 & -8.903265 \\
\hline $\mathrm{N}$ & -8.872639 & 1.529185 & -8.891127 \\
\hline$C$ & -9.426215 & 0.978437 & -7.815749 \\
\hline $\mathrm{N}$ & -9.158400 & 1.435841 & -6.605222 \\
\hline $\mathrm{N}$ & -10.266984 & -0.034923 & -7.964820 \\
\hline $\mathrm{H}$ & -7.492448 & 6.447501 & -6.720172 \\
\hline $\mathrm{H}$ & -6.080259 & 3.958401 & -7.295770 \\
\hline $\mathrm{H}$ & -7.496482 & 5.967443 & -9.079069 \\
\hline $\mathrm{H}$ & -6.472335 & 4.727412 & -9.743447 \\
\hline $\mathrm{H}$ & -8.865782 & 4.143041 & -7.943760 \\
\hline $\mathrm{H}$ & -8.937018 & 4.154881 & -9.679564 \\
\hline $\mathrm{H}$ & -7.248442 & 2.356428 & -9.822809 \\
\hline $\mathrm{H}$ & -7.107343 & 2.280401 & -8.102644 \\
\hline $\mathrm{H}$ & -9.143815 & 1.151075 & -9.771265 \\
\hline $\mathrm{H}$ & -8.629247 & 2.268681 & -6.487321 \\
\hline $\mathrm{H}$ & -9.631602 & 1.091880 & -5.783876 \\
\hline $\mathrm{H}$ & -10.325757 & -0.497121 & -8.845103 \\
\hline
\end{tabular}




\begin{tabular}{|c|c|c|c|}
\hline $\mathrm{H}$ & -10.582363 & -0.571456 & -7.170949 \\
\hline$N$ & -4.571419 & 6.757537 & -7.721986 \\
\hline $\mathrm{H}$ & -5.302695 & 7.370349 & -7.441242 \\
\hline C & -3.257792 & 7.352338 & -8.011302 \\
\hline $\mathrm{H}$ & -2.929240 & 7.090628 & -9.005007 \\
\hline $\mathrm{H}$ & -3.360549 & 8.426645 & -7.939570 \\
\hline C & -2.202231 & 6.873958 & -7.024718 \\
\hline O & -1.073852 & 6.638604 & -7.377578 \\
\hline$N$ & -2.633783 & 6.758811 & -5.763283 \\
\hline C & -1.761007 & 6.448293 & -4.656574 \\
\hline C & -1.491000 & 4.961024 & -4.524131 \\
\hline $\mathrm{O}$ & -0.363792 & 4.576977 & -4.319024 \\
\hline C & -2.349255 & 7.048560 & -3.373588 \\
\hline C & -1.762508 & 6.354340 & -2.145654 \\
\hline C & -2.111635 & 8.564410 & -3.410714 \\
\hline $\mathrm{H}$ & -3.557144 & 7.065119 & -5.546989 \\
\hline $\mathrm{H}$ & -0.788051 & 6.881331 & -4.838967 \\
\hline $\mathrm{H}$ & -3.417824 & 6.867712 & -3.387781 \\
\hline $\mathrm{H}$ & -1.979380 & 6.901791 & -1.247581 \\
\hline $\mathrm{H}$ & -2.172663 & 5.356412 & -2.033629 \\
\hline $\mathrm{H}$ & -0.689873 & 6.283703 & -2.215540 \\
\hline $\mathrm{H}$ & -2.836841 & 9.090480 & -2.806099 \\
\hline $\mathrm{H}$ & -1.116882 & 8.805684 & -3.051328 \\
\hline $\mathrm{H}$ & -2.213132 & 8.950231 & -4.418533 \\
\hline $\mathrm{N}$ & -2.517723 & 4.100220 & -4.645904 \\
\hline$C$ & -2.183874 & 2.694098 & -4.750258 \\
\hline$C$ & -1.220401 & 2.507869 & -5.924342 \\
\hline 0 & -0.294163 & 1.732064 & -5.842626 \\
\hline$C$ & -3.407847 & 1.776927 & -4.871499 \\
\hline$c$ & -4.364888 & 1.887265 & -3.676696 \\
\hline
\end{tabular}




\begin{tabular}{|c|c|c|c|}
\hline C & -5.406675 & 0.763417 & -3.611904 \\
\hline$N$ & -4.879475 & -0.427261 & -2.949793 \\
\hline C & -5.583840 & -1.519150 & -2.748491 \\
\hline$N$ & -6.813258 & -1.655527 & -3.295771 \\
\hline$N$ & -5.155159 & -2.493137 & -1.972250 \\
\hline $\mathrm{H}$ & -3.439230 & 4.421566 & -4.850950 \\
\hline $\mathrm{H}$ & -1.629052 & 2.394610 & -3.874428 \\
\hline $\mathrm{H}$ & -3.944330 & 1.990896 & -5.793998 \\
\hline $\mathrm{H}$ & -3.028660 & 0.764904 & -4.951115 \\
\hline $\mathrm{H}$ & -3.802055 & 1.899610 & -2.756841 \\
\hline $\mathrm{H}$ & -4.904106 & 2.820331 & -3.734069 \\
\hline $\mathrm{H}$ & -6.276908 & 1.113315 & -3.066024 \\
\hline $\mathrm{H}$ & -5.727354 & 0.511292 & -4.619620 \\
\hline $\mathrm{H}$ & -3.984955 & -0.324272 & -2.455368 \\
\hline $\mathrm{H}$ & -6.972757 & -1.178773 & -4.153531 \\
\hline $\mathrm{H}$ & -7.208521 & -2.578273 & -3.267051 \\
\hline $\mathrm{H}$ & -4.301483 & -2.472079 & -1.439477 \\
\hline $\mathrm{H}$ & -5.665078 & -3.351236 & -1.949303 \\
\hline$N$ & -1.459790 & 3.193298 & -7.035093 \\
\hline $\mathrm{H}$ & -2.284850 & 3.739868 & -7.148495 \\
\hline C & -0.596621 & 2.997529 & -8.183469 \\
\hline $\mathrm{H}$ & -0.949748 & 3.640778 & -8.979139 \\
\hline $\mathrm{H}$ & -0.611196 & 1.972323 & -8.524722 \\
\hline C & 0.855924 & 3.344619 & -7.871933 \\
\hline $\mathrm{O}$ & 1.781357 & 2.710999 & -8.313423 \\
\hline$N$ & 1.005385 & 4.404934 & -7.063953 \\
\hline $\mathrm{H}$ & 0.212185 & 4.964248 & -6.846845 \\
\hline C & 2.282350 & 4.880661 & -6.640316 \\
\hline $\mathrm{H}$ & 2.918995 & 5.137222 & -7.477606 \\
\hline $\mathrm{H}$ & 2.128365 & 5.777235 & -6.053918 \\
\hline
\end{tabular}




\begin{tabular}{|c|c|c|c|}
\hline C & 3.092850 & 3.928537 & -5.775022 \\
\hline $\mathrm{O}$ & 4.297527 & 4.059879 & -5.687843 \\
\hline$N$ & 2.428015 & 2.983364 & -5.101894 \\
\hline C & 3.096637 & 2.136984 & -4.148030 \\
\hline C & 4.270120 & 1.327443 & -4.710931 \\
\hline $\mathrm{O}$ & 5.183441 & 1.025017 & -3.969758 \\
\hline C & 2.008539 & 1.259742 & -3.526825 \\
\hline C & 2.372558 & 0.440796 & -2.294456 \\
\hline C & 1.056393 & 0.029098 & -1.643842 \\
\hline$N$ & 1.236414 & -1.007170 & -0.648781 \\
\hline C & 0.299013 & -1.927750 & -0.373780 \\
\hline$N$ & -0.855343 & -1.920458 & -0.991016 \\
\hline$N$ & 0.587706 & -2.880258 & 0.490821 \\
\hline $\mathrm{H}$ & 1.438768 & 2.900947 & -5.211376 \\
\hline $\mathrm{H}$ & 3.557572 & 2.746662 & -3.380300 \\
\hline $\mathrm{H}$ & 1.210866 & 1.943186 & -3.256602 \\
\hline $\mathrm{H}$ & 1.592799 & 0.612104 & -4.289713 \\
\hline $\mathrm{H}$ & 2.948408 & -0.439377 & -2.559505 \\
\hline $\mathrm{H}$ & 2.976597 & 1.023650 & -1.602782 \\
\hline $\mathrm{H}$ & 0.560578 & 0.894051 & -1.216187 \\
\hline $\mathrm{H}$ & 0.400052 & -0.365038 & -2.404392 \\
\hline $\mathrm{H}$ & 1.942194 & -0.877371 & 0.040877 \\
\hline $\mathrm{H}$ & -1.297768 & -1.062276 & -1.309198 \\
\hline $\mathrm{H}$ & -1.528394 & -2.645016 & -0.775507 \\
\hline $\mathrm{H}$ & 1.506491 & -2.992819 & 0.850273 \\
\hline $\mathrm{H}$ & -0.162067 & -3.479780 & 0.822424 \\
\hline$N$ & 4.280031 & 0.941778 & -5.998578 \\
\hline $\mathrm{H}$ & 3.541252 & 1.225888 & -6.608101 \\
\hline C & 5.363424 & 0.116638 & -6.504622 \\
\hline $\mathrm{H}$ & 5.137067 & -0.137711 & -7.532661 \\
\hline
\end{tabular}




\begin{tabular}{|c|c|c|c|}
\hline $\mathrm{H}$ & 5.442848 & -0.800432 & -5.942754 \\
\hline C & 6.736144 & 0.755908 & -6.476882 \\
\hline $\mathrm{O}$ & 7.738718 & 0.089366 & -6.368969 \\
\hline$N$ & 6.766124 & 2.085339 & -6.608293 \\
\hline $\mathrm{H}$ & 5.911948 & 2.604339 & -6.566385 \\
\hline C & 8.005021 & 2.823956 & -6.549017 \\
\hline $\mathrm{H}$ & 8.710228 & 2.440517 & -7.272781 \\
\hline $\mathrm{H}$ & 7.799820 & 3.854828 & -6.804955 \\
\hline C & 8.746724 & 2.826456 & -5.210435 \\
\hline $\mathrm{O}$ & 9.887175 & 3.237466 & -5.157199 \\
\hline$N$ & 8.101429 & 2.335406 & -4.144636 \\
\hline C & 8.681535 & 2.170358 & -2.822752 \\
\hline C & 9.947628 & 1.303165 & -2.830509 \\
\hline $\mathrm{O}$ & 10.765101 & 1.385821 & -1.928230 \\
\hline C & 7.577232 & 1.500558 & -1.974588 \\
\hline C & 7.771989 & 1.380062 & -0.463379 \\
\hline C & 7.427640 & 2.651039 & 0.322537 \\
\hline C & 7.315792 & 2.387413 & 1.820281 \\
\hline$N$ & 8.655413 & 2.356332 & 2.479120 \\
\hline $\mathrm{O}$ & 11.033153 & 1.615196 & 0.896933 \\
\hline $\mathrm{H}$ & 10.884685 & 1.825393 & -0.025412 \\
\hline $\mathrm{H}$ & 11.511050 & 0.788172 & 0.847886 \\
\hline $\mathrm{H}$ & 7.155893 & 2.032135 & -4.258577 \\
\hline $\mathrm{H}$ & 8.978832 & 3.127729 & -2.415574 \\
\hline $\mathrm{H}$ & 6.659843 & 2.042620 & -2.160986 \\
\hline $\mathrm{H}$ & 7.411669 & 0.512780 & -2.388883 \\
\hline $\mathrm{H}$ & 7.092628 & 0.600517 & -0.132528 \\
\hline $\mathrm{H}$ & 8.767415 & 1.028857 & -0.225864 \\
\hline $\mathrm{H}$ & 8.139802 & 3.452371 & 0.146214 \\
\hline & 6.462347 & 3.020645 & -0.008273 \\
\hline
\end{tabular}




\begin{tabular}{|c|c|c|c|}
\hline $\mathrm{H}$ & 6.754126 & 3.160288 & 2.321462 \\
\hline $\mathrm{H}$ & 6.835166 & 1.441414 & 2.016282 \\
\hline $\mathrm{H}$ & 9.366719 & 1.891048 & 1.923897 \\
\hline $\mathrm{H}$ & 8.976059 & 3.326528 & 2.632108 \\
\hline $\mathrm{H}$ & 8.601182 & 1.880366 & 3.367772 \\
\hline$N$ & 10.062468 & 0.448216 & -3.837538 \\
\hline C & 11.109366 & -0.537544 & -4.069419 \\
\hline C & 12.486372 & 0.023143 & -4.427254 \\
\hline $\mathrm{O}$ & 13.444004 & -0.705739 & -4.301638 \\
\hline C & 10.701363 & -1.443263 & -5.253397 \\
\hline C & 9.563117 & -2.441846 & -4.999769 \\
\hline C & 9.132182 & -3.065331 & -6.331061 \\
\hline C & 9.989883 & -3.533367 & -4.018140 \\
\hline $\mathrm{H}$ & 9.324246 & 0.445928 & -4.510632 \\
\hline $\mathrm{H}$ & 11.264674 & -1.125458 & -3.176633 \\
\hline $\mathrm{H}$ & 10.435185 & -0.796967 & -6.084494 \\
\hline $\mathrm{H}$ & 11.584298 & -1.995497 & -5.552179 \\
\hline $\mathrm{H}$ & 8.706457 & -1.911239 & -4.595973 \\
\hline $\mathrm{H}$ & 8.330940 & -3.781801 & -6.177422 \\
\hline $\mathrm{H}$ & 8.775111 & -2.307920 & -7.017149 \\
\hline $\mathrm{H}$ & 9.959527 & -3.592977 & -6.799164 \\
\hline $\mathrm{H}$ & 9.199757 & -4.263157 & -3.878961 \\
\hline $\mathrm{H}$ & 10.859908 & -4.059608 & -4.396973 \\
\hline $\mathrm{H}$ & 10.242747 & -3.137364 & -3.039386 \\
\hline$N$ & 12.574050 & 1.261829 & -4.919242 \\
\hline C & 13.814453 & 1.845370 & -5.393357 \\
\hline C & 14.958485 & 2.034739 & -4.378827 \\
\hline $\mathrm{O}$ & 16.012412 & 2.417529 & -4.811576 \\
\hline C & 13.496419 & 3.171929 & -6.086597 \\
\hline 4 & 12.917141 & 4.088890 & -5.202404 \\
\hline
\end{tabular}




\begin{tabular}{|c|c|c|c|}
\hline $\mathrm{H}$ & 11.756570 & 1.833767 & -4.915474 \\
\hline $\mathrm{H}$ & 14.248723 & 1.178604 & -6.128872 \\
\hline $\mathrm{H}$ & 14.419368 & 3.591232 & -6.455377 \\
\hline $\mathrm{H}$ & 12.845628 & 2.977723 & -6.933290 \\
\hline $\mathrm{H}$ & 11.969675 & 4.078408 & -5.265672 \\
\hline$N$ & 14.792294 & 1.722326 & -3.074279 \\
\hline C & 15.927992 & 1.647230 & -2.176435 \\
\hline C & 16.804495 & 0.409874 & -2.402229 \\
\hline O & 17.934274 & 0.393859 & -1.987681 \\
\hline C & 15.494230 & 1.576952 & -0.707148 \\
\hline C & 14.636405 & 2.733139 & -0.197767 \\
\hline$C$ & 14.280846 & 2.484613 & 1.268455 \\
\hline$N$ & 13.255547 & 3.379242 & 1.790687 \\
\hline C & 13.433452 & 4.530654 & 2.417606 \\
\hline$N$ & 14.651388 & 5.006220 & 2.653408 \\
\hline 0 & 15.028043 & 7.462722 & 4.207124 \\
\hline $\mathrm{H}$ & 14.745070 & 8.349635 & 3.968965 \\
\hline $\mathrm{H}$ & 15.748001 & 7.567698 & 4.816085 \\
\hline$N$ & 12.383758 & 5.219596 & 2.814043 \\
\hline $\mathrm{H}$ & 13.901416 & 1.417906 & -2.758955 \\
\hline $\mathrm{H}$ & 16.561534 & 2.504336 & -2.345514 \\
\hline $\mathrm{H}$ & 14.954330 & 0.648557 & -0.552268 \\
\hline $\mathrm{H}$ & 16.405896 & 1.508159 & -0.123829 \\
\hline $\mathrm{H}$ & 15.153949 & 3.681221 & -0.312268 \\
\hline $\mathrm{H}$ & 13.717995 & 2.814690 & -0.767353 \\
\hline $\mathrm{H}$ & 13.883321 & 1.485481 & 1.373081 \\
\hline $\mathrm{H}$ & 15.159484 & 2.525723 & 1.901468 \\
\hline $\mathrm{H}$ & 12.315325 & 3.083056 & 1.620748 \\
\hline $\mathrm{H}$ & 15.459068 & 4.512343 & 2.356887 \\
\hline $\mathrm{H}$ & 14.787188 & 5.861152 & 3.16489 \\
\hline
\end{tabular}




\begin{tabular}{|c|c|c|c|}
\hline $\mathrm{H}$ & 11.428069 & 4.939741 & 2.656779 \\
\hline $\mathrm{H}$ & 12.476311 & 6.063013 & 3.333653 \\
\hline$N$ & 16.200238 & -0.648980 & -2.967242 \\
\hline C & 16.870485 & -1.911748 & -3.168205 \\
\hline C & 17.667865 & -1.889273 & -4.468826 \\
\hline $\mathrm{H}$ & 18.067003 & -0.910804 & -4.745289 \\
\hline $\mathrm{O}$ & 17.867599 & -2.851332 & -5.135873 \\
\hline C & 15.867590 & -3.070231 & -3.148676 \\
\hline C & 15.324123 & -3.399076 & -1.791036 \\
\hline$N$ & 14.402671 & -2.626651 & -1.112116 \\
\hline 0 & 12.506042 & -0.433892 & -0.462206 \\
\hline $\mathrm{H}$ & 13.143122 & -1.036871 & -0.849396 \\
\hline $\mathrm{H}$ & 12.024862 & -0.037603 & -1.180542 \\
\hline C & 15.619497 & -4.488899 & -1.047858 \\
\hline C & 14.171505 & -3.250081 & -0.003229 \\
\hline $\mathrm{N}$ & 14.882335 & -4.379818 & 0.104639 \\
\hline $\mathrm{H}$ & 15.285715 & -0.550257 & -3.352830 \\
\hline $\mathrm{H}$ & 17.596346 & -2.030782 & -2.371442 \\
\hline $\mathrm{H}$ & 15.050132 & -2.842573 & -3.824055 \\
\hline $\mathrm{H}$ & 16.368086 & -3.944387 & -3.542813 \\
\hline $\mathrm{H}$ & 16.275389 & -5.312474 & -1.232455 \\
\hline $\mathrm{H}$ & 13.492857 & -2.920320 & 0.757312 \\
\hline $\mathrm{H}$ & 14.781793 & -5.062261 & 0.823783 \\
\hline 0 & -15.020213 & -2.471018 & 4.973947 \\
\hline $\mathrm{H}$ & -15.212269 & -1.661971 & 4.507127 \\
\hline $\mathrm{H}$ & -14.526681 & -2.213501 & 5.745680 \\
\hline 0 & -12.902805 & -1.270687 & 6.931187 \\
\hline $\mathrm{H}$ & -12.213112 & -1.722239 & 7.405267 \\
\hline $\mathrm{H}$ & -13.132674 & -0.515337 & 7.458650 \\
\hline $\mathrm{O}$ & -12.055753 & -0.850867 & 4.158774 \\
\hline
\end{tabular}




\begin{tabular}{|c|c|c|c|}
\hline $\mathrm{H}$ & -12.176337 & -0.902832 & 5.104108 \\
\hline $\mathrm{H}$ & -12.868116 & -0.459842 & 3.834508 \\
\hline O & -13.393747 & -1.856946 & 0.710307 \\
\hline $\mathrm{H}$ & -12.618284 & -1.293680 & 0.766814 \\
\hline $\mathrm{H}$ & -13.315966 & -2.505070 & 1.414951 \\
\hline 0 & -7.286578 & 1.302460 & -0.239832 \\
\hline $\mathrm{H}$ & -7.498670 & 1.637792 & 0.634927 \\
\hline $\mathrm{H}$ & -6.357470 & 1.101754 & -0.204429 \\
\hline $\mathrm{O}$ & -7.886340 & 2.078825 & 2.455260 \\
\hline $\mathrm{H}$ & -8.797907 & 1.924358 & 2.690781 \\
\hline $\mathrm{H}$ & -7.559723 & 2.755410 & 3.042588 \\
\hline O & -9.340583 & -0.024714 & -1.607137 \\
\hline $\mathrm{H}$ & -8.578916 & 0.394601 & -1.186585 \\
\hline $\mathrm{H}$ & -9.089673 & -0.922547 & -1.804081 \\
\hline $\mathrm{O}$ & -12.844468 & -2.336472 & -2.019732 \\
\hline $\mathrm{H}$ & -13.120890 & -2.372994 & -1.100710 \\
\hline $\mathrm{H}$ & -13.457233 & -2.846557 & -2.536626 \\
\hline 0 & -14.029220 & 0.929631 & -0.581729 \\
\hline $\mathrm{H}$ & -14.374084 & 0.067218 & -0.375890 \\
\hline $\mathrm{H}$ & -13.118598 & 0.887120 & -0.273370 \\
\hline 0 & -14.252289 & 4.929717 & 4.735637 \\
\hline $\mathrm{H}$ & -13.682720 & 5.570161 & 4.315809 \\
\hline $\mathrm{H}$ & -14.289917 & 5.214909 & 5.644026 \\
\hline 0 & -15.228230 & 2.155337 & 1.701339 \\
\hline $\mathrm{H}$ & -15.850248 & 2.871084 & 1.687644 \\
\hline $\mathrm{H}$ & -14.941090 & 1.991837 & 0.804275 \\
\hline 0 & -13.151682 & 0.247400 & -3.244027 \\
\hline $\mathrm{H}$ & -13.600362 & 0.778017 & -2.588539 \\
\hline $\mathrm{H}$ & -12.933673 & -0.556693 & -2.770726 \\
\hline 0 & -13.770241 & -1.924623 & -4.98113 \\
\hline
\end{tabular}




\begin{tabular}{|c|c|c|c|}
\hline $\mathrm{H}$ & -13.935128 & -1.061137 & -4.609276 \\
\hline $\mathrm{H}$ & -14.496296 & -2.133600 & -5.554536 \\
\hline $\mathrm{O}$ & -10.158517 & -2.764133 & -2.953315 \\
\hline $\mathrm{H}$ & -10.299828 & -2.610238 & -3.881079 \\
\hline $\mathrm{H}$ & -11.020981 & -2.685373 & -2.547665 \\
\hline $\mathrm{O}$ & -11.483664 & 0.213499 & 0.371431 \\
\hline $\mathrm{H}$ & -10.756842 & 0.131628 & -0.247651 \\
\hline $\mathrm{H}$ & -11.143523 & 0.654620 & 1.157231 \\
\hline $\mathrm{O}$ & -10.713541 & 1.341352 & 2.831460 \\
\hline $\mathrm{H}$ & -10.879054 & 0.581125 & 3.386200 \\
\hline $\mathrm{H}$ & -11.394559 & 1.967053 & 3.094746 \\
\hline $\mathrm{O}$ & -13.056431 & 2.612688 & 3.638284 \\
\hline $\mathrm{H}$ & -13.358288 & 3.352888 & 4.167517 \\
\hline $\mathrm{H}$ & -13.711662 & 2.532419 & 2.952964 \\
\hline $\mathrm{O}$ & -10.465899 & 0.624989 & -4.110407 \\
\hline $\mathrm{H}$ & -11.370355 & 0.792546 & -3.839565 \\
\hline $\mathrm{H}$ & -9.979019 & 0.491443 & -3.289473 \\
\hline $\mathrm{O}$ & -14.689453 & -0.342247 & 3.042901 \\
\hline $\mathrm{H}$ & -15.010244 & 0.527555 & 2.814387 \\
\hline $\mathrm{H}$ & -14.562674 & -0.799930 & 2.218332 \\
\hline $\mathrm{O}$ & -13.086321 & -3.361499 & 3.147701 \\
\hline $\mathrm{H}$ & -13.858109 & -3.220234 & 3.705614 \\
\hline $\mathrm{H}$ & -12.459215 & -2.724240 & 3.488715 \\
\hline O & -11.004346 & -1.654020 & -5.646469 \\
\hline $\mathrm{H}$ & -11.934456 & -1.876332 & -5.563288 \\
\hline $\mathrm{H}$ & -10.845416 & -0.947014 & -5.01737 \\
\hline
\end{tabular}


PROTON CONFIGURATION, GIVEN BY NOMINAL CHARGES: R300(+),Y266(-),E183(0) POTENTIAL:0 mV

\begin{tabular}{|c|c|c|c|}
\hline $\mathrm{N}$ & 15.815911 & -6.171507 & 5.162723 \\
\hline $\mathrm{H}$ & 15.012115 & -5.956338 & 5.720342 \\
\hline$C$ & 15.718932 & -5.550103 & 3.839266 \\
\hline$C$ & 15.012365 & -6.475093 & 2.829898 \\
\hline O & 14.264709 & -6.039831 & 1.969796 \\
\hline$C$ & 17.116994 & -5.218940 & 3.303618 \\
\hline $\mathrm{H}$ & 16.617975 & -5.835149 & 5.658171 \\
\hline $\mathrm{H}$ & 15.128406 & -4.643710 & 3.858853 \\
\hline $\mathrm{H}$ & 17.068657 & -4.811800 & 2.300728 \\
\hline $\mathrm{H}$ & 17.583876 & -4.474228 & 3.938259 \\
\hline $\mathrm{H}$ & 17.749422 & -6.099719 & 3.289140 \\
\hline $\mathrm{N}$ & 15.298602 & -7.772676 & 2.926189 \\
\hline $\mathrm{H}$ & 15.890371 & -8.048875 & 3.680154 \\
\hline$C$ & 14.687521 & -8.778222 & 2.055239 \\
\hline $\mathrm{H}$ & 14.756666 & -8.484825 & 1.020508 \\
\hline $\mathrm{H}$ & 15.207847 & -9.717029 & 2.190850 \\
\hline$C$ & 13.208188 & -8.956166 & 2.399416 \\
\hline O & 12.377761 & -9.166801 & 1.554734 \\
\hline $\mathrm{N}$ & 12.882012 & -8.827389 & 3.699958 \\
\hline $\mathrm{H}$ & 13.603979 & -8.750648 & 4.379008 \\
\hline$C$ & 11.501007 & -8.990668 & 4.160085 \\
\hline $\mathrm{H}$ & 11.500864 & -9.001261 & 5.241886 \\
\hline $\mathrm{H}$ & 11.073110 & -9.912783 & 3.798654 \\
\hline$C$ & 10.607647 & -7.843954 & 3.669621 \\
\hline 0 & 9.465056 & -8.044802 & 3.355847 \\
\hline $\mathrm{N}$ & 11.183937 & -6.630558 & 3.646549 \\
\hline$C$ & 10.519402 & -5.456882 & 3.137645 \\
\hline C & 10.320011 & -5.486917 & 1.619588 \\
\hline
\end{tabular}




\begin{tabular}{|c|c|c|c|}
\hline $\mathrm{O}$ & 9.275433 & -5.077566 & 1.172390 \\
\hline C & 11.261767 & -4.199859 & 3.618586 \\
\hline $\mathrm{O}$ & 12.652266 & -4.343916 & 3.599424 \\
\hline $\mathrm{H}$ & 12.141561 & -6.538812 & 3.893979 \\
\hline $\mathrm{H}$ & 9.516113 & -5.412177 & 3.528535 \\
\hline $\mathrm{H}$ & 10.943084 & -3.347172 & 3.029667 \\
\hline $\mathrm{H}$ & 10.997165 & -4.015588 & 4.649510 \\
\hline $\mathrm{H}$ & 12.978203 & -4.499861 & 2.721504 \\
\hline $\mathrm{N}$ & 11.302309 & -5.952837 & 0.828231 \\
\hline C & 11.043830 & -6.125794 & -0.598581 \\
\hline C & 9.920193 & -7.142080 & -0.815398 \\
\hline $\mathrm{O}$ & 9.166260 & -7.039064 & -1.754875 \\
\hline C & 12.337943 & -6.461504 & -1.400249 \\
\hline C & 12.579909 & -7.955836 & -1.649226 \\
\hline C & 12.336292 & -5.708423 & -2.731942 \\
\hline $\mathrm{H}$ & 12.139273 & -6.323653 & 1.222652 \\
\hline $\mathrm{H}$ & 10.645791 & -5.194130 & -0.967833 \\
\hline $\mathrm{H}$ & 13.162736 & -6.080200 & -0.808697 \\
\hline $\mathrm{H}$ & 13.535225 & -8.081946 & -2.148189 \\
\hline $\mathrm{H}$ & 12.599157 & -8.540735 & -0.740568 \\
\hline $\mathrm{H}$ & 11.820913 & -8.370919 & -2.305224 \\
\hline $\mathrm{H}$ & 13.234740 & -5.935553 & -3.294916 \\
\hline $\mathrm{H}$ & 11.480231 & -5.991949 & -3.334339 \\
\hline $\mathrm{H}$ & 12.306844 & -4.636609 & -2.576471 \\
\hline N & 9.827345 & -8.118301 & 0.095592 \\
\hline $\mathrm{H}$ & 10.577542 & -8.244162 & 0.736091 \\
\hline C & 8.879798 & -9.223877 & -0.009149 \\
\hline $\mathrm{H}$ & 9.249095 & -10.026356 & 0.616674 \\
\hline $\mathrm{H}$ & 8.822607 & -9.584183 & -1.024712 \\
\hline 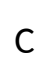 & 7.461395 & -8.899175 & 0.411818 \\
\hline
\end{tabular}




\begin{tabular}{|c|c|c|c|}
\hline $\mathrm{O}$ & 6.546433 & -9.371842 & -0.222127 \\
\hline$N$ & 7.267304 & -8.106214 & 1.471719 \\
\hline C & 5.922028 & -7.662010 & 1.759189 \\
\hline C & 5.467797 & -6.643661 & 0.719015 \\
\hline $\mathrm{O}$ & 4.288010 & -6.579464 & 0.447490 \\
\hline C & 5.664027 & -7.190546 & 3.204285 \\
\hline C & 5.843202 & -8.349467 & 4.190133 \\
\hline C & 6.476248 & -5.967681 & 3.634998 \\
\hline $\mathrm{H}$ & 8.041084 & -7.827455 & 2.034821 \\
\hline $\mathrm{H}$ & 5.261471 & -8.497403 & 1.586382 \\
\hline $\mathrm{H}$ & 4.615521 & -6.908950 & 3.209784 \\
\hline $\mathrm{H}$ & 5.551122 & -8.038826 & 5.187782 \\
\hline $\mathrm{H}$ & 5.225578 & -9.198378 & 3.915493 \\
\hline $\mathrm{H}$ & 6.874807 & -8.680547 & 4.232569 \\
\hline $\mathrm{H}$ & 6.133470 & -5.624046 & 4.605080 \\
\hline $\mathrm{H}$ & 7.526348 & -6.211817 & 3.728883 \\
\hline $\mathrm{H}$ & 6.375877 & -5.141572 & 2.939627 \\
\hline $\mathrm{N}$ & 6.363071 & -5.877460 & 0.087204 \\
\hline$C$ & 5.936976 & -5.048159 & -1.027868 \\
\hline$C$ & 5.525631 & -5.920726 & -2.221910 \\
\hline $\mathrm{O}$ & 4.493851 & -5.686874 & -2.806180 \\
\hline$C$ & 6.996928 & -3.989225 & -1.391640 \\
\hline$C$ & 7.172004 & -2.991739 & -0.232712 \\
\hline$C$ & 6.598023 & -3.262041 & -2.680452 \\
\hline$C$ & 8.441738 & -2.146489 & -0.335961 \\
\hline $\mathrm{H}$ & 7.326475 & -5.909064 & 0.342282 \\
\hline $\mathrm{H}$ & 5.024579 & -4.544371 & -0.746201 \\
\hline $\mathrm{H}$ & 7.941423 & -4.499968 & -1.555842 \\
\hline $\mathrm{H}$ & 6.298333 & -2.342640 & -0.198191 \\
\hline $\mathrm{H}$ & 7.201641 & -3.525092 & 0.708021 \\
\hline
\end{tabular}




\begin{tabular}{|c|c|c|c|}
\hline $\mathrm{H}$ & 7.279065 & -2.447758 & -2.886933 \\
\hline $\mathrm{H}$ & 6.612884 & -3.918794 & -3.540651 \\
\hline $\mathrm{H}$ & 5.598150 & -2.848743 & -2.599071 \\
\hline $\mathrm{H}$ & 8.528653 & -1.488305 & 0.521968 \\
\hline $\mathrm{H}$ & 9.321457 & -2.779131 & -0.354172 \\
\hline $\mathrm{H}$ & 8.455705 & -1.527074 & -1.225707 \\
\hline$N$ & 6.325141 & -6.934911 & -2.580644 \\
\hline $\mathrm{H}$ & 7.225240 & -7.038912 & -2.161767 \\
\hline C & 6.001247 & -7.762011 & -3.723308 \\
\hline $\mathrm{H}$ & 6.848899 & -8.409883 & -3.909055 \\
\hline $\mathrm{H}$ & 5.826972 & -7.163637 & -4.605121 \\
\hline C & 4.755989 & -8.628406 & -3.532713 \\
\hline $\mathrm{O}$ & 3.916813 & -8.694495 & -4.399884 \\
\hline$N$ & 4.667477 & -9.305886 & -2.381526 \\
\hline $\mathrm{H}$ & 5.405486 & -9.252845 & -1.712100 \\
\hline C & 3.503229 & -10.098282 & -2.062547 \\
\hline $\mathrm{H}$ & 3.279708 & -10.803426 & -2.849034 \\
\hline $\mathrm{H}$ & 3.713572 & -10.651382 & -1.155703 \\
\hline C & 2.248189 & -9.253726 & -1.853224 \\
\hline $\mathrm{O}$ & 1.171058 & -9.664212 & -2.221428 \\
\hline$N$ & 2.432037 & -8.060222 & -1.290929 \\
\hline C & 1.278730 & -7.174304 & -1.222456 \\
\hline C & 0.821087 & -6.680836 & -2.597439 \\
\hline $\mathrm{O}$ & -0.365668 & -6.562916 & -2.809270 \\
\hline C & 1.450110 & -5.958168 & -0.316435 \\
\hline $\mathrm{O}$ & 1.609903 & -6.302678 & 1.019995 \\
\hline $\mathrm{H}$ & 3.322655 & -7.755572 & -0.969563 \\
\hline $\mathrm{H}$ & 0.444643 & -7.739153 & -0.839360 \\
\hline $\mathrm{H}$ & 2.267481 & -5.338639 & -0.668220 \\
\hline $\mathrm{H}$ & 0.533492 & -5.397167 & -0.393286 \\
\hline
\end{tabular}




\begin{tabular}{|c|c|c|c|}
\hline $\mathrm{H}$ & 2.521394 & -6.515636 & 1.166869 \\
\hline$N$ & 1.735690 & -6.364452 & -3.523254 \\
\hline C & 1.322748 & -5.943973 & -4.850174 \\
\hline C & 0.580855 & -7.071626 & -5.563151 \\
\hline O & -0.413139 & -6.829197 & -6.216064 \\
\hline C & 2.517962 & -5.398415 & -5.666596 \\
\hline C & 2.889693 & -4.003825 & -5.135603 \\
\hline C & 2.218321 & -5.360277 & -7.170647 \\
\hline C & 4.199134 & -3.450508 & -5.695892 \\
\hline $\mathrm{H}$ & 2.708044 & -6.396674 & -3.303160 \\
\hline $\mathrm{H}$ & 0.584750 & -5.160708 & -4.754566 \\
\hline $\mathrm{H}$ & 3.356524 & -6.069514 & -5.507594 \\
\hline $\mathrm{H}$ & 2.079121 & -3.315016 & -5.367377 \\
\hline $\mathrm{H}$ & 2.968073 & -4.043740 & -4.056935 \\
\hline $\mathrm{H}$ & 3.064376 & -4.956828 & -7.712833 \\
\hline $\mathrm{H}$ & 2.027063 & -6.347486 & -7.573536 \\
\hline $\mathrm{H}$ & 1.354974 & -4.738850 & -7.386983 \\
\hline $\mathrm{H}$ & 4.471026 & -2.538718 & -5.174713 \\
\hline $\mathrm{H}$ & 5.009933 & -4.159439 & -5.561594 \\
\hline $\mathrm{H}$ & 4.131718 & -3.215916 & -6.752424 \\
\hline $\mathrm{N}$ & 1.051637 & -8.314455 & -5.427477 \\
\hline $\mathrm{H}$ & 1.903118 & -8.479454 & -4.931906 \\
\hline$C$ & 0.299633 & -9.430045 & -5.949333 \\
\hline $\mathrm{H}$ & 0.188985 & -9.370557 & -7.021905 \\
\hline $\mathrm{H}$ & 0.833390 & -10.339442 & -5.704314 \\
\hline$C$ & -1.106360 & -9.504557 & -5.359776 \\
\hline 0 & -2.060501 & -9.732088 & -6.069224 \\
\hline $\mathrm{N}$ & -1.211208 & -9.314421 & -4.045751 \\
\hline$C$ & -2.512865 & -9.372129 & -3.423385 \\
\hline$C$ & -3.439843 & -8.244078 & -3.877452 \\
\hline
\end{tabular}




$\begin{array}{llll}\mathrm{O} & -4.629907 & -8.477155 & -3.965478 \\ \mathrm{C} & -2.417153 & -9.359004 & -1.890916 \\ \mathrm{O} & -3.658129 & -9.655855 & -1.311407 \\ \mathrm{H} & -0.393945 & -9.226632 & -3.477870 \\ \mathrm{H} & -3.017615 & -10.280884 & -3.719320 \\ \mathrm{H} & -1.723131 & -10.124238 & -1.572312 \\ \mathrm{H} & -2.045592 & -8.399100 & -1.549005 \\ \mathrm{H} & -4.354114 & -9.250711 & -1.813396 \\ \mathrm{~N} & -2.907816 & -7.043418 & -4.102035 \\ \mathrm{C} & -3.672128 & -5.903629 & -4.584533 \\ \mathrm{C} & -4.250155 & -6.189476 & -5.969087 \\ \mathrm{O} & -5.419612 & -5.966818 & -6.216103 \\ \mathrm{C} & -2.740833 & -4.667344 & -4.583016 \\ \mathrm{C} & -3.413087 & -6.685176 & -6.880158 \\ \mathrm{C} & -3.140595 & -3.505856 & -5.477645 \\ \mathrm{C} & -1.672976 & -1.280946 & -7.570224 \\ \mathrm{C} & -4.452640 & -3.068153 & -5.613869 \\ \mathrm{C} & -2.129356 & -3.164030 & -6.111914 \\ \mathrm{C} & -2.151970 & -2.841425 & -6.196663 \\ \mathrm{C} & -4.768087 & -2.019308 & -6.463105 \\ \mathrm{C} & -2.457839 & -1.778325 & -7.029332 \\ \mathrm{H} & -3.773179 & -1.369202 & -7.173458 \\ \mathrm{H} & -1.940464 & -6.907594 & -3.891406 \\ \mathrm{H} & -2.623322 & -5.730873 & -3.944494 \\ \mathrm{H} & -0.559636 & -8.204042\end{array}$




\begin{tabular}{|c|c|c|c|}
\hline C & -5.017455 & -8.043767 & -8.143195 \\
\hline $\mathrm{O}$ & -5.995957 & -7.920163 & -8.835136 \\
\hline C & -2.771445 & -7.517283 & -9.105095 \\
\hline$S$ & -1.573702 & -6.249553 & -9.627714 \\
\hline $\mathrm{H}$ & -2.467960 & -6.887333 & -6.625516 \\
\hline $\mathrm{H}$ & -4.344116 & -6.129229 & -8.649026 \\
\hline $\mathrm{H}$ & -2.250491 & -8.342440 & -8.638619 \\
\hline $\mathrm{H}$ & -3.211211 & -7.881628 & -10.024132 \\
\hline $\mathrm{H}$ & -0.934757 & -6.111133 & -8.476292 \\
\hline$N$ & -4.844098 & -9.092525 & -7.315832 \\
\hline $\mathrm{H}$ & -3.986326 & -9.228331 & -6.823724 \\
\hline C & -5.823744 & -10.166269 & -7.361259 \\
\hline $\mathrm{H}$ & -5.998837 & -10.483794 & -8.378229 \\
\hline $\mathrm{H}$ & -5.423212 & -11.002472 & -6.800525 \\
\hline C & -7.201995 & -9.825979 & -6.773518 \\
\hline $\mathrm{O}$ & -8.216432 & -10.208508 & -7.288888 \\
\hline$N$ & -7.173193 & -9.142581 & -5.624140 \\
\hline C & -8.391642 & -8.823245 & -4.901199 \\
\hline C & -9.283781 & -7.797061 & -5.591752 \\
\hline $\mathrm{O}$ & -10.466389 & -7.765069 & -5.375929 \\
\hline C & -8.096228 & -8.332563 & -3.471062 \\
\hline C & -7.205135 & -7.086206 & -3.329419 \\
\hline$C$ & -7.800066 & -5.706614 & -3.216403 \\
\hline 0 & -9.097387 & -5.610557 & -3.211020 \\
\hline $\mathrm{H}$ & -9.355896 & -4.698419 & -3.016040 \\
\hline 0 & -7.078197 & -4.742800 & -3.109370 \\
\hline $\mathrm{H}$ & -6.290394 & -8.899911 & -5.226338 \\
\hline $\mathrm{H}$ & -9.000226 & -9.713712 & -4.828684 \\
\hline $\mathrm{H}$ & -9.047620 & -8.180115 & -2.980848 \\
\hline $\mathrm{H}$ & -7.596964 & -9.147277 & -2.957528 \\
\hline
\end{tabular}




\begin{tabular}{|c|c|c|c|}
\hline $\mathrm{H}$ & -6.608600 & -7.182011 & -2.429863 \\
\hline $\mathrm{H}$ & -6.487896 & -7.019283 & -4.128150 \\
\hline $\mathrm{N}$ & -8.660391 & -6.884845 & -6.361752 \\
\hline C & -9.415942 & -5.859912 & -7.059435 \\
\hline C & -10.431904 & -6.481777 & -8.011729 \\
\hline $\mathrm{H}$ & -10.231784 & -7.508021 & -8.323554 \\
\hline 0 & -11.380705 & -5.879493 & -8.400540 \\
\hline C & -8.417634 & -4.915254 & -7.759585 \\
\hline O & -7.705796 & -4.201511 & -6.767873 \\
\hline C & -9.062589 & -3.889129 & -8.679890 \\
\hline $\mathrm{H}$ & -7.723338 & -7.068918 & -6.648105 \\
\hline $\mathrm{H}$ & -9.984924 & -5.285577 & -6.339541 \\
\hline $\mathrm{H}$ & -7.716845 & -5.510514 & -8.335664 \\
\hline $\mathrm{H}$ & -6.960535 & -4.713374 & -6.461190 \\
\hline $\mathrm{H}$ & -8.291656 & -3.209303 & -9.024310 \\
\hline $\mathrm{H}$ & -9.524748 & -4.355751 & -9.538420 \\
\hline $\mathrm{H}$ & -9.826674 & -3.318814 & -8.162469 \\
\hline $\mathrm{N}$ & -12.648687 & -9.488839 & 3.058955 \\
\hline $\mathrm{H}$ & -12.898815 & -10.006846 & 2.242047 \\
\hline C & -11.501708 & -8.600016 & 2.799741 \\
\hline C & -10.502043 & -8.566087 & 3.937168 \\
\hline $\mathrm{O}$ & -9.378989 & -8.139091 & 3.740185 \\
\hline C & -11.938870 & -7.137400 & 2.575382 \\
\hline o & -12.528045 & -6.745581 & 3.788926 \\
\hline C & -12.915009 & -6.968443 & 1.416922 \\
\hline $\mathrm{H}$ & -13.445335 & -8.955729 & 3.348272 \\
\hline $\mathrm{H}$ & -10.957018 & -8.950087 & 1.934190 \\
\hline $\mathrm{H}$ & -11.048317 & -6.553161 & 2.384466 \\
\hline $\mathrm{H}$ & -12.575610 & -5.792938 & 3.839620 \\
\hline t & -13.114900 & -5.913419 & 1.263050 \\
\hline
\end{tabular}




\begin{tabular}{|c|c|c|c|}
\hline $\mathrm{H}$ & -12.519116 & -7.371986 & 0.492343 \\
\hline $\mathrm{H}$ & -13.859641 & -7.454439 & 1.629614 \\
\hline$N$ & -10.894750 & -8.995872 & 5.126307 \\
\hline $\mathrm{H}$ & -11.816196 & -9.373014 & 5.168304 \\
\hline C & -10.099020 & -8.986222 & 6.329155 \\
\hline $\mathrm{H}$ & -10.040548 & -7.993589 & 6.747950 \\
\hline $\mathrm{H}$ & -10.599214 & -9.614499 & 7.056662 \\
\hline$C$ & -8.649982 & -9.465316 & 6.235949 \\
\hline $\mathrm{O}$ & -7.827061 & -8.892465 & 6.906533 \\
\hline $\mathrm{N}$ & -8.309789 & -10.518610 & 5.470870 \\
\hline $\mathrm{H}$ & -9.009768 & -11.003230 & 4.955995 \\
\hline$C$ & -6.910425 & -10.909733 & 5.351390 \\
\hline $\mathrm{H}$ & -6.869764 & -11.878907 & 4.870802 \\
\hline $\mathrm{H}$ & -6.461186 & -10.995747 & 6.327774 \\
\hline$C$ & -6.060457 & -9.924029 & 4.525509 \\
\hline O & -4.895128 & -9.755453 & 4.776420 \\
\hline $\mathrm{N}$ & -6.740983 & -9.331139 & 3.550898 \\
\hline$C$ & -6.033225 & -8.278275 & 2.833869 \\
\hline$C$ & -6.007952 & -7.002819 & 3.669651 \\
\hline 0 & -5.030775 & -6.291139 & 3.650802 \\
\hline$C$ & -6.396261 & -8.084692 & 1.346360 \\
\hline$C$ & -7.568891 & -7.207952 & 0.955169 \\
\hline$C$ & -7.543264 & -5.834533 & 1.182797 \\
\hline$C$ & -8.642130 & -7.737862 & 0.251122 \\
\hline$C$ & -8.578963 & -5.022811 & 0.752165 \\
\hline$C$ & -9.666735 & -6.923897 & -0.205355 \\
\hline$C$ & -9.647577 & -5.564417 & 0.056905 \\
\hline $\mathrm{H}$ & -7.735512 & -9.354337 & 3.536299 \\
\hline $\mathrm{H}$ & -5.003903 & -8.591058 & 2.825407 \\
\hline $\mathrm{H}$ & -5.505074 & -7.672503 & 0.885742 \\
\hline
\end{tabular}




\begin{tabular}{|c|c|c|c|}
\hline $\mathrm{H}$ & -6.517106 & -9.076108 & 0.926010 \\
\hline $\mathrm{H}$ & -6.700272 & -5.388422 & 1.677479 \\
\hline $\mathrm{H}$ & -8.665919 & -8.791570 & 0.031549 \\
\hline $\mathrm{H}$ & -8.537277 & -3.963588 & 0.934797 \\
\hline $\mathrm{H}$ & -10.466721 & -7.347268 & -0.784215 \\
\hline $\mathrm{H}$ & -10.442741 & -4.930731 & -0.290120 \\
\hline$N$ & -7.049127 & -6.748929 & 4.464945 \\
\hline C & -7.024620 & -5.641532 & 5.388471 \\
\hline C & -5.905281 & -5.827862 & 6.411844 \\
\hline 0 & -5.303885 & -4.855587 & 6.816474 \\
\hline C & -8.412228 & -5.508044 & 6.057831 \\
\hline C & -8.770988 & -4.119843 & 6.543022 \\
\hline C & -9.060277 & -3.113744 & 5.622108 \\
\hline C & -8.880458 & -3.833445 & 7.895282 \\
\hline C & -9.444169 & -1.852038 & 6.042019 \\
\hline$C$ & -9.264792 & -2.569863 & 8.323131 \\
\hline$C$ & -9.545565 & -1.577011 & 7.400791 \\
\hline $\mathrm{H}$ & -7.898873 & -7.253945 & 4.337922 \\
\hline $\mathrm{H}$ & -6.778110 & -4.727777 & 4.867053 \\
\hline $\mathrm{H}$ & -9.148563 & -5.804355 & 5.319014 \\
\hline $\mathrm{H}$ & -8.483927 & -6.216503 & 6.873131 \\
\hline $\mathrm{H}$ & -8.976501 & -3.317597 & 4.567203 \\
\hline $\mathrm{H}$ & -8.664250 & -4.595398 & 8.623286 \\
\hline $\mathrm{H}$ & -9.661181 & -1.090200 & 5.315373 \\
\hline $\mathrm{H}$ & -9.334441 & -2.363724 & 9.376175 \\
\hline $\mathrm{H}$ & -9.816783 & -0.590633 & 7.732615 \\
\hline $\mathrm{N}$ & -5.619961 & -7.062417 & 6.820643 \\
\hline $\mathrm{H}$ & -6.245690 & -7.803215 & 6.594606 \\
\hline$C$ & -4.545704 & -7.337510 & 7.748180 \\
\hline $\mathrm{H}$ & -4.601650 & -6.706879 & 8.622582 \\
\hline
\end{tabular}




\begin{tabular}{|c|c|c|c|}
\hline $\mathrm{H}$ & -4.643967 & -8.368186 & 8.064548 \\
\hline$C$ & -3.148347 & -7.140437 & 7.163329 \\
\hline 0 & -2.289709 & -6.625997 & 7.839962 \\
\hline $\mathrm{N}$ & -2.927161 & -7.568375 & 5.915304 \\
\hline$C$ & -1.644480 & -7.363947 & 5.259385 \\
\hline$C$ & -1.417029 & -5.885553 & 4.915951 \\
\hline O & -0.325947 & -5.386531 & 5.090879 \\
\hline$C$ & -1.534355 & -8.285086 & 4.024036 \\
\hline$C$ & -0.365100 & -7.899908 & 3.114967 \\
\hline$C$ & -1.412866 & -9.747757 & 4.466376 \\
\hline $\mathrm{H}$ & -3.647561 & -8.058372 & 5.429539 \\
\hline $\mathrm{H}$ & -0.854950 & -7.604622 & 5.956511 \\
\hline $\mathrm{H}$ & -2.451610 & -8.172182 & 3.450721 \\
\hline $\mathrm{H}$ & -0.282651 & -8.616523 & 2.304696 \\
\hline $\mathrm{H}$ & -0.483529 & -6.922214 & 2.669145 \\
\hline $\mathrm{H}$ & 0.574127 & -7.904811 & 3.659889 \\
\hline $\mathrm{H}$ & -1.406955 & -10.400258 & 3.599250 \\
\hline $\mathrm{H}$ & -0.481722 & -9.904223 & 5.005130 \\
\hline $\mathrm{H}$ & -2.231111 & -10.057025 & 5.103644 \\
\hline$N$ & -2.462416 & -5.209014 & 4.428393 \\
\hline$C$ & -2.351452 & -3.823587 & 4.014602 \\
\hline$C$ & -2.255259 & -2.904640 & 5.230666 \\
\hline 0 & -1.384995 & -2.064571 & 5.292789 \\
\hline$C$ & -3.523300 & -3.394152 & 3.120749 \\
\hline$C$ & -3.698811 & -4.161346 & 1.800074 \\
\hline$C$ & -2.559594 & -4.000107 & 0.806157 \\
\hline 0 & -1.425530 & -4.298592 & 1.205748 \\
\hline 0 & -2.839077 & -3.594287 & -0.336331 \\
\hline $\mathrm{H}$ & -3.319862 & -5.689625 & 4.266155 \\
\hline $\mathrm{H}$ & -1.431504 & -3.693230 & 3.476674 \\
\hline
\end{tabular}




\begin{tabular}{|c|c|c|c|}
\hline $\mathrm{H}$ & -4.447910 & -3.478920 & 3.683518 \\
\hline $\mathrm{H}$ & -3.385731 & -2.337629 & 2.908573 \\
\hline $\mathrm{H}$ & -3.805050 & -5.217214 & 2.001896 \\
\hline $\mathrm{H}$ & -4.617668 & -3.823819 & 1.333817 \\
\hline$N$ & -3.159420 & -3.058434 & 6.216537 \\
\hline C & -2.965211 & -2.314132 & 7.438570 \\
\hline C & -1.638603 & -2.690243 & 8.107663 \\
\hline $\mathrm{O}$ & -0.994708 & -1.847714 & 8.694142 \\
\hline C & -4.101043 & -2.331440 & 8.472183 \\
\hline $\mathrm{O}$ & -4.279417 & -3.583414 & 9.065579 \\
\hline C & -5.398045 & -1.740572 & 7.929362 \\
\hline $\mathrm{H}$ & -3.822660 & -3.800511 & 6.181485 \\
\hline $\mathrm{H}$ & -2.832323 & -1.278631 & 7.166347 \\
\hline $\mathrm{H}$ & -3.744373 & -1.689655 & 9.268072 \\
\hline $\mathrm{H}$ & -4.713583 & -4.161683 & 8.450169 \\
\hline $\mathrm{H}$ & -6.135168 & -1.712113 & 8.723086 \\
\hline $\mathrm{H}$ & -5.238320 & -0.726688 & 7.575824 \\
\hline $\mathrm{H}$ & -5.800833 & -2.327256 & 7.114479 \\
\hline$N$ & -1.197653 & -3.951979 & 7.973464 \\
\hline $\mathrm{H}$ & -1.830960 & -4.682641 & 7.734735 \\
\hline C & 0.003080 & -4.342738 & 8.667209 \\
\hline $\mathrm{H}$ & -0.059057 & -4.157051 & 9.730255 \\
\hline $\mathrm{H}$ & 0.146161 & -5.402676 & 8.503573 \\
\hline C & 1.237435 & -3.607536 & 8.171552 \\
\hline $\mathrm{O}$ & 2.102829 & -3.265860 & 8.945185 \\
\hline$N$ & 1.325146 & -3.403013 & 6.841039 \\
\hline C & 2.486714 & -2.759176 & 6.293230 \\
\hline C & 2.387884 & -1.240921 & 6.125658 \\
\hline $\mathrm{O}$ & 3.434717 & -0.641171 & 5.966455 \\
\hline C & 3.049315 & -3.443415 & 5.048091 \\
\hline
\end{tabular}




\begin{tabular}{|c|c|c|c|}
\hline$S$ & 2.128357 & -3.210935 & 3.503743 \\
\hline $\mathrm{H}$ & 0.602257 & -3.757359 & 6.246744 \\
\hline $\mathrm{H}$ & 3.257834 & -2.853466 & 7.042792 \\
\hline $\mathrm{H}$ & 4.030048 & -3.030993 & 4.859988 \\
\hline $\mathrm{H}$ & 3.158248 & -4.500369 & 5.248178 \\
\hline $\mathrm{H}$ & 1.129924 & -4.055273 & 3.717552 \\
\hline $\mathrm{N}$ & 1.217735 & -0.611864 & 6.259419 \\
\hline C & 1.238052 & 0.824396 & 6.510019 \\
\hline C & 1.794097 & 1.110658 & 7.890819 \\
\hline 0 & 2.380680 & 2.139145 & 8.125306 \\
\hline C & -0.090012 & 1.560084 & 6.229855 \\
\hline C & -1.170786 & 1.337480 & 7.298077 \\
\hline C & -0.558518 & 1.263495 & 4.800381 \\
\hline C & -2.468403 & 2.103498 & 7.031414 \\
\hline $\mathrm{H}$ & 0.367182 & -1.136075 & 6.287271 \\
\hline $\mathrm{H}$ & 1.965612 & 1.245472 & 5.835012 \\
\hline $\mathrm{H}$ & 0.180966 & 2.609235 & 6.280873 \\
\hline $\mathrm{H}$ & -1.385652 & 0.283748 & 7.404624 \\
\hline $\mathrm{H}$ & -0.779411 & 1.661131 & 8.258154 \\
\hline $\mathrm{H}$ & -1.339599 & 1.949919 & 4.499413 \\
\hline $\mathrm{H}$ & 0.261485 & 1.378762 & 4.096987 \\
\hline $\mathrm{H}$ & -0.939427 & 0.256355 & 4.697867 \\
\hline $\mathrm{H}$ & -3.126271 & 2.039165 & 7.892814 \\
\hline $\mathrm{H}$ & -2.278363 & 3.156373 & 6.839105 \\
\hline $\mathrm{H}$ & -3.006759 & 1.703073 & 6.179312 \\
\hline $\mathrm{N}$ & 1.624535 & 0.134949 & 8.790803 \\
\hline $\mathrm{H}$ & 0.978993 & -0.601056 & 8.604012 \\
\hline C & 2.056048 & 0.307676 & 10.161042 \\
\hline $\mathrm{H}$ & 1.574375 & -0.455910 & 10.758663 \\
\hline $\mathrm{H}$ & 1.788502 & 1.281710 & 10.542231 \\
\hline
\end{tabular}




\begin{tabular}{|c|c|c|c|}
\hline C & 3.565620 & 0.174490 & 10.291442 \\
\hline $\mathrm{O}$ & 4.230222 & 0.975923 & 10.888992 \\
\hline$N$ & 4.109447 & -0.890960 & 9.658440 \\
\hline $\mathrm{H}$ & 3.517903 & -1.620984 & 9.320683 \\
\hline C & 5.539001 & -1.035248 & 9.619347 \\
\hline $\mathrm{H}$ & 5.776951 & -1.990977 & 9.166794 \\
\hline $\mathrm{H}$ & 5.975315 & -1.007508 & 10.607224 \\
\hline C & 6.235779 & 0.058236 & 8.812790 \\
\hline $\mathrm{O}$ & 7.365000 & 0.374501 & 9.095208 \\
\hline$N$ & 5.560517 & 0.564232 & 7.781950 \\
\hline C & 6.122095 & 1.732150 & 7.151180 \\
\hline C & 5.506677 & 3.022334 & 7.686752 \\
\hline $\mathrm{H}$ & 5.007124 & 2.947621 & 8.651217 \\
\hline $\mathrm{O}$ & 5.603153 & 4.057193 & 7.102411 \\
\hline C & 6.018820 & 1.678819 & 5.624689 \\
\hline C & 6.800787 & 0.546473 & 4.991476 \\
\hline C & 8.173076 & 0.399184 & 5.197993 \\
\hline C & 6.161161 & -0.364379 & 4.155921 \\
\hline C & 8.885539 & -0.612384 & 4.568227 \\
\hline C & 6.871254 & -1.373758 & 3.524991 \\
\hline C & 8.237285 & -1.497815 & 3.721170 \\
\hline $\mathrm{H}$ & 4.638390 & 0.260910 & 7.558519 \\
\hline $\mathrm{H}$ & 7.158684 & 1.768431 & 7.450170 \\
\hline $\mathrm{H}$ & 4.977120 & 1.590720 & 5.342930 \\
\hline $\mathrm{H}$ & 6.367648 & 2.632409 & 5.243407 \\
\hline $\mathrm{H}$ & 8.686791 & 1.057202 & 5.877802 \\
\hline $\mathrm{H}$ & 5.097511 & -0.297577 & 4.028059 \\
\hline $\mathrm{H}$ & 9.941252 & -0.711803 & 4.753095 \\
\hline $\mathrm{H}$ & 6.354816 & -2.068333 & 2.886670 \\
\hline $\mathrm{H}$ & 8.777869 & -2.284269 & 3.227130 \\
\hline
\end{tabular}




\begin{tabular}{|c|c|c|c|}
\hline$N$ & 12.361248 & 9.925824 & -1.111669 \\
\hline $\mathrm{H}$ & 11.607165 & 10.236416 & -1.695397 \\
\hline C & 12.093075 & 8.575192 & -0.609438 \\
\hline C & 11.064833 & 8.708373 & 0.506884 \\
\hline $\mathrm{O}$ & 10.076589 & 8.007466 & 0.528494 \\
\hline C & 13.372990 & 7.915314 & -0.077231 \\
\hline C & 14.457403 & 7.663971 & -1.130498 \\
\hline$S$ & 13.852162 & 6.602173 & -2.474096 \\
\hline C & 15.332976 & 6.516953 & -3.509331 \\
\hline $\mathrm{H}$ & 13.175987 & 9.936922 & -1.692771 \\
\hline $\mathrm{H}$ & 11.636210 & 7.929764 & -1.348324 \\
\hline $\mathrm{H}$ & 13.798365 & 8.534853 & 0.706508 \\
\hline $\mathrm{H}$ & 13.094939 & 6.969780 & 0.374010 \\
\hline $\mathrm{H}$ & 14.829618 & 8.596577 & -1.539453 \\
\hline $\mathrm{H}$ & 15.298874 & 7.183920 & -0.643812 \\
\hline $\mathrm{H}$ & 15.095814 & 5.872993 & -4.342489 \\
\hline $\mathrm{H}$ & 15.603069 & 7.497037 & -3.880799 \\
\hline $\mathrm{H}$ & 16.166126 & 6.091458 & -2.965280 \\
\hline$N$ & 11.273753 & 9.657624 & 1.435601 \\
\hline C & 10.366455 & 9.803216 & 2.556976 \\
\hline C & 9.028867 & 10.442990 & 2.182404 \\
\hline $\mathrm{O}$ & 8.044381 & 10.182399 & 2.827618 \\
\hline C & 11.035541 & 10.557243 & 3.712854 \\
\hline C & 12.231983 & 9.773158 & 4.220211 \\
\hline $\mathrm{O}$ & 13.357221 & 10.073305 & 3.879898 \\
\hline$N$ & 11.960963 & 8.744733 & 5.033726 \\
\hline $\mathrm{H}$ & 12.081004 & 10.237273 & 1.351557 \\
\hline $\mathrm{H}$ & 10.096464 & 8.817616 & 2.899843 \\
\hline $\mathrm{H}$ & 11.388413 & 11.531527 & 3.398664 \\
\hline 11 & 10.306347 & 10.688797 & 4.502252 \\
\hline
\end{tabular}




\begin{tabular}{|c|c|c|c|}
\hline $\mathrm{H}$ & 12.722525 & 8.163167 & 5.309693 \\
\hline $\mathrm{H}$ & 11.050654 & 8.332288 & 5.065346 \\
\hline$N$ & 8.986275 & 11.257116 & 1.110692 \\
\hline $\mathrm{H}$ & 9.824694 & 11.449963 & 0.612326 \\
\hline C & 7.710276 & 11.682666 & 0.574253 \\
\hline $\mathrm{H}$ & 7.882141 & 12.446668 & -0.173097 \\
\hline $\mathrm{H}$ & 7.088658 & 12.098225 & 1.350430 \\
\hline C & 6.920876 & 10.540892 & -0.068393 \\
\hline O & 5.719031 & 10.595894 & -0.100007 \\
\hline$N$ & 7.623050 & 9.519392 & -0.602366 \\
\hline C & 6.960358 & 8.323386 & -1.093860 \\
\hline C & 6.455159 & 7.440744 & 0.058105 \\
\hline O & 5.367844 & 6.921273 & -0.026341 \\
\hline C & 7.853574 & 7.566587 & -2.105710 \\
\hline C & 8.118482 & 8.453259 & -3.337450 \\
\hline C & 7.216500 & 6.232894 & -2.514202 \\
\hline C & 9.211617 & 7.929022 & -4.271868 \\
\hline $\mathrm{H}$ & 8.587225 & 9.437195 & -0.380283 \\
\hline $\mathrm{H}$ & 6.057923 & 8.635863 & -1.596173 \\
\hline $\mathrm{H}$ & 8.801229 & 7.354414 & -1.618956 \\
\hline $\mathrm{H}$ & 7.190092 & 8.567423 & -3.892641 \\
\hline $\mathrm{H}$ & 8.396868 & 9.449567 & -3.010333 \\
\hline $\mathrm{H}$ & 7.844015 & 5.723455 & -3.234362 \\
\hline $\mathrm{H}$ & 7.086215 & 5.570229 & -1.667335 \\
\hline $\mathrm{H}$ & 6.240564 & 6.380765 & -2.964738 \\
\hline $\mathrm{H}$ & 9.382972 & 8.631781 & -5.080816 \\
\hline $\mathrm{H}$ & 10.153718 & 7.796296 & -3.746844 \\
\hline $\mathrm{H}$ & 8.953630 & 6.977455 & -4.721733 \\
\hline$N$ & 7.218120 & 7.313118 & 1.162330 \\
\hline C & 6.737683 & 6.556151 & 2.306925 \\
\hline
\end{tabular}




\begin{tabular}{|c|c|c|c|}
\hline C & 5.372651 & 7.086484 & 2.765458 \\
\hline $\mathrm{O}$ & 4.444387 & 6.346364 & 2.983614 \\
\hline C & 7.696646 & 6.645266 & 3.504442 \\
\hline C & 9.131690 & 6.140408 & 3.323947 \\
\hline $\mathrm{O}$ & 10.009432 & 6.788324 & 3.887800 \\
\hline $\mathrm{O}$ & 9.330287 & 5.097239 & 2.652589 \\
\hline $\mathrm{H}$ & 8.170779 & 7.607712 & 1.140359 \\
\hline $\mathrm{H}$ & 6.569782 & 5.527992 & 2.026480 \\
\hline $\mathrm{H}$ & 7.757910 & 7.664687 & 3.855564 \\
\hline $\mathrm{H}$ & 7.259832 & 6.061540 & 4.312146 \\
\hline$N$ & 5.294712 & 8.419499 & 2.941340 \\
\hline $\mathrm{H}$ & 6.108013 & 8.982643 & 2.810320 \\
\hline C & 4.087638 & 9.040679 & 3.428277 \\
\hline $\mathrm{H}$ & 4.298045 & 10.086107 & 3.616558 \\
\hline $\mathrm{H}$ & 3.761212 & 8.596211 & 4.355205 \\
\hline C & 2.922311 & 8.979805 & 2.450004 \\
\hline $\mathrm{O}$ & 1.780343 & 9.070771 & 2.838296 \\
\hline$N$ & 3.253967 & 8.852155 & 1.162305 \\
\hline $\mathrm{H}$ & 4.213960 & 8.872983 & 0.909639 \\
\hline C & 2.295757 & 8.928673 & 0.103162 \\
\hline $\mathrm{H}$ & 2.829052 & 9.148479 & -0.812753 \\
\hline $\mathrm{H}$ & 1.600012 & 9.738683 & 0.273361 \\
\hline C & 1.420403 & 7.690813 & -0.149664 \\
\hline $\mathrm{O}$ & 0.380838 & 7.805684 & -0.738045 \\
\hline$N$ & 1.912066 & 6.546181 & 0.362212 \\
\hline C & 1.123506 & 5.352439 & 0.553946 \\
\hline C & 0.313178 & 5.409139 & 1.853539 \\
\hline $\mathrm{O}$ & -0.877361 & 5.209508 & 1.833230 \\
\hline C & 2.012984 & 4.110251 & 0.518352 \\
\hline $\mathrm{H}$ & 2.813662 & 6.590612 & 0.787695 \\
\hline
\end{tabular}




\begin{tabular}{|c|c|c|c|}
\hline $\mathrm{H}$ & 0.390839 & 5.297549 & -0.233497 \\
\hline $\mathrm{H}$ & 1.419564 & 3.220840 & 0.699178 \\
\hline $\mathrm{H}$ & 2.479393 & 4.029755 & -0.456658 \\
\hline $\mathrm{H}$ & 2.795442 & 4.161629 & 1.268589 \\
\hline $\mathrm{N}$ & 0.955799 & 5.660374 & 3.011342 \\
\hline$C$ & 0.239445 & 5.450441 & 4.260131 \\
\hline$C$ & -0.898248 & 6.451699 & 4.443906 \\
\hline 0 & -1.921349 & 6.116603 & 5.001188 \\
\hline$C$ & 1.170294 & 5.375023 & 5.495596 \\
\hline$C$ & 1.729503 & 6.726861 & 5.965015 \\
\hline$C$ & 2.284753 & 4.345685 & 5.253594 \\
\hline$C$ & 2.583608 & 6.626235 & 7.231326 \\
\hline $\mathrm{H}$ & 1.941700 & 5.807866 & 3.023563 \\
\hline $\mathrm{H}$ & -0.265507 & 4.497618 & 4.190218 \\
\hline $\mathrm{H}$ & 0.531177 & 5.000233 & 6.289362 \\
\hline $\mathrm{H}$ & 2.314368 & 7.172563 & 5.167081 \\
\hline $\mathrm{H}$ & 0.908238 & 7.408459 & 6.161388 \\
\hline $\mathrm{H}$ & 2.676532 & 3.981320 & 6.191941 \\
\hline $\mathrm{H}$ & 1.909816 & 3.491205 & 4.699318 \\
\hline $\mathrm{H}$ & 3.109279 & 4.772504 & 4.692781 \\
\hline $\mathrm{H}$ & 2.864117 & 7.618261 & 7.573014 \\
\hline $\mathrm{H}$ & 2.032374 & 6.146875 & 8.034925 \\
\hline $\mathrm{H}$ & 3.493833 & 6.061115 & 7.073924 \\
\hline $\mathrm{N}$ & -0.713871 & 7.698838 & 3.986985 \\
\hline $\mathrm{H}$ & 0.160725 & 7.965963 & 3.585406 \\
\hline$C$ & -1.707708 & 8.711512 & 4.224758 \\
\hline $\mathrm{H}$ & -1.322197 & 9.656200 & 3.862518 \\
\hline $\mathrm{H}$ & -1.918129 & 8.817721 & 5.279115 \\
\hline$C$ & -3.055908 & 8.418387 & 3.572063 \\
\hline 0 & -4.061986 & 8.598362 & 4.218495 \\
\hline
\end{tabular}




\begin{tabular}{|c|c|c|c|}
\hline$N$ & -3.085487 & 7.934665 & 2.316397 \\
\hline C & -4.424396 & 7.823301 & 1.734004 \\
\hline C & -5.116728 & 6.512006 & 2.064702 \\
\hline $\mathrm{O}$ & -6.328728 & 6.465419 & 2.052662 \\
\hline C & -4.227832 & 8.114139 & 0.239506 \\
\hline C & -2.986630 & 9.013024 & 0.255735 \\
\hline C & -2.106725 & 8.338901 & 1.296390 \\
\hline $\mathrm{H}$ & -5.062018 & 8.581303 & 2.161601 \\
\hline $\mathrm{H}$ & -4.041579 & 7.202242 & -0.312361 \\
\hline $\mathrm{H}$ & -5.105584 & 8.586759 & -0.182585 \\
\hline $\mathrm{H}$ & -2.486164 & 9.104999 & -0.690577 \\
\hline $\mathrm{H}$ & -3.260891 & 10.009845 & 0.592859 \\
\hline $\mathrm{H}$ & -1.610347 & 7.471344 & 0.900543 \\
\hline $\mathrm{H}$ & -1.355957 & 8.996173 & 1.703517 \\
\hline$N$ & -4.347909 & 5.461800 & 2.378965 \\
\hline$C$ & -4.936672 & 4.237281 & 2.892134 \\
\hline$C$ & -5.664171 & 4.491604 & 4.215738 \\
\hline 0 & -6.726167 & 3.954287 & 4.441223 \\
\hline$C$ & -3.879793 & 3.126534 & 3.083612 \\
\hline$C$ & -3.508391 & 2.339492 & 1.842038 \\
\hline$C$ & -3.840413 & 0.993666 & 1.727777 \\
\hline$C$ & -2.778502 & 2.895067 & 0.792263 \\
\hline$C$ & -3.488422 & 0.236764 & 0.619653 \\
\hline$C$ & -2.410634 & 2.155176 & -0.311044 \\
\hline$C$ & -2.756568 & 0.794211 & -0.450244 \\
\hline $\mathrm{O}$ & -2.435971 & 0.116882 & -1.507373 \\
\hline $\mathrm{H}$ & -3.358657 & 5.587871 & 2.429389 \\
\hline $\mathrm{H}$ & -5.699459 & 3.898649 & 2.208395 \\
\hline $\mathrm{H}$ & -3.000335 & 3.572773 & 3.539111 \\
\hline $\mathrm{H}$ & -4.281092 & 2.432575 & 3.814075 \\
\hline
\end{tabular}




\begin{tabular}{|c|c|c|c|}
\hline $\mathrm{H}$ & -4.389685 & 0.516311 & 2.523769 \\
\hline $\mathrm{H}$ & -2.469836 & 3.919371 & 0.842249 \\
\hline $\mathrm{H}$ & -3.741596 & -0.808619 & 0.575727 \\
\hline $\mathrm{H}$ & -1.840033 & 2.616058 & -1.099800 \\
\hline$N$ & -5.067567 & 5.302063 & 5.107633 \\
\hline $\mathrm{H}$ & -4.145253 & 5.644315 & 4.932713 \\
\hline C & -5.693050 & 5.616465 & 6.372271 \\
\hline $\mathrm{H}$ & -4.926728 & 5.981968 & 7.044812 \\
\hline $\mathrm{H}$ & -6.143195 & 4.737061 & 6.803590 \\
\hline C & -6.790404 & 6.676691 & 6.267850 \\
\hline $\mathrm{O}$ & -7.850558 & 6.542823 & 6.836045 \\
\hline$N$ & -6.502383 & 7.752530 & 5.528492 \\
\hline $\mathrm{H}$ & -5.606659 & 7.844328 & 5.098052 \\
\hline C & -7.450200 & 8.860410 & 5.330187 \\
\hline $\mathrm{H}$ & -6.985408 & 9.553054 & 4.640519 \\
\hline $\mathrm{H}$ & -7.641875 & 9.376803 & 6.259295 \\
\hline C & -8.807183 & 8.459359 & 4.784921 \\
\hline $\mathrm{O}$ & -9.801618 & 9.021967 & 5.170285 \\
\hline$N$ & -8.848541 & 7.481791 & 3.858232 \\
\hline C & -10.122203 & 7.081631 & 3.312035 \\
\hline C & -10.996107 & 6.294821 & 4.288441 \\
\hline $\mathrm{O}$ & -12.127110 & 6.014457 & 3.922102 \\
\hline$C$ & -9.957857 & 6.295381 & 1.992081 \\
\hline 0 & -11.202947 & 6.239333 & 1.319743 \\
\hline$C$ & -9.383135 & 4.898576 & 2.172762 \\
\hline $\mathrm{H}$ & -8.000713 & 7.085166 & 3.506572 \\
\hline $\mathrm{H}$ & -10.697469 & 7.970397 & 3.088210 \\
\hline $\mathrm{H}$ & -9.295231 & 6.875273 & 1.363152 \\
\hline $\mathrm{H}$ & -11.886911 & 6.182756 & 1.983503 \\
\hline $\mathrm{H}$ & -9.240611 & 4.434163 & 1.203771 \\
\hline
\end{tabular}




\begin{tabular}{|c|c|c|c|}
\hline $\mathrm{H}$ & -8.424859 & 4.917573 & 2.670240 \\
\hline $\mathrm{H}$ & -10.059779 & 4.274645 & 2.744698 \\
\hline$N$ & -10.493119 & 5.929455 & 5.458921 \\
\hline C & -11.290888 & 5.249506 & 6.455670 \\
\hline C & -12.227379 & 6.182969 & 7.228948 \\
\hline $\mathrm{O}$ & -13.145923 & 5.710381 & 7.846659 \\
\hline C & -10.417291 & 4.481507 & 7.457125 \\
\hline C & -9.518761 & 3.392849 & 6.853049 \\
\hline C & -8.677461 & 2.758808 & 7.965061 \\
\hline C & -10.308433 & 2.319055 & 6.099498 \\
\hline $\mathrm{H}$ & -9.557277 & 6.181496 & 5.702259 \\
\hline $\mathrm{H}$ & -11.953027 & 4.565534 & 5.948913 \\
\hline $\mathrm{H}$ & -9.795483 & 5.191083 & 7.991836 \\
\hline $\mathrm{H}$ & -11.093433 & 4.038694 & 8.180951 \\
\hline $\mathrm{H}$ & -8.833475 & 3.854093 & 6.152016 \\
\hline $\mathrm{H}$ & -7.992728 & 2.018660 & 7.561887 \\
\hline $\mathrm{H}$ & -8.089825 & 3.508385 & 8.485244 \\
\hline $\mathrm{H}$ & -9.307298 & 2.265549 & 8.702828 \\
\hline $\mathrm{H}$ & -9.636406 & 1.559004 & 5.713106 \\
\hline $\mathrm{H}$ & -11.032148 & 1.831378 & 6.750495 \\
\hline $\mathrm{H}$ & -10.850622 & 2.722898 & 5.251637 \\
\hline $\mathrm{N}$ & -11.934518 & 7.498613 & 7.234464 \\
\hline$C$ & -12.824643 & 8.434965 & 7.854322 \\
\hline$C$ & -13.986224 & 8.853713 & 6.977627 \\
\hline $\mathrm{H}$ & -14.699319 & 9.525147 & 7.469433 \\
\hline $\mathrm{O}$ & -14.140171 & 8.521188 & 5.850014 \\
\hline $\mathrm{H}$ & -11.256254 & 7.858362 & 6.599361 \\
\hline $\mathrm{H}$ & -13.222525 & 8.011962 & 8.767909 \\
\hline $\mathrm{H}$ & -12.274908 & 9.331477 & 8.126763 \\
\hline $\mathrm{N}$ & -11.802826 & 10.320195 & -2.707646 \\
\hline
\end{tabular}




\begin{tabular}{|c|c|c|c|}
\hline $\mathrm{H}$ & -11.574041 & 10.515984 & -1.753568 \\
\hline C & -12.080551 & 8.894437 & -2.897354 \\
\hline C & -11.661085 & 8.609736 & -4.333310 \\
\hline $\mathrm{O}$ & -10.593715 & 8.108261 & -4.591929 \\
\hline C & -13.499574 & 8.421126 & -2.515250 \\
\hline C & -13.765554 & 8.699174 & -1.024442 \\
\hline C & -13.687547 & 6.936446 & -2.853503 \\
\hline C & -15.187413 & 8.376057 & -0.561416 \\
\hline $\mathrm{H}$ & -12.598744 & 10.884176 & -2.939163 \\
\hline $\mathrm{H}$ & -11.367682 & 8.347624 & -2.295805 \\
\hline $\mathrm{H}$ & -14.224940 & 9.002043 & -3.089443 \\
\hline $\mathrm{H}$ & -13.052606 & 8.134577 & -0.427428 \\
\hline $\mathrm{H}$ & -13.577772 & 9.748484 & -0.819679 \\
\hline $\mathrm{H}$ & -14.711016 & 6.624705 & -2.689583 \\
\hline $\mathrm{H}$ & -13.451348 & 6.716628 & -3.890397 \\
\hline $\mathrm{H}$ & -13.048797 & 6.318288 & -2.230495 \\
\hline $\mathrm{H}$ & -15.326440 & 8.694612 & 0.465557 \\
\hline $\mathrm{H}$ & -15.927280 & 8.890201 & -1.168929 \\
\hline $\mathrm{H}$ & -15.400687 & 7.314264 & -0.601578 \\
\hline $\mathrm{N}$ & -12.482064 & 9.026362 & -5.328160 \\
\hline $\mathrm{H}$ & -13.415170 & 9.280236 & -5.095505 \\
\hline C & -12.231399 & 8.610726 & -6.691936 \\
\hline $\mathrm{H}$ & -12.263572 & 7.534536 & -6.795283 \\
\hline $\mathrm{H}$ & -13.001719 & 9.038466 & -7.321946 \\
\hline C & -10.872964 & 9.048477 & -7.245658 \\
\hline $\mathrm{O}$ & -10.244980 & 8.339256 & -7.978283 \\
\hline $\mathrm{N}$ & -10.472282 & 10.301224 & -6.896290 \\
\hline $\mathrm{H}$ & -11.035067 & 10.805894 & -6.250380 \\
\hline $\mathrm{C}$ & -9.219019 & 10.889862 & -7.340682 \\
\hline $\mathrm{H}$ & -9.115474 & 10.788962 & -8.409984 \\
\hline
\end{tabular}




\begin{tabular}{|c|c|c|c|}
\hline $\mathrm{H}$ & -9.227674 & 11.940526 & -7.081967 \\
\hline$C$ & -7.980405 & 10.233171 & -6.713787 \\
\hline $\mathrm{O}$ & -7.066038 & 9.820172 & -7.371600 \\
\hline$N$ & -7.951248 & 10.211886 & -5.346525 \\
\hline $\mathrm{H}$ & -8.771970 & 10.475474 & -4.849700 \\
\hline C & -6.773204 & 9.834574 & -4.573715 \\
\hline $\mathrm{H}$ & -5.890666 & 10.284242 & -5.000350 \\
\hline $\mathrm{H}$ & -6.899976 & 10.209176 & -3.565441 \\
\hline C & -6.501152 & 8.343717 & -4.490810 \\
\hline $\mathrm{O}$ & -5.355181 & 7.961999 & -4.529533 \\
\hline$N$ & -7.525544 & 7.484685 & -4.328211 \\
\hline C & -7.212550 & 6.104827 & -3.984309 \\
\hline C & -6.697719 & 5.300123 & -5.171359 \\
\hline $\mathrm{O}$ & -5.964196 & 4.357729 & -4.977792 \\
\hline C & -8.325959 & 5.343452 & -3.223307 \\
\hline C & -9.530425 & 4.945661 & -4.093763 \\
\hline C & -8.711969 & 6.118577 & -1.958772 \\
\hline C & -10.556737 & 4.074592 & -3.365414 \\
\hline $\mathrm{H}$ & -8.470676 & 7.811758 & -4.318455 \\
\hline $\mathrm{H}$ & -6.360554 & 6.132083 & -3.321863 \\
\hline $\mathrm{H}$ & -7.840997 & 4.425181 & -2.905434 \\
\hline $\mathrm{H}$ & -10.018557 & 5.828581 & -4.486623 \\
\hline $\mathrm{H}$ & -9.168986 & 4.382434 & -4.950137 \\
\hline $\mathrm{H}$ & -9.337015 & 5.516951 & -1.312643 \\
\hline $\mathrm{H}$ & -7.830679 & 6.392646 & -1.387496 \\
\hline $\mathrm{H}$ & -9.258152 & 7.024593 & -2.195671 \\
\hline $\mathrm{H}$ & -11.336698 & 3.762157 & -4.053138 \\
\hline $\mathrm{H}$ & -10.099766 & 3.177811 & -2.963036 \\
\hline $\mathrm{H}$ & -11.037854 & 4.604080 & -2.551566 \\
\hline$N$ & -7.073816 & 5.638270 & -6.420812 \\
\hline
\end{tabular}




\begin{tabular}{|c|c|c|c|}
\hline C & -6.450987 & 4.974479 & -7.548094 \\
\hline C & -5.026674 & 5.464849 & -7.812071 \\
\hline $\mathrm{O}$ & -4.194572 & 4.698191 & -8.233710 \\
\hline C & -7.341763 & 5.034464 & -8.797238 \\
\hline C & -8.478254 & 4.005581 & -8.715805 \\
\hline C & -7.972126 & 2.586652 & -9.006672 \\
\hline $\mathrm{N}$ & -8.874871 & 1.528173 & -8.557812 \\
\hline C & -8.929258 & 1.019825 & -7.329007 \\
\hline $\mathrm{N}$ & -8.167173 & 1.472639 & -6.346679 \\
\hline$N$ & -9.763834 & 0.035226 & -7.059737 \\
\hline $\mathrm{H}$ & -7.671243 & 6.424048 & -6.562250 \\
\hline $\mathrm{H}$ & -6.294403 & 3.947677 & -7.266612 \\
\hline $\mathrm{H}$ & -7.750978 & 6.032684 & -8.914300 \\
\hline $\mathrm{H}$ & -6.731258 & 4.842944 & -9.674116 \\
\hline $\mathrm{H}$ & -8.934965 & 4.045107 & -7.732555 \\
\hline $\mathrm{H}$ & -9.258920 & 4.255105 & -9.425698 \\
\hline $\mathrm{H}$ & -7.851080 & 2.449718 & -10.072283 \\
\hline $\mathrm{H}$ & -6.993488 & 2.411991 & -8.578136 \\
\hline $\mathrm{H}$ & -9.394778 & 1.045896 & -9.256042 \\
\hline $\mathrm{H}$ & -7.534446 & 2.227690 & -6.471548 \\
\hline $\mathrm{H}$ & -8.420750 & 1.224286 & -5.400433 \\
\hline $\mathrm{H}$ & -10.408646 & -0.319764 & -7.729813 \\
\hline $\mathrm{H}$ & -9.630508 & -0.532613 & -6.242181 \\
\hline$N$ & -4.779992 & 6.748345 & -7.543284 \\
\hline $\mathrm{H}$ & -5.522997 & 7.341551 & -7.251500 \\
\hline C & -3.485316 & 7.385726 & -7.827284 \\
\hline $\mathrm{H}$ & -3.157246 & 7.158327 & -8.829714 \\
\hline $\mathrm{H}$ & -3.616712 & 8.454655 & -7.727422 \\
\hline C & -2.411303 & 6.912320 & -6.860550 \\
\hline 0 & -1.281726 & 6.704121 & -7.226618 \\
\hline
\end{tabular}




\begin{tabular}{|c|c|c|c|}
\hline$N$ & -2.828457 & 6.767479 & -5.597011 \\
\hline C & -1.933862 & 6.465321 & -4.505927 \\
\hline C & -1.619439 & 4.984875 & -4.401593 \\
\hline $\mathrm{O}$ & -0.482104 & 4.629507 & -4.203840 \\
\hline C & -2.524255 & 7.029328 & -3.207201 \\
\hline C & -1.917662 & 6.322582 & -1.996582 \\
\hline C & -2.315398 & 8.550100 & -3.217773 \\
\hline $\mathrm{H}$ & -3.754329 & 7.056494 & -5.366174 \\
\hline $\mathrm{H}$ & -0.975713 & 6.928771 & -4.692138 \\
\hline $\mathrm{H}$ & -3.589324 & 6.829660 & -3.217866 \\
\hline $\mathrm{H}$ & -2.138346 & 6.848850 & -1.087086 \\
\hline $\mathrm{H}$ & -2.310547 & 5.315859 & -1.902646 \\
\hline $\mathrm{H}$ & -0.844625 & 6.271233 & -2.073744 \\
\hline $\mathrm{H}$ & -3.050147 & 9.052153 & -2.604354 \\
\hline $\mathrm{H}$ & -1.325181 & 8.804270 & -2.854846 \\
\hline $\mathrm{H}$ & -2.425205 & 8.951212 & -4.218869 \\
\hline$N$ & -2.622060 & 4.096465 & -4.535400 \\
\hline C & -2.249510 & 2.701983 & -4.666890 \\
\hline C & -1.300735 & 2.556267 & -5.857580 \\
\hline $\mathrm{O}$ & -0.352381 & 1.806056 & -5.797896 \\
\hline C & -3.452172 & 1.756963 & -4.766294 \\
\hline C & -4.371062 & 1.844521 & -3.540722 \\
\hline C & -5.377987 & 0.695911 & -3.437060 \\
\hline$N$ & -4.779198 & -0.493092 & -2.836825 \\
\hline C & -5.420980 & -1.630103 & -2.679624 \\
\hline$N$ & -6.662734 & -1.800277 & -3.160596 \\
\hline$N$ & -4.893685 & -2.637278 & -2.008631 \\
\hline $\mathrm{H}$ & -3.547101 & 4.396667 & -4.754613 \\
\hline $\mathrm{H}$ & -1.669259 & 2.409970 & -3.805424 \\
\hline $\mathrm{H}$ & -4.021020 & 1.966291 & -5.6701 \\
\hline
\end{tabular}




\begin{tabular}{|c|c|c|c|}
\hline $\mathrm{H}$ & -3.053329 & 0.754143 & -4.865686 \\
\hline $\mathrm{H}$ & -3.778806 & 1.873021 & -2.639593 \\
\hline $\mathrm{H}$ & -4.937775 & 2.762648 & -3.580318 \\
\hline $\mathrm{H}$ & -6.218814 & 1.015974 & -2.831531 \\
\hline $\mathrm{H}$ & -5.751777 & 0.452053 & -4.427706 \\
\hline $\mathrm{H}$ & -3.873939 & -0.374788 & -2.370380 \\
\hline $\mathrm{H}$ & -7.032973 & -1.165159 & -3.828895 \\
\hline $\mathrm{H}$ & -7.042367 & -2.728999 & -3.173415 \\
\hline $\mathrm{H}$ & -4.032180 & -2.598623 & -1.492245 \\
\hline $\mathrm{H}$ & -5.384573 & -3.504658 & -1.972268 \\
\hline$N$ & -1.566245 & 3.258454 & -6.951797 \\
\hline $\mathrm{H}$ & -2.408528 & 3.780791 & -7.050437 \\
\hline C & -0.705504 & 3.110063 & -8.109480 \\
\hline $\mathrm{H}$ & -1.081093 & 3.759599 & -8.889565 \\
\hline $\mathrm{H}$ & -0.694360 & 2.092078 & -8.471540 \\
\hline C & 0.738936 & 3.490488 & -7.797955 \\
\hline $\mathrm{O}$ & 1.678902 & 2.890814 & -8.255833 \\
\hline$N$ & 0.863994 & 4.538401 & -6.969587 \\
\hline $\mathrm{H}$ & 0.057483 & 5.074073 & -6.741814 \\
\hline C & 2.130051 & 5.040619 & -6.542744 \\
\hline $\mathrm{H}$ & 2.753786 & 5.334352 & -7.377563 \\
\hline $\mathrm{H}$ & 1.954385 & 5.918720 & -5.934853 \\
\hline C & 2.971154 & 4.092986 & -5.704450 \\
\hline $\mathrm{O}$ & 4.172380 & 4.256194 & -5.621505 \\
\hline$N$ & 2.338355 & 3.115574 & -5.046633 \\
\hline C & 3.044667 & 2.277830 & -4.112784 \\
\hline C & 4.231133 & 1.506149 & -4.702015 \\
\hline $\mathrm{O}$ & 5.158942 & 1.213471 & -3.974725 \\
\hline C & 1.993220 & 1.369435 & -3.474783 \\
\hline$C$ & 2.410727 & 0.551241 & -2.258610 \\
\hline
\end{tabular}




\begin{tabular}{|c|c|c|c|}
\hline$C$ & 1.124748 & 0.122338 & -1.560007 \\
\hline $\mathrm{N}$ & 1.348493 & -0.933938 & -0.594893 \\
\hline C & 0.468646 & -1.918857 & -0.361199 \\
\hline$N$ & -0.683847 & -1.954598 & -0.981799 \\
\hline$N$ & 0.812819 & -2.890773 & 0.461268 \\
\hline $\mathrm{H}$ & 1.351437 & 3.003230 & -5.150594 \\
\hline $\mathrm{H}$ & 3.503519 & 2.894102 & -3.349131 \\
\hline $\mathrm{H}$ & 1.186624 & 2.031845 & -3.178985 \\
\hline $\mathrm{H}$ & 1.573820 & 0.717178 & -4.231932 \\
\hline $\mathrm{H}$ & 2.985510 & -0.321632 & -2.548607 \\
\hline $\mathrm{H}$ & 3.035032 & 1.137878 & -1.588586 \\
\hline $\mathrm{H}$ & 0.645021 & 0.975608 & -1.092811 \\
\hline $\mathrm{H}$ & 0.436720 & -0.255535 & -2.299305 \\
\hline $\mathrm{H}$ & 2.060229 & -0.803538 & 0.088182 \\
\hline $\mathrm{H}$ & -1.169330 & -1.107744 & -1.267857 \\
\hline $\mathrm{H}$ & -1.315144 & -2.722055 & -0.791504 \\
\hline $\mathrm{H}$ & 1.725740 & -2.942064 & 0.847983 \\
\hline $\mathrm{H}$ & 0.092020 & -3.526417 & 0.788710 \\
\hline $\mathrm{N}$ & 4.240680 & 1.146385 & -5.997086 \\
\hline $\mathrm{H}$ & 3.489390 & 1.422198 & -6.595078 \\
\hline C & 5.342997 & 0.362579 & -6.528185 \\
\hline $\mathrm{H}$ & 5.116615 & 0.124591 & -7.560112 \\
\hline $\mathrm{H}$ & 5.451333 & -0.563932 & -5.986890 \\
\hline C & 6.697633 & 1.039094 & -6.494589 \\
\hline 0 & 7.719103 & 0.398703 & -6.406783 \\
\hline$N$ & 6.689816 & 2.371358 & -6.597599 \\
\hline $\mathrm{H}$ & 5.821817 & 2.865356 & -6.540036 \\
\hline C & 7.908114 & 3.142744 & -6.529888 \\
\hline $\mathrm{H}$ & 8.618848 & 2.794830 & -7.266098 \\
\hline $\mathrm{H}$ & 7.672690 & 4.172779 & -6.762091 \\
\hline
\end{tabular}




\begin{tabular}{|c|c|c|c|}
\hline C & 8.658375 & 3.137022 & -5.196083 \\
\hline 0 & 9.787596 & 3.577964 & -5.140840 \\
\hline $\mathrm{N}$ & 8.033768 & 2.605874 & -4.137287 \\
\hline C & 8.626709 & 2.428875 & -2.822709 \\
\hline C & 9.916197 & 1.597634 & -2.856545 \\
\hline 0 & 10.736975 & 1.683746 & -1.957497 \\
\hline C & 7.546867 & 1.710575 & -1.982732 \\
\hline C & 7.753961 & 1.563742 & -0.475517 \\
\hline C & 7.378842 & 2.807633 & 0.339139 \\
\hline C & 7.283515 & 2.509382 & 1.831527 \\
\hline $\mathrm{N}$ & 8.627504 & 2.502213 & 2.482073 \\
\hline 0 & 11.015629 & 1.860772 & 0.870565 \\
\hline $\mathrm{H}$ & 10.855107 & 2.085997 & -0.046208 \\
\hline $\mathrm{H}$ & 11.515347 & 1.048271 & 0.801235 \\
\hline $\mathrm{H}$ & 7.096247 & 2.278780 & -4.252134 \\
\hline $\mathrm{H}$ & 8.900012 & 3.385226 & -2.396740 \\
\hline $\mathrm{H}$ & 6.613581 & 2.230408 & -2.152548 \\
\hline $\mathrm{H}$ & 7.406374 & 0.727525 & -2.417021 \\
\hline $\mathrm{H}$ & 7.098581 & 0.758652 & -0.157753 \\
\hline $\mathrm{H}$ & 8.760160 & 1.235532 & -0.250773 \\
\hline $\mathrm{H}$ & 8.067175 & 3.632258 & 0.176046 \\
\hline $\mathrm{H}$ & 6.401623 & 3.156858 & 0.021479 \\
\hline $\mathrm{H}$ & 6.703350 & 3.255265 & 2.352169 \\
\hline $\mathrm{H}$ & 6.830952 & 1.546206 & 2.010013 \\
\hline $\mathrm{H}$ & 9.347976 & 2.068717 & 1.913160 \\
\hline $\mathrm{H}$ & 8.922083 & 3.477566 & 2.653864 \\
\hline $\mathrm{H}$ & 8.592148 & 2.006337 & 3.360750 \\
\hline $\mathrm{N}$ & 10.048263 & 0.767818 & -3.882324 \\
\hline C & 11.120660 & -0.183210 & -4.140789 \\
\hline$C$ & 12.479071 & 0.423004 & -4.495817 \\
\hline
\end{tabular}




\begin{tabular}{|c|c|c|c|}
\hline $\mathrm{O}$ & 13.457480 & -0.281435 & -4.392270 \\
\hline$C$ & 10.729413 & -1.076474 & -5.339706 \\
\hline C & 9.621079 & -2.111009 & -5.097777 \\
\hline C & 9.196927 & -2.719634 & -6.438086 \\
\hline C & 10.085480 & -3.209472 & -4.141253 \\
\hline $\mathrm{H}$ & 9.306122 & 0.759165 & -4.551083 \\
\hline $\mathrm{H}$ & 11.298615 & -0.784169 & -3.261008 \\
\hline $\mathrm{H}$ & 10.439224 & -0.421711 & -6.156008 \\
\hline $\mathrm{H}$ & 11.625048 & -1.597996 & -5.655393 \\
\hline $\mathrm{H}$ & 8.753347 & -1.612402 & -4.677127 \\
\hline $\mathrm{H}$ & 8.416907 & -3.460847 & -6.292979 \\
\hline $\mathrm{H}$ & 8.813745 & -1.958866 & -7.106148 \\
\hline $\mathrm{H}$ & 10.034753 & -3.214775 & -6.922789 \\
\hline $\mathrm{H}$ & 9.317113 & -3.963745 & -4.011255 \\
\hline $\mathrm{H}$ & 10.966963 & -3.703454 & -4.536675 \\
\hline $\mathrm{H}$ & 10.334560 & -2.826091 & -3.156495 \\
\hline$N$ & 12.529197 & 1.674109 & -4.960993 \\
\hline C & 13.749777 & 2.301832 & -5.429800 \\
\hline C & 14.895161 & 2.499829 & -4.418460 \\
\hline O & 15.936125 & 2.918497 & -4.849509 \\
\hline C & 13.390804 & 3.634240 & -6.091133 \\
\hline $\mathrm{O}$ & 12.792738 & 4.515035 & -5.183061 \\
\hline $\mathrm{H}$ & 11.696047 & 2.222647 & -4.940441 \\
\hline $\mathrm{H}$ & 14.197106 & 1.663837 & -6.182794 \\
\hline $\mathrm{H}$ & 14.299390 & 4.086863 & -6.456238 \\
\hline $\mathrm{H}$ & 12.739799 & 3.441029 & -6.937874 \\
\hline $\mathrm{H}$ & 11.845476 & 4.479890 & -5.240670 \\
\hline$N$ & 14.745759 & 2.156649 & -3.119625 \\
\hline C & 15.888817 & 2.094079 & -2.230321 \\
\hline C & 16.797862 & 0.886888 & -2.488267 \\
\hline
\end{tabular}




\begin{tabular}{|c|c|c|c|}
\hline O & 17.930596 & 0.893702 & -2.081380 \\
\hline$C$ & 15.466628 & 1.980130 & -0.760393 \\
\hline$C$ & 14.580191 & 3.100712 & -0.221206 \\
\hline$C$ & 14.240604 & 2.810898 & 1.241228 \\
\hline $\mathrm{N}$ & 13.194226 & 3.665261 & 1.788343 \\
\hline$C$ & 13.344071 & 4.807400 & 2.439160 \\
\hline $\mathrm{N}$ & 14.549949 & 5.311373 & 2.678313 \\
\hline O & 14.869775 & 7.744209 & 4.281061 \\
\hline $\mathrm{H}$ & 14.559394 & 8.627585 & 4.064773 \\
\hline $\mathrm{H}$ & 15.588619 & 7.856532 & 4.889987 \\
\hline $\mathrm{N}$ & 12.278327 & 5.458053 & 2.856558 \\
\hline $\mathrm{H}$ & 13.864843 & 1.823854 & -2.804898 \\
\hline $\mathrm{H}$ & 16.497298 & 2.971953 & -2.384381 \\
\hline $\mathrm{H}$ & 14.953772 & 1.033928 & -0.622501 \\
\hline $\mathrm{H}$ & 16.383573 & 1.924418 & -0.183972 \\
\hline $\mathrm{H}$ & 15.070472 & 4.065122 & -0.318019 \\
\hline $\mathrm{H}$ & 13.656352 & 3.168884 & -0.783708 \\
\hline $\mathrm{H}$ & 13.871479 & 1.799097 & 1.326258 \\
\hline $\mathrm{H}$ & 15.121645 & 2.862748 & 1.870126 \\
\hline $\mathrm{H}$ & 12.261500 & 3.346651 & 1.617495 \\
\hline $\mathrm{H}$ & 15.368948 & 4.847276 & 2.365265 \\
\hline $\mathrm{H}$ & 14.665354 & 6.158838 & 3.206931 \\
\hline $\mathrm{H}$ & 11.329638 & 5.155213 & 2.698915 \\
\hline $\mathrm{H}$ & 12.350647 & 6.292512 & 3.393565 \\
\hline$N$ & 16.219636 & -0.176222 & -3.072156 \\
\hline C & 16.923677 & -1.414983 & -3.303777 \\
\hline C & 17.713392 & -1.343009 & -4.607284 \\
\hline $\mathrm{H}$ & 18.076203 & -0.346683 & -4.869724 \\
\hline 0 & 17.944470 & -2.286793 & -5.290166 \\
\hline C & 15.953638 & -2.601351 & -3.303997 \\
\hline
\end{tabular}




\begin{tabular}{|c|c|c|c|}
\hline C & 15.427756 & -2.974136 & -1.950792 \\
\hline $\mathrm{N}$ & 14.489769 & -2.242001 & -1.250289 \\
\hline $\mathrm{O}$ & 12.538275 & -0.116070 & -0.541504 \\
\hline $\mathrm{H}$ & 13.188485 & -0.693087 & -0.945935 \\
\hline $\mathrm{H}$ & 12.040395 & 0.281344 & -1.247765 \\
\hline C & 15.757560 & -4.070970 & -1.232846 \\
\hline C & 14.282588 & -2.895072 & -0.153798 \\
\hline $\mathrm{N}$ & 15.024810 & -4.006840 & -0.074175 \\
\hline $\mathrm{H}$ & 15.299861 & -0.095232 & -3.449370 \\
\hline $\mathrm{H}$ & 17.657020 & -1.530904 & -2.513430 \\
\hline $\mathrm{H}$ & 15.126055 & -2.382251 & -3.969829 \\
\hline $\mathrm{H}$ & 16.476123 & -3.452464 & -3.719471 \\
\hline $\mathrm{H}$ & 16.434523 & -4.872121 & -1.438818 \\
\hline $\mathrm{H}$ & 13.599794 & -2.600431 & 0.617371 \\
\hline $\mathrm{H}$ & 14.947314 & -4.707149 & 0.630519 \\
\hline $\mathrm{O}$ & -14.615809 & -2.482410 & 4.768728 \\
\hline $\mathrm{H}$ & -14.852064 & -1.958458 & 4.005649 \\
\hline $\mathrm{H}$ & -14.381115 & -1.864405 & 5.453289 \\
\hline $\mathrm{O}$ & -13.191662 & -0.594781 & 6.670794 \\
\hline $\mathrm{H}$ & -12.500574 & -1.061104 & 7.126518 \\
\hline $\mathrm{H}$ & -13.560371 & 0.016906 & 7.296437 \\
\hline $\mathrm{O}$ & -12.192924 & -0.130395 & 3.899330 \\
\hline $\mathrm{H}$ & -12.475726 & -0.050048 & 4.803963 \\
\hline $\mathrm{H}$ & -12.977667 & -0.344870 & 3.394555 \\
\hline $\mathrm{O}$ & -12.405320 & -2.798951 & 1.211586 \\
\hline $\mathrm{H}$ & -11.728545 & -2.129098 & 1.172760 \\
\hline $\mathrm{H}$ & -12.301413 & -3.242307 & 2.053422 \\
\hline $\mathrm{O}$ & -6.815806 & 1.307713 & 0.056255 \\
\hline $\mathrm{H}$ & -7.122601 & 1.524476 & 0.940636 \\
\hline $\mathrm{H}$ & -5.886832 & 1.122256 & 0.145264 \\
\hline
\end{tabular}




\begin{tabular}{|c|c|c|c|}
\hline 0 & -7.842742 & 1.787559 & 2.655195 \\
\hline $\mathrm{H}$ & -8.795719 & 1.803286 & 2.617068 \\
\hline $\mathrm{H}$ & -7.570302 & 2.464721 & 3.269371 \\
\hline 0 & -8.640067 & -0.227926 & -1.283258 \\
\hline $\mathrm{H}$ & -7.940030 & 0.247482 & -0.813982 \\
\hline $\mathrm{H}$ & -8.308093 & -1.090753 & -1.508414 \\
\hline $\mathrm{O}$ & -12.290583 & -2.121506 & -1.641122 \\
\hline $\mathrm{H}$ & -12.795314 & -2.785666 & -1.184193 \\
\hline $\mathrm{H}$ & -11.987773 & -1.539874 & -0.945389 \\
\hline $\mathrm{O}$ & -12.763189 & 1.432073 & -1.313844 \\
\hline $\mathrm{H}$ & -12.139541 & 0.861008 & -0.866891 \\
\hline $\mathrm{H}$ & -12.664237 & 2.276669 & -0.873701 \\
\hline $\mathrm{O}$ & -13.848145 & 3.968839 & 2.991320 \\
\hline $\mathrm{H}$ & -13.378146 & 4.625144 & 3.502738 \\
\hline $\mathrm{H}$ & -14.765878 & 4.071430 & 3.203923 \\
\hline 0 & -15.159707 & 0.586181 & 0.082183 \\
\hline $\mathrm{H}$ & -15.883469 & 0.443557 & -0.511663 \\
\hline $\mathrm{H}$ & -14.418114 & 0.868655 & -0.452221 \\
\hline $\mathrm{O}$ & -12.217179 & -0.192514 & -3.771701 \\
\hline $\mathrm{H}$ & -12.546238 & 0.566212 & -3.293630 \\
\hline $\mathrm{H}$ & -12.356182 & -0.923388 & -3.170533 \\
\hline $\mathrm{O}$ & -12.037246 & -1.698877 & -6.309050 \\
\hline $\mathrm{H}$ & -12.302049 & -1.130879 & -5.586897 \\
\hline $\mathrm{H}$ & -12.774237 & -2.265124 & -6.500076 \\
\hline $\mathrm{O}$ & -9.712164 & -2.958409 & -2.642603 \\
\hline $\mathrm{H}$ & -9.649003 & -2.605256 & -3.529519 \\
\hline $\mathrm{H}$ & -10.590330 & -2.757793 & -2.325358 \\
\hline 0 & -10.989194 & -0.379079 & 0.185945 \\
\hline $\mathrm{H}$ & -10.137952 & -0.359434 & -0.261297 \\
\hline $\mathrm{H}$ & -10.902620 & 0.223367 & 0.933169 \\
\hline
\end{tabular}




$\begin{array}{llll}\mathrm{O} & -10.757759 & 1.531259 & 2.195718 \\ \mathrm{H} & -11.261943 & 1.136567 & 2.911674 \\ \mathrm{H} & -11.281357 & 2.236936 & 1.820157 \\ \mathrm{O} & -12.331550 & 3.511267 & 0.661366 \\ \mathrm{H} & -13.019697 & 3.628153 & 1.320285 \\ \mathrm{H} & -11.863933 & 4.342652 & 0.640087 \\ \mathrm{O} & -9.337734 & 0.612626 & -3.890324 \\ \mathrm{H} & -10.257950 & 0.355419 & -3.911572 \\ \mathrm{H} & -9.049652 & 0.439643 & -2.992154 \\ \mathrm{O} & -14.401300 & -1.015400 & 2.311194 \\ \mathrm{H} & -14.793411 & -0.426865 & 1.665242 \\ \mathrm{H} & -13.901486 & -1.656320 & 1.807755 \\ \mathrm{O} & -12.391809 & -3.876001 & 3.886340 \\ \mathrm{H} & -13.195693 & -3.458100 & 4.222416 \\ \mathrm{H} & -11.684883 & -3.529042 & 4.417399 \\ \mathrm{O} & -9.406459 & -2.362849 & -5.388474 \\ \mathrm{H} & -10.279235 & -2.531589 & -5.736960 \\ \mathrm{H} & -8.807301 & -2.996491 & -5.791534\end{array}$

PROTON CONFIGURATION, GIVEN BY NOMINAL CHARGES: R300(+),Y266(-),E183(0) POTENTIAL:+35mV

\begin{tabular}{|c|c|c|c|}
\hline $\mathrm{N}$ & 15.580942 & -6.451471 & 5.391415 \\
\hline $\mathrm{H}$ & 14.779104 & -6.204513 & 5.938620 \\
\hline C & 15.508191 & -5.856005 & 4.054570 \\
\hline & 14.783016 & -6.782958 & 3.060461 \\
\hline & 14.053577 & -6.345859 & 2.185899 \\
\hline & 16.917677 & -5.573721 & 3.520957 \\
\hline & 16.387759 & -6.124395 & 5.885359 \\
\hline & 14.941991 & -4.934023 & 4.051207 \\
\hline
\end{tabular}




\begin{tabular}{|c|c|c|c|}
\hline $\mathrm{H}$ & 16.885963 & -5.186741 & 2.509465 \\
\hline $\mathrm{H}$ & 17.400751 & -4.828476 & 4.142751 \\
\hline $\mathrm{H}$ & 17.526242 & -6.471247 & 3.528981 \\
\hline $\mathrm{N}$ & 15.032385 & -8.085607 & 3.186394 \\
\hline $\mathrm{H}$ & 15.610982 & -8.361763 & 3.950518 \\
\hline$C$ & 14.398655 & -9.092381 & 2.333234 \\
\hline $\mathrm{H}$ & 14.481873 & -8.823219 & 1.292970 \\
\hline $\mathrm{H}$ & 14.891987 & -10.042050 & 2.492097 \\
\hline$C$ & 12.912988 & -9.222114 & 2.671603 \\
\hline $\mathrm{O}$ & 12.082257 & -9.428517 & 1.826246 \\
\hline $\mathrm{N}$ & 12.582805 & -9.055890 & 3.966874 \\
\hline $\mathrm{H}$ & 13.302554 & -8.984003 & 4.648771 \\
\hline$C$ & 11.195103 & -9.170806 & 4.421393 \\
\hline $\mathrm{H}$ & 11.188255 & -9.158111 & 5.503149 \\
\hline $\mathrm{H}$ & 10.743824 & -10.088262 & 4.076954 \\
\hline$C$ & 10.336955 & -8.010603 & 3.900717 \\
\hline 0 & 9.191198 & -8.186526 & 3.583649 \\
\hline $\mathrm{N}$ & 10.946779 & -6.814294 & 3.855610 \\
\hline$C$ & 10.317527 & -5.633912 & 3.318096 \\
\hline$C$ & 10.126103 & -5.690555 & 1.799772 \\
\hline 0 & 9.096116 & -5.261786 & 1.337431 \\
\hline$C$ & 11.091289 & -4.387807 & 3.777159 \\
\hline 0 & 12.477603 & -4.569759 & 3.767472 \\
\hline $\mathrm{H}$ & 11.905030 & -6.743480 & 4.107374 \\
\hline $\mathrm{H}$ & 9.313636 & -5.553631 & 3.701699 \\
\hline $\mathrm{H}$ & 10.798291 & -3.538963 & 3.169683 \\
\hline $\mathrm{H}$ & 10.827436 & -4.175602 & 4.802882 \\
\hline $\mathrm{H}$ & 12.802544 & -4.752241 & 2.894343 \\
\hline $\mathrm{N}$ & 11.099667 & -6.200188 & 1.024514 \\
\hline$C$ & 10.844275 & -6.396400 & -0.399882 \\
\hline
\end{tabular}




\begin{tabular}{|c|c|c|c|}
\hline C & 9.694514 & -7.386178 & -0.602164 \\
\hline $\mathrm{O}$ & 8.948411 & -7.282241 & -1.547698 \\
\hline C & 12.133011 & L -6.783990 & -1.186532 \\
\hline C & 12.336014 & -8.289317 & -1.401796 \\
\hline C & 12.158579 & -6.060271 & -2.534161 \\
\hline $\mathrm{H}$ & 11.923084 & -6.586725 & 1.432080 \\
\hline $\mathrm{H}$ & 10.473896 & -5.462260 & -0.790979 \\
\hline $\mathrm{H}$ & 12.964634 & -6.412125 & -0.598542 \\
\hline $\mathrm{H}$ & 13.290340 & -8.451776 & -1.892105 \\
\hline $\mathrm{H}$ & 12.334419 & -8.854739 & -0.480691 \\
\hline $\mathrm{H}$ & 11.569745 & $5-8.698043$ & -2.053302 \\
\hline $\mathrm{H}$ & 13.053375 & -6.323813 & -3.087019 \\
\hline $\mathrm{H}$ & 11.298193 & $3-6.333473$ & -3.135146 \\
\hline $\mathrm{H}$ & 12.157470 & -4.984942 & -2.402011 \\
\hline$N$ & 9.570413 & -8.340330 & 0.328325 \\
\hline $\mathrm{H}$ & 10.313296 & -8.473119 & 0.975853 \\
\hline C & 8.593028 & -9.421195 & 0.241219 \\
\hline $\mathrm{H}$ & 8.934764 & -10.218801 & 0.888531 \\
\hline $\mathrm{H}$ & 8.533151 & -9.803240 & -0.766221 \\
\hline C & 7.181546 & -9.046150 & 0.643059 \\
\hline $\mathrm{O}$ & 6.257862 & -9.505041 & 0.011845 \\
\hline$N$ & 7.003139 & -8.225180 & 1.684377 \\
\hline C & 5.669236 & -7.737017 & 1.952534 \\
\hline C & 5.251026 & -6.727546 & 0.888869 \\
\hline $\mathrm{O}$ & 4.075011 & -6.634436 & 0.609248 \\
\hline C & 5.414749 & -7.229137 & 3.385884 \\
\hline C & 5.554761 & -8.372063 & 4.396420 \\
\hline C & 6.257705 & -6.020635 & 3.797779 \\
\hline $\mathrm{H}$ & 7.780632 & -7.957153 & 2.247562 \\
\hline $\mathrm{H}$ & 4.986299 & -8.556668 & 1.791680 \\
\hline
\end{tabular}




\begin{tabular}{|c|c|c|c|}
\hline $\mathrm{H}$ & 4.374383 & -6.918810 & 3.377975 \\
\hline $\mathrm{H}$ & 5.264683 & -8.032966 & 5.385334 \\
\hline $\mathrm{H}$ & 4.915597 & -9.208967 & 4.134554 \\
\hline $\mathrm{H}$ & 6.576433 & -8.730676 & 4.453191 \\
\hline $\mathrm{H}$ & 5.916925 & -5.646780 & 4.757341 \\
\hline $\mathrm{H}$ & 7.299661 & -6.292262 & 3.906033 \\
\hline $\mathrm{H}$ & 6.186856 & -5.207074 & 3.084168 \\
\hline $\mathrm{N}$ & 6.171317 & -6.000952 & 0.246571 \\
\hline$C$ & 5.774690 & -5.182491 & -0.887395 \\
\hline$C$ & 5.343127 & -6.067027 & -2.065410 \\
\hline $\mathrm{O}$ & 4.320846 & -5.815975 & -2.659082 \\
\hline$C$ & 6.866981 & -4.162911 & -1.267457 \\
\hline$C$ & 7.064539 & -3.145802 & -0.129354 \\
\hline$C$ & 6.496329 & -3.452744 & -2.574064 \\
\hline$C$ & 8.359106 & -2.340455 & -0.242737 \\
\hline $\mathrm{H}$ & 7.132068 & -6.054977 & 0.507757 \\
\hline $\mathrm{H}$ & 4.876392 & -4.646331 & -0.620583 \\
\hline $\mathrm{H}$ & 7.797090 & -4.704294 & -1.414889 \\
\hline $\mathrm{H}$ & 6.210170 & -2.470840 & -0.114628 \\
\hline $\mathrm{H}$ & 7.072970 & -3.658976 & 0.822973 \\
\hline $\mathrm{H}$ & 7.201636 & -2.662972 & -2.793820 \\
\hline $\mathrm{H}$ & 6.497032 & -4.128183 & -3.419846 \\
\hline $\mathrm{H}$ & 5.508375 & -3.009311 & -2.507758 \\
\hline $\mathrm{H}$ & 8.460015 & -1.666065 & 0.600945 \\
\hline $\mathrm{H}$ & 9.220074 & -2.998679 & -0.241061 \\
\hline $\mathrm{H}$ & 8.396599 & -1.741743 & -1.145871 \\
\hline $\mathrm{N}$ & 6.115468 & -7.110232 & -2.399910 \\
\hline $\mathrm{H}$ & 7.009414 & -7.232242 & -1.972867 \\
\hline$C$ & 5.773645 & -7.950643 & -3.527577 \\
\hline $\mathrm{H}$ & 6.604258 & -8.624483 & -3.697228 \\
\hline
\end{tabular}




\begin{tabular}{|c|c|c|c|}
\hline $\mathrm{H}$ & 5.618654 & -7.364976 & -4.421426 \\
\hline C & 4.504822 & -8.779444 & -3.325685 \\
\hline $\mathrm{O}$ & 3.667639 & -8.839480 & -4.195014 \\
\hline$N$ & 4.393582 & -9.432010 & -2.161998 \\
\hline $\mathrm{H}$ & 5.129956 & -9.385778 & -1.490355 \\
\hline C & 3.207574 & -10.187543 & -1.833506 \\
\hline $\mathrm{H}$ & 2.968501 & -10.900779 & -2.608026 \\
\hline $\mathrm{H}$ & 3.399860 & -10.729423 & -0.915948 \\
\hline C & 1.974222 & -9.306874 & -1.644766 \\
\hline $\mathrm{O}$ & 0.887704 & -9.697014 & -2.006487 \\
\hline$N$ & 2.187839 & -8.106992 & -1.107224 \\
\hline C & 1.056881 & -7.190771 & -1.061929 \\
\hline C & 0.613907 & -6.716952 & -2.448694 \\
\hline $\mathrm{O}$ & -0.568610 & -6.580259 & -2.671111 \\
\hline C & 1.259721 & -5.955130 & -0.190409 \\
\hline 0 & 1.407232 & -6.260833 & 1.156457 \\
\hline $\mathrm{H}$ & 3.085682 & -7.817705 & -0.792071 \\
\hline $\mathrm{H}$ & 0.208261 & -7.723964 & -0.665600 \\
\hline $\mathrm{H}$ & 2.093767 & -5.367945 & -0.558832 \\
\hline $\mathrm{H}$ & 0.358598 & -5.372731 & -0.287759 \\
\hline $\mathrm{H}$ & 2.310452 & -6.501650 & 1.311294 \\
\hline $\mathrm{N}$ & 1.541502 & -6.433051 & -3.372548 \\
\hline$C$ & 1.144612 & -6.026007 & -4.707974 \\
\hline$C$ & 0.383790 & -7.149795 & -5.406292 \\
\hline 0 & -0.599171 & -6.894962 & -6.070895 \\
\hline$C$ & 2.354340 & -5.514691 & -5.524623 \\
\hline$C$ & 2.747885 & -4.119911 & -5.010065 \\
\hline$C$ & 2.064279 & -5.491880 & -7.030704 \\
\hline$C$ & 4.068334 & -3.595300 & -5.571779 \\
\hline 11 & 2.511686 & -6.484301 & -3.146650 \\
\hline
\end{tabular}




\begin{tabular}{|c|c|c|c|}
\hline $\mathrm{H}$ & 0.420177 & -5.228082 & -4.628967 \\
\hline $\mathrm{H}$ & 3.179635 & -6.198616 & -5.350941 \\
\hline $\mathrm{H}$ & 1.949819 & -3.420754 & -5.254002 \\
\hline $\mathrm{H}$ & 2.821324 & -4.147221 & -3.930592 \\
\hline $\mathrm{H}$ & 2.921319 & -5.113249 & -7.573557 \\
\hline $\mathrm{H}$ & 1.855346 & -6.480629 & -7.420792 \\
\hline $\mathrm{H}$ & 1.214908 & -4.856255 & -7.260610 \\
\hline $\mathrm{H}$ & 4.352155 & -2.680824 & -5.061660 \\
\hline $\mathrm{H}$ & 4.867104 & -4.315145 & -5.424277 \\
\hline $\mathrm{H}$ & 4.008912 & -3.373972 & -6.631636 \\
\hline$N$ & 0.821975 & -8.401024 & -5.244109 \\
\hline $\mathrm{H}$ & 1.666597 & -8.577992 & -4.741098 \\
\hline C & 0.045228 & -9.507200 & -5.750358 \\
\hline $\mathrm{H}$ & -0.058497 & -9.463811 & -6.824338 \\
\hline $\mathrm{H}$ & 0.556132 & -10.424641 & -5.487227 \\
\hline$C$ & -1.365283 & -9.540071 & -5.166931 \\
\hline $\mathrm{O}$ & -2.319464 & -9.766564 & -5.875620 \\
\hline $\mathrm{N}$ & -1.472619 & -9.318902 & -3.858011 \\
\hline$C$ & -2.777211 & -9.341795 & -3.238828 \\
\hline$C$ & -3.683496 & -8.205639 & -3.713381 \\
\hline 0 & -4.881290 & -8.411371 & -3.773358 \\
\hline$C$ & -2.686235 & -9.304950 & -1.706232 \\
\hline $\mathrm{O}$ & -3.934364 & -9.568380 & -1.126274 \\
\hline $\mathrm{H}$ & -0.656792 & -9.234665 & -3.288050 \\
\hline $\mathrm{H}$ & -3.297513 & -10.246270 & -3.520891 \\
\hline $\mathrm{H}$ & -2.007118 & -10.077215 & -1.372750 \\
\hline $\mathrm{H}$ & -2.297956 & -8.346228 & -1.379699 \\
\hline $\mathrm{H}$ & -4.620589 & -9.163800 & -1.642351 \\
\hline $\mathrm{N}$ & -3.127097 & -7.027175 & -3.988495 \\
\hline C & -3.875858 & -5.902157 & -4.525077 \\
\hline
\end{tabular}




\begin{tabular}{|c|c|c|c|}
\hline C & -4.428434 & -6.235589 & -5.911243 \\
\hline $\mathrm{O}$ & -5.594526 & -6.042743 & -6.175320 \\
\hline C & -2.949786 & -4.666202 & -4.544227 \\
\hline C & -3.373636 & -3.509459 & -5.435319 \\
\hline C & -4.697947 & -3.120854 & -5.605552 \\
\hline C & -2.390312 & -2.795676 & -6.113831 \\
\hline C & -5.023269 & -2.068499 & -6.447795 \\
\hline C & -2.709386 & -1.733271 & -6.941919 \\
\hline C & -4.034305 & -1.370584 & -7.119442 \\
\hline $\mathrm{H}$ & -2.153793 & -6.905736 & -3.798098 \\
\hline $\mathrm{H}$ & -4.737823 & -5.708947 & -3.906157 \\
\hline $\mathrm{H}$ & -2.836409 & -4.330530 & -3.517808 \\
\hline $\mathrm{H}$ & -1.973752 & -4.990062 & -4.875339 \\
\hline $\mathrm{H}$ & -5.483859 & -3.656881 & -5.110437 \\
\hline $\mathrm{H}$ & -1.358959 & -3.079365 & -6.000281 \\
\hline $\mathrm{H}$ & -6.059029 & -1.808492 & -6.590088 \\
\hline $\mathrm{H}$ & -1.927143 & -1.199291 & -7.450686 \\
\hline $\mathrm{H}$ & -4.288225 & -0.560007 & -7.780133 \\
\hline$N$ & -3.561181 & -6.734650 & -6.798636 \\
\hline C & -4.008284 & -7.079042 & -8.128761 \\
\hline C & -5.137853 & -8.106774 & -8.097485 \\
\hline $\mathrm{O}$ & -6.023010 & -8.048974 & -8.911236 \\
\hline C & -2.862131 & -7.619587 & -8.987215 \\
\hline$S$ & -1.637704 & -6.375682 & -9.506124 \\
\hline $\mathrm{H}$ & -2.619240 & -6.918672 & -6.522129 \\
\hline $\mathrm{H}$ & -4.430127 & -6.208243 & -8.608134 \\
\hline $\mathrm{H}$ & -2.362916 & -8.439103 & -8.487970 \\
\hline $\mathrm{H}$ & -3.281972 & -7.998857 & -9.909110 \\
\hline $\mathrm{H}$ & -1.029712 & -6.214698 & -8.341195 \\
\hline $\mathrm{N}$ & -5.078530 & -9.066907 & -7.155626 \\
\hline
\end{tabular}




\begin{tabular}{|c|c|c|c|}
\hline $\mathrm{H}$ & -4.254666 & -9.191008 & -6.606637 \\
\hline C & -6.085055 & -10.116657 & $7 \quad-7.179838$ \\
\hline $\mathrm{H}$ & -6.256455 & -10.458573 & $\begin{array}{ll}3 & -8.188746\end{array}$ \\
\hline $\mathrm{H}$ & -5.713640 & -10.946041 & $1-6.589259$ \\
\hline C & -7.459153 & -9.722789 & -6.613043 \\
\hline $\mathrm{O}$ & -8.482093 & -10.083639 & $\begin{array}{ll}9 & -7.127753\end{array}$ \\
\hline$N$ & -7.417997 & -9.015713 & -5.478473 \\
\hline C & -8.635196 & -8.650708 & -4.780113 \\
\hline C & -9.497606 & -7.639375 & -5.527283 \\
\hline $\mathrm{O}$ & -10.691395 & -7.613337 & $7 \quad-5.381408$ \\
\hline C & -8.342053 & -8.119118 & -3.365579 \\
\hline C & -7.452402 & -6.868508 & -3.254031 \\
\hline C & -8.068524 & -5.494773 & -3.197106 \\
\hline $\mathrm{O}$ & -9.366324 & -5.425264 & -3.275547 \\
\hline $\mathrm{H}$ & -9.662172 & -4.515225 & -3.141118 \\
\hline $\mathrm{O}$ & -7.375167 & -4.513003 & -3.068626 \\
\hline $\mathrm{H}$ & -6.533173 & -8.780630 & -5.080914 \\
\hline $\mathrm{H}$ & -9.261610 & -9.526399 & -4.683142 \\
\hline $\mathrm{H}$ & -9.293825 & -7.952404 & -2.881705 \\
\hline $\mathrm{H}$ & -7.844659 & -8.919242 & -2.828358 \\
\hline $\mathrm{H}$ & -6.864343 & -6.931939 & -2.346430 \\
\hline $\mathrm{H}$ & -6.729514 & -6.823488 & -4.051081 \\
\hline $\mathrm{N}$ & -8.837321 & -6.734073 & -6.273860 \\
\hline$C$ & -9.562354 & -5.706193 & -6.995284 \\
\hline C & -10.453969 & -6.285518 & B -8.085558 \\
\hline $\mathrm{H}$ & -10.493803 & -7.369153 & $3-8.176161$ \\
\hline 0 & -11.080326 & -5.574130 & -8.814097 \\
\hline$C$ & -8.632453 & -4.606929 & -7.557429 \\
\hline 0 & -9.420594 & -3.476029 & -7.901149 \\
\hline 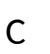 & -7.779220 & -5.029094 & -8.745836 \\
\hline
\end{tabular}




\begin{tabular}{|c|c|c|c|}
\hline $\mathrm{H}$ & -7.863216 & -6.859748 & -6.448780 \\
\hline $\mathrm{H}$ & -10.234601 & -5.219725 & -6.297852 \\
\hline $\mathrm{H}$ & -7.990170 & -4.289480 & -6.747269 \\
\hline $\mathrm{H}$ & -10.129530 & -3.784763 & -8.459484 \\
\hline $\mathrm{H}$ & -7.101539 & -4.221406 & -9.001021 \\
\hline $\mathrm{H}$ & -7.188732 & -5.909493 & -8.538909 \\
\hline $\mathrm{H}$ & -8.399370 & -5.232007 & -9.612380 \\
\hline$N$ & -12.952337 & -9.022034 & 3.174429 \\
\hline $\mathrm{H}$ & -13.221885 & -9.548043 & 2.368927 \\
\hline C & -11.777476 & -8.184402 & 2.899254 \\
\hline C & -10.786110 & -8.141422 & 4.045784 \\
\hline O & -9.651391 & -7.750012 & 3.844839 \\
\hline C & -12.138829 & -6.715798 & 2.593883 \\
\hline $\mathrm{O}$ & -12.680666 & -6.209730 & 3.786891 \\
\hline C & -13.123659 & -6.564348 & 1.440448 \\
\hline $\mathrm{H}$ & -13.731162 & -8.457526 & 3.453462 \\
\hline $\mathrm{H}$ & -11.234242 & -8.594444 & 2.059086 \\
\hline $\mathrm{H}$ & -11.219577 & -6.198933 & 2.354009 \\
\hline $\mathrm{H}$ & -12.705205 & -5.255890 & 3.753522 \\
\hline $\mathrm{H}$ & -13.277809 & -5.510731 & 1.232665 \\
\hline $\mathrm{H}$ & -12.760607 & -7.035071 & 0.534081 \\
\hline $\mathrm{H}$ & -14.087149 & -6.991933 & 1.690561 \\
\hline $\mathrm{N}$ & -11.197283 & -8.533693 & 5.241879 \\
\hline $\mathrm{H}$ & -12.125683 & -8.893751 & 5.282445 \\
\hline$C$ & -10.410859 & -8.533376 & 6.450870 \\
\hline $\mathrm{H}$ & -10.329987 & -7.538596 & 6.859769 \\
\hline $\mathrm{H}$ & -10.935023 & -9.139523 & 7.180325 \\
\hline$C$ & -8.975341 & -9.054819 & 6.376698 \\
\hline 0 & -8.142020 & -8.496097 & 7.046479 \\
\hline $\mathrm{N}$ & -8.658361 & -10.127845 & 5.62956 \\
\hline
\end{tabular}




\begin{tabular}{|c|c|c|c|}
\hline $\mathrm{H}$ & -9.367380 & -10.602437 & 5.117731 \\
\hline C & -7.269006 & -10.558267 & 5.527641 \\
\hline $\mathrm{H}$ & -7.250768 & -11.535588 & 5.062448 \\
\hline $\mathrm{H}$ & -6.829754 & -10.640724 & 6.508820 \\
\hline C & -6.387520 & -9.608326 & 4.692692 \\
\hline $\mathrm{O}$ & -5.220713 & -9.464642 & 4.951299 \\
\hline$N$ & -7.045371 & -9.017966 & 3.701161 \\
\hline C & -6.307965 & -7.993244 & 2.974822 \\
\hline C & -6.272142 & -6.694071 & 3.774969 \\
\hline 0 & -5.283028 & -5.999904 & 3.736324 \\
\hline C & -6.625165 & -7.840081 & 1.473997 \\
\hline C & -7.801927 & -7.000818 & 1.021176 \\
\hline C & -7.794152 & -5.617392 & 1.177428 \\
\hline C & -8.856280 & -7.580120 & 0.327262 \\
\hline C & -8.823327 & -4.839127 & 0.674747 \\
\hline$C$ & -9.876158 & -6.802235 & -0.197018 \\
\hline$C$ & -9.868183 & -5.429334 & -0.017212 \\
\hline $\mathrm{H}$ & -8.040034 & -9.015732 & 3.680471 \\
\hline $\mathrm{H}$ & -5.283750 & -8.321386 & 2.998909 \\
\hline $\mathrm{H}$ & -5.728280 & -7.418298 & 1.033763 \\
\hline $\mathrm{H}$ & -6.709424 & -8.843478 & 1.073800 \\
\hline $\mathrm{H}$ & -6.967682 & -5.138467 & 1.670053 \\
\hline $\mathrm{H}$ & -8.866379 & -8.644528 & 0.166524 \\
\hline $\mathrm{H}$ & -8.794761 & -3.771443 & 0.801947 \\
\hline $\mathrm{H}$ & -10.663426 & -7.264311 & -0.764062 \\
\hline $\mathrm{H}$ & -10.656637 & -4.826606 & -0.429629 \\
\hline $\mathrm{N}$ & -7.305307 & -6.401670 & 4.567842 \\
\hline$C$ & -7.244328 & -5.289935 & 5.486041 \\
\hline $\mathrm{C}$ & -6.123095 & -5.500187 & 6.503005 \\
\hline 0 & -5.483804 & -4.544173 & 6.886831 \\
\hline
\end{tabular}




\begin{tabular}{|c|c|c|c|}
\hline C & -8.621845 & -5.122928 & 6.162027 \\
\hline C & -8.871957 & -3.782293 & 6.818762 \\
\hline C & -9.076178 & -2.647527 & 6.038978 \\
\hline C & -8.965864 & -3.666117 & 8.198909 \\
\hline C & -9.355381 & -1.424999 & 6.623682 \\
\hline C & -9.253681 & -2.444987 & 8.791069 \\
\hline C & -9.446161 & -1.319898 & 8.006161 \\
\hline $\mathrm{H}$ & -8.166719 & -6.890540 & 4.455286 \\
\hline $\mathrm{H}$ & -6.979255 & -4.384090 & 4.959786 \\
\hline $\mathrm{H}$ & -9.370832 & -5.271045 & 5.390883 \\
\hline $\mathrm{H}$ & -8.751486 & -5.915482 & 6.886766 \\
\hline $\mathrm{H}$ & -9.017696 & -2.717718 & 4.966178 \\
\hline $\mathrm{H}$ & -8.815944 & -4.531878 & 8.819889 \\
\hline $\mathrm{H}$ & -9.498766 & -0.559608 & 6.003100 \\
\hline $\mathrm{H}$ & -9.315268 & -2.373487 & 9.862115 \\
\hline $\mathrm{H}$ & -9.642429 & -0.366151 & 8.463925 \\
\hline$N$ & -5.874695 & -6.736533 & 6.930564 \\
\hline $\mathrm{H}$ & -6.525231 & -7.461505 & 6.724341 \\
\hline C & -4.800131 & -7.025983 & 7.853808 \\
\hline $\mathrm{H}$ & -4.840700 & -6.391302 & 8.726234 \\
\hline $\mathrm{H}$ & -4.913440 & -8.053889 & 8.174044 \\
\hline C & -3.403101 & -6.854342 & 7.260234 \\
\hline 0 & -2.530344 & -6.355557 & 7.930301 \\
\hline $\mathrm{N}$ & -3.199966 & -7.288506 & 6.011338 \\
\hline$C$ & -1.918226 & -7.117567 & 5.344764 \\
\hline $\mathrm{C}$ & -1.662248 & -5.648412 & 4.981286 \\
\hline 0 & -0.560174 & -5.170187 & 5.147411 \\
\hline$C$ & -1.835701 & -8.057293 & 4.121485 \\
\hline $\mathrm{C}$ & -0.667103 & -7.706915 & 3.198450 \\
\hline C & -1.739142 & -9.516001 & 4.582692 \\
\hline
\end{tabular}




\begin{tabular}{|c|c|c|c|}
\hline $\mathrm{H}$ & -3.932129 & -7.771843 & 5.536955 \\
\hline $\mathrm{H}$ & -1.129030 & -7.365170 & 6.039841 \\
\hline $\mathrm{H}$ & -2.754959 & -7.933795 & 3.553529 \\
\hline $\mathrm{H}$ & -0.603814 & -8.436220 & 2.397891 \\
\hline $\mathrm{H}$ & -0.772183 & -6.733467 & 2.740400 \\
\hline $\mathrm{H}$ & 0.276309 & -7.720592 & 3.735539 \\
\hline $\mathrm{H}$ & -1.753429 & -10.180085 & 3.724485 \\
\hline $\mathrm{H}$ & -0.806460 & -9.683338 & 5.115514 \\
\hline $\mathrm{H}$ & -2.557433 & -9.800758 & 5.231252 \\
\hline$N$ & -2.693665 & -4.955534 & 4.488024 \\
\hline C & -2.553130 & -3.575509 & 4.065186 \\
\hline C & -2.426208 & -2.654326 & 5.277356 \\
\hline $\mathrm{O}$ & -1.537757 & -1.832859 & 5.330420 \\
\hline C & -3.719576 & -3.117929 & 3.177441 \\
\hline C & -3.943175 & -3.893126 & 1.868659 \\
\hline C & -2.803969 & -3.821723 & 0.864297 \\
\hline 0 & -1.683336 & -4.162278 & 1.268515 \\
\hline $\mathrm{O}$ & -3.070426 & -3.447865 & -0.292108 \\
\hline $\mathrm{H}$ & -3.562126 & -5.418546 & 4.333894 \\
\hline $\mathrm{H}$ & -1.634119 & -3.470633 & 3.520465 \\
\hline $\mathrm{H}$ & -4.640072 & -3.162042 & 3.751846 \\
\hline $\mathrm{H}$ & -3.547007 & -2.069801 & 2.949880 \\
\hline $\mathrm{H}$ & -4.110318 & -4.937451 & 2.087417 \\
\hline $\mathrm{H}$ & -4.844042 & -3.509906 & 1.402029 \\
\hline $\mathrm{N}$ & -3.326633 & -2.786991 & 6.269434 \\
\hline C & -3.116889 & -2.037329 & 7.484968 \\
\hline C & -1.793959 & -2.427212 & 8.154183 \\
\hline $\mathrm{O}$ & -1.130467 & -1.588153 & 8.723310 \\
\hline C & -4.246554 & -2.025444 & 8.524777 \\
\hline 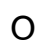 & -4.447038 & -3.268677 & 9.129345 \\
\hline
\end{tabular}




\begin{tabular}{|c|c|c|c|}
\hline C & -5.533431 & -1.412348 & 7.984810 \\
\hline $\mathrm{H}$ & -4.004323 & -3.515982 & 6.240661 \\
\hline $\mathrm{H}$ & -2.968153 & -1.005975 & 7.204737 \\
\hline $\mathrm{H}$ & -3.872128 & -1.384804 & 9.313464 \\
\hline $\mathrm{H}$ & -4.896877 & -3.842327 & 8.521013 \\
\hline $\mathrm{H}$ & -6.263581 & -1.358608 & 8.782951 \\
\hline $\mathrm{H}$ & -5.353504 & -0.406617 & 7.617726 \\
\hline $\mathrm{H}$ & -5.954608 & -1.999723 & 7.179927 \\
\hline$N$ & -1.380385 & -3.700437 & 8.043347 \\
\hline $\mathrm{H}$ & -2.029686 & -4.420959 & 7.816584 \\
\hline C & -0.188986 & -4.105194 & 8.744524 \\
\hline $\mathrm{H}$ & -0.243049 & -3.889309 & 9.802284 \\
\hline $\mathrm{H}$ & -0.075393 & -5.172767 & 8.609305 \\
\hline C & 1.063269 & -3.417761 & 8.226312 \\
\hline $\mathrm{O}$ & 1.939020 & -3.075595 & 8.988151 \\
\hline$N$ & 1.155018 & -3.255737 & 6.890573 \\
\hline C & 2.334927 & -2.660226 & 6.326820 \\
\hline C & 2.292759 & -1.139308 & 6.163427 \\
\hline $\mathrm{O}$ & 3.361594 & -0.575856 & 6.017663 \\
\hline C & 2.841729 & -3.357628 & 5.064677 \\
\hline$S$ & 1.925438 & -3.032480 & 3.534355 \\
\hline $\mathrm{H}$ & 0.422091 & -3.605818 & 6.305822 \\
\hline $\mathrm{H}$ & 3.115321 & -2.790801 & 7.061243 \\
\hline $\mathrm{H}$ & 3.845673 & -3.005743 & 4.875363 \\
\hline $\mathrm{H}$ & 2.882985 & -4.423240 & 5.242891 \\
\hline $\mathrm{H}$ & 0.882413 & -3.823131 & 3.739430 \\
\hline$N$ & 1.142366 & -0.471603 & 6.274272 \\
\hline C & 1.204810 & 0.968241 & 6.492101 \\
\hline C & 1.757084 & 1.271011 & 7.870353 \\
\hline 0 & 2.373300 & 2.286503 & 8.085460 \\
\hline
\end{tabular}




\begin{tabular}{|c|c|c|c|}
\hline C & -0.097879 & 1.735804 & 6.180395 \\
\hline C & -1.202986 & 1.553051 & 7.230812 \\
\hline C & -0.549988 & 1.433407 & 4.746657 \\
\hline C & -2.471638 & 2.356726 & 6.936894 \\
\hline $\mathrm{H}$ & 0.273967 & -0.966412 & 6.292942 \\
\hline $\mathrm{H}$ & 1.951090 & 1.351735 & 5.814852 \\
\hline $\mathrm{H}$ & 0.200239 & 2.777995 & 6.223840 \\
\hline $\mathrm{H}$ & -1.452136 & 0.506953 & 7.336813 \\
\hline $\mathrm{H}$ & -0.818983 & 1.869046 & 8.196355 \\
\hline $\mathrm{H}$ & -1.308318 & 2.135589 & 4.424419 \\
\hline $\mathrm{H}$ & 0.284236 & 1.518660 & 4.055742 \\
\hline $\mathrm{H}$ & -0.955856 & 0.435440 & 4.650550 \\
\hline $\mathrm{H}$ & -3.145709 & 2.318657 & 7.787290 \\
\hline $\mathrm{H}$ & -2.246349 & 3.401972 & 6.741016 \\
\hline $\mathrm{H}$ & -3.007395 & 1.966692 & 6.078484 \\
\hline$N$ & 1.549778 & 0.323284 & 8.791250 \\
\hline $\mathrm{H}$ & 0.886277 & -0.399186 & 8.614875 \\
\hline C & 1.977557 & 0.513535 & 10.160150 \\
\hline $\mathrm{H}$ & 1.469368 & -0.221659 & 10.771273 \\
\hline $\mathrm{H}$ & 1.736734 & 1.503327 & 10.517828 \\
\hline C & 3.481545 & 0.338407 & 10.305527 \\
\hline $\mathrm{O}$ & 4.163932 & 1.129242 & 10.897436 \\
\hline$N$ & 3.999309 & -0.752512 & 9.695188 \\
\hline $\mathrm{H}$ & 3.389881 & -1.465505 & 9.353007 \\
\hline C & 5.424263 & -0.937846 & 9.668203 \\
\hline $\mathrm{H}$ & 5.637572 & -1.908742 & 9.236097 \\
\hline $\mathrm{H}$ & 5.854510 & -0.903041 & 10.658459 \\
\hline C & 6.156892 & 0.119125 & 8.844833 \\
\hline $\mathrm{O}$ & 7.292547 & 0.410159 & 9.129102 \\
\hline IV & 5.502073 & 0.621264 & 7.799016 \\
\hline
\end{tabular}




\begin{tabular}{|c|c|c|c|}
\hline C & 6.105379 & 1.756770 & 7.150116 \\
\hline C & 5.529225 & 3.076874 & 7.656104 \\
\hline $\mathrm{H}$ & 5.019200 & 3.037006 & 8.617190 \\
\hline $\mathrm{O}$ & 5.664993 & 4.096776 & 7.053504 \\
\hline C & 6.008315 & 1.677414 & 5.624159 \\
\hline C & 6.757772 & 0.509176 & 5.017373 \\
\hline C & 8.124364 & 0.325373 & 5.232303 \\
\hline C & 6.094020 & -0.399426 & 4.198323 \\
\hline C & 8.808452 & -0.719816 & 4.626464 \\
\hline C & 6.775845 & -1.442301 & 3.591190 \\
\hline C & 8.136909 & -1.602992 & 3.795367 \\
\hline $\mathrm{H}$ & 4.573923 & 0.338581 & 7.573088 \\
\hline $\mathrm{H}$ & 7.140980 & 1.765288 & 7.454008 \\
\hline $\mathrm{H}$ & 4.965795 & 1.616528 & 5.338073 \\
\hline $\mathrm{H}$ & 6.388812 & 2.612093 & 5.226687 \\
\hline $\mathrm{H}$ & 8.655117 & 0.982033 & 5.900270 \\
\hline $\mathrm{H}$ & 5.033279 & -0.303711 & 4.064790 \\
\hline $\mathrm{H}$ & 9.860154 & -0.846617 & 4.817437 \\
\hline $\mathrm{H}$ & 6.241068 & -2.134057 & 2.965019 \\
\hline $\mathrm{H}$ & 8.655420 & -2.415347 & 3.319818 \\
\hline $\mathrm{N}$ & 12.613551 & 9.596234 & -1.248798 \\
\hline $\mathrm{H}$ & 11.870161 & 9.916292 & -1.841104 \\
\hline$C$ & 12.304851 & 8.264736 & -0.719492 \\
\hline$C$ & 11.274750 & 8.450359 & 0.387495 \\
\hline 0 & 10.268003 & 7.776899 & 0.418741 \\
\hline$C$ & 13.562814 & 7.581280 & -0.165146 \\
\hline$C$ & 14.645862 & 7.276992 & -1.205751 \\
\hline$S$ & 14.019241 & 6.202370 & -2.529191 \\
\hline C & 15.503228 & 6.052652 & -3.552379 \\
\hline П & 13.429440 & 9.571153 & -1.827880 \\
\hline
\end{tabular}




\begin{tabular}{|c|c|c|c|}
\hline $\mathrm{H}$ & 11.834244 & 7.616444 & -1.447171 \\
\hline $\mathrm{H}$ & 14.000777 & 8.205741 & 0.607669 \\
\hline $\mathrm{H}$ & 13.255924 & 6.653893 & 0.304704 \\
\hline $\mathrm{H}$ & 15.045770 & 8.189777 & -1.632910 \\
\hline $\mathrm{H}$ & 15.471079 & 6.785084 & -0.703294 \\
\hline $\mathrm{H}$ & 15.253026 & 5.397064 & -4.372564 \\
\hline $\mathrm{H}$ & 15.802981 & 7.016214 & -3.943838 \\
\hline $\mathrm{H}$ & 16.320884 & 5.616365 & -2.993521 \\
\hline$N$ & 11.504585 & 9.414161 & 1.296142 \\
\hline C & 10.595549 & 9.609526 & 2.408512 \\
\hline C & 9.278487 & 10.278160 & 2.012197 \\
\hline $\mathrm{O}$ & 8.284015 & 10.059880 & 2.657676 \\
\hline C & 11.279269 & 10.369172 & 3.552065 \\
\hline C & 12.449454 & 9.562313 & 4.084255 \\
\hline $\mathrm{O}$ & 13.585196 & 9.823151 & 3.746092 \\
\hline$N$ & 12.143757 & 8.559354 & 4.916978 \\
\hline $\mathrm{H}$ & 12.327488 & 9.969979 & 1.203551 \\
\hline $\mathrm{H}$ & 10.296014 & 8.639497 & 2.770696 \\
\hline $\mathrm{H}$ & 11.661916 & 11.325840 & 3.219140 \\
\hline ⺊ & 10.549503 & 10.538802 & 4.333631 \\
\hline $\mathrm{H}$ & 12.886454 & 7.961849 & 5.209714 \\
\hline $\mathrm{H}$ & 11.221999 & 8.173120 & 4.949741 \\
\hline$N$ & 9.264254 & 11.068704 & 0.922246 \\
\hline $\mathrm{H}$ & 10.110289 & 11.226689 & 0.424534 \\
\hline C & 8.003508 & 11.517469 & 0.368849 \\
\hline $\mathrm{H}$ & 8.200597 & 12.260284 & -0.393480 \\
\hline $\mathrm{H}$ & 7.389294 & 11.966561 & 1.132130 \\
\hline C & 7.186379 & 10.384433 & -0.254310 \\
\hline $\mathrm{O}$ & 5.986874 & 10.472141 & -0.295076 \\
\hline $\mathrm{N}$ & 7.863138 & 9.332291 & -0.761153 \\
\hline
\end{tabular}




\begin{tabular}{|c|c|c|c|}
\hline C & 7.170537 & 8.144459 & -1.230806 \\
\hline C & 6.635013 & 7.300996 & -0.063263 \\
\hline $\mathrm{O}$ & 5.535074 & 6.808463 & -0.143765 \\
\hline C & 8.048175 & 7.342014 & -2.220880 \\
\hline C & 8.343851 & 8.194449 & -3.469675 \\
\hline C & 7.377236 & 6.017618 & -2.604658 \\
\hline C & 9.427595 & 7.620879 & -4.385865 \\
\hline $\mathrm{H}$ & 8.823223 & 9.228423 & -0.530736 \\
\hline $\mathrm{H}$ & 6.279762 & 8.470571 & -1.745118 \\
\hline $\mathrm{H}$ & 8.987067 & 7.114702 & -1.723986 \\
\hline $\mathrm{H}$ & 7.422047 & 8.321513 & -4.032918 \\
\hline $\mathrm{H}$ & 8.647254 & 9.189740 & -3.162301 \\
\hline $\mathrm{H}$ & 7.994568 & 5.475988 & -3.309997 \\
\hline $\mathrm{H}$ & 7.224312 & 5.377117 & -1.744642 \\
\hline $\mathrm{H}$ & 6.408172 & 6.182146 & -3.064106 \\
\hline $\mathrm{H}$ & 9.622354 & 8.301070 & -5.208694 \\
\hline $\mathrm{H}$ & 10.362841 & 7.474253 & -3.852285 \\
\hline $\mathrm{H}$ & 9.146447 & 6.667087 & -4.816732 \\
\hline$N$ & 7.387521 & 7.177441 & 1.048751 \\
\hline C & 6.879214 & 6.459386 & 2.206267 \\
\hline C & 5.526964 & 7.037691 & 2.644201 \\
\hline $\mathrm{O}$ & 4.577394 & 6.328593 & 2.872994 \\
\hline C & 7.833035 & 6.547449 & 3.407935 \\
\hline C & 9.254186 & 5.998826 & 3.247571 \\
\hline $\mathrm{O}$ & 10.147089 & 6.635080 & 3.801055 \\
\hline $\mathrm{O}$ & 9.427266 & 4.935078 & 2.601900 \\
\hline $\mathrm{H}$ & 8.347933 & 7.445473 & 1.026788 \\
\hline $\mathrm{H}$ & 6.683997 & 5.430521 & 1.946962 \\
\hline $\mathrm{H}$ & 7.920845 & 7.572105 & 3.737535 \\
\hline $\mathrm{H}$ & 7.375101 & 5.993760 & 4.225031 \\
\hline
\end{tabular}




\begin{tabular}{|c|c|c|c|}
\hline $\mathrm{N}$ & 5.485400 & 8.375976 & 2.789462 \\
\hline $\mathrm{H}$ & 6.314906 & 8.913016 & 2.651009 \\
\hline$C$ & 4.293484 & 9.041756 & 3.254423 \\
\hline $\mathrm{H}$ & 4.532220 & 10.084899 & 3.420215 \\
\hline $\mathrm{H}$ & 3.949416 & 8.627985 & 4.189136 \\
\hline$C$ & 3.132432 & 8.991223 & 2.270507 \\
\hline $\mathrm{O}$ & 1.991571 & 9.123038 & 2.649828 \\
\hline $\mathrm{N}$ & 3.467298 & 8.824812 & 0.987996 \\
\hline $\mathrm{H}$ & 4.428824 & 8.814090 & 0.740710 \\
\hline$C$ & 2.517177 & 8.905263 & -0.078282 \\
\hline $\mathrm{H}$ & 3.061156 & 9.090910 & -0.995481 \\
\hline $\mathrm{H}$ & 1.843277 & 9.737570 & 0.070799 \\
\hline$C$ & 1.609143 & 7.687010 & -0.310217 \\
\hline $\mathrm{O}$ & 0.576548 & 7.817978 & -0.906932 \\
\hline $\mathrm{N}$ & 2.066075 & 6.540159 & 0.228970 \\
\hline$C$ & 1.242104 & 5.374512 & 0.442332 \\
\hline$C$ & 0.428577 & 5.482447 & 1.736686 \\
\hline O & -0.767067 & 5.314399 & 1.714675 \\
\hline$C$ & 2.093930 & 4.105641 & 0.436329 \\
\hline $\mathrm{H}$ & 2.965976 & 6.568616 & 0.659286 \\
\hline $\mathrm{H}$ & 0.511204 & 5.325335 & -0.347126 \\
\hline $\mathrm{H}$ & 1.473322 & 3.237799 & 0.630639 \\
\hline $\mathrm{H}$ & 2.562940 & 3.992232 & -0.534151 \\
\hline $\mathrm{H}$ & 2.873412 & 4.148239 & 1.190229 \\
\hline $\mathrm{N}$ & 1.071840 & 5.742054 & 2.891841 \\
\hline$C$ & 0.343362 & 5.580481 & 4.141115 \\
\hline$C$ & -0.767537 & 6.616369 & 4.295716 \\
\hline $\mathrm{O}$ & -1.801826 & 6.321542 & 4.854401 \\
\hline$C$ & 1.265038 & 5.506578 & 5.383483 \\
\hline$c$ & 1.859225 & 6.852475 & 5.826178 \\
\hline
\end{tabular}




\begin{tabular}{|c|c|c|c|}
\hline C & 2.351500 & 4.441264 & 5.171557 \\
\hline C & 2.703619 & 6.756488 & 7.099342 \\
\hline $\mathrm{H}$ & 2.061136 & 5.864514 & 2.906155 \\
\hline $\mathrm{H}$ & -0.187512 & 4.640745 & 4.089410 \\
\hline $\mathrm{H}$ & 0.611135 & 5.167601 & 6.181290 \\
\hline $\mathrm{H}$ & 2.460399 & 7.263935 & 5.021996 \\
\hline $\mathrm{H}$ & 1.056223 & 7.560748 & 6.002567 \\
\hline $\mathrm{H}$ & 2.727585 & 4.087181 & 6.120271 \\
\hline $\mathrm{H}$ & 1.955815 & 3.585271 & 4.634418 \\
\hline $\mathrm{H}$ & 3.190732 & 4.832107 & 4.606334 \\
\hline $\mathrm{H}$ & 3.009861 & 7.747706 & 7.420695 \\
\hline $\mathrm{H}$ & 2.135152 & 6.310542 & 7.910127 \\
\hline $\mathrm{H}$ & 3.598629 & 6.163010 & 6.959733 \\
\hline$N$ & -0.547055 & 7.847522 & 3.811660 \\
\hline $\mathrm{H}$ & 0.336411 & 8.082027 & 3.409318 \\
\hline C & -1.515086 & 8.891307 & 4.019976 \\
\hline $\mathrm{H}$ & -1.102635 & 9.816987 & 3.638872 \\
\hline $\mathrm{H}$ & -1.728707 & 9.026988 & 5.070294 \\
\hline C & -2.867019 & 8.618723 & 3.365914 \\
\hline O & -3.871382 & 8.837845 & 4.002830 \\
\hline$N$ & -2.902654 & 8.109030 & 2.120710 \\
\hline C & -4.238565 & 8.014256 & 1.531443 \\
\hline C & -4.961964 & 6.725338 & 1.881684 \\
\hline $\mathrm{O}$ & -6.174628 & 6.709525 & 1.859477 \\
\hline C & -4.026558 & 8.272467 & 0.032690 \\
\hline C & -2.762923 & 9.139713 & 0.039000 \\
\hline C & -1.907400 & 8.464717 & 1.098943 \\
\hline $\mathrm{H}$ & -4.863158 & 8.793103 & 1.940350 \\
\hline $\mathrm{H}$ & -3.860806 & 7.346274 & -0.501526 \\
\hline $\mathrm{H}$ & -4.889738 & 8.759425 & -0.402883 \\
\hline
\end{tabular}




\begin{tabular}{|c|c|c|c|}
\hline $\mathrm{H}$ & -2.254565 & 9.199306 & -0.905587 \\
\hline $\mathrm{H}$ & -3.013536 & 10.149855 & 0.354164 \\
\hline $\mathrm{H}$ & -1.432418 & 7.575956 & 0.724388 \\
\hline $\mathrm{H}$ & -1.141521 & 9.110072 & 1.496853 \\
\hline $\mathrm{N}$ & -4.225552 & 5.661050 & 2.224931 \\
\hline C & -4.858214 & 4.466274 & 2.755772 \\
\hline C & -5.577685 & 4.756789 & 4.076287 \\
\hline O & -6.635886 & 4.219495 & 4.318051 \\
\hline C & -3.848725 & 3.314934 & 2.960362 \\
\hline C & -3.521157 & 2.489217 & 1.732291 \\
\hline C & -4.003564 & 1.191796 & 1.596357 \\
\hline C & -2.681720 & 2.955760 & 0.723124 \\
\hline C & -3.676542 & 0.393874 & 0.510403 \\
\hline C & -2.344660 & 2.175323 & -0.362662 \\
\hline C & -2.828210 & 0.859856 & -0.516628 \\
\hline O & -2.524070 & 0.142696 & -1.553290 \\
\hline $\mathrm{H}$ & -3.232572 & 5.755249 & 2.273856 \\
\hline $\mathrm{H}$ & -5.633555 & 4.148264 & 2.076384 \\
\hline $\mathrm{H}$ & -2.946482 & 3.730438 & 3.399714 \\
\hline $\mathrm{H}$ & -4.274050 & 2.653899 & 3.707432 \\
\hline $\mathrm{H}$ & -4.645736 & 0.784605 & 2.361115 \\
\hline $\mathrm{H}$ & -2.264199 & 3.940005 & 0.792416 \\
\hline $\mathrm{H}$ & -4.031251 & -0.621635 & 0.459970 \\
\hline $\mathrm{H}$ & -1.690521 & 2.567564 & -1.123518 \\
\hline $\mathrm{N}$ & -4.978440 & 5.593135 & 4.942756 \\
\hline $\mathrm{H}$ & -4.053481 & 5.921603 & 4.756816 \\
\hline C & -5.596049 & 5.948456 & 6.200554 \\
\hline $\mathrm{H}$ & -4.820170 & 6.300490 & 6.869449 \\
\hline $\mathrm{H}$ & -6.073747 & 5.090239 & 6.644421 \\
\hline C & -6.660307 & 7.040830 & 6.077578 \\
\hline
\end{tabular}




\begin{tabular}{|c|c|c|c|}
\hline $\mathrm{O}$ & -7.721752 & 6.950751 & 6.651545 \\
\hline$N$ & -6.341994 & 8.091462 & 5.314146 \\
\hline $\mathrm{H}$ & -5.441929 & 8.148872 & 4.886995 \\
\hline C & -7.257699 & 9.220080 & 5.085083 \\
\hline $\mathrm{H}$ & -6.767747 & 9.891068 & 4.391851 \\
\hline $\mathrm{H}$ & -7.457203 & 9.753718 & 6.002575 \\
\hline C & -8.613235 & 8.823360 & 4.520535 \\
\hline $\mathrm{O}$ & -9.605046 & 9.420524 & 4.857066 \\
\hline$N$ & -8.648796 & 7.802988 & 3.639425 \\
\hline C & -9.906599 & 7.387763 & 3.064012 \\
\hline C & -10.814615 & 6.618253 & 4.025119 \\
\hline $\mathrm{O}$ & -11.921888 & 6.301996 & 3.625580 \\
\hline C & -9.699527 & 6.576916 & 1.766145 \\
\hline $\mathrm{O}$ & -10.918813 & 6.514769 & 1.049618 \\
\hline C & -9.142421 & 5.179765 & 1.996862 \\
\hline $\mathrm{H}$ & -7.796508 & 7.388185 & 3.322476 \\
\hline $\mathrm{H}$ & -10.475176 & 8.272116 & 2.806595 \\
\hline $\mathrm{H}$ & -9.008587 & 7.137973 & 1.151109 \\
\hline $\mathrm{H}$ & -11.629573 & 6.499085 & 1.686700 \\
\hline $\mathrm{H}$ & -8.977926 & 4.690673 & 1.043133 \\
\hline $\mathrm{H}$ & -8.198466 & 5.203156 & 2.518144 \\
\hline $\mathrm{H}$ & -9.837263 & 4.575605 & 2.567492 \\
\hline$N$ & -10.363122 & 6.325279 & 5.239884 \\
\hline C & -11.214402 & 5.721314 & 6.240613 \\
\hline C & -12.207051 & 6.706314 & 6.865822 \\
\hline $\mathrm{O}$ & -13.185155 & 6.280572 & 7.423061 \\
\hline C & -10.396555 & 5.060982 & 7.359045 \\
\hline C & -9.478101 & 3.911877 & 6.917843 \\
\hline C & -8.661192 & 3.427702 & 8.119731 \\
\hline C & -10.246801 & 2.746064 & 6.289385 \\
\hline
\end{tabular}




\begin{tabular}{|c|c|c|c|}
\hline $\mathrm{H}$ & -9.437516 & 6.588959 & 5.507287 \\
\hline $\mathrm{H}$ & -11.837314 & 4.985705 & 5.757037 \\
\hline $\mathrm{H}$ & -9.796264 & 5.822134 & 7.845268 \\
\hline $\mathrm{H}$ & -11.108399 & 4.696996 & 8.092892 \\
\hline $\mathrm{H}$ & -8.779802 & 4.285547 & 6.178014 \\
\hline $\mathrm{H}$ & -7.969882 & 2.641520 & 7.830025 \\
\hline $\mathrm{H}$ & -8.082754 & 4.239166 & 8.548896 \\
\hline $\mathrm{H}$ & -9.307119 & 3.032871 & 8.901334 \\
\hline $\mathrm{H}$ & -9.561149 & 1.949768 & 6.016503 \\
\hline $\mathrm{H}$ & -10.980636 & 2.338150 & 6.982615 \\
\hline $\mathrm{H}$ & -10.769388 & 3.037772 & 5.385163 \\
\hline$N$ & -11.895268 & 8.017538 & 6.820323 \\
\hline C & -12.827647 & 8.999955 & 7.287868 \\
\hline$C$ & -13.875021 & 9.388054 & 6.265499 \\
\hline $\mathrm{H}$ & -14.622480 & 10.098675 & 6.636203 \\
\hline $\mathrm{O}$ & -13.910679 & 8.993856 & 5.147651 \\
\hline $\mathrm{H}$ & -11.160970 & 8.329980 & 6.223270 \\
\hline $\mathrm{H}$ & -13.332800 & 8.632576 & 8.171896 \\
\hline $\mathrm{H}$ & -12.292981 & 9.901693 & 7.573044 \\
\hline$N$ & -11.427718 & 10.616303 & -3.022662 \\
\hline $\mathrm{H}$ & -11.181784 & 10.821002 & -2.074668 \\
\hline C & -11.805542 & 9.208255 & -3.171993 \\
\hline C & -11.411876 & 8.859853 & -4.600570 \\
\hline $\mathrm{O}$ & -10.372408 & 8.296781 & -4.847846 \\
\hline C & -13.251584 & 8.847022 & -2.769678 \\
\hline C & -13.485811 & 9.183007 & -1.285248 \\
\hline C & -13.546072 & 7.370428 & -3.064401 \\
\hline C & -14.920521 & 8.964482 & -0.801260 \\
\hline $\mathrm{H}$ & -12.185027 & 11.226758 & -3.265817 \\
\hline & -11.130048 & 8.630122 & -2.5572 \\
\hline
\end{tabular}




\begin{tabular}{|c|c|c|c|}
\hline$H$ & -13.938881 & 9.461455 & -3.355672 \\
\hline $\mathrm{H}$ & -12.805127 & 8.589818 & -0.678202 \\
\hline $\mathrm{H}$ & -13.228457 & 10.223205 & $5-1.111829$ \\
\hline $\mathrm{H}$ & -14.588386 & 7.137580 & -2.888916 \\
\hline $\mathrm{H}$ & -13.329917 & 7.104116 & -4.094842 \\
\hline $\mathrm{H}$ & -12.950770 & 6.727756 & -2.423252 \\
\hline $\mathrm{H}$ & -15.028799 & 9.322482 & 0.216146 \\
\hline $\mathrm{H}$ & -15.632448 & 9.504830 & -1.419559 \\
\hline $\mathrm{H}$ & -15.199251 & 7.917440 & -0.803647 \\
\hline$N$ & -12.213403 & 9.290245 & -5.603581 \\
\hline $\mathrm{H}$ & -13.132393 & 9.597055 & -5.378919 \\
\hline$C$ & -11.972873 & 8.843874 & -6.959238 \\
\hline $\mathrm{H}$ & -12.036120 & 7.767574 & -7.043632 \\
\hline $\mathrm{H}$ & -12.729694 & 9.282233 & -7.598216 \\
\hline$C$ & -10.601373 & 9.232962 & -7.517197 \\
\hline $\mathrm{O}$ & -9.995633 & 8.495660 & -8.239499 \\
\hline$N$ & -10.162522 & 10.477376 & $6-7.182169$ \\
\hline $\mathrm{H}$ & -10.712985 & 11.009959 & $9-6.548360$ \\
\hline C & -8.894347 & 11.024012 & -7.637087 \\
\hline $\mathrm{H}$ & -8.789649 & 10.891462 & -8.702767 \\
\hline $\mathrm{H}$ & -8.879068 & 12.081531 & -7.407689 \\
\hline C & -7.672369 & 10.359332 & -6.988502 \\
\hline $\mathrm{O}$ & -6.753696 & 9.933719 & -7.631003 \\
\hline$N$ & -7.659527 & 10.350076 & -5.619879 \\
\hline $\mathrm{H}$ & -8.480817 & 10.632301 & -5.134111 \\
\hline C & -6.495273 & 9.961231 & -4.832324 \\
\hline $\mathrm{H}$ & -5.599431 & 10.376548 & -5.265838 \\
\hline $\mathrm{H}$ & -6.615523 & 10.362142 & -3.833329 \\
\hline C & -6.266723 & 8.464845 & -4.710641 \\
\hline $\mathrm{O}$ & -5.132773 & 8.048098 & -4.719750 \\
\hline
\end{tabular}




\begin{tabular}{|c|c|c|c|}
\hline$N$ & -7.317132 & 7.640706 & -4.538962 \\
\hline C & -7.031730 & 6.283162 & -4.102747 \\
\hline C & -6.418100 & 5.430947 & -5.206525 \\
\hline $\mathrm{O}$ & -5.584341 & 4.608546 & -4.911717 \\
\hline C & -8.199209 & 5.564407 & -3.387622 \\
\hline C & -9.361970 & 5.169535 & -4.313467 \\
\hline C & -8.633670 & 6.382842 & -2.166096 \\
\hline C & -10.484162 & 4.409860 & -3.603906 \\
\hline $\mathrm{H}$ & -8.253340 & 7.991942 & -4.551234 \\
\hline $\mathrm{H}$ & -6.231077 & 6.343250 & -3.381332 \\
\hline $\mathrm{H}$ & -7.753917 & 4.644906 & -3.016860 \\
\hline $\mathrm{H}$ & -9.773862 & 6.044112 & -4.799706 \\
\hline $\mathrm{H}$ & -8.966171 & 4.535054 & -5.103163 \\
\hline $\mathrm{H}$ & -9.310817 & 5.819887 & -1.537922 \\
\hline $\mathrm{H}$ & -7.775406 & 6.646572 & -1.556464 \\
\hline $\mathrm{H}$ & -9.137245 & 7.297037 & -2.456407 \\
\hline $\mathrm{H}$ & -11.217872 & 4.059045 & -4.323485 \\
\hline $\mathrm{H}$ & -10.106686 & 3.542440 & -3.073613 \\
\hline $\mathrm{H}$ & -11.006150 & 5.037071 & -2.891818 \\
\hline $\mathrm{N}$ & -6.820746 & 5.582730 & -6.487696 \\
\hline$C$ & -6.124706 & 4.841502 & -7.520863 \\
\hline$C$ & -4.769596 & 5.446756 & -7.898048 \\
\hline 0 & -3.906166 & 4.707721 & -8.311091 \\
\hline$C$ & -6.992665 & 4.548821 & -8.753795 \\
\hline$C$ & -8.055759 & 3.457003 & -8.535877 \\
\hline$C$ & -7.451501 & 2.105427 & -8.124890 \\
\hline $\mathrm{N}$ & -8.381463 & 0.972383 & -8.170203 \\
\hline$C$ & -9.145668 & 0.545957 & -7.161522 \\
\hline $\mathrm{N}$ & -9.387147 & 1.353847 & -6.138685 \\
\hline$N$ & -9.650116 & -0.667484 & -7.17732 \\
\hline
\end{tabular}




\begin{tabular}{|c|c|c|c|}
\hline $\mathrm{H}$ & -7.464663 & 6.307747 & -6.721633 \\
\hline $\mathrm{H}$ & -5.829009 & 3.908699 & -7.075691 \\
\hline $\mathrm{H}$ & -7.482563 & 5.456711 & -9.092811 \\
\hline $\mathrm{H}$ & -6.328120 & 4.236451 & -9.552056 \\
\hline $\mathrm{H}$ & -8.783129 & 3.791013 & -7.801400 \\
\hline $\mathrm{H}$ & -8.601200 & 3.324068 & -9.464290 \\
\hline $\mathrm{H}$ & -6.640642 & 1.853746 & -8.795998 \\
\hline $\mathrm{H}$ & -7.028379 & 2.146125 & -7.130341 \\
\hline $\mathrm{H}$ & -8.324676 & 0.367552 & -8.959466 \\
\hline $\mathrm{H}$ & -9.187942 & 2.323896 & -6.209231 \\
\hline $\mathrm{H}$ & -9.908761 & 1.064717 & -5.327857 \\
\hline $\mathrm{H}$ & -9.309925 & -1.401990 & -7.769560 \\
\hline $\mathrm{H}$ & -10.323626 & -0.963698 & $\begin{array}{ll}8 & -6.488263\end{array}$ \\
\hline$N$ & -4.571401 & 6.757467 & -7.722150 \\
\hline $\mathrm{H}$ & -5.310884 & 7.354827 & -7.430331 \\
\hline$C$ & -3.257802 & 7.352376 & -8.011159 \\
\hline $\mathrm{H}$ & -2.929773 & 7.089171 & -9.004152 \\
\hline $\mathrm{H}$ & -3.359826 & 8.427210 & -7.941886 \\
\hline C & -2.200638 & 6.878496 & -7.024560 \\
\hline $\mathrm{O}$ & -1.071750 & 6.644367 & -7.377119 \\
\hline$N$ & -2.628281 & 6.767925 & -5.761127 \\
\hline C & -1.750491 & 6.461060 & -4.657481 \\
\hline C & -1.472709 & 4.974917 & -4.527491 \\
\hline $\mathrm{O}$ & -0.342171 & 4.593876 & -4.332972 \\
\hline C & -2.339329 & 7.057868 & -3.373273 \\
\hline C & -1.752748 & 6.360792 & -2.146915 \\
\hline C & -2.102396 & 8.574094 & -3.408792 \\
\hline $\mathrm{H}$ & -3.551036 & 7.073964 & -5.541396 \\
\hline $\mathrm{H}$ & -0.780207 & 6.899126 & -4.842254 \\
\hline & -3.407429 & 6.876217 & -3.388069 \\
\hline
\end{tabular}




\begin{tabular}{|c|c|c|c|}
\hline $\mathrm{H}$ & -1.966145 & 6.907704 & -1.247674 \\
\hline $\mathrm{H}$ & -2.166310 & 5.364057 & -2.035887 \\
\hline $\mathrm{H}$ & -0.680506 & 6.286294 & -2.218537 \\
\hline $\mathrm{H}$ & -2.833593 & 9.100400 & -2.811775 \\
\hline $\mathrm{H}$ & -1.110853 & 8.816640 & -3.041412 \\
\hline $\mathrm{H}$ & -2.195561 & 8.958917 & -4.417789 \\
\hline$N$ & -2.497811 & 4.112478 & -4.641194 \\
\hline C & -2.166732 & 2.706235 & -4.738411 \\
\hline C & -1.220364 & 2.507835 & -5.924366 \\
\hline $\mathrm{O}$ & -0.299613 & 1.724469 & -5.846519 \\
\hline C & -3.394698 & 1.789820 & -4.822878 \\
\hline C & -4.317762 & 1.918876 & -3.603384 \\
\hline$C$ & -5.382998 & 0.821051 & -3.504261 \\
\hline$N$ & -4.858956 & -0.390765 & -2.879134 \\
\hline C & -5.570174 & -1.480609 & -2.694283 \\
\hline$N$ & -6.815623 & -1.586270 & -3.199269 \\
\hline$N$ & -5.121262 & -2.493840 & -1.979728 \\
\hline $\mathrm{H}$ & -3.426274 & 4.432932 & -4.814255 \\
\hline $\mathrm{H}$ & -1.595729 & 2.414244 & -3.870318 \\
\hline r & -3.953136 & 1.996794 & -5.734046 \\
\hline $\mathrm{H}$ & -3.020653 & 0.775726 & -4.902400 \\
\hline $\mathrm{H}$ & -3.729304 & 1.919009 & -2.700063 \\
\hline $\mathrm{H}$ & -4.838359 & 2.862746 & -3.645256 \\
\hline $\mathrm{H}$ & -6.219089 & 1.187815 & -2.917838 \\
\hline $\mathrm{H}$ & -5.752181 & 0.587600 & -4.500392 \\
\hline $\mathrm{H}$ & -3.950919 & -0.310652 & -2.404335 \\
\hline $\mathrm{H}$ & -7.065169 & -0.991010 & -3.953347 \\
\hline $\mathrm{H}$ & -7.233590 & -2.498455 & -3.208162 \\
\hline $\mathrm{H}$ & -4.262415 & -2.495322 & -1.453764 \\
\hline & -5.653274 & -3.337196 & -1.951064 \\
\hline
\end{tabular}




\begin{tabular}{|c|c|c|c|}
\hline$N$ & -1.459796 & 3.193317 & -7.035069 \\
\hline $\mathrm{H}$ & -2.275109 & 3.755058 & -7.144634 \\
\hline C & -0.596734 & 2.996847 & -8.183415 \\
\hline $\mathrm{H}$ & -0.949731 & 3.640587 & -8.978656 \\
\hline $\mathrm{H}$ & -0.612418 & 1.971696 & -8.524569 \\
\hline C & 0.856438 & 3.342222 & -7.871335 \\
\hline O & 1.782013 & 2.705251 & -8.308357 \\
\hline$N$ & 1.006287 & 4.405059 & -7.066829 \\
\hline $\mathrm{H}$ & 0.213587 & 4.966413 & -6.852763 \\
\hline C & 2.282311 & 4.880660 & -6.640289 \\
\hline $\mathrm{H}$ & 2.920033 & 5.140173 & -7.475915 \\
\hline $\mathrm{H}$ & 2.126226 & 5.775339 & -6.051617 \\
\hline C & 3.092705 & 3.927578 & -5.776448 \\
\hline O & 4.297996 & 4.056843 & -5.690801 \\
\hline$N$ & 2.428042 & 2.983351 & -5.101928 \\
\hline$C$ & 3.098078 & 2.138002 & -4.148361 \\
\hline C & 4.271681 & 1.329667 & -4.712376 \\
\hline 0 & 5.187071 & 1.029301 & -3.972538 \\
\hline$C$ & 2.012622 & 1.258937 & -3.525104 \\
\hline$C$ & 2.383564 & 0.436981 & -2.296646 \\
\hline$C$ & 1.071700 & 0.014275 & -1.644960 \\
\hline $\mathrm{N}$ & 1.261570 & -1.019362 & -0.648220 \\
\hline$C$ & 0.329209 & -1.944122 & -0.369088 \\
\hline $\mathrm{N}$ & -0.822961 & -1.947915 & -0.990811 \\
\hline $\mathrm{N}$ & 0.620913 & -2.889800 & 0.501775 \\
\hline $\mathrm{H}$ & 1.438117 & 2.903912 & -5.207742 \\
\hline $\mathrm{H}$ & 3.559390 & 2.748218 & -3.381224 \\
\hline $\mathrm{H}$ & 1.215832 & 1.941299 & -3.249471 \\
\hline $\mathrm{H}$ & 1.593966 & 0.613242 & -4.288065 \\
\hline $\mathrm{H}$ & 2.964495 & -0.438463 & -2.566318 \\
\hline
\end{tabular}




\begin{tabular}{|c|c|c|c|}
\hline $\mathrm{H}$ & 2.985475 & 1.020988 & -1.603973 \\
\hline $\mathrm{H}$ & 0.567986 & 0.874811 & -1.218005 \\
\hline $\mathrm{H}$ & 0.418636 & -0.386703 & -2.404574 \\
\hline $\mathrm{H}$ & 1.961384 & -0.877409 & 0.045179 \\
\hline $\mathrm{H}$ & -1.270495 & -1.090194 & -1.304959 \\
\hline $\mathrm{H}$ & -1.492543 & -2.673986 & -0.771530 \\
\hline $\mathrm{H}$ & 1.537503 & -2.992026 & 0.869586 \\
\hline $\mathrm{H}$ & -0.129527 & -3.482597 & 0.842485 \\
\hline$N$ & 4.279931 & 0.941792 & -5.999239 \\
\hline $\mathrm{H}$ & 3.540628 & 1.225238 & -6.608640 \\
\hline C & 5.363429 & 0.116650 & -6.504621 \\
\hline $\mathrm{H}$ & 5.137543 & -0.138155 & -7.532654 \\
\hline $\mathrm{H}$ & 5.442790 & -0.800189 & -5.942286 \\
\hline$C$ & 6.736131 & 0.756021 & -6.476487 \\
\hline $\mathrm{O}$ & 7.739004 & 0.089700 & -6.367735 \\
\hline$N$ & 6.766121 & 2.085332 & -6.608316 \\
\hline $\mathrm{H}$ & 5.911829 & 2.604447 & -6.567405 \\
\hline C & 8.004966 & 2.823937 & -6.549436 \\
\hline $\mathrm{H}$ & 8.709861 & 2.441173 & -7.273888 \\
\hline $\mathrm{H}$ & 7.799420 & 3.854984 & -6.804432 \\
\hline C & 8.747309 & 2.825410 & -5.211196 \\
\hline O & 9.888641 & 3.234656 & -5.158081 \\
\hline $\mathrm{N}$ & 8.101388 & 2.335308 & -4.145513 \\
\hline C & 8.680327 & 2.169774 & -2.823268 \\
\hline C & 9.946635 & 1.302910 & -2.830344 \\
\hline $\mathrm{O}$ & 10.763704 & 1.385458 & -1.927473 \\
\hline C & 7.575211 & 1.499062 & -1.976701 \\
\hline C & 7.766324 & 1.380566 & -0.464926 \\
\hline$C$ & 7.417783 & 2.651966 & 0.318509 \\
\hline$C$ & 7.302687 & 2.390078 & 1.816278 \\
\hline
\end{tabular}




\begin{tabular}{|c|c|c|c|}
\hline$N$ & 8.641120 & 2.360680 & 2.477755 \\
\hline $\mathrm{O}$ & 11.024830 & 1.615144 & 0.899022 \\
\hline $\mathrm{H}$ & 10.877058 & 1.824607 & -0.023627 \\
\hline $\mathrm{H}$ & 11.500759 & 0.787047 & 0.851330 \\
\hline $\mathrm{H}$ & 7.155314 & 2.033455 & -4.259736 \\
\hline $\mathrm{H}$ & 8.976984 & 3.126966 & -2.415151 \\
\hline $\mathrm{H}$ & 6.657446 & 2.039390 & -2.165745 \\
\hline $\mathrm{H}$ & 7.411936 & 0.510684 & -2.390454 \\
\hline $\mathrm{H}$ & 7.087260 & 0.600396 & -0.134973 \\
\hline $\mathrm{H}$ & 8.761622 & 1.031072 & -0.224296 \\
\hline $\mathrm{H}$ & 8.129169 & 3.454182 & 0.142888 \\
\hline $\mathrm{H}$ & 6.452813 & 3.019640 & -0.015266 \\
\hline $\mathrm{H}$ & 6.739154 & 3.162927 & 2.315438 \\
\hline $\mathrm{H}$ & 6.822501 & 1.443850 & 2.012396 \\
\hline $\mathrm{H}$ & 9.353189 & 1.894266 & 1.924660 \\
\hline $\mathrm{H}$ & 8.962331 & 3.330852 & 2.630295 \\
\hline $\mathrm{H}$ & 8.585425 & 1.885879 & 3.366971 \\
\hline $\mathrm{N}$ & 10.062473 & 0.448236 & -3.837526 \\
\hline$C$ & 11.109423 & -0.537452 & -4.068766 \\
\hline C & 12.486378 & 0.023107 & -4.427190 \\
\hline $\mathrm{O}$ & 13.444372 & -0.705525 & -4.301692 \\
\hline C & 10.700996 & -1.444195 & -5.251842 \\
\hline C & 9.562735 & -2.442398 & -4.996623 \\
\hline C & 9.130515 & -3.066585 & -6.327159 \\
\hline C & 9.990124 & -3.533298 & -4.014557 \\
\hline $\mathrm{H}$ & 9.324421 & 0.445784 & -4.510916 \\
\hline $\mathrm{H}$ & 11.265041 & -1.124702 & -3.175583 \\
\hline $\mathrm{H}$ & 10.434318 & -0.798623 & -6.083327 \\
\hline $\mathrm{H}$ & 11.583739 & -1.996784 & -5.550593 \\
\hline & 8.706536 & -1.911341 & -4.592493 \\
\hline
\end{tabular}




\begin{tabular}{|c|c|c|c|}
\hline $\mathrm{H}$ & 8.329374 & -3.782884 & -6.172388 \\
\hline $\mathrm{H}$ & 8.772762 & -2.309477 & -7.013203 \\
\hline $\mathrm{H}$ & 9.957405 & -3.594522 & -6.795802 \\
\hline $\mathrm{H}$ & 9.200046 & -4.262903 & -3.874314 \\
\hline $\mathrm{H}$ & 10.859835 & $5-4.059880$ & -4.393709 \\
\hline $\mathrm{H}$ & 10.243703 & $3-3.136659$ & -3.036216 \\
\hline$N$ & 12.574040 & 1.261815 & -4.919241 \\
\hline C & 13.814365 & $\begin{array}{l}5.845027 \\
\end{array}$ & -5.393453 \\
\hline C & 14.959454 & 2.031091 & -4.379514 \\
\hline O & 16.015207 & 2.408283 & -4.813084 \\
\hline C & 13.496834 & 3.173240 & -6.083867 \\
\hline $\mathrm{O}$ & 12.919242 & 4.089092 & -5.197601 \\
\hline $\mathrm{H}$ & 11.755963 & 31.833054 & -4.917152 \\
\hline $\mathrm{H}$ & 14.247581 & 1.179477 & -6.130689 \\
\hline $\mathrm{H}$ & 14.419849 & 3.592385 & -6.452815 \\
\hline $\mathrm{H}$ & 12.845144 & 2.981116 & -6.930316 \\
\hline $\mathrm{H}$ & 11.971617 & 7.079044 & -5.259625 \\
\hline$N$ & 14.792294 & 1.722323 & -3.074273 \\
\hline C & 15.927449 & 1.647073 & -2.176062 \\
\hline C & 16.804345 & 50.410268 & -2.402952 \\
\hline $\mathrm{O}$ & 17.934867 & 0.394781 & -1.989920 \\
\hline C & 15.492578 & $\begin{array}{l}3.575149 \\
\end{array}$ & -0.707132 \\
\hline C & 14.633497 & 2.730365 & -0.197460 \\
\hline C & 14.275318 & 2.479761 & 1.267810 \\
\hline$N$ & 13.249920 & 3.374129 & 1.789875 \\
\hline C & 13.427861 & 4.525812 & 2.416443 \\
\hline$N$ & 14.646199 & 5.001311 & 2.651446 \\
\hline $\mathrm{O}$ & 15.028483 & 7.460479 & 4.200270 \\
\hline $\mathrm{H}$ & 14.742233 & 8.347246 & 3.965182 \\
\hline $\mathrm{H}$ & 15.744800 & 7.566168 & 4.813295 \\
\hline
\end{tabular}




\begin{tabular}{|c|c|c|c|}
\hline $\mathrm{N}$ & 12.378597 & 5.214426 & 2.813528 \\
\hline $\mathrm{H}$ & 13.899645 & 1.424374 & -2.757635 \\
\hline $\mathrm{H}$ & 16.561014 & 2.504510 & -2.343707 \\
\hline $\mathrm{H}$ & 14.953119 & 0.646336 & -0.553579 \\
\hline $\mathrm{H}$ & 16.403783 & 1.506482 & -0.123020 \\
\hline $\mathrm{H}$ & 15.150879 & 3.678802 & -0.309934 \\
\hline $\mathrm{H}$ & 13.715926 & 2.812463 & -0.768282 \\
\hline $\mathrm{H}$ & 13.876858 & 1.480758 & 1.369856 \\
\hline $\mathrm{H}$ & 15.153012 & 2.519180 & 1.902347 \\
\hline $\mathrm{H}$ & 12.309593 & 3.077599 & 1.620636 \\
\hline $\mathrm{H}$ & 15.453422 & 4.508399 & 2.352244 \\
\hline $\mathrm{H}$ & 14.782479 & 5.856907 & 3.161599 \\
\hline $\mathrm{H}$ & 11.422466 & 4.934747 & 2.656962 \\
\hline $\mathrm{H}$ & 12.470938 & 6.058752 & 3.331732 \\
\hline$N$ & 16.200243 & -0.648980 & -2.967248 \\
\hline C & 16.871169 & -1.911296 & -3.166856 \\
\hline C & 17.672697 & -1.890181 & -4.464988 \\
\hline $\mathrm{H}$ & 18.056001 & -0.908483 & -4.752290 \\
\hline $\mathrm{O}$ & 17.892522 & -2.857183 & -5.118531 \\
\hline C & 15.868545 & -3.070246 & -3.149067 \\
\hline C & 15.323575 & -3.399033 & -1.792010 \\
\hline$N$ & 14.402569 & -2.625551 & -1.113883 \\
\hline $\mathrm{O}$ & 12.505261 & -0.431587 & -0.461793 \\
\hline $\mathrm{H}$ & 13.140937 & -1.034460 & -0.851100 \\
\hline $\mathrm{H}$ & 12.021754 & -0.035064 & -1.178537 \\
\hline C & 15.617382 & -4.489055 & -1.048507 \\
\hline C & 14.169880 & -3.248580 & -0.005115 \\
\hline$N$ & 14.879583 & -4.379022 & 0.103505 \\
\hline $\mathrm{H}$ & 15.284663 & -0.551312 & -3.350771 \\
\hline $\mathrm{H}$ & 17.595216 & -2.030652 & -2.368481 \\
\hline
\end{tabular}




\begin{tabular}{|c|c|c|c|}
\hline $\mathrm{H}$ & 15.051744 & -2.842623 & -3.825294 \\
\hline $\mathrm{H}$ & 16.370118 & -3.944154 & -3.542418 \\
\hline $\mathrm{H}$ & 16.272300 & -5.313503 & -1.232667 \\
\hline $\mathrm{H}$ & 13.490877 & -2.918023 & 0.754754 \\
\hline $\mathrm{H}$ & 14.777596 & -5.061568 & 0.822398 \\
\hline $\mathrm{O}$ & -14.810668 & -2.300463 & 5.221065 \\
\hline $\mathrm{H}$ & -15.117954 & -1.614369 & 4.633438 \\
\hline $\mathrm{H}$ & -14.385842 & -1.854057 & 5.946330 \\
\hline O & -12.887354 & -0.749716 & 7.017608 \\
\hline $\mathrm{H}$ & -12.157408 & -1.240044 & 7.380299 \\
\hline $\mathrm{H}$ & -13.143846 & -0.123774 & 7.683721 \\
\hline $\mathrm{O}$ & -12.182389 & -0.219934 & 4.191620 \\
\hline $\mathrm{H}$ & -12.317790 & -0.215653 & 5.135005 \\
\hline $\mathrm{H}$ & -13.062350 & -0.213540 & 3.812117 \\
\hline O & -13.444610 & -2.081829 & 1.043386 \\
\hline $\mathrm{H}$ & -12.707094 & -1.469625 & 0.999055 \\
\hline $\mathrm{H}$ & -13.253806 & -2.667306 & 1.776452 \\
\hline 0 & -7.227548 & 1.027278 & -0.132813 \\
\hline $\mathrm{H}$ & -7.417762 & 1.422513 & 0.721308 \\
\hline $\mathrm{H}$ & -6.292804 & 0.853920 & -0.116698 \\
\hline 0 & -7.800419 & 2.004921 & 2.507516 \\
\hline $\mathrm{H}$ & -8.737229 & 1.938269 & 2.672960 \\
\hline $\mathrm{H}$ & -7.464331 & 2.681235 & 3.089814 \\
\hline 0 & -9.391463 & -0.255073 & -1.399411 \\
\hline $\mathrm{H}$ & -8.588874 & 0.107549 & -1.005860 \\
\hline $\mathrm{H}$ & -9.193998 & -1.131989 & -1.714374 \\
\hline 0 & -12.898664 & -2.342439 & -1.717073 \\
\hline $\mathrm{H}$ & -13.227643 & -2.467610 & -0.824356 \\
\hline $\mathrm{H}$ & -13.559134 & -2.657688 & -2.325338 \\
\hline 0 & -13.582322 & 1.728901 & -0.13945 \\
\hline
\end{tabular}




\begin{tabular}{|c|c|c|c|}
\hline $\mathrm{H}$ & -12.969970 & 1.047323 & 0.144676 \\
\hline $\mathrm{H}$ & -13.096574 & 2.541090 & 0.019456 \\
\hline $\mathrm{O}$ & -14.214222 & 4.925261 & 2.493490 \\
\hline $\mathrm{H}$ & -13.815871 & 5.427744 & 3.198927 \\
\hline $\mathrm{H}$ & -14.742625 & 4.237703 & 2.880913 \\
\hline $\mathrm{O}$ & -15.511899 & 2.131104 & 1.952915 \\
\hline $\mathrm{H}$ & -16.374601 & 2.337125 & 1.617116 \\
\hline $\mathrm{H}$ & -14.938951 & 2.043800 & 1.188553 \\
\hline $\mathrm{O}$ & -13.352006 & 0.380953 & -2.617827 \\
\hline $\mathrm{H}$ & -13.638571 & 0.979425 & -1.926569 \\
\hline $\mathrm{H}$ & -13.107532 & -0.426616 & -2.164391 \\
\hline $\mathrm{O}$ & -14.253928 & -1.710512 & -4.323675 \\
\hline $\mathrm{H}$ & -14.310430 & -0.848266 & -3.916051 \\
\hline $\mathrm{H}$ & -15.046048 & -1.836794 & -4.829677 \\
\hline $\mathrm{O}$ & -10.280927 & -2.825816 & -2.846643 \\
\hline $\mathrm{H}$ & -10.563576 & -2.516503 & -3.706541 \\
\hline $\mathrm{H}$ & -11.076214 & -2.783319 & -2.316075 \\
\hline 0 & -11.492292 & -0.111724 & 0.552159 \\
\hline $\mathrm{H}$ & -10.814978 & -0.221725 & -0.116904 \\
\hline $\mathrm{H}$ & -11.088207 & 0.400549 & 1.257364 \\
\hline 0 & -10.712479 & 1.650485 & 2.581335 \\
\hline $\mathrm{H}$ & -11.198742 & 1.216540 & 3.282895 \\
\hline $\mathrm{H}$ & -11.220941 & 2.398159 & 2.269761 \\
\hline 0 & -12.144953 & 3.759879 & 1.083310 \\
\hline $\mathrm{H}$ & -12.856805 & 4.177199 & 1.581844 \\
\hline $\mathrm{H}$ & -11.581387 & 4.476823 & 0.807283 \\
\hline $\mathrm{O}$ & -10.739823 & 0.825174 & -3.610766 \\
\hline $\mathrm{H}$ & -11.624638 & 0.967810 & -3.270608 \\
\hline $\mathrm{H}$ & -10.209406 & 0.558524 & -2.853536 \\
\hline O & -14.797356 & -0.395952 & 3.050048 \\
\hline
\end{tabular}




$\begin{array}{llll}\mathrm{H} & -15.180792 & 0.434158 & 2.765888 \\ \mathrm{H} & -14.625983 & -0.910082 & 2.263435 \\ \mathrm{O} & -12.786793 & -3.291148 & 3.584722 \\ \mathrm{H} & -13.540288 & -3.087120 & 4.150767 \\ \mathrm{H} & -12.146449 & -2.622532 & 3.809038 \\ \mathrm{O} & -11.464431 & -1.464384 & -5.064547 \\ \mathrm{H} & -12.394426 & -1.689741 & -5.061388 \\ \mathrm{H} & -11.372522 & -0.701816 & -4.494661\end{array}$

PROTON CONFIGURATION, GIVEN BY NOMINAL CHARGES: R300(+),Y266(-),E183(0) POTENTIAL:+70mV

$\mathrm{N}$

$\mathrm{H}$

C

C

0

C

$\mathrm{H}$

$\mathrm{H}$

$\mathrm{H}$

$\mathrm{H}$

$\mathrm{H}$

$\mathrm{N}$

$\mathrm{H}$

C

$\mathrm{H}$

$\mathrm{H}$

C

$\mathrm{O}$
$15.580942 \quad-6.451471 \quad 5.391415$

$14.780032-6.202450 \quad 5.939015$

$\begin{array}{lll}15.508936 & -5.856267 & 4.054381\end{array}$

$\begin{array}{lll}14.783016 & -6.782958 & 3.060461\end{array}$

$14.052927 \quad-6.345848 \quad 2.186351$

$\begin{array}{lll}16.918723 & -5.575267 & 3.520829\end{array}$

$\begin{array}{lll}16.388922 & -6.126445 & 5.884811\end{array}$

$\begin{array}{lll}14.943548 & -4.933783 & 4.050777\end{array}$

$\begin{array}{lll}16.887508 & -5.188602 & 2.509196\end{array}$

$17.402282-4.830204 \quad 4.142438$

$17.526634-6.473225 \quad 3.529213$

$\begin{array}{lll}15.032385 & -8.085607 & 3.186394\end{array}$

$\begin{array}{lll}15.611726 & -8.361773 \quad 3.949956\end{array}$

$\begin{array}{lll}14.398655 & -9.092381 & 2.333234\end{array}$

$\begin{array}{lll}14.482026 & -8.823411 & 1.292930\end{array}$

$14.891814-10.042109 \quad 2.492291$

$\begin{array}{lll}12.912941 & -9.221802 & 2.671549\end{array}$

$\begin{array}{lll}12.081991 & -9.427725 & 1.826222\end{array}$ 


\begin{tabular}{|c|c|c|c|}
\hline$N$ & 12.582805 & -9.055890 & 3.966874 \\
\hline $\mathrm{H}$ & 13.302601 & -8.984225 & 4.648771 \\
\hline C & 11.195103 & -9.170806 & 4.421393 \\
\hline $\mathrm{H}$ & 11.188268 & -9.158052 & 5.503150 \\
\hline $\mathrm{H}$ & 10.743814 & -10.088276 & 4.076999 \\
\hline C & 10.336955 & -8.010603 & 3.900717 \\
\hline 0 & 9.191008 & -8.186419 & 3.583982 \\
\hline$N$ & 10.946840 & -6.814402 & 3.855477 \\
\hline C & 10.317806 & -5.633946 & 3.317913 \\
\hline C & 10.125936 & -5.690944 & 1.799676 \\
\hline O & 9.095409 & -5.262914 & 1.337619 \\
\hline C & 11.091985 & -4.387878 & 3.776677 \\
\hline 0 & 12.478081 & -4.570518 & 3.768589 \\
\hline $\mathrm{H}$ & 11.905153 & -6.743734 & 4.107118 \\
\hline $\mathrm{H}$ & 9.314021 & -5.553346 & 3.701716 \\
\hline $\mathrm{H}$ & 10.799985 & -3.539354 & 3.168231 \\
\hline $\mathrm{H}$ & 10.827193 & -4.174714 & 4.801965 \\
\hline $\mathrm{H}$ & 12.804154 & -4.753549 & 2.895974 \\
\hline$N$ & 11.099427 & -6.200134 & 1.024233 \\
\hline C & 10.843906 & -6.396280 & -0.400142 \\
\hline C & 9.693932 & -7.385891 & -0.601949 \\
\hline $\mathrm{O}$ & 8.946846 & -7.281531 & -1.546722 \\
\hline C & 12.132581 & -6.784079 & -1.186875 \\
\hline C & 12.335091 & -8.289392 & -1.402676 \\
\hline C & 12.158507 & -6.059844 & -2.534220 \\
\hline $\mathrm{H}$ & 11.923592 & -6.585517 & 1.431446 \\
\hline $\mathrm{H}$ & 10.473599 & -5.462125 & -0.791291 \\
\hline $\mathrm{H}$ & 12.964299 & -6.412703 & -0.598709 \\
\hline $\mathrm{H}$ & 13.289243 & -8.451943 & -1.893254 \\
\hline $\mathrm{H}$ & 12.333626 & -8.855099 & -0.481749 \\
\hline
\end{tabular}




\begin{tabular}{|c|c|c|c|}
\hline $\mathrm{H}$ & 11.568572 & -8.697706 & -2.054165 \\
\hline $\mathrm{H}$ & 13.053324 & -6.323369 & -3.087025 \\
\hline $\mathrm{H}$ & 11.298163 & -6.332651 & -3.135467 \\
\hline $\mathrm{H}$ & 12.157646 & -4.984566 & -2.401658 \\
\hline$N$ & 9.570413 & -8.340330 & 0.328325 \\
\hline $\mathrm{H}$ & 10.313985 & -8.473695 & 0.975007 \\
\hline C & 8.593028 & -9.421195 & 0.241219 \\
\hline $\mathrm{H}$ & 8.934730 & -10.218716 & 0.888658 \\
\hline $\mathrm{H}$ & 8.533182 & -9.803389 & -0.766172 \\
\hline C & 7.181601 & -9.046055 & 0.643019 \\
\hline $\mathrm{O}$ & 6.257696 & -9.504303 & 0.011540 \\
\hline$N$ & 7.003254 & -8.225682 & 1.684795 \\
\hline C & 5.669337 & -7.737864 & 1.953248 \\
\hline C & 5.250683 & -6.728463 & 0.889688 \\
\hline $\mathrm{O}$ & 4.074489 & -6.635555 & 0.610319 \\
\hline C & 5.415062 & -7.230052 & 3.386666 \\
\hline C & 5.555804 & -8.372944 & 4.397153 \\
\hline C & 6.257693 & -6.021256 & 3.798335 \\
\hline $\mathrm{H}$ & 7.780786 & -7.958039 & 2.248181 \\
\hline $\mathrm{H}$ & 4.986498 & -8.557633 & 1.792534 \\
\hline $\mathrm{H}$ & 4.374560 & -6.920133 & 3.379027 \\
\hline $\mathrm{H}$ & 5.265824 & -8.033989 & 5.386151 \\
\hline $\mathrm{H}$ & 4.916955 & -9.210139 & 4.135403 \\
\hline $\mathrm{H}$ & 6.577632 & -8.731095 & 4.453691 \\
\hline $\mathrm{H}$ & 5.917252 & -5.647714 & 4.758148 \\
\hline $\mathrm{H}$ & 7.299799 & -6.292437 & 3.905981 \\
\hline $\mathrm{H}$ & 6.186132 & -5.207611 & 3.084889 \\
\hline$N$ & 6.170722 & -6.001783 & 0.247305 \\
\hline C & 5.774057 & -5.183188 & -0.886544 \\
\hline C & 5.342453 & -6.067614 & -2.064619 \\
\hline
\end{tabular}




\begin{tabular}{|c|c|c|c|}
\hline $\mathrm{O}$ & 4.319611 & -5.816967 & -2.657693 \\
\hline C & 6.866370 & -4.163568 & -1.266490 \\
\hline C & 7.064089 & -3.146778 & -0.128135 \\
\hline C & 6.495640 & -3.453091 & -2.572901 \\
\hline C & 8.358608 & -2.341364 & -0.241507 \\
\hline $\mathrm{H}$ & 7.131547 & -6.055767 & 0.508370 \\
\hline $\mathrm{H}$ & 4.875774 & -4.647055 & -0.619625 \\
\hline $\mathrm{H}$ & 7.796413 & -4.704961 & -1.414136 \\
\hline $\mathrm{H}$ & 6.209675 & -2.471852 & -0.113046 \\
\hline $\mathrm{H}$ & 7.072752 & -3.660261 & 0.824024 \\
\hline $\mathrm{H}$ & 7.201073 & -2.663439 & -2.792634 \\
\hline $\mathrm{H}$ & 6.496123 & -4.128372 & -3.418814 \\
\hline $\mathrm{H}$ & 5.507777 & -3.009450 & -2.506345 \\
\hline $\mathrm{H}$ & 8.459669 & -1.667279 & 0.602395 \\
\hline $\mathrm{H}$ & 9.219569 & -2.999574 & -0.240210 \\
\hline $\mathrm{H}$ & 8.395924 & -1.742331 & -1.144429 \\
\hline $\mathrm{N}$ & 6.115094 & -7.110267 & -2.399764 \\
\hline $\mathrm{H}$ & 7.009200 & -7.232182 & -1.972917 \\
\hline$C$ & 5.773166 & -7.950654 & -3.527407 \\
\hline $\mathrm{H}$ & 6.603773 & -8.624464 & -3.697164 \\
\hline $\mathrm{H}$ & 5.618088 & -7.364990 & -4.421243 \\
\hline$C$ & 4.504391 & -8.779437 & -3.325286 \\
\hline 0 & 3.666727 & -8.839217 & -4.194252 \\
\hline $\mathrm{N}$ & 4.393489 & -9.432237 & -2.161725 \\
\hline $\mathrm{H}$ & 5.130116 & -9.386274 & -1.490282 \\
\hline$C$ & 3.207509 & -10.187687 & -1.833100 \\
\hline $\mathrm{H}$ & 2.968457 & -10.901194 & -2.607387 \\
\hline $\mathrm{H}$ & 3.399729 & -10.729245 & -0.915337 \\
\hline$C$ & 1.974222 & -9.306874 & -1.644766 \\
\hline 0 & 0.887641 & -9.696716 & -2.006853 \\
\hline
\end{tabular}




\begin{tabular}{|c|c|c|c|}
\hline$N$ & 2.187839 & -8.106992 & -1.107224 \\
\hline C & 1.057293 & -7.190318 & -1.062587 \\
\hline C & 0.614393 & -6.717149 & -2.449618 \\
\hline $\mathrm{O}$ & -0.568222 & -6.580797 & -2.672229 \\
\hline C & 1.260884 & -5.953916 & -0.192301 \\
\hline $\mathrm{O}$ & 1.407207 & -6.258096 & 1.155035 \\
\hline $\mathrm{H}$ & 3.085630 & -7.817890 & -0.791642 \\
\hline $\mathrm{H}$ & 0.208391 & -7.722702 & -0.665750 \\
\hline $\mathrm{H}$ & 2.095744 & -5.368047 & -0.560913 \\
\hline $\mathrm{H}$ & 0.360409 & -5.370723 & -0.290954 \\
\hline $\mathrm{H}$ & 2.310209 & -6.499304 & 1.310800 \\
\hline$N$ & 1.541978 & -6.433219 & -3.373326 \\
\hline C & 1.145306 & -6.026444 & -4.708903 \\
\hline C & 0.383926 & -7.150161 & -5.406686 \\
\hline $\mathrm{O}$ & -0.599414 & -6.895377 & -6.070889 \\
\hline$C$ & 2.355364 & -5.516309 & -5.525830 \\
\hline$C$ & 2.750161 & -4.121661 & -5.011860 \\
\hline $\mathrm{C}$ & 2.065082 & -5.493782 & -7.031873 \\
\hline$C$ & 4.071113 & -3.598567 & -5.573819 \\
\hline $\mathrm{H}$ & 2.512179 & -6.484400 & -3.147282 \\
\hline $\mathrm{H}$ & 0.421352 & -5.228028 & -4.630260 \\
\hline $\mathrm{H}$ & 3.180097 & -6.200860 & -5.352025 \\
\hline $\mathrm{H}$ & 1.952712 & -3.421885 & -5.256114 \\
\hline $\mathrm{H}$ & 2.823616 & -4.148562 & -3.932382 \\
\hline $\mathrm{H}$ & 2.922287 & -5.115928 & -7.574977 \\
\hline $\mathrm{H}$ & 1.855414 & -6.482508 & -7.421643 \\
\hline $\mathrm{H}$ & 1.216128 & -4.857591 & -7.261829 \\
\hline $\mathrm{H}$ & 4.355835 & -2.684147 & -5.064138 \\
\hline $\mathrm{H}$ & 4.869191 & -4.319087 & -5.425944 \\
\hline $\mathrm{H}$ & 4.011957 & -3.377696 & -6.633782 \\
\hline
\end{tabular}




\begin{tabular}{|c|c|c|c|}
\hline $\mathrm{N}$ & 0.822029 & -8.401361 & -5.244419 \\
\hline $\mathrm{H}$ & 1.666961 & -8.578312 & -4.741837 \\
\hline C & 0.045183 & -9.507550 & -5.750396 \\
\hline $\mathrm{H}$ & -0.058547 & -9.464457 & -6.824391 \\
\hline $\mathrm{H}$ & 0.555968 & -10.424984 & -5.487021 \\
\hline C & -1.365283 & -9.540071 & -5.166931 \\
\hline $\mathrm{O}$ & -2.319660 & -9.766341 & -5.875532 \\
\hline$N$ & -1.472619 & -9.318902 & -3.858011 \\
\hline C & -2.777199 & -9.341695 & -3.238902 \\
\hline C & -3.683450 & -8.205506 & -3.713465 \\
\hline $\mathrm{O}$ & -4.881322 & -8.411248 & -3.773692 \\
\hline C & -2.686361 & -9.304845 & -1.706302 \\
\hline $\mathrm{O}$ & -3.934868 & -9.567478 & -1.126550 \\
\hline $\mathrm{H}$ & -0.656773 & -9.234632 & -3.288022 \\
\hline $\mathrm{H}$ & -3.297570 & -10.246125 & -3.521001 \\
\hline $\mathrm{H}$ & -2.007741 & -10.077477 & $7-1.372691$ \\
\hline $\mathrm{H}$ & -2.297630 & -8.346339 & -1.379736 \\
\hline $\mathrm{H}$ & -4.620706 & -9.163263 & -1.643416 \\
\hline$N$ & -3.127111 & -7.027015 & -3.988257 \\
\hline C & -3.875863 & -5.901980 & -4.524784 \\
\hline C & -4.428614 & -6.235544 & -5.910866 \\
\hline $\mathrm{O}$ & -5.594890 & -6.043062 & -6.174788 \\
\hline C & -2.949638 & -4.666160 & -4.544134 \\
\hline C & -3.373582 & -3.509162 & -5.434866 \\
\hline C & -4.697943 & -3.121138 & -5.605936 \\
\hline C & -2.390079 & -2.794466 & -6.112213 \\
\hline C & -5.023140 & -2.068528 & -6.447950 \\
\hline C & -2.709037 & -1.731822 & -6.940011 \\
\hline C & -4.034028 & -1.369769 & -7.118460 \\
\hline 1 & -2.153777 & -6.905563 & -3.79775 \\
\hline
\end{tabular}




\begin{tabular}{|c|c|c|c|}
\hline $\mathrm{H}$ & -4.737744 & -5.708640 & -3.905770 \\
\hline $\mathrm{H}$ & -2.835792 & -4.330664 & -3.517704 \\
\hline $\mathrm{H}$ & -1.973785 & -4.990141 & -4.875645 \\
\hline $\mathrm{H}$ & -5.483973 & -3.657794 & -5.111689 \\
\hline $\mathrm{H}$ & -1.358668 & -3.077613 & -5.997935 \\
\hline $\mathrm{H}$ & -6.058947 & -1.809003 & -6.590949 \\
\hline $\mathrm{H}$ & -1.926647 & -1.197125 & -7.447783 \\
\hline $\mathrm{H}$ & -4.287805 & -0.558995 & -7.778972 \\
\hline$N$ & -3.561441 & -6.734521 & -6.798272 \\
\hline C & -4.008508 & -7.078851 & -8.128429 \\
\hline C & -5.138203 & -8.106391 & -8.097030 \\
\hline 0 & -6.023786 & -8.048359 & -8.910373 \\
\hline C & -2.862308 & -7.619503 & -8.986796 \\
\hline S & -1.637985 & -6.375566 & -9.505778 \\
\hline $\mathrm{H}$ & -2.619303 & -6.918057 & -6.521968 \\
\hline $\mathrm{H}$ & -4.430210 & -6.208006 & -8.607851 \\
\hline $\mathrm{H}$ & -2.363073 & -8.438930 & -8.487425 \\
\hline $\mathrm{H}$ & -3.282114 & -7.998944 & -9.908641 \\
\hline $\mathrm{H}$ & -1.028311 & -6.216387 & -8.341476 \\
\hline $\mathrm{N}$ & -5.078530 & -9.066907 & -7.155626 \\
\hline $\mathrm{H}$ & -4.254448 & -9.191282 & -6.606960 \\
\hline$C$ & -6.085060 & -10.116602 & -7.179909 \\
\hline $\mathrm{H}$ & -6.256484 & -10.458535 & -8.188814 \\
\hline $\mathrm{H}$ & -5.713662 & -10.945999 & -6.589336 \\
\hline$C$ & -7.459153 & -9.722789 & -6.613043 \\
\hline 0 & -8.482202 & -10.083712 & -7.127603 \\
\hline $\mathrm{N}$ & -7.417997 & -9.015713 & -5.478473 \\
\hline$C$ & -8.635228 & -8.650794 & -4.780214 \\
\hline$C$ & -9.497583 & -7.639488 & -5.527515 \\
\hline 0 & -10.691523 & -7.613783 & -5.3822 \\
\hline
\end{tabular}




\begin{tabular}{|c|c|c|c|}
\hline C & -8.342204 & -8.119479 & -3.365555 \\
\hline C & -7.452656 & -6.868873 & -3.253586 \\
\hline C & -8.068928 & -5.495205 & -3.197281 \\
\hline 0 & -9.366890 & -5.425967 & -3.274947 \\
\hline $\mathrm{H}$ & -9.662569 & -4.515899 & -3.140575 \\
\hline $\mathrm{O}$ & -7.375855 & -4.513192 & -3.069779 \\
\hline $\mathrm{H}$ & -6.533145 & -8.780549 & -5.080936 \\
\hline $\mathrm{H}$ & -9.261654 & -9.526501 & -4.683426 \\
\hline $\mathrm{H}$ & -9.293990 & -7.952983 & -2.881624 \\
\hline $\mathrm{H}$ & -7.844713 & -8.919716 & -2.828593 \\
\hline $\mathrm{H}$ & -6.865359 & -6.932172 & -2.345462 \\
\hline $\mathrm{H}$ & -6.729038 & -6.824038 & -4.049967 \\
\hline$N$ & -8.837321 & -6.734073 & -6.273860 \\
\hline C & -9.562354 & -5.706193 & -6.995284 \\
\hline C & -10.454398 & -6.285823 & -8.085024 \\
\hline $\mathrm{H}$ & -10.492575 & -7.369440 & -8.176611 \\
\hline $\mathrm{O}$ & -11.082831 & -5.574761 & -8.812120 \\
\hline C & -8.632334 & -4.607486 & -7.558369 \\
\hline $\mathrm{O}$ & -9.420395 & -3.476450 & -7.901913 \\
\hline C & -7.779995 & -5.030258 & -8.747174 \\
\hline $\mathrm{H}$ & -7.862924 & -6.858983 & -6.447851 \\
\hline $\mathrm{H}$ & -10.234191 & -5.219167 & -6.297860 \\
\hline $\mathrm{H}$ & -7.989439 & -4.289969 & -6.748737 \\
\hline $\mathrm{H}$ & -10.129723 & -3.785046 & -8.459788 \\
\hline $\mathrm{H}$ & -7.102240 & -4.222849 & -9.003008 \\
\hline $\mathrm{H}$ & -7.189656 & -5.910795 & -8.540406 \\
\hline $\mathrm{H}$ & -8.400760 & -5.233196 & -9.613299 \\
\hline$N$ & -12.952337 & -9.022034 & 3.174429 \\
\hline $\mathrm{H}$ & -13.221898 & -9.547843 & 2.368800 \\
\hline C & -11.777476 & -8.184402 & 2.899254 \\
\hline
\end{tabular}




\begin{tabular}{|c|c|c|c|}
\hline C & -10.786206 & -8.141049 & 4.045833 \\
\hline 0 & -9.651794 & -7.748991 & 3.844940 \\
\hline C & -12.139204 & -6.715971 & 2.593616 \\
\hline $\mathrm{O}$ & -12.682500 & -6.210242 & 3.786160 \\
\hline C & -13.123190 & -6.564867 & 1.439408 \\
\hline $\mathrm{H}$ & -13.731053 & -8.457372 & 3.453447 \\
\hline $\mathrm{H}$ & -11.234128 & -8.594502 & 2.059193 \\
\hline $\mathrm{H}$ & -11.220030 & -6.198617 & 2.354573 \\
\hline $\mathrm{H}$ & -12.705580 & -5.256359 & 3.753617 \\
\hline $\mathrm{H}$ & -13.277812 & -5.511284 & 1.231776 \\
\hline $\mathrm{H}$ & -12.759142 & -7.035134 & 0.533219 \\
\hline $\mathrm{H}$ & -14.086619 & -6.993097 & 1.688714 \\
\hline $\mathrm{N}$ & -11.197283 & -8.533693 & 5.241879 \\
\hline $\mathrm{H}$ & -12.125441 & -8.894327 & 5.282310 \\
\hline$C$ & -10.410839 & -8.533143 & 6.450874 \\
\hline $\mathrm{H}$ & -10.329810 & -7.538243 & 6.859445 \\
\hline $\mathrm{H}$ & -10.935126 & -9.138969 & 7.180508 \\
\hline C & -8.975370 & -9.054834 & 6.377001 \\
\hline 0 & -8.142317 & -8.496461 & 7.047326 \\
\hline $\mathrm{N}$ & -8.658401 & -10.127681 & 5.629597 \\
\hline $\mathrm{H}$ & -9.367337 & -10.601801 & 5.117236 \\
\hline C & -7.269036 & -10.558230 & 5.527662 \\
\hline $\mathrm{H}$ & -7.250873 & -11.535566 & 5.062507 \\
\hline $\mathrm{H}$ & -6.829823 & -10.640686 & 6.508852 \\
\hline$C$ & -6.387520 & -9.608326 & 4.692692 \\
\hline 0 & -5.220778 & -9.464714 & 4.951348 \\
\hline$N$ & -7.045371 & -9.017966 & 3.701161 \\
\hline C & -6.307770 & -7.993249 & 2.974966 \\
\hline C & -6.271951 & -6.694110 & 3.775155 \\
\hline 0 & -5.283209 & -5.999603 & 3.73604 \\
\hline
\end{tabular}




\begin{tabular}{|c|c|c|c|}
\hline C & -6.624645 & -7.839933 & 1.474129 \\
\hline C & -7.801491 & -7.000779 & 1.021382 \\
\hline C & -7.793771 & -5.617349 & 1.177637 \\
\hline C & -8.855869 & -7.580140 & 0.327550 \\
\hline C & -8.823001 & -4.839135 & 0.674989 \\
\hline C & -9.875794 & -6.802301 & -0.196695 \\
\hline C & -9.867860 & -5.429397 & -0.016917 \\
\hline $\mathrm{H}$ & -8.039999 & -9.015706 & 3.680426 \\
\hline $\mathrm{H}$ & -5.283584 & -8.321480 & 2.999193 \\
\hline $\mathrm{H}$ & -5.727772 & -7.417896 & 1.034140 \\
\hline $\mathrm{H}$ & -6.708627 & -8.843258 & 1.073702 \\
\hline $\mathrm{H}$ & -6.967234 & -5.138390 & 1.670106 \\
\hline $\mathrm{H}$ & -8.865826 & -8.644534 & 0.166700 \\
\hline $\mathrm{H}$ & -8.794372 & -3.771435 & 0.802038 \\
\hline $\mathrm{H}$ & -10.662946 & -7.264403 & -0.763886 \\
\hline $\mathrm{H}$ & -10.656231 & -4.826671 & -0.429504 \\
\hline$N$ & -7.304887 & -6.402066 & 4.568621 \\
\hline C & -7.243633 & -5.290399 & 5.486837 \\
\hline C & -6.122529 & -5.500849 & 6.503923 \\
\hline O & -5.483919 & -4.544745 & 6.888491 \\
\hline C & -8.621133 & -5.123009 & 6.162735 \\
\hline C & -8.870978 & -3.782230 & 6.819262 \\
\hline C & -9.074329 & -2.647422 & 6.039317 \\
\hline C & -8.965227 & -3.665869 & 8.199371 \\
\hline C & -9.352878 & -1.424655 & 6.623845 \\
\hline C & -9.252495 & -2.444533 & 8.791351 \\
\hline C & -9.444026 & -1.319377 & 8.006301 \\
\hline $\mathrm{H}$ & -8.166278 & -6.890903 & 4.456207 \\
\hline $\mathrm{H}$ & -6.978275 & -4.384626 & 4.960604 \\
\hline $\mathrm{H}$ & -9.370110 & -5.271048 & 5.391559 \\
\hline
\end{tabular}




\begin{tabular}{|c|c|c|c|}
\hline $\mathrm{H}$ & -8.750975 & -5.915427 & 6.887595 \\
\hline $\mathrm{H}$ & -9.015326 & -2.717689 & 4.966554 \\
\hline $\mathrm{H}$ & -8.815744 & -4.531611 & 8.820473 \\
\hline $\mathrm{H}$ & -9.495167 & -0.559151 & 6.003164 \\
\hline $\mathrm{H}$ & -9.314106 & -2.372866 & 9.862383 \\
\hline $\mathrm{H}$ & -9.639479 & -0.365401 & 8.463951 \\
\hline$N$ & -5.873694 & -6.737403 & 6.930639 \\
\hline $\mathrm{H}$ & -6.523837 & -7.462461 & 6.723681 \\
\hline C & -4.798918 & -7.027219 & 7.853568 \\
\hline $\mathrm{H}$ & -4.839434 & -6.392910 & 8.726262 \\
\hline $\mathrm{H}$ & -4.912057 & -8.055293 & 8.173308 \\
\hline$C$ & -3.402041 & -6.855021 & 7.259756 \\
\hline $\mathrm{O}$ & -2.529453 & -6.356048 & 7.929815 \\
\hline$N$ & -3.199026 & -7.288853 & 6.010718 \\
\hline$C$ & -1.917440 & -7.117395 & 5.343880 \\
\hline$C$ & -1.661931 & -5.648043 & 4.980942 \\
\hline $\mathrm{O}$ & -0.560113 & -5.169528 & 5.147342 \\
\hline C & -1.834981 & -8.056581 & 4.120196 \\
\hline C & -0.666627 & -7.705511 & 3.197092 \\
\hline C & -1.737973 & -9.515476 & 4.580732 \\
\hline $\mathrm{H}$ & -3.931066 & -7.772440 & 5.536444 \\
\hline $\mathrm{H}$ & -1.128028 & -7.365099 & 6.038671 \\
\hline $\mathrm{H}$ & -2.754394 & -7.933020 & 3.552461 \\
\hline $\mathrm{H}$ & -0.603247 & -8.434485 & 2.396249 \\
\hline $\mathrm{H}$ & -0.772052 & -6.731927 & 2.739407 \\
\hline $\mathrm{H}$ & 0.276865 & -7.719144 & 3.734016 \\
\hline $\mathrm{H}$ & -1.752280 & -10.179174 & 3.722230 \\
\hline $\mathrm{H}$ & -0.805135 & -9.682838 & 5.113241 \\
\hline $\mathrm{H}$ & -2.556016 & -9.800751 & 5.229403 \\
\hline $\mathrm{N}$ & -2.693556 & -4.955263 & 4.487767 \\
\hline
\end{tabular}




\begin{tabular}{|c|c|c|c|}
\hline C & -2.553158 & -3.575182 & 4.065039 \\
\hline C & -2.426463 & -2.653977 & 5.277199 \\
\hline $\mathrm{O}$ & -1.538667 & -1.831911 & 5.330023 \\
\hline C & -3.719679 & -3.117509 & 3.177421 \\
\hline C & -3.943574 & -3.892489 & 1.868582 \\
\hline C & -2.804542 & -3.821061 & 0.863953 \\
\hline $\mathrm{O}$ & -1.683850 & -4.161187 & 1.268101 \\
\hline $\mathrm{O}$ & -3.071448 & -3.447741 & -0.292538 \\
\hline $\mathrm{H}$ & -3.561739 & -5.418545 & 4.333127 \\
\hline $\mathrm{H}$ & -1.634155 & -3.470126 & 3.520324 \\
\hline $\mathrm{H}$ & -4.640090 & -3.161549 & 3.752004 \\
\hline $\mathrm{H}$ & -3.547026 & -2.069386 & 2.949929 \\
\hline $\mathrm{H}$ & -4.110693 & -4.936857 & 2.087166 \\
\hline $\mathrm{H}$ & -4.844544 & -3.509167 & 1.402187 \\
\hline$N$ & -3.326750 & -2.787077 & 6.269499 \\
\hline C & -3.116889 & -2.037329 & 7.484968 \\
\hline C & -1.794237 & -2.427359 & 8.154644 \\
\hline $\mathrm{O}$ & -1.131337 & -1.588396 & 8.724486 \\
\hline C & -4.246694 & -2.024466 & 8.524552 \\
\hline O & -4.447835 & -3.267125 & 9.130153 \\
\hline C & -5.533301 & -1.411439 & 7.983889 \\
\hline $\mathrm{H}$ & -4.003544 & -3.516870 & 6.241112 \\
\hline $\mathrm{H}$ & -2.967713 & -1.006143 & 7.204427 \\
\hline $\mathrm{H}$ & -3.872217 & -1.383373 & 9.312827 \\
\hline $\mathrm{H}$ & -4.896526 & -3.841595 & 8.521772 \\
\hline $\mathrm{H}$ & -6.263504 & -1.356701 & 8.781924 \\
\hline $\mathrm{H}$ & -5.353002 & -0.406131 & 7.615824 \\
\hline $\mathrm{H}$ & -5.954533 & -1.999485 & 7.179516 \\
\hline$N$ & -1.380385 & -3.700437 & 8.043347 \\
\hline II & -2.029276 & -4.420959 & 7.815592 \\
\hline
\end{tabular}




\begin{tabular}{|c|c|c|c|}
\hline C & -0.188931 & -4.105369 & 8.744389 \\
\hline $\mathrm{H}$ & -0.243085 & -3.889884 & 9.802219 \\
\hline $\mathrm{H}$ & -0.075224 & -5.172869 & 8.608749 \\
\hline C & 1.063302 & -3.417566 & 8.226548 \\
\hline $\mathrm{O}$ & 1.938496 & -3.074993 & 8.988764 \\
\hline$N$ & 1.155534 & -3.255591 & 6.890819 \\
\hline$C$ & 2.335628 & -2.659989 & 6.327401 \\
\hline C & 2.293225 & -1.139129 & 6.163586 \\
\hline $\mathrm{O}$ & 3.361827 & -0.575540 & 6.017522 \\
\hline C & 2.843026 & -3.357441 & 5.065548 \\
\hline$S$ & 1.926576 & -3.033088 & 3.535114 \\
\hline $\mathrm{H}$ & 0.423034 & -3.606058 & 6.305800 \\
\hline $\mathrm{H}$ & 3.115762 & -2.790277 & 7.062151 \\
\hline $\mathrm{H}$ & 3.846755 & -3.004983 & 4.876236 \\
\hline $\mathrm{H}$ & 2.884937 & -4.422992 & 5.243934 \\
\hline $\mathrm{H}$ & 0.884375 & -3.824861 & 3.739961 \\
\hline$N$ & 1.142633 & -0.471584 & 6.274413 \\
\hline C & 1.204810 & 0.968241 & 6.492101 \\
\hline C & 1.755916 & 1.271616 & 7.870712 \\
\hline $\mathrm{O}$ & 2.370061 & 2.288207 & 8.086287 \\
\hline C & -0.097734 & 1.735605 & 6.179432 \\
\hline C & -1.203364 & 1.552573 & 7.229246 \\
\hline C & -0.548937 & 1.433114 & 4.745437 \\
\hline C & -2.472122 & 2.355834 & 6.934670 \\
\hline $\mathrm{H}$ & 0.274371 & -0.966553 & 6.293137 \\
\hline $\mathrm{H}$ & 1.951539 & 1.351706 & 5.815329 \\
\hline $\mathrm{H}$ & 0.200172 & 2.777829 & 6.223077 \\
\hline $\mathrm{H}$ & -1.452254 & 0.506401 & 7.335103 \\
\hline $\mathrm{H}$ & -0.819965 & 1.868670 & 8.194982 \\
\hline $\mathrm{H}$ & -1.307234 & 2.135135 & 4.422711 \\
\hline
\end{tabular}




\begin{tabular}{|c|c|c|c|}
\hline $\mathrm{H}$ & 0.285689 & 1.518562 & 4.055049 \\
\hline $\mathrm{H}$ & -0.954514 & 0.435057 & 4.649078 \\
\hline $\mathrm{H}$ & -3.146623 & 2.317607 & 7.784741 \\
\hline $\mathrm{H}$ & -2.247035 & 3.401138 & 6.738861 \\
\hline $\mathrm{H}$ & -3.007297 & 1.965581 & 6.075985 \\
\hline$N$ & 1.549778 & 0.323284 & 8.791250 \\
\hline $\mathrm{H}$ & 0.887646 & -0.400248 & 8.614365 \\
\hline C & 1.977411 & 0.513277 & 10.160240 \\
\hline $\mathrm{H}$ & 1.469297 & -0.222138 & 10.771158 \\
\hline $\mathrm{H}$ & 1.736371 & 1.502965 & 10.518037 \\
\hline C & 3.481464 & 0.338437 & 10.305644 \\
\hline O & 4.163590 & 1.129611 & 10.897343 \\
\hline $\mathrm{N}$ & 3.999374 & -0.752513 & 9.695502 \\
\hline $\mathrm{H}$ & 3.390026 & -1.465660 & 9.353573 \\
\hline C & 5.424384 & -0.937776 & 9.668387 \\
\hline $\mathrm{H}$ & 5.637697 & -1.908748 & 9.236466 \\
\hline $\mathrm{H}$ & 5.854709 & -0.902773 & 10.658595 \\
\hline C & 6.156892 & 0.119125 & 8.844833 \\
\hline O & 7.292422 & 0.410310 & 9.129215 \\
\hline$N$ & 5.502073 & 0.621264 & 7.799016 \\
\hline C & 6.105379 & 1.756770 & 7.150116 \\
\hline C & 5.527844 & 3.076528 & 7.655357 \\
\hline $\mathrm{H}$ & 5.019807 & 3.036813 & 8.617533 \\
\hline O & 5.660218 & 4.095982 & 7.051290 \\
\hline C & 6.009871 & 1.677000 & 5.624112 \\
\hline C & 6.759574 & 0.508461 & 5.018261 \\
\hline C & 8.125859 & 0.324149 & 5.234675 \\
\hline C & 6.096367 & -0.399896 & 4.198540 \\
\hline C & 8.810160 & -0.721369 & 4.629662 \\
\hline C & 6.778403 & -1.443104 & 3.592198 \\
\hline
\end{tabular}




\begin{tabular}{|c|c|c|c|}
\hline C & 8.139152 & -1.604335 & 3.797882 \\
\hline $\mathrm{H}$ & 4.574258 & 0.337994 & 7.572539 \\
\hline $\mathrm{H}$ & 7.140676 & 1.765946 & 7.455081 \\
\hline $\mathrm{H}$ & 4.967618 & 1.616394 & 5.336959 \\
\hline $\mathrm{H}$ & 6.390954 & 2.611487 & 5.226767 \\
\hline $\mathrm{H}$ & 8.656185 & 0.980627 & 5.903151 \\
\hline $\mathrm{H}$ & 5.035834 & -0.303646 & 4.063675 \\
\hline $\mathrm{H}$ & 9.861591 & -0.848598 & 4.821775 \\
\hline $\mathrm{H}$ & 6.244060 & -2.134648 & 2.965419 \\
\hline $\mathrm{H}$ & 8.657876 & -2.416890 & 3.322938 \\
\hline$N$ & 12.613551 & 9.596234 & -1.248798 \\
\hline $\mathrm{H}$ & 11.871436 & 9.915304 & -1.843204 \\
\hline$C$ & 12.304851 & 8.264736 & -0.719492 \\
\hline C & 11.274408 & 8.450403 & 0.387212 \\
\hline $\mathrm{O}$ & 10.267132 & 7.777553 & 0.417750 \\
\hline C & 13.562748 & 7.581214 & -0.165073 \\
\hline C & 14.645710 & 7.276794 & -1.205745 \\
\hline$S$ & 14.018807 & 6.202678 & -2.529470 \\
\hline C & 15.502614 & 6.053319 & -3.553061 \\
\hline $\mathrm{H}$ & 13.431108 & 9.571901 & -1.825531 \\
\hline $\mathrm{H}$ & 11.834411 & 7.616446 & -1.447272 \\
\hline $\mathrm{H}$ & 14.000797 & 8.205633 & 0.607734 \\
\hline $\mathrm{H}$ & 13.255768 & 6.653868 & 0.304789 \\
\hline $\mathrm{H}$ & 15.045970 & 8.189500 & -1.632722 \\
\hline $\mathrm{H}$ & 15.470760 & 6.784540 & -0.703374 \\
\hline $\mathrm{H}$ & 15.252313 & 5.397949 & -4.373385 \\
\hline $\mathrm{H}$ & 15.802203 & 7.017027 & -3.944248 \\
\hline $\mathrm{H}$ & 16.320373 & 5.616909 & -2.994482 \\
\hline$N$ & 11.504217 & 9.413659 & 1.296390 \\
\hline C & 10.594885 & 9.608715 & 2.408553 \\
\hline
\end{tabular}




\begin{tabular}{|c|c|c|c|}
\hline C & 9.277870 & 10.277202 & 2.011867 \\
\hline 0 & 8.282950 & 10.058359 & 2.656562 \\
\hline C & 11.278152 & 10.368526 & 3.552276 \\
\hline C & 12.449004 & 9.562346 & 4.084095 \\
\hline $\mathrm{O}$ & 13.584327 & 9.823627 & 3.745137 \\
\hline$N$ & 12.144247 & 8.559449 & 4.917297 \\
\hline $\mathrm{H}$ & 12.327524 & 9.969043 & 1.204660 \\
\hline $\mathrm{H}$ & 10.295446 & 8.638638 & 2.770692 \\
\hline $\mathrm{H}$ & 11.660153 & 11.325520 & 3.219551 \\
\hline $\mathrm{H}$ & 10.548333 & 10.537494 & 4.333937 \\
\hline $\mathrm{H}$ & 12.887462 & 7.962683 & 5.210285 \\
\hline $\mathrm{H}$ & 11.222606 & 8.173133 & 4.951167 \\
\hline$N$ & 9.264065 & 11.068466 & 0.922439 \\
\hline $\mathrm{H}$ & 10.110333 & 11.226927 & 0.425236 \\
\hline C & 8.003508 & 11.517469 & 0.368849 \\
\hline $\mathrm{H}$ & 8.200883 & 12.260155 & -0.393534 \\
\hline $\mathrm{H}$ & 7.389207 & 11.966707 & 1.131974 \\
\hline$C$ & 7.186379 & 10.384433 & -0.254310 \\
\hline 0 & 5.986762 & 10.471967 & -0.294680 \\
\hline $\mathrm{N}$ & 7.862975 & 9.332412 & -0.761422 \\
\hline$C$ & 7.170216 & 8.144717 & -1.231180 \\
\hline$C$ & 6.634121 & 7.301583 & -0.063672 \\
\hline 0 & 5.533542 & 6.810234 & -0.143954 \\
\hline$C$ & 8.047953 & 7.341826 & -2.220812 \\
\hline$C$ & 8.344214 & 8.193868 & -3.469735 \\
\hline C & 7.376724 & 6.017526 & -2.604410 \\
\hline$C$ & 9.427799 & 7.619595 & -4.385676 \\
\hline $\mathrm{H}$ & 8.823171 & 9.228493 & -0.531400 \\
\hline $\mathrm{H}$ & 6.279689 & 8.470973 & -1.745832 \\
\hline $\mathrm{H}$ & 8.986622 & 7.114373 & -1.723614 \\
\hline
\end{tabular}




\begin{tabular}{|c|c|c|c|}
\hline $\mathrm{H}$ & 7.422527 & 8.321304 & -4.033115 \\
\hline $\mathrm{H}$ & 8.648074 & 9.189061 & -3.162501 \\
\hline $\mathrm{H}$ & 7.994074 & 5.475544 & -3.309450 \\
\hline $\mathrm{H}$ & 7.223435 & 5.377267 & -1.744262 \\
\hline $\mathrm{H}$ & 6.407815 & 6.182227 & -3.064151 \\
\hline $\mathrm{H}$ & 9.623064 & 8.299583 & -5.208546 \\
\hline $\mathrm{H}$ & 10.362875 & 7.472532 & -3.851957 \\
\hline $\mathrm{H}$ & 9.146213 & 6.665900 & -4.816478 \\
\hline$N$ & 7.386803 & 7.177010 & 1.048001 \\
\hline C & 6.878471 & 6.458966 & 2.205533 \\
\hline C & 5.526387 & 7.037544 & 2.643594 \\
\hline $\mathrm{O}$ & 4.576348 & 6.328842 & 2.872050 \\
\hline C & 7.832431 & 6.546973 & 3.407115 \\
\hline C & 9.253720 & 5.998440 & 3.246793 \\
\hline O & 10.146174 & 6.634099 & 3.801494 \\
\hline $\mathrm{O}$ & 9.426915 & 4.935452 & 2.599915 \\
\hline $\mathrm{H}$ & 8.347402 & 7.444505 & 1.025873 \\
\hline 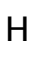 & 6.683101 & 5.430114 & 1.946241 \\
\hline $\mathrm{H}$ & 7.920235 & 7.571604 & 3.736778 \\
\hline r & 7.374599 & 5.993215 & 4.224226 \\
\hline$N$ & 5.485186 & 8.375799 & 2.789221 \\
\hline $\mathrm{H}$ & 6.314855 & 8.912710 & 2.651013 \\
\hline C & 4.293396 & 9.041619 & 3.254297 \\
\hline $\mathrm{H}$ & 4.532121 & 10.084823 & 3.419754 \\
\hline $\mathrm{H}$ & 3.949486 & 8.628088 & 4.189184 \\
\hline C & 3.132251 & 8.990779 & 2.270521 \\
\hline $\mathrm{O}$ & 1.991221 & 9.122025 & 2.649825 \\
\hline$N$ & 3.467175 & 8.824996 & 0.988015 \\
\hline $\mathrm{H}$ & 4.428731 & 8.814459 & 0.740715 \\
\hline 6 & 2.517177 & 8.905263 & -0.078282 \\
\hline
\end{tabular}




\begin{tabular}{|c|c|c|c|}
\hline $\mathrm{H}$ & 3.061296 & 9.090783 & -0.995428 \\
\hline $\mathrm{H}$ & 1.843247 & 9.737601 & 0.070557 \\
\hline C & 1.609143 & 7.687010 & -0.310217 \\
\hline O & 0.575961 & 7.817995 & -0.906057 \\
\hline$N$ & 2.066603 & 6.540113 & 0.228298 \\
\hline C & 1.243128 & 5.374178 & 0.441778 \\
\hline C & 0.429102 & 5.482160 & 1.735839 \\
\hline $\mathrm{O}$ & -0.766627 & 5.314105 & 1.713365 \\
\hline C & 2.095741 & 4.105816 & 0.436590 \\
\hline $\mathrm{H}$ & 2.966990 & 6.568467 & 0.657682 \\
\hline $\mathrm{H}$ & 0.512481 & 5.324257 & -0.347877 \\
\hline $\mathrm{H}$ & 1.475672 & 3.237653 & 0.631216 \\
\hline $\mathrm{H}$ & 2.565032 & 3.992230 & -0.533730 \\
\hline $\mathrm{H}$ & 2.875060 & 4.149297 & 1.190578 \\
\hline$N$ & 1.071867 & 5.741877 & 2.891149 \\
\hline C & 0.343102 & 5.580151 & 4.140230 \\
\hline C & -0.767934 & 6.615908 & 4.294558 \\
\hline $\mathrm{O}$ & -1.802740 & 6.320914 & 4.852384 \\
\hline C & 1.264523 & 5.506261 & 5.382840 \\
\hline C & 1.858524 & 6.852144 & 5.825842 \\
\hline C & 2.351129 & 4.441076 & 5.171011 \\
\hline C & 2.702732 & 6.755957 & 7.099120 \\
\hline $\mathrm{H}$ & 2.061139 & 5.864756 & 2.905732 \\
\hline $\mathrm{H}$ & -0.187624 & 4.640344 & 4.088332 \\
\hline $\mathrm{H}$ & 0.610483 & 5.167122 & 6.180480 \\
\hline $\mathrm{H}$ & 2.459789 & 7.263805 & 5.021839 \\
\hline $\mathrm{H}$ & 1.055437 & 7.560337 & 6.002264 \\
\hline $\mathrm{H}$ & 2.727135 & 4.087057 & 6.119765 \\
\hline $\mathrm{H}$ & 1.955619 & 3.585054 & 4.633775 \\
\hline $\mathrm{H}$ & 3.190411 & 4.832017 & 4.605953 \\
\hline
\end{tabular}




\begin{tabular}{|c|c|c|c|}
\hline $\mathrm{H}$ & 3.008906 & 7.747130 & 7.420653 \\
\hline $\mathrm{H}$ & 2.134150 & 6.309856 & 7.909746 \\
\hline $\mathrm{H}$ & 3.597757 & 6.162523 & 6.959547 \\
\hline$N$ & -0.547166 & 7.847269 & 3.811235 \\
\hline $\mathrm{H}$ & 0.336647 & 8.082052 & 3.409699 \\
\hline C & -1.515070 & 8.891045 & 4.019963 \\
\hline $\mathrm{H}$ & -1.102613 & 9.816787 & 3.639001 \\
\hline $\mathrm{H}$ & -1.728571 & 9.026558 & 5.070337 \\
\hline C & -2.867019 & 8.618723 & 3.365914 \\
\hline $\mathrm{O}$ & -3.871542 & 8.838073 & 4.002641 \\
\hline$N$ & -2.902654 & 8.109030 & 2.120710 \\
\hline C & -4.238446 & 8.014455 & 1.531311 \\
\hline C & -4.962235 & 6.725911 & 1.882105 \\
\hline O & -6.175023 & 6.710540 & 1.860509 \\
\hline C & -4.026033 & 8.271837 & 0.032465 \\
\hline C & -2.762245 & 9.138872 & 0.038703 \\
\hline C & -1.907041 & 8.464089 & 1.099009 \\
\hline $\mathrm{H}$ & -4.862895 & 8.793693 & 1.939718 \\
\hline $\mathrm{H}$ & -3.860235 & 7.345333 & -0.501208 \\
\hline $\mathrm{H}$ & -4.889032 & 8.758703 & -0.403610 \\
\hline $\mathrm{H}$ & -2.253665 & 9.198035 & -0.905772 \\
\hline $\mathrm{H}$ & -3.012754 & 10.149174 & 0.353458 \\
\hline $\mathrm{H}$ & -1.432187 & 7.575084 & 0.724914 \\
\hline $\mathrm{H}$ & -1.141107 & 9.109403 & 1.496836 \\
\hline$N$ & -4.226116 & 5.661452 & 2.225155 \\
\hline C & -4.858905 & 4.466859 & 2.756249 \\
\hline C & -5.578253 & 4.757896 & 4.076637 \\
\hline $\mathrm{O}$ & -6.637196 & 4.221784 & 4.318215 \\
\hline C & -3.849571 & 3.315415 & 2.961167 \\
\hline$C$ & -3.522554 & 2.489227 & 1.733310 \\
\hline
\end{tabular}




\begin{tabular}{|c|c|c|c|}
\hline C & -4.006256 & 1.192327 & 1.597330 \\
\hline C & -2.682196 & 2.954873 & 0.724458 \\
\hline C & -3.679641 & 0.393976 & 0.511541 \\
\hline C & -2.345570 & 2.174039 & -0.361123 \\
\hline C & -2.830320 & 0.859002 & -0.515103 \\
\hline $\mathrm{O}$ & -2.526300 & 0.141424 & -1.551460 \\
\hline $\mathrm{H}$ & -3.233060 & 5.755324 & 2.273732 \\
\hline $\mathrm{H}$ & -5.634268 & 4.148768 & 2.076922 \\
\hline $\mathrm{H}$ & -2.947150 & 3.730906 & 3.400116 \\
\hline $\mathrm{H}$ & -4.274834 & 2.654773 & 3.708629 \\
\hline $\mathrm{H}$ & -4.648977 & 0.785788 & 2.361985 \\
\hline $\mathrm{H}$ & -2.263497 & 3.938608 & 0.793964 \\
\hline $\mathrm{H}$ & -4.035128 & -0.621264 & 0.461151 \\
\hline $\mathrm{H}$ & -1.690545 & 2.565435 & -1.121639 \\
\hline$N$ & -4.978249 & 5.593349 & 4.943342 \\
\hline $\mathrm{H}$ & -4.052878 & 5.920958 & 4.757670 \\
\hline C & -5.595830 & 5.948867 & 6.201061 \\
\hline $\mathrm{H}$ & -4.820004 & 6.301440 & 6.869721 \\
\hline $\mathrm{H}$ & -6.073260 & 5.090711 & 6.645348 \\
\hline C & -6.660373 & 7.040856 & 6.077506 \\
\hline $\mathrm{O}$ & -7.722216 & 6.950555 & 6.650877 \\
\hline$N$ & -6.341994 & 8.091462 & 5.314146 \\
\hline $\mathrm{H}$ & -5.441694 & 8.149171 & 4.887399 \\
\hline C & -7.257699 & 9.220080 & 5.085083 \\
\hline $\mathrm{H}$ & -6.767935 & 9.890855 & 4.391520 \\
\hline $\mathrm{H}$ & -7.457015 & 9.753941 & 6.002489 \\
\hline C & -8.613245 & 8.822905 & 4.520945 \\
\hline $\mathrm{O}$ & -9.605397 & 9.419352 & 4.858020 \\
\hline$N$ & -8.648646 & 7.802868 & 3.639474 \\
\hline C & -9.906427 & 7.387730 & 3.064070 \\
\hline
\end{tabular}




\begin{tabular}{|c|c|c|c|}
\hline C & -10.814615 & 6.618253 & 4.025119 \\
\hline $\mathrm{O}$ & -11.922342 & 6.303047 & 3.625709 \\
\hline C & -9.699456 & 6.577154 & 1.766027 \\
\hline $\mathrm{O}$ & -10.918960 & 6.515377 & 1.049630 \\
\hline C & -9.142513 & 5.179909 & 1.996305 \\
\hline $\mathrm{H}$ & -7.796253 & 7.388578 & 3.321987 \\
\hline $\mathrm{H}$ & -10.475007 & 8.272123 & 2.806771 \\
\hline $\mathrm{H}$ & -9.008548 & 7.138293 & 1.151057 \\
\hline $\mathrm{H}$ & -11.629541 & 6.500089 & 1.686895 \\
\hline $\mathrm{H}$ & -8.978219 & 4.691000 & 1.042450 \\
\hline $\mathrm{H}$ & -8.198473 & 5.203106 & 2.517425 \\
\hline $\mathrm{H}$ & -9.837331 & 4.575701 & 2.566939 \\
\hline$N$ & -10.362995 & 6.324476 & 5.239523 \\
\hline C & -11.214402 & 5.721314 & 6.240613 \\
\hline C & -12.204465 & 6.707703 & 6.867696 \\
\hline $\mathrm{O}$ & -13.182138 & 6.283487 & 7.426952 \\
\hline C & -10.396506 & 5.058836 & 7.357807 \\
\hline C & -9.479288 & 3.909496 & 6.914686 \\
\hline C & -8.662618 & 3.422662 & 8.115655 \\
\hline C & -10.249252 & 2.745435 & 6.284559 \\
\hline$r$ & -9.437061 & 6.587469 & 5.506673 \\
\hline $\mathrm{H}$ & -11.839051 & 4.987107 & 5.757154 \\
\hline $\mathrm{H}$ & -9.795195 & 5.818862 & 7.844521 \\
\hline $\mathrm{H}$ & -11.108262 & 4.694674 & 8.091654 \\
\hline $\mathrm{H}$ & -8.780726 & 4.283617 & 6.175345 \\
\hline $\mathrm{H}$ & -7.972056 & 2.636351 & 7.824574 \\
\hline $\mathrm{H}$ & -8.083338 & 4.232886 & 8.546012 \\
\hline $\mathrm{H}$ & -9.308772 & 3.027201 & 8.896768 \\
\hline $\mathrm{H}$ & -9.564390 & 1.949162 & 6.009681 \\
\hline & -10.982936 & 2.336746 & 6.977519 \\
\hline
\end{tabular}




\begin{tabular}{|c|c|c|c|}
\hline $\mathrm{H}$ & -10.772233 & 3.039237 & 5.381240 \\
\hline$N$ & -11.890969 & 8.018420 & 6.821510 \\
\hline C & -12.820046 & 9.002006 & 7.292926 \\
\hline C & -13.872848 & 9.389999 & 6.276136 \\
\hline $\mathrm{H}$ & -14.617563 & 10.101552 & 6.650702 \\
\hline $\mathrm{O}$ & -13.915547 & 8.994959 & 5.158858 \\
\hline $\mathrm{H}$ & -11.156942 & 8.330169 & 6.223743 \\
\hline $\mathrm{H}$ & -13.320760 & 8.636217 & 8.180174 \\
\hline $\mathrm{H}$ & -12.283011 & 9.903589 & 7.574076 \\
\hline$N$ & -11.428782 & 10.616585 & -3.022953 \\
\hline $\mathrm{H}$ & -11.181466 & 10.821440 & -2.075361 \\
\hline C & -11.805542 & 9.208255 & -3.171993 \\
\hline C & -11.412068 & 8.859747 & -4.600590 \\
\hline $\mathrm{O}$ & -10.372868 & 8.296374 & -4.847938 \\
\hline C & -13.251221 & 8.846068 & -2.769246 \\
\hline$C$ & -13.485124 & 9.181872 & -1.284733 \\
\hline C & -13.544905 & 7.369304 & -3.063914 \\
\hline C & -14.919638 & 8.962969 & -0.800354 \\
\hline $\mathrm{H}$ & -12.186808 & 11.226537 & -3.265071 \\
\hline $\mathrm{H}$ & -11.129483 & 8.630685 & -2.557341 \\
\hline $\mathrm{H}$ & -13.939062 & 9.460100 & -3.355054 \\
\hline $\mathrm{H}$ & -12.804141 & 8.588809 & -0.677922 \\
\hline $\mathrm{H}$ & -13.227943 & 10.222109 & -1.111296 \\
\hline $\mathrm{H}$ & -14.586961 & 7.135733 & -2.887775 \\
\hline $\mathrm{H}$ & -13.329214 & 7.103259 & -4.094512 \\
\hline $\mathrm{H}$ & -12.948756 & 6.726968 & -2.423236 \\
\hline $\mathrm{H}$ & -15.027701 & 9.320778 & 0.217149 \\
\hline $\mathrm{H}$ & -15.631859 & 9.503270 & -1.418386 \\
\hline $\mathrm{H}$ & -15.198148 & 7.915864 & -0.802825 \\
\hline$N$ & -12.213547 & 9.290342 & -5.603633 \\
\hline
\end{tabular}




\begin{tabular}{|c|c|c|c|}
\hline $\mathrm{H}$ & -13.132406 & 9.597526 & -5.379013 \\
\hline C & -11.972873 & 8.843874 & -6.959238 \\
\hline $\mathrm{H}$ & -12.036058 & 7.767562 & -7.043539 \\
\hline $\mathrm{H}$ & -12.729630 & 9.282181 & -7.598332 \\
\hline C & -10.601373 & 9.232962 & -7.517197 \\
\hline $\mathrm{O}$ & -9.996079 & 8.495776 & -8.239965 \\
\hline$N$ & -10.162529 & 10.477325 & $5-7.182123$ \\
\hline $\mathrm{H}$ & -10.712336 & 11.009297 & $7-6.547235$ \\
\hline C & -8.894347 & 11.024012 & -7.637087 \\
\hline $\mathrm{H}$ & -8.789739 & 10.891343 & -8.702753 \\
\hline $\mathrm{H}$ & -8.879150 & 12.081555 & -7.407803 \\
\hline C & -7.672369 & 10.359332 & -6.988502 \\
\hline $\mathrm{O}$ & -6.753965 & 9.933366 & -7.631082 \\
\hline$N$ & -7.659524 & 10.350113 & -5.619891 \\
\hline $\mathrm{H}$ & -8.480443 & 10.633312 & -5.134100 \\
\hline C & -6.495273 & 9.961231 & -4.832324 \\
\hline $\mathrm{H}$ & -5.599400 & 10.376510 & -5.265816 \\
\hline $\mathrm{H}$ & -6.615483 & 10.362178 & -3.833336 \\
\hline$C$ & -6.266723 & 8.464845 & -4.710641 \\
\hline $\mathrm{O}$ & -5.132833 & 8.048154 & -4.719743 \\
\hline$N$ & -7.317133 & 7.640563 & -4.539039 \\
\hline C & -7.031573 & 6.283107 & -4.102678 \\
\hline C & -6.418931 & 5.430126 & -5.206427 \\
\hline $\mathrm{O}$ & -5.586728 & 4.606355 & -4.911260 \\
\hline C & -8.198463 & 5.564610 & -3.386362 \\
\hline C & -9.361756 & 5.169035 & -4.311225 \\
\hline C & -8.632279 & 6.383551 & -2.164964 \\
\hline C & -10.482793 & 4.408545 & -3.600702 \\
\hline $\mathrm{H}$ & -8.253318 & 7.991768 & -4.551204 \\
\hline $\mathrm{H}$ & -6.230345 & 6.343370 & -3.38192 \\
\hline
\end{tabular}




\begin{tabular}{|c|c|c|c|}
\hline $\mathrm{H}$ & -7.752766 & 4.645382 & -3.015459 \\
\hline $\mathrm{H}$ & -9.774614 & 6.043361 & -4.797090 \\
\hline $\mathrm{H}$ & -8.966287 & 4.534851 & -5.101317 \\
\hline $\mathrm{H}$ & -9.309101 & 5.820863 & -1.536192 \\
\hline $\mathrm{H}$ & -7.773699 & 6.647537 & -1.555899 \\
\hline $\mathrm{H}$ & -9.135988 & 7.297652 & -2.455395 \\
\hline $\mathrm{H}$ & -11.216954 & 4.057318 & -4.319632 \\
\hline $\mathrm{H}$ & -10.104193 & 3.541312 & -3.070904 \\
\hline $\mathrm{H}$ & -11.004489 & 5.035287 & -2.887965 \\
\hline$N$ & -6.820884 & 5.582674 & -6.487785 \\
\hline C & -6.124627 & 4.841492 & -7.520874 \\
\hline C & -4.769750 & 5.446888 & -7.898563 \\
\hline $\mathrm{O}$ & -3.906737 & 4.707881 & -8.312407 \\
\hline C & -6.992446 & 4.547912 & -8.753666 \\
\hline C & -8.054183 & 3.454884 & -8.535516 \\
\hline C & -7.448181 & 2.104001 & -8.124832 \\
\hline$N$ & -8.377144 & 0.970074 & -8.169473 \\
\hline C & -9.141568 & 0.544168 & -7.160846 \\
\hline$N$ & -9.383153 & 1.352333 & -6.138252 \\
\hline$N$ & -9.646514 & -0.669132 & -7.176514 \\
\hline $\mathrm{H}$ & -7.463236 & 6.309046 & -6.721803 \\
\hline $\mathrm{H}$ & -5.828534 & 3.909038 & -7.075290 \\
\hline $\mathrm{H}$ & -7.483445 & 5.455266 & -9.092532 \\
\hline $\mathrm{H}$ & -6.327709 & 4.236324 & -9.552057 \\
\hline $\mathrm{H}$ & -8.781678 & 3.788049 & -7.800766 \\
\hline $\mathrm{H}$ & -8.599796 & 3.321296 & -9.463741 \\
\hline $\mathrm{H}$ & -6.637600 & 1.853063 & -8.796527 \\
\hline $\mathrm{H}$ & -7.024312 & 2.145133 & -7.130623 \\
\hline $\mathrm{H}$ & -8.319372 & 0.364483 & -8.958076 \\
\hline & -9.181981 & 2.322015 & -6.208071 \\
\hline
\end{tabular}




\begin{tabular}{|c|c|c|c|}
\hline $\mathrm{H}$ & -9.904768 & 1.063414 & -5.327421 \\
\hline $\mathrm{H}$ & -9.306774 & -1.403720 & -7.768900 \\
\hline $\mathrm{H}$ & -10.319989 & -0.965052 & -6.487346 \\
\hline$N$ & -4.571401 & 6.757467 & -7.722150 \\
\hline $\mathrm{H}$ & -5.310566 & 7.354659 & -7.429234 \\
\hline C & -3.257802 & 7.352376 & -8.011159 \\
\hline $\mathrm{H}$ & -2.929895 & 7.089195 & -9.004186 \\
\hline $\mathrm{H}$ & -3.359766 & 8.427208 & -7.941863 \\
\hline C & -2.200566 & 6.878360 & -7.024721 \\
\hline $\mathrm{O}$ & -1.071914 & 6.643833 & -7.377519 \\
\hline$N$ & -2.627994 & 6.768045 & -5.761132 \\
\hline C & -1.749940 & 6.461258 & -4.657643 \\
\hline C & -1.472401 & 4.975118 & -4.527434 \\
\hline $\mathrm{O}$ & -0.342035 & 4.593898 & -4.332800 \\
\hline C & -2.338366 & 7.058405 & -3.373402 \\
\hline C & -1.752062 & 6.361076 & -2.147056 \\
\hline C & -2.100690 & 8.574533 & -3.409071 \\
\hline $\mathrm{H}$ & -3.550440 & 7.074758 & -5.541178 \\
\hline $\mathrm{H}$ & -0.779628 & 6.899122 & -4.842729 \\
\hline $\mathrm{H}$ & -3.406570 & 6.877236 & -3.388107 \\
\hline $\mathrm{H}$ & -1.965353 & 6.907943 & -1.247781 \\
\hline $\mathrm{H}$ & -2.165885 & 5.364428 & -2.036170 \\
\hline $\mathrm{H}$ & -0.679842 & 6.286334 & -2.218551 \\
\hline $\mathrm{H}$ & -2.831816 & 9.101340 & -2.812388 \\
\hline $\mathrm{H}$ & -1.109168 & 8.816643 & -3.041388 \\
\hline $\mathrm{H}$ & -2.193268 & 8.959248 & -4.418178 \\
\hline$N$ & -2.497671 & 4.112723 & -4.641093 \\
\hline C & -2.166523 & 2.706506 & -4.738287 \\
\hline C & -1.220364 & 2.507835 & -5.924366 \\
\hline 4 & -0.300102 & 1.723969 & -5.846625 \\
\hline
\end{tabular}




\begin{tabular}{|c|c|c|c|}
\hline C & -3.394415 & 1.790021 & -4.822486 \\
\hline C & -4.317072 & 1.918766 & -3.602696 \\
\hline C & -5.382627 & 0.821190 & -3.503935 \\
\hline$N$ & -4.858872 & -0.390743 & -2.878874 \\
\hline C & -5.570099 & -1.480473 & -2.694072 \\
\hline$N$ & -6.815576 & -1.586145 & -3.199646 \\
\hline$N$ & -5.121736 & -2.493378 & -1.978831 \\
\hline $\mathrm{H}$ & -3.426010 & 4.433213 & -4.814557 \\
\hline $\mathrm{H}$ & -1.595337 & 2.414685 & -3.870258 \\
\hline $\mathrm{H}$ & -3.953161 & 1.997088 & -5.733460 \\
\hline $\mathrm{H}$ & -3.020314 & 0.775975 & -4.902296 \\
\hline $\mathrm{H}$ & -3.728329 & 1.918131 & -2.699561 \\
\hline $\mathrm{H}$ & -4.837326 & 2.862844 & -3.643857 \\
\hline $\mathrm{H}$ & -6.218791 & 1.188026 & -2.917636 \\
\hline $\mathrm{H}$ & -5.751709 & 0.588009 & -4.500175 \\
\hline $\mathrm{H}$ & -3.951037 & -0.310539 & -2.403352 \\
\hline $\mathrm{H}$ & -7.063115 & -0.992515 & -3.955708 \\
\hline $\mathrm{H}$ & -7.232775 & -2.498664 & -3.208983 \\
\hline $\mathrm{H}$ & -4.262812 & -2.495015 & -1.452853 \\
\hline $\mathrm{H}$ & -5.653361 & -3.336980 & -1.950745 \\
\hline$N$ & -1.459796 & 3.193317 & -7.035069 \\
\hline $\mathrm{H}$ & -2.274663 & 3.755702 & -7.144273 \\
\hline C & -0.596806 & 2.996902 & -8.183514 \\
\hline $\mathrm{H}$ & -0.949862 & 3.640673 & -8.978700 \\
\hline $\mathrm{H}$ & -0.612568 & 1.971759 & -8.524674 \\
\hline C & 0.856426 & 3.342190 & -7.871520 \\
\hline $\mathrm{O}$ & 1.781779 & 2.705154 & -8.308745 \\
\hline$N$ & 1.006300 & 4.404904 & -7.066843 \\
\hline $\mathrm{H}$ & 0.213643 & 4.966346 & -6.852996 \\
\hline C & 2.282311 & 4.880660 & -6.640289 \\
\hline
\end{tabular}




\begin{tabular}{|c|c|c|c|}
\hline $\mathrm{H}$ & 2.920019 & 5.140127 & -7.475932 \\
\hline $\mathrm{H}$ & 2.126148 & 5.775415 & -6.051758 \\
\hline C & 3.092715 & 3.927752 & -5.776302 \\
\hline $\mathrm{O}$ & 4.297866 & 4.057240 & -5.690502 \\
\hline$N$ & 2.428042 & 2.983351 & -5.101928 \\
\hline C & 3.098132 & 2.138091 & -4.148255 \\
\hline C & 4.271477 & 1.329244 & -4.712126 \\
\hline $\mathrm{O}$ & 5.186206 & 1.028106 & -3.971893 \\
\hline C & 2.012665 & 1.259397 & -3.524524 \\
\hline C & 2.383938 & 0.437214 & -2.296295 \\
\hline C & 1.072250 & 0.014581 & -1.644206 \\
\hline$N$ & 1.262215 & -1.019085 & -0.647482 \\
\hline C & 0.330250 & -1.943988 & -0.368225 \\
\hline$N$ & -0.822216 & -1.947790 & -0.989648 \\
\hline$N$ & 0.622138 & -2.889888 & 0.502311 \\
\hline $\mathrm{H}$ & 1.438168 & 2.903902 & -5.207745 \\
\hline $\mathrm{H}$ & 3.559695 & 2.748451 & -3.381394 \\
\hline $\mathrm{H}$ & 1.216212 & 1.942011 & -3.248467 \\
\hline $\mathrm{H}$ & 1.593516 & 0.613829 & -4.287331 \\
\hline $\mathrm{H}$ & 2.964670 & -0.438230 & -2.566336 \\
\hline $\mathrm{H}$ & 2.986166 & 1.021031 & -1.603772 \\
\hline $\mathrm{H}$ & 0.568823 & 0.875153 & -1.216969 \\
\hline $\mathrm{H}$ & 0.418899 & -0.386302 & -2.403636 \\
\hline $\mathrm{H}$ & 1.963553 & -0.878296 & 0.044610 \\
\hline $\mathrm{H}$ & -1.269966 & -1.090214 & -1.303507 \\
\hline $\mathrm{H}$ & -1.491568 & -2.673986 & -0.770534 \\
\hline $\mathrm{H}$ & 1.538744 & -2.992293 & 0.870067 \\
\hline $\mathrm{H}$ & -0.128173 & -3.482747 & 0.842903 \\
\hline$N$ & 4.279927 & 0.941794 & -5.999119 \\
\hline $\mathrm{H}$ & 3.541075 & 1.225977 & -6.608687 \\
\hline
\end{tabular}




\begin{tabular}{|c|c|c|c|}
\hline C & 5.363429 & 0.116650 & -6.504621 \\
\hline $\mathrm{H}$ & 5.137453 & -0.138134 & -7.532634 \\
\hline $\mathrm{H}$ & 5.442774 & -0.800174 & -5.942267 \\
\hline C & 6.736143 & 0.756003 & -6.476543 \\
\hline 0 & 7.738854 & 0.089545 & -6.367895 \\
\hline$N$ & 6.766121 & 2.085332 & -6.608316 \\
\hline $\mathrm{H}$ & 5.911874 & 2.604440 & -6.567369 \\
\hline C & 8.004996 & 2.823954 & -6.549403 \\
\hline $\mathrm{H}$ & 8.709975 & 2.440909 & -7.273615 \\
\hline $\mathrm{H}$ & 7.799551 & 3.854914 & -6.804810 \\
\hline C & 8.747153 & 2.825883 & -5.211064 \\
\hline O & 9.888176 & 3.235697 & -5.157967 \\
\hline $\mathrm{N}$ & 8.101513 & 2.335547 & -4.145228 \\
\hline C & 8.681066 & 2.170330 & -2.823189 \\
\hline C & 9.947171 & 1.303177 & -2.830526 \\
\hline O & 10.764433 & 1.385764 & -1.927914 \\
\hline C & 7.576317 & 1.500246 & -1.975725 \\
\hline C & 7.769196 & 1.380775 & -0.464218 \\
\hline C & 7.422867 & 2.651993 & 0.320468 \\
\hline C & 7.309433 & 2.389262 & 1.818243 \\
\hline $\mathrm{N}$ & 8.648470 & 2.359147 & 2.478369 \\
\hline O & 11.028966 & 1.615400 & 0.897874 \\
\hline $\mathrm{H}$ & 10.880843 & 1.825153 & -0.024643 \\
\hline $\mathrm{H}$ & 11.505773 & 0.787783 & 0.849620 \\
\hline $\mathrm{H}$ & 7.155623 & 2.033171 & -4.259232 \\
\hline $\mathrm{H}$ & 8.978157 & 3.127611 & -2.415614 \\
\hline $\mathrm{H}$ & 6.658798 & 2.041514 & -2.163486 \\
\hline $\mathrm{H}$ & 7.411794 & 0.512166 & -2.389712 \\
\hline $\mathrm{H}$ & 7.089851 & 0.601019 & -0.133836 \\
\hline $\mathrm{H}$ & 8.764499 & 1.030272 & -0.225085 \\
\hline
\end{tabular}




\begin{tabular}{|c|c|c|c|}
\hline $\mathrm{H}$ & 8.134690 & 3.453719 & 0.144483 \\
\hline $\mathrm{H}$ & 6.457767 & 3.020719 & -0.011818 \\
\hline $\mathrm{H}$ & 6.746791 & 3.162101 & 2.318405 \\
\hline $\mathrm{H}$ & 6.829088 & 1.443113 & 2.014349 \\
\hline $\mathrm{H}$ & 9.360186 & 1.893353 & 1.924196 \\
\hline $\mathrm{H}$ & 8.969331 & 3.329393 & 2.631134 \\
\hline $\mathrm{H}$ & 8.593611 & 1.883776 & 3.367311 \\
\hline$N$ & 10.062473 & 0.448236 & -3.837526 \\
\hline C & 11.109385 & -0.537499 & -4.068892 \\
\hline C & 12.486318 & 0.023018 & -4.427498 \\
\hline $\mathrm{O}$ & 13.444102 & -0.705865 & -4.302265 \\
\hline C & 10.700752 & -1.444326 & -5.251778 \\
\hline C & 9.562247 & -2.442193 & -4.996372 \\
\hline C & 9.129842 & -3.066510 & -6.326790 \\
\hline C & 9.989374 & -3.533054 & -4.014143 \\
\hline $\mathrm{H}$ & 9.324430 & 0.445949 & -4.510888 \\
\hline $\mathrm{H}$ & 11.265082 & -1.124645 & -3.175660 \\
\hline $\mathrm{H}$ & 10.434267 & -0.798849 & -6.083402 \\
\hline $\mathrm{H}$ & 11.583366 & -1.997163 & -5.550421 \\
\hline $\mathrm{H}$ & 8.706186 & -1.910840 & -4.592279 \\
\hline $\mathrm{H}$ & 8.328479 & -3.782558 & -6.171896 \\
\hline $\mathrm{H}$ & 8.772359 & -2.309432 & -7.013020 \\
\hline $\mathrm{H}$ & 9.956567 & -3.594776 & -6.795320 \\
\hline $\mathrm{H}$ & 9.199147 & -4.262500 & -3.873818 \\
\hline $\mathrm{H}$ & 10.858975 & -4.059856 & -4.393195 \\
\hline $\mathrm{H}$ & 10.243041 & -3.136330 & -3.035863 \\
\hline$N$ & 12.574040 & 1.261815 & -4.919241 \\
\hline C & 13.814402 & 1.845124 & -5.393451 \\
\hline C & 14.959101 & 2.032366 & -4.379294 \\
\hline 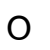 & 16.014162 & 2.411657 & -4.812553 \\
\hline
\end{tabular}




\begin{tabular}{|c|c|c|c|}
\hline C & 13.496699 & 3.172760 & -6.084835 \\
\hline $\mathrm{O}$ & 12.918485 & 4.088908 & -5.199199 \\
\hline $\mathrm{H}$ & 11.756268 & 1.833430 & -4.916309 \\
\hline $\mathrm{H}$ & 14.247968 & 1.179129 & -6.130075 \\
\hline $\mathrm{H}$ & 14.419688 & 3.591979 & -6.453707 \\
\hline $\mathrm{H}$ & 12.845327 & 2.979937 & -6.931375 \\
\hline $\mathrm{H}$ & 11.970943 & 4.079074 & -5.262046 \\
\hline$N$ & 14.792294 & 1.722323 & -3.074273 \\
\hline C & 15.927719 & 1.647188 & -2.176263 \\
\hline C & 16.804389 & 0.410085 & -2.402579 \\
\hline $\mathrm{O}$ & 17.934553 & 0.394315 & -1.988782 \\
\hline C & 15.493418 & 1.576199 & -0.707149 \\
\hline C & 14.635029 & 2.731997 & -0.197714 \\
\hline C & 14.278053 & 2.482535 & 1.268023 \\
\hline$N$ & 13.252762 & 3.377149 & 1.790066 \\
\hline C & 13.430756 & 4.528612 & 2.416967 \\
\hline$N$ & 14.648942 & 5.004016 & 2.652422 \\
\hline $\mathrm{O}$ & 15.028528 & 7.461937 & 4.203427 \\
\hline $\mathrm{H}$ & 14.743647 & 8.348737 & 3.966953 \\
\hline $\mathrm{H}$ & 15.746372 & 7.567396 & 4.814740 \\
\hline$N$ & 12.381341 & 5.217339 & 2.813920 \\
\hline $\mathrm{H}$ & 13.900310 & 1.421993 & -2.758069 \\
\hline $\mathrm{H}$ & 16.561324 & 2.504420 & -2.344669 \\
\hline $\mathrm{H}$ & 14.953683 & 0.647645 & -0.552865 \\
\hline $\mathrm{H}$ & 16.404853 & 1.507479 & -0.123427 \\
\hline $\mathrm{H}$ & 15.152565 & 3.680213 & -0.311232 \\
\hline $\mathrm{H}$ & 13.717071 & 2.813876 & -0.767959 \\
\hline $\mathrm{H}$ & 13.879966 & 1.483506 & 1.371369 \\
\hline $\mathrm{H}$ & 15.156187 & 2.522763 & 1.901849 \\
\hline & 12.312466 & 3.080894 & 1.620435 \\
\hline
\end{tabular}




\begin{tabular}{|c|c|c|c|}
\hline $\mathrm{H}$ & 15.456331 & 4.510925 & 2.353898 \\
\hline $\mathrm{H}$ & 14.785053 & 5.859333 & 3.163140 \\
\hline $\mathrm{H}$ & 11.425393 & 4.937628 & 2.657052 \\
\hline $\mathrm{H}$ & 12.473853 & 6.061264 & 3.332730 \\
\hline$N$ & 16.200243 & -0.648980 & -2.967248 \\
\hline C & 16.870894 & -1.911462 & -3.167466 \\
\hline C & 17.670755 & -1.889822 & -4.466601 \\
\hline $\mathrm{H}$ & 18.060349 & -0.909366 & -4.749612 \\
\hline O & 17.882413 & -2.854921 & -5.125587 \\
\hline C & 15.868150 & -3.070216 & -3.149002 \\
\hline C & 15.323809 & -3.399061 & -1.791707 \\
\hline$N$ & 14.402539 & -2.626099 & -1.113257 \\
\hline 0 & 12.506099 & -0.432151 & -0.462430 \\
\hline $\mathrm{H}$ & 13.141962 & -1.035492 & -0.850861 \\
\hline $\mathrm{H}$ & 12.023646 & -0.035558 & -1.179803 \\
\hline$C$ & 15.618413 & -4.488933 & -1.048296 \\
\hline $\mathrm{C}$ & 14.170588 & -3.249263 & -0.004407 \\
\hline $\mathrm{N}$ & 14.880878 & -4.379339 & 0.103926 \\
\hline $\mathrm{H}$ & 15.285173 & -0.550797 & -3.351781 \\
\hline $\mathrm{H}$ & 17.595677 & -2.030721 & -2.369751 \\
\hline $\mathrm{H}$ & 15.051075 & -2.842552 & -3.824865 \\
\hline $\mathrm{H}$ & 16.369280 & -3.944217 & -3.542697 \\
\hline $\mathrm{H}$ & 16.273858 & -5.312929 & -1.232605 \\
\hline $\mathrm{H}$ & 13.491731 & -2.919058 & 0.755750 \\
\hline $\mathrm{H}$ & 14.779630 & -5.061789 & 0.822979 \\
\hline 0 & -14.807923 & -2.300201 & 5.225778 \\
\hline $\mathrm{H}$ & -15.114833 & -1.614500 & 4.637480 \\
\hline $\mathrm{H}$ & -14.381301 & -1.853417 & 5.949779 \\
\hline 0 & -12.881033 & -0.751105 & 7.019263 \\
\hline 11 & -12.148450 & -1.240895 & 7.3774 \\
\hline
\end{tabular}




\begin{tabular}{|c|c|c|c|}
\hline $\mathrm{H}$ & -13.133124 & -0.124765 & 7.686669 \\
\hline $\mathrm{O}$ & -12.181285 & -0.220132 & 4.192191 \\
\hline $\mathrm{H}$ & -12.315488 & -0.215257 & 5.135732 \\
\hline $\mathrm{H}$ & -13.061658 & -0.214532 & 3.813686 \\
\hline 0 & -13.444348 & -2.081950 & 1.045259 \\
\hline $\mathrm{H}$ & -12.705775 & -1.471167 & 0.998793 \\
\hline $\mathrm{H}$ & -13.252860 & -2.667409 & 1.778155 \\
\hline 0 & -7.228125 & 1.029560 & -0.130575 \\
\hline $\mathrm{H}$ & -7.417577 & 1.424375 & 0.723910 \\
\hline $\mathrm{H}$ & -6.293291 & 0.856415 & -0.115512 \\
\hline 0 & -7.800955 & 2.006537 & 2.509943 \\
\hline $\mathrm{H}$ & -8.737809 & 1.940497 & 2.675116 \\
\hline $\mathrm{H}$ & -7.464497 & 2.683073 & 3.091808 \\
\hline 0 & -9.389751 & -0.254834 & -1.397153 \\
\hline $\mathrm{H}$ & -8.587735 & 0.109151 & -1.003540 \\
\hline $\mathrm{H}$ & -9.190521 & -1.131292 & -1.712271 \\
\hline 0 & -12.900404 & -2.343357 & -1.716558 \\
\hline $\mathrm{H}$ & -13.229366 & -2.469208 & -0.823999 \\
\hline $\mathrm{H}$ & -13.560377 & -2.659081 & -2.325070 \\
\hline 0 & -13.579449 & 1.728351 & -0.139810 \\
\hline $\mathrm{H}$ & -12.966518 & 1.047048 & 0.143924 \\
\hline $\mathrm{H}$ & -13.094119 & 2.540930 & 0.018491 \\
\hline 0 & -14.214072 & 4.927385 & 2.494770 \\
\hline $\mathrm{H}$ & -13.812294 & 5.429762 & 3.198406 \\
\hline $\mathrm{H}$ & -14.737755 & 4.237706 & 2.884783 \\
\hline 0 & -15.506305 & 2.129796 & 1.954476 \\
\hline $\mathrm{H}$ & -16.367951 & 2.339354 & 1.618209 \\
\hline $\mathrm{H}$ & -14.932993 & 2.042234 & 1.190377 \\
\hline 0 & -13.350841 & 0.380707 & -2.618185 \\
\hline 11 & -13.636177 & 0.979357 & -1.9265 \\
\hline
\end{tabular}




\begin{tabular}{|c|c|c|c|}
\hline $\mathrm{H}$ & -13.106681 & -0.427018 & -2.164882 \\
\hline O & -14.252655 & -1.708972 & -4.324807 \\
\hline $\mathrm{H}$ & -14.308315 & -0.847013 & -3.916393 \\
\hline $\mathrm{H}$ & -15.043869 & -1.833161 & -4.832700 \\
\hline $\mathrm{O}$ & -10.281317 & -2.826376 & -2.845590 \\
\hline $\mathrm{H}$ & -10.563412 & -2.516751 & -3.705510 \\
\hline $\mathrm{H}$ & -11.076653 & -2.783768 & -2.315210 \\
\hline $\mathrm{O}$ & -11.491070 & -0.112620 & 0.552589 \\
\hline $\mathrm{H}$ & -10.813061 & -0.222667 & -0.115844 \\
\hline $\mathrm{H}$ & -11.087654 & 0.399932 & 1.257990 \\
\hline $\mathrm{O}$ & -10.714101 & 1.651582 & 2.580969 \\
\hline $\mathrm{H}$ & -11.200103 & 1.217835 & 3.282801 \\
\hline $\mathrm{H}$ & -11.222541 & 2.399231 & 2.269407 \\
\hline $\mathrm{O}$ & -12.145597 & 3.761965 & 1.081496 \\
\hline $\mathrm{H}$ & -12.857387 & 4.179540 & 1.579790 \\
\hline $\mathrm{H}$ & -11.581051 & 4.478575 & 0.806527 \\
\hline $\mathrm{O}$ & -10.736540 & 0.823996 & -3.609666 \\
\hline $\mathrm{H}$ & -11.621294 & 0.967500 & -3.269918 \\
\hline $\mathrm{H}$ & -10.206422 & 0.557538 & -2.852110 \\
\hline $\mathrm{O}$ & -14.797200 & -0.398360 & 3.052640 \\
\hline $\mathrm{H}$ & -15.179082 & 0.432347 & 2.768201 \\
\hline $\mathrm{H}$ & -14.623868 & -0.911687 & 2.265877 \\
\hline $\mathrm{O}$ & -12.785738 & -3.291256 & 3.586549 \\
\hline $\mathrm{H}$ & -13.538329 & -3.087115 & 4.153694 \\
\hline $\mathrm{H}$ & -12.144591 & -2.623232 & 3.810321 \\
\hline $\mathrm{O}$ & -11.462580 & -1.464169 & -5.064183 \\
\hline $\mathrm{H}$ & -12.392795 & -1.688487 & -5.061272 \\
\hline $\mathrm{H}$ & -11.369844 & -0.701874 & -4.494078 \\
\hline
\end{tabular}


Y266F MUTATION, NOMINAL CHARGES R300(0),E183(0),R303(0),E226(0) POTENTIAL:-70mV
$\mathrm{N}$
$15.734428-6.579114 \quad 5.125688$
$\mathrm{H}$
$14.935254-6.345574 \quad 5.679949$
C
$\begin{array}{lll}15.663766 & -5.947871 & 3.801799\end{array}$
C
$\begin{array}{lll}14.924083 & -6.843526 & 2.790318\end{array}$
O
$14.137314-6.393073 \quad 1.979350$
C
$\begin{array}{lll}17.067515 & -5.651914 & 3.264043\end{array}$
$\mathrm{H}$
$16.545461 \quad-6.273878 \quad 5.624071$
$\begin{array}{llll}\text { H } & 15.097268 & -5.025097 & 3.826552\end{array}$
H $\quad 17.021880-5.251728 \quad 2.257692$
H $\quad 17.555511-4.9172323 .895203$
H $\quad 17.678273-6.548455 \quad 3.251274$
$\begin{array}{llll}N & 15.183645 & -8.147600 & 2.874109\end{array}$
H $\quad 15.812038-8.441270 \quad 3.588240$
$\begin{array}{llll}\text { C } & 14.551997 & -9.131969 & 1.993656\end{array}$
H $\quad 14.618276-8.825103 \quad 0.962065$
H $\quad 15.059006-10.081025 \quad 2.111374$
C $\quad 13.072865 \quad-9.304805 \quad 2.341309$
O $\quad 12.239818-9.535517 \quad 1.503012$
$\begin{array}{llll}N & 12.746616 & -9.160368 & 3.638917\end{array}$
H $\quad \begin{array}{llll}13.468050 & -9.047439 & 4.311918\end{array}$
C $\quad 11.362687 \quad-9.300198 \quad 4.098282$
H $\quad 11.359000 \quad-9.321395 \quad 5.180363$
H $\quad 10.915805-10.2090893 .725583$
$\begin{array}{llll}\text { C } & 10.492661 & -8.130735 & 3.619561\end{array}$
O $\quad 9.333534 \quad-8.296645 \quad 3.346510$
$\begin{array}{llll}N & 11.108959 & -6.936495 & 3.561788\end{array}$
$\begin{array}{llll}\text { C } & 10.468564 & -5.754384 & 3.042716\end{array}$ 


\begin{tabular}{|c|c|c|c|}
\hline C & 10.273124 & -5.791375 & 1.524809 \\
\hline 0 & 9.216339 & -5.408953 & 1.081969 \\
\hline C & 11.202635 & -4.492515 & 3.520844 \\
\hline 0 & 12.593790 & -4.605603 & 3.503073 \\
\hline $\mathrm{H}$ & 12.079375 & -6.881782 & 3.761312 \\
\hline $\mathrm{H}$ & 9.460862 & -5.700239 & 3.421512 \\
\hline $\mathrm{H}$ & 10.856869 & -3.645436 & 2.933705 \\
\hline $\mathrm{H}$ & 10.932159 & -4.310734 & 4.552038 \\
\hline $\mathrm{H}$ & 12.935320 & -4.899351 & 2.669664 \\
\hline$N$ & 11.258316 & -6.233650 & 0.721734 \\
\hline C & 10.969028 & -6.403910 & -0.699968 \\
\hline C & 9.822409 & -7.398711 & -0.887994 \\
\hline $\mathrm{O}$ & 9.063810 & -7.295830 & -1.824003 \\
\hline C & 12.230978 & -6.757809 & -1.535496 \\
\hline C & 12.584381 & -8.248178 & -1.573730 \\
\hline$C$ & 12.078967 & -6.232904 & -2.964780 \\
\hline $\mathrm{H}$ & 12.119307 & -6.570457 & 1.096096 \\
\hline $\mathrm{H}$ & 10.575587 & -5.468529 & -1.068377 \\
\hline $\mathrm{H}$ & 13.052102 & -6.221241 & -1.068483 \\
\hline $\mathrm{H}$ & 13.537937 & -8.374531 & -2.075635 \\
\hline $\mathrm{H}$ & 12.657548 & -8.696123 & -0.594311 \\
\hline $\mathrm{H}$ & 11.847694 & -8.809194 & -2.141886 \\
\hline $\mathrm{H}$ & 12.965778 & -6.467325 & -3.543263 \\
\hline $\mathrm{H}$ & 11.223696 & -6.681056 & -3.458266 \\
\hline $\mathrm{H}$ & 11.951431 & -5.158936 & -2.977010 \\
\hline $\mathrm{N}$ & 9.705481 & -8.354397 & 0.043515 \\
\hline $\mathrm{H}$ & 10.468656 & -8.511759 & 0.660989 \\
\hline C & 8.735562 & -9.439358 & -0.071419 \\
\hline $\mathrm{H}$ & 9.091687 & -10.259833 & 0.539699 \\
\hline $\mathrm{H}$ & 8.663238 & -9.782248 & -1.092406 \\
\hline
\end{tabular}




\begin{tabular}{|c|c|c|c|}
\hline$C$ & 7.324929 & -9.099481 & 0.364060 \\
\hline O & 6.397058 & -9.541147 & -0.274745 \\
\hline $\mathrm{N}$ & 7.148873 & -8.330482 & 1.444373 \\
\hline$C$ & 5.812196 & -7.871865 & 1.748015 \\
\hline$C$ & 5.361342 & -6.831864 & 0.727546 \\
\hline 0 & 4.173387 & -6.711385 & 0.506318 \\
\hline$C$ & 5.576744 & -7.412180 & 3.200591 \\
\hline$C$ & 5.752293 & -8.581761 & 4.173994 \\
\hline$C$ & 6.409763 & -6.202712 & 3.630146 \\
\hline $\mathrm{H}$ & 7.929778 & -8.072703 & 2.006497 \\
\hline $\mathrm{H}$ & 5.136694 & -8.695607 & 1.570160 \\
\hline $\mathrm{H}$ & 4.532105 & -7.115865 & 3.220218 \\
\hline $\mathrm{H}$ & 5.475956 & -8.275885 & 5.177783 \\
\hline $\mathrm{H}$ & 5.119861 & -9.419926 & 3.899113 \\
\hline $\mathrm{H}$ & 6.779930 & -8.926424 & 4.201189 \\
\hline $\mathrm{H}$ & 6.081818 & -5.862945 & 4.606955 \\
\hline $\mathrm{H}$ & 7.457540 & -6.461435 & 3.709960 \\
\hline $\mathrm{H}$ & 6.309904 & -5.370600 & 2.941199 \\
\hline $\mathrm{N}$ & 6.270367 & -6.114515 & 0.063468 \\
\hline$C$ & 5.865140 & -5.265807 & -1.044656 \\
\hline$C$ & 5.431983 & -6.119352 & -2.240748 \\
\hline $\mathrm{O}$ & 4.381978 & -5.887230 & -2.793874 \\
\hline$C$ & 6.961024 & -4.250923 & -1.415719 \\
\hline$C$ & 7.208475 & -3.282162 & -0.248922 \\
\hline$C$ & 6.559048 & -3.483558 & -2.679999 \\
\hline$C$ & 8.543332 & -2.550411 & -0.352344 \\
\hline $\mathrm{H}$ & 7.235054 & -6.188776 & 0.299836 \\
\hline $\mathrm{H}$ & 4.974397 & -4.727162 & -0.758058 \\
\hline $\mathrm{H}$ & 7.876945 & -4.801518 & -1.614063 \\
\hline $\mathrm{H}$ & 6.389464 & -2.568502 & -0.218978 \\
\hline
\end{tabular}




\begin{tabular}{|c|c|c|c|}
\hline $\mathrm{H}$ & 7.192574 & -3.818152 & 0.690817 \\
\hline $\mathrm{H}$ & 7.261995 & -2.687084 & -2.884064 \\
\hline $\mathrm{H}$ & 6.533210 & -4.121872 & -3.554164 \\
\hline $\mathrm{H}$ & 5.576167 & -3.039126 & -2.564070 \\
\hline $\mathrm{H}$ & 8.678310 & -1.887996 & 0.495334 \\
\hline $\mathrm{H}$ & 9.362484 & -3.259059 & -0.350815 \\
\hline $\mathrm{H}$ & 8.614436 & -1.952255 & -1.252682 \\
\hline$N$ & 6.232837 & -7.115268 & -2.641992 \\
\hline $\mathrm{H}$ & 7.137921 & -7.231340 & -2.239385 \\
\hline C & 5.881109 & -7.920762 & -3.790628 \\
\hline $\mathrm{H}$ & 6.710642 & -8.587611 & -3.992826 \\
\hline $\mathrm{H}$ & 5.709115 & -7.310275 & -4.665389 \\
\hline C & 4.618612 & -8.756614 & -3.592763 \\
\hline O & 3.770149 & -8.800844 & -4.452367 \\
\hline$N$ & 4.519383 & -9.430515 & -2.440080 \\
\hline $\mathrm{H}$ & 5.265057 & -9.406598 & -1.778221 \\
\hline C & 3.335206 & -10.192163 & -2.125304 \\
\hline $\mathrm{H}$ & 3.089594 & -10.886879 & -2.915121 \\
\hline $\mathrm{H}$ & 3.526940 & -10.754672 & -1.219543 \\
\hline C & 2.103914 & -9.316649 & -1.911364 \\
\hline $\mathrm{O}$ & 1.015038 & -9.699226 & -2.274819 \\
\hline$N$ & 2.312311 & -8.132395 & -1.338198 \\
\hline C & 1.175222 & -7.233178 & -1.240518 \\
\hline C & 0.723258 & -6.681989 & -2.596244 \\
\hline $\mathrm{O}$ & -0.460736 & -6.490415 & -2.772606 \\
\hline C & 1.385414 & -6.071698 & -0.275070 \\
\hline $\mathrm{O}$ & 1.478788 & -6.486420 & 1.046498 \\
\hline $\mathrm{H}$ & 3.209933 & -7.848270 & -1.020684 \\
\hline $\mathrm{H}$ & 0.329325 & -7.798048 & -0.881689 \\
\hline & 2.260883 & -5.506869 & -0.574747 \\
\hline
\end{tabular}




\begin{tabular}{|c|c|c|c|}
\hline $\mathrm{H}$ & 0.512575 & -5.437934 & -0.354416 \\
\hline $\mathrm{H}$ & 2.374942 & -6.730464 & 1.221180 \\
\hline$N$ & 1.639027 & -6.412901 & -3.530924 \\
\hline C & 1.242916 & -5.965056 & -4.853814 \\
\hline C & 0.493000 & -7.070430 & -5.592398 \\
\hline O & -0.488190 & -6.796592 & -6.252459 \\
\hline C & 2.452484 & -5.416064 & -5.646619 \\
\hline C & 2.825007 & -4.033720 & -5.085452 \\
\hline C & 2.175984 & -5.355505 & -7.153922 \\
\hline C & 4.128312 & -3.462694 & -5.640915 \\
\hline $\mathrm{H}$ & 2.608740 & -6.505571 & -3.320968 \\
\hline $\mathrm{H}$ & 0.512818 & -5.175515 & -4.748934 \\
\hline $\mathrm{H}$ & 3.284222 & -6.096537 & -5.486151 \\
\hline $\mathrm{H}$ & 2.011540 & -3.341215 & -5.294716 \\
\hline $\mathrm{H}$ & 2.908371 & -4.098537 & -4.007822 \\
\hline $\mathrm{H}$ & 3.038291 & -4.962351 & -7.677695 \\
\hline $\mathrm{H}$ & 1.973036 & -6.334729 & -7.571041 \\
\hline $\mathrm{H}$ & 1.328318 & -4.715154 & -7.377211 \\
\hline $\mathrm{H}$ & 4.403741 & -2.568254 & -5.092377 \\
\hline r & 4.941783 & -4.174736 & -5.539983 \\
\hline $\mathrm{H}$ & 4.048597 & -3.193629 & -6.688610 \\
\hline$N$ & 0.929810 & -8.326151 & -5.465463 \\
\hline $\mathrm{H}$ & 1.777858 & -8.518738 & -4.976438 \\
\hline C & 0.151353 & -9.414407 & -6.007253 \\
\hline $\mathrm{H}$ & 0.039898 & -9.333713 & -7.078762 \\
\hline $\mathrm{H}$ & 0.662186 & -10.341571 & -5.778435 \\
\hline C & -1.256369 & -9.464904 & -5.418388 \\
\hline $\mathrm{O}$ & -2.214341 & -9.662164 & -6.129982 \\
\hline$N$ & -1.356880 & -9.285561 & -4.102538 \\
\hline$C$ & -2.663912 & -9.299065 & -3.486583 \\
\hline
\end{tabular}




\begin{tabular}{|c|c|c|c|}
\hline C & -3.548273 & -8.128837 & -3.922705 \\
\hline $\mathrm{O}$ & -4.748987 & -8.309373 & -3.981657 \\
\hline C & -2.570434 & -9.310726 & -1.954853 \\
\hline $\mathrm{O}$ & -3.824839 & -9.532940 & -1.374478 \\
\hline $\mathrm{H}$ & -0.538487 & -9.239443 & -3.533101 \\
\hline $\mathrm{H}$ & -3.205141 & -10.182020 & -3.798706 \\
\hline $\mathrm{H}$ & -1.917264 & -10.117294 & -1.647246 \\
\hline $\mathrm{H}$ & -2.140220 & -8.375805 & -1.607954 \\
\hline $\mathrm{H}$ & -4.504799 & -9.153250 & -1.911169 \\
\hline$N$ & -2.969516 & -6.953238 & -4.162320 \\
\hline C & -3.694148 & -5.792893 & -4.655742 \\
\hline C & -4.275556 & -6.061957 & -6.041239 \\
\hline $\mathrm{O}$ & -5.427710 & -5.776089 & -6.294822 \\
\hline C & -2.731107 & -4.581602 & -4.665542 \\
\hline C & -3.128597 & -3.429235 & -5.569550 \\
\hline C & -4.372525 & -2.815808 & -5.484079 \\
\hline C & -2.228362 & -2.966104 & -6.521984 \\
\hline C & -4.710883 & -1.780043 & -6.337740 \\
\hline C & -2.555454 & -1.917809 & -7.366506 \\
\hline C & -3.802631 & -1.324458 & -7.279727 \\
\hline $\mathrm{H}$ & -1.996474 & -6.855031 & -3.966955 \\
\hline $\mathrm{H}$ & -4.545063 & -5.590006 & -4.023169 \\
\hline $\mathrm{H}$ & -2.617976 & -4.243289 & -3.640037 \\
\hline $\mathrm{H}$ & -1.763723 & -4.935587 & -4.987697 \\
\hline $\mathrm{H}$ & -5.093967 & -3.157088 & -4.766530 \\
\hline $\mathrm{H}$ & -1.265457 & -3.436817 & -6.614114 \\
\hline $\mathrm{H}$ & -5.693520 & -1.348276 & -6.273287 \\
\hline $\mathrm{H}$ & -1.842540 & -1.577897 & -8.096601 \\
\hline $\mathrm{H}$ & -4.066027 & -0.522238 & -7.947395 \\
\hline$T$ & -3.461900 & -6.613021 & -6.942978 \\
\hline
\end{tabular}




\begin{tabular}{|c|c|c|c|}
\hline C & -3.955607 & -6.916805 & -8.267295 \\
\hline C & -5.112058 & -7.914757 & -8.211968 \\
\hline $\mathrm{O}$ & -6.072898 & -7.765640 & -8.921549 \\
\hline C & -2.849462 & -7.475423 & -9.165219 \\
\hline$S$ & -1.581895 & -6.268720 & -9.668131 \\
\hline $\mathrm{H}$ & -2.528391 & -6.851580 & -6.684717 \\
\hline $\mathrm{H}$ & -4.368101 & -6.024960 & -8.715719 \\
\hline $\mathrm{H}$ & -2.374474 & -8.330602 & -8.702731 \\
\hline $\mathrm{H}$ & -3.301576 & -7.807825 & -10.090659 \\
\hline $\mathrm{H}$ & -0.944387 & -6.174731 & -8.509822 \\
\hline$N$ & -4.985866 & -8.958052 & -7.368072 \\
\hline $\mathrm{H}$ & -4.133959 & -9.116239 & -6.875719 \\
\hline C & -5.986871 & -10.013369 & -7.421773 \\
\hline $\mathrm{H}$ & -6.170895 & -10.316619 & -8.442040 \\
\hline $\mathrm{H}$ & -5.601492 & -10.862824 & -6.869374 \\
\hline C & -7.357871 & -9.648606 & -6.831503 \\
\hline $\mathrm{O}$ & -8.377195 & -9.998608 & -7.358893 \\
\hline$N$ & -7.314770 & -8.977255 & -5.675499 \\
\hline C & -8.544952 & -8.517550 & -5.057979 \\
\hline C & -9.297290 & -7.451890 & -5.851299 \\
\hline $\mathrm{O}$ & -10.490730 & -7.355628 & -5.744975 \\
\hline C & -8.321981 & -7.999661 & -3.629324 \\
\hline C & -7.380692 & -6.799644 & -3.458580 \\
\hline C & -7.990028 & -5.422695 & -3.445239 \\
\hline $\mathrm{O}$ & -9.153324 & -5.154854 & -3.520801 \\
\hline $\mathrm{O}$ & -7.050116 & -4.505829 & -3.295547 \\
\hline $\mathrm{H}$ & -7.362665 & -3.594269 & -3.303819 \\
\hline $\mathrm{H}$ & -6.433324 & -8.721206 & -5.287572 \\
\hline $\mathrm{H}$ & -9.233929 & -9.348650 & -5.005564 \\
\hline $\mathrm{H}$ & -9.294970 & -7.762051 & -3.224590 \\
\hline
\end{tabular}




\begin{tabular}{|c|c|c|c|}
\hline $\mathrm{H}$ & -7.920827 & -8.823614 & -3.049228 \\
\hline $\mathrm{H}$ & -6.860814 & -6.882727 & -2.510001 \\
\hline $\mathrm{H}$ & -6.602939 & -6.782339 & -4.203912 \\
\hline$N$ & -8.561657 & -6.582238 & -6.574357 \\
\hline C & -9.221307 & -5.523565 & -7.301533 \\
\hline C & -10.091055 & -6.097012 & -8.406984 \\
\hline $\mathrm{H}$ & -9.918882 & -7.149569 & -8.646442 \\
\hline $\mathrm{O}$ & -10.909253 & -5.451779 & -8.980030 \\
\hline C & -8.169818 & -4.507747 & -7.783909 \\
\hline $\mathrm{O}$ & -7.629235 & -3.860779 & -6.651963 \\
\hline C & -8.720997 & -3.428395 & -8.703125 \\
\hline $\mathrm{H}$ & -7.611955 & -6.795459 & -6.781359 \\
\hline $\mathrm{H}$ & -9.898091 & -5.011665 & -6.628330 \\
\hline $\mathrm{H}$ & -7.378257 & -5.040490 & -8.302311 \\
\hline $\mathrm{H}$ & -6.895251 & -4.361819 & -6.319946 \\
\hline $\mathrm{H}$ & -7.938409 & -2.695830 & -8.870431 \\
\hline $\mathrm{H}$ & -9.037352 & -3.832952 & -9.653889 \\
\hline $\mathrm{H}$ & -9.575473 & -2.939798 & -8.248607 \\
\hline$N$ & -12.792515 & -9.296717 & 3.007085 \\
\hline $\mathrm{H}$ & -13.047198 & -9.828823 & 2.202940 \\
\hline C & -11.617026 & -8.454549 & 2.744241 \\
\hline C & -10.570030 & -8.439548 & 3.891280 \\
\hline $\mathrm{O}$ & -9.437123 & -8.056103 & 3.704473 \\
\hline C & -11.972922 & -6.984115 & 2.458914 \\
\hline $\mathrm{O}$ & -12.487405 & -6.478661 & 3.661153 \\
\hline C & -12.973491 & -6.821367 & 1.320084 \\
\hline $\mathrm{H}$ & -13.573980 & -8.737443 & 3.283884 \\
\hline $\mathrm{H}$ & -11.084555 & -8.847603 & 1.888380 \\
\hline $\mathrm{H}$ & -11.051906 & -6.473796 & 2.198916 \\
\hline & -12.629561 & -5.541309 & 3.5757 \\
\hline
\end{tabular}




\begin{tabular}{|c|c|c|c|}
\hline $\mathrm{H}$ & -13.120105 & -5.766689 & 1.117109 \\
\hline $\mathrm{H}$ & -12.627061 & -7.294204 & 0.408659 \\
\hline $\mathrm{H}$ & -13.937547 & -7.240655 & 1.582952 \\
\hline $\mathrm{N}$ & -11.028029 & -8.859522 & 5.078157 \\
\hline $\mathrm{H}$ & -11.958160 & -9.213996 & 5.077471 \\
\hline$C$ & -10.257489 & -8.909544 & 6.296271 \\
\hline $\mathrm{H}$ & -10.212778 & -7.941929 & 6.774682 \\
\hline $\mathrm{H}$ & -10.757934 & -9.586680 & 6.979804 \\
\hline$C$ & -8.803643 & -9.366939 & 6.182595 \\
\hline 0 & -7.969399 & -8.775166 & 6.823989 \\
\hline $\mathrm{N}$ & -8.471588 & -10.431366 & 5.430205 \\
\hline $\mathrm{H}$ & -9.178650 & -10.931302 & 4.942703 \\
\hline$C$ & -7.076785 & -10.832684 & 5.312652 \\
\hline $\mathrm{H}$ & -7.042082 & -11.812933 & 4.853346 \\
\hline $\mathrm{H}$ & -6.622237 & -10.899704 & 6.288791 \\
\hline$C$ & -6.214046 & -9.880005 & 4.465346 \\
\hline 0 & -5.037717 & -9.758043 & 4.699401 \\
\hline $\mathrm{N}$ & -6.882601 & -9.263783 & 3.496980 \\
\hline$C$ & -6.119714 & -8.298276 & 2.706289 \\
\hline$C$ & -6.072070 & -6.924243 & 3.379480 \\
\hline 0 & -5.082742 & -6.231627 & 3.264179 \\
\hline$C$ & -6.415184 & -8.267276 & 1.196158 \\
\hline$C$ & -7.651627 & -7.559282 & 0.687933 \\
\hline$C$ & -7.717479 & -6.167516 & 0.666841 \\
\hline$C$ & -8.698925 & -8.278639 & 0.130560 \\
\hline $\mathrm{C}$ & -8.802986 & -5.515353 & 0.108846 \\
\hline$C$ & -9.783380 & -7.628827 & -0.438666 \\
\hline$C$ & -9.839008 & -6.246624 & -0.452647 \\
\hline $\mathrm{H}$ & -7.875695 & -9.270237 & 3.467349 \\
\hline $\mathrm{H}$ & -5.098788 & -8.632853 & 2.773311 \\
\hline
\end{tabular}




\begin{tabular}{|c|c|c|c|}
\hline $\mathrm{H}$ & -5.545087 & -7.806445 & 0.741501 \\
\hline $\mathrm{H}$ & -6.414982 & -9.297404 & 0.859945 \\
\hline $\mathrm{H}$ & -6.901575 & -5.589324 & 1.065202 \\
\hline $\mathrm{H}$ & -8.653863 & -9.354523 & 0.108769 \\
\hline $\mathrm{H}$ & -8.830832 & -4.440325 & 0.079653 \\
\hline $\mathrm{H}$ & -10.568643 & -8.202782 & -0.898060 \\
\hline $\mathrm{H}$ & -10.664020 & -5.743302 & -0.921222 \\
\hline$N$ & -7.114528 & -6.576169 & 4.135348 \\
\hline C & -7.087315 & -5.455957 & 5.043634 \\
\hline C & -6.014429 & -5.670767 & 6.109343 \\
\hline $\mathrm{O}$ & -5.334698 & -4.734874 & 6.475045 \\
\hline C & -8.497224 & -5.310356 & 5.653656 \\
\hline C & -8.732960 & -4.086806 & 6.511610 \\
\hline C & -9.035090 & -2.862833 & 5.924300 \\
\hline C & -8.714217 & -4.173930 & 7.898165 \\
\hline C & -9.306388 & -1.751171 & 6.703672 \\
\hline C & -8.989295 & -3.064839 & 8.683046 \\
\hline C & -9.286353 & -1.849582 & 8.087762 \\
\hline $\mathrm{H}$ & -7.948348 & -7.117645 & 4.086254 \\
\hline $\mathrm{H}$ & -6.805357 & -4.547486 & 4.529391 \\
\hline $\mathrm{H}$ & -9.196310 & -5.302203 & 4.824523 \\
\hline $\mathrm{H}$ & -8.707211 & -6.201031 & 6.232107 \\
\hline $\mathrm{H}$ & -9.074049 & -2.776667 & 4.851831 \\
\hline $\mathrm{H}$ & -8.492782 & -5.115355 & 8.371594 \\
\hline $\mathrm{H}$ & -9.536390 & -0.814241 & 6.230216 \\
\hline $\mathrm{H}$ & -8.968078 & -3.150858 & 9.754933 \\
\hline $\mathrm{H}$ & -9.488247 & -0.983903 & 8.694162 \\
\hline$N$ & -5.850491 & -6.898095 & 6.597982 \\
\hline $\mathrm{H}$ & -6.529764 & -7.598743 & 6.403526 \\
\hline$C$ & -4.843933 & -7.201416 & 7.589364 \\
\hline
\end{tabular}




\begin{tabular}{|c|c|c|c|}
\hline $\mathrm{H}$ & -4.905526 & -6.544745 & 8.444878 \\
\hline $\mathrm{H}$ & -5.014193 & -8.216586 & 7.925902 \\
\hline C & -3.414375 & -7.095084 & 7.070354 \\
\hline 0 & -2.560718 & -6.605043 & 7.769916 \\
\hline$N$ & -3.155186 & -7.566908 & 5.843120 \\
\hline C & -1.817807 & -7.467920 & 5.286318 \\
\hline C & -1.474804 & -6.020372 & 4.915733 \\
\hline $\mathrm{O}$ & -0.365029 & -5.594459 & 5.146867 \\
\hline C & -1.647948 & -8.452996 & 4.108666 \\
\hline C & -0.361328 & -8.198032 & 3.319871 \\
\hline C & -1.668375 & -9.897047 & 4.623804 \\
\hline $\mathrm{H}$ & -3.854014 & -8.082925 & 5.353670 \\
\hline $\mathrm{H}$ & -1.099876 & -7.713552 & 6.056512 \\
\hline $\mathrm{H}$ & -2.491096 & -8.310705 & 3.435290 \\
\hline $\mathrm{H}$ & -0.258815 & -8.946040 & 2.540973 \\
\hline $\mathrm{H}$ & -0.339217 & -7.229877 & 2.839575 \\
\hline $\mathrm{H}$ & 0.510119 & -8.265064 & 3.964806 \\
\hline $\mathrm{H}$ & -1.617524 & -10.589203 & 3.789566 \\
\hline $\mathrm{H}$ & -0.806852 & -10.081612 & 5.260694 \\
\hline $\mathrm{H}$ & -2.564563 & -10.125845 & 5.183531 \\
\hline$N$ & -2.427412 & -5.271047 & 4.337174 \\
\hline C & -2.174156 & -3.887165 & 3.977125 \\
\hline C & -2.122583 & -2.965714 & 5.202634 \\
\hline $\mathrm{O}$ & -1.227464 & -2.154481 & 5.286751 \\
\hline C & -3.204932 & -3.380795 & 2.960928 \\
\hline C & -3.109078 & -4.035004 & 1.565971 \\
\hline C & -2.185566 & -3.336882 & 0.594486 \\
\hline $\mathrm{O}$ & -0.937302 & -3.271209 & 0.993631 \\
\hline $\mathrm{H}$ & -0.385782 & -2.841943 & 0.333159 \\
\hline 4 & -2.547720 & -2.892465 & -0.457167 \\
\hline
\end{tabular}




\begin{tabular}{|c|c|c|c|}
\hline $\mathrm{H}$ & -3.324677 & -5.667636 & 4.152986 \\
\hline $\mathrm{H}$ & -1.187431 & -3.811404 & 3.550638 \\
\hline $\mathrm{H}$ & -4.200688 & -3.564254 & 3.347331 \\
\hline $\mathrm{H}$ & -3.092226 & -2.305401 & 2.875981 \\
\hline $\mathrm{H}$ & -2.784935 & -5.065933 & 1.657024 \\
\hline $\mathrm{H}$ & -4.085175 & -4.042765 & 1.102355 \\
\hline$N$ & -3.070267 & -3.077549 & 6.145874 \\
\hline C & -2.905144 & -2.335285 & 7.382761 \\
\hline C & -1.625638 & -2.763495 & 8.101559 \\
\hline $\mathrm{O}$ & -0.978373 & -1.950203 & 8.720969 \\
\hline C & -4.096680 & -2.334917 & 8.350219 \\
\hline $\mathrm{O}$ & -4.374962 & -3.604983 & 8.858256 \\
\hline C & -5.326409 & -1.652390 & 7.762158 \\
\hline $\mathrm{H}$ & -3.770787 & -3.781058 & 6.075021 \\
\hline $\mathrm{H}$ & -2.727626 & -1.303132 & 7.123219 \\
\hline $\mathrm{H}$ & -3.754947 & -1.750331 & 9.196833 \\
\hline $\mathrm{H}$ & -4.828354 & -4.109868 & 8.199437 \\
\hline $\mathrm{H}$ & -6.108344 & -1.621351 & 8.510646 \\
\hline $\mathrm{H}$ & -5.094945 & -0.633840 & 7.466477 \\
\hline $\mathrm{H}$ & -5.707347 & -2.182612 & 6.898580 \\
\hline$N$ & -1.229338 & -4.041788 & 7.968292 \\
\hline $\mathrm{H}$ & -1.896363 & -4.741797 & 7.729828 \\
\hline C & -0.082562 & -4.478931 & 8.725203 \\
\hline $\mathrm{H}$ & -0.183262 & -4.274429 & 9.782286 \\
\hline $\mathrm{H}$ & 0.022540 & -5.546762 & 8.580419 \\
\hline C & 1.199695 & -3.803975 & 8.271916 \\
\hline $\mathrm{O}$ & 2.052173 & -3.488763 & 9.069313 \\
\hline$N$ & 1.336791 & -3.621659 & 6.942798 \\
\hline C & 2.532253 & -3.021760 & 6.424637 \\
\hline C & 2.501297 & -1.500222 & 6.258787 \\
\hline
\end{tabular}




\begin{tabular}{|c|c|c|c|}
\hline O & 3.581480 & -0.951597 & 6.201302 \\
\hline$C$ & 3.110006 & -3.738065 & 5.206102 \\
\hline$S$ & 2.139126 & -3.695157 & 3.674400 \\
\hline $\mathrm{H}$ & 0.605042 & -3.924835 & 6.336202 \\
\hline $\mathrm{H}$ & 3.279203 & -3.138591 & 7.195430 \\
\hline $\mathrm{H}$ & 4.051419 & -3.264949 & 4.963963 \\
\hline $\mathrm{H}$ & 3.305323 & -4.768019 & 5.473819 \\
\hline $\mathrm{H}$ & 1.189391 & -4.557244 & 4.007373 \\
\hline $\mathrm{N}$ & 1.355719 & -0.811842 & 6.286386 \\
\hline$C$ & 1.429671 & 0.628940 & 6.499796 \\
\hline$C$ & 1.897658 & 0.944470 & 7.933108 \\
\hline 0 & 2.466151 & 1.978946 & 8.181778 \\
\hline$C$ & 0.146281 & 1.401220 & 6.133311 \\
\hline$C$ & -0.992307 & 1.201311 & 7.145028 \\
\hline$C$ & -0.247411 & 1.115980 & 4.679396 \\
\hline$C$ & -2.275036 & 1.958772 & 6.803399 \\
\hline $\mathrm{H}$ & 0.480445 & -1.288201 & 6.228225 \\
\hline $\mathrm{H}$ & 2.218185 & 1.003872 & 5.866097 \\
\hline $\mathrm{H}$ & 0.440810 & 2.443671 & 6.200561 \\
\hline $\mathrm{H}$ & -1.212455 & 0.148864 & 7.256969 \\
\hline $\mathrm{H}$ & -0.649730 & 1.539098 & 8.118652 \\
\hline $\mathrm{H}$ & -0.993771 & 1.821355 & 4.334580 \\
\hline $\mathrm{H}$ & 0.615178 & 1.209908 & 4.025252 \\
\hline $\mathrm{H}$ & -0.647408 & 0.118258 & 4.554798 \\
\hline $\mathrm{H}$ & -2.977392 & 1.900669 & 7.629482 \\
\hline $\mathrm{H}$ & -2.080241 & 3.011068 & 6.611791 \\
\hline $\mathrm{H}$ & -2.763787 & 1.544400 & 5.928648 \\
\hline$N$ & 1.675656 & -0.021227 & 8.823607 \\
\hline $\mathrm{H}$ & 1.041234 & -0.757479 & 8.611033 \\
\hline C & 2.099907 & 0.128901 & 10.199499 \\
\hline
\end{tabular}




\begin{tabular}{|c|c|c|c|}
\hline $\mathrm{H}$ & 1.600441 & -0.631549 & 10.787203 \\
\hline $\mathrm{H}$ & 1.849915 & 1.105047 & 10.587964 \\
\hline C & 3.608282 & -0.031473 & 10.341724 \\
\hline $\mathrm{O}$ & 4.277766 & 0.752964 & 10.955584 \\
\hline$N$ & 4.142582 & -1.100012 & 9.704939 \\
\hline $\mathrm{H}$ & 3.542911 & -1.818918 & 9.363522 \\
\hline C & 5.570805 & -1.264076 & 9.662300 \\
\hline $\mathrm{H}$ & 5.794465 & -2.230978 & 9.225399 \\
\hline $\mathrm{H}$ & 6.010856 & -1.226471 & 10.648884 \\
\hline C & 6.284382 & -0.191778 & 8.842048 \\
\hline $\mathrm{O}$ & 7.419073 & 0.120280 & 9.112560 \\
\hline$N$ & 5.619219 & 0.337788 & 7.816482 \\
\hline C & 6.190541 & 1.508390 & 7.178754 \\
\hline C & 6.228487 & 2.682421 & 8.168714 \\
\hline $\mathrm{H}$ & 5.786670 & 2.499973 & 9.149271 \\
\hline $\mathrm{O}$ & 6.703652 & 3.732332 & 7.872241 \\
\hline C & 5.439387 & 1.948294 & 5.920355 \\
\hline C & 5.597046 & 1.123163 & 4.656468 \\
\hline C & 6.069757 & -0.183708 & 4.627820 \\
\hline C & 5.198454 & 1.703591 & 3.453500 \\
\hline C & 6.105178 & -0.900315 & 3.440237 \\
\hline C & 5.226762 & 0.991122 & 2.268371 \\
\hline C & 5.672036 & -0.323335 & 2.257195 \\
\hline $\mathrm{H}$ & 4.700987 & 0.035449 & 7.582641 \\
\hline $\mathrm{H}$ & 7.226526 & 1.303013 & 6.933021 \\
\hline $\mathrm{H}$ & 4.383885 & 2.014198 & 6.158999 \\
\hline $\mathrm{H}$ & 5.773437 & 2.955234 & 5.702935 \\
\hline $\mathrm{H}$ & 6.364756 & -0.662754 & 5.541148 \\
\hline $\mathrm{H}$ & 4.863494 & 2.726169 & 3.448406 \\
\hline & 6.457967 & -1.917936 & 3.443550 \\
\hline
\end{tabular}




\begin{tabular}{|c|c|c|c|}
\hline $\mathrm{H}$ & 4.915186 & 1.466886 & 1.353507 \\
\hline $\mathrm{H}$ & 5.696949 & -0.882430 & 1.338875 \\
\hline$N$ & 12.605097 & 9.645450 & -0.989694 \\
\hline $\mathrm{H}$ & 11.811783 & 10.019842 & -1.474196 \\
\hline C & 12.309724 & 8.295662 & -0.500503 \\
\hline C & 11.358265 & 8.485133 & 0.678935 \\
\hline $\mathrm{O}$ & 10.372706 & 7.800191 & 0.819562 \\
\hline C & 13.587572 & 7.559801 & -0.084249 \\
\hline C & 14.603137 & 7.347577 & -1.212949 \\
\hline S & 13.871191 & 6.440892 & -2.606847 \\
\hline C & 15.302958 & 6.285570 & -3.700731 \\
\hline $\mathrm{H}$ & 13.344312 & 9.626370 & -1.663160 \\
\hline $\mathrm{H}$ & 11.767178 & 7.691727 & -1.217401 \\
\hline $\mathrm{H}$ & 14.075953 & 8.105162 & 0.718255 \\
\hline $\mathrm{H}$ & 13.293052 & 6.594618 & 0.306785 \\
\hline $\mathrm{H}$ & 15.009460 & 8.292104 & -1.558461 \\
\hline $\mathrm{H}$ & 15.438321 & 6.780091 & -0.817339 \\
\hline $\mathrm{H}$ & 14.962768 & 5.735005 & -4.565047 \\
\hline $\mathrm{H}$ & 15.663189 & 7.258508 & -4.009459 \\
\hline $\mathrm{H}$ & 16.101016 & 5.731878 & -3.223557 \\
\hline$N$ & 11.633132 & 9.508483 & 1.512139 \\
\hline C & 10.803356 & 9.774034 & 2.666178 \\
\hline C & 9.391406 & 10.231556 & 2.272859 \\
\hline $\mathrm{O}$ & 8.436194 & 9.887888 & 2.918276 \\
\hline C & 11.428851 & 10.834619 & 3.573117 \\
\hline C & 12.711544 & 10.385284 & 4.254282 \\
\hline $\mathrm{O}$ & 13.081048 & 9.235746 & 4.241846 \\
\hline$N$ & 13.391670 & 11.358819 & 4.873700 \\
\hline $\mathrm{H}$ & 12.423569 & 10.072287 & 1.295811 \\
\hline $\mathrm{H}$ & 10.663716 & 8.862403 & 3.226757 \\
\hline
\end{tabular}




\begin{tabular}{|c|c|c|c|}
\hline $\mathrm{H}$ & 11.616905 & 11.753676 & 3.024205 \\
\hline $\mathrm{H}$ & 10.715465 & 11.078993 & 4.355091 \\
\hline $\mathrm{H}$ & 14.206576 & 11.123246 & 5.392662 \\
\hline $\mathrm{H}$ & 13.056525 & 12.291776 & 4.926002 \\
\hline $\mathrm{N}$ & 9.287052 & 11.051569 & 1.205792 \\
\hline $\mathrm{H}$ & 10.106082 & 11.286990 & 0.696468 \\
\hline$C$ & 7.991458 & 11.479987 & 0.716012 \\
\hline $\mathrm{H}$ & 8.137413 & 12.263269 & -0.016973 \\
\hline $\mathrm{H}$ & 7.390674 & 11.873672 & 1.521104 \\
\hline$C$ & 7.178835 & 10.360642 & 0.062912 \\
\hline $\mathrm{O}$ & 5.979704 & 10.443531 & 0.030976 \\
\hline $\mathrm{N}$ & 7.865769 & 9.339949 & -0.490158 \\
\hline$C$ & 7.191788 & 8.190879 & -1.073552 \\
\hline$C$ & 6.625966 & 7.261505 & 0.008298 \\
\hline 0 & 5.498903 & 6.840692 & -0.095648 \\
\hline$C$ & 8.111684 & 7.466039 & -2.082293 \\
\hline$C$ & 8.420425 & 8.393461 & -3.272500 \\
\hline$C$ & 7.481414 & 6.149042 & -2.549284 \\
\hline$C$ & 9.527276 & 7.891299 & -4.201712 \\
\hline $\mathrm{H}$ & 8.826334 & 9.237613 & -0.264597 \\
\hline $\mathrm{H}$ & 6.316868 & 8.553744 & -1.592320 \\
\hline $\mathrm{H}$ & 9.043678 & 7.230564 & -1.570557 \\
\hline $\mathrm{H}$ & 7.506500 & 8.541067 & -3.844085 \\
\hline $\mathrm{H}$ & 8.702307 & 9.373779 & -2.900030 \\
\hline $\mathrm{H}$ & 8.124465 & 5.657859 & -3.267801 \\
\hline $\mathrm{H}$ & 7.328958 & 5.461878 & -1.725467 \\
\hline $\mathrm{H}$ & 6.517580 & 6.315649 & -3.019360 \\
\hline $\mathrm{H}$ & 9.726183 & 8.623326 & -4.977731 \\
\hline $\mathrm{H}$ & 10.455904 & 7.722689 & -3.663082 \\
\hline $\mathrm{H}$ & 9.264745 & 6.963142 & -4.695654 \\
\hline
\end{tabular}




\begin{tabular}{|c|c|c|c|}
\hline$N$ & 7.396810 & 6.977211 & 1.073194 \\
\hline$C$ & 6.870667 & 6.219922 & 2.199020 \\
\hline$C$ & 5.597065 & 6.863244 & 2.756489 \\
\hline 0 & 4.620878 & 6.194666 & 3.003143 \\
\hline$C$ & 7.954245 & 6.087259 & 3.287149 \\
\hline$C$ & 8.877118 & 4.900205 & 3.014230 \\
\hline 0 & 8.312746 & 3.792572 & 3.091685 \\
\hline 0 & 10.070105 & 5.073631 & 2.745069 \\
\hline $\mathrm{H}$ & 8.347648 & 7.274285 & 1.101118 \\
\hline $\mathrm{H}$ & 6.558354 & 5.235417 & 1.884578 \\
\hline $\mathrm{H}$ & 8.512843 & 7.009524 & 3.380568 \\
\hline $\mathrm{H}$ & 7.469175 & 5.878821 & 4.234051 \\
\hline $\mathrm{N}$ & 5.618631 & 8.193709 & 2.957647 \\
\hline $\mathrm{H}$ & 6.466170 & 8.702491 & 2.833407 \\
\hline$C$ & 4.455927 & 8.873968 & 3.476710 \\
\hline $\mathrm{H}$ & 4.714977 & 9.913383 & 3.638085 \\
\hline $\mathrm{H}$ & 4.141412 & 8.457332 & 4.421641 \\
\hline$C$ & 3.249080 & 8.846680 & 2.543859 \\
\hline 0 & 2.128519 & 8.961806 & 2.984634 \\
\hline $\mathrm{N}$ & 3.519946 & 8.724741 & 1.241347 \\
\hline $\mathrm{H}$ & 4.467098 & 8.717820 & 0.947172 \\
\hline$C$ & 2.521876 & 8.841272 & 0.221535 \\
\hline $\mathrm{H}$ & 3.021555 & 9.088019 & -0.706909 \\
\hline $\mathrm{H}$ & 1.840596 & 9.651214 & 0.445584 \\
\hline$C$ & 1.621509 & 7.623939 & -0.042854 \\
\hline 0 & 0.566567 & 7.765487 & -0.595952 \\
\hline $\mathrm{N}$ & 2.114075 & 6.465600 & 0.435020 \\
\hline$C$ & 1.325052 & 5.275905 & 0.632023 \\
\hline$C$ & 0.521051 & 5.332272 & 1.934991 \\
\hline 0 & -0.665222 & 5.097145 & 1.919975 \\
\hline
\end{tabular}




\begin{tabular}{|c|c|c|c|}
\hline C & 2.221393 & 4.038554 & 0.605973 \\
\hline $\mathrm{H}$ & 3.032880 & 6.490074 & 0.820726 \\
\hline $\mathrm{H}$ & 0.586544 & 5.215106 & -0.151268 \\
\hline $\mathrm{H}$ & 1.642221 & 3.148854 & 0.825399 \\
\hline $\mathrm{H}$ & 2.667555 & 3.936222 & -0.376202 \\
\hline $\mathrm{H}$ & 3.018817 & 4.116945 & 1.337540 \\
\hline$N$ & 1.160734 & 5.612581 & 3.086106 \\
\hline C & 0.445753 & 5.417564 & 4.338052 \\
\hline C & -0.688432 & 6.421301 & 4.513939 \\
\hline $\mathrm{O}$ & -1.715591 & 6.086282 & 5.064691 \\
\hline C & 1.365414 & 5.358355 & 5.583335 \\
\hline C & 1.903350 & 6.719532 & 6.053495 \\
\hline C & 2.490602 & 4.338333 & 5.365721 \\
\hline C & 2.784614 & 6.631051 & 7.301416 \\
\hline $\mathrm{H}$ & 2.140161 & 5.794735 & 3.090161 \\
\hline $\mathrm{H}$ & -0.060458 & 4.464400 & 4.278000 \\
\hline $\mathrm{H}$ & 0.719388 & 4.980710 & 6.371214 \\
\hline $\mathrm{H}$ & 2.457740 & 7.191993 & 5.248597 \\
\hline $\mathrm{H}$ & 1.069038 & 7.377438 & 6.275109 \\
\hline $\mathrm{H}$ & 2.862727 & 3.983365 & 6.316314 \\
\hline $\mathrm{H}$ & 2.134669 & 3.475300 & 4.812842 \\
\hline $\mathrm{H}$ & 3.318926 & 4.770017 & 4.813867 \\
\hline $\mathrm{H}$ & 3.036049 & 7.626730 & 7.653890 \\
\hline $\mathrm{H}$ & 2.271645 & 6.116420 & 8.108369 \\
\hline $\mathrm{H}$ & 3.713557 & 6.104963 & 7.114495 \\
\hline$N$ & -0.496270 & 7.670065 & 4.067139 \\
\hline $\mathrm{H}$ & 0.383206 & 7.935521 & 3.677294 \\
\hline C & -1.482307 & 8.685751 & 4.329394 \\
\hline $\mathrm{H}$ & -1.098743 & 9.633836 & 3.972608 \\
\hline $\mathrm{H}$ & -1.680826 & 8.780515 & 5.387719 \\
\hline
\end{tabular}




\begin{tabular}{|c|c|c|c|}
\hline C & -2.837640 & 8.406102 & 3.688546 \\
\hline $\mathrm{O}$ & -3.836751 & 8.589636 & 4.342416 \\
\hline$N$ & -2.877446 & 7.935275 & 2.428240 \\
\hline C & -4.220403 & 7.836635 & 1.852529 \\
\hline C & -4.943742 & 6.549192 & 2.211117 \\
\hline O & -6.153734 & 6.532863 & 2.198041 \\
\hline C & -4.022959 & 8.098999 & 0.354399 \\
\hline C & -2.778189 & 8.992138 & 0.358059 \\
\hline C & -1.898729 & 8.326332 & 1.403918 \\
\hline $\mathrm{H}$ & -4.843944 & 8.613510 & 2.269745 \\
\hline $\mathrm{H}$ & -3.838470 & 7.176028 & -0.180426 \\
\hline $\mathrm{H}$ & -4.898583 & 8.567297 & -0.076716 \\
\hline $\mathrm{H}$ & -2.279065 & 9.071736 & -0.589617 \\
\hline $\mathrm{H}$ & -3.047887 & 9.993701 & 0.685654 \\
\hline $\mathrm{H}$ & -1.403037 & 7.453915 & 1.014892 \\
\hline $\mathrm{H}$ & -1.146531 & 8.987927 & 1.802593 \\
\hline $\mathrm{N}$ & -4.206530 & 5.481044 & 2.558121 \\
\hline C & -4.850493 & 4.325074 & 3.154800 \\
\hline C & -5.508386 & 4.673999 & 4.494185 \\
\hline O & -6.601718 & 4.233176 & 4.768981 \\
\hline C & -3.863422 & 3.167891 & 3.402179 \\
\hline C & -3.562234 & 2.258476 & 2.227574 \\
\hline C & -4.265584 & 1.066274 & 2.074534 \\
\hline C & -2.532678 & 2.543873 & 1.339500 \\
\hline C & -3.919670 & 0.162834 & 1.080029 \\
\hline C & -2.184467 & 1.639896 & 0.349163 \\
\hline C & -2.859756 & 0.437984 & 0.227599 \\
\hline $\mathrm{H}$ & -2.543981 & -0.291743 & -0.496033 \\
\hline $\mathrm{H}$ & -3.216320 & 5.582454 & 2.618493 \\
\hline $\mathrm{H}$ & -5.656491 & 4.000385 & 2.514629 \\
\hline
\end{tabular}




\begin{tabular}{|c|c|c|c|}
\hline $\mathrm{H}$ & -2.939961 & 3.577867 & 3.796986 \\
\hline $\mathrm{H}$ & -4.288460 & 2.557371 & 4.189729 \\
\hline $\mathrm{H}$ & -5.079725 & 0.837899 & 2.740978 \\
\hline $\mathrm{H}$ & -1.978071 & 3.455548 & 1.437522 \\
\hline $\mathrm{H}$ & -4.463735 & -0.759548 & 0.978246 \\
\hline $\mathrm{H}$ & -1.367648 & 1.867138 & -0.313212 \\
\hline$N$ & -4.798586 & 5.430586 & 5.345921 \\
\hline $\mathrm{H}$ & -3.870490 & 5.714825 & 5.114979 \\
\hline C & -5.328718 & 5.798705 & 6.638834 \\
\hline $\mathrm{H}$ & -4.514798 & 6.194203 & 7.234486 \\
\hline $\mathrm{H}$ & -5.743650 & 4.940936 & 7.145710 \\
\hline C & -6.436975 & 6.846136 & 6.574470 \\
\hline $\mathrm{O}$ & -7.387562 & 6.793074 & 7.317466 \\
\hline$N$ & -6.296069 & 7.791340 & 5.640483 \\
\hline $\mathrm{H}$ & -5.436517 & 7.847229 & 5.140913 \\
\hline C & -7.221224 & 8.920197 & 5.453612 \\
\hline $\mathrm{H}$ & -6.754149 & 9.597407 & 4.749698 \\
\hline r & -7.387747 & 9.444795 & 6.383351 \\
\hline C & -8.589411 & 8.538047 & 4.928208 \\
\hline $\mathrm{O}$ & -9.573026 & 9.111548 & 5.328641 \\
\hline$N$ & -8.646072 & 7.566085 & 4.000131 \\
\hline C & -9.923313 & 7.209078 & 3.427515 \\
\hline C & -10.818965 & 6.437408 & 4.389075 \\
\hline $\mathrm{O}$ & -11.965708 & 6.220912 & 4.055364 \\
\hline C & -9.769950 & 6.442831 & 2.093494 \\
\hline $\mathrm{O}$ & -10.958766 & 6.545684 & 1.354121 \\
\hline C & -9.349884 & 4.987637 & 2.259810 \\
\hline $\mathrm{H}$ & -7.805360 & 7.150863 & 3.657528 \\
\hline $\mathrm{H}$ & -10.471970 & 8.115611 & 3.210235 \\
\hline & -9.013575 & 6.967865 & 1.522689 \\
\hline
\end{tabular}




\begin{tabular}{|c|c|c|c|}
\hline $\mathrm{H}$ & -11.677645 & 6.275186 & 1.910390 \\
\hline $\mathrm{H}$ & -9.153965 & 4.551097 & 1.287678 \\
\hline $\mathrm{H}$ & -8.450668 & 4.897403 & 2.854885 \\
\hline $\mathrm{H}$ & -10.141339 & 4.411991 & 2.729064 \\
\hline$N$ & -10.294538 & 6.001274 & 5.537719 \\
\hline C & -11.127360 & 5.378333 & 6.548334 \\
\hline C & -12.164075 & 6.334298 & 7.142836 \\
\hline $\mathrm{O}$ & -13.196767 & 5.888203 & 7.586332 \\
\hline C & -10.277972 & 4.818918 & 7.699419 \\
\hline C & -9.297418 & 3.696681 & 7.327125 \\
\hline C & -8.454933 & 3.339284 & 8.555516 \\
\hline C & -10.000673 & 2.453496 & 6.776122 \\
\hline $\mathrm{H}$ & -9.347014 & 6.207149 & 5.758566 \\
\hline $\mathrm{H}$ & -11.705909 & 4.592408 & 6.089703 \\
\hline $\mathrm{H}$ & -9.719568 & 5.636987 & 8.143712 \\
\hline $\mathrm{H}$ & -10.970068 & 4.455782 & 8.453996 \\
\hline $\mathrm{H}$ & -8.617869 & 4.061365 & 6.564274 \\
\hline $\mathrm{H}$ & -7.723664 & 2.572768 & 8.314265 \\
\hline $\mathrm{H}$ & -7.921380 & 4.208676 & 8.924371 \\
\hline $\mathrm{H}$ & -9.078011 & 2.960388 & 9.363199 \\
\hline $\mathrm{H}$ & -9.270844 & 1.678242 & 6.561906 \\
\hline $\mathrm{H}$ & -10.712497 & 2.055543 & 7.497675 \\
\hline $\mathrm{H}$ & -10.536074 & 2.652778 & 5.854776 \\
\hline$N$ & -11.837819 & 7.634435 & 7.225023 \\
\hline C & -12.776765 & 8.587947 & 7.740207 \\
\hline C & -13.830077 & 9.025782 & 6.745453 \\
\hline $\mathrm{H}$ & -14.569777 & 9.723269 & 7.159464 \\
\hline $\mathrm{O}$ & -13.881203 & 8.680917 & 5.612976 \\
\hline $\mathrm{H}$ & -11.057023 & 7.981713 & 6.712469 \\
\hline $\mathrm{H}$ & -13.283260 & 8.176506 & 8.605351 \\
\hline
\end{tabular}




\begin{tabular}{|c|c|c|c|}
\hline $\mathrm{H}$ & -12.243322 & 9.475036 & 8.069918 \\
\hline$N$ & -11.987120 & 10.578869 & -2.764592 \\
\hline $\mathrm{H}$ & -11.751692 & 10.959577 & -1.870987 \\
\hline C & -11.843999 & 9.130699 & -2.771738 \\
\hline C & -11.371899 & 8.816543 & -4.181802 \\
\hline $\mathrm{O}$ & -10.303173 & 8.308824 & -4.429956 \\
\hline C & -13.097681 & 8.344887 & -2.324940 \\
\hline C & -13.437935 & 8.683343 & -0.862077 \\
\hline C & -12.911609 & 6.838396 & -2.524534 \\
\hline C & -14.763413 & 8.100056 & -0.369997 \\
\hline $\mathrm{H}$ & -12.927479 & 10.865466 & -2.954239 \\
\hline $\mathrm{H}$ & -11.017331 & 8.866942 & -2.124678 \\
\hline $\mathrm{H}$ & -13.934325 & 8.672613 & -2.947305 \\
\hline $\mathrm{H}$ & -12.630986 & 8.332787 & -0.222995 \\
\hline $\mathrm{H}$ & -13.483546 & 9.762280 & -0.745381 \\
\hline $\mathrm{H}$ & -13.831210 & 6.304279 & -2.322028 \\
\hline $\mathrm{H}$ & -12.617755 & 6.591521 & -3.540032 \\
\hline $\mathrm{H}$ & -12.151435 & 6.458645 & -1.850543 \\
\hline $\mathrm{H}$ & -14.977283 & 8.453926 & 0.632331 \\
\hline $\mathrm{H}$ & -15.589909 & 8.398488 & -1.010071 \\
\hline $\mathrm{H}$ & -14.743791 & 7.017034 & -0.331259 \\
\hline $\mathrm{N}$ & -12.192657 & 9.240443 & -5.161369 \\
\hline $\mathrm{H}$ & -13.089576 & 9.577737 & -4.903483 \\
\hline$C$ & -12.011414 & 8.884787 & -6.567479 \\
\hline $\mathrm{H}$ & -12.092493 & 7.817828 & -6.724477 \\
\hline $\mathrm{H}$ & -12.784505 & 9.383186 & -7.138846 \\
\hline$C$ & -10.646468 & 9.301893 & -7.121043 \\
\hline 0 & -10.007899 & 8.570545 & -7.823006 \\
\hline $\mathrm{N}$ & -10.257651 & 10.558634 & -6.809092 \\
\hline H & -10.800853 & 11.059272 & -6.144133 \\
\hline
\end{tabular}




\begin{tabular}{|c|c|c|c|}
\hline C & -8.953652 & 11.108373 & -7.195869 \\
\hline $\mathrm{H}$ & -8.831025 & 11.030151 & -8.265539 \\
\hline $\mathrm{H}$ & -8.942953 & 12.154723 & -6.917771 \\
\hline C & -7.725399 & 10.427883 & -6.575522 \\
\hline $\mathrm{O}$ & -6.814041 & 10.019586 & -7.242355 \\
\hline$N$ & -7.700740 & 10.405557 & -5.227497 \\
\hline $\mathrm{H}$ & -8.524363 & 10.658582 & -4.733220 \\
\hline C & -6.528681 & 9.976762 & -4.440793 \\
\hline $\mathrm{H}$ & -5.634309 & 10.408124 & -4.860922 \\
\hline $\mathrm{H}$ & -6.656793 & 10.343746 & -3.429741 \\
\hline C & -6.295542 & 8.478017 & -4.374212 \\
\hline $\mathrm{O}$ & -5.155197 & 8.078206 & -4.433512 \\
\hline$N$ & -7.325650 & 7.636682 & -4.183102 \\
\hline C & -7.006433 & 6.262528 & -3.820324 \\
\hline C & -6.445041 & 5.466269 & -4.990811 \\
\hline 0 & -5.642453 & 4.585405 & -4.780604 \\
\hline$C$ & -8.129967 & 5.491217 & -3.086373 \\
\hline$C$ & -9.325974 & 5.105625 & -3.972640 \\
\hline$C$ & -8.534021 & 6.248381 & -1.816398 \\
\hline$C$ & -10.308935 & 4.151738 & -3.291972 \\
\hline $\mathrm{H}$ & -8.268149 & 7.968720 & -4.160340 \\
\hline $\mathrm{H}$ & -6.171691 & 6.302029 & -3.135698 \\
\hline $\mathrm{H}$ & -7.647053 & 4.568492 & -2.774381 \\
\hline $\mathrm{H}$ & -9.845674 & 5.996226 & -4.304365 \\
\hline $\mathrm{H}$ & -8.955628 & 4.612924 & -4.868172 \\
\hline $\mathrm{H}$ & -9.177354 & 5.644811 & -1.191102 \\
\hline $\mathrm{H}$ & -7.661213 & 6.506537 & -1.224368 \\
\hline $\mathrm{H}$ & -9.068568 & 7.161985 & -2.050857 \\
\hline $\mathrm{H}$ & -11.105463 & 3.883409 & -3.979581 \\
\hline $\mathrm{H}$ & -9.822482 & 3.232768 & -2.990920 \\
\hline
\end{tabular}




\begin{tabular}{|c|c|c|c|}
\hline $\mathrm{H}$ & -10.770062 & 4.594278 & -2.418601 \\
\hline$N$ & -6.859329 & 5.742481 & -6.243030 \\
\hline C & -6.234516 & 5.059284 & -7.357275 \\
\hline C & -4.828417 & 5.581984 & -7.656692 \\
\hline $\mathrm{O}$ & -3.982411 & 4.820249 & -8.062590 \\
\hline C & -7.134704 & 5.060918 & -8.601441 \\
\hline C & -8.262462 & 4.022738 & -8.506346 \\
\hline C & -7.743880 & 2.597429 & -8.724700 \\
\hline$N$ & -8.740287 & 1.555550 & -8.491788 \\
\hline C & -9.000257 & 0.964199 & -7.324725 \\
\hline$N$ & -8.438537 & 1.378135 & -6.203035 \\
\hline$N$ & -9.841373 & -0.051720 & -7.280511 \\
\hline $\mathrm{H}$ & -7.508781 & 6.481850 & -6.397153 \\
\hline $\mathrm{H}$ & -6.046038 & 4.045094 & -7.045388 \\
\hline $\mathrm{H}$ & -7.557448 & 6.050080 & -8.749381 \\
\hline $\mathrm{H}$ & -6.520256 & 4.852806 & -9.472209 \\
\hline $\mathrm{H}$ & -8.758643 & 4.100559 & -7.543578 \\
\hline $\mathrm{H}$ & -9.017262 & 4.236462 & -9.255855 \\
\hline $\mathrm{H}$ & -7.417772 & 2.482929 & -9.750306 \\
\hline $\mathrm{H}$ & -6.874296 & 2.385570 & -8.115233 \\
\hline $\mathrm{H}$ & -9.160883 & 1.147081 & -9.294192 \\
\hline $\mathrm{H}$ & -7.879848 & 2.196908 & -6.192469 \\
\hline $\mathrm{H}$ & -8.803682 & 1.059342 & -5.311888 \\
\hline $\mathrm{H}$ & -10.200969 & -0.427562 & $2-8.127368$ \\
\hline $\mathrm{H}$ & -9.831710 & -0.683540 & -6.486956 \\
\hline$N$ & -4.599868 & 6.879742 & -7.441550 \\
\hline $\mathrm{H}$ & -5.338150 & 7.475669 & -7.146867 \\
\hline C & -3.292587 & 7.493378 & -7.719943 \\
\hline $\mathrm{H}$ & -2.965478 & 7.263319 & -8.722586 \\
\hline $\mathrm{H}$ & -3.402807 & 8.564912 & -7.616375 \\
\hline
\end{tabular}




\begin{tabular}{|c|c|c|c|}
\hline$C$ & -2.225526 & 6.999452 & -6.757111 \\
\hline 0 & -1.096518 & 6.788030 & -7.123961 \\
\hline $\mathrm{N}$ & -2.643483 & 6.845473 & -5.495203 \\
\hline$C$ & -1.750230 & 6.515475 & -4.412904 \\
\hline$C$ & -1.466826 & 5.028340 & -4.317252 \\
\hline 0 & -0.337836 & 4.647952 & -4.116999 \\
\hline$C$ & -2.320776 & 7.083777 & -3.107291 \\
\hline$C$ & -1.730066 & 6.349823 & -1.904999 \\
\hline$C$ & -2.071008 & 8.598762 & -3.107966 \\
\hline $\mathrm{H}$ & -3.566562 & 7.137295 & -5.260576 \\
\hline $\mathrm{H}$ & -0.781883 & 6.957604 & -4.602048 \\
\hline $\mathrm{H}$ & -3.391560 & 6.913361 & -3.117818 \\
\hline $\mathrm{H}$ & -1.938470 & 6.868730 & -0.988943 \\
\hline $\mathrm{H}$ & -2.144658 & 5.349974 & -1.824047 \\
\hline $\mathrm{H}$ & -0.658230 & 6.275333 & -1.983775 \\
\hline $\mathrm{H}$ & -2.797229 & 9.119510 & -2.499711 \\
\hline $\mathrm{H}$ & -1.076789 & 8.824830 & -2.736374 \\
\hline $\mathrm{H}$ & -2.162209 & 9.005373 & -4.108651 \\
\hline $\mathrm{N}$ & -2.488829 & 4.163540 & -4.455760 \\
\hline$C$ & -2.145750 & 2.764357 & -4.598988 \\
\hline$C$ & -1.205798 & 2.601630 & -5.798724 \\
\hline $\mathrm{O}$ & -0.272770 & 1.833217 & -5.736683 \\
\hline$C$ & -3.376006 & 1.849256 & -4.673163 \\
\hline$C$ & -4.248226 & 1.933749 & -3.411393 \\
\hline$C$ & -5.382896 & 0.900075 & -3.345627 \\
\hline $\mathrm{N}$ & -4.929348 & -0.344445 & -2.747862 \\
\hline$C$ & -5.747122 & -1.420432 & -2.548132 \\
\hline $\mathrm{N}$ & -6.893250 & -1.628524 & -3.082175 \\
\hline $\mathrm{N}$ & -5.258358 & -2.336573 & -1.662564 \\
\hline $\mathrm{H}$ & -3.407356 & 4.484360 & -4.671915 \\
\hline
\end{tabular}




\begin{tabular}{|c|c|c|c|}
\hline $\mathrm{H}$ & -1.546848 & 2.466137 & -3.750200 \\
\hline $\mathrm{H}$ & -3.975485 & 2.106345 & -5.543557 \\
\hline $\mathrm{H}$ & -3.020789 & 0.834990 & -4.821411 \\
\hline $\mathrm{H}$ & -3.625260 & 1.835217 & -2.526098 \\
\hline $\mathrm{H}$ & -4.696058 & 2.915112 & -3.357638 \\
\hline $\mathrm{H}$ & -6.209970 & 1.314217 & -2.772438 \\
\hline $\mathrm{H}$ & -5.751500 & 0.697258 & -4.345562 \\
\hline $\mathrm{H}$ & -4.226947 & -0.233303 & -2.053460 \\
\hline $\mathrm{H}$ & -7.191214 & -0.886824 & -3.680633 \\
\hline $\mathrm{H}$ & -4.276785 & -2.394682 & -1.497374 \\
\hline $\mathrm{H}$ & -5.720509 & -3.216774 & -1.691743 \\
\hline$N$ & -1.457497 & 3.319608 & -6.885943 \\
\hline $\mathrm{H}$ & -2.291982 & 3.853544 & -6.983906 \\
\hline C & -0.598165 & 3.160353 & -8.043207 \\
\hline $\mathrm{H}$ & -0.961826 & 3.818251 & -8.822576 \\
\hline $\mathrm{H}$ & -0.600798 & 2.142494 & -8.407259 \\
\hline C & 0.851686 & 3.516011 & -7.730445 \\
\hline $\mathrm{O}$ & 1.780674 & 2.904809 & -8.195302 \\
\hline$N$ & 0.996656 & 4.554288 & -6.892936 \\
\hline 1 & 0.203311 & 5.107681 & -6.665839 \\
\hline C & 2.274462 & 5.022316 & -6.461804 \\
\hline $\mathrm{H}$ & 2.905535 & 5.311163 & -7.293504 \\
\hline r & 2.120529 & 5.897033 & -5.842318 \\
\hline C & 3.091684 & 4.049398 & -5.637497 \\
\hline $\mathrm{O}$ & 4.296290 & 4.190888 & -5.555332 \\
\hline$N$ & 2.444256 & 3.078839 & -4.984585 \\
\hline C & 3.169500 & 2.252660 & -4.047876 \\
\hline C & 4.332543 & 1.454285 & -4.656013 \\
\hline O & 5.280287 & 1.168028 & -3.952517 \\
\hline$C$ & 2.163836 & 1.375436 & -3.289371 \\
\hline
\end{tabular}




\begin{tabular}{|c|c|c|c|}
\hline C & 2.749656 & 0.608835 & -2.103567 \\
\hline C & 1.669330 & 0.247987 & -1.082802 \\
\hline$N$ & 2.190891 & -0.557952 & 0.003125 \\
\hline C & 2.200503 & -1.916450 & -0.085782 \\
\hline$N$ & 1.352456 & -2.526080 & -0.816778 \\
\hline$N$ & 3.198236 & -2.529743 & 0.643994 \\
\hline $\mathrm{H}$ & 1.456315 & 2.973931 & -5.077369 \\
\hline $\mathrm{H}$ & 3.673956 & 2.893927 & -3.334803 \\
\hline $\mathrm{H}$ & 1.398539 & 2.059496 & -2.932722 \\
\hline $\mathrm{H}$ & 1.665654 & 0.699803 & -3.976702 \\
\hline $\mathrm{H}$ & 3.240518 & -0.298593 & -2.439337 \\
\hline $\mathrm{H}$ & 3.514344 & 1.208616 & -1.614259 \\
\hline $\mathrm{H}$ & 1.239877 & 1.151988 & -0.664603 \\
\hline $\mathrm{H}$ & 0.871836 & -0.310411 & -1.547965 \\
\hline $\mathrm{H}$ & 2.922651 & -0.147838 & 0.536419 \\
\hline $\mathrm{H}$ & 1.544917 & -3.504969 & -0.838053 \\
\hline $\mathrm{H}$ & 3.530574 & -2.007729 & 1.425612 \\
\hline $\mathrm{H}$ & 2.978562 & -3.460754 & 0.924206 \\
\hline$N$ & 4.305833 & 1.076221 & -5.946783 \\
\hline $\mathrm{H}$ & 3.541654 & 1.348138 & -6.527734 \\
\hline C & 5.391670 & 0.279995 & -6.494689 \\
\hline $\mathrm{H}$ & 5.167743 & 0.077252 & -7.535045 \\
\hline $\mathrm{H}$ & 5.480890 & -0.663157 & -5.977803 \\
\hline C & 6.757888 & 0.931485 & -6.434324 \\
\hline $\mathrm{O}$ & 7.768019 & 0.273311 & -6.333310 \\
\hline$N$ & 6.778946 & 2.261487 & -6.545564 \\
\hline $\mathrm{H}$ & 5.922709 & 2.774430 & -6.498806 \\
\hline C & 8.016502 & 3.004101 & -6.531004 \\
\hline $\mathrm{H}$ & 8.694264 & 2.628585 & -7.285161 \\
\hline $\mathrm{H}$ & 7.796154 & 4.036262 & -6.771917 \\
\hline
\end{tabular}




\begin{tabular}{|c|c|c|c|}
\hline C & 8.821096 & 3.005479 & -5.230147 \\
\hline $\mathrm{O}$ & 9.983291 & 3.357917 & -5.251229 \\
\hline$N$ & 8.212780 & 2.591092 & -4.112407 \\
\hline C & 8.894197 & 2.414330 & -2.837976 \\
\hline C & 10.082265 & 1.454809 & -2.928749 \\
\hline $\mathrm{O}$ & 10.989464 & 1.522137 & -2.117237 \\
\hline C & 7.827250 & 1.902777 & -1.839362 \\
\hline C & 8.280908 & 1.116249 & -0.609186 \\
\hline C & 8.963161 & 1.884152 & 0.522139 \\
\hline C & 9.059499 & 0.960858 & 1.733999 \\
\hline$N$ & 9.654847 & 1.632783 & 2.916402 \\
\hline $\mathrm{O}$ & 12.140230 & 0.180777 & 2.408583 \\
\hline $\mathrm{H}$ & 12.211275 & -0.097933 & 1.499226 \\
\hline $\mathrm{H}$ & 12.889977 & -0.162855 & 2.866114 \\
\hline $\mathrm{H}$ & 7.253580 & 2.316407 & -4.165557 \\
\hline $\mathrm{H}$ & 9.321772 & 3.351139 & -2.506671 \\
\hline $\mathrm{H}$ & 7.227707 & 2.755343 & -1.536396 \\
\hline $\mathrm{H}$ & 7.161750 & 1.251262 & -2.390773 \\
\hline $\mathrm{H}$ & 7.379609 & 0.663318 & -0.207728 \\
\hline $\mathrm{H}$ & 8.914095 & 0.292348 & -0.913219 \\
\hline $\mathrm{H}$ & 9.947687 & 2.221659 & 0.213277 \\
\hline $\mathrm{H}$ & 8.381253 & 2.756424 & 0.792576 \\
\hline $\mathrm{H}$ & 8.073196 & 0.645046 & 2.030254 \\
\hline $\mathrm{H}$ & 9.656071 & 0.085738 & 1.520292 \\
\hline $\mathrm{H}$ & 9.465307 & 1.099347 & 3.746996 \\
\hline $\mathrm{H}$ & 10.655773 & 1.683169 & 2.829951 \\
\hline $\mathrm{H}$ & 9.195363 & 2.597245 & 3.034014 \\
\hline$N$ & 10.045486 & 0.555561 & -3.903113 \\
\hline C & 11.054577 & -0.458050 & -4.167584 \\
\hline 0 & 12.458859 & 0.070120 & -4.457551 \\
\hline
\end{tabular}




\begin{tabular}{|c|c|c|c|}
\hline 0 & 13.394173 & -0.679474 & -4.281970 \\
\hline C & 10.647497 & -1.293354 & -5.402092 \\
\hline C & 9.518532 & -2.315633 & -5.203194 \\
\hline C & 9.071673 & -2.848811 & -6.567791 \\
\hline C & 9.964305 & -3.471556 & -4.307153 \\
\hline $\mathrm{H}$ & 9.262387 & 0.565409 & -4.522440 \\
\hline $\mathrm{H}$ & 11.170287 & -1.089936 & -3.297889 \\
\hline $\mathrm{H}$ & 10.371270 & -0.600664 & -6.190696 \\
\hline $\mathrm{H}$ & 11.532247 & -1.822960 & -5.738476 \\
\hline $\mathrm{H}$ & 8.665163 & -1.819383 & -4.750726 \\
\hline $\mathrm{H}$ & 8.276841 & -3.579578 & -6.452551 \\
\hline $\mathrm{H}$ & 8.700873 & -2.047111 & -7.193313 \\
\hline $\mathrm{H}$ & 9.895313 & -3.337769 & -7.082910 \\
\hline $\mathrm{H}$ & 9.177456 & -4.210029 & -4.201141 \\
\hline $\mathrm{H}$ & 10.826255 & -3.971259 & -4.738764 \\
\hline $\mathrm{H}$ & 10.233144 & -3.142019 & -3.307543 \\
\hline$N$ & 12.588893 & 1.304731 & -4.952551 \\
\hline C & 13.849531 & 1.853749 & -5.423478 \\
\hline C & 14.976789 & 2.048844 & -4.390829 \\
\hline $\mathrm{O}$ & 16.044063 & 2.430178 & -4.788097 \\
\hline C & 13.570293 & 3.172441 & -6.147976 \\
\hline $\mathrm{O}$ & 13.001336 & 4.121992 & -5.292539 \\
\hline $\mathrm{H}$ & 11.777949 & 1.881614 & -5.004548 \\
\hline $\mathrm{H}$ & 14.284296 & 1.163158 & -6.138136 \\
\hline $\mathrm{H}$ & 14.510029 & 3.560619 & -6.511250 \\
\hline $\mathrm{H}$ & 12.927284 & 2.975085 & -7.000702 \\
\hline $\mathrm{H}$ & 12.056672 & 4.113499 & -5.344854 \\
\hline $\mathrm{N}$ & 14.757352 & 1.720377 & -3.100444 \\
\hline C & 15.786701 & 1.762329 & -2.093018 \\
\hline C & 16.680932 & 0.520907 & -2.138428 \\
\hline
\end{tabular}




\begin{tabular}{|c|c|c|c|}
\hline 0 & 17.711102 & 0.515347 & -1.515339 \\
\hline$C$ & 15.189815 & 1.836161 & -0.677466 \\
\hline C & 14.431515 & 3.118938 & -0.306089 \\
\hline C & 13.784025 & 2.925383 & 1.070417 \\
\hline$N$ & 13.059768 & 4.078874 & 1.613899 \\
\hline C & 13.541495 & 4.949851 & 2.495175 \\
\hline$N$ & 14.843590 & 5.014112 & 2.753055 \\
\hline O & 14.894697 & 7.137387 & 4.801448 \\
\hline $\mathrm{H}$ & 14.482511 & 7.988064 & 4.692499 \\
\hline $\mathrm{H}$ & 14.982766 & 6.988206 & 5.729444 \\
\hline$N$ & 12.723811 & 5.776198 & 3.110769 \\
\hline $\mathrm{H}$ & 13.820841 & 1.539888 & -2.823320 \\
\hline $\mathrm{H}$ & 16.437986 & 2.606168 & -2.276151 \\
\hline $\mathrm{H}$ & 14.527330 & 0.983927 & -0.544953 \\
\hline $\mathrm{H}$ & 16.015596 & 1.692187 & 0.009824 \\
\hline $\mathrm{H}$ & 15.116211 & 3.963607 & -0.294413 \\
\hline $\mathrm{H}$ & 13.664471 & 3.354230 & -1.034976 \\
\hline $\mathrm{H}$ & 13.070170 & 2.118565 & 1.018358 \\
\hline $\mathrm{H}$ & 14.526558 & 2.615696 & 1.798166 \\
\hline $\mathrm{H}$ & 12.067240 & 4.109991 & 1.502346 \\
\hline $\mathrm{H}$ & 15.501804 & 4.509638 & 2.210846 \\
\hline $\mathrm{H}$ & 15.168996 & 5.688788 & 3.416121 \\
\hline $\mathrm{H}$ & 11.721887 & 5.665133 & 3.034145 \\
\hline $\mathrm{H}$ & 13.103474 & 6.420942 & 3.767874 \\
\hline $\mathrm{N}$ & 16.216802 & -0.533115 & -2.833306 \\
\hline C & 16.961137 & -1.777538 & -2.878589 \\
\hline C & 17.929767 & -1.762363 & -4.052072 \\
\hline $\mathrm{H}$ & 18.394101 & -0.791204 & -4.248536 \\
\hline o & 18.185868 & -2.717043 & -4.710147 \\
\hline C & 16.037036 & -2.995273 & -2.926916 \\
\hline
\end{tabular}




\begin{tabular}{|c|c|c|c|}
\hline C & 15.157691 & -3.107083 & -1.721257 \\
\hline C & 13.809394 & -3.031340 & -1.667136 \\
\hline $\mathrm{O}$ & 12.153260 & -0.381310 & -0.378832 \\
\hline $\mathrm{H}$ & 12.852693 & -0.785217 & -0.869672 \\
\hline $\mathrm{H}$ & 11.739333 & 0.252799 & -0.958635 \\
\hline$N$ & 15.662929 & -3.309576 & -0.457652 \\
\hline$N$ & 13.479516 & -3.196227 & -0.339149 \\
\hline C & 14.640106 & -3.352362 & 0.325705 \\
\hline $\mathrm{H}$ & 15.371597 & -0.457642 & -3.355937 \\
\hline $\mathrm{H}$ & 17.565527 & -1.817809 & -1.979621 \\
\hline $\mathrm{H}$ & 15.421091 & -2.951937 & -3.818950 \\
\hline $\mathrm{H}$ & 16.657852 & -3.877953 & -3.014321 \\
\hline $\mathrm{H}$ & 13.083085 & -2.877444 & -2.434624 \\
\hline $\mathrm{H}$ & 12.567873 & -3.164785 & 0.051104 \\
\hline $\mathrm{H}$ & 14.674147 & -3.495071 & 1.385915 \\
\hline 0 & -14.991273 & -2.648063 & 4.801082 \\
\hline $\mathrm{H}$ & -15.123215 & -1.854114 & 4.296179 \\
\hline $\mathrm{H}$ & -14.566571 & -2.383588 & 5.604624 \\
\hline 0 & -12.965961 & -1.514342 & 6.948920 \\
\hline $\mathrm{H}$ & -12.329483 & -2.018626 & 7.433631 \\
\hline $\mathrm{H}$ & -13.211906 & -0.785417 & 7.497401 \\
\hline 0 & -11.972385 & -1.083850 & 4.217796 \\
\hline $\mathrm{H}$ & -12.172637 & -1.130686 & 5.144785 \\
\hline $\mathrm{H}$ & -12.757303 & -0.723459 & 3.810072 \\
\hline 0 & -13.204411 & -2.028782 & 0.755849 \\
\hline $\mathrm{H}$ & -12.410169 & -1.499284 & 0.776903 \\
\hline $\mathrm{H}$ & -13.095257 & -2.730149 & 1.389933 \\
\hline 0 & -7.138286 & 1.738246 & 0.266100 \\
\hline $\mathrm{H}$ & -7.393087 & 1.879711 & 1.176203 \\
\hline 11 & -6.206847 & 1.586701 & 0.292864 \\
\hline
\end{tabular}




\begin{tabular}{|c|c|c|c|}
\hline 0 & -7.703944 & 1.935504 & 3.062238 \\
\hline $\mathrm{H}$ & -8.619666 & 1.703470 & 3.181042 \\
\hline $\mathrm{H}$ & -7.509107 & 2.654848 & 3.649504 \\
\hline 0 & -8.853326 & -0.035200 & -1.102322 \\
\hline $\mathrm{H}$ & -8.206100 & 0.507128 & -0.652533 \\
\hline $\mathrm{H}$ & -8.443908 & -0.855855 & -1.346181 \\
\hline $\mathrm{O}$ & -12.784135 & -2.296437 & -2.142829 \\
\hline $\mathrm{H}$ & -13.089903 & -2.422389 & -1.253268 \\
\hline $\mathrm{H}$ & -11.851970 & -2.508970 & -2.144071 \\
\hline $\mathrm{O}$ & -13.619059 & 0.856110 & -0.718252 \\
\hline $\mathrm{H}$ & -14.008768 & -0.005264 & -0.756084 \\
\hline $\mathrm{H}$ & -12.748168 & 0.708502 & -0.350683 \\
\hline $\mathrm{O}$ & -14.182794 & 4.592813 & 5.045993 \\
\hline $\mathrm{H}$ & -13.687977 & 5.307461 & 4.663901 \\
\hline $\mathrm{H}$ & -14.211650 & 4.796021 & 5.971362 \\
\hline $\mathrm{O}$ & -14.856714 & 2.012773 & 1.552126 \\
\hline $\mathrm{H}$ & -15.584550 & 2.602099 & 1.440016 \\
\hline $\mathrm{H}$ & -14.524580 & 1.777574 & 0.688958 \\
\hline $\mathrm{O}$ & -12.457334 & 0.205109 & -3.431325 \\
\hline $\mathrm{H}$ & -12.922846 & 0.860194 & -2.931464 \\
\hline $\mathrm{H}$ & -12.566874 & -0.597990 & -2.926209 \\
\hline $\mathrm{O}$ & -12.479251 & -2.293797 & -5.147075 \\
\hline $\mathrm{H}$ & -12.684135 & -1.386307 & -4.971561 \\
\hline $\mathrm{H}$ & -12.849305 & -2.770288 & -4.417400 \\
\hline $\mathrm{O}$ & -10.035475 & -2.676707 & -2.420023 \\
\hline $\mathrm{H}$ & -9.779161 & -2.116989 & -3.141961 \\
\hline $\mathrm{H}$ & -9.835892 & -3.553714 & -2.731861 \\
\hline $\mathrm{O}$ & -11.212563 & -0.011304 & 0.514993 \\
\hline $\mathrm{H}$ & -10.448995 & -0.103824 & -0.050706 \\
\hline & -10.919618 & 0.426968 & 1.310908 \\
\hline
\end{tabular}




$\begin{array}{llll}\mathrm{O} & -10.494974 & 1.114503 & 3.075921 \\ \mathrm{H} & -10.754218 & 0.342087 & 3.566999 \\ \mathrm{H} & -11.182765 & 1.754074 & 3.260670 \\ \mathrm{O} & -12.812174 & 2.564019 & 3.588412 \\ \mathrm{H} & -13.201631 & 3.171086 & 4.211108 \\ \mathrm{H} & -13.435753 & 2.495197 & 2.877570 \\ \mathrm{O} & -9.639602 & 0.761074 & -3.731082 \\ \mathrm{H} & -10.579565 & 0.602202 & -3.708888 \\ \mathrm{H} & -9.323687 & 0.573992 & -2.851963 \\ \mathrm{O} & -14.474076 & -0.511211 & 2.917565 \\ \mathrm{H} & -14.736609 & 0.369408 & 2.674722 \\ \mathrm{H} & -14.287208 & -0.967790 & 2.104876 \\ \mathrm{O} & -12.899288 & -3.661300 & 3.199433 \\ \mathrm{H} & -13.714054 & -3.493330 & 3.672936 \\ \mathrm{H} & -12.305584 & -3.001790 & 3.542445 \\ \mathrm{O} & -9.670116 & -2.113511 & -5.368169 \\ \mathrm{H} & -10.553945 & -2.470830 & -5.326220 \\ \mathrm{H} & -9.063762 & -2.791258 & -5.649430\end{array}$

Y266F MUTATION NOMINAL CHARGES R300(+),E183(0),R303(0),E226(-) POTENTIAL: OmV

$\mathrm{N}$

$15.734446-6.579103 \quad 5.125638$

H $\quad 14.931649-6.353023 \quad 5.677865$

C $\quad 15.661662 \quad-5.947200 \quad 3.802385$

C $\quad 14.923831-6.843598 \quad 2.790620$

O $\quad 14.139199-6.3927301 .978291$

C $\quad 17.064897 \quad-5.648497 \quad 3.264923$

$\begin{array}{llll}H & 16.541049 & -6.266248 & 5.626483\end{array}$ 


\begin{tabular}{|c|c|c|c|}
\hline $\mathrm{H}$ & 15.093129 & -5.025646 & 3.827586 \\
\hline $\mathrm{H}$ & 17.018970 & -5.248939 & 2.258349 \\
\hline $\mathrm{H}$ & 17.551240 & -4.912493 & 3.895886 \\
\hline $\mathrm{H}$ & 17.677258 & -6.543975 & 3.252840 \\
\hline$N$ & 15.183877 & -8.147583 & 2.873907 \\
\hline $\mathrm{H}$ & 15.809732 & -8.441283 & 3.590215 \\
\hline C & 14.551980 & -9.131983 & 1.993674 \\
\hline $\mathrm{H}$ & 14.616367 & -8.823507 & 0.962479 \\
\hline $\mathrm{H}$ & 15.061295 & -10.080105 & 2.109084 \\
\hline C & 13.073760 & -9.309267 & 2.342201 \\
\hline O & 12.243063 & -9.547018 & 1.503973 \\
\hline$N$ & 12.746600 & -9.160379 & 3.638928 \\
\hline $\mathrm{H}$ & 13.467371 & -9.042208 & 4.311587 \\
\hline C & 11.362681 & -9.300155 & 4.098291 \\
\hline $\mathrm{H}$ & 11.359041 & -9.322084 & 5.180351 \\
\hline $\mathrm{H}$ & 10.915754 & -10.208846 & 3.725223 \\
\hline $\mathrm{C}$ & 10.492663 & -8.130745 & 3.619542 \\
\hline 0 & 9.332854 & -8.293281 & 3.351750 \\
\hline $\mathrm{N}$ & 11.114743 & -6.938173 & 3.549358 \\
\hline$C$ & 10.477621 & -5.757277 & 3.023762 \\
\hline$C$ & 10.299871 & -5.785069 & 1.502522 \\
\hline 0 & 9.259601 & -5.372175 & 1.049528 \\
\hline$C$ & 11.198957 & -4.493355 & 3.512933 \\
\hline 0 & 12.592027 & -4.591417 & 3.490757 \\
\hline $\mathrm{H}$ & 12.085464 & -6.886182 & 3.746810 \\
\hline $\mathrm{H}$ & 9.465402 & -5.708224 & 3.390585 \\
\hline $\mathrm{H}$ & 10.843748 & -3.645008 & 2.933221 \\
\hline $\mathrm{H}$ & 10.928553 & -4.323848 & 4.546334 \\
\hline $\mathrm{H}$ & 12.931237 & -4.894532 & 2.659886 \\
\hline T & 11.282651 & -6.252271 & 0.7094 \\
\hline
\end{tabular}




\begin{tabular}{|c|c|c|c|}
\hline C & 11.002366 & -6.429556 & -0.712957 \\
\hline C & 9.858589 & -7.425679 & -0.909835 \\
\hline $\mathrm{O}$ & 9.135539 & -7.345657 & -1.875183 \\
\hline C & 12.268340 & -6.786562 & -1.540904 \\
\hline C & 12.612627 & -8.279226 & -1.579060 \\
\hline C & 12.128292 & -6.259282 & -2.970905 \\
\hline $\mathrm{H}$ & 12.132467 & -6.604473 & 1.094162 \\
\hline $\mathrm{H}$ & 10.611173 & -5.495949 & -1.087040 \\
\hline $\mathrm{H}$ & 13.089141 & -6.255237 & -1.067370 \\
\hline $\mathrm{H}$ & 13.570422 & -8.411742 & -2.071521 \\
\hline $\mathrm{H}$ & 12.671410 & -8.729681 & -0.599726 \\
\hline $\mathrm{H}$ & 11.877329 & -8.833117 & -2.155821 \\
\hline $\mathrm{H}$ & 13.020380 & -6.492469 & -3.541992 \\
\hline $\mathrm{H}$ & 11.277354 & -6.707472 & -3.471306 \\
\hline $\mathrm{H}$ & 12.000227 & -5.185110 & -2.983531 \\
\hline$N$ & 9.705480 & -8.354399 & 0.043523 \\
\hline $\mathrm{H}$ & 10.451131 & -8.499461 & 0.684311 \\
\hline C & 8.735566 & -9.439355 & -0.071429 \\
\hline $\mathrm{H}$ & 9.105432 & -10.271026 & 0.516423 \\
\hline $\mathrm{H}$ & 8.651511 & -9.765429 & -1.096717 \\
\hline C & 7.329417 & -9.124903 & 0.389069 \\
\hline $\mathrm{O}$ & 6.408579 & -9.627280 & -0.214489 \\
\hline$N$ & 7.148455 & -8.314875 & 1.435518 \\
\hline C & 5.809786 & -7.827170 & 1.688613 \\
\hline C & 5.391248 & -6.855552 & 0.588773 \\
\hline $\mathrm{O}$ & 4.222954 & -6.782715 & 0.299499 \\
\hline C & 5.547587 & -7.275015 & 3.102243 \\
\hline C & 5.675841 & -8.385415 & 4.149556 \\
\hline C & 6.393085 & -6.058258 & 3.483788 \\
\hline $\mathrm{H}$ & 7.928998 & -8.011386 & 1.973223 \\
\hline
\end{tabular}




\begin{tabular}{|c|c|c|c|}
\hline $\mathrm{H}$ & 5.131260 & -8.655102 & 1.552664 \\
\hline $\mathrm{H}$ & 4.508260 & -6.961529 & 3.069932 \\
\hline $\mathrm{H}$ & 5.387766 & -8.012741 & 5.127323 \\
\hline $\mathrm{H}$ & 5.029135 & -9.224236 & 3.912821 \\
\hline $\mathrm{H}$ & 6.694537 & -8.751625 & 4.218395 \\
\hline $\mathrm{H}$ & 6.040509 & -5.648074 & 4.424433 \\
\hline $\mathrm{H}$ & 7.431510 & -6.334598 & 3.618221 \\
\hline $\mathrm{H}$ & 6.339471 & -5.270810 & 2.739893 \\
\hline$N$ & 6.325083 & -6.142825 & -0.064679 \\
\hline C & 5.939527 & -5.353804 & -1.221322 \\
\hline C & 5.540616 & -6.259635 & -2.391410 \\
\hline $\mathrm{O}$ & 4.553515 & -5.999908 & -3.040890 \\
\hline C & 7.034312 & -4.346036 & -1.621158 \\
\hline C & 7.292386 & -3.340260 & -0.487167 \\
\hline C & 6.631055 & -3.613505 & -2.905808 \\
\hline C & 8.633928 & -2.623214 & -0.616652 \\
\hline $\mathrm{H}$ & 7.277710 & -6.176012 & 0.220450 \\
\hline $\mathrm{H}$ & 5.038561 & -4.810167 & -0.980284 \\
\hline $\mathrm{H}$ & 7.951113 & -4.899617 & -1.806620 \\
\hline r & 6.482996 & -2.613462 & -0.475565 \\
\hline $\mathrm{H}$ & 7.274762 & -3.844448 & 0.469134 \\
\hline $\mathrm{H}$ & 7.320010 & -2.807123 & -3.117837 \\
\hline $\mathrm{H}$ & 6.623211 & -4.269291 & -3.766396 \\
\hline $\mathrm{H}$ & 5.637019 & -3.188977 & -2.808120 \\
\hline $\mathrm{H}$ & 8.795071 & -1.962583 & 0.227469 \\
\hline $\mathrm{H}$ & 9.445061 & -3.340806 & -0.628396 \\
\hline $\mathrm{H}$ & 8.696841 & -2.026493 & -1.519389 \\
\hline$N$ & 6.298920 & -7.327737 & -2.672084 \\
\hline $\mathrm{H}$ & 7.187265 & -7.438561 & -2.234407 \\
\hline$C$ & 5.956066 & -8.199733 & -3.773375 \\
\hline
\end{tabular}




\begin{tabular}{|c|c|c|c|}
\hline $\mathrm{H}$ & 6.769034 & -8.904118 & -3.903412 \\
\hline $\mathrm{H}$ & 5.828574 & -7.647476 & -4.693116 \\
\hline C & 4.662393 & -8.983554 & -3.554003 \\
\hline $\mathrm{O}$ & 3.837293 & -9.067461 & -4.433699 \\
\hline$N$ & 4.519394 & -9.569109 & -2.359664 \\
\hline $\mathrm{H}$ & 5.244453 & -9.496715 & -1.679407 \\
\hline C & 3.302975 & -10.255820 & -1.999113 \\
\hline $\mathrm{H}$ & 3.048342 & -11.015891 & -2.723239 \\
\hline $\mathrm{H}$ & 3.459818 & -10.730217 & -1.037866 \\
\hline C & 2.103907 & -9.316648 & -1.911366 \\
\hline 0 & 1.037264 & -9.649217 & -2.382579 \\
\hline$N$ & 2.312313 & -8.132393 & -1.338193 \\
\hline C & 1.268660 & -7.118196 & -1.434126 \\
\hline C & 0.881652 & -6.762026 & -2.869876 \\
\hline $\mathrm{O}$ & -0.292141 & -6.599506 & -3.130041 \\
\hline$C$ & 1.615422 & -5.769003 & -0.803964 \\
\hline 0 & 1.415214 & -5.759574 & 0.574055 \\
\hline $\mathrm{H}$ & 3.168055 & -7.897418 & -0.886194 \\
\hline $\mathrm{H}$ & 0.360202 & -7.484344 & -0.981226 \\
\hline $\mathrm{H}$ & 2.632605 & -5.505962 & -1.055397 \\
\hline $\mathrm{H}$ & 0.962312 & -5.039529 & -1.263128 \\
\hline $\mathrm{H}$ & 1.043708 & -4.910320 & 0.784478 \\
\hline $\mathrm{N}$ & 1.835210 & -6.535679 & -3.779197 \\
\hline$C$ & 1.471669 & -6.139287 & -5.128694 \\
\hline$C$ & 0.630588 & -7.229391 & -5.778646 \\
\hline 0 & -0.342505 & -6.943901 & -6.447000 \\
\hline$C$ & 2.713707 & -5.764003 & -5.972135 \\
\hline$C$ & 3.222476 & -4.378214 & -5.540076 \\
\hline $\mathrm{C}$ & 2.413898 & -5.800102 & -7.476570 \\
\hline C & 4.575169 & -3.997135 & -6.141756 \\
\hline
\end{tabular}




\begin{tabular}{|c|c|c|c|}
\hline $\mathrm{H}$ & 2.798122 & -6.586043 & -3.524396 \\
\hline $\mathrm{H}$ & 0.810876 & -5.283546 & -5.084106 \\
\hline $\mathrm{H}$ & 3.483417 & -6.501408 & -5.762954 \\
\hline $\mathrm{H}$ & 2.479700 & -3.632667 & -5.822010 \\
\hline $\mathrm{H}$ & 3.305911 & -4.351096 & -4.461110 \\
\hline $\mathrm{H}$ & 3.292544 & -5.516489 & -8.042218 \\
\hline $\mathrm{H}$ & 2.129382 & -6.790237 & -7.811664 \\
\hline $\mathrm{H}$ & 1.611625 & -5.116186 & -7.736233 \\
\hline $\mathrm{H}$ & 4.943666 & -3.080772 & -5.692293 \\
\hline $\mathrm{H}$ & 5.313656 & -4.771277 & -5.956656 \\
\hline $\mathrm{H}$ & 4.522444 & -3.835778 & -7.212755 \\
\hline$N$ & 1.000923 & -8.494082 & -5.569732 \\
\hline $\mathrm{H}$ & 1.817985 & -8.699413 & -5.034681 \\
\hline C & 0.144747 & -9.564730 & -6.008547 \\
\hline $\mathrm{H}$ & 0.029019 & -9.569641 & -7.082582 \\
\hline $\mathrm{H}$ & 0.588444 & -10.502304 & -5.697754 \\
\hline$C$ & -1.256373 & -9.464911 & -5.418391 \\
\hline 0 & -2.228049 & -9.600033 & -6.130526 \\
\hline $\mathrm{N}$ & -1.356876 & -9.285557 & -4.102538 \\
\hline$C$ & -2.656415 & -9.224190 & -3.479530 \\
\hline$C$ & -3.512677 & -8.041625 & -3.941161 \\
\hline 0 & -4.721407 & -8.175808 & -3.960870 \\
\hline$C$ & -2.525815 & -9.184050 & -1.951248 \\
\hline 0 & -3.763628 & -9.393055 & -1.333248 \\
\hline $\mathrm{H}$ & -0.535209 & -9.232612 & -3.537204 \\
\hline $\mathrm{H}$ & -3.232730 & -10.099964 & -3.748030 \\
\hline $\mathrm{H}$ & -1.862556 & -9.978212 & -1.633795 \\
\hline $\mathrm{H}$ & -2.089582 & -8.238142 & -1.644957 \\
\hline $\mathrm{H}$ & -4.456281 & -9.013864 & -1.853179 \\
\hline $\mathrm{N}$ & -2.891715 & -6.903402 & -4.244263 \\
\hline
\end{tabular}




\begin{tabular}{|c|c|c|c|}
\hline C & -3.563364 & -5.738139 & -4.801275 \\
\hline C & -4.210727 & -6.106298 & -6.132385 \\
\hline $\mathrm{O}$ & -5.383739 & -5.889025 & -6.333913 \\
\hline C & -2.491113 & -4.634131 & -4.922000 \\
\hline C & -2.702484 & -3.385350 & -5.773531 \\
\hline C & -3.768780 & -3.131581 & -6.627341 \\
\hline C & -1.660497 & -2.454996 & -5.746473 \\
\hline C & -3.767646 & -2.010518 & -7.452302 \\
\hline C & -1.659993 & -1.335991 & -6.556235 \\
\hline C & -2.717986 & -1.113871 & -7.428484 \\
\hline $\mathrm{H}$ & -1.906912 & -6.851020 & -4.087555 \\
\hline $\mathrm{H}$ & -4.374819 & -5.427063 & -4.157910 \\
\hline $\mathrm{H}$ & -2.245852 & -4.329029 & -3.909846 \\
\hline $\mathrm{H}$ & -1.601929 & -5.107105 & -5.317025 \\
\hline $\mathrm{H}$ & -4.603403 & -3.799488 & -6.682900 \\
\hline $\mathrm{H}$ & -0.826192 & -2.623708 & -5.086201 \\
\hline $\mathrm{H}$ & -4.591350 & -1.860987 & -8.129821 \\
\hline $\mathrm{H}$ & -0.843248 & -0.638905 & -6.509749 \\
\hline $\mathrm{H}$ & -2.712103 & -0.259733 & -8.083100 \\
\hline $\mathrm{N}$ & -3.405487 & -6.641760 & -7.056355 \\
\hline C & -3.936979 & -6.972395 & -8.357133 \\
\hline C & -5.106718 & -7.945818 & -8.250594 \\
\hline 0 & -6.070095 & -7.815618 & -8.960856 \\
\hline C & -2.859536 & -7.568445 & -9.265958 \\
\hline$S$ & -1.587525 & -6.388285 & -9.819378 \\
\hline $\mathrm{H}$ & -2.462330 & -6.871148 & -6.824332 \\
\hline $\mathrm{H}$ & -4.346981 & -6.086459 & -8.821320 \\
\hline $\mathrm{H}$ & -2.384966 & -8.417128 & -8.791314 \\
\hline $\mathrm{H}$ & -3.335488 & -7.916238 & -10.173543 \\
\hline - & -0.898692 & -6.309710 & -8.689150 \\
\hline
\end{tabular}




\begin{tabular}{|c|c|c|c|}
\hline$N$ & -4.985869 & -8.958046 & -7.368054 \\
\hline $\mathrm{H}$ & -4.128558 & -9.114360 & -6.882804 \\
\hline C & -5.986846 & -10.010389 & -7.418493 \\
\hline $\mathrm{H}$ & -6.167157 & -10.322292 & -8.436795 \\
\hline $\mathrm{H}$ & -5.609339 & -10.857030 & -6.856071 \\
\hline C & -7.357892 & -9.648668 & -6.831488 \\
\hline $\mathrm{O}$ & -8.376649 & -10.028113 & -7.341729 \\
\hline$N$ & -7.314713 & -8.977257 & -5.675582 \\
\hline C & -8.539003 & -8.672110 & -4.957869 \\
\hline C & -9.357763 & -7.497931 & -5.510322 \\
\hline 0 & -10.486054 & -7.331305 & -5.116251 \\
\hline C & -8.290639 & -8.487059 & -3.453379 \\
\hline C & -7.526633 & -7.212268 & -3.051675 \\
\hline C & -8.342377 & -6.000544 & -2.687109 \\
\hline $\mathrm{O}$ & -9.406299 & -6.238191 & -1.959825 \\
\hline $\mathrm{H}$ & -9.852561 & -5.406856 & -1.782302 \\
\hline 0 & -8.002612 & -4.878129 & -2.959655 \\
\hline $\mathrm{H}$ & -6.434865 & -8.677541 & -5.312341 \\
\hline $\mathrm{H}$ & -9.200018 & -9.519429 & -5.077325 \\
\hline $\mathrm{H}$ & -9.248341 & -8.512622 & -2.953149 \\
\hline $\mathrm{H}$ & -7.727301 & -9.348214 & -3.115591 \\
\hline $\mathrm{H}$ & -6.947587 & -7.425530 & -2.156894 \\
\hline $\mathrm{H}$ & -6.816306 & -6.900789 & -3.800536 \\
\hline $\mathrm{N}$ & -8.756093 & -6.682539 & -6.390137 \\
\hline$C$ & -9.449810 & -5.618960 & -7.091863 \\
\hline$C$ & -10.091065 & -6.097038 & -8.407010 \\
\hline $\mathrm{H}$ & -9.693586 & -7.027185 & -8.823577 \\
\hline 0 & -10.975582 & -5.499119 & -8.930600 \\
\hline $\mathrm{C}$ & -8.483397 & -4.429212 & -7.306241 \\
\hline O & -8.132258 & -3.873691 & -6.068871 \\
\hline
\end{tabular}




\begin{tabular}{|c|c|c|c|}
\hline C & -9.028285 & -3.333498 & -8.221265 \\
\hline $\mathrm{H}$ & -7.811518 & -6.870849 & -6.645556 \\
\hline $\mathrm{H}$ & -10.273472 & -5.290761 & -6.470283 \\
\hline $\mathrm{H}$ & -7.561081 & -4.811738 & -7.727014 \\
\hline $\mathrm{H}$ & -8.913886 & -3.627592 & -5.589802 \\
\hline $\mathrm{H}$ & -8.319071 & -2.511368 & -8.214035 \\
\hline $\mathrm{H}$ & -9.160252 & -3.668993 & -9.240921 \\
\hline $\mathrm{H}$ & -9.986631 & -2.969701 & -7.861711 \\
\hline $\mathrm{N}$ & -12.792520 & -9.296693 & 3.007091 \\
\hline $\mathrm{H}$ & -13.053843 & -9.901263 & 2.258280 \\
\hline C & -11.560355 & -8.559359 & 2.703028 \\
\hline C & -10.570027 & -8.439517 & 3.891301 \\
\hline O & -9.457962 & -7.997154 & 3.718391 \\
\hline C & -11.820892 & -7.127508 & 2.203349 \\
\hline 0 & -12.545608 & -6.521881 & 3.236258 \\
\hline$C$ & -12.567295 & -7.078403 & 0.876280 \\
\hline $\mathrm{H}$ & -13.541493 & -8.661600 & 3.197896 \\
\hline $\mathrm{H}$ & -11.005970 & -9.102244 & 1.946562 \\
\hline $\mathrm{H}$ & -10.857373 & -6.641604 & 2.077330 \\
\hline $\mathrm{H}$ & -12.617941 & -5.581644 & 3.112478 \\
\hline $\mathrm{H}$ & -12.745930 & -6.043597 & 0.601059 \\
\hline $\mathrm{H}$ & -11.988831 & -7.537610 & 0.080273 \\
\hline $\mathrm{H}$ & -13.529146 & -7.574184 & 0.943486 \\
\hline $\mathrm{N}$ & -11.028025 & -8.859560 & 5.078145 \\
\hline $\mathrm{H}$ & -11.943536 & -9.252037 & 5.076584 \\
\hline$C$ & -10.250171 & -8.822886 & 6.294467 \\
\hline $\mathrm{H}$ & -10.219736 & -7.827204 & 6.713655 \\
\hline $\mathrm{H}$ & -10.729142 & -9.472035 & 7.018694 \\
\hline $\mathrm{C}$ & -8.787581 & -9.251333 & 6.171110 \\
\hline $\mathrm{O}$ & -7.945875 & -8.595872 & 6.731446 \\
\hline
\end{tabular}




\begin{tabular}{|c|c|c|c|}
\hline$N$ & -8.466225 & -10.364368 & 5.484504 \\
\hline $\mathrm{H}$ & -9.182771 & -10.917115 & 5.074428 \\
\hline C & -7.074892 & -10.775374 & 5.369479 \\
\hline $\mathrm{H}$ & -7.049652 & -11.778214 & 4.961470 \\
\hline $\mathrm{H}$ & -6.604193 & -10.792695 & 6.340562 \\
\hline C & -6.214055 & -9.879991 & 4.465363 \\
\hline $\mathrm{O}$ & -5.029202 & -9.781271 & 4.652703 \\
\hline$N$ & -6.882602 & -9.263796 & 3.496967 \\
\hline C & -6.217934 & -8.418763 & 2.524220 \\
\hline C & -6.045081 & -7.003683 & 3.079642 \\
\hline $\mathrm{O}$ & -4.997907 & -6.412749 & 2.961163 \\
\hline C & -6.957957 & -8.386859 & 1.176200 \\
\hline C & -7.235969 & -9.754138 & 0.581079 \\
\hline C & -8.526715 & -10.093774 & 0.196686 \\
\hline C & -6.218362 & -10.682542 & 0.380846 \\
\hline $\mathrm{C}$ & -8.805671 & -11.333570 & -0.360351 \\
\hline $\mathrm{C}$ & -6.494106 & -11.920125 & -0.173474 \\
\hline$C$ & -7.789273 & -12.253869 & -0.540826 \\
\hline $\mathrm{H}$ & -7.870439 & -9.344362 & 3.457341 \\
\hline $\mathrm{H}$ & -5.217959 & -8.796997 & 2.383782 \\
\hline $\mathrm{H}$ & -7.894027 & -7.851871 & 1.290782 \\
\hline $\mathrm{H}$ & -6.340997 & -7.801651 & 0.500442 \\
\hline $\mathrm{H}$ & -9.317761 & -9.372283 & 0.305547 \\
\hline $\mathrm{H}$ & -5.202372 & -10.434136 & 0.625581 \\
\hline $\mathrm{H}$ & -9.810957 & -11.572764 & -0.660207 \\
\hline $\mathrm{H}$ & -5.693086 & -12.620741 & -0.328306 \\
\hline $\mathrm{H}$ & -7.998724 & -13.215255 & -0.974925 \\
\hline $\mathrm{N}$ & -7.104760 & -6.499082 & 3.723628 \\
\hline $\mathrm{C}$ & -7.033740 & -5.332603 & 4.566744 \\
\hline$c$ & -6.031202 & -5.524384 & 5.703650 \\
\hline
\end{tabular}




\begin{tabular}{|c|c|c|c|}
\hline $\mathrm{O}$ & -5.439710 & -4.556084 & 6.129842 \\
\hline C & -8.455233 & -5.038676 & 5.088071 \\
\hline C & -8.627053 & -3.736335 & 5.836996 \\
\hline C & -8.536821 & -2.517990 & 5.169872 \\
\hline C & -8.920092 & -3.732791 & 7.193587 \\
\hline C & -8.728280 & -1.324189 & 5.841369 \\
\hline C & -9.117797 & -2.537846 & 7.873045 \\
\hline C & -9.025320 & -1.331897 & 7.197796 \\
\hline $\mathrm{H}$ & -7.929949 & -7.050286 & 3.780396 \\
\hline $\mathrm{H}$ & -6.664232 & -4.486654 & 4.005673 \\
\hline $\mathrm{H}$ & -9.109033 & -5.041272 & 4.221981 \\
\hline $\mathrm{H}$ & -8.768728 & -5.864689 & 5.715687 \\
\hline $\mathrm{H}$ & -8.313829 & -2.495530 & 4.116702 \\
\hline $\mathrm{H}$ & -8.984862 & -4.664112 & 7.729882 \\
\hline $\mathrm{H}$ & -8.632950 & -0.398373 & 5.303730 \\
\hline $\mathrm{H}$ & -9.319640 & -2.552417 & 8.930318 \\
\hline $\mathrm{H}$ & -9.162084 & -0.406045 & 7.726476 \\
\hline$N$ & -5.822899 & -6.754803 & 6.169766 \\
\hline $\mathrm{H}$ & -6.485046 & -7.467899 & 5.969514 \\
\hline C & -4.817864 & -7.045681 & 7.168560 \\
\hline $\mathrm{H}$ & -4.890140 & -6.389778 & 8.023464 \\
\hline $\mathrm{H}$ & -4.974879 & -8.062398 & 7.504068 \\
\hline C & -3.385961 & -6.921577 & 6.656150 \\
\hline 0 & -2.517866 & -6.560198 & 7.414889 \\
\hline$N$ & -3.153146 & -7.224271 & 5.375235 \\
\hline C & -1.863644 & -6.983860 & 4.755718 \\
\hline C & -1.655728 & -5.486102 & 4.489280 \\
\hline 0 & -0.590666 & -4.976515 & 4.769117 \\
\hline$C$ & -1.692838 & -7.863606 & 3.499384 \\
\hline$C$ & -0.465533 & -7.456785 & 2.683181 \\
\hline
\end{tabular}




\begin{tabular}{|c|c|c|c|}
\hline C & -1.604696 & -9.340698 & 3.899387 \\
\hline $\mathrm{H}$ & -3.890055 & -7.593991 & 4.821243 \\
\hline $\mathrm{H}$ & -1.090600 & -7.231286 & 5.468843 \\
\hline $\mathrm{H}$ & -2.572701 & -7.722240 & 2.876241 \\
\hline $\mathrm{H}$ & -0.336120 & -8.135634 & 1.847830 \\
\hline $\mathrm{H}$ & -0.550127 & -6.460379 & 2.273075 \\
\hline $\mathrm{H}$ & 0.439815 & -7.491125 & 3.281370 \\
\hline $\mathrm{H}$ & -1.538603 & -9.961482 & 3.011074 \\
\hline $\mathrm{H}$ & -0.711841 & -9.520078 & 4.493623 \\
\hline $\mathrm{H}$ & -2.465331 & -9.667028 & 4.467652 \\
\hline$N$ & -2.674565 & -4.780348 & 3.984062 \\
\hline C & -2.579754 & -3.338328 & 3.793979 \\
\hline C & -2.297768 & -2.639184 & 5.130320 \\
\hline $\mathrm{O}$ & -1.428120 & -1.802048 & 5.221675 \\
\hline C & -3.884007 & -2.768408 & 3.212832 \\
\hline C & -4.263978 & -3.193978 & 1.783063 \\
\hline C & -3.597047 & -2.366651 & 0.677111 \\
\hline $\mathrm{O}$ & -2.381980 & -2.303581 & 0.662580 \\
\hline $\mathrm{O}$ & -4.391192 & -1.825138 & -0.152484 \\
\hline $\mathrm{H}$ & -3.510431 & -5.253999 & 3.718299 \\
\hline $\mathrm{H}$ & -1.756396 & -3.098210 & 3.139031 \\
\hline $\mathrm{H}$ & -4.693038 & -3.035909 & 3.883987 \\
\hline $\mathrm{H}$ & -3.805747 & -1.686202 & 3.251008 \\
\hline $\mathrm{H}$ & -4.011198 & -4.236358 & 1.616536 \\
\hline $\mathrm{H}$ & -5.339602 & -3.104761 & 1.679518 \\
\hline$N$ & -3.095464 & -2.963400 & 6.160954 \\
\hline C & -2.939464 & -2.327720 & 7.447609 \\
\hline C & -1.754562 & -2.850886 & 8.242134 \\
\hline $\mathrm{O}$ & -1.241009 & -2.100527 & 9.045434 \\
\hline C & -4.197170 & -2.310161 & 8.32888 \\
\hline
\end{tabular}




\begin{tabular}{|c|c|c|c|}
\hline $\mathrm{O}$ & -4.620339 & -3.597947 & 8.675449 \\
\hline C & -5.321121 & -1.479793 & 7.724471 \\
\hline $\mathrm{H}$ & -3.801935 & -3.650884 & 6.032435 \\
\hline $\mathrm{H}$ & -2.679131 & -1.297475 & 7.270561 \\
\hline $\mathrm{H}$ & -3.880550 & -1.845169 & 9.253913 \\
\hline $\mathrm{H}$ & -5.070986 & -3.986002 & 7.940195 \\
\hline $\mathrm{H}$ & -6.152630 & -1.447313 & 8.417708 \\
\hline $\mathrm{H}$ & -4.991918 & -0.462595 & 7.536520 \\
\hline $\mathrm{H}$ & -5.675170 & -1.902275 & 6.792044 \\
\hline$N$ & -1.229334 & -4.041802 & 7.968335 \\
\hline $\mathrm{H}$ & -1.776797 & -4.742324 & 7.521109 \\
\hline C & -0.048500 & -4.466073 & 8.670904 \\
\hline $\mathrm{H}$ & -0.108968 & -4.261011 & 9.729127 \\
\hline $\mathrm{H}$ & 0.068784 & -5.532345 & 8.519936 \\
\hline C & 1.194551 & -3.764911 & 8.148284 \\
\hline $\mathrm{O}$ & 2.074951 & -3.407444 & 8.897363 \\
\hline$N$ & 1.264785 & -3.600261 & 6.813297 \\
\hline C & 2.442242 & -3.008798 & 6.237847 \\
\hline C & 2.461564 & -1.482093 & 6.254595 \\
\hline $\mathrm{O}$ & 3.549102 & -0.950088 & 6.304727 \\
\hline C & 2.796441 & -3.561288 & 4.858146 \\
\hline$S$ & 1.856644 & -2.927833 & 3.444988 \\
\hline $\mathrm{H}$ & 0.516743 & -3.920869 & 6.234324 \\
\hline $\mathrm{H}$ & 3.266645 & -3.264517 & 6.887056 \\
\hline $\mathrm{H}$ & 3.820492 & -3.279272 & 4.652281 \\
\hline $\mathrm{H}$ & 2.740558 & -4.641809 & 4.885742 \\
\hline $\mathrm{H}$ & 0.711327 & -3.553972 & 3.667683 \\
\hline$N$ & 1.323449 & -0.776812 & 6.279895 \\
\hline C & 1.425629 & 0.655966 & 6.506219 \\
\hline C & 1.897655 & 0.944464 & 7.933109 \\
\hline
\end{tabular}




\begin{tabular}{|c|c|c|c|}
\hline $\mathrm{O}$ & 2.465547 & 1.978221 & 8.191566 \\
\hline C & 0.159048 & 1.462605 & 6.169105 \\
\hline C & -1.005324 & 1.198233 & 7.135269 \\
\hline C & -0.200326 & 1.289570 & 4.691505 \\
\hline C & -2.267635 & 2.004817 & 6.828855 \\
\hline $\mathrm{H}$ & 0.448196 & -1.213816 & 6.077260 \\
\hline $\mathrm{H}$ & 2.219718 & 1.031980 & 5.878836 \\
\hline $\mathrm{H}$ & 0.461655 & 2.493324 & 6.321192 \\
\hline $\mathrm{H}$ & -1.245379 & 0.144317 & 7.142360 \\
\hline $\mathrm{H}$ & -0.682194 & 1.443746 & 8.142196 \\
\hline $\mathrm{H}$ & -0.929570 & 2.028536 & 4.385489 \\
\hline $\mathrm{H}$ & 0.676787 & 1.423126 & 4.064750 \\
\hline $\mathrm{H}$ & -0.604264 & 0.307850 & 4.486027 \\
\hline $\mathrm{H}$ & -2.984983 & 1.902927 & 7.637671 \\
\hline $\mathrm{H}$ & -2.048709 & 3.063745 & 6.715263 \\
\hline $\mathrm{H}$ & -2.751204 & 1.664480 & 5.919709 \\
\hline $\mathrm{N}$ & 1.675654 & -0.021225 & 8.823611 \\
\hline $\mathrm{H}$ & 1.081328 & -0.782670 & 8.593150 \\
\hline$C$ & 2.100401 & 0.120562 & 10.198982 \\
\hline $\mathrm{H}$ & 1.591164 & -0.634630 & 10.785030 \\
\hline $\mathrm{H}$ & 1.857308 & 1.098667 & 10.586244 \\
\hline$C$ & 3.606223 & -0.053001 & 10.353721 \\
\hline 0 & 4.268511 & 0.708011 & 11.006098 \\
\hline $\mathrm{N}$ & 4.143030 & -1.107136 & 9.700757 \\
\hline $\mathrm{H}$ & 3.547921 & -1.775669 & 9.263300 \\
\hline$C$ & 5.570377 & -1.269428 & 9.654130 \\
\hline $\mathrm{H}$ & 5.793819 & -2.232706 & 9.209312 \\
\hline $\mathrm{H}$ & 6.006571 & -1.240482 & 10.642328 \\
\hline$C$ & 6.284386 & -0.191779 & 8.842046 \\
\hline $\mathrm{O}$ & 7.419465 & 0.119159 & 9.118286 \\
\hline
\end{tabular}




\begin{tabular}{|c|c|c|c|}
\hline $\mathrm{N}$ & 5.619218 & 0.337787 & 7.816479 \\
\hline$C$ & 6.241448 & 1.489915 & 7.202196 \\
\hline$C$ & 6.228486 & 2.682421 & 8.168718 \\
\hline $\mathrm{H}$ & 5.777148 & 2.503484 & 9.145550 \\
\hline 0 & 6.691157 & 3.736387 & 7.864473 \\
\hline$C$ & 5.642712 & 1.823159 & 5.839548 \\
\hline$C$ & 5.811401 & 0.738469 & 4.785865 \\
\hline$C$ & 6.701032 & -0.324780 & 4.914108 \\
\hline$C$ & 5.021215 & 0.789274 & 3.640030 \\
\hline$C$ & 6.781269 & -1.309513 & 3.938649 \\
\hline$C$ & 5.092867 & -0.192543 & 2.667386 \\
\hline$C$ & 5.976243 & -1.252944 & 2.814673 \\
\hline $\mathrm{H}$ & 4.710941 & 0.029664 & 7.550120 \\
\hline $\mathrm{H}$ & 7.295865 & 1.276196 & 7.078122 \\
\hline $\mathrm{H}$ & 4.585226 & 2.030496 & 5.953273 \\
\hline $\mathrm{H}$ & 6.109289 & 2.738601 & 5.490140 \\
\hline $\mathrm{H}$ & 7.299068 & -0.426662 & 5.800375 \\
\hline $\mathrm{H}$ & 4.319450 & 1.596570 & 3.522113 \\
\hline $\mathrm{H}$ & 7.458747 & -2.135893 & 4.074043 \\
\hline $\mathrm{H}$ & 4.445774 & -0.137842 & 1.809074 \\
\hline $\mathrm{H}$ & 6.038094 & -2.024940 & 2.068497 \\
\hline $\mathrm{N}$ & 12.605068 & 9.645440 & -0.989737 \\
\hline $\mathrm{H}$ & 11.813281 & 10.016706 & -1.479100 \\
\hline$C$ & 12.309752 & 8.295709 & -0.500458 \\
\hline$C$ & 11.349969 & 8.484824 & 0.672897 \\
\hline 0 & 10.360067 & 7.804504 & 0.804190 \\
\hline$C$ & 13.584182 & 7.561164 & -0.070729 \\
\hline$C$ & 14.607928 & 7.340736 & -1.190260 \\
\hline$S$ & 13.887607 & 6.422022 & -2.582409 \\
\hline C & 15.327726 & 6.262514 & -3.664527 \\
\hline
\end{tabular}




\begin{tabular}{|c|c|c|c|}
\hline $\mathrm{H}$ & 13.348016 & 9.626479 & -1.659191 \\
\hline $\mathrm{H}$ & 11.773230 & 7.689484 & -1.219885 \\
\hline $\mathrm{H}$ & 14.067469 & 8.110838 & 0.731909 \\
\hline $\mathrm{H}$ & 13.285673 & 6.598935 & 0.325657 \\
\hline $\mathrm{H}$ & 15.015158 & 8.283148 & -1.540702 \\
\hline $\mathrm{H}$ & 15.440854 & 6.777465 & -0.784009 \\
\hline $\mathrm{H}$ & 14.996658 & 5.702644 & -4.526420 \\
\hline $\mathrm{H}$ & 15.686405 & 7.234046 & -3.979646 \\
\hline $\mathrm{H}$ & 16.124669 & 5.716710 & -3.176492 \\
\hline$N$ & 11.623863 & 9.503381 & 1.512871 \\
\hline C & 10.793076 & 9.760309 & 2.668178 \\
\hline C & 9.384915 & 10.232748 & 2.279268 \\
\hline O & 8.429509 & 9.901988 & 2.930855 \\
\hline C & 11.422586 & 10.805418 & 3.589993 \\
\hline C & 12.699208 & 10.339467 & 4.271024 \\
\hline 0 & 13.069918 & 9.191107 & 4.233533 \\
\hline $\mathrm{N}$ & 13.374167 & 11.298161 & 4.919474 \\
\hline $\mathrm{H}$ & 12.420720 & 10.062194 & . 1.308047 \\
\hline $\mathrm{H}$ & 10.645917 & 8.842435 & 3.216703 \\
\hline $\mathrm{H}$ & 11.619980 & 11.729276 & 3.052357 \\
\hline $\mathrm{H}$ & 10.707138 & 11.045996 & 4.371178 \\
\hline $\mathrm{H}$ & 14.184737 & 11.049682 & 5.439106 \\
\hline $\mathrm{H}$ & 13.036382 & 12.228584 & 4.994210 \\
\hline $\mathrm{N}$ & 9.285172 & 11.051262 & 1.210542 \\
\hline $\mathrm{H}$ & 10.104064 & 11.267946 & 0.692782 \\
\hline$C$ & 7.991434 & 11.480001 & 0.715991 \\
\hline $\mathrm{H}$ & 8.140196 & 12.261200 & -0.018629 \\
\hline $\mathrm{H}$ & 7.389548 & 11.876449 & 1.518812 \\
\hline C & 7.178859 & 10.360599 & 0.062947 \\
\hline 0 & 5.981168 & 10.454168 & 0.01610 \\
\hline
\end{tabular}




\begin{tabular}{|c|c|c|c|}
\hline$N$ & 7.863887 & 9.332233 & -0.478635 \\
\hline C & 7.182932 & 8.185716 & -1.058783 \\
\hline C & 6.598702 & 7.270955 & 0.025460 \\
\hline $\mathrm{O}$ & 5.467799 & 6.862709 & -0.085760 \\
\hline C & 8.101138 & 7.443713 & -2.056301 \\
\hline C & 8.428034 & 8.359684 & -3.250458 \\
\hline C & 7.457238 & 6.131675 & -2.518701 \\
\hline C & 9.532497 & 7.837674 & -4.171596 \\
\hline $\mathrm{H}$ & 8.818953 & 9.217199 & -0.236022 \\
\hline $\mathrm{H}$ & 6.315492 & 8.554139 & -1.586024 \\
\hline $\mathrm{H}$ & 9.026987 & 7.200532 & -1.537622 \\
\hline $\mathrm{H}$ & 7.518860 & 8.516285 & -3.827407 \\
\hline $\mathrm{H}$ & 8.721115 & 9.338268 & -2.882036 \\
\hline $\mathrm{H}$ & 8.097622 & 5.627863 & -3.230882 \\
\hline $\mathrm{H}$ & 7.291596 & 5.451779 & -1.691505 \\
\hline $\mathrm{H}$ & 6.497885 & 6.307560 & -2.994655 \\
\hline $\mathrm{H}$ & 9.744433 & 8.562158 & -4.951387 \\
\hline $\mathrm{H}$ & 10.456465 & 7.660635 & -3.627844 \\
\hline $\mathrm{H}$ & 9.260662 & 6.909637 & -4.660775 \\
\hline $\mathrm{N}$ & 7.357108 & 6.986982 & 1.098993 \\
\hline$C$ & 6.815976 & 6.238775 & 2.224872 \\
\hline$C$ & 5.549349 & 6.898414 & 2.777604 \\
\hline O & 4.562334 & 6.244312 & 3.014203 \\
\hline$C$ & 7.895158 & 6.091733 & 3.314277 \\
\hline$C$ & 8.805886 & 4.897444 & 3.029837 \\
\hline 0 & 8.229648 & 3.797016 & 3.101866 \\
\hline 0 & 9.997757 & 5.066103 & 2.752565 \\
\hline $\mathrm{H}$ & 8.312249 & 7.269571 & 1.129777 \\
\hline $\mathrm{H}$ & 6.491197 & 5.258292 & 1.910871 \\
\hline $\mathrm{H}$ & 8.461856 & 7.008758 & 3.413910 \\
\hline
\end{tabular}




\begin{tabular}{|c|c|c|c|}
\hline $\mathrm{H}$ & 7.406423 & 5.881754 & 4.258845 \\
\hline$N$ & 5.585351 & 8.228790 & 2.983940 \\
\hline $\mathrm{H}$ & 6.438384 & 8.729912 & 2.867563 \\
\hline C & 4.422178 & 8.916159 & 3.493140 \\
\hline $\mathrm{H}$ & 4.682273 & 9.956718 & 3.645609 \\
\hline $\mathrm{H}$ & 4.101804 & 8.509010 & 4.440327 \\
\hline C & 3.220967 & 8.882064 & 2.553035 \\
\hline $\mathrm{O}$ & 2.097607 & 9.015018 & 2.981958 \\
\hline$N$ & 3.504285 & 8.737718 & 1.255799 \\
\hline $\mathrm{H}$ & 4.453831 & 8.702315 & 0.973429 \\
\hline C & 2.521915 & 8.841264 & 0.221505 \\
\hline $\mathrm{H}$ & 3.033992 & 9.074810 & -0.703424 \\
\hline $\mathrm{H}$ & 1.837858 & 9.653799 & 0.426236 \\
\hline C & 1.621484 & 7.623962 & -0.042825 \\
\hline $\mathrm{O}$ & 0.586656 & 7.778614 & -0.634227 \\
\hline$N$ & 2.071079 & 6.463016 & 0.463371 \\
\hline C & 1.251511 & 5.281579 & 0.615059 \\
\hline C & 0.451563 & 5.305277 & 1.922259 \\
\hline $\mathrm{O}$ & -0.730932 & 5.065495 & 1.914218 \\
\hline C & 2.105844 & 4.017403 & 0.527039 \\
\hline $\mathrm{H}$ & 2.978515 & 6.468890 & 0.876635 \\
\hline $\mathrm{H}$ & 0.510918 & 5.275409 & -0.167321 \\
\hline $\mathrm{H}$ & 1.486619 & 3.138632 & 0.668164 \\
\hline $\mathrm{H}$ & 2.573373 & 3.965514 & -0.449346 \\
\hline $\mathrm{H}$ & 2.887857 & 4.014286 & 1.279726 \\
\hline$N$ & 1.109127 & 5.577553 & 3.070463 \\
\hline C & 0.415708 & 5.379089 & 4.331647 \\
\hline C & -0.707157 & 6.392744 & 4.526066 \\
\hline $\mathrm{O}$ & -1.733999 & 6.072899 & 5.085785 \\
\hline$C$ & 1.361997 & 5.312196 & 5.557357 \\
\hline
\end{tabular}




\begin{tabular}{|c|c|c|c|}
\hline C & 1.925978 & 6.667971 & 6.011986 \\
\hline C & 2.473662 & 4.280871 & 5.315631 \\
\hline C & 2.828038 & 6.571785 & 7.244499 \\
\hline $\mathrm{H}$ & 2.090427 & 5.746642 & 3.063712 \\
\hline $\mathrm{H}$ & -0.099334 & 4.430756 & 4.278367 \\
\hline $\mathrm{H}$ & 0.731278 & 4.942840 & 6.361221 \\
\hline $\mathrm{H}$ & 2.474118 & 7.127350 & 5.195421 \\
\hline $\mathrm{H}$ & 1.105070 & 7.338854 & 6.245244 \\
\hline $\mathrm{H}$ & 2.860202 & 3.919252 & 6.258487 \\
\hline $\mathrm{H}$ & 2.095894 & 3.424283 & 4.768474 \\
\hline $\mathrm{H}$ & 3.296118 & 4.703643 & 4.748276 \\
\hline $\mathrm{H}$ & 3.099764 & 7.565200 & 7.588960 \\
\hline $\mathrm{H}$ & 2.322599 & 6.066935 & 8.062302 \\
\hline $\mathrm{H}$ & 3.745880 & 6.031953 & 7.043023 \\
\hline$N$ & -0.506922 & 7.641164 & 4.076779 \\
\hline $\mathrm{H}$ & 0.371603 & 7.899590 & 3.680796 \\
\hline C & -1.483815 & 8.662817 & 4.341826 \\
\hline $\mathrm{H}$ & -1.089445 & 9.610803 & 3.995981 \\
\hline $\mathrm{H}$ & -1.689393 & 8.750272 & 5.399407 \\
\hline C & -2.837595 & 8.406077 & 3.688540 \\
\hline $\mathrm{O}$ & -3.840913 & 8.610537 & 4.331020 \\
\hline$N$ & -2.877489 & 7.935289 & 2.428242 \\
\hline C & -4.217339 & 7.889276 & 1.842381 \\
\hline C & -4.972031 & 6.625367 & 2.206688 \\
\hline $\mathrm{O}$ & -6.184175 & 6.618353 & 2.162603 \\
\hline C & -3.995910 & 8.135921 & 0.345216 \\
\hline C & -2.726562 & 8.993938 & 0.360502 \\
\hline C & -1.877307 & 8.302582 & 1.414507 \\
\hline $\mathrm{H}$ & -4.816047 & 8.690752 & 2.250191 \\
\hline $\mathrm{H}$ & -3.827093 & 7.204981 & -0.182070 \\
\hline
\end{tabular}




\begin{tabular}{|c|c|c|c|}
\hline $\mathrm{H}$ & -4.853231 & 8.624982 & -0.100236 \\
\hline $\mathrm{H}$ & -2.214433 & 9.060076 & -0.581439 \\
\hline $\mathrm{H}$ & -2.971845 & 10.002564 & 0.685970 \\
\hline $\mathrm{H}$ & -1.402809 & 7.416646 & 1.029578 \\
\hline $\mathrm{H}$ & -1.111457 & 8.942579 & 1.821539 \\
\hline$N$ & -4.245826 & 5.567959 & 2.582585 \\
\hline C & -4.881610 & 4.372656 & 3.097316 \\
\hline$C$ & -5.515294 & 4.625903 & 4.471481 \\
\hline 0 & -6.575699 & 4.116845 & 4.750087 \\
\hline$C$ & -3.893615 & 3.209489 & 3.203628 \\
\hline$C$ & -3.364350 & 2.621443 & 1.907506 \\
\hline$C$ & -2.656125 & 1.425261 & 1.993822 \\
\hline$C$ & -3.505375 & 3.212799 & 0.662206 \\
\hline$C$ & -2.062839 & 0.856829 & 0.885432 \\
\hline$C$ & -2.910216 & 2.646449 & -0.456530 \\
\hline$C$ & -2.177527 & 1.481074 & -0.347383 \\
\hline $\mathrm{H}$ & -1.714579 & 1.040843 & -1.212230 \\
\hline $\mathrm{H}$ & -3.250906 & 5.643276 & 2.602052 \\
\hline $\mathrm{H}$ & -5.705185 & 4.103909 & 2.451687 \\
\hline $\mathrm{H}$ & -3.049544 & 3.529628 & 3.808715 \\
\hline $\mathrm{H}$ & -4.389473 & 2.421932 & 3.761846 \\
\hline $\mathrm{H}$ & -2.566238 & 0.930907 & 2.944315 \\
\hline $\mathrm{H}$ & -4.058972 & 4.124440 & 0.548929 \\
\hline $\mathrm{H}$ & -1.558202 & -0.085400 & 0.971031 \\
\hline $\mathrm{H}$ & -3.024736 & 3.128425 & -1.411784 \\
\hline$N$ & -4.828447 & 5.408443 & 5.322804 \\
\hline $\mathrm{H}$ & -3.910601 & 5.718047 & 5.084171 \\
\hline$C$ & -5.352588 & 5.769196 & 6.619635 \\
\hline $\mathrm{H}$ & -4.530968 & 6.132360 & 7.225434 \\
\hline 1 & -5.790440 & 4.914144 & 7.111073 \\
\hline
\end{tabular}




\begin{tabular}{|c|c|c|c|}
\hline$C$ & -6.436971 & 6.846135 & 6.574466 \\
\hline O & -7.383478 & 6.805059 & 7.324530 \\
\hline $\mathrm{N}$ & -6.296072 & 7.791340 & 5.640484 \\
\hline $\mathrm{H}$ & -5.437689 & 7.845459 & 5.138067 \\
\hline$C$ & -7.221222 & 8.920186 & 5.453602 \\
\hline $\mathrm{H}$ & -6.753342 & 9.600000 & 4.752853 \\
\hline $\mathrm{H}$ & -7.394675 & 9.441931 & 6.383607 \\
\hline$C$ & -8.586084 & 8.529464 & 4.920432 \\
\hline $\mathrm{O}$ & -9.576464 & 9.101216 & 5.306484 \\
\hline $\mathrm{N}$ & -8.632744 & 7.543786 & 4.004680 \\
\hline$C$ & -9.900814 & 7.173284 & 3.419814 \\
\hline$C$ & -10.818979 & 6.437427 & 4.389095 \\
\hline $\mathrm{O}$ & -11.966881 & 6.229851 & 4.052049 \\
\hline$C$ & -9.716080 & 6.341626 & 2.129525 \\
\hline $\mathrm{O}$ & -10.890552 & 6.401399 & 1.360717 \\
\hline$C$ & -9.302375 & 4.894493 & 2.373394 \\
\hline $\mathrm{H}$ & -7.785100 & 7.150718 & 3.652281 \\
\hline $\mathrm{H}$ & -10.441889 & 8.070021 & 3.148137 \\
\hline $\mathrm{H}$ & -8.948466 & 6.838128 & 1.549510 \\
\hline $\mathrm{H}$ & -11.621970 & 6.175429 & 1.920630 \\
\hline $\mathrm{H}$ & -9.084141 & 4.418316 & 1.424093 \\
\hline $\mathrm{H}$ & -8.422336 & 4.821232 & 2.999236 \\
\hline $\mathrm{H}$ & -10.109587 & 4.340743 & 2.842202 \\
\hline $\mathrm{N}$ & -10.294540 & 6.001277 & 5.537721 \\
\hline$C$ & -11.131185 & 5.393282 & 6.554142 \\
\hline$C$ & -12.169270 & 6.356053 & 7.134058 \\
\hline O & -13.210485 & 5.916253 & 7.563802 \\
\hline$C$ & -10.282718 & 4.839370 & 7.707426 \\
\hline$C$ & -9.289711 & 3.732198 & 7.325051 \\
\hline C & -8.476636 & 3.335915 & 8.560948 \\
\hline
\end{tabular}




\begin{tabular}{|c|c|c|c|}
\hline C & -9.975299 & 2.509395 & 6.712118 \\
\hline $\mathrm{H}$ & -9.346560 & 6.206947 & 5.756711 \\
\hline $\mathrm{H}$ & -11.710438 & 4.603654 & 6.103268 \\
\hline $\mathrm{H}$ & -9.734684 & 5.659674 & 8.160453 \\
\hline $\mathrm{H}$ & -10.974572 & 4.462483 & 8.455547 \\
\hline $\mathrm{H}$ & -8.592127 & 4.120989 & 6.591416 \\
\hline $\mathrm{H}$ & -7.733358 & 2.584081 & 8.310923 \\
\hline $\mathrm{H}$ & -7.957680 & 4.194433 & 8.973752 \\
\hline $\mathrm{H}$ & -9.116854 & 2.925100 & 9.339115 \\
\hline $\mathrm{H}$ & -9.237375 & 1.747953 & 6.484081 \\
\hline $\mathrm{H}$ & -10.703365 & 2.081032 & 7.399252 \\
\hline $\mathrm{H}$ & -10.488533 & 2.741816 & 5.785849 \\
\hline$N$ & -11.837511 & 7.654650 & 7.216421 \\
\hline C & -12.776032 & 8.614181 & 7.720921 \\
\hline C & -13.819828 & 9.054470 & 6.717451 \\
\hline $\mathrm{H}$ & -14.558364 & 9.757156 & 7.124778 \\
\hline $\mathrm{O}$ & -13.866038 & 8.706826 & 5.585445 \\
\hline $\mathrm{H}$ & -11.048320 & 7.996576 & 6.713104 \\
\hline $\mathrm{H}$ & -13.291339 & 8.208116 & 8.583451 \\
\hline $\mathrm{H}$ & -12.241010 & 9.499657 & 8.052314 \\
\hline$N$ & -11.987120 & 10.578869 & -2.764592 \\
\hline $\mathrm{H}$ & -11.139682 & 11.102772 & -2.676643 \\
\hline C & -11.737367 & 9.147430 & -2.773520 \\
\hline C & -11.361431 & 8.791069 & -4.206373 \\
\hline $\mathrm{O}$ & -10.359481 & 8.165733 & -4.477956 \\
\hline C & -12.975329 & 8.367330 & -2.266495 \\
\hline C & -13.129235 & 8.550420 & -0.742548 \\
\hline C & -12.898791 & 6.881089 & -2.631403 \\
\hline C & -14.480890 & 8.096924 & -0.188093 \\
\hline $\mathrm{H}$ & -12.574648 & 10.851296 & -2.00462 \\
\hline
\end{tabular}




\begin{tabular}{|c|c|c|c|}
\hline $\mathrm{H}$ & -10.882923 & 8.861425 & -2.166123 \\
\hline $\mathrm{H}$ & -13.846959 & 8.798736 & -2.758167 \\
\hline $\mathrm{H}$ & -12.330786 & 8.012691 & -0.239519 \\
\hline $\mathrm{H}$ & -13.000741 & 9.596235 & -0.476320 \\
\hline $\mathrm{H}$ & -13.783720 & 6.359803 & -2.289763 \\
\hline $\mathrm{H}$ & -12.831628 & 6.724392 & -3.703062 \\
\hline $\mathrm{H}$ & -12.038266 & 6.414534 & -2.164161 \\
\hline $\mathrm{H}$ & -14.539261 & 8.311762 & 0.873026 \\
\hline $\mathrm{H}$ & -15.302950 & 8.612982 & -0.676992 \\
\hline $\mathrm{H}$ & -14.636770 & 7.030777 & -0.309175 \\
\hline$N$ & -12.192658 & 9.240443 & -5.161369 \\
\hline $\mathrm{H}$ & -12.937053 & 9.837603 & -4.884379 \\
\hline C & -12.011414 & 8.884787 & -6.567478 \\
\hline $\mathrm{H}$ & -12.085161 & 7.816396 & -6.710172 \\
\hline $\mathrm{H}$ & -12.792692 & 9.368464 & -7.140618 \\
\hline C & -10.649820 & 9.302759 & -7.126326 \\
\hline $\mathrm{O}$ & -10.006084 & 8.572941 & -7.824409 \\
\hline$N$ & -10.257651 & 10.558633 & $3-6.809092$ \\
\hline $\mathrm{H}$ & -10.833141 & 11.091346 & $6-6.200194$ \\
\hline C & -8.953654 & 11.108373 & -7.195869 \\
\hline $\mathrm{H}$ & -8.829082 & 11.038793 & -8.266124 \\
\hline $\mathrm{H}$ & -8.936298 & 12.151967 & -6.907837 \\
\hline C & -7.736646 & 10.405276 & -6.576984 \\
\hline $\mathrm{O}$ & -6.847009 & 9.949786 & -7.240820 \\
\hline$N$ & -7.700741 & 10.405557 & -5.227497 \\
\hline $\mathrm{H}$ & -8.491737 & 10.745233 & -4.734724 \\
\hline C & -6.528678 & 9.976769 & -4.440791 \\
\hline $\mathrm{H}$ & -5.631644 & 10.397924 & -4.866069 \\
\hline $\mathrm{H}$ & -6.650898 & 10.354226 & -3.432867 \\
\hline C & -6.298977 & 8.478461 & -4.356947 \\
\hline
\end{tabular}




\begin{tabular}{|c|c|c|c|}
\hline $\mathrm{O}$ & -5.160100 & 8.079944 & -4.429601 \\
\hline$N$ & -7.321731 & 7.634119 & -4.128670 \\
\hline C & -6.973982 & 6.274899 & -3.734636 \\
\hline C & -6.414448 & 5.452652 & -4.888680 \\
\hline $\mathrm{O}$ & -5.629780 & 4.563804 & -4.654157 \\
\hline C & -8.059456 & 5.493070 & -2.955784 \\
\hline$C$ & -9.261839 & 5.034157 & -3.798194 \\
\hline C & -8.469207 & 6.271075 & -1.700925 \\
\hline C & -10.110615 & 3.962412 & -3.108893 \\
\hline $\mathrm{H}$ & -8.270205 & 7.950115 & -4.120408 \\
\hline $\mathrm{H}$ & -6.129581 & 6.350490 & -3.065266 \\
\hline $\mathrm{H}$ & -7.533480 & 4.599086 & -2.630608 \\
\hline $\mathrm{H}$ & -9.881408 & 5.882869 & -4.059661 \\
\hline $\mathrm{H}$ & -8.899814 & 4.623037 & -4.738209 \\
\hline $\mathrm{H}$ & -9.115743 & 5.680862 & -1.065616 \\
\hline $\mathrm{H}$ & -7.600095 & 6.543018 & -1.110208 \\
\hline $\mathrm{H}$ & -9.005529 & 7.178328 & -1.954826 \\
\hline $\mathrm{H}$ & -10.923071 & 3.648057 & -3.757542 \\
\hline $\mathrm{H}$ & -9.521107 & 3.082391 & -2.866881 \\
\hline $\mathrm{H}$ & -10.554718 & 4.320020 & -2.188119 \\
\hline$N$ & -6.822678 & 5.718329 & -6.146765 \\
\hline C & -6.203981 & 5.029879 & -7.261290 \\
\hline C & -4.813200 & 5.571762 & -7.606212 \\
\hline $\mathrm{O}$ & -3.964984 & 4.810359 & -8.007131 \\
\hline C & -7.119648 & 5.009309 & -8.492052 \\
\hline C & -8.344139 & 4.097115 & -8.336593 \\
\hline C & -7.978738 & 2.611117 & -8.268580 \\
\hline$N$ & -9.148160 & 1.732216 & -8.268408 \\
\hline C & -9.728394 & 1.155628 & -7.219459 \\
\hline $\mathrm{N}$ & -9.398739 & 1.477169 & -5.984088 \\
\hline
\end{tabular}




\begin{tabular}{|c|c|c|c|}
\hline$N$ & -10.665539 & 0.247291 & -7.435587 \\
\hline $\mathrm{H}$ & -7.445531 & 6.479320 & -6.305991 \\
\hline $\mathrm{H}$ & -5.993971 & 4.022517 & -6.940476 \\
\hline $\mathrm{H}$ & -7.456961 & 6.017337 & -8.715548 \\
\hline $\mathrm{H}$ & -6.530497 & 4.682666 & -9.343524 \\
\hline $\mathrm{H}$ & -8.919322 & 4.380364 & -7.460042 \\
\hline $\mathrm{H}$ & -8.995436 & 4.250949 & -9.191741 \\
\hline $\mathrm{H}$ & -7.384030 & 2.334928 & -9.130720 \\
\hline $\mathrm{H}$ & -7.378081 & 2.374407 & -7.401333 \\
\hline $\mathrm{H}$ & -9.556507 & 1.538081 & -9.154444 \\
\hline $\mathrm{H}$ & -8.800778 & 2.247705 & -5.804070 \\
\hline $\mathrm{H}$ & -9.709367 & 0.939426 & -5.188834 \\
\hline $\mathrm{H}$ & -10.769877 & -0.153603 & -8.338713 \\
\hline $\mathrm{H}$ & -11.234283 & -0.139405 & -6.704079 \\
\hline$N$ & -4.599878 & 6.879766 & -7.441561 \\
\hline $\mathrm{H}$ & -5.334680 & 7.474332 & -7.135570 \\
\hline C & -3.292582 & 7.493362 & -7.719931 \\
\hline $\mathrm{H}$ & -2.967402 & 7.266071 & -8.723802 \\
\hline $\mathrm{H}$ & -3.402951 & 8.564609 & -7.614227 \\
\hline C & -2.224332 & 6.995185 & -6.760772 \\
\hline $\mathrm{O}$ & -1.100230 & 6.772264 & -7.134219 \\
\hline$N$ & -2.637167 & 6.848483 & -5.496649 \\
\hline C & -1.742436 & 6.511690 & -4.416874 \\
\hline C & -1.466378 & 5.021090 & -4.331742 \\
\hline $\mathrm{O}$ & -0.336995 & 4.630764 & -4.159685 \\
\hline C & -2.305604 & 7.082488 & -3.110315 \\
\hline C & -1.694144 & 6.358794 & -1.914519 \\
\hline C & -2.064747 & 8.598925 & -3.116508 \\
\hline $\mathrm{H}$ & -3.553638 & 7.155104 & -5.256772 \\
\hline $\mathrm{H}$ & -0.772057 & 6.948699 & -4.607607 \\
\hline
\end{tabular}




\begin{tabular}{|c|c|c|c|}
\hline $\mathrm{H}$ & -3.375847 & 6.904101 & -3.110124 \\
\hline $\mathrm{H}$ & -1.905998 & 6.870274 & -0.995136 \\
\hline $\mathrm{H}$ & -2.083374 & 5.351210 & -1.830057 \\
\hline $\mathrm{H}$ & -0.621861 & 6.308243 & -2.001031 \\
\hline $\mathrm{H}$ & -2.781763 & 9.114687 & -2.493088 \\
\hline $\mathrm{H}$ & -1.065662 & 8.829011 & -2.761351 \\
\hline $\mathrm{H}$ & -2.175193 & 9.006998 & -4.114860 \\
\hline$N$ & -2.493846 & 4.160668 & -4.464856 \\
\hline C & -2.165514 & 2.758006 & -4.617042 \\
\hline C & -1.205775 & 2.601595 & -5.798724 \\
\hline $\mathrm{O}$ & -0.280099 & 1.822621 & -5.736851 \\
\hline C & -3.427376 & 1.870774 & -4.769353 \\
\hline C & -3.455939 & 0.691703 & -3.786939 \\
\hline C & -4.653004 & -0.215545 & -4.051698 \\
\hline$N$ & -4.765857 & -1.267278 & -3.049887 \\
\hline$C$ & -5.617151 & -2.282415 & -3.146375 \\
\hline$N$ & -6.315761 & -2.494605 & -4.262325 \\
\hline$N$ & -5.794567 & -3.080984 & -2.117814 \\
\hline $\mathrm{H}$ & -3.424001 & 4.482421 & -4.617107 \\
\hline $\mathrm{H}$ & -1.594930 & 2.439507 & -3.756879 \\
\hline $\mathrm{H}$ & -4.306640 & 2.483108 & -4.611594 \\
\hline $\mathrm{H}$ & -3.488302 & 1.495246 & -5.786434 \\
\hline $\mathrm{H}$ & -2.545853 & 0.108077 & -3.873219 \\
\hline $\mathrm{H}$ & -3.510632 & 1.069389 & -2.773408 \\
\hline $\mathrm{H}$ & -5.566885 & 0.376698 & -4.064610 \\
\hline $\mathrm{H}$ & -4.528521 & -0.660949 & -5.029704 \\
\hline $\mathrm{H}$ & -4.347477 & -1.151486 & -2.140414 \\
\hline $\mathrm{H}$ & -6.004641 & -2.102166 & -5.117485 \\
\hline $\mathrm{H}$ & -6.832339 & -3.345272 & -4.363246 \\
\hline & -5.354188 & -2.832263 & -1.2408 \\
\hline
\end{tabular}




\begin{tabular}{|c|c|c|c|}
\hline $\mathrm{H}$ & -6.470670 & -3.809268 & -2.191192 \\
\hline$N$ & -1.457551 & 3.319595 & -6.885953 \\
\hline $\mathrm{H}$ & -2.281871 & 3.870894 & -6.972275 \\
\hline C & -0.599719 & 3.167663 & -8.045740 \\
\hline $\mathrm{H}$ & -0.963171 & 3.833200 & -8.818581 \\
\hline $\mathrm{H}$ & -0.605689 & 2.153522 & -8.420370 \\
\hline C & 0.851838 & 3.516468 & -7.732641 \\
\hline $\mathrm{O}$ & 1.776862 & 2.899237 & -8.196469 \\
\hline$N$ & 0.998040 & 4.554871 & -6.896949 \\
\hline $\mathrm{H}$ & 0.205008 & 5.107891 & -6.668979 \\
\hline C & 2.274483 & 5.022348 & -6.461770 \\
\hline $\mathrm{H}$ & 2.906919 & 5.313785 & -7.291461 \\
\hline $\mathrm{H}$ & 2.118574 & 5.895484 & -5.840594 \\
\hline C & 3.092094 & 4.048117 & -5.639735 \\
\hline $\mathrm{O}$ & 4.297124 & 4.182955 & -5.566668 \\
\hline$N$ & 2.444250 & 3.078801 & -4.984622 \\
\hline C & 3.172094 & 2.245621 & -4.056294 \\
\hline C & 4.354513 & 1.495130 & -4.680445 \\
\hline $\mathrm{O}$ & 5.323292 & 1.242551 & -3.993584 \\
\hline C & 2.202121 & 1.280820 & -3.353116 \\
\hline C & 2.870995 & 0.495152 & -2.228958 \\
\hline C & 1.939159 & -0.457640 & -1.488107 \\
\hline$N$ & 2.753905 & -1.242905 & -0.578572 \\
\hline C & 2.306191 & -2.430662 & -0.037338 \\
\hline$N$ & 1.121486 & -2.843526 & 0.073690 \\
\hline$N$ & 3.375944 & -3.216324 & 0.388331 \\
\hline $\mathrm{H}$ & 1.452622 & 2.996693 & -5.052042 \\
\hline $\mathrm{H}$ & 3.646414 & 2.872067 & -3.310030 \\
\hline $\mathrm{H}$ & 1.390225 & 1.882654 & -2.956186 \\
\hline & 1.761786 & 0.611286 & -4.08544 \\
\hline
\end{tabular}




\begin{tabular}{|c|c|c|c|}
\hline $\mathrm{H}$ & 3.689207 & -0.086693 & -2.639310 \\
\hline $\mathrm{H}$ & 3.314534 & 1.181407 & -1.512411 \\
\hline $\mathrm{H}$ & 1.186493 & 0.090634 & -0.931326 \\
\hline $\mathrm{H}$ & 1.418221 & -1.099918 & -2.197625 \\
\hline $\mathrm{H}$ & 3.694272 & -1.334332 & -0.892237 \\
\hline $\mathrm{H}$ & 0.416185 & -2.236068 & -0.292920 \\
\hline $\mathrm{H}$ & 3.984840 & -2.706656 & 0.993501 \\
\hline $\mathrm{H}$ & 3.052967 & -4.036440 & 0.858793 \\
\hline$N$ & 4.307489 & 1.085164 & -5.959499 \\
\hline $\mathrm{H}$ & 3.536205 & 1.340891 & -6.538162 \\
\hline C & 5.391673 & 0.280012 & -6.494636 \\
\hline $\mathrm{H}$ & 5.170432 & 0.061584 & -7.532282 \\
\hline $\mathrm{H}$ & 5.477397 & -0.656325 & -5.962956 \\
\hline C & 6.758118 & 0.931300 & -6.437819 \\
\hline $\mathrm{O}$ & 7.766735 & 0.271236 & -6.340521 \\
\hline$N$ & 6.778946 & 2.261504 & -6.545618 \\
\hline $\mathrm{H}$ & 5.924112 & 2.775814 & -6.499020 \\
\hline C & 8.018536 & 3.002914 & -6.530038 \\
\hline $\mathrm{H}$ & 8.695485 & 2.626639 & -7.284514 \\
\hline r & 7.799375 & 4.035359 & -6.770782 \\
\hline C & 8.823915 & 3.002762 & -5.228361 \\
\hline $\mathrm{O}$ & 9.986383 & 3.354173 & -5.249894 \\
\hline$N$ & 8.210480 & 2.586977 & -4.115147 \\
\hline C & 8.872139 & 2.397050 & -2.830976 \\
\hline C & 10.060106 & 1.434922 & -2.909824 \\
\hline $\mathrm{O}$ & 10.949801 & 1.489322 & -2.078824 \\
\hline C & 7.775150 & 1.887658 & -1.863268 \\
\hline C & 8.166759 & 1.084199 & -0.624239 \\
\hline C & 8.826805 & 1.818252 & 0.543318 \\
\hline$C$ & 8.676790 & 0.942788 & 1.783083 \\
\hline
\end{tabular}




\begin{tabular}{|c|c|c|c|}
\hline$N$ & 9.353110 & 1.508769 & 2.974516 \\
\hline $\mathrm{O}$ & 11.850475 & 0.120031 & 2.393514 \\
\hline $\mathrm{H}$ & 11.961694 & -0.156113 & 1.486953 \\
\hline $\mathrm{H}$ & 12.556414 & -0.258241 & 2.891386 \\
\hline $\mathrm{H}$ & 7.249749 & 2.319689 & -4.177599 \\
\hline $\mathrm{H}$ & 9.297559 & 3.329103 & -2.483504 \\
\hline $\mathrm{H}$ & 7.175055 & 2.744626 & -1.574551 \\
\hline $\mathrm{H}$ & 7.121009 & 1.248385 & -2.439815 \\
\hline $\mathrm{H}$ & 7.235918 & 0.653338 & -0.263543 \\
\hline $\mathrm{H}$ & 8.786395 & 0.243510 & -0.909851 \\
\hline $\mathrm{H}$ & 9.872036 & 2.014397 & 0.325216 \\
\hline $\mathrm{H}$ & 8.343458 & 2.770184 & 0.727011 \\
\hline $\mathrm{H}$ & 7.634747 & 0.847952 & 2.040484 \\
\hline $\mathrm{H}$ & 9.081880 & -0.045824 & 1.619120 \\
\hline $\mathrm{H}$ & 9.048310 & 1.015018 & 3.796349 \\
\hline $\mathrm{H}$ & 10.352221 & 1.396664 & 2.901554 \\
\hline $\mathrm{H}$ & 9.042821 & 2.521920 & 3.078578 \\
\hline$N$ & 10.045455 & 0.555560 & -3.903137 \\
\hline C & 11.055414 & -0.456381 & -4.175724 \\
\hline C & 12.460763 & 0.073762 & -4.448352 \\
\hline $\mathrm{O}$ & 13.397061 & -0.672095 & -4.263016 \\
\hline C & 10.666097 & -1.263271 & -5.436647 \\
\hline C & 9.572250 & -2.327485 & -5.271047 \\
\hline C & 9.134402 & -2.823666 & -6.652443 \\
\hline C & 10.060894 & -3.501054 & -4.421047 \\
\hline $\mathrm{H}$ & 9.266776 & 0.570106 & -4.527586 \\
\hline $\mathrm{H}$ & 11.161147 & -1.106401 & -3.318498 \\
\hline $\mathrm{H}$ & 10.366937 & -0.553119 & -6.200761 \\
\hline $\mathrm{H}$ & 11.564289 & -1.755168 & -5.794046 \\
\hline $\mathrm{H}$ & 8.707150 & -1.874614 & -4.796037 \\
\hline
\end{tabular}




\begin{tabular}{|c|c|c|c|}
\hline $\mathrm{H}$ & 8.365137 & -3.584271 & -6.560718 \\
\hline $\mathrm{H}$ & 8.734140 & -2.011901 & -7.246188 \\
\hline $\mathrm{H}$ & 9.970774 & -3.265635 & -7.189151 \\
\hline $\mathrm{H}$ & 9.297819 & -4.266431 & -4.335734 \\
\hline $\mathrm{H}$ & 10.934822 & -3.958519 & -4.875817 \\
\hline $\mathrm{H}$ & 10.327783 & $3-3.199195$ & -3.412883 \\
\hline$N$ & 12.588928 & 1.304758 & -4.952501 \\
\hline C & 13.850354 & 1.856476 & -5.420953 \\
\hline C & 14.978463 & 2.052987 & -4.388079 \\
\hline O & 16.044296 & 2.440013 & -4.784073 \\
\hline C & 13.569668 & 3.175792 & -6.143669 \\
\hline $\mathrm{O}$ & 13.004361 & 4.125775 & -5.286644 \\
\hline $\mathrm{H}$ & 11.776208 & $\begin{array}{l}3.879199 \\
\end{array}$ & -5.007782 \\
\hline $\mathrm{H}$ & 14.286201 & 1.167378 & -6.136519 \\
\hline $\mathrm{H}$ & 14.508655 & 3.563587 & -6.509255 \\
\hline $\mathrm{H}$ & 12.924090 & 2.979451 & -6.994809 \\
\hline $\mathrm{H}$ & 12.059273 & 3.112757 & -5.330134 \\
\hline$N$ & 14.757274 & 1.720286 & -3.100410 \\
\hline C & 15.774445 & 1.754063 & -2.081167 \\
\hline C & 16.679174 & 0.521349 & -2.138103 \\
\hline $\mathrm{O}$ & 17.713561 & 0.520832 & -1.522010 \\
\hline C & 15.154506 & 1.792404 & -0.673159 \\
\hline C & 14.371939 & 3.056995 & -0.290247 \\
\hline C & 13.704646 & 2.837052 & 1.073677 \\
\hline$N$ & 12.979244 & 3.985428 & 1.624910 \\
\hline C & 13.470017 & 7.863015 & 2.493686 \\
\hline$N$ & 14.774724 & 4.924626 & 2.741787 \\
\hline $\mathrm{O}$ & 14.848226 & 7.058436 & 4.784771 \\
\hline $\mathrm{H}$ & 14.445677 & 7.913525 & 4.674631 \\
\hline 11 & 14.920431 & 6.903404 & 5.713152 \\
\hline
\end{tabular}




\begin{tabular}{|c|c|c|c|}
\hline$N$ & 12.661501 & 5.699637 & 3.107158 \\
\hline $\mathrm{H}$ & 13.821356 & 1.526529 & -2.831286 \\
\hline $\mathrm{H}$ & 16.419723 & 2.607809 & -2.237975 \\
\hline $\mathrm{H}$ & 14.502693 & 0.927958 & -0.567732 \\
\hline $\mathrm{H}$ & 15.970942 & 1.646163 & 0.024936 \\
\hline $\mathrm{H}$ & 15.044541 & 3.910725 & -0.255502 \\
\hline $\mathrm{H}$ & 13.612502 & 3.294593 & -1.026231 \\
\hline $\mathrm{H}$ & 12.986517 & 2.035371 & 0.997628 \\
\hline $\mathrm{H}$ & 14.436527 & 2.510935 & 1.805612 \\
\hline $\mathrm{H}$ & 11.984659 & 4.007982 & 1.529886 \\
\hline $\mathrm{H}$ & 15.426419 & 4.398990 & 2.212346 \\
\hline $\mathrm{H}$ & 15.107795 & 5.601431 & 3.398440 \\
\hline $\mathrm{H}$ & 11.657478 & 5.600070 & 3.032458 \\
\hline $\mathrm{H}$ & 13.049848 & 6.343563 & 3.759743 \\
\hline$N$ & 16.216815 & -0.533118 & -2.833292 \\
\hline$C$ & 16.969897 & -1.771894 & -2.888631 \\
\hline$C$ & 17.940836 & -1.738371 & -4.059716 \\
\hline $\mathrm{H}$ & 18.396046 & -0.761191 & -4.247669 \\
\hline 0 & 18.208513 & -2.685268 & -4.724605 \\
\hline$C$ & 16.053653 & -2.994764 & -2.949957 \\
\hline$C$ & 15.172515 & -3.121165 & -1.747224 \\
\hline$C$ & 13.824406 & -3.045846 & -1.694092 \\
\hline 0 & 12.076126 & -0.451516 & -0.368918 \\
\hline $\mathrm{H}$ & 12.809847 & -0.826571 & -0.831080 \\
\hline $\mathrm{H}$ & 11.674414 & 0.185693 & -0.953917 \\
\hline $\mathrm{N}$ & 15.676509 & -3.337053 & -0.485314 \\
\hline $\mathrm{N}$ & 13.493045 & -3.223613 & -0.368373 \\
\hline$C$ & 14.652594 & -3.388805 & 0.296157 \\
\hline $\mathrm{H}$ & 15.367553 & -0.461618 & -3.349901 \\
\hline $\mathrm{H}$ & 17.572765 & -1.817104 & -1.988814 \\
\hline
\end{tabular}




\begin{tabular}{|c|c|c|c|}
\hline $\mathrm{H}$ & 15.439536 & -2.947447 & -3.843095 \\
\hline $\mathrm{H}$ & 16.679945 & -3.872944 & -3.043338 \\
\hline $\mathrm{H}$ & 13.099127 & -2.882465 & -2.460459 \\
\hline $\mathrm{H}$ & 12.579478 & -3.210483 & 0.018739 \\
\hline $\mathrm{H}$ & 14.685009 & -3.544388 & 1.354404 \\
\hline 0 & -14.785306 & -2.853004 & 5.042749 \\
\hline $\mathrm{H}$ & -15.042258 & -2.023152 & 4.661043 \\
\hline $\mathrm{H}$ & -14.255184 & -2.645040 & 5.799988 \\
\hline 0 & -12.545104 & -1.840413 & 6.941536 \\
\hline $\mathrm{H}$ & -11.772675 & -2.304524 & 7.232415 \\
\hline $\mathrm{H}$ & -12.696860 & -1.154137 & 7.572938 \\
\hline $\mathrm{O}$ & -11.981242 & -1.151500 & 4.149820 \\
\hline $\mathrm{H}$ & -11.991757 & -1.254641 & 5.093654 \\
\hline $\mathrm{H}$ & -12.824778 & -0.768318 & 3.925013 \\
\hline 0 & -13.461874 & -2.005687 & 0.887427 \\
\hline $\mathrm{H}$ & -12.667188 & -1.472041 & 0.876074 \\
\hline $\mathrm{H}$ & -13.334323 & -2.678622 & 1.555932 \\
\hline 0 & -7.007523 & -0.750818 & 0.541836 \\
\hline $\mathrm{H}$ & -7.007741 & -0.116551 & 1.247866 \\
\hline $\mathrm{H}$ & -6.103202 & -1.016015 & 0.380226 \\
\hline 0 & -7.662875 & 1.233100 & 2.940078 \\
\hline $\mathrm{H}$ & -8.611301 & 1.283941 & 2.915736 \\
\hline $\mathrm{H}$ & -7.369096 & 1.976154 & 3.447035 \\
\hline 0 & -9.236623 & -1.030641 & -1.128112 \\
\hline $\mathrm{H}$ & -8.431234 & -0.932376 & -0.611337 \\
\hline $\mathrm{H}$ & -9.422161 & -1.960310 & -1.201142 \\
\hline 0 & -13.386941 & -2.981249 & -1.707811 \\
\hline $\mathrm{H}$ & -13.511753 & -2.742852 & -0.788205 \\
\hline $\mathrm{H}$ & -14.029476 & -3.637309 & -1.927577 \\
\hline 0 & -13.934675 & 0.647455 & -0.617564 \\
\hline
\end{tabular}




\begin{tabular}{|c|c|c|c|}
\hline $\mathrm{H}$ & -14.332668 & -0.152190 & -0.302717 \\
\hline $\mathrm{H}$ & -13.034733 & 0.601961 & -0.293346 \\
\hline $\mathrm{O}$ & -14.165092 & 4.583169 & 5.025512 \\
\hline $\mathrm{H}$ & -13.656100 & 5.293940 & 4.653903 \\
\hline $\mathrm{H}$ & -14.209403 & 4.787666 & 5.950333 \\
\hline $\mathrm{O}$ & -15.068736 & 1.968808 & 1.715866 \\
\hline $\mathrm{H}$ & -15.724696 & 2.647249 & 1.702588 \\
\hline $\mathrm{H}$ & -14.804343 & 1.791693 & 0.820338 \\
\hline $\mathrm{O}$ & -12.988103 & -0.498343 & -3.105002 \\
\hline $\mathrm{H}$ & -13.428207 & 0.098742 & -2.508674 \\
\hline $\mathrm{H}$ & -13.083601 & -1.361671 & -2.710440 \\
\hline $\mathrm{O}$ & -12.678692 & -1.100021 & -5.803588 \\
\hline $\mathrm{H}$ & -12.964079 & -0.788839 & -4.946636 \\
\hline $\mathrm{H}$ & -13.443852 & -1.407167 & -6.263975 \\
\hline $\mathrm{O}$ & -10.547578 & -3.663152 & -1.872988 \\
\hline $\mathrm{H}$ & -10.366108 & -3.544033 & -2.800161 \\
\hline $\mathrm{H}$ & -11.483896 & -3.533861 & -1.778936 \\
\hline 0 & -11.439731 & -0.082422 & 0.460583 \\
\hline $\mathrm{H}$ & -10.685585 & -0.346610 & -0.062402 \\
\hline $\mathrm{H}$ & -11.102243 & 0.358908 & 1.241279 \\
\hline 0 & -10.597966 & 1.022779 & 2.907114 \\
\hline $\mathrm{H}$ & -10.826694 & 0.259383 & 3.428410 \\
\hline $\mathrm{H}$ & -11.233572 & 1.687581 & 3.168448 \\
\hline 0 & -12.866486 & 2.483491 & 3.621170 \\
\hline $\mathrm{H}$ & -13.209788 & 3.123077 & 4.239438 \\
\hline $\mathrm{H}$ & -13.520481 & 2.421865 & 2.938981 \\
\hline 0 & -10.164796 & -0.023702 & -3.615688 \\
\hline $\mathrm{H}$ & -11.077711 & -0.044086 & -3.343141 \\
\hline $\mathrm{H}$ & -9.657647 & -0.222090 & -2.827999 \\
\hline O & -14.656102 & -0.532202 & 3.23676 \\
\hline
\end{tabular}




$\begin{array}{llll}\mathrm{H} & -14.903039 & 0.361756 & 3.034546 \\ \mathrm{H} & -14.550338 & -0.967883 & 2.401822 \\ \mathrm{O} & -12.930023 & -3.670491 & 3.101963 \\ \mathrm{H} & -13.664187 & -3.585565 & 3.712393 \\ \mathrm{H} & -12.297689 & -3.041474 & 3.436580 \\ \mathrm{O} & -10.438575 & -2.724507 & -4.653410 \\ \mathrm{H} & -11.258910 & -2.593408 & -5.108073 \\ \mathrm{H} & -10.158823 & -1.854756 & -4.391222\end{array}$

R303cit MUTATION: NOMINAL CHARGES, Y266(0), E183(0) POTENTIAL: -70mV

$\mathrm{N}$

$15.580944-6.451469 \quad 5.391416$

$\mathrm{H}$

$14.778721-6.221506 \quad 5.942956$

C

$15.496182 \quad-5.852360 \quad 4.058535$

C

$14.783013-6.7829613 .060453$

O $\quad 14.059772-6.346221 \quad 2.181659$

C $\quad 16.898622 \quad-5.543534 \quad 3.522670$

H $\quad 16.387053-6.123736 \quad 5.883073$

H $\quad 14.912587 \quad-4.940419 \quad 4.059364$

H $\quad \begin{array}{lll}16.856096 & -5.149452 & 2.514323\end{array}$

H $\quad \begin{array}{llll}17.371781 & -4.795364 & 4.148834\end{array}$

H $\quad 17.520987-6.432039 \quad 3.521605$

N $\quad \begin{array}{lll}15.032379 & -8.085604 & 3.186404\end{array}$

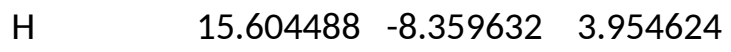

C $\quad \begin{array}{llll}14.398661 & -9.092386 & 2.333235\end{array}$

H $\quad \begin{array}{lll}14.473079 & -8.817195 & 1.293410\end{array}$

H $\quad 14.898004-10.040740 \quad 2.484013$

C $\quad 12.915566 \quad-9.237106 \quad 2.674696$ 


\begin{tabular}{|c|c|c|c|}
\hline $\mathrm{O}$ & 12.091047 & -9.472910 & 1.831600 \\
\hline$N$ & 12.582804 & -9.055884 & 3.966877 \\
\hline $\mathrm{H}$ & 13.300401 & -8.952342 & 4.644778 \\
\hline C & 11.195108 & -9.170814 & 4.421376 \\
\hline $\mathrm{H}$ & 11.186121 & -9.158618 & 5.503549 \\
\hline $\mathrm{H}$ & 10.743031 & -10.087116 & 4.073834 \\
\hline C & 10.336944 & -8.010595 & 3.900734 \\
\hline $\mathrm{O}$ & 9.189623 & -8.187785 & 3.593898 \\
\hline$N$ & 10.947888 & -6.814647 & 3.844867 \\
\hline C & 10.307915 & -5.635464 & 3.316218 \\
\hline$C$ & 10.122054 & -5.677140 & 1.796732 \\
\hline $\mathrm{O}$ & 9.101972 & -5.229344 & 1.334258 \\
\hline C & 11.066256 & -4.386316 & 3.786757 \\
\hline 0 & 12.455638 & -4.545396 & 3.745752 \\
\hline $\mathrm{H}$ & 11.906296 & -6.741332 & 4.089670 \\
\hline $\mathrm{H}$ & 9.301194 & -5.569087 & 3.696350 \\
\hline $\mathrm{H}$ & 10.744266 & -3.531790 & 3.201132 \\
\hline $\mathrm{H}$ & 10.816125 & -4.198601 & 4.821679 \\
\hline $\mathrm{H}$ & 12.762907 & -4.720594 & 2.869165 \\
\hline $\mathrm{N}$ & 11.093922 & -6.194189 & 1.021791 \\
\hline$C$ & 10.834858 & -6.389726 & -0.401802 \\
\hline$C$ & 9.692850 & -7.388519 & -0.606448 \\
\hline 0 & 8.955961 & -7.295761 & -1.558787 \\
\hline$C$ & 12.123852 & -6.767009 & -1.190596 \\
\hline$C$ & 12.355672 & -8.272145 & -1.373227 \\
\hline $\mathrm{C}$ & 12.126409 & -6.071633 & -2.552874 \\
\hline $\mathrm{H}$ & 11.907714 & -6.594654 & 1.431659 \\
\hline $\mathrm{H}$ & 10.454906 & -5.457753 & -0.790548 \\
\hline $\mathrm{H}$ & 12.951582 & -6.365225 & -0.615949 \\
\hline $\mathrm{H}$ & 13.314463 & -8.427505 & -1.857860 \\
\hline
\end{tabular}




\begin{tabular}{|c|c|c|c|}
\hline $\mathrm{H}$ & 12.359749 & -8.818307 & -0.440328 \\
\hline $\mathrm{H}$ & 11.598129 & -8.708184 & -2.017473 \\
\hline $\mathrm{H}$ & 13.018871 & -6.336159 & -3.109741 \\
\hline $\mathrm{H}$ & 11.262212 & -6.366445 & -3.138180 \\
\hline $\mathrm{H}$ & 12.114070 & -4.994064 & -2.441309 \\
\hline$N$ & 9.570431 & -8.340344 & 0.328319 \\
\hline $\mathrm{H}$ & 10.307980 & -8.465745 & 0.980619 \\
\hline$C$ & 8.593019 & -9.421180 & 0.241223 \\
\hline $\mathrm{H}$ & 8.936112 & -10.222208 & 0.884479 \\
\hline $\mathrm{H}$ & 8.528603 & -9.797854 & -0.768425 \\
\hline$C$ & 7.182085 & -9.050582 & 0.647345 \\
\hline $\mathrm{O}$ & 6.260842 & -9.524975 & 0.024190 \\
\hline $\mathrm{N}$ & 7.005575 & -8.223203 & 1.682776 \\
\hline$C$ & 5.668735 & -7.741118 & 1.951776 \\
\hline C & 5.235307 & -6.754245 & 0.873617 \\
\hline 0 & 4.064959 & -6.719838 & 0.570307 \\
\hline$C$ & 5.415152 & -7.215973 & 3.378629 \\
\hline$C$ & 5.562643 & -8.344244 & 4.404104 \\
\hline$C$ & 6.247746 & -5.995348 & 3.773988 \\
\hline $\mathrm{H}$ & 7.784102 & -7.941227 & 2.234165 \\
\hline $\mathrm{H}$ & 4.992284 & -8.569225 & 1.804641 \\
\hline $\mathrm{H}$ & 4.372370 & -6.912641 & 3.368443 \\
\hline $\mathrm{H}$ & 5.273821 & -7.992184 & 5.389018 \\
\hline $\mathrm{H}$ & 4.924983 & -9.186424 & 4.155111 \\
\hline $\mathrm{H}$ & 6.586332 & -8.698508 & 4.461968 \\
\hline $\mathrm{H}$ & 5.901885 & -5.610026 & 4.727236 \\
\hline $\mathrm{H}$ & 7.291890 & -6.258982 & 3.887928 \\
\hline $\mathrm{H}$ & 6.169958 & -5.193840 & 3.048309 \\
\hline $\mathrm{N}$ & 6.132684 & -5.989047 & 0.244728 \\
\hline$C$ & 5.696855 & -5.158945 & -0.870091 \\
\hline
\end{tabular}




\begin{tabular}{|c|c|c|c|}
\hline C & 5.296702 & -6.033333 & -2.065134 \\
\hline $\mathrm{O}$ & 4.302267 & -5.776276 & -2.699429 \\
\hline C & 6.770930 & -4.116630 & -1.241972 \\
\hline C & 6.976245 & -3.122041 & -0.086118 \\
\hline C & 6.376579 & -3.383046 & -2.528637 \\
\hline C & 8.267475 & -2.311858 & -0.201250 \\
\hline $\mathrm{H}$ & 7.089896 & -6.000462 & 0.517461 \\
\hline $\mathrm{H}$ & 4.785693 & -4.650049 & -0.585442 \\
\hline $\mathrm{H}$ & 7.707106 & -4.644429 & -1.413025 \\
\hline $\mathrm{H}$ & 6.115579 & -2.457998 & -0.041052 \\
\hline $\mathrm{H}$ & 7.000815 & -3.654869 & 0.855292 \\
\hline $\mathrm{H}$ & 7.060830 & -2.569160 & -2.731544 \\
\hline $\mathrm{H}$ & 6.390446 & -4.037683 & -3.390735 \\
\hline $\mathrm{H}$ & 5.376000 & -2.973446 & -2.445487 \\
\hline $\mathrm{H}$ & 8.371653 & -1.641675 & 0.645580 \\
\hline $\mathrm{H}$ & 9.129858 & -2.969217 & -0.207601 \\
\hline $\mathrm{H}$ & 8.299973 & -1.708681 & -1.102124 \\
\hline $\mathrm{N}$ & 6.077711 & -7.079449 & -2.385043 \\
\hline $\mathrm{H}$ & 6.959866 & -7.205789 & -1.940342 \\
\hline$C$ & 5.752414 & -7.908953 & -3.523653 \\
\hline $\mathrm{H}$ & 6.591369 & -8.571842 & -3.700329 \\
\hline $\mathrm{H}$ & 5.587881 & -7.314560 & -4.410563 \\
\hline$C$ & 4.495136 & -8.757486 & -3.333983 \\
\hline 0 & 3.664721 & -8.828911 & -4.209262 \\
\hline$N$ & 4.393710 & -9.421895 & -2.176055 \\
\hline $\mathrm{H}$ & 5.115840 & -9.348134 & -1.493595 \\
\hline$C$ & 3.210276 & -10.180583 & -1.848728 \\
\hline $\mathrm{H}$ & 2.968650 & -10.885962 & -2.630339 \\
\hline $\mathrm{H}$ & 3.406768 & -10.731622 & -0.936782 \\
\hline C & 1.974185 & -9.306852 & -1.644812 \\
\hline
\end{tabular}




\begin{tabular}{|c|c|c|c|}
\hline 0 & 0.890526 & -9.709539 & -2.006858 \\
\hline $\mathrm{N}$ & 2.187876 & -8.107002 & -1.107175 \\
\hline C & 1.012886 & -7.252376 & -0.965832 \\
\hline C & 0.545710 & -6.702050 & -2.313977 \\
\hline O & -0.642021 & -6.600070 & -2.533184 \\
\hline C & 1.163378 & -6.146269 & 0.075942 \\
\hline 0 & 1.421534 & -6.707870 & 1.327333 \\
\hline $\mathrm{H}$ & 3.065164 & -7.832942 & -0.730040 \\
\hline $\mathrm{H}$ & 0.197103 & -7.876550 & -0.638504 \\
\hline $\mathrm{H}$ & 1.933350 & -5.442946 & -0.200343 \\
\hline $\mathrm{H}$ & 0.220235 & -5.622796 & 0.131930 \\
\hline $\mathrm{H}$ & 2.356605 & -6.752795 & 1.430579 \\
\hline $\mathrm{N}$ & 1.458863 & -6.375799 & -3.241601 \\
\hline C & 1.040325 & -5.948927 & -4.561843 \\
\hline C & 0.321333 & -7.075681 & -5.302065 \\
\hline 0 & -0.658091 & -6.824655 & -5.976063 \\
\hline C & 2.227309 & -5.367300 & -5.366608 \\
\hline C & 2.554205 & -3.967123 & -4.821865 \\
\hline$C$ & 1.946409 & -5.329650 & -6.873968 \\
\hline C & 3.829406 & -3.354337 & -5.397458 \\
\hline $\mathrm{H}$ & 2.429656 & -6.387940 & -3.016204 \\
\hline $\mathrm{H}$ & 0.283541 & -5.183419 & -4.460645 \\
\hline $\mathrm{H}$ & 3.083019 & -6.015458 & -5.200400 \\
\hline $\mathrm{H}$ & 1.713446 & -3.307480 & -5.032170 \\
\hline $\mathrm{H}$ & 2.651703 & -4.016589 & -3.745072 \\
\hline $\mathrm{H}$ & 2.794532 & -4.913266 & -7.403438 \\
\hline $\mathrm{H}$ & 1.774907 & -6.318716 & -7.281928 \\
\hline $\mathrm{H}$ & 1.077492 & -4.719480 & -7.100870 \\
\hline $\mathrm{H}$ & 4.072811 & -2.440208 & -4.866028 \\
\hline $\mathrm{H}$ & 4.670031 & -4.032369 & -5.289350 \\
\hline
\end{tabular}




\begin{tabular}{|c|c|c|c|}
\hline $\mathrm{H}$ & 3.730978 & -3.105296 & -6.448950 \\
\hline$N$ & 0.787067 & -8.321965 & -5.181476 \\
\hline $\mathrm{H}$ & 1.629515 & -8.498347 & -4.676282 \\
\hline C & 0.045256 & -9.425831 & -5.745357 \\
\hline $\mathrm{H}$ & -0.060609 & -9.330635 & -6.816446 \\
\hline $\mathrm{H}$ & 0.586385 & -10.338900 & -5.529478 \\
\hline C & -1.365274 & -9.540101 & -5.166933 \\
\hline 0 & -2.306887 & -9.811308 & -5.877044 \\
\hline$N$ & -1.472642 & -9.318870 & -3.858023 \\
\hline C & -2.775758 & -9.368202 & -3.235321 \\
\hline C & -3.698644 & -8.231569 & -3.681698 \\
\hline $\mathrm{O}$ & -4.892287 & -8.459293 & -3.766303 \\
\hline C & -2.685085 & -9.371581 & -1.702540 \\
\hline 0 & -3.930809 & -9.672021 & -1.134250 \\
\hline $\mathrm{H}$ & -0.658585 & -9.198186 & -3.293558 \\
\hline $\mathrm{H}$ & -3.288101 & -10.270066 & -3.541002 \\
\hline $\mathrm{H}$ & -1.991739 & -10.141827 & $7-1.391851$ \\
\hline $\mathrm{H}$ & -2.310860 & -8.416028 & -1.349806 \\
\hline $\mathrm{H}$ & -4.619006 & -9.252255 & -1.627732 \\
\hline $\mathrm{N}$ & -3.166169 & -7.033680 & -3.913437 \\
\hline$C$ & -3.942967 & -5.920828 & -4.435541 \\
\hline$C$ & -4.476161 & -6.248018 & -5.830667 \\
\hline 0 & -5.641613 & -6.070728 & -6.108422 \\
\hline$C$ & -3.061297 & -4.651732 & -4.438918 \\
\hline$C$ & -3.544043 & -3.522635 & -5.333580 \\
\hline $\mathrm{C}$ & -4.871491 & -3.106840 & -5.366701 \\
\hline$C$ & -2.631681 & -2.871704 & -6.155345 \\
\hline$C$ & -5.268512 & -2.084391 & -6.211351 \\
\hline $\mathrm{C}$ & -3.022446 & -1.837478 & -6.990360 \\
\hline C & -4.348248 & -1.444785 & -7.025850 \\
\hline
\end{tabular}




\begin{tabular}{|c|c|c|c|}
\hline $\mathrm{H}$ & -2.203200 & -6.886787 & -3.691052 \\
\hline $\mathrm{H}$ & -4.815495 & -5.760781 & -3.821248 \\
\hline $\mathrm{H}$ & -2.965740 & -4.309135 & -3.412733 \\
\hline $\mathrm{H}$ & -2.072591 & -4.936163 & -4.769910 \\
\hline $\mathrm{H}$ & -5.604410 & -3.595238 & -4.752880 \\
\hline $\mathrm{H}$ & -1.601039 & -3.180188 & -6.149539 \\
\hline $\mathrm{H}$ & -6.305927 & -1.795034 & -6.236729 \\
\hline $\mathrm{H}$ & -2.293378 & -1.349711 & -7.612756 \\
\hline $\mathrm{H}$ & -4.658954 & -0.652154 & -7.685253 \\
\hline$N$ & -3.592131 & -6.727714 & -6.713717 \\
\hline C & -4.015210 & -7.045249 & -8.057310 \\
\hline C & -5.138068 & -8.080755 & -8.068498 \\
\hline 0 & -6.012741 & -8.009181 & -8.893646 \\
\hline C & -2.854374 & -7.568232 & -8.906784 \\
\hline$S$ & -1.618410 & -6.317742 & -9.381269 \\
\hline $\mathrm{H}$ & -2.645681 & -6.876065 & -6.434715 \\
\hline $\mathrm{H}$ & -4.434719 & -6.167874 & -8.528775 \\
\hline $\mathrm{H}$ & -2.364209 & -8.397281 & -8.413644 \\
\hline $\mathrm{H}$ & -3.259501 & -7.928939 & -9.843102 \\
\hline $\mathrm{H}$ & -1.023930 & -6.185661 & -8.204100 \\
\hline $\mathrm{N}$ & -5.078558 & -9.066914 & -7.155618 \\
\hline $\mathrm{H}$ & -4.269966 & -9.185399 & -6.585169 \\
\hline$C$ & -6.089593 & -10.108532 & -7.194207 \\
\hline $\mathrm{H}$ & -6.270510 & -10.427377 & -8.209590 \\
\hline $\mathrm{H}$ & -5.720335 & -10.953218 & -6.623276 \\
\hline$C$ & -7.459140 & -9.722569 & -6.613184 \\
\hline 0 & -8.482033 & -10.093281 & -7.124002 \\
\hline $\mathrm{N}$ & -7.417942 & -9.016208 & -5.478355 \\
\hline$C$ & -8.648379 & -8.662987 & -4.796849 \\
\hline 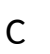 & -9.505958 & -7.661910 & -5.56468 \\
\hline
\end{tabular}




\begin{tabular}{|c|c|c|c|}
\hline $\mathrm{O}$ & -10.706016 & -7.670725 & -5.474841 \\
\hline C & -8.384496 & -8.139675 & -3.375761 \\
\hline C & -7.525130 & -6.872785 & -3.235898 \\
\hline C & -8.183013 & -5.515585 & -3.207105 \\
\hline $\mathrm{O}$ & -9.481231 & -5.502387 & -3.330568 \\
\hline $\mathrm{H}$ & -9.835700 & -4.614888 & -3.215774 \\
\hline $\mathrm{O}$ & -7.531239 & -4.513280 & -3.057680 \\
\hline $\mathrm{H}$ & -6.537742 & -8.775970 & -5.075678 \\
\hline $\mathrm{H}$ & -9.269992 & -9.544580 & -4.717237 \\
\hline $\mathrm{H}$ & -9.346082 & -7.994797 & -2.905184 \\
\hline $\mathrm{H}$ & -7.880184 & -8.936557 & -2.839395 \\
\hline $\mathrm{H}$ & -6.974302 & -6.920610 & -2.303748 \\
\hline $\mathrm{H}$ & -6.773404 & -6.815661 & -4.005850 \\
\hline$N$ & -8.837305 & -6.733964 & -6.273853 \\
\hline C & -9.555190 & -5.702612 & -7.000840 \\
\hline C & -10.380280 & -6.277583 & -8.142491 \\
\hline $\mathrm{H}$ & -10.392379 & -7.361959 & -8.250254 \\
\hline $\mathrm{O}$ & -10.986939 & -5.567424 & -8.889810 \\
\hline C & -8.627868 & -4.567856 & -7.490331 \\
\hline $\mathrm{O}$ & -9.428216 & -3.446960 & -7.840235 \\
\hline C & -7.714316 & -4.938660 & -8.650180 \\
\hline $\mathrm{H}$ & -7.850313 & -6.816355 & -6.387291 \\
\hline $\mathrm{H}$ & -10.271827 & -5.251866 & -6.322526 \\
\hline $\mathrm{H}$ & -8.028611 & -4.260170 & -6.643559 \\
\hline $\mathrm{H}$ & -10.101156 & -3.752726 & -8.436041 \\
\hline $\mathrm{H}$ & -7.033710 & -4.116418 & -8.843519 \\
\hline $\mathrm{H}$ & -7.125703 & -5.820183 & -8.442640 \\
\hline $\mathrm{H}$ & -8.291137 & -5.118858 & -9.551435 \\
\hline$N$ & -12.952117 & -9.022533 & 3.174166 \\
\hline $\mathrm{H}$ & -13.212203 & -9.555727 & 2.372042 \\
\hline
\end{tabular}




\begin{tabular}{|c|c|c|c|}
\hline C & -11.773529 & -8.180033 & 2.898782 \\
\hline C & -10.792793 & -8.116127 & 4.050703 \\
\hline $\mathrm{O}$ & -9.669187 & -7.695095 & 3.859858 \\
\hline C & -12.153157 & -6.724244 & 2.562295 \\
\hline $\mathrm{O}$ & -12.723325 & -6.208781 & 3.737452 \\
\hline C & -13.124624 & -6.608058 & 1.393589 \\
\hline $\mathrm{H}$ & -13.729904 & -8.455274 & 3.445915 \\
\hline $\mathrm{H}$ & -11.219375 & -8.597694 & 2.069698 \\
\hline $\mathrm{H}$ & -11.238230 & -6.195980 & 2.323962 \\
\hline $\mathrm{H}$ & -12.860811 & -5.272517 & 3.643058 \\
\hline $\mathrm{H}$ & -13.297627 & -5.559704 & 1.174797 \\
\hline $\mathrm{H}$ & -12.738583 & -7.078720 & 0.497661 \\
\hline $\mathrm{H}$ & -14.082253 & -7.054128 & 1.635469 \\
\hline$N$ & -11.197265 & -8.533586 & 5.241923 \\
\hline $\mathrm{H}$ & -12.114878 & -8.917725 & 5.275612 \\
\hline C & -10.414054 & -8.496029 & 6.449928 \\
\hline $\mathrm{H}$ & -10.332269 & -7.489763 & 6.832138 \\
\hline $\mathrm{H}$ & -10.934868 & -9.083468 & 7.197584 \\
\hline C & -8.979364 & -9.016494 & 6.388596 \\
\hline $\mathrm{O}$ & -8.152341 & -8.455039 & 7.063068 \\
\hline$N$ & -8.662777 & -10.094113 & 5.648233 \\
\hline $\mathrm{H}$ & -9.369412 & -10.557222 & 5.125428 \\
\hline C & -7.274548 & -10.528739 & 5.553415 \\
\hline $\mathrm{H}$ & -7.256904 & -11.519385 & 5.115981 \\
\hline $\mathrm{H}$ & -6.833488 & -10.582569 & 6.536179 \\
\hline C & -6.387408 & -9.608374 & 4.692653 \\
\hline $\mathrm{O}$ & -5.216063 & -9.491131 & 4.937578 \\
\hline$N$ & -7.045394 & -9.018027 & 3.701138 \\
\hline C & -6.281229 & -8.044831 & 2.926661 \\
\hline$C$ & -6.253481 & -6.681700 & 3.61657 \\
\hline
\end{tabular}




\begin{tabular}{|c|c|c|c|}
\hline $\mathrm{O}$ & -5.277898 & -5.980226 & 3.501055 \\
\hline C & -6.554224 & -7.997241 & 1.413761 \\
\hline C & -7.770313 & -7.255633 & 0.907918 \\
\hline C & -7.800398 & -5.863374 & 0.911688 \\
\hline C & -8.834615 & -7.940773 & 0.338571 \\
\hline C & -8.876105 & -5.176643 & 0.376460 \\
\hline C & -9.904410 & -7.255451 & -0.213846 \\
\hline C & -9.931750 & -5.871913 & -0.190922 \\
\hline $\mathrm{H}$ & -8.038231 & -9.006758 & 3.677930 \\
\hline $\mathrm{H}$ & -5.259464 & -8.373646 & 3.004627 \\
\hline $\mathrm{H}$ & -5.671805 & -7.543731 & 0.976290 \\
\hline $\mathrm{H}$ & -6.569828 & -9.023644 & 1.066034 \\
\hline $\mathrm{H}$ & -6.965550 & -5.311779 & 1.307104 \\
\hline $\mathrm{H}$ & -8.812053 & -9.016725 & 0.293425 \\
\hline $\mathrm{H}$ & -8.873356 & -4.101167 & 0.370812 \\
\hline $\mathrm{H}$ & -10.700827 & -7.800970 & -0.688164 \\
\hline $\mathrm{H}$ & -10.750407 & -5.341772 & -0.641330 \\
\hline$N$ & -7.286215 & -6.346127 & 4.398547 \\
\hline C & -7.231011 & -5.196327 & 5.266954 \\
\hline C & -6.174944 & -5.392765 & 6.353500 \\
\hline $\mathrm{O}$ & -5.552435 & -4.433941 & 6.752902 \\
\hline C & -8.635410 & -4.967568 & 5.860679 \\
\hline C & -8.832236 & -3.673185 & 6.620149 \\
\hline C & -8.878552 & -2.455145 & 5.947342 \\
\hline C & -9.022153 & -3.678344 & 7.995597 \\
\hline C & -9.097850 & -1.273200 & 6.631889 \\
\hline C & -9.249177 & -2.495711 & 8.687335 \\
\hline C & -9.287765 & -1.290147 & 8.006929 \\
\hline $\mathrm{H}$ & -8.139320 & -6.853184 & 4.328992 \\
\hline $\mathrm{H}$ & -6.909320 & -4.322168 & 4.718249 \\
\hline
\end{tabular}




\begin{tabular}{|c|c|c|c|}
\hline $\mathrm{H}$ & -9.335619 & -5.002465 & 5.032151 \\
\hline $\mathrm{H}$ & -8.869900 & -5.802679 & 6.508530 \\
\hline $\mathrm{H}$ & -8.744451 & -2.427953 & 4.879607 \\
\hline $\mathrm{H}$ & -8.985396 & -4.608427 & 8.536438 \\
\hline $\mathrm{H}$ & -9.121671 & -0.343685 & 6.092499 \\
\hline $\mathrm{H}$ & -9.377058 & -2.519061 & 9.755470 \\
\hline $\mathrm{H}$ & -9.447539 & -0.370421 & 8.541589 \\
\hline$N$ & -5.968943 & -6.622483 & 6.822068 \\
\hline $\mathrm{H}$ & -6.616773 & -7.342709 & 6.602262 \\
\hline C & -4.936727 & -6.909935 & 7.791769 \\
\hline $\mathrm{H}$ & -4.985848 & -6.246334 & 8.642485 \\
\hline $\mathrm{H}$ & -5.087668 & -7.924222 & 8.140212 \\
\hline C & -3.520611 & -6.791823 & 7.233634 \\
\hline $\mathrm{O}$ & -2.647363 & -6.327873 & 7.927344 \\
\hline$N$ & -3.313612 & -7.225201 & 5.986939 \\
\hline$C$ & -2.023899 & -7.080235 & 5.331026 \\
\hline C & -1.727440 & -5.611796 & 4.999885 \\
\hline $\mathrm{O}$ & -0.642418 & -5.158598 & 5.283452 \\
\hline C & -1.947857 & -8.008121 & 4.101670 \\
\hline C & -0.782326 & -7.644813 & 3.183260 \\
\hline C & -1.845430 & -9.470236 & 4.550164 \\
\hline $\mathrm{H}$ & -4.047453 & -7.693078 & 5.505117 \\
\hline $\mathrm{H}$ & -1.246428 & -7.350840 & 6.031699 \\
\hline $\mathrm{H}$ & -2.869370 & -7.879982 & 3.537413 \\
\hline $\mathrm{H}$ & -0.717256 & -8.358455 & 2.370046 \\
\hline $\mathrm{H}$ & -0.883956 & -6.662057 & 2.743314 \\
\hline $\mathrm{H}$ & 0.162211 & -7.668069 & 3.716065 \\
\hline $\mathrm{H}$ & -1.881878 & -10.128440 & 3.687663 \\
\hline $\mathrm{H}$ & -0.898477 & -9.641550 & 5.056619 \\
\hline & -2.647551 & -9.757208 & 5.217736 \\
\hline
\end{tabular}




\begin{tabular}{|c|c|c|c|}
\hline$N$ & -2.697994 & -4.887143 & 4.428764 \\
\hline C & -2.466689 & -3.509800 & 4.021656 \\
\hline C & -2.398438 & -2.589531 & 5.241026 \\
\hline $\mathrm{O}$ & -1.569728 & -1.709008 & 5.303561 \\
\hline C & -3.556754 & -3.001271 & 3.064987 \\
\hline C & -3.679714 & -3.710336 & 1.704639 \\
\hline C & -2.464778 & -3.543636 & 0.783731 \\
\hline 0 & -1.410756 & -4.044011 & 1.206315 \\
\hline $\mathrm{O}$ & -2.625470 & -2.943721 & -0.289097 \\
\hline $\mathrm{H}$ & -3.541901 & -5.335782 & 4.150844 \\
\hline $\mathrm{H}$ & -1.505798 & -3.439917 & 3.541250 \\
\hline $\mathrm{H}$ & -4.519089 & -3.059012 & 3.569678 \\
\hline $\mathrm{H}$ & -3.356747 & -1.946732 & 2.897048 \\
\hline $\mathrm{H}$ & -3.833950 & -4.771418 & 1.857844 \\
\hline $\mathrm{H}$ & -4.561270 & -3.316238 & 1.208713 \\
\hline$N$ & -3.291291 & -2.769819 & 6.236592 \\
\hline C & -3.094021 & -2.024920 & 7.456986 \\
\hline C & -1.786791 & -2.428838 & 8.150580 \\
\hline 0 & -1.130556 & -1.592446 & 8.734475 \\
\hline C & -4.242891 & -1.992992 & 8.472703 \\
\hline 0 & -4.510070 & -3.238765 & 9.045472 \\
\hline$C$ & -5.493579 & -1.325861 & 7.914669 \\
\hline $\mathrm{H}$ & -3.938971 & -3.522276 & 6.199064 \\
\hline $\mathrm{H}$ & -2.922578 & -0.996869 & 7.179416 \\
\hline $\mathrm{H}$ & -3.863446 & -1.380191 & 9.282717 \\
\hline $\mathrm{H}$ & -4.949560 & -3.782419 & 8.408517 \\
\hline $\mathrm{H}$ & -6.244984 & -1.270510 & 8.693232 \\
\hline $\mathrm{H}$ & -5.272676 & -0.317350 & 7.578128 \\
\hline $\mathrm{H}$ & -5.905376 & -1.881915 & 7.083152 \\
\hline$N$ & -1.380380 & -3.700423 & 8.043288 \\
\hline
\end{tabular}




\begin{tabular}{|c|c|c|c|}
\hline $\mathrm{H}$ & -2.005425 & -4.420007 & 7.762130 \\
\hline C & -0.131383 & -4.094491 & 8.640062 \\
\hline $\mathrm{H}$ & -0.100608 & -3.880771 & 9.699635 \\
\hline $\mathrm{H}$ & -0.018107 & -5.160627 & 8.490034 \\
\hline C & 1.071994 & -3.389833 & 8.026149 \\
\hline $\mathrm{O}$ & 1.978890 & -2.997871 & 8.730124 \\
\hline$N$ & 1.088060 & -3.259934 & 6.690709 \\
\hline C & 2.236638 & -2.668978 & 6.053454 \\
\hline C & 2.270063 & -1.144384 & 6.153809 \\
\hline O & 3.355672 & -0.594733 & 6.163659 \\
\hline C & 2.440696 & -3.150150 & 4.612360 \\
\hline$S$ & 1.368543 & -2.312481 & 3.402094 \\
\hline $\mathrm{H}$ & 0.367655 & -3.683296 & 6.139395 \\
\hline $\mathrm{H}$ & 3.112261 & -2.971438 & 6.609986 \\
\hline $\mathrm{H}$ & 3.474707 & -2.967510 & 4.352720 \\
\hline $\mathrm{H}$ & 2.256195 & -4.215830 & 4.580498 \\
\hline $\mathrm{H}$ & 1.799554 & -2.978468 & 2.339088 \\
\hline $\mathrm{N}$ & 1.126092 & -0.463369 & 6.261850 \\
\hline$C$ & 1.204069 & 0.968501 & 6.491363 \\
\hline$C$ & 1.761855 & 1.265767 & 7.869776 \\
\hline $\mathrm{O}$ & 2.372713 & 2.286572 & 8.087135 \\
\hline$C$ & -0.093933 & 1.751377 & 6.198872 \\
\hline$C$ & -1.193707 & 1.550710 & 7.251800 \\
\hline C & -0.553896 & 1.488869 & 4.759637 \\
\hline$C$ & -2.470932 & 2.344346 & 6.971336 \\
\hline $\mathrm{H}$ & 0.249518 & -0.931163 & 6.150316 \\
\hline $\mathrm{H}$ & 1.952272 & 1.357753 & 5.816338 \\
\hline $\mathrm{H}$ & 0.212971 & 2.790517 & 6.266463 \\
\hline $\mathrm{H}$ & -1.431127 & 0.501322 & 7.351322 \\
\hline 1 & -0.807476 & 1.861995 & 8.218303 \\
\hline
\end{tabular}




\begin{tabular}{|c|c|c|c|}
\hline $\mathrm{H}$ & -1.300533 & 2.213126 & 4.457739 \\
\hline $\mathrm{H}$ & 0.279673 & 1.573372 & 4.068374 \\
\hline $\mathrm{H}$ & -0.978650 & 0.501693 & 4.639085 \\
\hline $\mathrm{H}$ & -3.142173 & 2.289393 & 7.823588 \\
\hline $\mathrm{H}$ & -2.256835 & 3.394750 & 6.787147 \\
\hline $\mathrm{H}$ & -3.004586 & 1.957990 & 6.109793 \\
\hline$N$ & 1.549785 & 0.323276 & 8.791268 \\
\hline $\mathrm{H}$ & 0.924729 & -0.426893 & 8.596421 \\
\hline C & 1.986100 & 0.500666 & 10.156042 \\
\hline $\mathrm{H}$ & 1.462325 & -0.222220 & 10.769782 \\
\hline $\mathrm{H}$ & 1.768585 & 1.496342 & 10.513755 \\
\hline C & 3.484960 & 0.295346 & 10.316365 \\
\hline $\mathrm{O}$ & 4.161388 & 1.050551 & 10.963138 \\
\hline$N$ & 4.001659 & -0.777718 & 9.680608 \\
\hline $\mathrm{H}$ & 3.398860 & -1.426421 & 9.220989 \\
\hline C & 5.426736 & -0.958277 & 9.643657 \\
\hline $\mathrm{H}$ & 5.641165 & -1.918858 & 9.188737 \\
\hline $\mathrm{H}$ & 5.856108 & -0.943200 & 10.634972 \\
\hline C & 6.156884 & 0.119116 & 8.844831 \\
\hline $\mathrm{O}$ & 7.283485 & 0.430639 & 9.147134 \\
\hline$N$ & 5.502075 & 0.621284 & 7.799030 \\
\hline C & 6.098618 & 1.759274 & 7.146316 \\
\hline C & 5.535843 & 3.077053 & 7.670198 \\
\hline $\mathrm{H}$ & 5.003130 & 3.023929 & 8.619908 \\
\hline $\mathrm{O}$ & 5.700657 & 4.107370 & 7.091428 \\
\hline C & 5.964102 & 1.686659 & 5.622494 \\
\hline C & 6.677912 & 0.506854 & 4.995244 \\
\hline C & 8.044724 & 0.295989 & 5.182068 \\
\hline C & 5.977747 & -0.387754 & 4.191070 \\
\hline 6 & 8.693856 & -0.763895 & 4.563679 \\
\hline
\end{tabular}




\begin{tabular}{|c|c|c|c|}
\hline C & 6.625182 & -1.445010 & 3.571495 \\
\hline C & 7.986595 & -1.634263 & 3.749286 \\
\hline $\mathrm{H}$ & 4.595003 & 0.300639 & 7.538209 \\
\hline $\mathrm{H}$ & 7.141665 & 1.764832 & 7.428581 \\
\hline $\mathrm{H}$ & 4.913698 & 1.641632 & 5.362190 \\
\hline $\mathrm{H}$ & 6.348743 & 2.618160 & 5.219726 \\
\hline $\mathrm{H}$ & 8.602098 & 0.941641 & 5.840004 \\
\hline $\mathrm{H}$ & 4.915916 & -0.274367 & 4.077565 \\
\hline $\mathrm{H}$ & 9.747028 & -0.912451 & 4.733408 \\
\hline $\mathrm{H}$ & 6.060426 & -2.123407 & 2.957097 \\
\hline $\mathrm{H}$ & 8.477856 & -2.459462 & 3.265491 \\
\hline$N$ & 12.613550 & 9.596234 & -1.248802 \\
\hline $\mathrm{H}$ & 11.875371 & 9.913983 & -1.846612 \\
\hline C & 12.304853 & 8.264740 & -0.719488 \\
\hline C & 11.274242 & 8.447550 & 0.387609 \\
\hline $\mathrm{O}$ & 10.270539 & 7.770142 & 0.419851 \\
\hline C & 13.561105 & 7.577803 & -0.166669 \\
\hline C & 14.640145 & 7.267853 & -1.209184 \\
\hline$S$ & 14.005701 & 6.195739 & -2.530673 \\
\hline C & 15.483639 & 6.038081 & -3.560022 \\
\hline $\mathrm{H}$ & 13.436386 & 9.575614 & -1.815741 \\
\hline $\mathrm{H}$ & 11.832859 & 7.616957 & -1.447424 \\
\hline $\mathrm{H}$ & 14.003238 & 8.201298 & 0.605228 \\
\hline $\mathrm{H}$ & 13.250621 & 6.651952 & 0.304501 \\
\hline $\mathrm{H}$ & 15.043433 & 8.178707 & -1.638947 \\
\hline $\mathrm{H}$ & 15.464443 & 6.771810 & -0.708456 \\
\hline $\mathrm{H}$ & 15.222801 & 5.383944 & -4.378108 \\
\hline $\mathrm{H}$ & 15.786540 & 7.000480 & -3.952794 \\
\hline $\mathrm{H}$ & 16.301059 & 5.595840 & -3.005150 \\
\hline $\mathrm{N}$ & 11.501599 & 9.413469 & 1.295150 \\
\hline
\end{tabular}




\begin{tabular}{|c|c|c|c|}
\hline C & 10.592248 & 9.606047 & 2.407507 \\
\hline C & 9.279666 & 10.287585 & 2.017629 \\
\hline $\mathrm{O}$ & 8.289452 & 10.086012 & 2.674527 \\
\hline C & 11.279972 & 10.350479 & 3.558211 \\
\hline C & 12.451052 & 9.538400 & 4.079766 \\
\hline $\mathrm{O}$ & 13.587794 & 9.816673 & 3.756854 \\
\hline$N$ & 12.148272 & 8.513112 & 4.883471 \\
\hline $\mathrm{H}$ & 12.320437 & 9.972402 & 1.198453 \\
\hline $\mathrm{H}$ & 10.284423 & 8.634277 & 2.760936 \\
\hline $\mathrm{H}$ & 11.664064 & 11.310587 & 3.235747 \\
\hline $\mathrm{H}$ & 10.551426 & 10.510527 & 4.343261 \\
\hline $\mathrm{H}$ & 12.896017 & 7.923884 & 5.174477 \\
\hline $\mathrm{H}$ & 11.228061 & 8.125410 & 4.909339 \\
\hline$N$ & 9.265352 & 11.070902 & 0.921775 \\
\hline $\mathrm{H}$ & 10.107337 & 11.207311 & 0.413431 \\
\hline C & 8.003503 & 11.517468 & 0.368862 \\
\hline $\mathrm{H}$ & 8.196834 & 12.261331 & -0.393975 \\
\hline $\mathrm{H}$ & 7.387903 & 11.963042 & 1.133693 \\
\hline C & 7.186383 & 10.384438 & -0.254326 \\
\hline O & 5.988474 & 10.483090 & -0.309205 \\
\hline$N$ & 7.860524 & 9.323653 & -0.745051 \\
\hline C & 7.162093 & 8.138573 & -1.212865 \\
\hline C & 6.623560 & 7.303803 & -0.041159 \\
\hline $\mathrm{O}$ & 5.517599 & 6.824139 & -0.115549 \\
\hline C & 8.033450 & 7.325747 & -2.199734 \\
\hline C & 8.345605 & 8.174069 & -3.447106 \\
\hline C & 7.346039 & 6.010593 & -2.585652 \\
\hline C & 9.423005 & 7.585403 & -4.360416 \\
\hline $\mathrm{H}$ & 8.817926 & 9.215641 & -0.510349 \\
\hline $\mathrm{H}$ & 6.271104 & 8.467798 & -1.726386 \\
\hline
\end{tabular}




\begin{tabular}{|c|c|c|c|}
\hline $\mathrm{H}$ & 8.968023 & 7.086894 & -1.698317 \\
\hline $\mathrm{H}$ & 7.426845 & 8.314760 & -4.012990 \\
\hline $\mathrm{H}$ & 8.662508 & 9.165172 & -3.138097 \\
\hline $\mathrm{H}$ & 7.961327 & 5.456951 & -3.283239 \\
\hline $\mathrm{H}$ & 7.173736 & 5.375467 & -1.725469 \\
\hline $\mathrm{H}$ & 6.384126 & 6.188718 & -3.055437 \\
\hline $\mathrm{H}$ & 9.626960 & 8.261504 & -5.184671 \\
\hline $\mathrm{H}$ & 10.355911 & 7.428425 & -3.825425 \\
\hline $\mathrm{H}$ & 9.130994 & 6.633998 & -4.789345 \\
\hline$N$ & 7.378441 & 7.176113 & 1.068804 \\
\hline C & 6.865001 & 6.461093 & 2.226701 \\
\hline C & 5.515601 & 7.045285 & 2.664267 \\
\hline $\mathrm{O}$ & 4.566882 & 6.338845 & 2.900772 \\
\hline C & 7.818996 & 6.544802 & 3.427786 \\
\hline C & 9.234998 & 5.988958 & 3.259695 \\
\hline $\mathrm{O}$ & 10.139158 & 6.633744 & 3.787157 \\
\hline $\mathrm{O}$ & 9.396311 & 4.913683 & 2.632075 \\
\hline 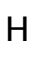 & 8.341397 & 7.429992 & 1.040426 \\
\hline $\mathrm{H}$ & 6.663211 & 5.432694 & 1.968582 \\
\hline r & 7.913693 & 7.569808 & 3.756351 \\
\hline $\mathrm{H}$ & 7.360165 & 5.991522 & 4.244777 \\
\hline$N$ & 5.475394 & 8.385157 & 2.800808 \\
\hline $\mathrm{H}$ & 6.304186 & 8.919766 & 2.658608 \\
\hline C & 4.282462 & 9.052707 & 3.260188 \\
\hline $\mathrm{H}$ & 4.522217 & 10.094631 & 3.434622 \\
\hline $\mathrm{H}$ & 3.928259 & 8.633895 & 4.189280 \\
\hline C & 3.125895 & 9.015483 & 2.270588 \\
\hline $\mathrm{O}$ & 1.987567 & 9.179845 & 2.644986 \\
\hline$N$ & 3.462275 & 8.827414 & 0.992053 \\
\hline & 4.422548 & 8.780775 & 0.750309 \\
\hline
\end{tabular}




\begin{tabular}{|c|c|c|c|}
\hline C & 2.517195 & 8.905257 & -0.078303 \\
\hline $\mathrm{H}$ & 3.063976 & 9.089531 & -0.994780 \\
\hline $\mathrm{H}$ & 1.841813 & 9.737275 & 0.068108 \\
\hline C & 1.609130 & 7.687023 & -0.310195 \\
\hline $\mathrm{O}$ & 0.568239 & 7.818812 & -0.891663 \\
\hline$N$ & 2.082674 & 6.540758 & 0.214374 \\
\hline C & 1.265121 & 5.376763 & 0.458801 \\
\hline C & 0.458403 & 5.531164 & 1.751648 \\
\hline $\mathrm{O}$ & -0.745011 & 5.431769 & 1.724406 \\
\hline C & 2.121409 & 4.111815 & 0.480560 \\
\hline $\mathrm{H}$ & 2.984319 & 6.576380 & 0.637504 \\
\hline $\mathrm{H}$ & 0.527066 & 5.306962 & -0.323636 \\
\hline $\mathrm{H}$ & 1.510397 & 3.244212 & 0.703312 \\
\hline $\mathrm{H}$ & 2.581753 & 3.973798 & -0.490833 \\
\hline $\mathrm{H}$ & 2.908039 & 4.178233 & 1.225514 \\
\hline$N$ & 1.103380 & 5.766666 & 2.910707 \\
\hline C & 0.359919 & 5.601859 & 4.152269 \\
\hline C & -0.753277 & 6.635729 & 4.301341 \\
\hline $\mathrm{O}$ & -1.787484 & 6.338089 & 4.859958 \\
\hline C & 1.268752 & 5.522648 & 5.403488 \\
\hline C & 1.867566 & 6.865444 & 5.848299 \\
\hline C & 2.349872 & 4.449223 & 5.205032 \\
\hline C & 2.698719 & 6.767947 & 7.129597 \\
\hline $\mathrm{H}$ & 2.097415 & 5.824520 & 2.934193 \\
\hline $\mathrm{H}$ & -0.173209 & 4.662680 & 4.094578 \\
\hline $\mathrm{H}$ & 0.603494 & 5.188930 & 6.194680 \\
\hline $\mathrm{H}$ & 2.480416 & 7.267876 & 5.047847 \\
\hline $\mathrm{H}$ & 1.068097 & 7.581322 & 6.013013 \\
\hline $\mathrm{H}$ & 2.705719 & 4.086686 & 6.158550 \\
\hline $\mathrm{H}$ & 1.956054 & 3.598741 & 4.658550 \\
\hline
\end{tabular}




\begin{tabular}{|c|c|c|c|}
\hline $\mathrm{H}$ & 3.201779 & 4.836539 & 4.655611 \\
\hline $\mathrm{H}$ & 3.011170 & 7.758047 & 7.449572 \\
\hline $\mathrm{H}$ & 2.118517 & 6.330220 & 7.936793 \\
\hline $\mathrm{H}$ & 3.589461 & 6.165249 & 7.001109 \\
\hline$N$ & -0.537734 & 7.868344 & 3.819129 \\
\hline $\mathrm{H}$ & 0.340631 & 8.105230 & 3.410001 \\
\hline C & -1.517047 & 8.902494 & 4.019313 \\
\hline $\mathrm{H}$ & -1.113762 & 9.831023 & 3.633584 \\
\hline $\mathrm{H}$ & -1.735897 & 9.041250 & 5.068751 \\
\hline C & -2.866969 & 8.618760 & 3.365891 \\
\hline $\mathrm{O}$ & -3.873013 & 8.835243 & 4.002903 \\
\hline$N$ & -2.902694 & 8.108965 & 2.120754 \\
\hline C & -4.240747 & 8.006926 & 1.538818 \\
\hline C & -4.985005 & 6.744200 & 1.937892 \\
\hline $\mathrm{O}$ & -6.197583 & 6.757516 & 1.931133 \\
\hline C & -4.025738 & 8.210828 & 0.034049 \\
\hline C & -2.777443 & 9.099328 & 0.019839 \\
\hline C & -1.911386 & 8.462277 & 1.093741 \\
\hline $\mathrm{H}$ & -4.857319 & 8.807367 & 1.920528 \\
\hline r & -3.837180 & 7.267635 & -0.462653 \\
\hline $\mathrm{H}$ & -4.894798 & 8.664946 & -0.425313 \\
\hline $\mathrm{H}$ & -2.268609 & 9.143979 & -0.924756 \\
\hline $\mathrm{H}$ & -3.046329 & 10.112660 & 0.310987 \\
\hline $\mathrm{H}$ & -1.419707 & 7.575449 & 0.736816 \\
\hline $\mathrm{H}$ & -1.158349 & 9.130327 & 1.480642 \\
\hline$N$ & -4.272419 & 5.671195 & 2.306342 \\
\hline C & -4.944660 & 4.508958 & 2.862948 \\
\hline C & -5.649229 & 4.863615 & 4.175098 \\
\hline O & -6.762293 & 4.446846 & 4.412822 \\
\hline$C$ & -3.984662 & 3.328790 & 3.119143 \\
\hline
\end{tabular}




\begin{tabular}{|c|c|c|c|}
\hline C & -3.566245 & 2.524101 & 1.904560 \\
\hline C & -4.156942 & 1.297363 & 1.616047 \\
\hline C & -2.530891 & 2.947897 & 1.083945 \\
\hline C & -3.733907 & 0.521921 & 0.547046 \\
\hline C & -2.103023 & 2.189271 & 0.011706 \\
\hline C & -2.696939 & 0.968417 & -0.267370 \\
\hline $\mathrm{O}$ & -2.242895 & 0.273562 & -1.321877 \\
\hline $\mathrm{H}$ & -2.952690 & -0.231068 & -1.719485 \\
\hline $\mathrm{H}$ & -3.278257 & 5.741763 & 2.345946 \\
\hline $\mathrm{H}$ & -5.728252 & 4.202213 & 2.187212 \\
\hline $\mathrm{H}$ & -3.108952 & 3.707165 & 3.637974 \\
\hline $\mathrm{H}$ & -4.489221 & 2.664240 & 3.811690 \\
\hline $\mathrm{H}$ & -4.954290 & 0.931394 & 2.240961 \\
\hline $\mathrm{H}$ & -2.039409 & 3.876870 & 1.278128 \\
\hline $\mathrm{H}$ & -4.172302 & -0.439677 & 0.359116 \\
\hline $\mathrm{H}$ & -1.304354 & 2.533385 & -0.621596 \\
\hline$N$ & -4.975134 & 5.619849 & 5.057657 \\
\hline $\mathrm{H}$ & -4.033333 & 5.895763 & 4.874980 \\
\hline C & -5.590535 & 6.005783 & 6.305803 \\
\hline $\mathrm{H}$ & -4.816268 & 6.383175 & 6.963109 \\
\hline $\mathrm{H}$ & -6.068038 & 5.161270 & 6.777593 \\
\hline C & -6.653547 & 7.089065 & 6.140512 \\
\hline 0 & -7.707896 & 7.042151 & 6.734549 \\
\hline $\mathrm{N}$ & -6.342148 & 8.091247 & 5.313908 \\
\hline $\mathrm{H}$ & -5.447310 & 8.133900 & 4.875928 \\
\hline$C$ & -7.257687 & 9.220268 & 5.085303 \\
\hline $\mathrm{H}$ & -6.780977 & 9.874221 & 4.365771 \\
\hline $\mathrm{H}$ & -7.425267 & 9.773907 & 5.998204 \\
\hline$C$ & -8.631028 & 8.841954 & 4.565103 \\
\hline 0 & -9.609634 & 9.436787 & 4.949337 \\
\hline
\end{tabular}




\begin{tabular}{|c|c|c|c|}
\hline$N$ & -8.689665 & 7.852504 & 3.658311 \\
\hline C & -9.953936 & 7.451761 & 3.088773 \\
\hline C & -10.769603 & 6.546741 & 3.997573 \\
\hline $\mathrm{O}$ & -11.817458 & 6.083081 & 3.565386 \\
\hline C & -9.759684 & 6.810649 & 1.692333 \\
\hline $\mathrm{O}$ & -10.952324 & 6.896525 & 0.954858 \\
\hline C & -9.237764 & 5.377501 & 1.739069 \\
\hline $\mathrm{H}$ & -7.846122 & 7.423083 & 3.337882 \\
\hline $\mathrm{H}$ & -10.571911 & 8.331416 & 2.960916 \\
\hline $\mathrm{H}$ & -9.040690 & 7.431820 & 1.172827 \\
\hline $\mathrm{H}$ & -11.637837 & 6.496969 & 1.472067 \\
\hline $\mathrm{H}$ & -8.969437 & 5.050165 & 0.742518 \\
\hline $\mathrm{H}$ & -8.358262 & 5.288511 & 2.361358 \\
\hline $\mathrm{H}$ & -9.998394 & 4.702991 & 2.115269 \\
\hline$N$ & -10.353337 & 6.327942 & 5.240899 \\
\hline C & -11.263937 & 5.755933 & 6.221480 \\
\hline C & -12.430178 & 6.704185 & 6.510430 \\
\hline $\mathrm{O}$ & -13.521427 & 6.260257 & 6.782760 \\
\hline C & -10.540844 & 5.423961 & 7.534519 \\
\hline$c$ & -9.544386 & 4.255508 & 7.460538 \\
\hline C & -8.723890 & 4.208798 & 8.752885 \\
\hline C & -10.237545 & 2.911150 & 7.217389 \\
\hline 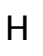 & -9.456611 & 6.643768 & 5.544060 \\
\hline $\mathrm{H}$ & -11.721310 & 4.869439 & 5.813842 \\
\hline $\mathrm{H}$ & -10.022944 & 6.313219 & 7.877814 \\
\hline $\mathrm{H}$ & -11.305429 & 5.193593 & 8.272050 \\
\hline $\mathrm{H}$ & -8.857468 & 4.436276 & 6.639877 \\
\hline $\mathrm{H}$ & -8.000651 & 3.398671 & 8.724251 \\
\hline $\mathrm{H}$ & -8.183136 & 5.136740 & 8.900808 \\
\hline & -9.364036 & 4.048091 & 9.618118 \\
\hline
\end{tabular}




\begin{tabular}{|c|c|c|c|}
\hline $\mathrm{H}$ & -9.500781 & 2.113113 & 7.199477 \\
\hline $\mathrm{H}$ & -10.947203 & 2.691551 & 8.013377 \\
\hline $\mathrm{H}$ & -10.770374 & 2.881265 & 6.274128 \\
\hline$N$ & -12.161548 & 8.022371 & 6.508919 \\
\hline C & -13.213370 & 8.981115 & 6.674726 \\
\hline C & -13.981245 & 9.276037 & 5.404614 \\
\hline $\mathrm{H}$ & -14.806318 & 9.987713 & 5.534383 \\
\hline $\mathrm{O}$ & -13.739401 & 8.809974 & 4.340737 \\
\hline $\mathrm{H}$ & -11.296639 & 8.348955 & 6.135131 \\
\hline $\mathrm{H}$ & -13.917311 & 8.632615 & 7.421011 \\
\hline $\mathrm{H}$ & -12.797121 & 9.917795 & 7.034850 \\
\hline$N$ & -11.441241 & 10.613163 & -3.010180 \\
\hline $\mathrm{H}$ & -11.171665 & 10.805432 & -2.067594 \\
\hline C & -11.816457 & 9.206991 & -3.172692 \\
\hline C & -11.416593 & 8.871301 & -4.602200 \\
\hline $\mathrm{O}$ & -10.367825 & 8.327599 & -4.850535 \\
\hline C & -13.263658 & 8.844362 & -2.777744 \\
\hline C & -13.505983 & 9.186420 & -1.295966 \\
\hline C & -13.551523 & 7.366187 & -3.069339 \\
\hline$c$ & -14.938026 & 8.950945 & -0.813600 \\
\hline $\mathrm{H}$ & -12.199822 & 11.226667 & -3.235231 \\
\hline $\mathrm{H}$ & -11.144625 & 8.621479 & -2.559970 \\
\hline $\mathrm{H}$ & -13.950309 & 9.455497 & -3.370072 \\
\hline $\mathrm{H}$ & -12.816999 & 8.610086 & -0.682292 \\
\hline $\mathrm{H}$ & -13.265469 & 10.232331 & -1.130718 \\
\hline $\mathrm{H}$ & -14.596292 & 7.132332 & -2.908960 \\
\hline $\mathrm{H}$ & -13.318975 & 7.095146 & -4.095053 \\
\hline $\mathrm{H}$ & -12.964200 & 6.728604 & -2.415942 \\
\hline $\mathrm{H}$ & -15.050112 & 9.306023 & 0.204720 \\
\hline $\mathrm{H}$ & -15.657067 & 9.483055 & -1.431648 \\
\hline
\end{tabular}




\begin{tabular}{|c|c|c|c|}
\hline $\mathrm{H}$ & -15.205308 & 7.900551 & -0.818003 \\
\hline$N$ & -12.221605 & 9.293884 & -5.606697 \\
\hline $\mathrm{H}$ & -13.144303 & 9.585644 & -5.383198 \\
\hline C & -11.972872 & 8.843880 & -6.959258 \\
\hline $\mathrm{H}$ & -12.030826 & 7.766437 & -7.041610 \\
\hline $\mathrm{H}$ & -12.727332 & 9.277620 & -7.605113 \\
\hline C & -10.601635 & 9.233244 & -7.517693 \\
\hline $\mathrm{O}$ & -10.001429 & 8.500085 & -8.247884 \\
\hline$N$ & -10.163627 & 10.478873 & $\begin{array}{ll}3 & -7.182432\end{array}$ \\
\hline $\mathrm{H}$ & -10.705002 & 11.000804 & $4 \quad-6.533973$ \\
\hline C & -8.894341 & 11.023967 & -7.637100 \\
\hline $\mathrm{H}$ & -8.788447 & 10.888364 & -8.702796 \\
\hline $\mathrm{H}$ & -8.876005 & 12.081928 & -7.407754 \\
\hline C & -7.672564 & 10.359017 & -6.988311 \\
\hline $\mathrm{O}$ & -6.750720 & 9.944631 & -7.632766 \\
\hline$N$ & -7.659883 & 10.351601 & -5.619712 \\
\hline $\mathrm{H}$ & -8.489577 & 10.606490 & -5.135691 \\
\hline C & -6.495254 & 9.961264 & -4.832349 \\
\hline $\mathrm{H}$ & -5.598896 & 10.375427 & -5.266935 \\
\hline 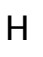 & -6.614392 & 10.359253 & -3.831602 \\
\hline C & -6.268291 & 8.463911 & -4.714277 \\
\hline $\mathrm{O}$ & -5.133276 & 8.049574 & -4.714449 \\
\hline$N$ & -7.319505 & 7.640709 & -4.554014 \\
\hline C & -7.041022 & 6.286202 & -4.100339 \\
\hline C & -6.399273 & 5.427597 & -5.182974 \\
\hline $\mathrm{O}$ & -5.555778 & 4.625980 & -4.865138 \\
\hline C & -8.230441 & 5.578173 & -3.412387 \\
\hline C & -9.372729 & 5.197623 & -4.369143 \\
\hline C & -8.686920 & 6.403628 & -2.204162 \\
\hline & -10.520284 & 4.447573 & -3.6926 \\
\hline
\end{tabular}




\begin{tabular}{|c|c|c|c|}
\hline $\mathrm{H}$ & -8.252791 & 7.995231 & -4.566114 \\
\hline $\mathrm{H}$ & -6.257136 & 6.350501 & -3.359957 \\
\hline $\mathrm{H}$ & -7.804402 & 4.654646 & -3.027832 \\
\hline $\mathrm{H}$ & -9.763026 & 6.078375 & -4.863312 \\
\hline $\mathrm{H}$ & -8.964088 & 4.563062 & -5.151621 \\
\hline $\mathrm{H}$ & -9.402396 & 5.859321 & -1.603640 \\
\hline $\mathrm{H}$ & -7.843656 & 6.639114 & -1.562733 \\
\hline $\mathrm{H}$ & -9.152445 & 7.333392 & -2.508425 \\
\hline $\mathrm{H}$ & -11.234943 & 4.101822 & -4.434147 \\
\hline $\mathrm{H}$ & -10.161814 & 3.579350 & -3.151526 \\
\hline $\mathrm{H}$ & -11.057496 & 5.078299 & -2.995191 \\
\hline$N$ & -6.791003 & 5.554791 & -6.471692 \\
\hline C & -6.079039 & 4.805068 & -7.486926 \\
\hline C & -4.754681 & 5.444973 & -7.908168 \\
\hline $\mathrm{O}$ & -3.894153 & 4.728963 & -8.364366 \\
\hline C & -6.944630 & 4.429443 & -8.697814 \\
\hline C & -7.972902 & 3.318009 & -8.426847 \\
\hline C & -7.335488 & 1.999228 & -7.961437 \\
\hline$N$ & -8.248833 & 0.854884 & -7.954140 \\
\hline C & -9.058522 & 0.500921 & -6.954248 \\
\hline$N$ & -9.287055 & 1.345625 & -5.960773 \\
\hline$N$ & -9.639824 & -0.678275 & -6.960157 \\
\hline $\mathrm{H}$ & -7.450829 & 6.258333 & -6.720152 \\
\hline $\mathrm{H}$ & -5.738748 & 3.902187 & -7.010489 \\
\hline $\mathrm{H}$ & -7.463536 & 5.306941 & -9.073984 \\
\hline $\mathrm{H}$ & -6.271884 & 4.102933 & -9.483573 \\
\hline $\mathrm{H}$ & -8.709286 & 3.661953 & -7.705928 \\
\hline $\mathrm{H}$ & -8.516934 & 3.129205 & -9.347103 \\
\hline $\mathrm{H}$ & -6.525225 & 1.733545 & -8.628448 \\
\hline & -6.900295 & 2.090101 & -6.974335 \\
\hline
\end{tabular}




\begin{tabular}{|c|c|c|c|}
\hline $\mathrm{H}$ & -8.192204 & 0.217291 & -8.715211 \\
\hline $\mathrm{H}$ & -8.928770 & 2.268224 & -5.991742 \\
\hline $\mathrm{H}$ & -9.780791 & 1.079953 & -5.126944 \\
\hline $\mathrm{H}$ & -9.336528 & -1.443270 & -7.533272 \\
\hline $\mathrm{H}$ & -10.356064 & -0.895104 & -6.288737 \\
\hline$N$ & -4.571382 & 6.757518 & -7.721942 \\
\hline $\mathrm{H}$ & -5.298999 & 7.337833 & -7.376967 \\
\hline C & -3.257813 & 7.352386 & -8.011369 \\
\hline $\mathrm{H}$ & -2.929746 & 7.080324 & -9.002182 \\
\hline $\mathrm{H}$ & -3.360944 & 8.428528 & -7.952678 \\
\hline C & -2.193219 & 6.896491 & -7.027526 \\
\hline $\mathrm{O}$ & -1.061215 & 6.684714 & -7.383873 \\
\hline$N$ & -2.614922 & 6.781484 & -5.763570 \\
\hline C & -1.730110 & 6.466737 & -4.669021 \\
\hline C & -1.453232 & 4.979585 & -4.555254 \\
\hline 0 & -0.320391 & 4.598179 & -4.370159 \\
\hline C & -2.311053 & 7.055944 & -3.378256 \\
\hline C & -1.724824 & 6.346597 & -2.158742 \\
\hline C & -2.067587 & 8.571886 & -3.408271 \\
\hline $\mathrm{r}$ & -3.541599 & 7.067290 & -5.539318 \\
\hline $\mathrm{H}$ & -0.760260 & 6.905461 & -4.858082 \\
\hline $\mathrm{H}$ & -3.380917 & 6.879752 & -3.391464 \\
\hline $\mathrm{H}$ & -1.936497 & 6.883760 & -1.253143 \\
\hline $\mathrm{H}$ & -2.138679 & 5.348614 & -2.058455 \\
\hline $\mathrm{H}$ & -0.652297 & 6.271921 & -2.232441 \\
\hline $\mathrm{H}$ & -2.806351 & 9.102365 & -2.824136 \\
\hline $\mathrm{H}$ & -1.080458 & 8.810160 & -3.025697 \\
\hline $\mathrm{H}$ & -2.143649 & 8.955910 & -4.419238 \\
\hline$N$ & -2.480494 & 4.124610 & -4.674163 \\
\hline & -2.164161 & 2.717524 & -4.734154 \\
\hline
\end{tabular}




\begin{tabular}{|c|c|c|c|}
\hline C & -1.272670 & 2.436910 & -5.935556 \\
\hline $\mathrm{O}$ & -0.408422 & 1.595377 & -5.885649 \\
\hline C & -3.392490 & 1.790010 & -4.722420 \\
\hline C & -4.258321 & 1.918120 & -3.461106 \\
\hline C & -5.303866 & 0.801541 & -3.300541 \\
\hline$N$ & -4.771050 & -0.372108 & -2.635142 \\
\hline C & -5.548932 & -1.357423 & -2.405906 \\
\hline$N$ & -6.887078 & -1.413741 & -2.801361 \\
\hline$N$ & -5.164690 & -2.418476 & -1.664709 \\
\hline $\mathrm{H}$ & -3.415252 & 4.445085 & -4.798683 \\
\hline $\mathrm{H}$ & -1.555259 & 2.458807 & -3.879215 \\
\hline $\mathrm{H}$ & -4.000706 & 1.972948 & -5.607935 \\
\hline $\mathrm{H}$ & -3.015117 & 0.776604 & -4.803676 \\
\hline $\mathrm{H}$ & -3.623573 & 1.924969 & -2.587144 \\
\hline $\mathrm{H}$ & -4.777778 & 2.866653 & -3.485464 \\
\hline $\mathrm{H}$ & -6.144568 & 1.211814 & -2.738029 \\
\hline $\mathrm{H}$ & -5.696595 & 0.561343 & -4.295261 \\
\hline $\mathrm{H}$ & -7.071232 & -0.913658 & -3.641916 \\
\hline $\mathrm{H}$ & -7.273165 & -2.337867 & -2.840603 \\
\hline $\mathrm{H}$ & -4.229898 & -2.489266 & -1.295686 \\
\hline $\mathrm{H}$ & -5.683003 & -3.262984 & -1.740791 \\
\hline$N$ & -1.459779 & 3.193346 & -7.035076 \\
\hline $\mathrm{H}$ & -2.264018 & 3.767364 & -7.143528 \\
\hline C & -0.596738 & 2.980726 & -8.177683 \\
\hline $\mathrm{H}$ & -0.945588 & 3.613755 & -8.984380 \\
\hline $\mathrm{H}$ & -0.605399 & 1.950878 & -8.505996 \\
\hline C & 0.856772 & 3.335685 & -7.872241 \\
\hline $\mathrm{O}$ & 1.784264 & 2.709096 & -8.321503 \\
\hline$N$ & 1.007506 & 4.397742 & -7.065992 \\
\hline & 0.209314 & 4.934998 & -6.822421 \\
\hline
\end{tabular}




\begin{tabular}{|c|c|c|c|}
\hline C & 2.282349 & 4.880604 & -6.640357 \\
\hline $\mathrm{H}$ & 2.921372 & 5.136843 & -7.476466 \\
\hline $\mathrm{H}$ & 2.123623 & 5.776550 & -6.053644 \\
\hline C & 3.090814 & 3.928324 & -5.777631 \\
\hline $\mathrm{O}$ & 4.297674 & 4.051600 & -5.702333 \\
\hline$N$ & 2.428078 & 2.983428 & -5.101794 \\
\hline C & 3.113691 & 2.135740 & -4.164098 \\
\hline C & 4.318215 & 1.403883 & -4.753426 \\
\hline $\mathrm{O}$ & 5.285285 & 1.187363 & -4.050692 \\
\hline C & 2.103548 & 1.144773 & -3.576669 \\
\hline C & 2.725784 & 0.179901 & -2.582098 \\
\hline C & 1.699092 & -0.716628 & -1.914718 \\
\hline$N$ & 2.418546 & -1.721620 & -1.171285 \\
\hline C & 1.868896 & -2.613520 & -0.294751 \\
\hline$N$ & 0.545579 & -2.727780 & -0.299407 \\
\hline $\mathrm{O}$ & 2.621483 & -3.260498 & 0.423983 \\
\hline $\mathrm{H}$ & 1.432989 & 2.943196 & -5.156082 \\
\hline $\mathrm{H}$ & 3.544148 & 2.732272 & -3.367565 \\
\hline $\mathrm{H}$ & 1.325049 & 1.728995 & -3.095183 \\
\hline $\mathrm{H}$ & 1.622291 & 0.603034 & -4.383402 \\
\hline $\mathrm{H}$ & 3.452177 & -0.452627 & -3.080198 \\
\hline $\mathrm{H}$ & 3.270475 & 0.731223 & -1.817586 \\
\hline $\mathrm{H}$ & 1.040213 & -0.131031 & -1.277087 \\
\hline $\mathrm{H}$ & 1.076015 & -1.189557 & -2.668227 \\
\hline $\mathrm{H}$ & 3.371363 & -1.540542 & -0.959886 \\
\hline $\mathrm{H}$ & -0.050824 & -2.153350 & -0.847864 \\
\hline $\mathrm{H}$ & 0.049119 & -3.332430 & 0.338568 \\
\hline$N$ & 4.284556 & 0.951822 & -6.016429 \\
\hline $\mathrm{H}$ & 3.508524 & 1.169822 & -6.604581 \\
\hline C & 5.363401 & 0.116580 & -6.504631 \\
\hline
\end{tabular}




\begin{tabular}{|c|c|c|c|}
\hline $\mathrm{H}$ & 5.141461 & -0.157579 & -7.529028 \\
\hline $\mathrm{H}$ & 5.444896 & -0.791660 & -5.926553 \\
\hline C & 6.735556 & 0.755735 & -6.484745 \\
\hline $\mathrm{O}$ & 7.739593 & 0.088468 & -6.387531 \\
\hline$N$ & 6.766097 & 2.085339 & -6.608338 \\
\hline $\mathrm{H}$ & 5.914177 & 2.605595 & -6.566033 \\
\hline C & 8.008019 & 2.818112 & -6.552164 \\
\hline $\mathrm{H}$ & 8.713131 & 2.428925 & -7.273911 \\
\hline $\mathrm{H}$ & 7.808806 & 3.850147 & -6.810441 \\
\hline C & 8.752414 & 2.822116 & -5.214784 \\
\hline $\mathrm{O}$ & 9.896112 & 3.229690 & -5.165797 \\
\hline$N$ & 8.104621 & 2.336859 & -4.150538 \\
\hline C & 8.678169 & 2.169284 & -2.826204 \\
\hline C & 9.942813 & 1.301192 & -2.829377 \\
\hline $\mathrm{O}$ & 10.756994 & 1.383099 & -1.923027 \\
\hline C & 7.567816 & 1.496961 & -1.986522 \\
\hline C & 7.740156 & 1.380299 & -0.472963 \\
\hline C & 7.361160 & 2.645127 & 0.306255 \\
\hline C & 7.227282 & 2.380697 & 1.801701 \\
\hline$N$ & 8.556181 & 2.360637 & 2.481696 \\
\hline $\mathrm{O}$ & 10.975135 & 1.600057 & 0.910496 \\
\hline $\mathrm{H}$ & 10.836546 & 1.807037 & -0.009990 \\
\hline $\mathrm{H}$ & 11.440399 & 0.771211 & 0.877074 \\
\hline $\mathrm{H}$ & 7.154458 & 2.045838 & -4.264762 \\
\hline $\mathrm{H}$ & 8.976417 & 3.124846 & -2.412874 \\
\hline $\mathrm{H}$ & 6.650361 & 2.032676 & -2.188140 \\
\hline $\mathrm{H}$ & 7.412051 & 0.507304 & -2.401124 \\
\hline $\mathrm{H}$ & 7.067158 & 0.590988 & -0.153072 \\
\hline $\mathrm{H}$ & 8.736734 & 1.043884 & -0.217908 \\
\hline & 8.063206 & 3.458725 & 0.140984 \\
\hline
\end{tabular}




\begin{tabular}{|c|c|c|c|}
\hline $\mathrm{H}$ & 6.394783 & 2.996917 & -0.041234 \\
\hline $\mathrm{H}$ & 6.648879 & 3.148266 & 2.293578 \\
\hline $\mathrm{H}$ & 6.750010 & 1.430569 & 1.989859 \\
\hline $\mathrm{H}$ & 9.271622 & 1.890043 & 1.940201 \\
\hline $\mathrm{H}$ & 8.876580 & 3.331280 & 2.632790 \\
\hline $\mathrm{H}$ & 8.485699 & 1.887566 & 3.369657 \\
\hline$N$ & 10.062459 & 0.448217 & -3.837523 \\
\hline C & 11.108353 & -0.538444 & -4.063358 \\
\hline C & 12.484382 & 0.019845 & -4.433381 \\
\hline $\mathrm{O}$ & 13.443189 & -0.709995 & -4.317075 \\
\hline C & 10.690947 & -1.458251 & -5.232494 \\
\hline C & 9.531307 & -2.429423 & -4.966306 \\
\hline C & 9.094242 & -3.067810 & -6.288311 \\
\hline C & 9.926922 & -3.509712 & -3.960361 \\
\hline $\mathrm{H}$ & 9.324155 & 0.443221 & -4.509301 \\
\hline $\mathrm{H}$ & 11.270825 & -1.117051 & -3.164859 \\
\hline $\mathrm{H}$ & 10.438866 & -0.821597 & -6.076204 \\
\hline $\mathrm{H}$ & 11.566371 & -2.031151 & -5.516049 \\
\hline $\mathrm{H}$ & 8.682480 & -1.874320 & -4.578335 \\
\hline $\mathrm{H}$ & 8.279027 & -3.765900 & -6.124898 \\
\hline $\mathrm{H}$ & 8.753571 & -2.314937 & -6.987849 \\
\hline $\mathrm{H}$ & 9.913735 & -3.619496 & -6.743547 \\
\hline $\mathrm{H}$ & 9.117215 & -4.214699 & -3.808339 \\
\hline $\mathrm{H}$ & 10.784145 & -4.065934 & -4.325834 \\
\hline $\mathrm{H}$ & 10.185820 & -3.099001 & -2.989098 \\
\hline$N$ & 12.574048 & 1.261825 & -4.919242 \\
\hline C & 13.814758 & 1.840629 & -5.394045 \\
\hline C & 14.958271 & 2.031677 & -4.379289 \\
\hline $\mathrm{O}$ & 16.013181 & 2.413631 & -4.812566 \\
\hline C & 13.501679 & 3.164654 & -6.094152 \\
\hline
\end{tabular}




\begin{tabular}{|c|c|c|c|}
\hline $\mathrm{O}$ & 12.926293 & 4.090133 & -5.218451 \\
\hline $\mathrm{H}$ & 11.755408 & 1.831045 & -4.920136 \\
\hline $\mathrm{H}$ & 14.251680 & 1.170192 & -6.125856 \\
\hline $\mathrm{H}$ & 14.428563 & 3.575930 & -6.465265 \\
\hline $\mathrm{H}$ & 12.851126 & 2.965494 & -6.940836 \\
\hline $\mathrm{H}$ & 11.981521 & 4.069876 & -5.263840 \\
\hline$N$ & 14.792298 & 1.722328 & -3.074282 \\
\hline C & 15.930482 & 1.649333 & -2.180230 \\
\hline C & 16.805629 & 0.411917 & -2.408434 \\
\hline $\mathrm{O}$ & 17.938979 & 0.397411 & -2.001491 \\
\hline C & 15.502896 & 1.578241 & -0.709013 \\
\hline C & 14.629921 & 2.722246 & -0.198775 \\
\hline C & 14.253766 & 2.452893 & 1.258476 \\
\hline$N$ & 13.218520 & 3.336161 & 1.778853 \\
\hline C & 13.386550 & 4.493213 & 2.396111 \\
\hline $\mathrm{N}$ & 14.602013 & 4.980632 & 2.623990 \\
\hline $\mathrm{O}$ & 15.039639 & 7.450799 & 4.160770 \\
\hline $\mathrm{H}$ & 14.748831 & 8.335504 & 3.942971 \\
\hline $\mathrm{H}$ & 15.759562 & 7.540610 & 4.764607 \\
\hline $\mathrm{N}$ & 12.332054 & 5.174672 & 2.789703 \\
\hline $\mathrm{H}$ & 13.902607 & 1.418488 & -2.757875 \\
\hline $\mathrm{H}$ & 16.565136 & 2.505622 & -2.354128 \\
\hline $\mathrm{H}$ & 14.974155 & 0.643993 & -0.551634 \\
\hline $\mathrm{H}$ & 16.418127 & 1.522193 & -0.129001 \\
\hline $\mathrm{H}$ & 15.141677 & 3.676153 & -0.296016 \\
\hline $\mathrm{H}$ & 13.718456 & 2.803224 & -0.779860 \\
\hline $\mathrm{H}$ & 13.856413 & 1.451108 & 1.338634 \\
\hline $\mathrm{H}$ & 15.123908 & 2.485351 & 1.905478 \\
\hline $\mathrm{H}$ & 12.281509 & 3.028699 & 1.615279 \\
\hline $\mathrm{H}$ & 15.407527 & 4.489738 & 2.322747 \\
\hline
\end{tabular}




\begin{tabular}{|c|c|c|c|}
\hline $\mathrm{H}$ & 14.732423 & 5.837673 & 3.129132 \\
\hline $\mathrm{H}$ & 11.380330 & 4.880723 & 2.640349 \\
\hline $\mathrm{H}$ & 12.414666 & 6.024369 & 3.297594 \\
\hline $\mathrm{N}$ & 16.200237 & -0.648976 & -2.967239 \\
\hline$C$ & 16.873232 & -1.911333 & -3.155405 \\
\hline$C$ & 17.690170 & -1.897370 & -4.443379 \\
\hline $\mathrm{H}$ & 18.039344 & -0.909140 & -4.756666 \\
\hline 0 & 17.957088 & -2.875825 & -5.061927 \\
\hline$C$ & 15.871409 & -3.071161 & -3.142299 \\
\hline$C$ & 15.317297 & -3.394693 & -1.788203 \\
\hline $\mathrm{N}$ & 14.402693 & -2.609434 & -1.116482 \\
\hline $\mathrm{O}$ & 12.562337 & -0.372519 & -0.476237 \\
\hline $\mathrm{H}$ & 13.157748 & -1.001902 & -0.875328 \\
\hline $\mathrm{H}$ & 12.043072 & 0.012939 & -1.168529 \\
\hline$C$ & 15.595997 & -4.485851 & -1.040674 \\
\hline$C$ & 14.157981 & -3.226642 & -0.006971 \\
\hline $\mathrm{N}$ & 14.854271 & -4.364863 & 0.107724 \\
\hline $\mathrm{H}$ & 15.281178 & -0.557338 & -3.341499 \\
\hline $\mathrm{H}$ & 17.591357 & -2.028102 & -2.350123 \\
\hline $\mathrm{H}$ & 15.058108 & -2.844791 & -3.824032 \\
\hline $\mathrm{H}$ & 16.377719 & -3.945471 & -3.529396 \\
\hline $\mathrm{H}$ & 16.241966 & -5.318000 & -1.222201 \\
\hline $\mathrm{H}$ & 13.478569 & -2.885218 & 0.748276 \\
\hline $\mathrm{H}$ & 14.742258 & -5.043928 & 0.827194 \\
\hline 0 & -14.960527 & -2.469725 & 5.344596 \\
\hline $\mathrm{H}$ & -15.102336 & -1.594511 & 5.005496 \\
\hline $\mathrm{H}$ & -14.426088 & -2.371683 & 6.120447 \\
\hline 0 & -12.680208 & -1.808938 & 7.362317 \\
\hline $\mathrm{H}$ & -11.956090 & -2.343237 & 7.656457 \\
\hline П & -12.783232 & -1.130069 & 8.011127 \\
\hline
\end{tabular}




\begin{tabular}{|c|c|c|c|}
\hline 0 & -11.943052 & -0.969095 & 4.626111 \\
\hline $\mathrm{H}$ & -11.999187 & -1.156846 & 5.554799 \\
\hline $\mathrm{H}$ & -12.763460 & -0.538730 & 4.398975 \\
\hline $\mathrm{O}$ & -13.427561 & -1.434041 & 1.204091 \\
\hline $\mathrm{H}$ & -12.556946 & -1.039012 & 1.184793 \\
\hline $\mathrm{H}$ & -13.381570 & -2.183735 & 1.792252 \\
\hline $\mathrm{O}$ & -7.254553 & 2.154569 & 0.018657 \\
\hline $\mathrm{H}$ & -7.410356 & 2.204124 & 0.961019 \\
\hline $\mathrm{H}$ & -6.339914 & 1.929911 & -0.071088 \\
\hline $\mathrm{O}$ & -7.592412 & 2.119624 & 2.821497 \\
\hline $\mathrm{H}$ & -8.353679 & 1.648725 & 3.136834 \\
\hline $\mathrm{H}$ & -7.455797 & 2.865375 & 3.393781 \\
\hline $\mathrm{O}$ & -8.959903 & 0.258077 & -1.133245 \\
\hline $\mathrm{H}$ & -8.391808 & 0.940214 & -0.767266 \\
\hline $\mathrm{H}$ & -8.374413 & -0.427159 & -1.440636 \\
\hline $\mathrm{O}$ & -13.031259 & -1.860031 & -1.615205 \\
\hline $\mathrm{H}$ & -13.333741 & -1.922675 & -0.714345 \\
\hline $\mathrm{H}$ & -13.753684 & -2.059220 & -2.194057 \\
\hline $\mathrm{O}$ & -13.671463 & 1.576011 & 0.463493 \\
\hline $\mathrm{H}$ & -14.093183 & 0.744781 & 0.629069 \\
\hline $\mathrm{H}$ & -12.750601 & 1.404956 & 0.645641 \\
\hline $\mathrm{O}$ & -14.672397 & 5.278923 & 4.160493 \\
\hline $\mathrm{H}$ & -13.982154 & 5.830178 & 3.817334 \\
\hline $\mathrm{H}$ & -14.612989 & 5.395416 & 5.099982 \\
\hline $\mathrm{O}$ & -14.717613 & 2.679824 & 3.054362 \\
\hline $\mathrm{H}$ & -14.899455 & 3.558542 & 3.385524 \\
\hline $\mathrm{H}$ & -14.552860 & 2.728958 & 2.122264 \\
\hline $\mathrm{O}$ & -13.172071 & 0.927910 & -2.304205 \\
\hline $\mathrm{H}$ & -13.457425 & 1.424996 & -1.545266 \\
\hline $\mathrm{H}$ & -12.968144 & 0.059200 & -1.967183 \\
\hline
\end{tabular}




\begin{tabular}{|c|c|c|c|}
\hline $\mathrm{O}$ & -14.434016 & -0.889227 & -4.096995 \\
\hline $\mathrm{H}$ & -14.305837 & -0.064049 & -3.641581 \\
\hline $\mathrm{H}$ & -15.232280 & -0.825088 & -4.595505 \\
\hline 0 & -10.644895 & -3.038237 & -2.964691 \\
\hline $\mathrm{H}$ & -10.923718 & -2.629968 & -3.774432 \\
\hline $\mathrm{H}$ & -11.313554 & -2.811417 & -2.332035 \\
\hline $\mathrm{O}$ & -11.141332 & 0.223138 & 0.862571 \\
\hline $\mathrm{H}$ & -10.463164 & 0.115265 & 0.201670 \\
\hline $\mathrm{H}$ & -10.703109 & 0.478736 & 1.673257 \\
\hline O & -10.187929 & 0.963592 & 3.413774 \\
\hline $\mathrm{H}$ & -10.599986 & 0.280385 & 3.932086 \\
\hline $\mathrm{H}$ & -10.696201 & 1.758444 & 3.566935 \\
\hline O & -11.945023 & 3.112422 & 3.762167 \\
\hline $\mathrm{H}$ & -11.899126 & 4.055844 & 3.675573 \\
\hline $\mathrm{H}$ & -12.857440 & 2.879980 & 3.640466 \\
\hline 0 & -10.521877 & 0.898844 & -3.359850 \\
\hline $\mathrm{H}$ & -11.345589 & 1.219059 & -3.006754 \\
\hline $\mathrm{H}$ & -9.958729 & 0.697615 & -2.609779 \\
\hline 0 & -14.503463 & -0.058467 & 3.756269 \\
\hline $\mathrm{H}$ & -14.716174 & 0.866892 & 3.663925 \\
\hline $\mathrm{H}$ & -14.425442 & -0.410955 & 2.879894 \\
\hline 0 & -13.121954 & -3.336938 & 3.387866 \\
\hline $\mathrm{H}$ & -13.869905 & -3.201636 & 3.969829 \\
\hline $\mathrm{H}$ & -12.449569 & -2.774306 & 3.757953 \\
\hline 0 & -11.636768 & -1.169560 & -4.915529 \\
\hline $\mathrm{H}$ & -12.586803 & -1.181519 & -4.885835 \\
\hline $\mathrm{H}$ & -11.3466665 & -0.549056 & -4.2534 \\
\hline
\end{tabular}


R303cit MUTATION: NOMINAL CHARGES, Y266(0), E183(0) POTENTIAL: OmV

\begin{tabular}{|c|c|c|c|}
\hline $\mathrm{N}$ & 15.811987 & -6.063897 & 5.314056 \\
\hline $\mathrm{H}$ & 15.005750 & -5.858786 & 5.869538 \\
\hline C & 15.703106 & -5.466092 & 3.982355 \\
\hline C & 15.011770 & -6.415695 & 2.986877 \\
\hline 0 & 14.271932 & -5.998632 & 2.112517 \\
\hline C & 17.093375 & -5.116519 & 3.439914 \\
\hline $\mathrm{H}$ & 16.610099 & -5.712546 & 5.802433 \\
\hline $\mathrm{H}$ & 15.093416 & -4.571364 & 3.987180 \\
\hline $\mathrm{H}$ & 17.034516 & -4.722581 & 2.432342 \\
\hline $\mathrm{H}$ & 17.548103 & -4.355873 & 4.064725 \\
\hline $\mathrm{H}$ & 17.740892 & -5.986851 & 3.434534 \\
\hline $\mathrm{N}$ & 15.298586 & -7.710857 & 3.110135 \\
\hline $\mathrm{H}$ & 15.882089 & -7.969294 & 3.875166 \\
\hline C & 14.689227 & -8.734397 & 2.259151 \\
\hline $\mathrm{H}$ & 14.750118 & -8.455942 & 1.219324 \\
\hline $\mathrm{H}$ & 15.216488 & -9.668167 & 2.405900 \\
\hline C & 13.212818 & -8.922313 & 2.608213 \\
\hline $\mathrm{O}$ & 12.391403 & -9.181811 & 1.769230 \\
\hline$N$ & 12.881523 & -8.750874 & 3.902113 \\
\hline $\mathrm{H}$ & 13.599293 & -8.627004 & 4.576374 \\
\hline C & 11.499986 & -8.905480 & 4.363628 \\
\hline $\mathrm{H}$ & 11.496386 & -8.894461 & 5.445847 \\
\hline $\mathrm{H}$ & 11.072283 & -9.833972 & 4.017562 \\
\hline C & 10.606664 & -7.769565 & 3.848581 \\
\hline 0 & 9.463166 & -7.976763 & 3.547347 \\
\hline$N$ & 11.184934 & -6.556843 & 3.789943 \\
\hline C & 10.508735 & -5.396226 & 3.266180 \\
\hline
\end{tabular}




\begin{tabular}{|c|c|c|c|}
\hline C & 10.321283 & -5.436208 & 1.746623 \\
\hline 0 & 9.292961 & -5.003164 & 1.288125 \\
\hline C & 11.229132 & -4.126011 & 3.738713 \\
\hline 0 & 12.622781 & -4.239507 & 3.685649 \\
\hline $\mathrm{H}$ & 12.142285 & -6.457206 & 4.029248 \\
\hline $\mathrm{H}$ & 9.501137 & -5.362203 & 3.648581 \\
\hline $\mathrm{H}$ & 10.874504 & -3.279167 & 3.160749 \\
\hline $\mathrm{H}$ & 10.981460 & -3.952756 & 4.776768 \\
\hline $\mathrm{H}$ & 12.927445 & -4.406230 & 2.806495 \\
\hline$N$ & 11.300464 & -5.933899 & 0.968546 \\
\hline C & 11.043277 & -6.135289 & -0.454640 \\
\hline C & 9.933729 & -7.169723 & -0.661122 \\
\hline $\mathrm{O}$ & 9.205593 & -7.106529 & -1.622420 \\
\hline C & 12.340873 & -6.469959 & -1.248270 \\
\hline C & 12.619208 & -7.966964 & -1.432681 \\
\hline$C$ & 12.316198 & -5.773844 & -2.609987 \\
\hline $\mathrm{H}$ & 12.122861 & -6.318009 & 1.376748 \\
\hline $\mathrm{H}$ & 10.633314 & -5.214763 & -0.839894 \\
\hline $\mathrm{H}$ & 13.157265 & -6.042539 & -0.675981 \\
\hline $\mathrm{H}$ & 13.582257 & -8.092405 & -1.917656 \\
\hline $\mathrm{H}$ & 12.639765 & -8.513595 & -0.500230 \\
\hline $\mathrm{H}$ & 11.875062 & -8.425228 & -2.077011 \\
\hline $\mathrm{H}$ & 13.214629 & -6.009306 & -3.170381 \\
\hline $\mathrm{H}$ & 11.459597 & -6.095648 & -3.192097 \\
\hline $\mathrm{H}$ & 12.269939 & -4.697235 & -2.497540 \\
\hline $\mathrm{N}$ & 9.831339 & -8.117556 & 0.279877 \\
\hline $\mathrm{H}$ & 10.566330 & -8.215520 & 0.939380 \\
\hline$C$ & 8.884542 & -9.225591 & 0.196546 \\
\hline $\mathrm{H}$ & 9.259721 & -10.021441 & 0.828458 \\
\hline $\mathrm{H}$ & 8.822932 & -9.596883 & -0.81513 \\
\hline
\end{tabular}




\begin{tabular}{|c|c|c|c|}
\hline C & 7.466191 & -8.909913 & 0.617296 \\
\hline $\mathrm{O}$ & 6.563459 & -9.436300 & 0.007963 \\
\hline$N$ & 7.261555 & -8.077627 & 1.641684 \\
\hline C & 5.910133 & -7.614860 & 1.879577 \\
\hline C & 5.480697 & -6.671643 & 0.761453 \\
\hline $\mathrm{O}$ & 4.339514 & -6.713459 & 0.384756 \\
\hline C & 5.622610 & -7.056094 & 3.285621 \\
\hline C & 5.776985 & -8.153007 & 4.343732 \\
\hline C & 6.424100 & -5.809441 & 3.663982 \\
\hline $\mathrm{H}$ & 8.032151 & -7.747256 & 2.176536 \\
\hline $\mathrm{H}$ & 5.252883 & -8.459763 & 1.747362 \\
\hline $\mathrm{H}$ & 4.573773 & -6.776682 & 3.243801 \\
\hline $\mathrm{H}$ & 5.464977 & -7.782337 & 5.314863 \\
\hline $\mathrm{H}$ & 5.162350 & -9.015544 & 4.106806 \\
\hline $\mathrm{H}$ & 6.807162 & -8.483538 & 4.427004 \\
\hline $\mathrm{H}$ & 6.064210 & -5.414870 & 4.608470 \\
\hline $\mathrm{H}$ & 7.474050 & -6.045622 & 3.789505 \\
\hline $\mathrm{H}$ & 6.332613 & -5.023641 & 2.923243 \\
\hline$N$ & 6.369416 & -5.852603 & 0.173178 \\
\hline C & 5.919052 & -5.051594 & -0.953623 \\
\hline C & 5.531874 & -5.952799 & -2.134618 \\
\hline $\mathrm{O}$ & 4.547277 & -5.705407 & -2.790549 \\
\hline C & 6.967731 & -3.988483 & -1.338923 \\
\hline C & 7.144766 & -2.973407 & -0.195125 \\
\hline C & 6.560021 & -3.278846 & -2.634533 \\
\hline C & 8.415624 & -2.132018 & -0.313537 \\
\hline $\mathrm{H}$ & 7.307901 & -5.787383 & 0.496889 \\
\hline $\mathrm{H}$ & 4.997401 & -4.555940 & -0.679072 \\
\hline $\mathrm{H}$ & 7.916713 & -4.494995 & -1.501945 \\
\hline $\mathrm{H}$ & 6.268339 & -2.329489 & -0.161717 \\
\hline
\end{tabular}




\begin{tabular}{|c|c|c|c|}
\hline $\mathrm{H}$ & 7.177014 & -3.491583 & 0.753832 \\
\hline $\mathrm{H}$ & 7.224715 & -2.450918 & -2.844092 \\
\hline $\mathrm{H}$ & 6.589098 & -3.941761 & -3.489474 \\
\hline $\mathrm{H}$ & 5.549948 & -2.890577 & -2.558139 \\
\hline $\mathrm{H}$ & 8.501088 & -1.454358 & 0.529601 \\
\hline $\mathrm{H}$ & 9.294621 & -2.767008 & -0.313706 \\
\hline $\mathrm{H}$ & 8.435069 & -1.533196 & -1.217869 \\
\hline$N$ & 6.314468 & -7.007042 & -2.418208 \\
\hline $\mathrm{H}$ & 7.192921 & -7.113820 & -1.961461 \\
\hline C & 6.006173 & -7.872766 & -3.533949 \\
\hline $\mathrm{H}$ & 6.859015 & -8.522264 & -3.693131 \\
\hline $\mathrm{H}$ & 5.831682 & -7.304976 & -4.436126 \\
\hline C & 4.766767 & -8.745168 & -3.327817 \\
\hline $\mathrm{O}$ & 3.940451 & -8.850797 & -4.204678 \\
\hline$N$ & 4.677913 & -9.391187 & -2.159729 \\
\hline $\mathrm{H}$ & 5.389309 & -9.275280 & -1.471742 \\
\hline C & 3.506322 & -10.158937 & -1.810717 \\
\hline $\mathrm{H}$ & 3.282337 & -10.898499 & -2.565688 \\
\hline $\mathrm{H}$ & 3.708120 & -10.669311 & -0.876606 \\
\hline C & 2.255477 & -9.297031 & -1.655394 \\
\hline $\mathrm{O}$ & 1.186606 & -9.701469 & -2.061393 \\
\hline$N$ & 2.437859 & -8.092144 & -1.117479 \\
\hline C & 1.301238 & -7.181561 & -1.081027 \\
\hline C & 0.832358 & -6.784518 & -2.480004 \\
\hline $\mathrm{O}$ & -0.354425 & -6.727332 & -2.718646 \\
\hline C & 1.557608 & -5.871176 & -0.351131 \\
\hline $\mathrm{O}$ & 1.666550 & -6.083433 & 1.018962 \\
\hline $\mathrm{H}$ & 3.284514 & -7.822316 & -0.667940 \\
\hline $\mathrm{H}$ & 0.455967 & -7.674223 & -0.624156 \\
\hline & 2.446603 & -5.402590 & -0.745406 \\
\hline
\end{tabular}




\begin{tabular}{|c|c|c|c|}
\hline $\mathrm{H}$ & 0.708121 & -5.233380 & -0.567453 \\
\hline $\mathrm{H}$ & 2.005366 & -5.278480 & 1.384316 \\
\hline$N$ & 1.747642 & -6.457087 & -3.402413 \\
\hline C & 1.327376 & -6.056997 & -4.731587 \\
\hline C & 0.592123 & -7.199011 & -5.424613 \\
\hline $\mathrm{O}$ & -0.399409 & -6.972511 & -6.089652 \\
\hline C & 2.516615 & -5.514008 & -5.558564 \\
\hline C & 2.860130 & -4.099929 & -5.061671 \\
\hline C & 2.228138 & -5.522078 & -7.064903 \\
\hline C & 4.156170 & -3.532316 & -5.636859 \\
\hline $\mathrm{H}$ & 2.715822 & -6.429553 & -3.163695 \\
\hline $\mathrm{H}$ & 0.581157 & -5.278417 & -4.647505 \\
\hline $\mathrm{H}$ & 3.365679 & -6.166074 & -5.374684 \\
\hline $\mathrm{H}$ & 2.034152 & -3.434055 & -5.307937 \\
\hline $\mathrm{H}$ & 2.942457 & -4.114730 & -3.982560 \\
\hline $\mathrm{H}$ & 3.076034 & -5.128000 & -7.611616 \\
\hline $\mathrm{H}$ & 2.047386 & -6.522462 & -7.440017 \\
\hline $\mathrm{H}$ & 1.362178 & -4.913258 & -7.306361 \\
\hline $\mathrm{H}$ & 4.407045 & -2.602864 & -5.136454 \\
\hline $\mathrm{H}$ & 4.981894 & -4.219974 & -5.485276 \\
\hline $\mathrm{H}$ & 4.082574 & -3.322892 & -6.698895 \\
\hline$N$ & 1.066470 & -8.436456 & -5.262601 \\
\hline $\mathrm{H}$ & 1.903959 & -8.589886 & -4.741458 \\
\hline C & 0.311296 & -9.562967 & -5.751662 \\
\hline $\mathrm{H}$ & 0.197138 & -9.532664 & -6.825669 \\
\hline $\mathrm{H}$ & 0.842280 & -10.467377 & -5.481525 \\
\hline C & -1.094210 & -9.621495 & -5.160577 \\
\hline O & -2.047506 & -9.869825 & -5.866798 \\
\hline$N$ & -1.200902 & -9.404752 & -3.850754 \\
\hline$C$ & -2.505780 & -9.442079 & -3.231062 \\
\hline
\end{tabular}




\begin{tabular}{|c|c|c|c|}
\hline C & -3.436521 & -8.315636 & -3.688828 \\
\hline $\mathrm{O}$ & -4.631692 & -8.543545 & -3.747522 \\
\hline C & -2.409783 & -9.417849 & -1.698523 \\
\hline 0 & -3.652703 & -9.703242 & -1.116714 \\
\hline $\mathrm{H}$ & -0.387201 & -9.276761 & -3.286551 \\
\hline $\mathrm{H}$ & -3.016955 & -10.350401 & -3.519953 \\
\hline $\mathrm{H}$ & -1.715743 & -10.183252 & -1.377196 \\
\hline $\mathrm{H}$ & -2.030884 & -8.456667 & -1.366552 \\
\hline $\mathrm{H}$ & -4.344397 & -9.308365 & -1.625819 \\
\hline$N$ & -2.907718 & -7.121938 & -3.947207 \\
\hline C & -3.687861 & -6.001300 & -4.445128 \\
\hline C & -4.253921 & -6.312512 & -5.829036 \\
\hline $\mathrm{O}$ & -5.420071 & -6.089996 & -6.093073 \\
\hline C & -2.787662 & -4.740829 & -4.456753 \\
\hline C & -3.214561 & -3.632982 & -5.402415 \\
\hline C & -4.501208 & -3.105953 & -5.397751 \\
\hline C & -2.296328 & -3.118017 & -6.309044 \\
\hline C & -4.859339 & -2.106654 & -6.285188 \\
\hline C & -2.644778 & -2.104178 & -7.186759 \\
\hline C & -3.932271 & -1.599037 & -7.181051 \\
\hline $\mathrm{H}$ & -1.937117 & -6.982029 & -3.755264 \\
\hline $\mathrm{H}$ & -4.548470 & -5.838659 & -3.813345 \\
\hline $\mathrm{H}$ & -2.725777 & -4.368925 & -3.438637 \\
\hline $\mathrm{H}$ & -1.792292 & -5.051450 & -4.739275 \\
\hline $\mathrm{H}$ & -5.233482 & -3.489987 & -4.712426 \\
\hline $\mathrm{H}$ & -1.297894 & -3.517595 & -6.335629 \\
\hline $\mathrm{H}$ & -5.868782 & -1.734593 & -6.282756 \\
\hline $\mathrm{H}$ & -1.913743 & -1.719649 & -7.875569 \\
\hline $\mathrm{H}$ & -4.210348 & -0.821084 & -7.871226 \\
\hline$N$ & -3.409280 & -6.833648 & -6.719482 \\
\hline
\end{tabular}




\begin{tabular}{|c|c|c|c|}
\hline C & -3.877422 & -7.177754 & -8.042300 \\
\hline C & -4.992113 & -8.220776 & -7.981720 \\
\hline O & -5.954482 & -8.119815 & -8.699683 \\
\hline C & -2.740821 & -7.705605 & -8.921105 \\
\hline$S$ & -1.530090 & -6.452642 & -9.449271 \\
\hline $\mathrm{H}$ & -2.465870 & -7.023500 & -6.453047 \\
\hline $\mathrm{H}$ & -4.322751 & -6.311337 & -8.509184 \\
\hline $\mathrm{H}$ & -2.227295 & -8.523199 & -8.432740 \\
\hline $\mathrm{H}$ & -3.171697 & -8.084203 & -9.839009 \\
\hline $\mathrm{H}$ & -0.884940 & -6.322265 & -8.298675 \\
\hline$N$ & -4.829289 & -9.251610 & -7.129472 \\
\hline $\mathrm{H}$ & -3.980523 & -9.372875 & -6.619846 \\
\hline C & -5.811687 & -10.322426 & -7.162249 \\
\hline $\mathrm{H}$ & -5.990778 & -10.648701 & -8.176289 \\
\hline $\mathrm{H}$ & -5.413752 & -11.155295 & -6.593528 \\
\hline C & -7.189253 & -9.976452 & -6.575336 \\
\hline O & -8.202787 & -10.379393 & -7.077825 \\
\hline$N$ & -7.159766 & -9.266859 & -5.440989 \\
\hline C & -8.389918 & -8.950158 & -4.743239 \\
\hline C & -9.281417 & -7.964883 & -5.492732 \\
\hline O & -10.476759 & -7.986715 & -5.365030 \\
\hline C & -8.109551 & -8.426473 & -3.325710 \\
\hline C & -7.328256 & -7.108834 & -3.202788 \\
\hline C & -8.083707 & -5.804871 & -3.212528 \\
\hline $\mathrm{O}$ & -9.382894 & -5.905354 & -3.273801 \\
\hline $\mathrm{H}$ & -9.797889 & -5.046105 & -3.177912 \\
\hline O & -7.517431 & -4.744590 & -3.133842 \\
\hline $\mathrm{H}$ & -6.282254 & -9.009661 & -5.042295 \\
\hline $\mathrm{H}$ & -8.986906 & -9.848088 & -4.657777 \\
\hline$H$ & -9.059484 & -8.343522 & -2.818405 \\
\hline
\end{tabular}




\begin{tabular}{|c|c|c|c|}
\hline $\mathrm{H}$ & -7.537715 & -9.195093 & -2.816432 \\
\hline $\mathrm{H}$ & -6.797636 & -7.101987 & -2.256985 \\
\hline $\mathrm{H}$ & -6.564296 & -7.020411 & -3.957704 \\
\hline $\mathrm{N}$ & -8.647837 & -7.027884 & -6.226384 \\
\hline$C$ & -9.403915 & -6.017282 & -6.945316 \\
\hline$C$ & -10.395649 & -6.668173 & -7.903164 \\
\hline $\mathrm{H}$ & -10.166205 & -7.692806 & -8.204030 \\
\hline 0 & -11.349074 & -6.088089 & -8.315033 \\
\hline$C$ & -8.401504 & -5.085310 & -7.657832 \\
\hline 0 & -7.707016 & -4.339748 & -6.682817 \\
\hline$C$ & -9.046356 & -4.093491 & -8.615290 \\
\hline $\mathrm{H}$ & -7.680911 & -7.149608 & -6.429986 \\
\hline $\mathrm{H}$ & -9.989947 & -5.431685 & -6.247059 \\
\hline $\mathrm{H}$ & -7.693940 & -5.696475 & -8.210664 \\
\hline $\mathrm{H}$ & -6.940634 & -4.809013 & -6.372559 \\
\hline $\mathrm{H}$ & -8.279586 & -3.408383 & -8.959829 \\
\hline $\mathrm{H}$ & -9.485234 & -4.588514 & -9.470478 \\
\hline $\mathrm{H}$ & -9.829694 & -3.529784 & -8.120065 \\
\hline $\mathrm{N}$ & -12.647666 & -9.440103 & 3.241040 \\
\hline $\mathrm{H}$ & -12.898347 & -9.972873 & 2.435603 \\
\hline$C$ & -11.501621 & -8.556151 & 2.963498 \\
\hline$C$ & -10.512041 & -8.480381 & 4.105006 \\
\hline 0 & -9.397927 & -8.033106 & 3.908978 \\
\hline$C$ & -11.930212 & -7.101151 & 2.677307 \\
\hline 0 & -12.490136 & -6.641577 & 3.880507 \\
\hline$C$ & -12.926191 & -6.971009 & 1.531694 \\
\hline $\mathrm{H}$ & -13.442415 & -8.903620 & 3.525970 \\
\hline $\mathrm{H}$ & -10.947995 & -8.936515 & 2.116694 \\
\hline $\mathrm{H}$ & -11.034277 & -6.540372 & 2.439664 \\
\hline $\mathrm{H}$ & -12.532916 & -5.691736 & 3.8779 \\
\hline
\end{tabular}




\begin{tabular}{|c|c|c|c|}
\hline $\mathrm{H}$ & -13.128211 & -5.921188 & 1.347006 \\
\hline $\mathrm{H}$ & -12.547187 & -7.404494 & 0.613942 \\
\hline $\mathrm{H}$ & -13.868502 & -7.446589 & 1.776741 \\
\hline $\mathrm{N}$ & -10.896810 & -8.903383 & 5.300230 \\
\hline $\mathrm{H}$ & -11.809741 & -9.295757 & 5.353085 \\
\hline$C$ & -10.101206 & -8.807718 & 6.497020 \\
\hline $\mathrm{H}$ & -10.047376 & -7.788763 & 6.851158 \\
\hline $\mathrm{H}$ & -10.588786 & -9.394465 & 7.267177 \\
\hline$C$ & -8.650595 & -9.277564 & 6.416904 \\
\hline 0 & -7.823871 & -8.663640 & 7.044143 \\
\hline $\mathrm{N}$ & -8.315248 & -10.371080 & 5.707132 \\
\hline $\mathrm{H}$ & -9.017894 & -10.878963 & 5.221841 \\
\hline$C$ & -6.917801 & -10.769106 & 5.606705 \\
\hline $\mathrm{H}$ & -6.876480 & -11.765410 & 5.183885 \\
\hline $\mathrm{H}$ & -6.464933 & -10.796766 & 6.585472 \\
\hline$C$ & -6.061356 & -9.841470 & 4.724496 \\
\hline 0 & -4.887190 & -9.700855 & 4.950271 \\
\hline $\mathrm{N}$ & -6.741060 & -9.268556 & 3.737273 \\
\hline$C$ & -6.005690 & -8.317886 & 2.913195 \\
\hline$C$ & -5.987681 & -6.944049 & 3.572170 \\
\hline $\mathrm{O}$ & -5.019466 & -6.232993 & 3.444479 \\
\hline$C$ & -6.331144 & -8.310133 & 1.408023 \\
\hline$C$ & -7.540760 & -7.542144 & 0.923625 \\
\hline$C$ & -7.541335 & -6.149372 & 0.923395 \\
\hline$C$ & -8.632682 & -8.204595 & 0.380481 \\
\hline$C$ & -8.619201 & -5.440556 & 0.422036 \\
\hline$C$ & -9.703166 & -7.497413 & -0.142519 \\
\hline$C$ & -9.705833 & -6.113611 & -0.112789 \\
\hline $\mathrm{H}$ & -7.734119 & -9.291786 & 3.715097 \\
\hline H & -4.978522 & -8.634627 & 2.971353 \\
\hline
\end{tabular}




\begin{tabular}{|c|c|c|c|}
\hline $\mathrm{H}$ & -5.450405 & -7.903028 & 0.924040 \\
\hline $\mathrm{H}$ & -6.393890 & -9.345799 & 1.094746 \\
\hline $\mathrm{H}$ & -6.684969 & -5.613442 & 1.292739 \\
\hline $\mathrm{H}$ & -8.633818 & -9.280784 & 0.334884 \\
\hline $\mathrm{H}$ & -8.594671 & -4.365222 & 0.418132 \\
\hline $\mathrm{H}$ & -10.521641 & -8.025995 & -0.597627 \\
\hline $\mathrm{H}$ & -10.528382 & -5.565783 & -0.533868 \\
\hline$N$ & -7.027445 & -6.607658 & 4.340758 \\
\hline C & -6.989933 & -5.440679 & 5.182317 \\
\hline C & -5.951015 & -5.605925 & 6.291840 \\
\hline $\mathrm{O}$ & -5.406780 & -4.618758 & 6.737722 \\
\hline C & -8.405674 & -5.178018 & 5.737984 \\
\hline C & -8.653892 & -3.772714 & 6.242775 \\
\hline C & -8.610355 & -2.689775 & 5.365788 \\
\hline C & -8.970415 & -3.538527 & 7.573006 \\
\hline C & -8.852478 & -1.402212 & 5.811715 \\
\hline C & -9.225034 & -2.252004 & 8.024141 \\
\hline$C$ & -9.159031 & -1.180672 & 7.149193 \\
\hline $\mathrm{H}$ & -7.866336 & -7.141076 & 4.296945 \\
\hline $\mathrm{H}$ & -6.652454 & -4.586028 & 4.614222 \\
\hline $\mathrm{H}$ & -9.100321 & -5.387394 & 4.931164 \\
\hline $\mathrm{H}$ & -8.615106 & -5.893646 & 6.524150 \\
\hline $\mathrm{H}$ & -8.365527 & -2.848542 & 4.328962 \\
\hline $\mathrm{H}$ & -9.009290 & -4.360511 & 8.266398 \\
\hline $\mathrm{H}$ & -8.775936 & -0.578427 & 5.123902 \\
\hline $\mathrm{H}$ & -9.451863 & -2.087457 & 9.062924 \\
\hline $\mathrm{H}$ & -9.312184 & -0.179073 & 7.508953 \\
\hline$N$ & -5.665572 & -6.835936 & 6.713391 \\
\hline $\mathrm{H}$ & -6.283055 & -7.576812 & 6.477611 \\
\hline C & -4.629301 & -7.109633 & 7.683142 \\
\hline
\end{tabular}




\begin{tabular}{|c|c|c|c|}
\hline $\mathrm{H}$ & -4.689737 & -6.449326 & 8.535214 \\
\hline $\mathrm{H}$ & -4.765074 & -8.127200 & 8.027754 \\
\hline C & -3.214993 & -6.964580 & 7.124584 \\
\hline $\mathrm{O}$ & -2.362465 & -6.438607 & 7.800172 \\
\hline$N$ & -2.978486 & -7.435541 & 5.895767 \\
\hline C & -1.702198 & -7.208287 & 5.235206 \\
\hline C & -1.503304 & -5.719783 & 4.933584 \\
\hline $\mathrm{O}$ & -0.434088 & -5.201017 & 5.162215 \\
\hline C & -1.584272 & -8.089231 & 3.972900 \\
\hline C & -0.421501 & -7.656935 & 3.075148 \\
\hline C & -1.440956 & -9.563224 & 4.368458 \\
\hline $\mathrm{H}$ & -3.687449 & -7.949656 & 5.422200 \\
\hline $\mathrm{H}$ & -0.902276 & -7.453219 & 5.920307 \\
\hline $\mathrm{H}$ & -2.504420 & -7.968883 & 3.404427 \\
\hline $\mathrm{H}$ & -0.326909 & -8.348182 & 2.244844 \\
\hline $\mathrm{H}$ & -0.554881 & -6.668752 & 2.655582 \\
\hline $\mathrm{H}$ & 0.519034 & -7.662658 & 3.617241 \\
\hline $\mathrm{H}$ & -1.433675 & -10.187913 & 3.480848 \\
\hline $\mathrm{H}$ & -0.502044 & -9.723371 & 4.892898 \\
\hline $\mathrm{H}$ & -2.248959 & -9.902830 & 5.004003 \\
\hline$N$ & -2.546547 & -5.047006 & 4.432279 \\
\hline C & -2.412220 & -3.659895 & 4.030916 \\
\hline C & -2.312557 & -2.744475 & 5.250489 \\
\hline $\mathrm{O}$ & -1.473099 & -1.867330 & 5.280469 \\
\hline C & -3.567913 & -3.210495 & 3.126760 \\
\hline C & -3.633715 & -3.875210 & 1.739154 \\
\hline C & -2.448864 & -3.528633 & 0.833428 \\
\hline O & -1.348443 & -3.933164 & 1.205225 \\
\hline $\mathrm{O}$ & -2.695607 & -2.865465 & -0.198973 \\
\hline & -3.370288 & -5.543114 & 4.174300 \\
\hline
\end{tabular}




\begin{tabular}{|c|c|c|c|}
\hline $\mathrm{H}$ & -1.487647 & -3.543575 & 3.493605 \\
\hline $\mathrm{H}$ & -4.508981 & -3.382612 & 3.641977 \\
\hline $\mathrm{H}$ & -3.472734 & -2.135938 & 3.004823 \\
\hline $\mathrm{H}$ & -3.666500 & -4.950928 & 1.850853 \\
\hline $\mathrm{H}$ & -4.554946 & -3.560770 & 1.260069 \\
\hline$N$ & -3.170251 & -2.925742 & 6.267478 \\
\hline C & -2.974287 & -2.171410 & 7.485231 \\
\hline C & -1.646980 & -2.536353 & 8.159257 \\
\hline $\mathrm{O}$ & -1.006610 & -1.676658 & 8.725501 \\
\hline C & -4.115598 & -2.192800 & 8.511501 \\
\hline $\mathrm{O}$ & -4.341385 & -3.462658 & 9.047428 \\
\hline C & -5.389764 & -1.545075 & 7.982436 \\
\hline $\mathrm{H}$ & -3.828186 & -3.670672 & 6.237470 \\
\hline $\mathrm{H}$ & -2.840601 & -1.136870 & 7.208056 \\
\hline $\mathrm{H}$ & -3.746104 & -1.592162 & 9.335004 \\
\hline $\mathrm{H}$ & -4.800474 & -3.990394 & 8.410614 \\
\hline $\mathrm{H}$ & -6.136481 & -1.537002 & 8.767951 \\
\hline $\mathrm{H}$ & -5.201028 & -0.519707 & 7.679601 \\
\hline $\mathrm{H}$ & -5.793443 & -2.083929 & 7.135389 \\
\hline$N$ & -1.206549 & -3.796924 & 8.055904 \\
\hline $\mathrm{H}$ & -1.817723 & -4.537873 & 7.796708 \\
\hline C & 0.062188 & -4.144138 & 8.643552 \\
\hline $\mathrm{H}$ & 0.099596 & -3.914717 & 9.699595 \\
\hline $\mathrm{H}$ & 0.208001 & -5.208421 & 8.507489 \\
\hline C & 1.231615 & -3.408852 & 7.999780 \\
\hline $\mathrm{O}$ & 2.149485 & -2.994528 & 8.673355 \\
\hline$N$ & 1.195881 & -3.276215 & 6.665068 \\
\hline C & 2.299613 & -2.658816 & 5.971783 \\
\hline C & 2.327646 & -1.141441 & 6.129368 \\
\hline $\mathrm{O}$ & 3.403889 & -0.577093 & 6.129324 \\
\hline
\end{tabular}




\begin{tabular}{|c|c|c|c|}
\hline C & 2.355580 & -3.079035 & 4.492778 \\
\hline$S$ & 1.432122 & -2.063482 & 3.302752 \\
\hline $\mathrm{H}$ & 0.478485 & -3.740366 & 6.146609 \\
\hline $\mathrm{H}$ & 3.217282 & -2.985866 & 6.441584 \\
\hline $\mathrm{H}$ & 3.381601 & -3.009826 & 4.157443 \\
\hline $\mathrm{H}$ & 2.047747 & -4.112708 & 4.413307 \\
\hline $\mathrm{H}$ & 0.249061 & -2.079107 & 3.898112 \\
\hline$N$ & 1.174584 & -0.481707 & 6.279328 \\
\hline C & 1.228527 & 0.949825 & 6.498120 \\
\hline C & 1.800391 & 1.255515 & 7.870039 \\
\hline $\mathrm{O}$ & 2.395847 & 2.285750 & 8.083778 \\
\hline C & -0.091113 & 1.707767 & 6.235951 \\
\hline C & -1.159109 & 1.503768 & 7.321489 \\
\hline C & -0.592220 & 1.425527 & 4.814999 \\
\hline C & -2.454798 & 2.276063 & 7.065919 \\
\hline $\mathrm{H}$ & 0.305811 & -0.967311 & 6.210681 \\
\hline $\mathrm{H}$ & 1.956049 & 1.350919 & 5.807870 \\
\hline $\mathrm{H}$ & 0.200749 & 2.751891 & 6.284079 \\
\hline $\mathrm{H}$ & -1.379458 & 0.452035 & 7.442640 \\
\hline $\mathrm{H}$ & -0.752017 & 1.833238 & 8.273063 \\
\hline $\mathrm{H}$ & -1.342760 & 2.148921 & 4.523085 \\
\hline $\mathrm{H}$ & 0.220326 & 1.490467 & 4.097766 \\
\hline $\mathrm{H}$ & -1.029982 & 0.440460 & 4.725231 \\
\hline $\mathrm{H}$ & -3.102102 & 2.222166 & 7.936414 \\
\hline $\mathrm{H}$ & -2.260750 & 3.326698 & 6.863207 \\
\hline $\mathrm{H}$ & -3.006270 & 1.872691 & 6.223449 \\
\hline$N$ & 1.612303 & 0.307477 & 8.793116 \\
\hline $\mathrm{H}$ & 1.005984 & -0.457617 & 8.600248 \\
\hline C & 2.051429 & 0.495691 & 10.155553 \\
\hline $\mathrm{H}$ & 1.548493 & -0.239040 & 10.772753 \\
\hline
\end{tabular}




\begin{tabular}{|c|c|c|c|}
\hline $\mathrm{H}$ & 1.811237 & 1.485779 & 10.514377 \\
\hline C & 3.555334 & 0.327731 & 10.310887 \\
\hline $\mathrm{O}$ & 4.211958 & 1.094060 & 10.964528 \\
\hline$N$ & 4.099120 & -0.725840 & 9.665137 \\
\hline $\mathrm{H}$ & 3.514583 & -1.380496 & 9.191176 \\
\hline C & 5.528692 & -0.865960 & 9.622083 \\
\hline $\mathrm{H}$ & 5.768664 & -1.818866 & 9.163905 \\
\hline $\mathrm{H}$ & 5.960450 & -0.840893 & 10.612006 \\
\hline C & 6.223546 & 0.233873 & 8.822826 \\
\hline $\mathrm{O}$ & 7.341890 & 0.577654 & 9.119791 \\
\hline$N$ & 5.549411 & 0.718265 & 7.780935 \\
\hline C & 6.111205 & 1.873315 & 7.127100 \\
\hline C & 5.522793 & 3.174903 & 7.663004 \\
\hline $\mathrm{H}$ & 5.005725 & 3.105642 & 8.620349 \\
\hline $\mathrm{O}$ & 5.652608 & 4.210582 & 7.085251 \\
\hline C & 5.960156 & 1.802705 & 5.604558 \\
\hline C & 6.694008 & 0.644067 & 4.960117 \\
\hline C & 8.065147 & 0.455529 & 5.138927 \\
\hline C & 6.008233 & -0.250563 & 4.143552 \\
\hline C & 8.731421 & -0.581788 & 4.500667 \\
\hline C & 6.672692 & -1.285127 & 3.503641 \\
\hline C & 8.038127 & -1.451727 & 3.673651 \\
\hline $\mathrm{H}$ & 4.647519 & 0.377507 & 7.529041 \\
\hline $\mathrm{H}$ & 7.156838 & 1.902658 & 7.397750 \\
\hline $\mathrm{H}$ & 4.907873 & 1.733522 & 5.357041 \\
\hline $\mathrm{H}$ & 6.316005 & 2.745140 & 5.200827 \\
\hline $\mathrm{H}$ & 8.613397 & 1.100674 & 5.804907 \\
\hline $\mathrm{H}$ & 4.944291 & -0.154215 & 4.034227 \\
\hline $\mathrm{H}$ & 9.787813 & -0.713210 & 4.664437 \\
\hline & 6.118562 & -1.963078 & 2.878846 \\
\hline
\end{tabular}




\begin{tabular}{|c|c|c|c|}
\hline $\mathrm{H}$ & 8.543439 & -2.259629 & 3.175069 \\
\hline $\mathrm{N}$ & 12.357062 & 9.899479 & -1.293582 \\
\hline $\mathrm{H}$ & 11.607207 & 10.196712 & -1.887394 \\
\hline$C$ & 12.088942 & 8.559269 & -0.764141 \\
\hline$C$ & 11.059086 & 8.711851 & 0.348237 \\
\hline 0 & 10.074730 & 8.006776 & 0.384374 \\
\hline$C$ & 13.366957 & 7.907687 & -0.218484 \\
\hline$C$ & 14.448915 & 7.629383 & -1.266891 \\
\hline$S$ & 13.838112 & 6.541308 & -2.586471 \\
\hline$C$ & 15.314815 & 6.425848 & -3.623170 \\
\hline $\mathrm{H}$ & 13.177333 & 9.902767 & -1.864587 \\
\hline $\mathrm{H}$ & 11.631813 & 7.899043 & -1.490385 \\
\hline $\mathrm{H}$ & 13.795251 & 8.542722 & 0.551806 \\
\hline $\mathrm{H}$ & 13.085242 & 6.972969 & 0.253247 \\
\hline $\mathrm{H}$ & 14.824240 & 8.551708 & -1.697521 \\
\hline $\mathrm{H}$ & 15.289382 & 7.156142 & -0.770996 \\
\hline $\mathrm{H}$ & 15.068447 & 5.765136 & -4.440452 \\
\hline $\mathrm{H}$ & 15.588667 & 7.396632 & -4.016637 \\
\hline $\mathrm{H}$ & 16.147002 & 6.006346 & -3.072613 \\
\hline $\mathrm{N}$ & 11.263823 & 9.682568 & 1.256036 \\
\hline$C$ & 10.354863 & 9.848184 & 2.373039 \\
\hline$C$ & 9.021522 & 10.492385 & 1.990254 \\
\hline 0 & 8.040516 & 10.261618 & 2.651403 \\
\hline$C$ & 11.026786 & 10.610903 & 3.521095 \\
\hline$C$ & 12.223068 & 9.832010 & 4.036032 \\
\hline 0 & 13.349817 & 10.142999 & 3.708052 \\
\hline $\mathrm{N}$ & 11.953547 & 8.797517 & 4.839799 \\
\hline $\mathrm{H}$ & 12.066207 & 10.264347 & 1.156185 \\
\hline $\mathrm{H}$ & 10.076559 & 8.867743 & 2.726993 \\
\hline П & 11.381799 & 11.581837 & 3.19773 \\
\hline
\end{tabular}




\begin{tabular}{|c|c|c|c|}
\hline $\mathrm{H}$ & 10.297897 & 10.749469 & 4.309906 \\
\hline $\mathrm{H}$ & 12.719213 & 8.229685 & 5.126712 \\
\hline $\mathrm{H}$ & 11.044851 & 8.383851 & 4.869871 \\
\hline$N$ & 8.979602 & 11.276431 & 0.895672 \\
\hline $\mathrm{H}$ & 9.814963 & 11.438031 & 0.383786 \\
\hline C & 7.702859 & 11.687848 & 0.349739 \\
\hline $\mathrm{H}$ & 7.871252 & 12.437565 & -0.413280 \\
\hline $\mathrm{H}$ & 7.078839 & 12.115166 & 1.118155 \\
\hline C & 6.914954 & 10.532689 & -0.270426 \\
\hline O & 5.714316 & 10.596800 & -0.317902 \\
\hline$N$ & 7.616172 & 9.492344 & -0.766803 \\
\hline C & 6.948911 & 8.288608 & -1.232522 \\
\hline C & 6.439666 & 7.437884 & -0.059161 \\
\hline $\mathrm{O}$ & 5.347318 & 6.927296 & -0.128766 \\
\hline C & 7.837817 & 7.501380 & -2.224482 \\
\hline C & 8.120747 & 8.359475 & -3.472146 \\
\hline C & 7.185017 & 6.168296 & -2.608988 \\
\hline C & 9.209022 & 7.801308 & -4.391693 \\
\hline $\mathrm{H}$ & 8.577622 & 9.411286 & -0.537806 \\
\hline $\mathrm{H}$ & 6.046566 & 8.593243 & -1.741381 \\
\hline $\mathrm{H}$ & 8.780919 & 7.287723 & -1.727691 \\
\hline $\mathrm{H}$ & 7.195699 & 8.476142 & -4.033243 \\
\hline $\mathrm{H}$ & 8.412367 & 9.358347 & -3.163284 \\
\hline $\mathrm{H}$ & 7.812053 & 5.632504 & -3.310034 \\
\hline $\mathrm{H}$ & 7.034004 & 5.527673 & -1.748892 \\
\hline $\mathrm{H}$ & 6.216500 & 6.320636 & -3.074291 \\
\hline $\mathrm{H}$ & 9.390821 & 8.483976 & -5.215713 \\
\hline $\mathrm{H}$ & 10.148388 & 7.668414 & -3.861522 \\
\hline $\mathrm{H}$ & 8.940410 & 6.843166 & -4.820928 \\
\hline 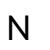 & 7.203398 & 7.330444 & 1.046884 \\
\hline
\end{tabular}




\begin{tabular}{|c|c|c|c|}
\hline$C$ & 6.716303 & 6.600319 & 2.206722 \\
\hline$C$ & 5.353578 & 7.146568 & 2.652028 \\
\hline 0 & 4.425488 & 6.414257 & 2.892053 \\
\hline$C$ & 7.674095 & 6.709649 & 3.402766 \\
\hline$C$ & 9.104395 & 6.193990 & 3.226964 \\
\hline $\mathrm{O}$ & 9.992370 & 6.862756 & 3.752067 \\
\hline O & 9.292833 & 5.124963 & 2.596386 \\
\hline $\mathrm{H}$ & 8.158666 & 7.611408 & 1.013922 \\
\hline $\mathrm{H}$ & 6.541785 & 5.566946 & 1.948613 \\
\hline $\mathrm{H}$ & 7.741694 & 7.736583 & 3.731974 \\
\hline $\mathrm{H}$ & 7.235388 & 6.142974 & 4.221632 \\
\hline $\mathrm{N}$ & 5.277230 & 8.484587 & 2.791054 \\
\hline $\mathrm{H}$ & 6.090069 & 9.042301 & 2.645414 \\
\hline$C$ & 4.068208 & 9.117886 & 3.256706 \\
\hline $\mathrm{H}$ & 4.278351 & 10.166900 & 3.426882 \\
\hline $\mathrm{H}$ & 3.732133 & 8.691443 & 4.189078 \\
\hline$C$ & 2.907686 & 9.043868 & 2.273864 \\
\hline $\mathrm{O}$ & 1.766416 & 9.169049 & 2.654824 \\
\hline $\mathrm{N}$ & 3.243034 & 8.870134 & 0.993151 \\
\hline $\mathrm{H}$ & 4.203026 & 8.855292 & 0.746156 \\
\hline$C$ & 2.290477 & 8.921686 & -0.072058 \\
\hline $\mathrm{H}$ & 2.826773 & 9.122092 & -0.991279 \\
\hline $\mathrm{H}$ & 1.591938 & 9.733605 & 0.078476 \\
\hline$C$ & 1.416146 & 7.678398 & -0.300637 \\
\hline O & 0.370924 & 7.783061 & -0.880569 \\
\hline $\mathrm{N}$ & 1.916743 & 6.544070 & 0.223913 \\
\hline$C$ & 1.128936 & 5.356414 & 0.454411 \\
\hline$C$ & 0.320164 & 5.469890 & 1.750471 \\
\hline $\mathrm{O}$ & -0.877636 & 5.311962 & 1.731128 \\
\hline$c$ & 2.017953 & 4.113775 & 0.461532 \\
\hline
\end{tabular}




\begin{tabular}{|c|c|c|c|}
\hline $\mathrm{H}$ & 2.820270 & 6.597875 & 0.641250 \\
\hline $\mathrm{H}$ & 0.393349 & 5.276716 & -0.329298 \\
\hline $\mathrm{H}$ & 1.430600 & 3.227883 & 0.675280 \\
\hline $\mathrm{H}$ & 2.481063 & 3.998832 & -0.511471 \\
\hline $\mathrm{H}$ & 2.803071 & 4.192480 & 1.206870 \\
\hline$N$ & 0.964323 & 5.731963 & 2.904258 \\
\hline C & 0.241257 & 5.544979 & 4.153640 \\
\hline C & -0.896347 & 6.549330 & 4.317259 \\
\hline $\mathrm{O}$ & -1.917255 & 6.224071 & 4.884868 \\
\hline C & 1.166726 & 5.488477 & 5.393997 \\
\hline C & 1.735929 & 6.845831 & 5.833538 \\
\hline C & 2.272888 & 4.443656 & 5.181227 \\
\hline C & 2.581023 & 6.769172 & 7.107110 \\
\hline $\mathrm{H}$ & 1.954546 & 5.837634 & 2.917427 \\
\hline $\mathrm{H}$ & -0.266853 & 4.591985 & 4.099810 \\
\hline $\mathrm{H}$ & 0.519604 & 5.137637 & 6.192821 \\
\hline $\mathrm{H}$ & 2.331136 & 7.263379 & 5.027691 \\
\hline $\mathrm{H}$ & 0.920372 & 7.541352 & 6.006076 \\
\hline $\mathrm{H}$ & 2.651501 & 4.091706 & 6.130101 \\
\hline $\mathrm{H}$ & 1.894626 & 3.582821 & 4.639752 \\
\hline $\mathrm{H}$ & 3.106505 & 4.853063 & 4.620106 \\
\hline $\mathrm{H}$ & 2.870810 & 7.766867 & 7.424666 \\
\hline $\mathrm{H}$ & 2.019585 & 6.316766 & 7.919492 \\
\hline $\mathrm{H}$ & 3.485835 & 6.189742 & 6.970471 \\
\hline $\mathrm{N}$ & -0.716685 & 7.787511 & 3.835132 \\
\hline $\mathrm{H}$ & 0.152658 & 8.048162 & 3.421109 \\
\hline$C$ & -1.720054 & 8.796192 & 4.046304 \\
\hline $\mathrm{H}$ & -1.342853 & 9.736264 & 3.662289 \\
\hline $\mathrm{H}$ & -1.935887 & 8.924681 & 5.097625 \\
\hline$C$ & -3.065581 & 8.479544 & 3.399396 \\
\hline
\end{tabular}




\begin{tabular}{|c|c|c|c|}
\hline $\mathrm{O}$ & -4.073417 & 8.668598 & 4.041715 \\
\hline$N$ & -3.093200 & 7.970199 & 2.153867 \\
\hline C & -4.428845 & 7.829750 & 1.574117 \\
\hline C & -5.119888 & 6.531579 & 1.954580 \\
\hline $\mathrm{O}$ & -6.331916 & 6.496599 & 1.963569 \\
\hline C & -4.228965 & 8.065691 & 0.071098 \\
\hline C & -3.000308 & 8.981249 & 0.060560 \\
\hline C & -2.115385 & 8.352627 & 1.124114 \\
\hline $\mathrm{H}$ & -5.072997 & 8.599181 & 1.972961 \\
\hline $\mathrm{H}$ & -4.025101 & 7.135806 & -0.444113 \\
\hline $\mathrm{H}$ & -5.111277 & 8.508861 & -0.373429 \\
\hline $\mathrm{H}$ & -2.497549 & 9.046621 & -0.886543 \\
\hline $\mathrm{H}$ & -3.289238 & 9.985412 & 0.363674 \\
\hline $\mathrm{H}$ & -1.607693 & 7.479019 & 0.756282 \\
\hline $\mathrm{H}$ & -1.373518 & 9.032202 & 1.512033 \\
\hline$N$ & -4.359732 & 5.481725 & 2.293074 \\
\hline C & -4.968660 & 4.290482 & 2.860305 \\
\hline C & -5.695395 & 4.615970 & 4.168757 \\
\hline 0 & -6.774949 & 4.123481 & 4.413715 \\
\hline$C$ & -3.932482 & 3.184798 & 3.151335 \\
\hline$C$ & -3.496027 & 2.295837 & 2.002336 \\
\hline$C$ & -3.988137 & 0.999691 & 1.889907 \\
\hline$C$ & -2.508084 & 2.680869 & 1.103534 \\
\hline$C$ & -3.492204 & 0.107074 & 0.953795 \\
\hline$C$ & -1.989938 & 1.797109 & 0.176041 \\
\hline $\mathrm{C}$ & -2.445702 & 0.485742 & 0.116018 \\
\hline 0 & -1.849363 & -0.353492 & -0.732860 \\
\hline $\mathrm{H}$ & -3.368518 & 5.592084 & 2.319685 \\
\hline $\mathrm{H}$ & -5.729044 & 3.921346 & 2.187998 \\
\hline $\mathrm{H}$ & -3.065831 & 3.649815 & 3.611252 \\
\hline
\end{tabular}




\begin{tabular}{|c|c|c|c|}
\hline $\mathrm{H}$ & -4.368754 & 2.545903 & 3.911372 \\
\hline $\mathrm{H}$ & -4.762663 & 0.664525 & 2.561021 \\
\hline $\mathrm{H}$ & -2.103055 & 3.670692 & 1.145898 \\
\hline $\mathrm{H}$ & -3.880490 & -0.889505 & 0.883567 \\
\hline $\mathrm{H}$ & -1.204707 & 2.103109 & -0.492680 \\
\hline $\mathrm{H}$ & -2.176898 & -1.255360 & -0.634712 \\
\hline$N$ & -5.068854 & 5.423468 & 5.041382 \\
\hline $\mathrm{H}$ & -4.141332 & 5.742582 & 4.856208 \\
\hline C & -5.686618 & 5.770323 & 6.300796 \\
\hline $\mathrm{H}$ & -4.918552 & 6.163048 & 6.956485 \\
\hline $\mathrm{H}$ & -6.129316 & 4.902435 & 6.763777 \\
\hline$C$ & -6.788528 & 6.820077 & 6.167600 \\
\hline $\mathrm{O}$ & -7.836106 & 6.718951 & 6.765161 \\
\hline$N$ & -6.514331 & 7.852000 & 5.364461 \\
\hline $\mathrm{H}$ & -5.623702 & 7.926344 & 4.922486 \\
\hline C & -7.462506 & 8.954972 & 5.142173 \\
\hline $\mathrm{H}$ & -7.002497 & 9.628543 & 4.429907 \\
\hline $\mathrm{H}$ & -7.649073 & 9.497015 & 6.058385 \\
\hline C & -8.819936 & 8.541166 & 4.615540 \\
\hline $\mathrm{O}$ & -9.813855 & 9.115377 & 4.986428 \\
\hline$N$ & -8.852379 & 7.540652 & 3.717576 \\
\hline C & -10.112923 & 7.117423 & 3.163297 \\
\hline C & -10.956051 & 6.267129 & 4.105949 \\
\hline $\mathrm{O}$ & -12.050357 & 5.897268 & 3.714210 \\
\hline C & -9.913370 & 6.383780 & 1.818754 \\
\hline $\mathrm{O}$ & -11.149346 & 6.316142 & 1.133997 \\
\hline C & -9.298352 & 4.999363 & 1.970431 \\
\hline $\mathrm{H}$ & -7.999608 & 7.135850 & 3.391528 \\
\hline $\mathrm{H}$ & -10.719956 & 7.994171 & 2.974885 \\
\hline & -9.258635 & 7.001762 & 1.216652 \\
\hline
\end{tabular}




\begin{tabular}{|c|c|c|c|}
\hline $\mathrm{H}$ & -11.830240 & 6.204142 & 1.788127 \\
\hline $\mathrm{H}$ & -9.128021 & 4.561865 & 0.993415 \\
\hline $\mathrm{H}$ & -8.347178 & 5.036425 & 2.479380 \\
\hline $\mathrm{H}$ & -9.961743 & 4.344429 & 2.521080 \\
\hline$N$ & -10.474378 & 5.976392 & 5.310489 \\
\hline C & -11.318450 & 5.358173 & 6.316616 \\
\hline C & -12.342330 & 6.320807 & 6.925022 \\
\hline $\mathrm{O}$ & -13.306537 & 5.870352 & 7.487333 \\
\hline C & -10.482005 & 4.742649 & 7.446114 \\
\hline C & -9.459980 & 3.686226 & 7.004058 \\
\hline C & -8.698714 & 3.171522 & 8.229059 \\
\hline$C$ & -10.096671 & 2.529348 & 6.233115 \\
\hline $\mathrm{H}$ & -9.563170 & 6.281028 & 5.577938 \\
\hline $\mathrm{H}$ & -11.915105 & 4.596306 & 5.840341 \\
\hline $\mathrm{H}$ & -9.956035 & 5.538145 & 7.964604 \\
\hline $\mathrm{H}$ & -11.183572 & 4.307975 & 8.151692 \\
\hline $\mathrm{H}$ & -8.738158 & 4.156288 & 6.347875 \\
\hline $\mathrm{H}$ & -7.930307 & 2.460054 & 7.939807 \\
\hline $\mathrm{H}$ & -8.214492 & 3.987102 & 8.756684 \\
\hline $\mathrm{H}$ & -9.367011 & 2.675056 & 8.930446 \\
\hline $\mathrm{H}$ & -9.338052 & 1.807621 & 5.949355 \\
\hline $\mathrm{H}$ & -10.848628 & 2.019476 & 6.832613 \\
\hline $\mathrm{H}$ & -10.572528 & 2.860979 & 5.317619 \\
\hline$N$ & -12.070920 & 7.640112 & 6.858893 \\
\hline C & -13.023350 & 8.608950 & 7.310968 \\
\hline C & -13.928471 & 9.134993 & 6.219267 \\
\hline $\mathrm{H}$ & -14.684838 & 9.848831 & 6.571524 \\
\hline $\mathrm{O}$ & -13.853372 & 8.842805 & 5.072412 \\
\hline $\mathrm{H}$ & -11.329212 & 7.966051 & 6.279992 \\
\hline $\mathrm{H}$ & -13.640194 & 8.171312 & 8.085705 \\
\hline
\end{tabular}




\begin{tabular}{|c|c|c|c|}
\hline $\mathrm{H}$ & -12.510341 & 9.462456 & 7.748699 \\
\hline$N$ & -11.803319 & 10.245201 & -2.923053 \\
\hline $\mathrm{H}$ & -11.555021 & 10.454220 & -1.978245 \\
\hline C & -12.081744 & 8.817744 & -3.090505 \\
\hline C & -11.659906 & 8.509079 & -4.520736 \\
\hline $\mathrm{O}$ & -10.586906 & 8.014797 & -4.768819 \\
\hline C & -13.502304 & 8.355143 & -2.702014 \\
\hline C & -13.767855 & 8.659113 & -1.216178 \\
\hline C & -13.696579 & 6.866364 & -3.016239 \\
\hline C & -15.188580 & 8.343963 & -0.745757 \\
\hline $\mathrm{H}$ & -12.599529 & 10.806940 & -3.153309 \\
\hline $\mathrm{H}$ & -11.371313 & 8.276768 & -2.479770 \\
\hline $\mathrm{H}$ & -14.225833 & 8.930594 & -3.285990 \\
\hline $\mathrm{H}$ & -13.053850 & 8.105173 & -0.609878 \\
\hline $\mathrm{H}$ & -13.579361 & 9.712197 & -1.030206 \\
\hline $\mathrm{H}$ & -14.722396 & 6.562690 & -2.851981 \\
\hline $\mathrm{H}$ & -13.456228 & 6.627238 & -4.047920 \\
\hline $\mathrm{H}$ & -13.064529 & 6.255522 & -2.379467 \\
\hline $\mathrm{H}$ & -15.324903 & 8.678460 & 0.276578 \\
\hline $\mathrm{H}$ & -15.929526 & 8.848974 & -1.360356 \\
\hline $\mathrm{H}$ & -15.402746 & 7.281845 & -0.768103 \\
\hline$N$ & -12.483337 & 8.899582 & -5.524731 \\
\hline $\mathrm{H}$ & -13.418222 & 9.146350 & -5.296968 \\
\hline C & -12.227292 & 8.456234 & -6.878662 \\
\hline $\mathrm{H}$ & -12.256120 & 7.377532 & -6.961182 \\
\hline $\mathrm{H}$ & -12.995244 & 8.869968 & -7.521675 \\
\hline C & -10.868344 & 8.883357 & -7.439409 \\
\hline $\mathrm{O}$ & -10.242872 & 8.163305 & -8.162683 \\
\hline$N$ & -10.469649 & 10.144592 & -7.114873 \\
\hline $\mathrm{H}$ & -11.026873 & 10.654117 & -6.469800 \\
\hline
\end{tabular}




\begin{tabular}{|c|c|c|c|}
\hline C & -9.215260 & 10.723366 & -7.569981 \\
\hline $\mathrm{H}$ & -9.108942 & 10.597982 & -8.636948 \\
\hline $\mathrm{H}$ & -9.221439 & 11.779500 & -7.332374 \\
\hline C & -7.977128 & 10.080286 & -6.928057 \\
\hline $\mathrm{O}$ & -7.055078 & 9.673747 & -7.578108 \\
\hline$N$ & -7.952448 & 10.083289 & -5.560306 \\
\hline $\mathrm{H}$ & -8.784216 & 10.322256 & -5.071871 \\
\hline C & -6.773002 & 9.726201 & -4.778822 \\
\hline $\mathrm{H}$ & -5.891433 & 10.166685 & -5.217771 \\
\hline $\mathrm{H}$ & -6.899798 & 10.121366 & -3.777930 \\
\hline C & -6.499696 & 8.237642 & -4.664834 \\
\hline $\mathrm{O}$ & -5.353052 & 7.857152 & -4.702496 \\
\hline$N$ & -7.520102 & 7.383617 & -4.468411 \\
\hline C & -7.193719 & 6.020597 & -4.070931 \\
\hline C & -6.607821 & 5.203961 & -5.215411 \\
\hline 0 & -5.793724 & 4.344706 & -4.977393 \\
\hline$C$ & -8.330608 & 5.263700 & -3.343414 \\
\hline$C$ & -9.503545 & 4.859533 & -4.253385 \\
\hline$C$ & -8.760118 & 6.052051 & -2.101336 \\
\hline$C$ & -10.585309 & 4.039525 & -3.548048 \\
\hline $\mathrm{H}$ & -8.464859 & 7.707284 & -4.462119 \\
\hline $\mathrm{H}$ & -6.371742 & 6.080033 & -3.372434 \\
\hline $\mathrm{H}$ & -7.856604 & 4.348836 & -2.997457 \\
\hline $\mathrm{H}$ & -9.952508 & 5.739093 & -4.699410 \\
\hline $\mathrm{H}$ & -9.114570 & 4.259714 & -5.071975 \\
\hline $\mathrm{H}$ & -9.409067 & 5.458198 & -1.472546 \\
\hline $\mathrm{H}$ & -7.898088 & 6.329086 & -1.502572 \\
\hline $\mathrm{H}$ & -9.295376 & 6.957053 & -2.366296 \\
\hline $\mathrm{H}$ & -11.337793 & 3.721796 & -4.263100 \\
\hline $\mathrm{H}$ & -10.175665 & 3.144314 & -3.095498 \\
\hline
\end{tabular}




\begin{tabular}{|c|c|c|c|}
\hline $\mathrm{H}$ & -11.090765 & 4.609942 & -2.777743 \\
\hline$N$ & -7.018956 & 5.442207 & -6.479222 \\
\hline C & -6.375230 & 4.738827 & -7.568791 \\
\hline C & -4.984277 & 5.280357 & -7.895096 \\
\hline $\mathrm{O}$ & -4.139657 & 4.532989 & -8.327171 \\
\hline C & -7.270416 & 4.676722 & -8.814215 \\
\hline C & -8.352936 & 3.596343 & -8.697094 \\
\hline C & -7.763189 & 2.191292 & -8.845627 \\
\hline$N$ & -8.724080 & 1.117248 & -8.613085 \\
\hline C & -9.051315 & 0.591755 & -7.434458 \\
\hline$N$ & -8.560123 & 1.049910 & -6.301582 \\
\hline$N$ & -9.911964 & -0.415168 & -7.387958 \\
\hline $\mathrm{H}$ & -7.674880 & 6.169605 & -6.658059 \\
\hline $\mathrm{H}$ & -6.161660 & 3.742131 & -7.220149 \\
\hline $\mathrm{H}$ & -7.732397 & 5.644394 & -8.985578 \\
\hline $\mathrm{H}$ & -6.645587 & 4.472954 & -9.678338 \\
\hline $\mathrm{H}$ & -8.869705 & 3.689209 & -7.746735 \\
\hline $\mathrm{H}$ & -9.100904 & 3.742865 & -9.469663 \\
\hline $\mathrm{H}$ & -7.394494 & 2.054531 & -9.853825 \\
\hline $\mathrm{H}$ & -6.908313 & 2.038464 & -8.199403 \\
\hline $\mathrm{H}$ & -9.078991 & 0.649826 & -9.414654 \\
\hline $\mathrm{H}$ & -7.907183 & 1.796195 & -6.284347 \\
\hline $\mathrm{H}$ & -8.972179 & 0.764234 & -5.419823 \\
\hline $\mathrm{H}$ & -10.240826 & -0.810670 & -8.238036 \\
\hline $\mathrm{H}$ & -9.855844 & -1.045558 & -6.600293 \\
\hline $\mathrm{N}$ & -4.773232 & 6.580669 & -7.681188 \\
\hline $\mathrm{H}$ & -5.511241 & 7.160217 & -7.356719 \\
\hline$C$ & -3.479244 & 7.213377 & -7.976907 \\
\hline $\mathrm{H}$ & -3.148364 & 6.956564 & -8.971586 \\
\hline $\mathrm{H}$ & -3.613098 & 8.285337 & -7.910482 \\
\hline
\end{tabular}




\begin{tabular}{|c|c|c|c|}
\hline$C$ & -2.396876 & 6.780364 & -7.001585 \\
\hline O & -1.260917 & 6.604381 & -7.364954 \\
\hline $\mathrm{N}$ & -2.810535 & 6.641800 & -5.737302 \\
\hline$C$ & -1.911758 & 6.357890 & -4.645441 \\
\hline$C$ & -1.587278 & 4.880549 & -4.526083 \\
\hline $\mathrm{O}$ & -0.443484 & 4.536072 & -4.336778 \\
\hline$C$ & -2.507278 & 6.930903 & -3.353422 \\
\hline$C$ & -1.895038 & 6.244968 & -2.133528 \\
\hline$C$ & -2.313976 & 8.453548 & -3.384154 \\
\hline $\mathrm{H}$ & -3.744900 & 6.899987 & -5.510563 \\
\hline $\mathrm{H}$ & -0.957172 & 6.827155 & -4.838964 \\
\hline $\mathrm{H}$ & -3.571250 & 6.720637 & -3.363244 \\
\hline $\mathrm{H}$ & -2.125525 & 6.776768 & -1.229151 \\
\hline $\mathrm{H}$ & -2.272396 & 5.232875 & -2.030859 \\
\hline $\mathrm{H}$ & -0.820723 & 6.209180 & -2.208350 \\
\hline $\mathrm{H}$ & -3.059981 & 8.957718 & -2.785981 \\
\hline $\mathrm{H}$ & -1.329149 & 8.722952 & -3.016457 \\
\hline $\mathrm{H}$ & -2.419273 & 8.837067 & -4.392592 \\
\hline $\mathrm{N}$ & -2.587924 & 3.993530 & -4.636211 \\
\hline$C$ & -2.233138 & 2.594004 & -4.706746 \\
\hline$C$ & -1.337556 & 2.351590 & -5.914538 \\
\hline $\mathrm{O}$ & -0.434867 & 1.551922 & -5.868306 \\
\hline$C$ & -3.450208 & 1.654090 & -4.690641 \\
\hline$C$ & -4.290911 & 1.760634 & -3.410594 \\
\hline$C$ & -5.398685 & 0.697148 & -3.305987 \\
\hline $\mathrm{N}$ & -4.926102 & -0.476639 & -2.609323 \\
\hline$C$ & -5.666337 & -1.493477 & -2.480364 \\
\hline $\mathrm{N}$ & -6.957043 & -1.636130 & -3.006573 \\
\hline $\mathrm{N}$ & -5.305663 & -2.545214 & -1.692861 \\
\hline 11 & -3.525271 & 4.286172 & -4.803538 \\
\hline
\end{tabular}




\begin{tabular}{|c|c|c|c|}
\hline $\mathrm{H}$ & -1.612208 & 2.349389 & -3.856661 \\
\hline $\mathrm{H}$ & -4.075892 & 1.855003 & -5.559699 \\
\hline $\mathrm{H}$ & -3.073719 & 0.642243 & -4.794689 \\
\hline $\mathrm{H}$ & -3.651130 & 1.652647 & -2.542979 \\
\hline $\mathrm{H}$ & -4.743259 & 2.743582 & -3.366194 \\
\hline $\mathrm{H}$ & -6.246342 & 1.139946 & -2.779740 \\
\hline $\mathrm{H}$ & -5.758231 & 0.470252 & -4.316799 \\
\hline $\mathrm{H}$ & -7.125956 & -1.111620 & -3.835887 \\
\hline $\mathrm{H}$ & -7.254382 & -2.585590 & -3.115539 \\
\hline $\mathrm{H}$ & -4.379607 & -2.552533 & -1.309577 \\
\hline $\mathrm{H}$ & -5.710337 & -3.432777 & -1.886834 \\
\hline$N$ & -1.558189 & 3.105534 & -7.012690 \\
\hline $\mathrm{H}$ & -2.397025 & 3.627955 & -7.124192 \\
\hline C & -0.696793 & 2.918943 & -8.161218 \\
\hline $\mathrm{H}$ & -1.068304 & 3.543008 & -8.964881 \\
\hline $\mathrm{H}$ & -0.677086 & 1.889770 & -8.491552 \\
\hline C & 0.747189 & 3.315896 & -7.863486 \\
\hline $\mathrm{O}$ & 1.689898 & 2.717834 & -8.319990 \\
\hline$N$ & 0.871729 & 4.380384 & -7.056137 \\
\hline 1 & 0.059674 & 4.894286 & -6.807924 \\
\hline C & 2.135358 & 4.897749 & -6.637158 \\
\hline $\mathrm{H}$ & 2.761409 & 5.174181 & -7.476623 \\
\hline $\mathrm{H}$ & 1.955303 & 5.787526 & -6.047131 \\
\hline C & 2.972650 & 3.968185 & -5.782665 \\
\hline $\mathrm{O}$ & 4.175886 & 4.127039 & -5.712864 \\
\hline$N$ & 2.343282 & 3.004398 & -5.101701 \\
\hline C & 3.082909 & 2.200275 & -4.161229 \\
\hline C & 4.293212 & 1.491719 & -4.766737 \\
\hline O & 5.275628 & 1.300658 & -4.077229 \\
\hline$C$ & 2.144710 & 1.184385 & -3.49827 \\
\hline
\end{tabular}




\begin{tabular}{|c|c|c|c|}
\hline C & 2.824884 & 0.382876 & -2.393708 \\
\hline C & 1.879878 & -0.603570 & -1.732623 \\
\hline$N$ & 2.611518 & -1.353011 & -0.732828 \\
\hline C & 2.094158 & -2.437907 & -0.069997 \\
\hline$N$ & 0.766031 & -2.554933 & -0.097491 \\
\hline $\mathrm{O}$ & 2.852250 & -3.226536 & 0.472494 \\
\hline $\mathrm{H}$ & 1.350306 & 2.921762 & -5.148931 \\
\hline $\mathrm{H}$ & 3.517737 & 2.837630 & -3.399778 \\
\hline $\mathrm{H}$ & 1.308128 & 1.740634 & -3.086792 \\
\hline $\mathrm{H}$ & 1.729974 & 0.527065 & -4.255623 \\
\hline $\mathrm{H}$ & 3.671877 & -0.163099 & -2.795768 \\
\hline $\mathrm{H}$ & 3.222933 & 1.055759 & -1.637632 \\
\hline $\mathrm{H}$ & 1.058703 & -0.069951 & -1.267061 \\
\hline $\mathrm{H}$ & 1.446201 & -1.260658 & -2.484840 \\
\hline $\mathrm{H}$ & 3.588309 & -1.456850 & -0.883422 \\
\hline $\mathrm{H}$ & 0.189227 & -1.819627 & -0.432040 \\
\hline $\mathrm{H}$ & 0.284064 & -3.216017 & 0.483347 \\
\hline$N$ & 4.253844 & 1.030071 & -6.025822 \\
\hline $\mathrm{H}$ & 3.466315 & 1.226168 & -6.606404 \\
\hline C & 5.351309 & 0.223258 & -6.522314 \\
\hline $\mathrm{H}$ & 5.129754 & -0.056814 & -7.545123 \\
\hline $\mathrm{H}$ & 5.461963 & -0.681811 & -5.944437 \\
\hline C & 6.705026 & 0.900869 & -6.509706 \\
\hline $\mathrm{O}$ & 7.727769 & 0.261864 & -6.419081 \\
\hline$N$ & 6.697146 & 2.230905 & -6.631135 \\
\hline $\mathrm{H}$ & 5.831462 & 2.727313 & -6.585484 \\
\hline C & 7.918740 & 2.998098 & -6.582245 \\
\hline $\mathrm{H}$ & 8.629585 & 2.629347 & -7.309062 \\
\hline $\mathrm{H}$ & 7.688987 & 4.024319 & -6.838349 \\
\hline C & 8.672153 & 3.022380 & -5.249869 \\
\hline
\end{tabular}




\begin{tabular}{|c|c|c|c|}
\hline $\mathrm{O}$ & 9.804905 & 3.460380 & -5.209453 \\
\hline$N$ & 8.044646 & 2.521019 & -4.181151 \\
\hline C & 8.630117 & 2.368503 & -2.860015 \\
\hline C & 9.918372 & 1.536097 & -2.871021 \\
\hline O & 10.737055 & 1.641839 & -1.971384 \\
\hline C & 7.544155 & 1.663412 & -2.015304 \\
\hline C & 7.728359 & 1.549798 & -0.503042 \\
\hline C & 7.315745 & 2.802351 & 0.278853 \\
\hline C & 7.196209 & 2.532985 & 1.774340 \\
\hline$N$ & 8.528656 & 2.547721 & 2.447902 \\
\hline O & 10.961441 & 1.858936 & 0.863104 \\
\hline $\mathrm{H}$ & 10.813017 & 2.064500 & -0.056144 \\
\hline $\mathrm{H}$ & 11.450561 & 1.044050 & 0.824787 \\
\hline $\mathrm{H}$ & 7.102208 & 2.203794 & -4.289780 \\
\hline $\mathrm{H}$ & 8.903340 & 3.331647 & -2.446992 \\
\hline $\mathrm{H}$ & 6.610547 & 2.172672 & -2.210748 \\
\hline $\mathrm{H}$ & 7.414946 & 0.670244 & -2.430555 \\
\hline $\mathrm{H}$ & 7.081011 & 0.740390 & -0.180292 \\
\hline $\mathrm{H}$ & 8.736087 & 1.243538 & -0.254023 \\
\hline $\mathrm{H}$ & 7.992663 & 3.636475 & 0.110961 \\
\hline $\mathrm{H}$ & 6.337646 & 3.125224 & -0.063817 \\
\hline $\mathrm{H}$ & 6.600120 & 3.284433 & 2.269946 \\
\hline $\mathrm{H}$ & 6.745277 & 1.570408 & 1.963264 \\
\hline $\mathrm{H}$ & 9.253932 & 2.097692 & 1.902043 \\
\hline $\mathrm{H}$ & 8.822837 & 3.526477 & 2.599058 \\
\hline $\mathrm{H}$ & 8.475148 & 2.071358 & 3.335197 \\
\hline$N$ & 10.052776 & 0.685290 & -3.879050 \\
\hline C & 11.124839 & -0.270655 & -4.116735 \\
\hline C & 12.483579 & 0.328506 & -4.482879 \\
\hline 0 & 13.463853 & -0.372010 & -4.365049 \\
\hline
\end{tabular}




\begin{tabular}{|c|c|c|c|}
\hline C & 10.734981 & -1.185970 & -5.299525 \\
\hline C & 9.620503 & -2.210654 & -5.043630 \\
\hline C & 9.198672 & -2.840509 & -6.374658 \\
\hline C & 10.072201 & -3.293028 & -4.063743 \\
\hline $\mathrm{H}$ & 9.309109 & 0.659564 & -4.544358 \\
\hline $\mathrm{H}$ & 11.303920 & -0.855778 & -3.225656 \\
\hline $\mathrm{H}$ & 10.450050 & -0.544946 & -6.129229 \\
\hline $\mathrm{H}$ & 11.630774 & -1.717040 & -5.600421 \\
\hline $\mathrm{H}$ & 8.752900 & -1.699643 & -4.637167 \\
\hline $\mathrm{H}$ & 8.415320 & -3.575774 & -6.218644 \\
\hline $\mathrm{H}$ & 8.820263 & -2.089050 & -7.056071 \\
\hline $\mathrm{H}$ & 10.036642 & -3.347816 & -6.847538 \\
\hline $\mathrm{H}$ & 9.296025 & -4.036312 & -3.919957 \\
\hline $\mathrm{H}$ & 10.950276 & -3.803244 & -4.447042 \\
\hline $\mathrm{H}$ & 10.321211 & -2.891240 & -3.086368 \\
\hline$N$ & 12.534695 & 1.570690 & -4.972734 \\
\hline C & 13.756147 & 2.185168 & -5.452963 \\
\hline C & 14.898598 & 2.408413 & -4.443794 \\
\hline $\mathrm{O}$ & 15.939404 & $\quad 2.822324$ & -4.881903 \\
\hline C & 13.402134 & 3.500041 & -6.150830 \\
\hline $\mathrm{O}$ & 12.806333 & 34.409277 & -5.271881 \\
\hline $\mathrm{H}$ & 11.699158 & 2.114820 & -4.973814 \\
\hline $\mathrm{H}$ & 14.208600 & 1.527940 & -6.187400 \\
\hline $\mathrm{H}$ & 14.315082 & 3.936966 & -6.527076 \\
\hline $\mathrm{H}$ & 12.752572 & 3.282950 & -6.993898 \\
\hline $\mathrm{H}$ & 11.862275 & 5.360574 & -5.309807 \\
\hline$N$ & 14.748508 & 2.092146 & -3.138682 \\
\hline C & 15.893161 & 2.050653 & -2.250858 \\
\hline C & 16.801777 & 7 0.838735 & -2.484974 \\
\hline O & 17.937397 & 0.856235 & -2.084437 \\
\hline
\end{tabular}




\begin{tabular}{|c|c|c|c|}
\hline C & 15.475829 & 1.966498 & -0.777396 \\
\hline C & 14.573729 & 3.085121 & -0.261597 \\
\hline C & 14.213816 & 2.804857 & 1.197686 \\
\hline$N$ & 13.156301 & 3.657748 & 1.724047 \\
\hline C & 13.293983 & 4.818355 & 2.342015 \\
\hline$N$ & 14.495975 & 5.340167 & 2.565072 \\
\hline $\mathrm{O}$ & 14.870543 & 7.819484 & 4.103502 \\
\hline $\mathrm{H}$ & 14.553875 & 8.695778 & 3.887774 \\
\hline $\mathrm{H}$ & 15.590698 & 7.929147 & 4.703776 \\
\hline$N$ & 12.222161 & 5.468921 & 2.741322 \\
\hline $\mathrm{H}$ & 13.870526 & 1.759596 & -2.818606 \\
\hline $\mathrm{H}$ & 16.502268 & 2.924731 & -2.427484 \\
\hline $\mathrm{H}$ & 14.974516 & 1.017600 & -0.617883 \\
\hline $\mathrm{H}$ & 16.395437 & 1.935887 & -0.202419 \\
\hline $\mathrm{H}$ & 15.057633 & 4.053208 & -0.361309 \\
\hline $\mathrm{H}$ & 13.657060 & 3.140262 & -0.837484 \\
\hline $\mathrm{H}$ & 13.846134 & 1.791968 & 1.279957 \\
\hline $\mathrm{H}$ & 15.086267 & 2.862480 & 1.839783 \\
\hline $\mathrm{H}$ & 12.227744 & 3.323750 & 1.564312 \\
\hline $\mathrm{H}$ & 15.313839 & 4.872904 & 2.259548 \\
\hline $\mathrm{H}$ & 14.604139 & 6.199890 & 3.070917 \\
\hline $\mathrm{H}$ & 11.278627 & 5.148035 & 2.595864 \\
\hline $\mathrm{H}$ & 12.282795 & 6.319877 & 3.250195 \\
\hline$N$ & 16.223616 & -0.238416 & -3.041419 \\
\hline C & 16.931013 & -1.481233 & -3.233577 \\
\hline C & 17.739114 & -1.444028 & -4.526610 \\
\hline $\mathrm{H}$ & 18.060199 & -0.446438 & -4.840326 \\
\hline $\mathrm{O}$ & 18.027721 & -2.414057 & -5.148701 \\
\hline C & 15.962807 & -2.669110 & -3.214066 \\
\hline C & 15.426494 & -3.008018 & -1.856578 \\
\hline
\end{tabular}




\begin{tabular}{|c|c|c|c|}
\hline$N$ & 14.495168 & -2.248224 & -1.178604 \\
\hline O & 12.592125 & -0.068118 & -0.532443 \\
\hline $\mathrm{H}$ & 13.205196 & -0.679567 & -0.932809 \\
\hline $\mathrm{H}$ & 12.059910 & 0.301046 & -1.223705 \\
\hline C & 15.739323 & -4.091624 & -1.111593 \\
\hline C & 14.274117 & -2.872577 & -0.068106 \\
\hline$N$ & 15.001824 & -3.991424 & 0.041482 \\
\hline $\mathrm{H}$ & 15.299838 & -0.172983 & -3.409508 \\
\hline $\mathrm{H}$ & 17.657207 & -1.577252 & -2.432792 \\
\hline $\mathrm{H}$ & 15.139155 & -2.466239 & -3.890761 \\
\hline $\mathrm{H}$ & 16.491342 & -3.528771 & -3.604260 \\
\hline $\mathrm{H}$ & 16.406300 & -4.905966 & -1.297824 \\
\hline $\mathrm{H}$ & 13.590197 & -2.550369 & 0.691500 \\
\hline $\mathrm{H}$ & 14.911300 & -4.674518 & 0.760216 \\
\hline O & -14.263520 & -2.279454 & 5.036254 \\
\hline $\mathrm{H}$ & -14.661796 & -1.757324 & 4.347938 \\
\hline $\mathrm{H}$ & -13.925328 & -1.662476 & 5.670572 \\
\hline $\mathrm{O}$ & -12.705352 & -0.285101 & 6.665424 \\
\hline $\mathrm{H}$ & -11.840735 & -0.641906 & 6.811742 \\
\hline $\mathrm{H}$ & -12.996088 & 0.076252 & 7.488570 \\
\hline 0 & -12.882577 & 1.207988 & 4.065773 \\
\hline $\mathrm{H}$ & -12.801768 & 0.926833 & 4.967629 \\
\hline $\mathrm{H}$ & -13.437193 & 0.561187 & 3.627719 \\
\hline 0 & -12.791843 & -2.334923 & 1.314238 \\
\hline $\mathrm{H}$ & -12.061805 & -1.770307 & 1.069737 \\
\hline $\mathrm{H}$ & -12.507682 & -2.900907 & 2.022523 \\
\hline 0 & -7.031903 & 1.655996 & 0.279357 \\
\hline $\mathrm{H}$ & -7.231878 & 1.642531 & 1.212548 \\
\hline $\mathrm{H}$ & -6.125816 & 1.396592 & 0.196984 \\
\hline 0 & -7.829237 & 1.683990 & 3.051748 \\
\hline
\end{tabular}




\begin{tabular}{|c|c|c|c|}
\hline $\mathrm{H}$ & -8.768624 & 1.743037 & 2.920923 \\
\hline $\mathrm{H}$ & -7.562676 & 2.461487 & 3.528575 \\
\hline $\mathrm{O}$ & -8.717730 & 0.008436 & -1.252479 \\
\hline $\mathrm{H}$ & -8.166693 & 0.610558 & -0.749631 \\
\hline $\mathrm{H}$ & -8.132409 & -0.646518 & -1.622549 \\
\hline $\mathrm{O}$ & -13.069448 & -2.747541 & -1.520904 \\
\hline $\mathrm{H}$ & -13.250723 & -2.687834 & -0.590830 \\
\hline $\mathrm{H}$ & -12.212512 & -3.143257 & -1.593245 \\
\hline O & -13.028741 & 1.284014 & -0.893261 \\
\hline $\mathrm{H}$ & -12.437088 & 0.660145 & -0.482262 \\
\hline $\mathrm{H}$ & -12.662909 & 2.136839 & -0.686430 \\
\hline $\mathrm{O}$ & -13.791133 & 3.644190 & 2.810443 \\
\hline $\mathrm{H}$ & -13.560248 & 4.442493 & 3.269336 \\
\hline $\mathrm{H}$ & -13.553427 & 2.923543 & 3.385485 \\
\hline O & -15.289890 & 1.547186 & 1.053870 \\
\hline $\mathrm{H}$ & -15.101177 & 2.364711 & 1.492816 \\
\hline $\mathrm{H}$ & -14.674900 & 1.472088 & 0.332642 \\
\hline 0 & -12.596678 & -0.496353 & -3.164347 \\
\hline $\mathrm{H}$ & -12.917570 & 0.291831 & -2.741701 \\
\hline $\mathrm{H}$ & -12.776503 & -1.196593 & -2.536956 \\
\hline $\mathrm{O}$ & -12.283320 & -2.581802 & -5.057630 \\
\hline $\mathrm{H}$ & -12.581425 & -1.770628 & -4.655023 \\
\hline $\mathrm{H}$ & -13.047893 & -3.101007 & -5.252479 \\
\hline 0 & -10.616172 & -3.451517 & -2.867206 \\
\hline $\mathrm{H}$ & -9.929234 & -2.811838 & -2.992993 \\
\hline $\mathrm{H}$ & -11.255400 & -3.271743 & -3.550003 \\
\hline 0 & -10.985858 & -0.391705 & 0.416285 \\
\hline $\mathrm{H}$ & -10.238419 & -0.411343 & -0.177086 \\
\hline $\mathrm{H}$ & -10.755351 & 0.212503 & 1.117153 \\
\hline 0 & -10.662813 & 1.652359 & 2.34712 \\
\hline
\end{tabular}




$\begin{array}{llll}\mathrm{H} & -11.343623 & 1.510024 & 3.000148 \\ \mathrm{H} & -10.986283 & 2.327191 & 1.758999 \\ \mathrm{O} & -12.064326 & 3.543173 & 0.577354 \\ \mathrm{H} & -12.727825 & 3.627324 & 1.260141 \\ \mathrm{H} & -11.668434 & 4.400600 & 0.486943 \\ \mathrm{O} & -9.864109 & 0.509767 & -3.824055 \\ \mathrm{H} & -10.735226 & 0.149809 & -3.698077 \\ \mathrm{H} & -9.446130 & 0.475274 & -2.968160 \\ \mathrm{O} & -14.559623 & -0.593415 & 2.740235 \\ \mathrm{H} & -15.027353 & 0.034846 & 2.192865 \\ \mathrm{H} & -14.068455 & -1.163529 & 2.154433 \\ \mathrm{O} & -12.254859 & -3.757407 & 3.830499 \\ \mathrm{H} & -12.977498 & -3.311268 & 4.281571 \\ \mathrm{H} & -11.462978 & -3.491924 & 4.272535 \\ \mathrm{O} & -9.400406 & -2.417981 & -5.450020 \\ \mathrm{H} & -10.257943 & -2.819795 & -5.430550 \\ \mathrm{H} & -8.776261 & -3.075039 & -5.752754\end{array}$



